Annette Schönherr

\title{
Vereinigungsbedingte Dimensionen regionaler Arbeitsmobilität
}




\section{Vereinigungsbedingte Dimensionen regionaler Arbeitsmobilität}

Nach der Öffnung der Mauer im Jahr 1989 setzten massive Wanderungen aus der ehemaligen DDR nach Westdeutschland ein. Zur Beurteilung der ökonomischen Konsequenzen solcher vereinigungsbedingter Arbeitsmobilität wird zunächst ein Referenzmodell entwickelt, das später durch Variationen der Annahmen ergänzt bzw. modifiziert wird. Das deutsche Migrationspotential und seine Ausschöpfung werden empirisch geschätzt und die für die Zukunft zu erwartenden Erträge und Kosten aus regionalen Unterschieden monetär bewertet. Verschiedene sich hinsichtlich der Angleichsdauer von Löhnen und Mieten unterscheidende Varianten werden durchgespielt. Die individuellen Kosten-Nutzen-Rechnungen zeigen, daß die möglichen Wanderungserträge von vielen Beschäftigten deutlich unterschätzt wurden.

Annette Schönherr studierte Volkswirtschaftslehre an der Universität Hamburg. Sie arbeitete bei der Deutschen Bank im Bereich der regionalen Marktanalyse, als wissenschaftliche Mitarbeiterin am Institut für Finanzwissenschaft der Universität der Bundeswehr Hamburg und in den Bereichen Marketing, Öffentlichkeitsarbeit und Unternehmensfinanzierung der Deutschen Hypothekenbank FrankfurtHamburg AG, Promotion 1999. 
Vereinigungsbedingte Dimensionen

regionaler Arbeitsmobilität 


\title{
SCHRIFTEN ZUR \\ WIRTSCHAFTSTHEORIE UND WIRTSCHAFTSPOLITIK
}

\author{
Herausgegeben von \\ Rolf Hasse, Jörn Kruse, Wolf Schäfer, Thomas Straubhaar \\ und Klaus W. Zimmermann
}

Band 16

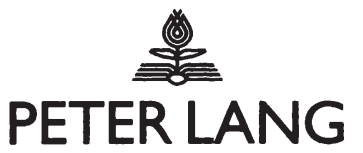

Frankfurt am Main - Berlin - Bern - Bruxelles - New York - Wien 


\section{Annette Schönherr}

\section{Vereinigungsbedingte Dimensionen regionaler Arbeitsmobilität}

Wirkungen unter analytisch einfachen Bedingungen und potentielle individuelle Migrationsgewinne in Deutschland nach der Vereinigung

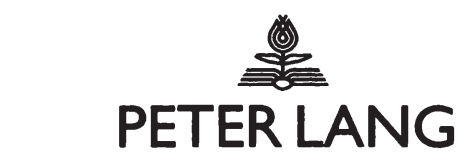

Europäischer Verlag der Wissenschaften 
Die Deutsche Bibliothek - CIP-Einheitsaufnahme

Schönherr, Annette:

Vereinigungsbedingte Dimensionen regionaler Arbeitsmobilität : Wirkungen unter analytisch einfachen Bedingungen und potentielle individuelle Migrationsgewinne in Deutschland nach der Vereinigung / Annette Schönherr. - Frankfurt am Main ; Berlin ; Bern ; Bruxelles ; New York ; Wien : Lang, 1999

(Schriften zur Wirtschaftstheorie und Wirtschaftspolitik ; Bd. 16)

Zugl.: Hamburg, Univ. der Bundeswehr, Diss., 1999

ISBN 3-631-35137-2

Open Access: The online version of this publication is published on www.peterlang.com and www.econstor.eu under the international Creative Commons License CC-BY 4.0. Learn more on how you can use and share this work: http://creativecommons. org/licenses/by/4.0.

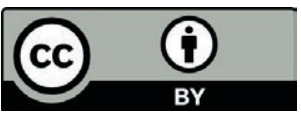

This book is available Open Access thanks to the kind support of ZBW - Leibniz-Informationszentrum Wirtschaft.

Gedruckt mit Unterstützung der Universität der Bundeswehr Hamburg.

Gedruckt auf alterungsbeständigem, säurefreiem Papier.

D 705

ISSN 1433-1519

ISBN 3-631-35137-2

ISBN 978-3-631-75008-7 (eBook)

(C) Peter Lang GmbH

Europäischer Verlag der Wissenschaften

Frankfurt am Main 1999

Alle Rechte vorbehalten.

Das Werk einschließlich aller seiner Teile ist urheberrechtlich geschützt. Jede Verwertung außerhalb der engen Grenzen des

Urheberrechtsgesetzes ist ohne Zustimmung des Verlages unzulässig und strafbar. Das gilt insbesondere für

Vervielfältigungen, Übersetzungen, Mikroverfilmungen und die Einspeicherung und Verarbeitung in elektronischen Systemen.

Printed in Germany 124567 


\section{Inhaltsverzeichnis}

Abbildungsverzeichnis 12

Tabellenverzeichnis 15

1 Einführung 19

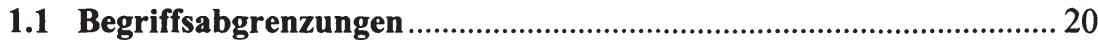

1.2 Themenabgrenzungen und Fragestellungen ..................................... 23

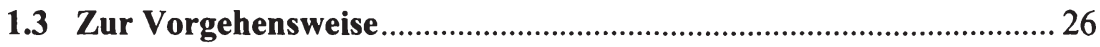

2 Wirkungen regionaler Arbeitsmobilität unter analytisch einfachen Bedingungen.

2.1 Regionale Arbeitsmobilität bei Zugrundelegung eines einfachen neoklassischen Vollbeschäftigungsmodells

2.1.1 Lohnniveau, Beschäftigung und Einkommen mit und ohne Vereinigung. 32

2.1.1.1 Der Modellrahmen: Das neoklassische Arbeitsmarktmodell......... 32

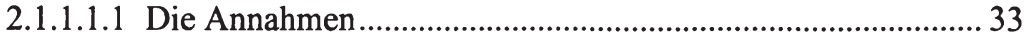

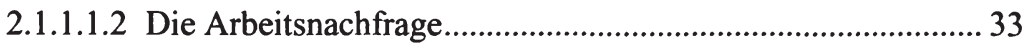

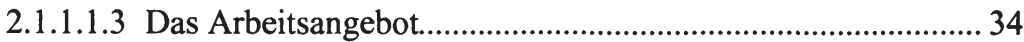

2.1.1.1.4 Gleichgewicht auf dem Arbeitsmarkt......................................36

2.1.1.2 Lohnniveau und Beschäftigung..................................................... 37

2.1.1.2.1 Lohnhöhe und Beschäftigung bei vollkommenen regionalen Mobilitätsbarrieren.

2.1.1.2.2 Lohnhöhe und Beschäftigung nach Aufhebung regionaler Mobilitätsbarrieren.

2.1.1.2.3 Vergleich der Beschäftigungshöhe ohne und mit Vereinigung 
2.1.1.2.4 Die Wirkungen von Arbeitsmobilität infolge einer Vereinigung bei extremen regionalen Unterschieden

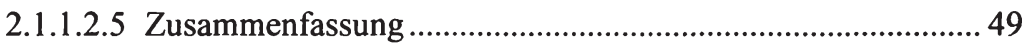

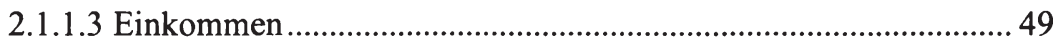

2.1.1.3.1 Bestimmung der funktionalen Einkommensverteilung und der Arbeitnehmerrenten................................................... 50

2.1.1.3.1.1 Die funktionale Einkommensverteilung nach der Grenzproduktivitätstheorie............................................... 50

2.1.1.3.1.2 Übertragung auf den Modellrahmen................................. 52

2.1.1.3.1.3 Die Ermittlung der Arbeitnehmerrente.............................. 54

2.1.1.3.2 Vergleich der Einkommen mit und ohne Vereinigung........... 56

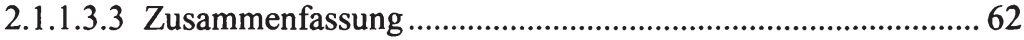

2.1.2 Modellmäßig zu erwartende Entwicklungen der regionalen deutsch-deutschen Arbeitsmobilität....................................................63

2.1.2.1 Stilisierung der west- und ostdeutschen Arbeitsmärkte vor

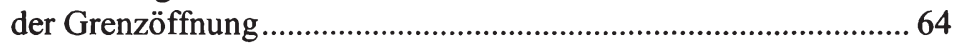

2.1.2.2 Modellmäßig zu erwartende Anpassungsprozesse nach

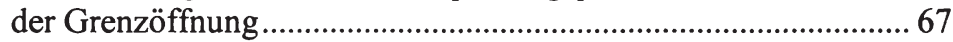

2.1.2.3 Modifikationen und Vergleich mit den tatsächlichen

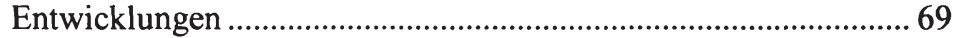

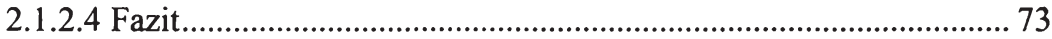

2.2 Die Berücksichtigung von Arbeitslosigkeit............................................. 74

2.2.1 Potentielle Gründe für Arbeitslosigkeit............................................ 74

2.2.2 Beschäftigung und Einkommen - insbesondere Arbeitnehmerrenten - bei rigiden Lohnsätzen....................................................... 77

2.2.3 Vergleich der Beschäftigung und der Einkommen mit und

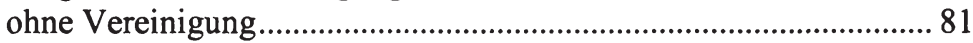

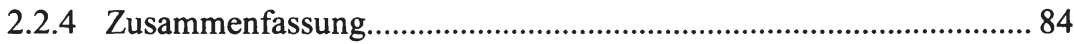




\subsection{Die Berücksichtigung von Besonderheiten auf der}

Produktionsseite

2.3.1 Die Bedeutung der regionalen Güternachfrage für die

Arbeitsnachfrage

2.3.2 Die Bedeutung von Qualifikationsunterschieden

2.3.2.1 Das Humankapital als Teil des (die Arbeitsnachfrage determinierenden) Kapitalstocks

2.3.2.2 Komplementär einsetzbare Qualifikationen 90

2.3.2.3 Die Tragweite von selektiven Wanderungen für die Entwicklung der Regionen 94

2.3.3 Zusammenfassung. 96

2.4 Die Berücksichtigung regionaler Annehmlichkeiten, regionaler Preisunterschiede und Transaktionskosten.

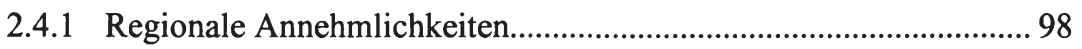

2.4.1.1 Definition des Begriffs regionale Annehmlichkeiten.................... 98

2.4.1.2 Regionale Annehmlichkeiten und ihre allgemeine Bedeutung für Arbeitsmärkte. 99

2.4.1.2.1 Einfluß regionaler Annehmlichkeiten auf die Lohnforderungen der Arbeitsanbieter.

2.4.1.2.2 Einfluß regionaler Annehmlichkeiten auf die Kosten der Unternehmen

2.4.1.2.3 Das Arbeitsmarktgleichgewicht 104

2.4.1.3 Vereinigungsbedingte Auswirkungen regionaler Annehmlichkeiten. 106

2.4.1.3.1 Bedingungen, unter denen Wanderungen in die Hochlohnregion induziert werden

2.4.1.3.2 Bedingungen, unter denen Wanderungen in die Niedriglohnregion bzw. keine Wanderungen induziert werden.

2.4.1.3.3 Einfluß der Präferenzstärke für Annehmlichkeiten 


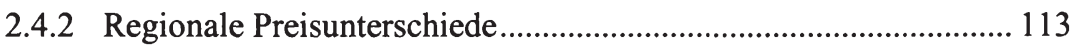

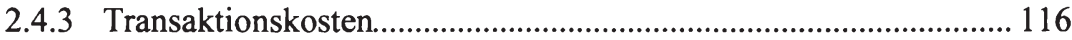

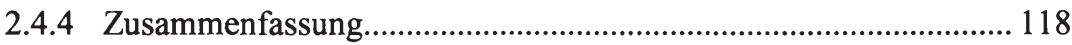

3 Potentielle individuelle Migrationsgewinne und tatsächlicher

Umfang der Wanderungen in Deutschland nach der Vereinigung........ 121

\subsection{Die empirische Berücksichtigung von}

Wanderungsdeterminanten.

3.1.1 Wanderungsdeterminanten in ausgewählten empirisch ausgerichteten Analysen.

3.1.2 Zur empirischen Erfassung und Erfaßbarkeit von Wanderungsdeterminanten in einer Rechnung zur Messung der Vorteilhaftigkeit einer Migration

3.1.2.1 Regionale Einkommens- und Arbeitslosigkeitsunterschiede. 127

3.1.2.1.1 Einkommensbestandteile 128

3.1.2.1.2 Das Arbeitslosigkeitsrisiko. 129

3.1.2.2 Regionale Preisunterschiede 133

3.1.2.3 Regionale Annehmlichkeiten. 134

3.1.2.4 Informationskosten und -probleme. 135

3.1.2.5 Transaktionskosten 137

3.1.2.6 Die Länge der Entfernung zwischen Quell- und Zielregion 138

3.1.2.7 Die Bedeutung früherer Wanderungen und Rückwanderungen.

3.1.2.8 Psychische Kosten und Erträge. 141

3.1.2.9 Sonstige personenspezifische Wanderungsgründe. 143

3.1.3 Zusammenfassung. 146 


\subsection{Modellrechnungen zur Beurteilung individueller Vorteilhaftigkeit einer Wanderung aus den neuen in die alten}

Länder

3.2.1 Migrationserträge aus regionalen Lohn- und Preisunterschieden

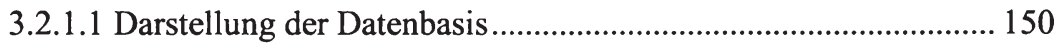

3.2.1.1.1 Regionale Brutto- und Nettolohneinkommen........................ 150

3.2.1.1.2 Regionale (Miet-)Preisniveaus............................................... 151

3.2.1.2 Im Jahr 1990 erwartbare Migrationserträge aus regionalen Lohn- und Mietunterschieden..................................................... 154

3.2.1.2.1 Erwartbare Migrationserträge gesplittet nach Branchen....... 155

3.2.1.2.2 Erwartbare Migrationserträge gesplittet nach Status und Geschlecht

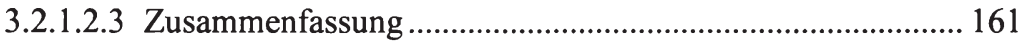

3.2.1.3 Im Jahr 1990 erwartbare Migrationserträge aus regionalen Lohn- und Mietunterschieden unter Berücksichtigung der tatsächlichen Entwicklung bis 1994.

3.2.1.3.1 Korrigierte erwartbare Migrationserträge aus regionalen Lohn- und Mietunterschieden bis 1994

3.2.1.3.1.1 Migrationserträge gesplittet nach Branchen...................... 164

3.2.1.3.1.2 Migrationserträge gesplittet nach Status und Geschlecht

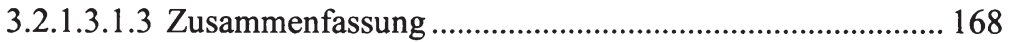

3.2.1.3.2 Simulation der korrigierten erwartbaren Migrationserträge aus regionalen Lohn- und Mietunterschieden nach 1994

3.2.1.3.2.1 Simulation gesplittet nach Branchen................................ 170

3.2.1.3.2.2 Simulation gesplittet nach Status und Geschlecht............ 172

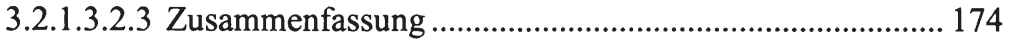


3.2.1.3.3 Korrigierte erwartbare Migrationserträge aus regionalen Lohn- und Mietunterschieden insgesamt unter Berücksichtigung der tatsächlichen Entwicklung bis 1994.

3.2.1.3.3.1 Migrationserträge nach Branchen................................... 175

3.2.1.3.3.2 Migrationserträge nach Status und Geschlecht................. 177

3.2.1.3.3.3 Zusammenfassung ....................................................... 179

3.2.1.4 Vergleich der 1990 erwartbaren mit den korrigierten erwartbaren Migrationserträgen aus regionalen Lohn- und Preisunterschieden

3.2.2 Bewertung und Berücksichtigung weiterer Migrationskosten und -erträge 184

3.2.2.1 Monetäre Bewertung weiterer Migrationskosten und -erträge.... 184

3.2.2.1.1 Mehrkosten durch Neuanmietung einer Wohnung............... 185

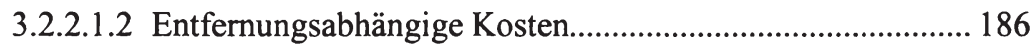

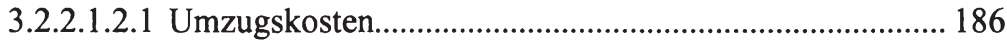

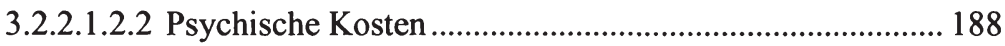

3.2.2.1.3 Arbeitsplatzabhängige Kosten und Erträge........................... 190

3.2.2.1.3.1 Differenzen der regionalen Jahresarbeitszeiten................ 190

3.2.2.1.3.2 Verschlechterung der beruflichen Stellung...................... 193

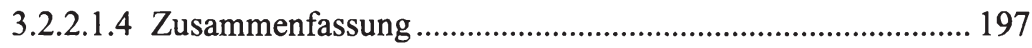

3.2.2.2 Erwartbare Migrationserträge nach Berücksichtigung weiterer Kosten und Erträge.............................................................. 200

3.2.2.2.1 Erwartbare Migrationserträge insgesamt.............................. 201

3.2.2.2.2 Korrgierte erwartbare Migrationserträge insgesamt............. 206

3.2.2.3 Zusammenfassung und Vergleich................................................. 209

3.2.3 Migrationserträge nach Berücksichtigung von Arbeitslosigkeit..... 213

3.2.4 Zusammenfassung und Schlußfolgerungen.................................... 222 


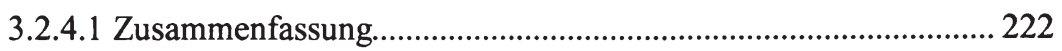

3.2.4.2 Schlußfolgerungen und Weiterführendes.................................... 226

3.2.4.2.1 Fazit aus der Modellrechnung ................................................ 226

3.2.4.2.2 War ein Umzug aus der Perspektive von 1994 noch lohnend?

\subsection{Ausmaß der Wanderungen zwischen den neuen und alten} Bundesländern 233

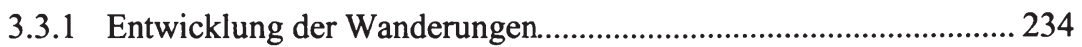

3.3.2 Zusammenfassende Schlußfolgerungen......................................... 242

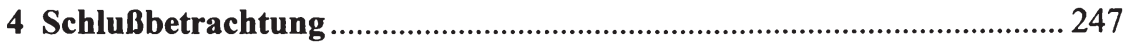

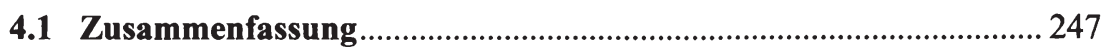

4.2 Abschließende Bemerkungen ....................................................... 252

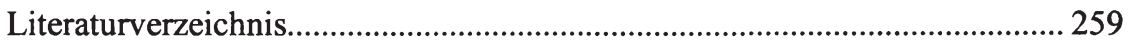

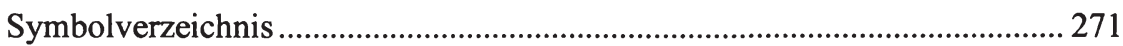

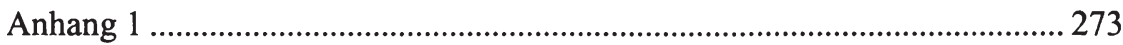

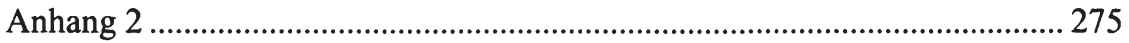

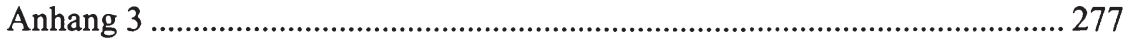

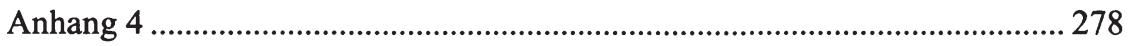




\section{Abbildungsverzeichnis}

Abbildung 1: Arbeitsnachfrage und -angebot in Abhängigkeit vom Reallohnsatz

Abbildung 2: Lohnniveau und Beschäftigung bei Immobilität............................38

Abbildung 3: Lohnniveau und Beschäftigung bei Mobilität............................. 41

Abbildung 4: Partielle Produktionsfunktionen des Kapitals............................. 52

Abbildung 5: Die funktionale Einkommensverteilung..................................... 53

Abbildung 6: Individueller Nutzen bei alternativem Arbeitsangebot.............. 55

Abbildung 7: Einkommensveränderung durch Vereinigung............................. 57

Abbildung 8: Gewinnänderung bei parallel verlaufenden Arbeitsnachfrage- und -angebotskurven

Abbildung 9: Der ost- und der westdeutsche Arbeitsmarkt ohne Vereinigung.

Abbildung 10: Der deutsch-deutsche Arbeitsmarkt vor und nach Vereinigung.

Abbildung 11: Ostdeutscher Arbeitsmarkt nach ersten Modifikationen............ 70

Abbildung 12: Ostdeutscher Arbeitsmarkt nach weiteren Modifikationen........ 72

Abbildung 13: Arbeitnehmerrente bei rigiden Löhnen....................................... 78

Abbildung 14: Kapitaleinkommen bei Zahlung von Effizienzlöhnen................ 80

Abbildung 15: Migration bei Einfluß von Humankapital auf die Arbeitsnachfrage

Abbildung 16: Regionale Beschäftigungsentwicklung bei Zuwanderung unqualifizierter Arbeitskräfte.

Abbildung 17: Beschäftigungsentwicklung bei Berücksichtigung zweier Arbeitnehmergruppen und Komplementarität.

Abbildung 18: Indifferenzkurven für Einkommen und regionale Annehmlichkeiten 
Abbildung 19: Kostenverläufe bei alternativen Wirkungsrichtungen der regionalen Annehmlichkeiten.................................................. 102

Abbildung 20: Ableitung von Lohn-Angebotskurven.................................... 103

Abbildung 21: Gleichgewichtssituationen bei alternativen Lohn-Attraktivitätsniveaus.......................................................................... 104

Abbildung 22: Gleichgewicht bei durchgängig kostensenkendem Einfluß der regionalen Annehmlichkeiten............................................ 106

Abbildung 23: Wanderungen in die Hochlohnregion bei kostensenkenden regionalen Annehmlichkeiten................................................ 108

Abbildung 24: Wanderungen in die Hochlohnregion bei kostenerhöhenden regionalen Annehmlichkeiten.

Abbildung 25: Wanderungen bei interindividuellen Unterschieden in den Präferenzen

Abbildung 26: Indifferenzkurven bei alternativen Löhnen, Preisen und regionalen Annehmlichkeiten.

Abbildung 27: Gleichgewicht bei Berücksichtigung von Wanderungskosten

Abbildung 28: Erwartbare Erträge aus regionalen Lohn- und Mietpreisdifferenzen eines durchschnittlichen Beschäftigten

Abbildung 29: Ausgewählte erwartbare Nettomigrationserträge nach Mietzahlung bei alternativen Zeitpräferenzraten und 10jähriger Lohnanpassungsdauer.

Abbildung 30: Korrigierte erwartbare Migrationserträge für unterschiedliche Beschäftigte bis 1994 .

Abbildung 31: Korrigierte erwartbare Überschüsse aus regionalen Lohnund Mietdifferenzen nach 1994

Abbildung 32: Ausgewählte korrigierte erwartbare Migrationserträge aus Lohn- und Mietunterschieden insgesamt.

Abbildung 33: Zusammenfassung der weiteren Migrationskosten und -erträge 
Abbildung 34: Ausgewählte erwartbare Migrationserträge bei alternativen Angleichungszeiträumen und Wanderungsdistanzen..... 210

Abbildung 35: Ausgewählte erwartbare Migrationserträge unter alternativen Angleichungsverläufen in ausgewählten Branchen bei einer Wanderungsdistanz von $400 \mathrm{~km}$.

Abbildung 36: (Korrigierte) erwartbare Migrationserträge bei alternativen Angleichungsverläufen nach Status und Geschlecht bei einer Wanderungsdistanz von $400 \mathrm{~km}$.

Abbildung 37: Anteil der verschiedenen Ertrags- und Kostenkomponenten am Gesamtergebnis

Abbildung 38: Verteilung der Abwanderungen aus den neuen Ländern auf Regionen der alten Länder. 238 


\section{Tabellenverzeichnis}

Tabelle 1: Entwicklung der ostdeutschen Mietpreise.................................... 152

Tabelle 2: Erwartbare Erträge aus regionalen Lohn- und Mietpreisunterschieden nach Branchen bei einer 3jährigen Angleichungsdauer

Tabelle 3: Erwartbare Erträge aus regionalen Lohn- und Mietpreisunterschieden nach Branchen bei einer 5jährigen Angleichungsdauer.

Tabelle 4: Erwartbare Erträge aus regionalen Lohn- und Mietpreisunterschieden nach Branchen bei einer 10jährigen Angleichungsdauer.

Tabelle 5: Erwartbare Erträge aus regionalen Lohn- und Mietpreisunterschieden nach Status und Geschlecht bei einer 3jährigen Angleichungsdauer

Tabelle 6: Erwartbare Erträge aus regionalen Lohn- und Mietpreisunterschieden nach Status und Geschlecht bei einer 5jährigen Angleichungsdauer

Tabelle 7: Erwartbare Erträge aus regionalen Lohn- und Mietpreisunterschieden nach Status und Geschlecht bei einer 10jährigen Angleichungsdauer

Tabelle 8: Durchschnittliches Jahreseinkommen von Beschäftigten in Ost- und Westdeutschland

Tabelle 9: Korrigierte erwartbare Erträge aus regionalen Lohn- und Mietpreisunterschieden bis 1994 nach Branchen.

Tabelle 10: Korrigierte erwartbare Erträge aus regionalen Lohn- und Mietpreisunterschieden bis 1994 nach Status und Geschlecht..... 168

Tabelle 11: Korrigierte erwartbare Erträge aus regionalen Lohn- und Mietpreisunterschieden nach 1994 nach Branchen bei einer Angleichung bis 1999.

Tabelle 12: Korrigierte erwartbare Erträge aus regionalen Lohn- und Mietpreisunterschieden nach 1994 nach Branchen bei einer Anglei-chung bis 2004 
Tabelle 13: Korrigierte erwartbare Erträge aus regionalen Lohn- und Mietpreisunterschieden nach 1994 nach Status und Geschlecht bei einer Angleichung bis 1999.

Tabelle 14: Korrigierte erwartbare Erträge aus regionalen Lohn- und Mietpreisunterschieden nach 1994 nach Status und Geschlecht bei einer Angleichung bis 2004.

Tabelle 15: Korrigierte erwartbare Erträge aus regionalen Lohn- und Mietpreisunterschieden insgesamt nach Branchen bei einer Anglei-chung bis 1999.

Tabelle 16: Korrigierte erwartbare Erträge aus regionalen Lohn- und Mietpreisunterschieden insgesamt nach Branchen bei einer Angleichung bis 2004 . 176

Tabelle 17: Korrigierte erwartbare Erträge aus regionalen Lohn- und Mietpreisunterschieden insgesamt nach Status und Geschlecht bei einer Angleichung bis 1999.

Tabelle 18: Korrigierte erwartbare Erträge aus regionalen Lohn- und Mietpreisunterschieden insgesamt nach Status und Geschlecht bei einer Angleichung bis 2004. 178

Tabelle 19: Mehrkosten bei Neuanmietung einer Wohnung. 186

Tabelle 20: Umzugskosten 187

Tabelle 21: Psychische Kosten einer Migration bewertet mit der Reisekostenmethode.

Tabelle 22: Abweichung der ostdeutschen Jahresarbeitszeit von der westdeutschen

Tabelle 23: Monetäre Bewertung der geringeren Arbeitszeiten in den alten Ländern 1991-1994 nach Branchen.

Tabelle 24: Monetäre Bewertung der geringeren Arbeitszeiten in den alten Ländern 1991-1994 nach Status und Geschlecht

Tabelle 25: Umfrageergebnis: Inwieweit entspricht der Arbeitsplatz der Qualifikation der Übersiedler?

Tabelle 26: Monetäre Bewertung der Verschlechterung der beruflichen Stellung 1991-1994 nach Branchen. 
Tabelle 27: Monetäre Bewertung der Verschlechterung der beruflichen Stellung 1991-1994 nach Status und Geschlecht.......................... 196

Tabelle 28: Zusammenfassung der entfernungsabhängigen Kosten................ 197

Tabelle 29: Zusammenfassung der Kosten und Erträge durch Verschlechterung der beruflichen Stellung und Arbeitszeitdifferenzen nach Branchen.

Tabelle 30: Zusammenfassung der Kosten und Erträge durch Verschlechterung der beruflichen Stellung und Arbeitszeitdifferenzen nach Status und Geschlecht

Tabelle 31: Zusammenfassung der weiteren Migrationskosten und -erträge nach Branchen

Tabelle 32: Zusammenfassung der weiteren Migrationskosten- und -erträge nach Status und Geschlecht.

Tabelle 33: Erwartbare Migrationserträge insgesamt bei einer 3jährigen Angleichungsdauer nach Branchen 202

Tabelle 34: Erwartbare Migrationserträge insgesamt bei einer 5jährigen Angleichungsdauer nach Branchen 202

Tabelle 35: Erwartbare Migrationserträge insgesamt bei einer 10jährigen Angleichungsdauer nach Branchen

Tabelle 36: Erwartbare Migrationserträge insgesamt bei einer 3jährigen Angleichungsdauer nach Status und Geschlecht. 204

Tabelle 37: Erwartbare Migrationserträge insgesamt bei einer 5jährigen Angleichungsdauer nach Status und Geschlecht. 204

Tabelle 38: Erwartbare Migrationserträge insgesamt bei einer 10jährigen Angleichungsdauer nach Status und Geschlecht.

Tabelle 39: Korrigierte erwartbare Migrationserträge insgesamt nach Branchen bei einer Angleichung bis 1999 207

Tabelle 40: Korrigierte erwartbare Migrationserträge insgesamt nach Branchen bei einer Angleichung bis 2004 . 207

Tabelle 41: Korrigierte erwartbare Migrationserträge insgesamt nach Status und Geschlecht bei einer Angleichung bis 1999. 208 
Tabelle 42: Korrigierte erwartbare Migrationserträge insgesamt nach Status und Geschlecht bei einer Angleichung bis 2004.

Tabelle 43: Entwicklung der Beschäftigung und der Arbeitslosigkeit in Ostdeutschland.

Tabelle 44: Entwicklung der Beschäftigung und der Arbeitslosigkeit in Westdeutschland

Tabelle 45: Erwartungswerte für das Nettoeinkommen 1991-1994 bei Berücksichtigung von Arbeitslosigkeit.

Tabelle 46: Vergleich der erwartbaren Überschüsse aus regionalen Lohndifferenzen mit und ohne Berücksichtigung von Arbeitslosigkeit

Tabelle 47: Differenzen der Erträge aus regionalen Lohndifferenzen bei alternativen Annahmen über die Zahlung von Lohnersatzleistungen im Vergleich zum Ohne-Arbeitslosigkeits-Fall.

Tabelle 48: 1994 erwartbare Erträge aus regionalen Lohn- und Mietunterschiede nach Branchen bei einer Angleichung bis 1999....... 230

Tabelle 49: 1994 erwartbare Erträge aus regionalen Lohn- und Mietunterschiede nach Branchen bei einer Angleichung bis 2004.

Tabelle 50: 1994 erwartbare Erträge aus regionalen Lohn- und Mietunterschieden nach Status und Geschlecht bei einer Angleichung bis zum Jahr 1999

Tabelle 51: 1994 erwartbare Erträge aus regionalen Lohn- und Mietunterschieden nach Status und Geschlecht bei einer Angleichung bis zum Jahr 2004 232

Tabelle 52: Wanderungen zwischen den neuen und alten Bundesländern...... 235

Tabelle 53: Fort- und Zuzüge zwischen den neuen und den alten Länder je 1.000 der Bevölkerung.

Tabelle 54: Verteilung der Wanderungen auf Regionen der alten Länder....... 236

Tabelle 55: Wanderungen 1989 in die alten Bundesländer nach Alter 239 


\section{Einführung}

Knapp dreißig Jahre lang war es den Bürgern der ehemaligen DDR' nahezu unmöglich in die alte Bundesrepublik auszureisen ${ }^{2}$. Sie konnten zwar Ausreiseanträge stellen, dies führte jedoch zumeist zu vielfältigen Sanktionen durch den Staat. Zudem wurde die Ausreise selten bewilligt. Andere Möglichkeiten der Abwanderung wie eine Flucht waren lebensgefährlich (man denke etwa an den Schießbefehl); außerdem drohte den Flüchtlingen Gefängnisstrafen, wenn sie ertappt wurden. Darüber hinaus waren Nachteile für die im Osten verbliebenen Verwandten zu erwarten.

Im Zuge der politischen Reformen in Osteuropa änderten sich die Ausreisemöglichkeiten. Als Ungarn im Frühjahr 1989 als erstes „sozialistisches“ Land den „eisernen“ Vorhang öffnete, setzte eine Fluchtwelle von DDR-Bürgern über Ungarn ein. Auch in anderen osteuropäischen Ländern schritt der Reformprozeß schneller als in der DDR voran, wodurch eine Flucht über diese Länder möglich wurde. Durch den hieraus entstandenen Druck auf das politische System öffnete schließlich auch die DDR im November 1989 ihre Grenzen. Ein weiterer Massenexodus war die Folge: Täglich siedelten Tausende von DDR-Bürgern in die Bundesrepublik über und ließen eine Entleerung Ostdeutschlands befürchten.

Vor allem um die Abwanderungswelle zu stoppen wurde die D-Mark innerhalb kürzester Zeit in der ehemaligen DDR eingeführt: Am 1.7.1990 trat die Wirtschafts-, Währungs- und Sozialunion in Kraft - entgegen gravierenden ökonomischen Bedenken. An diesem Datum wurde die ostdeutsche Wirtschaft quasi von einem Tag auf den anderen der internationalen Konkurrenz ausgesetzt, was erhebliche Konsequenzen für die ökonomische Lage mit sich brachte und massive Anpassungsreaktionen auf vielen Gebieten hervorrief. Die Produktionsbedingungen und Standortfaktoren in Ostdeutschland waren denkbar ungünstig: die Infrastruktur war desolat, die Produktionsanlagen veraltet und abgenutzt, die Angebotspalette bei einem $1 \mathrm{zu} 1$ von Mark der deutschen Notenbank in DM überführten Lohnniveau nicht wettbewerbsfähig und vielen Arbeitskräften fehlte die Erfahrung im Umgang mit modernen Technologien. Hinzu kam der Zusammenbruch des Osthandels, durch den die neuen Bundesländer ihre traditionellen Absatzmärkte verloren. Innerhalb eines Jahres ging die Industrieproduktion um etwa $30 \%$ zurück.

I Statt von ehemalige DDR wird im Folgenden auch von den neue Länder, dem Osten oder Ostdeutschland gesprochen. Mit früherer Bundesrepublik, Westdeutschland oder alte Länder wird das Gebiet der Bundesrepublik Deutschland vor dem 3. Oktober 1990 bezeichnet. Mit Deutschland bzw. Bundesrepublik ist das Territorium nach Vereinigung gemeint.

2 Lediglich Personen im Rentenalter konnten frei ausreisen. 
Die Mehrheit der ostdeutschen Bevölkerung empfand es als ungerecht, daß sie für eine von ihnen als gleich empfundene Arbeit weniger Lohn als in Westdeutschland erhielt. Daher verfolgte die Arbeitnehmerseite das Ziel, das Lohnniveau möglichst schnell anzugleichen. Die von den Tarifparteien ausgehandelten Lohnabschlüsse waren außerordentlich hoch: Bereits vor dem 1. Juli 1990 stiegen die Bruttoverdienste in Ostdeutschland gegenüber dem ersten Halbjahr der entsprechenden Vorjahreszeit um etwa 18\%; dies war sehr viel mehr als in den Jahren zuvor ${ }^{3}$. Durch die hohen Lohnsteigerungen sollte der Anreiz für die Beschäftigten vermindert werden, in die alten Bundesländer abzuwandern, denn insbesondere durch die Abwanderung qualifizierter Arbeitskräfte (brain drain) wurde eine weitere Verschlechterung der prekären wirtschaftlichen Situation Ostdeutschlands erwartet.

Die durch die Vereinigung zu erwartenden und die tatsächlich ausgelösten Mobilitätsprozesse und ihre Bedeutung für die ökonomischen Perspektiven der neuen Länder gaben den Anstoß für diese Arbeit.

\subsection{Begriffsabgrenzungen}

Da im Mittelpunkt dieser Arbeit das Thema „Mobilität“ steht, soll zunächst abgegrenzt werden, was unter Mobilität verstanden wird und welche Form von Mobilität im Folgenden untersucht wird.

In der ökonomischen Diskussion wird der Mobilitätsbegriff für Mengenanpassungsprozesse auf dem Arbeitsmarkt verwendet, während Flexibilität für das Ausmaß und die Schnelligkeit der Anpassung von Preisen und Mengen an eine veränderte Nachfrage steht ${ }^{4}$. Mobilität wird also im Sinne von Arbeitsmobilität gebraucht und beschreibt verschiedene Phänomene. Folgende Formen von Mobilitätsprozessen werden unterschieden:

- Fluktuationen: zwischenbetriebliche Arbeitsplatzwechsel innerhalb eines Wirtschaftszweiges ${ }^{5}$

- zwischensektorale Mobilität: Wechsel zwischen zwei Arbeitgebern mit unterschiedlicher Branchenzugehörigkeit ${ }^{6}$

- Statusmobilität: Ausscheiden oder Eintreten in eine Beschäftigung ${ }^{7,8}$

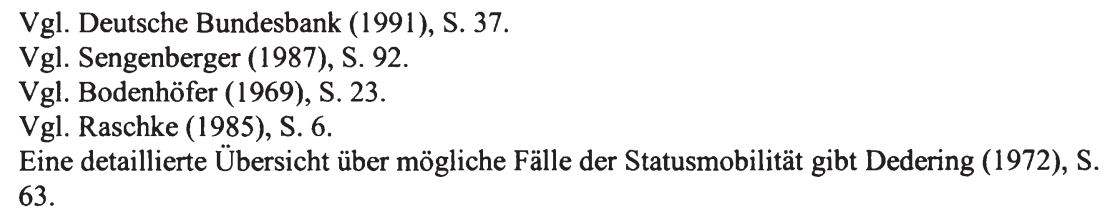


- berufliche Mobilität: Wechsel des Berufs, bzw. der Funktion durch den Beschäftigten

- regionale Mobilität: Wechsel des Arbeitsplatzes mit Änderung der regionalen Lokalisierung?.

Umbesetzungen innerhalb einer Unternehmung werden z.T. ebenfalls als Arbeitsmobilität bezeichnet (z.B. Bodenhöfer (1969), S. 23 und Dedering (1972), S. 62f), z.T. jedoch nicht (vgl. etwa Molitor (1969), S. 88ff).

Im Zentrum dieser Arbeit steht die regionale Mobilität. Daher wird im Folgenden Mobilität als Synonym für „regionale Mobilität" benutzt. Alle anderen Formen von Mobilität werden nicht betrachtet.

Nach Parnes (1954, S. 13ff) ${ }^{10}$ werden mit Mobilität nicht nur tatsächlich stattfindende Reallokationsvorgänge der Arbeit bezeichnet. Er unterscheidet drei Konzepte der Arbeitsmobilität:

- Mobilitätsfähigkeit

- Mobilitätsbereitschaft

- realisierte Mobilität.

Die Mobilitätsfähigkeit ist die Fähigkeit, eine Veränderung zu vollziehen. Sie hängt $\mathrm{ab}$ von der beruflichen Qualifikation, den wirtschaftlichen Gegebenheiten und den Anforderungen, die an einen Arbeitsplatzbewerber gestellt werden. Die Summe aller Mobilitätsfähigkeiten sind die objektiv vorhandenen Möglichkeiten zum Wechseln, daher kann sie als maximale potentielle Flexibilität bei der Allokation der menschlichen Arbeitskraft bezeichnet werden". Die Bereitschaft zur Mobilität wird auch latente Mobilität genannt und ist die Neigung der Arbeitskräfte unter bestimmten Bedingungen einen Wechsel vorzunehmen. Realisiert wird Mobilität, wenn ein Umzug vollzogen wird. Die Entscheidung zur Realisation fällt nicht grundsätzlich, sobald die Fähigkeit und die Möglichkeit vorhan-

8 Eine Form von Statusmobilität ist die Erwerbsmobilität (d.h. Bewegungen zwischen dem Erwerbs- und dem Nichterwerbssektor). Es gibt verschiedene Auffassungen darüber, ob die Erwerbsmobilität überhaupt zur Arbeitsmobilität zählt. Bei Raschke ((1985), S. 7) etwa steht Arbeitsmobilität für intersektorale Mobilität und Statusmobilität nur für Bewegungen innerhalb des Erwerbssektors (etwa Wechsel aus der Arbeitslosigkeit in eine Stelle). Für Bodenhöfer dagegen ist Erwerbsmobilität eine Form der Arbeitsmobilität (vgl. Bodenhöfer (1969), S. 23, Fußnote 9).

9 Definition nach Bodenhöfer (1969), S. 24.

10 Vgl. hierzu auch Dedering (1972), S. 55ff. Außer den drei Mobilitätskonzepten von Parnes nennt Dedering noch ein viertes, nämlich die Mobilitätseigenschaften. Diese lassen sich jedoch nicht sauber von den Mobilitätsfähigkeiten trennen. Vgl. Dedering (1972), S. 56f.

1 Vgl. Parnes (1954), S.13, vgl. hierzu auch Sheldon (1985), S. 58ff, Röder (1974), S. $51 f$. 
den sind, sondern die Durchführung ist von weiteren Faktoren wie z.B. dem Informationsstand und der Risikofreude der Individuen abhängig ${ }^{12}$.

Diese Unterscheidung dürfte besonders im Hinblick auf die deutschen Mobilitätsprozesse von Bedeutung sein, wo aufgrund von fehlenden Arbeitsplätzen auch bei einer bestehenden Mobilitätsbereitschaft ein Umzug manchmal nicht durchgeführt wurde.

Welche Grenzen überschritten bzw. Distanzen überwunden werden müssen, um als regionale Mobilität bezeichnet zu werden, wird höchst unterschiedlich abgegrenzt:

"Die Möglichkeiten reichen von der Abgrenzung von Wohngrundstücken, Stadtbezirken, Gemeinden usw. bis zu den Planungsregionen verschiedener raumordnungspolitischer Programme."13

Die jeweils verwendete Abgrenzung ist abhängig vom Untersuchungsziel und gegenstand, zum Teil aber auch von den verfügbaren Statistiken. Da der Untersuchungsgegenstand dieser Arbeit Wanderungen zwischen zwei Regionen ist, werden im Folgenden als Wanderungen nur Wohnortwechsel bezeichnet (und untersucht), bei denen Regionsgrenzen überschritten werden.

Im Folgenden wird ausschließlich Mobilität von Erwerbspersonen betrachtet, wobei ein Erwerbstätiger immer dort arbeiten soll, wo er wohnt. Daher schließt die Verwendung der Begriffe Umzug, Wanderung oder Migration stets einen gleichzeitigen Arbeitsplatzwechsel in die Zugangsregion mit ein. Ebenso gilt, daß ein Arbeitsplatzwechsel in die andere Region immer mit einem Wohnsitzwechsel verbunden sein soll.

Im Folgenden wird davon ausgegangen, daß eine Vereinigung zweier Regionen mit dem Fehlen jeglicher Mobilitätsbarrieren für den Faktor Arbeit gleichzusetzen ist. Daher wird „Abbau von Mobilitätsbarrieren“ im Folgenden synonym zu „mit Vereinigung“ gebraucht.

Im folgenden werden i.d.R. zwei Regionen mit und ohne Vereinigung miteinander verglichen. Im Prinzip verschmelzen durch eine Vereinigung zwei Regionen zu einer. Wenn jedoch noch Unterschiede zwischen den Gebieten bestehen bleiben, wie eine unterschiedliche Ausstattung mit natürlichen Ressourcen oder eine differierende Kapitalausstattung, kann es Sinn machen, weiterhin von zwei

12 Da die Individuen meist nur über unvollkommene Informationen verfügen, wird das Potential an Mobilität die realisierte Mobilität i.d.R. übertreffen. Vgl. dazu Sheldon (1985), S. $59 \mathrm{ff}$.

13 Mackensen u.a. (1975), S. 9. 
Regionen zu sprechen. Zudem ist es aus analytischen Zwecken notwendig, auch die beiden Gebieten mit und ohne Vereinigung einzeln $\mathrm{zu}$ betrachten. Wenn beide Regionen gemeinsam betrachtet werden, wird dies im folgenden stets explizit entsprechend ausgedrückt.

Mit regionaler Mobilität werden teilweise nicht nur Wanderungen von Individuen bezeichnet, sondern auch die räumliche Umverteilung von Arbeitsplätzen. Üblich ist aber der Begriff der Standortmobilität, wenn ein solcher Kapitaltransfer in andere Regionen betrachtet wird.

\subsection{Themenabgrenzungen und Fragestellungen}

Über die Ursachen und Wirkungen von Mobilität ist bereits viel geforscht worden. Die Forschungsansätze sind ebenso vielfältig wie die Wanderungsmotive und die -wirkungen:

"Einerseits geht es um stationäre Prozesse (des Güteraustausches, der Pendlerbewegungen aller Art) und ihre begrifflichen Elemente wie Aktionsreichweiten, Interaktionsfelder, Einzugsgebiete, räumliche Kommunikationskreise. Andererseits wird den raumstrukturverändernden Bewegungen der Produktionsfaktoren, ihren Gründen oder Hindernissen, große Aufmerksamkeit gewidmet, d.h. den (meist selektiven) Wanderungsvorgängen (bzw. umgekehrt der jeweiligen partiellen Immobilität) von Bevölkerung, Unternehmungen und Kapital sowie der Ausbreitung technischen und sonstigen Wissens und den Folgewirkungen solcher Wanderungen auf die sozio-ökonomische Struktur der Herkunfts- und Zielstandorte."14

Ökonomische Mobilitätsmodelle befassen sich hauptsächlich mit Allokationsprozessen am Arbeitsmarkt. Wanderungen werden als Anpassungsbewegungen des Produktionsfaktors Arbeit mit dem Ziel seiner optimalen Allokation interpretiert. Migrationen führen zu einem Ausgleich regionaler Ungleichgewichtssituationen auf dem Arbeitsmarkt. Ausgelöst werden Wanderungen nach ökonomischen Vorstellungen durch regionale Lohnunterschiede und Arbeitslosigkeit.

Die massiven Übersiedlungen der ostdeutschen Bevölkerung direkt nach der Öffnung der Grenze waren nicht allein ökonomisch motiviert, sondern politische Gründe überwogen vielfach. Spätestens jedoch nach der Vereinigung am 3.

${ }^{14}$ Bartels (1982), S. 51. 
Oktober 1990 dürften politisch motivierte Wanderungen zurück gegangen sein. Ökonomische Gründe traten mehr und mehr in den Vordergrund.

Ostdeutschland wies in fast allen Lebensbereichen einen Rückstand gegenüber dem früheren Bundesgebiet auf. Die Wohnungen waren in einem schlechten $\mathrm{Zu}-$ stand, die Infrastruktur war nahezu durchgängig desolat, die Umweltverschmutzung hoch und die Einkommen gering. All diese Faktoren können die Abwanderungsneigung positiv beeinflußt haben. Besonderes Gewicht wurde in der öffentlichen Diskussion dem Lohnrückstand gegeben. Gemäß ökonomischer Vorstellungen hätten Wanderungen in einem Ausmaß einsetzen müssen, bis die Lohndifferenzen beseitigt sind. Ohne alle Eingriffe in das Marktgeschehen hätten auf diesem Weg die Einkommensunterschiede zum Verschwinden gebracht werden können.

In der öffentlichen Diskussion wurde aber immer wieder gefordert, Bedingungen zu schaffen, die geeignet sind, Massenabwanderungen zu verhindern. Zahlreiche Maßnahmen wurden durchgeführt, um die Lebensbedingungen in Ostdeutschland zu verbessern. Die Löhne in Ostdeutschland wurden in den Tarifverhandlungen auf ein Niveau weit oberhalb des Vollbeschäftigungslohns festgesetzt. Ferner wurden Lohnangleichungen innerhalb weniger Jahre vereinbart, obwohl dies ökonomisch nicht zu rechtfertigen war.

Die Massenabwanderungen und die damit einhergehenden Befürchtungen werfen eine Vielzahl von Fragen auf, wie beispielsweise folgende: Welche ökonomischen Konsequenzen bringt ein massiver Abwanderungsstrom mit sich? Warum wurden Eingriffe zur Verhinderung von Umzügen gefordert und vorgenommen, wenn doch Wanderungen gemäß der ökonomischen Theorie zu einer optimalen Allokation der Arbeit führen? Verringerte sich die Abwanderung durch die schnelle Lohnangleichung oder erhöhte sie sich sogar infolge der durch die hohen Löhne verursachten Arbeitslosigkeit? War tatsächlich zu erwarten gewesen, daß der Abwanderungsstrom zur Entleerung ganzer Landstriche führt? Welche Ostdeutschen sind abgewandert und welche waren immobil? Was waren ihre Gründe? War ihre Einschätzung der Konsequenzen über ihre Mobilität bzw. Immobilität zutreffend? Hätte eine Wanderung nicht angesichts der regionalen Lohnunterschiede von jedem Ostdeutschen erwogen werden müssen?

Einigen dieser Fragen soll im Folgenden nachgegangen werden. Zunächst werden einige vereinigungsbedingte Wirkungen aufgezeigt. Aufgrund der vielfältigen Interdependenzen und der Komplexität des Phänomens vereinigungsbedingter Wanderungen werden die Wirkungen von regionaler Mobilität jedoch nur aus theoretischer Sicht und radikal vereinfacht abgehandelt. Auf die Beur- 
teilung der Maßnahmen der öffentlichen Hand hinsichtlich ihrer Wirksamkeit in Bezug auf die regionale Mobilität nach der deutsch-deutschen Vereinigung wird verzichtet, da die Eingriffe in das Marktgeschehen nicht gezielt zur Verhinderung von Wanderungen ergriffen wurden, sondern primär auf andere Ziele wie die Angleichung der Lebensbedingungen ausgerichtet waren und ihre Wirkungen hinsichtlich regionaler Mobilität daher kaum isoliert zu beurteilen sind. Ferner wird untersucht, wie hoch die Erträge einer Wanderung kurz nach der Vereinigung zwischen den neuen und alten Ländern gewesen wären und welchen Einfluß die verschiedenen Wanderungsfaktoren hatten. Außerdem wird geprüft, ob Wanderungsanreize für alle Ostdeutschen vorhanden waren und für welchen Teil der Bevölkerung die Anreize besonders hoch waren. Bevor nun der Gang der Untersuchung beschrieben wird, soll noch eine Abgrenzung der Arbeit vorgenommen werden.

Wanderungen finden nicht nur aus ökonomischen Gründen statt. Die Soziologen gehen davon aus, daß die (soziale) Funktion räumlicher Mobilität im Ausgleich sozialer Spannungen und der Beseitigung sozialer Konflikte liegt ${ }^{15}$. Starke Ungleichgewichte wie zwischen den alten und neuen Ländern im Jahr 1989 führen zu enormen sozialen Spannungen. Die Behandlung derartiger Fragen geht über das Thema hinaus, da bereits die ökonomischen Ursachen und Wirkungen so vielfältig sind, daß sie nicht alle in dieser Arbeit berücksichtigt werden können.

Beim geographischen Ansatz wird traditionell der Einfluß des Klimas, des Bodens und der Landwirtschaft auf die Migration untersucht. Der Schwerpunkt der Untersuchungsgebiete der Geographen hat sich in jüngerer Zeit auf Fragen der Verstädterung, zentraler Orte ${ }^{16}$ und Infrastruktur verschoben ${ }^{17}$. Die Bedeutung des Klimas und der Infrastruktur als Wanderungsursachen werden in einigen Abschnitten dieser Arbeit aufgegriffen.

Demographen interessieren Wanderungen im Hinblick auf die Zusammensetzung der Bevölkerung und deren Veränderung. Demographische Untersuchungen zielen darauf ab, Erkenntnisse für die Bevölkerungspolitik, speziell für die Wanderungspolitik, zu gewinnen ${ }^{18}$. Lange Zeit wurde angenommen, daß Wanderungen durch einen „Bevölkerungsdruck“ ausgelöst werden. Demnach finden Wanderungen aus Gebieten mit einem hohen Geburtenüberschuß in dünner

15 Vgl. Szell (1972), S.18 und Röder (1974), S. 38.

16 Ein "zentraler Ort" ist der Mittelpunkt einer Region, der die Versorgung des Umlandes mit übernimmt. Die zentralen Funktionen sind insbesondere die Leistungen des tertiären Sektors. Der Zentrale-Orte-Ansatz geht zurück auf Christaller (1933). Vgl. hierzu auch Müller (1977), S. 457 und Fürst/Hesse (1981), S. $27 \mathrm{ff.}$

7 Vgl. Szell (1972), S. 19.

18 Vgl. Szell (1972), S. 15 f und Röder (1974), S. 37. 
besiedelte Regionen statt und vermindern so den Bevölkerungsdruck, der infolge eines starken natürlichen Bevölkerungswachstums entsteht. Da das natürliche Bevölkerungswachstum in den Industrieländern zurückgeht und die Sterbefälle sogar die Zahl der Geburten in einigen Ländern übersteigen, bestimmen Wanderungsbewegungen zunehmend die Entwicklung der Bevölkerungsgröße. Ein Bevölkerungsdruck bestand nach der Vereinigung nicht, die neuen Länder waren sogar weniger dicht besiedelt als die alten.

Eine mögliche Alternative zu einer Wanderung aus Ost- nach Westdeutschland wäre ein Umzug ins Ausland gewesen. Die Barrieren wären aber ungleich höher gewesen, z. B. wegen der fremden Sprache oder institutioneller Hemmnisse. Diese Arbeit beschäftigt sich ausschließlich mit Binnenwanderungen, die speziellen Probleme von Außenwanderungen werden nicht betrachtet.

Dadurch, daß ein Ohne- und ein Mit-Vereinigungsfall betrachtet wird, soll ausdrücklich ausgeschlossen werden, daß potentielle Mobilitätsursachen, wie Unterschiede des politischen Systems oder der Sozialversicherung betrachtet werden müssen.

Der Rahmen dieser Arbeit erlaubte es nicht, die Bedeutung von vereinigungsbedingter Kapitalmobilität zu untersuchen. Es bleibt unbestritten, daß Kapitalmobilität stärkere wirtschaftliche Veränderungen hervorrufen kann als Arbeitsmobilität. Auch Rücküberweisungen von Migranten in ihre Heimatregion, die vor allem bei internationaler Wanderungen bedeutsam sein können, werden nicht berücksichtigt.

\subsection{Zur Vorgehensweise}

Im ersten Teil der Arbeit werden aus partialanalytisch komparativ-statischer Sicht Ausmaß und Folgen von Wanderungsbewegungen auf den Arbeitsmarkt untersucht, die aus einer Beseitigung von Mobilitätshindernissen zwischen zwei Gebieten aufgrund einer Vereinigung resultieren. Dabei werden die Auswirkungen auf Beschäftigung, Lohnniveau und Einkommen unter analytisch einfachen Bedingungen dargestellt, wobei der Bedingungsrahmen schrittweise modifiziert wird.

In Kapitel 2.1 wird angenommen, daß die einzige Wanderungsursache regionale reale Lohnunterschiede sind. Nach der Beschreibung des neoklassischen Modellrahmens wird gezeigt, welche Lohnhöhe und Beschäftigung sich bei vollkommener regionaler Immobilität in einer 2-Regionen-Welt ergeben. Danach wird untersucht, welche Veränderungen von Lohn und Beschäftigung sich nach einer 
Aufhebung der Mobilitätsbarrieren für Arbeit einstellen. Im anschließenden Abschnitt wird das Einkommen vor und nach Vereinigung bestimmt. Zunächst wird die funktionale Einkommensverteilung beschrieben. Danach wird gezeigt, wie die Höhe der Arbeitnehmerrente bei gegebenem Lohnniveau bestimmt werden kann. Auf Basis dieser Grundlagen werden verschiedene Einkommensgrößen mit und ohne Vereinigung dargestellt. Außerdem werden Umverteilungswirkungen zwischen den Regionen und Veränderungen der funktionalen Einkommensumverteilung untersucht. Hierbei wird auch gezeigt, daß das Sozialprodukt der Regionen insgesamt durch eine Vereinigung nicht grundsätzlich steigen wird, obwohl Mobilität einen produktiveren Einsatz von Arbeit bewirkt.

Im Anschluß werden die sich ergebenden Erkenntnisse über die zu erwartende Lohnentwicklung und das Wanderungsverhalten übertragen auf den deutschdeutschen Arbeitsmarkt nach der politischen Wende in der ehemaligen DDR. Dabei wird auch erläutert, wie die tatsächliche Entwicklung der Arbeitsmobilität und der regionalen Lohnsätze von der modelltheoretischen abweicht und nach Gründen für die Abweichung gesucht.

Aufgrund dieser Überlegungen werden in den anschließenden Kapiteln verschiedene Annahmen modifiziert. In Kapitel 2.2 wird die Möglichkeit von Arbeitslosigkeit zugelassen. Die Annahme flexibler Löhne wird aufgegeben und gezeigt, welche Lohnhöhe, Beschäftigung und Einkommen sich unter den geänderten Voraussetzungen ergeben. Anschließend wird die Wirkung alternativer Lohnabschlüsse zum Zeitpunkt der Vereinigung im Hinblick auf die dadurch ausgelösten Mobilitätsprozesse untersucht.

In Kapitel 2.3 werden die Annahmen bezüglich des Einsatzes von Arbeit und der Bedeutung der regionalen Nachfrage nach Gütern und Dienstleistungen für die Arbeitsnachfrageseite modifiziert. Zunächst wird berücksichtigt, daß bei der Produktion unterschiedlich qualifizierte Arbeitnehmer eingesetzt werden können. Im ersten Unterabschnitt wird Humankapital als Teil des Kapitalstocks betrachtet, der die Arbeitsnachfrage determiniert, so daß sich durch Arbeitskräftewanderungen nicht nur die Arbeitsangebotskurven verändern, sondern auch die Arbeitsnachfragekurven. Im zweiten Unterabschnitt von Kapitel 2.3 werden die Wirkungen untersucht, wenn wandernde Arbeitskräfte komplementär zu heimischen Arbeitnehmer eingesetzt werden können. Im darauf folgenden Abschnitt wird die Bedeutung selektiver Wanderungen mit Blick auf die Zukunftsperspektiven einer Region beleuchtet. Im Anschluß werden die Wirkungen von Wanderungen dargestellt, wenn sie Einfluß auf die regionale Nachfrage nach Gütern und Dienstleistungen haben. 
In Kapitel 2.4 werden weitere Wanderungsgründe berücksichtigt, nämlich Unterschiede in der Attraktivität von Regionen und der regionalen Preisniveaus sowie Transaktionskosten. Dabei soll gezeigt werden, daß unter solchen Bedingungen auch mit Vereinigung regionale Reallohnunterschiede bestehen können und eine Vereinigung unter bestimmten Bedingungen sogar zu einer Umkehrung der interregionalen Lohnverhältnisse führen kann.

Während im ersten Teil der Arbeit einige vereinigungsbedingte Wirkungen regionaler Arbeitsmobilität aus theoretischer Sicht aufgezeigt werden, steht im Mittelpunkt des anschließenden Teils die individuelle Wanderungsentscheidung ostdeutscher Beschäftigter kurz nach der Vereinigung.

In Kapitel 3 wird untersucht, welche Beschäftigten einen Wanderungsgewinn erzielt hätten, wenn sie sich für einen Umzug in die alten Länder (mit gleichzeitigem Arbeitsplatzwechsel) entschieden hätten. Dafür sollen alle relevanten Wanderungsfaktoren einbezogen werden. Daher wird zunächst eine Übersicht darüber gegeben, welche Wanderungsdeterminanten generell in verschiedenen empirischen Ansätzen berücksichtigt werden. Die einzelnen Faktoren werden dann eingehend dahingehend beleuchtet, wie sie monetär bewertet werden können, um sie in eine individuelle Modellrechnung zur Beurteilung der Vorteilhaftigkeit einer Wanderung einzubeziehen. Dabei wird erläutert, welche zusätzlichen Einkommensbestandteile neben den Löhnen berücksichtigt werden sollten und wie regional unterschiedliche Risiken, arbeitslos zu werden, bewertet werden könnten. Daneben werden regionale Preisunterschiede, die Standortqualität, die Bedeutung von Informationen und ihrer Beschaffung betrachtet. Darüber hinaus werden die Bewertung der Umzugskosten, der Entfernung und der psychischen Kosten und Erträge behandelt, sowie die Möglichkeit wiederholter Wanderungen bzw. Rückwanderungen und sonstige personenspezifische Wanderungsgründe erörtert.

Auf dieser Grundlage werden im anschließenden Kapitel 3.2 die Faktoren, die für die Wanderungsüberlegungen eines ostdeutschen Arbeitnehmers am Ende des Jahres 1990 bedeutsam gewesen sein dürften, quantifiziert. In Modellrechnungen wird geschätzt, welche Wanderungserträge für Erwerbstätige aus den neuen Bundesländern nach der Vereinigung erwartbar waren und welche Erträge bei Zugrundelegung der tatsächlichen Entwicklung erwartbar gewesen wären. Berücksichtigt werden die regionalen Löhne, die Mietpreise, verschiedene Erwartungen über den ökonomischen Angleichungsverlauf zwischen den neuen und alten Bundesländern, Branchenzugehörigkeit sowie Status und Geschlecht der Beschäftigten. In einem weiteren Schritt werden die Umzugskosten, die psychische Kosten der Wanderung, die unterschiedlichen regionalen Jahresarbeitszeiten, die Mehrkosten bei Neuanmietung einer Wohnung im Vergleich zur Be- 
standsmiete sowie die Lohneinbußen durch eine Verschlechterung der beruflichen Stellung berücksichtigt. Im Anschluß wird die monetäre Bedeutung der regional unterschiedlich hohen Arbeitslosigkeit untersucht. Nach einer Zusammenfassung der wichtigsten Ergebnisse wird dann geschätzt, welches Wanderungspotential sich auf Grundlage der durchgeführten Rechnung ergeben hätte. Abschließend wird berechnet, wie lohnend eine Wanderung fünf Jahre nach der Wende in Ostdeutschland noch gewesen wäre.

Im Anschluß wird in Kapitel 3.3 gezeigt, wie sich die deutsch-deutschen Wanderungen seit 1989 tatsächlich entwickelt haben und dies verglichen mit den Ergebnissen aus Kapitel 3.2.

In der Schlußbetrachtung werden zunächst die wichtigsten Ergebnisse dieser Arbeit zusammengefaßt. Abschließend wird kurz auf die wichtigsten Maßnahmen der öffentlichen Hand und der Tarifparteien, die Einfluß auf die ostdeutsche Mobilitätsneigung gehabt haben könnten, hingewiesen. 
Annette Schönherr - 978-3-631-75008-7

Downloaded from PubFactory at 01/11/2019 09:19:06AM

via free access 


\section{Wirkungen regionaler Arbeitsmobilität unter analytisch einfachen Bedingungen}

Um die grundlegenden Wirkungsmechanismen einer Vereinigung zweier Regionen zu zeigen, wird zunächst von einem einfaches neoklassisches Vollbeschäftigungsmodell ausgegangen, um das neoklassisch zu erwartende Ausmaß regionaler Mobilität sowie Lohnhöhe, Beschäftigung und Einkommen vor und nach Vereinigung theoretisch zu bestimmen. Anhand der Entwicklung in Deutschland vor und nach der Vereinigung wird dann erörtert, ob die Vereinigungsproblematik sich auf diese Weise problemadäquat abbilden läßt und welche Modifikation das Modell gegebenenfalls erfahren müßte. Aufgrund dieser Überlegungen wird das Modell schrittweise erweitert, und zwar um Arbeitslosigkeit, Besonderheiten der Arbeitsnachfrage, regionale Annehmlichkeiten, Preisniveauunterschiede und Transaktionskosten.

\subsection{Regionale Arbeitsmobilität bei Zugrundelegung eines einfachen neoklassischen Vollbeschäftigungsmodells}

Ziel dieses Abschnittes ist es, Beschäftigungs- und Wohlfahrtswirkungen regionaler Arbeitsmobilität aufzuzeigen. Als Referenzmodell wird das neoklassische Modell zugrunde gelegt, auch wenn dieses insbesondere den ostdeutschen Arbeitsmarkt nicht widerspiegelt. Dieses Modell kann aber als Meßlatte dafür dienen, welche Unterschiede sich idealerweise ergeben würden. Es werden aus partialanalytischer komparativ-statischer Sicht die Folgen von Wanderungsbewegungen auf den Arbeitsmarkt untersucht, die aus einer Beseitigung von Mobilitätshindernissen infolge einer Vereinigung zweier Gebiete resultieren. Rückwirkungen auf andere Märkte als Folge von Migrationen werden nicht berücksichtigt. Es werden nur kurzfristige Effekte betrachtet, die Bedeutung von Wanderungen für Entwicklungsperspektiven wird zunächst ausgeklammert.

Um die Voraussetzung für die Untersuchung zu schaffen, werden zunächst der neoklassische Modellrahmen sowie die Annahmen über das Arbeitsangebot und die -nachfrage skizziert. Für den Fall vollkommener interregionaler Immobilität (z.B. infolge politischer Maßnahmen (wie Mauerbau) oder unendlich hoher Mobilitätskosten) und den Fall vollkommener regionaler Arbeitsmobilität zwischen zwei Gebieten wird jeweils das Lohnniveau und die Beschäftigung bestimmt. Beschäftigung und Lohnsätze vor und nach Vereinigung werden miteinander verglichen. Nach einer allgemeingültigen Betrachtung werden auch Sonderfälle mit extrem hohen regionalen Unterschieden dargestellt. 
Im anschließenden Abschnitt 2.1.1.3 wird beschrieben, wie die funktionale Einkommensverteilung nach der Grenzproduktivitätstheorie und die Höhe der Arbeitnehmerrente bei gegebenem Lohnniveau bestimmt werden können. Danach werden die verschiedenen Einkommensgrößen Sozialprodukt, Gewinn, Lohnsumme und Arbeitnehmerrenten mit und ohne Vereinigung dargestellt. Außerdem werden Umverteilungswirkungen zwischen den Regionen sowie Veränderungen der funktionalen Einkommensumverteilung untersucht.

Im dann folgenden Abschnitt werden die sich ergebenden Erkenntnisse über das Wanderungsverhalten und die zu erwartende Lohnentwicklung auf den deutschdeutschen Arbeitsmarkt nach der politischen Wende in der ehemaligen DDR übertragen. Daran anschließend wird erläutert, wie die tatsächliche Entwicklung der Arbeitsmobilität und der Löhne von der modelltheoretischen abwich und nach Gründen für die Abweichung gesucht.

Zur Vereinfachung wird im Folgenden regionale Preisunterschiedslosigkeit unterstellt. Diese Annahme läßt sich damit begründen, daß zunächst nur handelbare Güter produziert werden, die überall zu Weltmarktpreisen gekauft werden können. Die Nominallohndifferenzen entsprechen dann den Reallohndifferenzen.

\subsubsection{Lohnniveau, Beschäftigung und Einkommen mit und ohne Vereinigung}

\subsubsection{Der Modellrahmen: Das neoklassische Arbeitsmarktmodell}

Referenzsystem des Arbeitsmarktes ist üblicherweise das neoklassische Arbeitsmarktmodell. Zentrales Element der Neoklassik ist die Allgemeine Gleichgewichtstheorie. Sie unterstellt ein Verhalten, welches zur Beseitigung von Ungleichgewichten und damit auf dem Arbeitsmarkt zu Vollbeschäftigung und unterschiedsloser Entlohnung führt. Alle Marktteilnehmer sind in ihrer Entscheidungsfreiheit nicht eingeschränkt und verhalten sich stets rational.

Für die Einordnung der Schlußfolgerungen aus den nächsten Abschnitten ist eine ausführliche Erläuterung des zugrundeliegenden Modells notwendig. Dieser Abschnitt operiert mit einem 1-Regionen-Modell, während im Folgenden immer 2 Regionen betrachtet werden. Der Modellrahmen gilt aber für jede Region, daher ist die zunächst vorgenommene Einschränkung auf eine Region unproblematisch. 


\subsection{Die Annahmen}

Folgende Annahmen liegen dem neoklassischen Arbeitsmarktmodell und anfänglich der nachfolgenden Untersuchung zugrunde ${ }^{19}$ :

(1) Es gibt sehr viele Anbieter und Nachfrager.

(2) Die angebotene Arbeit ist homogen.

(3) Es gibt außer Lohn- und Produktivitätspräferenzen keine weiteren Präferenzen. Unternehmen bevorzugen oder diskriminieren keinen der Arbeitsanbieter; für die Arbeitskräfte bedeutet ein Wechsel des Arbeitgebers oder Arbeitsplatzes keine Nutzenänderung, wenn der Wechsel nicht mit Lohnänderungen verbunden ist.

(4) Es besteht vollkommene Markttransparenz. Arbeitsanbieter und -nachfrager sind vollständig über die jetzige und zukünftige Arbeitsmarktsituation informiert.

(5) Es gibt keine Transaktions- und Informationskosten ${ }^{20}$.

(6) Der Lohnsatz ist vollkommen flexibel, es existieren somit keine Rigiditäten.

(7) Die Arbeitsanbieter sind intraregional vollkommen mobil ${ }^{21}$.

(8) Die Arbeitsmärkte sind Konkurrenzmärkte.

Diese Annahmen sind durchweg realitätsfern. Trotzdem wird zunächst mit ihnen gearbeitet, um die grundsätzlichen Wirkungszusammenhänge als Folge von Wanderungsbewegungen auf den Arbeitsmarkt zu zeigen. Die Problemadäquanz des Modells in Hinsicht auf die deutschen Verhältnisse wird in einem späteren Abschnitt diskutiert.

\subsection{Die Arbeitsnachfrage}

Die Arbeitsnachfrage wird von den Unternehmen entfaltet. Der Produktpreis sei für sie stets ein Datum.

Unterstellt wird eine Produktionsfunktion vom neoklassischen Typ mit $\mathrm{Y}=\mathrm{Y}$ $(\mathrm{K}, \mathrm{L})$. Kapitalstock $(\mathrm{K})$ und Technologie seien gegeben; kurzfristig ist die $\mathrm{Ar}-$

19 Vgl. etwa Sesselmeier/Blauermel (1990), S. 20.

${ }^{20}$ Mit Blick auf die Absatz- und Beschaffungsmärkte schließt das die Abwesenheit von „Transportkosten“ ein. Das bedeutet zugleich eine (interregionale) transaktionskostenlose Handelbarkeit aller Güter.

21 Diese Annahme ergibt sich im Prinzip bereits aus Annahme (3) und (5), sofern es keine institutionellen Hindernisse gibt. 
beitseinsatzmenge ${ }^{22}$ (L) der variable Faktor. Die Ertragskurve der Arbeit entspricht unter dieser Bedingung der Produktionsfunktion. Es wird angenommen, daß sich die Unternehmen als Gewinnmaximierer verhalten. Aus dem Gewinnkalkül der Unternehmen ergibt sich, daß sie solange Arbeit nachfragen und einsetzen, bis das Grenzwertprodukt der Arbeit dem Reallohnsatz (w) entspricht. Die Grenzwertproduktkurve entspricht daher der Nachfrage nach Arbeit bei alternativen Lohnsätzen (unter der Annahme, daß der Einsatz aller anderen Faktoren konstant ist). Die Marktnachfrage nach Arbeit ergibt sich als Aggregation der einzelwirtschaftlichen Nachfrage.

Die Grenzproduktivität der Arbeit ist immer positiv, sinkt jedoch mit zunehmendem Arbeitseinsatz, so daß die Arbeitsnachfragekurve in Abhängigkeit vom Reallohnsatz eine negative Steigung aufweist.

Im allgemeinen wird die langfristige Abhängigkeit der Arbeitsnachfrage vom Lohn nicht bestritten. Jedoch wird häufig die These vertreten, daß die Arbeitsnachfrage kurzfristig völlig lohnunelastisch is ${ }^{23}$. Dieser Auffassung wird hier nicht gefolgt, sondern im folgenden wird angenommen, daß die kurzfristige Arbeitsnachfragekurve zwar steiler als die langfristige verlaufen wird, aber nicht völlig unelastisch ist. Es mag zwar in einer Volkswirtschaft für Teile des Produktionsapparates quasi-fixe Faktoreinsatzverhältnisse geben ${ }^{24}$, jedoch wohl nicht für alle Produktionsmöglichkeiten, sondern bei jedem Lohnniveau dürfte es einige (Grenz-) Unternehmen geben, die auf Lohnänderungen mit Anpassungen der Beschäftigungsmenge reagieren ${ }^{25}$.

\subsection{Das Arbeitsangebot}

Das regionale bzw. gesamtwirtschaftliche Arbeitsangebot setzt sich aus der Summe der individuellen Arbeitsangebote zusammen. Dabei steht zunächst jeder einzelne Arbeitsanbieter vor der Entscheidung wieviel Arbeit er für welchen

${ }^{22} \mathrm{~L}=$ Arbeitsstunden.

23 So äußert sich etwa Neubäumer (1991), S. 104: "Dagegen läßt sich kurzfristig keine mit dem Reallohn fallende Arbeitsnachfrage beobachten...Eine wesentliche Ursache dafür ist, $\mathrm{da} ß$ sich die vorhandenen Produktionsmittel oft nicht mit mehr oder weniger Arbeit kombinieren lassen, d.h. keine Ex post-Substituierbarkeit besteht.". Auch Priewe (1984), S. 20 geht von limitationalen Faktoreinsatzverhältnissen aus.

${ }^{24}$ Zur Begründung, warum Arbeit zunehmend ein quasi-fixer Faktor ist vgl. etwa Bombach (1988).

${ }^{25}$ Die Ansicht, daß auch die kurzfristige Arbeitsnachfrage elastisch ist, vertritt z.B. auch Hoffman (1986), S. 37: "There is no such thing as a fixed number of jobs in an economy", vgl. hierzu auch ebda S. 60 . 
Lohn anbieten soll und über wieviel Freizeit er verfügen will. Für diese Arbeitszeit-Freizeit-Entscheidung verhalte sich jeder als Nutzenmaximierer. Arbeit sei immer mit "Arbeitsleid" verbunden. Die Opportunitätskosten einer Stunde Freizeit entsprechen dem Stundenlohnsatz. Der einzelne wird bei gegebenem Lohn soviel Arbeit anbieten, bis die Grenzrate der Substitution von Einkommen durch Freizeit gleich dem Lohnsatz ist. Ein steigender Lohnsatz verteuert die Opportunitätskosten der Freizeit und führt zu einer Substitution von Freizeit durch Arbeit. Gleichzeitig ergibt sich ein gegenläufiger Effekt: durch den höheren Lohnsatz wird ein höheres Einkommen erzielt. Ist Freizeit ein superiores Gut, würde dieser Einkommenseffekt zu einer Mehrnachfrage nach Freizeit und dementsprechend einer Verringerung des Arbeitsangebots führen. Ob das Individuum mehr oder weniger Arbeit bei einem höheren (Stunden-) Lohn anbietet, hängt davon ab, ob der Einkommenseffekt den Substitutionseffekt übersteigt. Es sind drei Reaktionsweisen denkbar:

- der Einkommenseffekt ist geringer als der Substitutionseffekt: das Arbeitsangebot erhöht sich mit steigendem Lohnsatz (dies wird auch als "normale" Reaktion bezeichnet),

- der Einkommenseffekt übersteigt den Substitutionseffekt: das Arbeitsangebot geht mit steigendem Lohnsatz zurück ("anomale" Reaktion),

- der Einkommenseffekt entspricht dem Substitutionseffekt: das Arbeitsangebot bleibt bei Lohnvariationen konstant

Die individuellen Arbeitsangebotskurven werden in der Regel nicht nur durch eine einzelne dieser drei Reaktionsweisen gekennzeichnet sein, sondern prinzipiell sind z.B. auch Kurven mit zurückbiegendem Verlauf oder einem in Form eines Fragezeichens denkbar ${ }^{26}$.

Das Marktangebot ergibt sich aus einer Horizontalaggregation der individuellen Arbeitsangebotskurven ${ }^{27}$. Trotz der unterschiedlichen individuellen Kurven wird sich eine Marktangebotskurve mit überwiegend "normalem" Verlauf (= positive Steigung) ergeben, wenn etwa die individuellen Arbeitsangebotskurven mit "normalem" Verlauf die anderen überkompensieren. Außerdem erhöht sich mit steigendem Lohnsatz die Zahl der Arbeitsanbieter, und zwar um diejenigen mit einem höheren Reservationslohn, was zu einem "normalen" Verlauf beiträgt ${ }^{28}$.

26 Vgl. hierzu etwa Sesselmeier/Blauermel (1990), S. 26f oder Brinkmann (1981), S. 46ff.

$27 \mathrm{Zu}$ den hierbei entstehenden Aggregationsproblemen vgl. Pencavel (1986), S. $31 \mathrm{ff}$ und Priewe (1984), S. 15f.

28 Vgl. Hoffman (1986), S. $97 \mathrm{f}$. 
In der Literatur findet sich z.T. die Auffassung, daß das Arbeitsangebot in bezug auf Lohnsatzänderungen relativ starr $\operatorname{sei}^{29}$. Auch sei die Möglichkeit der Arbeitsanbieter gering, beliebig kleine Einheiten an Arbeit anzubieten, da zum einen die Nachfrage hierfür fehle und zum anderen institutionelle Bedingungen wie Tarifverträge etc. dem entgegenstünden ${ }^{30}$. Für einen unelastischen Bereich der Arbeitsangebotskurve spräche auch, daß Arbeit - entgegen der oben getroffenen Annahme - nicht immer mit Arbeitsleid verbunden sein muß. Eine gewisse Arbeitsmenge kann Nutzen stiften und würde vielleicht auch angeboten werden, wenn der Lohn sehr niedrig ist oder sogar bei Null liegt (solche Fälle sind in der Realität durchaus beobachtbar, so könnten etwa ehrenamtliche Tätigkeiten als Beispiel hierfür gelten). Für einen "Workaholic" könnte Freizeit sogar mit Unbehagen verbunden sein, so daß fürihn zusätzliche Freizeit mit einem höheren Einkommen ausgeglichen werden müßte um sein Wohlfahrtsniveau konstant zu halten ${ }^{31}$.

Im Folgenden werden daher neben "normal" verlaufenden Arbeitsangebotsfunktionen auch solche mit unelastischen Verläufen berücksichtigt. Anomale Verläufe werden jedoch ausgeschlossen.

\subsection{Gleichgewicht auf dem Arbeitsmarkt}

Arbeitsangebots- (LS) und -nachfragekurve (LD) lassen sich unter Zugrundelegung der bisherigen Annahmen in dem üblichen Arbeitsmarktdiagramm darstellen:

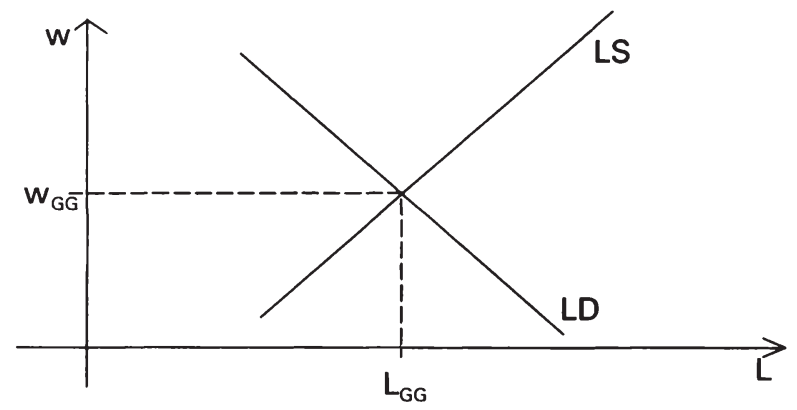

Abbildung 1: Arbeitsnachfrage und -angebot in Abhängigkeit vom Reallohnsatz

29 Vgl. etwa Priewe (1984), S. 16.

${ }^{30}$ Vgl. etwa Brinkmann (1981), S. 55 oder Sesselmeier/Blauermel (1990), S. 28.

31 Vgl. Franz (1991), S. 37. 
Im Schnittpunkt von Angebots- und Nachfragekurve befindet sich der Arbeitsmarkt im Gleichgewicht. Zum Lohnsatz $\mathrm{w}_{\mathrm{GG}}$ fragen die Unternehmen Arbeit in Höhe von $\mathrm{L}_{\mathrm{GG}}$ nach, und die Arbeiter bieten genau diese Menge zu diesem Lohnsatz an. Jeder, der bereit ist, zu dem Lohnsatz $\mathrm{w}_{\mathrm{GG}}$ zu arbeiten, findet eine Beschäftigung; unfreiwillige Arbeitslosigkeit gibt es nicht. Jede Situation, die vom Gleichgewicht abweicht, löst Anpassungsprozesse aus, die zu einem neuen Gleichgewicht führen. Da angenommen wird, daß die Arbeit homogen ist, muß der Lohnsatz im Gleichgewicht für alle Arbeiter gleich hoch sein ${ }^{32}$.

\subsubsection{Lohnniveau und Beschäftigung}

Es soll nun gezeigt werden, wie sich bei Immobilität im Vergleich zu regionaler Mobilität die Beschäftigungsmenge und der Lohnsatz voneinander unterscheiden. Daher werden im Folgenden zwei Regionen betrachtet. Zunächst wird analysiert, welche Ergebnisse sich bei Annahme vollständiger regionaler Immobilität einstellen. Im Anschluß werden Beschäftigung und Lohnsatz bei vollkommener regionaler Mobilität bestimmt und danach ein Vergleich der Ergebnisse vorgenommen. Dabei wird zunächst derallgemeine Fall betrachtet und daran anschließend Sonderfälle, wie sie sich durch besonders hohe regionale Unterschiede der Arbeitsnachfrage und des -angebots ergeben.

\subsection{Lohnhöhe und Beschäftigung bei vollkommenen regionalen Mobilitätsbarrieren}

Für die Analyse werden zwei regionale Arbeitsmärkte betrachtet. Annahme 6 (Die Arbeitnehmer sind vollkommen mobil) wird dahingehend modifiziert, daß nur innerhalb des jeweiligen Arbeitsmarkts Mobilität gegeben sei, jedoch nicht über die jeweiligen Gebietsgrenzen hinaus. Die regionalen Arbeitsmärkte werden als Region $\mathrm{x}$ und Region $\mathrm{y}$ bezeichnet. Jeder der beiden regionalen Arbeitsmärkte wird in Abbildung 2 durch ein Lohn-Arbeits-Diagramm - analog zu Abbildung 1 - dargestellt:

Arbeitsangebot und -nachfrage in den beiden Gebieten sind durch die LD- und LS- Kurven dargestellt. In dem abgebildeten Fall unterscheiden sich Arbeitsangebots- und -nachfragefunktionen der beiden Regionen voneinander. Die Arbeitsangebotskurve einer Region ergibt sich annahmegemäß ausschließlich aus dem Arbeitsangebot der in diesem Gebiet ansässigen Bevölkerung. Die Arbeitsnachfrage in einem Gebiet entfalten die dort tätigen Unternehmen entsprechend

${ }^{32}$ Vgl. Barro (1986), S. 269. 
der regionalen Produktionsfunktionen. Die jeweiligen Arbeitsmarktgleichgewichte ergeben sich wiederum im Schnittpunkt von Arbeitsangebots- und nachfragekurven. In dem gewählten Beispiel unterscheiden sich die Gleichgewichtslöhne der beiden Regionen und ihre Beschäftigungshöhe.

Es gibt keinen Anpassungsmechanismus, der auf eine Angleichung der regionalen Lohnniveaus hinwirkt.
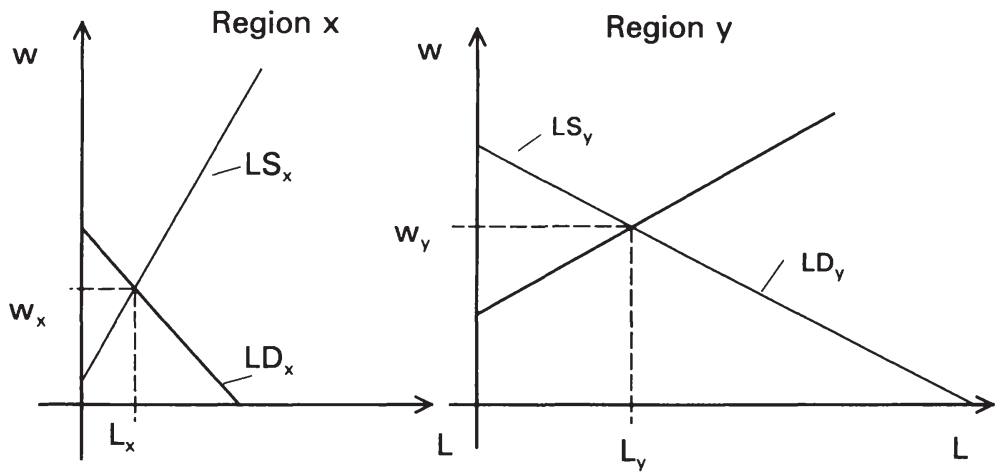

Abbildung 2: Lohnniveau und Beschäftigung bei Immobilität

Für einem späteren Vergleich der Resultate ist es sinnvoll, die Gleichgewichtsbeschäftigung und den -lohn auch mathematisch zu bestimmen. Zur Vereinfachung werden - wie auch in der Graphik - Arbeitsnachfrage- und -angebotsfunktionen mit linearen Verlauf unterstellt:

$\underline{\text { Region } \mathrm{x}:}$

(1a) $L_{D_{X}}: w_{X}=A_{X}-a_{X} L_{X}$

Arbeitsnachfrage

(2a) $\operatorname{LS}_{\mathbf{X}}: w_{X}=B_{X}+b_{X} L_{X}$

Arbeitsangebot

mit $\mathrm{a}_{\mathrm{X}}, \mathrm{b}_{\mathrm{X}} \geq 0$ und $0 \leq \mathrm{B}_{\mathrm{X}} \leq \mathrm{A}_{\mathrm{X}}$

$A_{X}$ ist der maximale Lohnsatz, den die Unternehmen in $\mathbf{x}$ für die erste Einheit Arbeit zu zahlen bereit sind; $a_{x}$ zeigt an, in welchem Ausmaß die Arbeitsnachfrage der Unternehmen steigt, wenn der Lohnsatz sinkt. Analoge Bedeutung haben $B_{X}$ und $b_{X}: B_{X}$ ist der Reservationslohn bzw. Anspruchslohn, unterhalb dessen kein $\mathrm{x}$-er bereit ist $\mathrm{zu}$ arbeiten und $\mathrm{b}_{\mathbf{X}}$ zeigt an, wie stark sich das Arbeitsangebot bei steigenden Löhnen erhöht. Es wird ein "normaler" Verlauf von Angebots- und Nachfragekurve unterstellt, daher müssen die Werte für $a$ und $b$ je- 
weils positiv sein. Der Reservationslohn $B_{x}$ ist jeweils kleiner als der potentielle Höchstlohn $A_{X}$. Je größer a und $b$ sind, desto unelastischer reagieren Angebot und Nachfrage auf Lohnänderungen.

Im Gleichgewicht stimmen Arbeitsangebot und -nachfrage überein $\left(\operatorname{LD}_{\mathbf{X}}=\right.$ $\mathrm{LS}_{\mathbf{X})}$.

Durch Einsetzen von (1a) und (2a) in die Gleichgewichtsbedingung ergibt sich (nach Umformung) für den Gleichgewichtslohn (3a) und die -beschäftigung (4a):

(3a) $w_{x G G}=\frac{A_{x} b_{x}-B_{x} a_{x}}{a_{x}+b_{x}}$

(4a) $L_{x G G}=\frac{A_{x}-B_{x}}{a_{x}+b_{x}}$

Unter den getroffenen Annahmen bezüglich der Determinanten von Arbeitsangebot und -nachfrage existiert ein Gleichgewicht mit einem Beschäftigungsniveau größer als Null. Ist dieses Gleichgewicht erreicht, so gibt es ohne eine exogene Störung keine Anpassungsreaktionen mehr, sondern das System wird in diesem Gleichgewicht verharren.

Für Region y ergibt sich analog:

(1b) $L D_{y}: w_{y}=A_{y}-a_{y} L_{y}$

(2b) $L S_{y}: w_{y}=B_{y}+b_{y} L_{y}$

(3b) $w_{y G G}=\frac{A_{y} b_{y}-B_{y} a_{y}}{a_{y}+b_{y}}$

(4b) $L_{y G G}=\frac{A_{y}-B_{y}}{a_{y}+b_{y}}$

mit $a_{y}$, by $\geq 0$ und $0 \leq B_{y} \leq A_{y}$

Lohnsatz und Beschäftigungsniveau der beiden Regionen unterscheiden sich in der Regel, eine Übereinstimmung wäre rein zufällig. 
Die Beschäftigungsmenge für beide Regionen insgesamt berechnet sich als Summe aus den gleichgewichtigen Beschäftigungsvolumina (4a) und (4b):

(5) $L=L_{x}+L_{y}=\frac{A_{x}-B_{x}}{a_{x}+b_{x}}+\frac{A_{y}-B_{y}}{a_{y}+b_{y}}$

Im Gleichgewicht ist - lineare Verläufe vorausgesetzt - die Beschäftigung c.p. um so größer, je stärker Arbeitsangebot und -nachfrage auf Lohnsatzänderungen reagieren (je geringer $a$ und $b$ sind), je geringer der „Reservationslohn“ $B$ und je größer der Prohibitivlohn (A) ist.

\subsection{Lohnhöhe und Beschäftigung nach Aufhebung regionaler Mobilitäts- barrieren}

Nachdem Lohnhöhe und Beschäftigung ohne Vereinigung bestimmt wurden, wird nun angenommen, daß durch eine Vereinigung alle Mobilitätsbarrieren für die Arbeitnehmer wegfallen. Es werden die Höhe der Wanderung sowie die Änderungen von Lohnniveau und Beschäftigung analysiert. Außerdem wird gezeigt, daß die Richtung der vereinigungsbedingten Beschäftigungsänderung nicht eindeutig, sondern abhängig von den Bedingungen am Arbeitsmarkt ist. Abschließend werden diese Bedingungen - soweit möglich - formuliert. Doch zunächst werden die sich durch eine Vereinigung ergebenden Anpassungsreaktionen erläutert.

Ausgangspunkt der Betrachtung sind wiederum die beiden Regionen $\mathrm{x}$ und $\mathrm{y}$, die im vorhergehenden Abschnitt durch vollkommene interregionale Immobilität der Arbeit gekennzeichnet waren. Die beiden Gebiete beschließen nun dieVereinigung ihrer Regionen. Im hierdurch entstandenen Territorium gelten die in Abschnitt 2.1.1.1 formulierten Annahmen bezüglich des neoklassischen Arbeitsmarktmodells. Sollte sich das ohne Vereinigung herrschende Lohnniveau der Regionen unterscheiden, werden die Erwerbspersonen der Niedriglohnregion ihre Arbeitskraft in der Hochlohnregion anbieten. Hierdurch entsteht in der Hochlohnregion ein Druck auf die Löhne. Es wird dort zu Lohnsenkungen kommen. $\mathrm{Zu}$ dem geringeren Lohn stellen die Arbeitgeber in der Zuwanderungsregion - gemäß ihrer Nachfragekurve - mehr Arbeiter an, so daß dort die Beschäftigung steigt.

Durch die Abwanderungen aus der Niedriglohnregion in Beschäftigungen in der Hochlohnregion verringert sich in der Abwanderungsregion das Angebot an Arbeitskräften. Bei höheren Löhnen wird nicht mehr soviel Arbeit wie bisher 
nachgefragt, die regionale Beschäftigung sinkt. Die Anpassungsprozesse setzen sich solange fort, bis sich das Lohnniveau zwischen den beiden Regionen angeglichen hat und damit die Wanderungsanreize verschwunden sind.

Wie hoch der Gleichgewichtslohn und die -beschäftigung nach der Vereinigung sind, soll nun graphisch illustriert werden. Abbildung 2 muß dafür um ein weiteres Diagramm ergänzt werden. Nach der Vereinigung bietet ein Arbeiter unter den gegebenen Annahmen - keine Präferenzen für einen bestimmten Arbeitsanbieter und Abwesenheit von regionenspezifischen Präferenzen - seine Arbeit auf beiden regionalen Teilmärkten simultan an. Daher kann es im Prinzip nur eine gemeinsame Arbeitsangebotskurve für beide Gebiete geben. Diese kann nicht in die Arbeitsmarktgraphiken der Regionen eingetragen werden, sondern nur in die Darstellung des Gesamtmarktes. Für die Arbeitsnachfrage sei hingegen eine kurzfristige Immobilität des Kapitalstocks angenommen ${ }^{33}$, so daß es weiterhin zwei regionale Arbeitsnachfragefunktionen gibt.

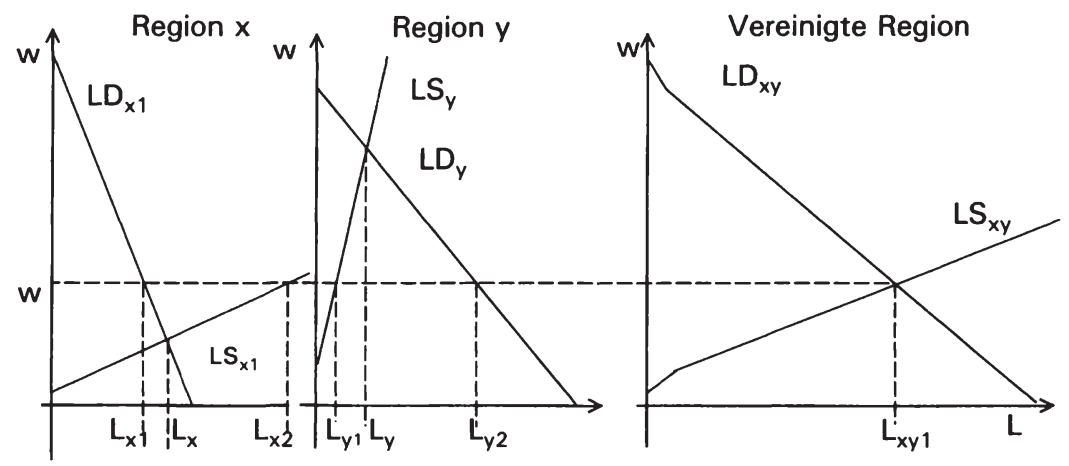

Abbildung 3: Lohnniveau und Beschäftigung bei Mobilität

Für die graphische Darstellung sind mithin drei Arbeitsmärkte abzubilden, die beiden regionalen und der Gesamtarbeitsmarkt (Region xy). In den Arbeitsmärkten der Regionen $\mathrm{x}$ und $\mathrm{y}$ wird die jeweilige Arbeitsnachfragefunktion - die weiterhin derjenigen ohne Vereinigung entspricht - eingetragen. In der Darstellung des vereinten Arbeitsmarktes werden die aggregierten Arbeitsangebots- und -nachfragekurven ( $\left(\mathrm{LS}_{\mathrm{xy}}\right.$ und $\left.\mathrm{LD}_{\mathrm{xy}}\right)$ abgebildet, die sich aus einer Horrizontaladdition der regionalen Kurven ergeben. In deren Schnittpunkt liegt das

${ }^{33}$ Langfristig gibt es keine lohnbedingten Gründe für eine Verlegung des Kapitalstocks, wenn es bei vollkommener Arbeitsmobilität immer zu regionalen Lohnnivellierungen kommt. 
gleichgewichtige Arbeitsvolumen $\left(\mathrm{L}_{\mathrm{xyl}}\right)$ und der dazu gehörige Lohnsatz (w) für das vereinigte Gebiet. Der Lohnsatz $\mathrm{w}_{1}$ stellt sich nach allen Anpassungsprozessen in beiden Regionen ein. Bei einem Lohnniveau von $w_{1}$ liegt ein Gleichgewicht auf beiden Arbeitsmärkten und zwischen den Arbeitsmarktregionen vor. Es gibt weder Überschußnachfrage noch -angebot an Arbeit. Kein Arbeitsanbieter hat einen Anreiz, seine Arbeitsmarktregion zu wechseln, da er in dem jeweils anderen Gebiet kein höheres Lohneinkommen erzielen kann.

Das Beschäftigungsvolumen in den einzelnen Regionen kann (graphisch) durch einen Rückschluß über den neuen Gleichgewichtslohnsatz bestimmt werden. Der Schnittpunkt einer Horizontalen in Höhe des Gleichgewichtslohns mit den regionalen Arbeitsnachfragekurven ergibt die jeweilige Höhe der Beschäftigung in den beiden Regionen. In Region $\mathrm{x}$ würden $\mathrm{L}_{\mathrm{x} 1}$ und in $\mathrm{y} \mathrm{L}_{\mathrm{y} 2}$ Arbeitseinheiten beschäftigt. $\mathrm{Zu}$ welcher regionalen Umschichtung es in der Beschäftigung kommt, kann ebenfalls aus Abbildung 3 abgelesen werden. Die ursprünglichen Arbeitsangebotskurven werden so eingetragen, wie sie sich vor der Vereinigung dargestellt haben. Ihr Schnittpunkt mit der Horizontalen zeigt die Höhe der Beschäftigten nach Herkunftsregion an; es sind $L_{x 2} x$-er und $L_{y 1} y$-er Arbeitseinheiten beschäftigt. Aus der Region $x$ sind $\mathrm{L}_{\mathrm{x} 2}-\mathrm{L}_{\mathrm{x}}$ Arbeitseinheiten mehr und aus der Region y $L_{y}-L_{y 1}$ weniger beschäftigt. Dabei sind $L_{x 2}-L_{x 1}$ Einheiten von der Region $\mathrm{x}$ in die Region y gewandert.

Mathematisch lassen sich Beschäftigungsmenge und Lohnsatz im Mit-Vereinigungsfall wie folgt ermitteln:

Die aggregierten Arbeitsangebots- und Nachfragekurven ergeben sich als Summe der regionalen Funktionen:

(6) $L D_{x y}=L D_{x}+L D_{y}=\frac{A_{x}-w}{a_{x}}+\frac{A_{y}-w}{a_{y}}$

(7) $\mathrm{LS}_{\mathrm{xy}}=\mathrm{LS}_{\mathrm{x}}+\mathrm{LS}_{\mathrm{y}}=\frac{\mathrm{w}-\mathrm{B}_{\mathrm{x}}}{b_{\mathrm{x}}}+\frac{\mathrm{w}-\mathrm{B}_{\mathrm{y}}}{b_{\mathrm{y}}}$

Die Funktionen (6) und (7) gelten jedoch nur für den „gemeinsamen" Bereich der Arbeitsnachfrage und des -angebots, mit anderen Worten dem Lohnbereich, bei dem allen Regionen eine positive Beschäftigungsmenge zugeordnet werden kann. Im ,nicht-gemeinsamen“ Bereich gilt eine der regionalen Funktionen. Ist etwa $A_{x}>A_{y}$, gilt für LDxy zunächst nur LDxy = LDx, und zwar solange, bis $A_{x}-a_{x} w=A_{y}$. Ist dagegen $A_{x}<A_{y}$, lautet die Nachfragefunktion 
bis zu einer Lohnhöhe von $A_{x}: L D_{x y}=A_{x}-a_{x} w$. Gleiches gilt auch im übertragenen Sinn für die Nachfrage.

Aus (6) und (7) kann die Gleichgewichtsbedingung gebildet werden:

$$
\frac{A_{x}-W}{a_{x}}+\frac{A_{y}-w}{a_{y}}=\frac{W-B_{x}}{b_{x}}+\frac{W-B_{y}}{b_{y}}
$$

Aufgelöst nach Lohnsatz und Beschäftigung ergibt sich hieraus der Gleichgewichtslohn (8) und die -beschäftigung (9):

(8) $w=\frac{\frac{A_{x}}{a_{x}}+\frac{A_{y}}{a_{y}}+\frac{B_{x}}{b_{x}}+\frac{B_{y}}{b_{y}}}{\frac{1}{a_{x}}+\frac{1}{a_{y}}+\frac{1}{b_{x}}+\frac{1}{b_{y}}}$

(9) $L_{x y}=\frac{A_{x}}{a_{x}}+\frac{A_{y}}{a_{y}}-\frac{\frac{A_{x}}{a_{x}}+\frac{A_{y}}{a_{y}}+\frac{B_{x}}{b_{x}}+\frac{B_{y}}{b_{y}}}{\frac{1}{a_{x}}+\frac{1}{a_{y}}+\frac{1}{b_{x}}+\frac{1}{b_{y}}}\left(\frac{1}{a_{x}}+\frac{1}{a_{y}}\right)$

Allgemeinere Aussagen über den Einfluß der Parameter wie für den Zwei-Regionen-Fall können hier nicht formuliert werden, da die Formel (9) wesentlich komplexer ist. Da die Angebots- und Nachfragefunktionen aber Aggregationen der regionalen Kurven sind, dürfte das gleiche gelten, wie in Abschnitt 2.1.1.2.1 heraus gearbeitet. Damit steigt die Beschäftigung c.p. mit sinkendem a, b oder B und c.p. bei steigendem A.

\subsection{Vergleich der Beschäftigungshöhe ohne und mit Vereinigung}

Die Beschäftigungshöhe ohne und mit Vereinigung ist die Differenz zwischen den jeweiligen gleichgewichtigen Arbeitsmengen gemäß Gleichungen (9) und (5): 
(10) $L_{x y}=\frac{A_{x}}{a_{x}}+\frac{A_{y}}{a_{y}}-\frac{\frac{A_{x}}{a_{x}}+\frac{A_{y}}{a_{y}}+\frac{B_{x}}{b_{x}}+\frac{B_{y}}{b_{y}}}{\frac{1}{a_{x}}+\frac{1}{a_{y}}+\frac{1}{b_{x}}+\frac{1}{b_{y}}}\left(\frac{1}{a_{x}}+\frac{1}{a_{y}}\right)-\left(\frac{A_{x}-B_{x}}{a_{x}+b_{x}}+\frac{A_{y}-B_{y}}{a_{y}+b_{y}}\right)$

In Gleichung (10) zeigt sich, daß die Differenz zwischen der Beschäftigung ohne und mit Vereinigung nicht zwingend positiv oder negativ ist und damit das gleichgewichtige Beschäftigungsvolumen im Fall vollkommener Mobilität sowohl höher als auch niedriger sein kann als bei Immobilität. Sämtliche Faktoren der Arbeitsangebots- und -nachfragefunktionen haben Einfluß auf die Differenz des Beschäftigungsniveaus vor und nach Vereinigung.

Der Einfluß der Arbeitsangebotsseite läßt sich verdeutlichen, wenn zwei Regionen mit gleichen Nachfragekurven, aber unterschiedlichen Angebotsfunktionen betrachtet werden. Ist etwa die Niedriglohnregion durch ein vollkommen unelastisches und die Hochlohnregion durch ein elastisches Arbeitsangebot gekennzeichnet, so reduziert sich bei Abwanderung die Beschäftigung um das gesamte Arbeitsangebot der Auswanderer - vorausgesetzt der Arbeitsmarkt ist so aufnahmefähig, daß der Lohn in der Hochlohnregion nicht unter den der anderen Region sinkt. In der Zuwanderungsregion steigt die Beschäftigung zwar, jedoch nicht in vollem Umfang des zugewanderten Arbeitspotentials, da durch das Sinken der Löhnen einige der bisherigen Arbeitsanbieter der Zuwanderungsregion nicht mehr bereit sind zu arbeiten.

Es können jedoch keine allgemeinen Tendenzen ausgemacht werden, in welche Richtung die c.p. Erhöhung eines Koeffizienten wirkt. Für bestimmte Konstellationen der Arbeitsnachfrage- und -angebotskurven können Aussagen getroffen werden. Die entsprechenden Szenarien und die dort wirksamen Zusammenhänge sind in den folgenden Fällen a-d beschrieben.

a) Zunächst sei der Fall betrachtet, daß die Arbeitsanbieter in beiden Regionen auf die Erhöhung des Lohnsatzes um eine Einheit mit einer identischen Angebotserhöhung reagieren, die Reservationslöhne sich jedoch unterscheiden. Gleiches gelte im übertragenen Sinn für die Unternehmen.

Bei einer solchen Konstellation unterscheiden sich die Steigungen der $\mathrm{Ar}$ beitsangebots- und -nachfragekurven in den beiden Gebieten nicht, d.h. $a_{x}=a_{y}$ und $b_{x}=b_{y}$. Die gleichgewichtige Beschäftigungsmenge ohne und mit Vereinigung ist dann identisch, unabhängig davon, ob die Reservationslöhne und die Grenzwertprodukte für die erste eingesetzte Arbeitseinheit voneinander abweichen. 
Diese Aussage läßt sich durch einen kurzen Beweis einfach zeigen. Dabei wird nachgewiesen, daß unter der Annahme $a_{x}=a_{y}, b_{x}=b_{y}$ die Gleichungen (6) und (9) übereinstimmen. Zunächst wird Gleichung (6) und im Anschluß Gleichung (9) aufgelöst.

(6) $L=\frac{A_{x}-B_{x}}{a_{x}+b_{x}}+\frac{A_{y}-B_{y}}{a_{y}+b_{y}}$

Durch Ersetzen von $a_{x}$ für $a_{y}$ und von $b_{x}$ für $b_{y}$ in Gleichung (6) und Umformen ergibt sich als Summe für die Beschäftigung bei zwei Regionen:

$$
L=\frac{A_{x}-B_{x}}{a_{x}+b_{x}}+\frac{A_{y}-B_{y}}{a_{x}+b_{x}}
$$

bzw.

$$
L=\frac{A x+A y}{a x+b x}-\frac{B x+B y}{a x+b x}
$$

Die gleichgewichtige Beschäftigung nach Vereinigung war:

$$
\text { (9) } L_{x y}=\frac{A_{x}}{a_{x}}+\frac{A_{y}}{a_{y}}-\frac{\frac{A_{x}}{a_{x}}+\frac{A_{y}}{a_{y}}+\frac{B_{x}}{b_{x}}+\frac{B_{y}}{b_{y}}}{\frac{1}{a_{x}}+\frac{1}{a_{y}}+\frac{1}{b_{x}}+\frac{1}{b_{y}}}\left(\frac{1}{a_{x}}+\frac{1}{a_{y}}\right)
$$

Durch Einsetzen von $a_{x}$ und $a_{y}$ und $b_{x}$ für $b_{y}$ in (9) ergibt sich:

$$
L_{x y}=\frac{A_{x}}{a_{x}}+\frac{A_{y}}{a_{y}}-\frac{\frac{A_{x}}{a_{x}}+\frac{A_{y}}{a_{x}}+\frac{B_{x}}{b_{x}}+\frac{B_{y}}{b_{x}}}{\frac{2}{a_{x}}+\frac{2}{b_{x}}} \cdot \frac{2}{a_{x}}
$$

Dies läßt sich umformen in: 


$$
L_{x y}=\frac{A_{x}}{a_{x}}\left(1-\frac{\frac{1}{a_{x}}}{\frac{1}{a_{x}}+\frac{1}{b_{x}}}\right)+\frac{A_{y}}{a_{y}}\left(1-\frac{\frac{1}{a_{x}}}{\frac{1}{a_{x}}+\frac{1}{b_{x}}}\right)-\frac{\frac{B_{x}+B_{y}}{a_{x} b_{x}}}{\frac{1}{a_{x}}+\frac{1}{b_{x}}}
$$

$$
\operatorname{Lxy}=\frac{A_{x}}{a_{x}}\left(1-\frac{\frac{1}{a_{x}}}{\frac{1}{a_{x}}+\frac{1}{b_{x}}}\right)+\frac{A_{y}}{a_{y}}\left(1-\frac{1}{\frac{1}{a_{x}}+\frac{1}{b_{x}}}\right)-\frac{\frac{B_{x}+B_{y}}{a_{x} b_{x}}}{\frac{a_{x}+b_{x}}{a_{x} b_{x}}}
$$

und kürzen in:

$$
L x y=\frac{A_{x}\left(a_{x}+b_{x}-b_{x}\right)}{a_{x}\left(a_{x}+b_{x}\right)}+\frac{A_{y}\left(a_{x}+b_{x}-b_{x}\right)}{a_{x}\left(a_{x}+b_{x}\right)}-\frac{B_{x}+B_{y}}{a_{x}+b_{x}}
$$

Wird die Gleichung weiter zusammengefaßt, zeigt sich, daß (6) und (9) unter den gegebenen Annahmen zu dem gleichen Ergebnis führen:

$$
\frac{A_{x}+A_{y}}{a_{x}+b_{x}}-\frac{B_{x}+B_{y}}{a_{x}+b_{x}}=\frac{\left(A_{x}+A_{y}\right) a_{x}}{a_{x}\left(a_{x}+b_{x}\right)}-\frac{B_{x}+B_{y}}{a_{x}+b_{x}}
$$

Die Höhe der Beschäftigung stimmt unter dieser Annahme mit und ohne Vereinigung überein. Allerdings würden die bei Immobilität bestehenden Lohnunterschiede zwischen den Regionen durch die Beseitigung der Mobilitätsbarrieren und der damit verursachten regionalen Umverteilung einer konstanten Menge an Arbeit zum Verschwinden gebracht werden.

b) Wenn sich die individuellen Angebots- und Nachfragekurven in den einzelnen Regionen nicht voneinander unterscheiden, die Gebiete folglich die gleichen Produktionsbedingungen und Arbeitswilligkeiten der einzelnen Individuen aufweisen, die eine Region jedoch größer ist als die andere, unterscheiden sich die regionalen Arbeitsangebots- und -nachfragefunktionen trotzdem voneinander ${ }^{34}$.

${ }^{34}$ In der mathematischen Darstellung bedeutet das, daß in den beiden Regionen die gleichen Achsenabschnitte vorliegen und sich die Koeffizienten a und b nur dahingehend unterscheiden, daß sie mit einem konstanten Faktor multipliziert zu den Koeffizienten der an- 
Entspricht das Verhältnis von Kapital und Arbeit in der einen Region dem in der anderen Region, so ergibt sich jedoch ein identischer Vollbeschäftigungslohnsatz. In diesem Fall ist das Lohnniveau und das Gesamtbeschäftigungsvolumen bei Mobilität ebenso hoch wie bei Immobilität. Dies bedeutet gleichzeitig, daß sich die regionalen Beschäftigungsmengen bei einer Vereinigung nicht ändern. Die Größen der sich vereinigenden Regionen haben dann als solche keine Bedeutung für die sich ergebenden Gesamtbeschäftigungseffekte. Andere Umstände müssen hinzukommen.

c) Ist das Arbeitsangebot in beiden Regionen unelastisch, so kann sich zwar der Lohnsatz durch eine Vereinigung ändern, das Beschäftigungsniveau bleibt jedoch konstant.

d) Auch wenn sich die Arbeitsangebotsfunktionen der beiden Regionen nicht voneinander unterscheiden, sondern nur die Nachfragefunktionen, führt die Vereinigung zu veränderter Gesamtbeschäftigung. Dies gilt auch für den Fall, daß sich die Nachfragefunktionen nicht unterscheiden, aber die Angebotsfunktionen.

\subsection{Die Wirkungen von Arbeitsmobilität infolge einer Vereinigung bei extremen regionalen Unterschieden}

Oben wurde bereits erwähnt, daß die durch Gleichung (9) bestimmte gleichgewichtige Beschäftigungsmenge nur gilt, wenn sich Angebots- und Nachfragekurven in den "gemeinsamen" ${ }^{35}$ Bereichen schneiden. Trifft dies nicht zu, kann eine Vereinigung - nach der hier vorgenommenen ,graphischen Analyse“ - in dem Sinn zu kuriosen Ergebnissen führen, daß in einer Region oder von einer Arbeitergruppe quasi gar nicht mehr gearbeitet wird. Dieser Fall kann eintreten zum einen, wenn der Gleichgewichtslohn $\left(\mathrm{w}_{\mathrm{GG}}\right)$ unter einem der regionalen $\mathrm{Re}-$ servationslöhne liegt (Fall b) und zum anderen, wenn $\mathrm{w}_{\mathrm{GG}}$ über dem Höchstlohn liegt, den die Unternehmen in einer Region höchstens für die erste Einheit Arbeit zu zahlen bereit sind (Fall a). Diese Möglichkeiten sind Extremfälle. Da ihr Auftreten aber nicht von vornherein ausgeschlossen werden kann und sie darüber hinaus geeignet sind aufzuzeigen, in welchen Dimensionen Beschäftigungsänderungen auftreten können, wenn sich die vereinigenden Regionen stark unterscheiden, werden sie im folgenden betrachtet. Folgende Resultate können sich bei den unterschiedlichen extremen Konstellationen ergeben:

deren Region führen.

35 Mit „gemeinsamer" Bereich wurde der Lohnbereich bezeichnet, in dem sich für beide Regionen eine positive Beschäftigungsmenge ergibt. 
Situation a) Der Gleichgewichtslohn liegt über einem regionalen Höchstlohn

Fall a1) Ist $\mathrm{w}_{\mathrm{GG}}>\mathrm{A}_{\mathrm{X}}$, wird nur in der Region y gearbeitet.

Fall a2) Ist $\mathrm{w}_{\mathrm{GG}}>\mathrm{A}_{\mathrm{y}}$, wird nur in der Region $\mathrm{x}$ gearbeitet.

Situation b) Der Gleichgewichtslohn liegt unter einem regionalen Reservationslohn

Fall b1) Ist $\mathrm{w}_{\mathrm{GG}}<\mathrm{B}_{\mathrm{X}}$, arbeitet niemand, der aus der Region $\mathrm{x}$ stammt.

Fall b2) Ist $\mathrm{w}_{\mathrm{GG}}<\mathrm{B}_{\mathrm{y}}$, arbeitet niemand, der aus der Region y stammt.

Im Fall a1) liegt der Kapitalstock in Region $\mathrm{x}$ brach, denn die Produktionsfunktion gibt zu dem Vollbeschäftigungslohn nichts mehr her. Alle Arbeitnehmer, die bereit sind, zu dem Gleichgewichtslohn nach der Vereinigung zu arbeiten, sind in die Region y abgewandert, bzw. dort geblieben. Keiner, der in Region $\mathbf{x}$ ansässig ist, arbeitet oder ist unter den gegebenen Bedingungen dazu bereit. In der Zuwanderungsregion y entsteht ein Druck auf den Lohnsatz; die Unternehmen werden durch das sinkende Lohnniveau zu einer erhöhten Arbeitsnachfrage veranlaßt. Die Beschäftigungszunahme besteht ausschließlich aus ehemaligen $\mathrm{x}$ Bürgern. Einige gebürtige y-er scheiden aus der Erwerbstätigkeit aus (bzw. schränken ihr Arbeitsangebot ein), da sie zu dem gesunkenen Lohnsatz nicht mehr bereit sind (soviel) zu arbeiten. Analoges gilt für Fall a2). Fazit: Schließen sich zwei Gebiete mit sehr unterschiedlichen Produktionsfunktionen zusammen, so kann dies theoretisch (aus partialanalytischer statischer Sicht) dazu führen, daß in der unproduktiven Region gar nicht mehr gearbeitet wird.

Die Ergebnisse des Falls a), daß der neue Gleichgewichtslohn über dem maximalen Grenzprodukt des Kapitals einer Region liegt und daher in dieser Region nicht mehr gearbeitet wird, erscheint unrealistisch. Der Kapitalstock dieser Region wäre so gering, daß sich nicht einmal die Beschäftigung einer einzigen Arbeitseinheit in Kombination mit dem gesamten Kapitalstock (!)lohnen würde, wenn die Löhne auf ein bestimmtes Niveau steigen. Die Ausführungen sind daher eher von theoretischem Interesse als von praktischer Relevanz.

Trifft Konstellation b1) zu, so wird vielleicht noch in Region $\mathrm{x}$ gearbeitet, aber nur von gebürtigen y-ern. Auch in der Region y würden nur Einheimische arbeiten, da der Gleichgewichtslohn unterhalb des Mindestlohns liegt, zu dem überhaupt ein gebürtiger x-er bereit wäre zu arbeiten. Analog gilt dies auch für b2). Aber auch dieser Fall ist in der Realität kaum vorstellbar.

Aus diesen Überlegungen läßt sich als notwendige Bedingung für den Fall, daß in jeder Region und von mindestens einem Arbeiter jeder Region nach Wegfall 
der Mobilitätsbarrieren gearbeitet wird, formulieren:

$A_{x}>B_{y}$ und $A_{y}>B_{x}$

d.h. der Reservationslohn muß für jede Region kleiner sein als der potentielle Höchstlohnsatz der jeweils anderen Region.

Hinreichende Bedingung für die Erfüllung der Forderung ist:

$A_{x}, A_{y}>w_{G G}>B_{x}, B_{y}$

Liegt der Gleichgewichtslohn über allen Reservationslöhnen und ist er gleichzeitig niedriger als die maximale Grenzproduktivität in allen Regionen, so wird überall und von Beschäftigten aller Regionen gearbeitet.

\subsection{Zusammenfassung}

Durch die Beseitigung von Mobilitätsbarrieren entsteht eine Tendenz zum regionalen Lohnausgleich. Das gleichgewichtige Lohnniveau liegt immer zwischen den beiden ursprünglichen regionalen Löhnen. Das Lohnniveau in der Abwanderungsregion steigt, während es in der Zuwanderungsregion sinkt. Die Beschäftigung in der Abwanderungsregion nimmt zu, die in der Zuwanderungsregion nimmt ab. Die Richtung der Beschäftigungsänderung insgesamt ist nicht eindeutig, sondern hängt von den jeweiligen Arbeitsangebots- und nachfragebedingungen $a b$. Besonders hohe Unterschiede in den regionalen Gegebenheiten können bewirken, daß es nach einer Vereinigung zu einer weitgehenden (produktionsmäßigen) Entleerung von Räumen (wie Industrien mit interregional handelbaren Gütern) kommt oder aber eine der Bevölkerungsgruppen zu dem neuen Gleichgewichtslohn keine Arbeit mehr anbietet.

\subsubsection{Einkommen}

In dem vorangegangenen Abschnitt wurde gezeigt, wie sich Lohnhöhe und Beschäftigung durch Beseitigung von Mobilitätsbarrieren ändern. Im folgenden wird unter Zugrundelegung dieser Ergebnisse untersucht, wie eine Vereinigung die Wohlfahrt beeinflußt.

Die Wohlfahrt einer Gesellschaft kann nicht durch einen eindimensionalen monetären Indikator abgebildet werden kann, da sie sich aus vielschichtigen Komponenten zusammensetzt. Sie wird $u$. a. bestimmt durch die äußere und innere Sicherheit, die Umweltsituation und nicht zuletzt auch durch die Höhe der Kon- 
summöglichkeiten (welche sich in der Einkommenshöhe widerspiegelt). Alle Elemente, die die Wohlfahrt beeinflussen, können hier nicht berücksichtigt werden, sondern dieser Abschnitt konzentriert sich auf einen Teilbereich, nämlich auf Einkommen und Arbeitnehmerrenten.

Die ökonomische Wohlfahrt hängt vor allem von der Einkommenssituation einer Gesellschaft ab. Höhere Einkommen werden in der Regel mit einem höheren Nutzenniveau gleichgesetzt. Trotzdem ist es problematisch davon auszugehen, daß mit der Produktion des größtmöglichen Sozialprodukts der größte Nutzen einer Volkswirtschaft realisiert wird. Zusätzlich müßte die personale Einkommensverteilung berücksichtigt werden. Eine Behandlung von Verteilungsfragen dieser Art würde den Rahmen dieser Arbeit sprengen. Als Ausdruck für Wohlfahrtsänderungen stehen hier nur Änderungen des Sozialprodukts. Es wird davon ausgegangen, daß - unter den im übrigen vorausgesetzten Bedingungen - ein steigendes Sozialprodukt eine erhöhte Wohlfahrt anzeigt; die Vereinigung ist dann aus ökonomischer Sicht zu befürworten. Als weiterer Indikator zur Beurteilung der Vorteilhaftigkeit von Mobilität wird das um das Arbeitsleid bereinigte Sozialprodukt herangezogen. Dazu ist eine Betrachtung der funktionalen Einkommensverteilung erforderlich.

\subsection{Bestimmung der funktionalen Einkommensverteilung und der Arbeitnehmerrenten}

Im Folgenden wird zunächst gezeigt, wie die funktionale Einkommensverteilung, also die Distribution des Volkseinkommens auf die an seiner Entstehung beteiligten Produktionsfaktoren, bestimmt werden kann, um in dem nächsten Schritt die Einkommensveränderung von Arbeit und Kapital infolge einer Vereinigung darstellen zu können. Hierfür bietet es sich an, auf die makroökonomische Grenzproduktivitätstheorie der Verteilung zurückzugreifen ${ }^{36}$, da hiermit die Faktoranteile am Sozialprodukt erklärt werden können. Für die Bestimmung der Einkommensgrößen wird aus Vereinfachungsgründen zunächst ein 1-RegionenArbeitsmarktmodell betrachtet.

\subsection{Die funktionale Einkommensverteilung nach der Grenzpro- duktivitätstheorie}

Es gelten weiterhin die in Abschnitt 2.1.1.1.3 getroffenen Annahmen über die gesamtwirtschaftliche Produktionsfunktion, die aber noch um weitere Annahmen

${ }^{36}$ Vgl. zum folgenden Blümle (1975), S. $126 f f$ und Schmitt-Rink (1978), S.16ff. 
ergänzt werden. Die Produktionsfunktion sei homogen vom Grade eins. Die beiden Produktionsfaktoren seien substituierbar und weisen bei partieller Faktorvariation abnehmende Grenzzuwächse auf. Die Faktoren werden bei vollkommener Konkurrenz und Gewinnmaximierungsverhalten der Unternehmen gemäß ihrem Grenzprodukt entlohnt. Die Konkurrenz führt bei freiem und vollkommenem Wettbewerb zu einem langfristig gewinnlosen Gleichgewicht. Hieraus folgt, daß die Faktoren bei Entlohnung nach ihren Grenzprodukten das gesamte Produkt (Y) ausschöpfen:

(11) $\mathrm{Y}=\mathrm{L} * \frac{\mathrm{Y}}{\mathrm{d}}+\mathrm{K} * \frac{\mathrm{Y}}{\mathrm{K}}$

(11) gilt nur dann, wenn die Produktionsfunktion linear-homogen ist ${ }^{37}$. Im Gewinnmaximum stimmen die realen Faktorpreise mit den partiellen Grenzproduktivitäten, die Einkommensquoten mit den partiellen Produktionselastizitäten überein. ${ }^{38}$ Die Summe der partiellen Produktionselastizitäten (totale Produktionselastizität) ist gleich eins.

Es erfolgt eine Entlohnung nach der Knappheit. Der Preis eines Faktors ist c.p. um so höher, je größer sein Grenzprodukt und je geringer der Faktoreinsatz ist. Wegen der Entlohnung nach dem Grenzprodukt und der fallenden Ertragszuwächse sinkt der Faktorpreis mit zunehmendem Einsatz. Steigende Faktoreinsätze können daher bei gegebener Produktionsfunktion sowohl zu einer zuwie auch zu einer abnehmenden Summe der Faktoreinkommen führen.

Unter diesen Umständen gilt für die Änderung der Arbeitseinkommen bei alternativen Faktoreinsätzen folgendes: Ist die Lohnelastizität der Nachfrage größer als eins, führt eine relative Erhöhung des Arbeitseinsatzes zu einer relativ geringen Abnahme des Lohnsatzes; die Lohneinkommen steigen insgesamt. Ist die Elastizität dagegen kleiner als eins, sinkt die Lohnsumme bei steigendem Arbeitseinsatz ${ }^{39}$.

${ }^{37}$ Zunehmende Skalenerträge würden bedeuten, daß bei einer Produktionsausweitung die partiellen Grenzprodukte der zusätzlichen Faktoren die der bisherigen übersteigen und daher die Ausbringung nicht für die Grenzproduktentlohnung aller Faktoren ausreicht. Bei abnehmende Skalenerträge hingegen verbleibt bei einer Entlohnung nach dem Grenzprodukt ein Rest (vgl. Blümle (1975), S. 130).

38 Vgl. Schmitt-Rink (1978), S. 17.

39 Vgl. Blümle (1975), S. 127. 


\subsection{2 Übertragung auf den Modellrahmen}

Die Erkenntnisse der makroökonomischen Grenzproduktivitätstheorie sollen nun auf das oben beschriebene Arbeitsmarktmodell übertragen werden.

Es könnte zunächst vermutet werden, daß das Grenzwertprodukt des Kapitals aufgrund des gegebenen Kapitalstocks immer konstant sein müßte. Das Grenzprodukt kann sich jedoch ändern: Bei konstantem Kapitaleinsatz hängt die Grenzproduktivität des Kapitals von der Höhe des Arbeitseinsatzes ab:

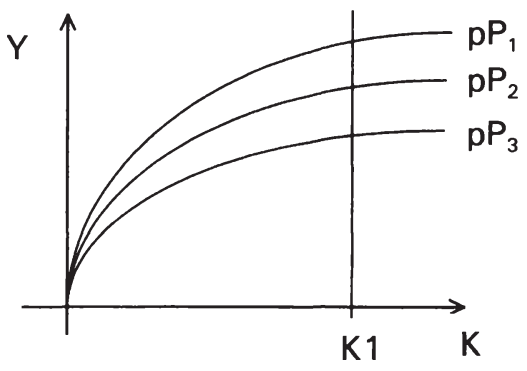

Abbildung 4: Partielle Produktionsfunktionen des Kapitals

Abbildung 4 zeigt partielle Produktionsfunktionen $\left(\mathrm{pP}_{\mathrm{i}}\right.$ ) des Kapitals bei alternativen Arbeitseinsatzmengen. Ein höherer Einsatz von Arbeit führt zu einer steiler verlaufenden partiellen Produktionsfunktion und höherer Grenzproduktivität des Kapitals. Mit wachsendem Arbeitseinsatz wird eine größere Ausbringungsmenge produziert. Das bedeutet, daß bei konstantem Kapitaleinsatz die Entlohnung des Kapitals mit zunehmendem Arbeitseinsatz steigen muß.

Der Zusammenhang ist eindeutig: steigender Arbeitseinsatz bedingt höhere Kapitalrenten und damit eine höhere Kapitalentlohnung. Für den Produktionsfaktor Arbeit sind die Konsequenzen eines erhöhten Arbeitseinsatzes auf die Entlohnung (Lohnsumme) hingegen nicht eindeutig, da bei konstantem Kapital das Grenzprodukt der Arbeit mit zunehmenden Arbeitseinsatz sinkt. Dadurch ist die Wirkung auf das Grenzwertprodukt unbestimmt, es kann sowohl zu- als auch abnehmen.

Da der Output durch einen Mehreinsatz von Arbeit steigt (bei positiven partiellen Grenzerträgen und effizienten Faktoreinsätzen), muß die Summe der Veränderungen der Kapital- und Arbeitsentlohnung positiv sein. Das Grenzwertprodukt der Arbeit kann daher nicht stärker sinken, als das Grenzwertprodukt des Kapitals steigt. 
Anhand des üblichen Arbeitsmarktdiagramms in Abbildung 5 kann illustriert werden, wie das Sozialprodukt und die Faktoreinkommen bei verschiedenen Lohnniveaus graphisch bestimmt werden können.

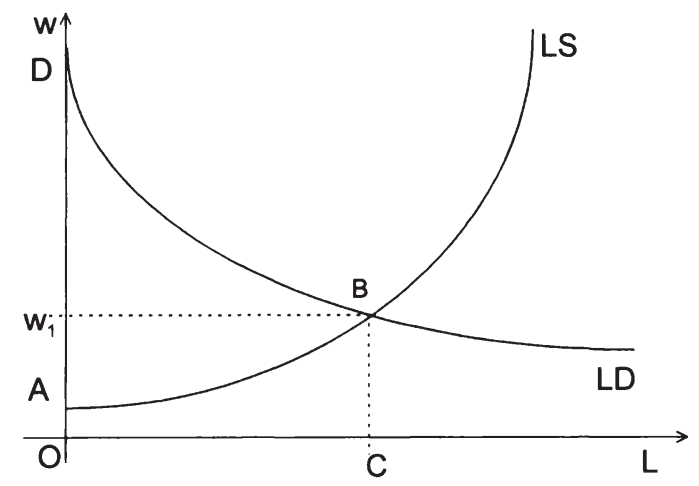

Abbildung 5: Die funktionale Einkommensverteilung

Die Grenzwertproduktkurve der Arbeit entspricht der Nachfrage nach Arbeit bei alternativen Lohnsätzen. Daher beschreibt das Integral unter dieser Kurve den Wert des Produktes ${ }^{40}$.

Das jeweils produzierte Sozialprodukt ergibt sich als Integral der Arbeitsnachfragekurve vom Ordinatenabschnitt bis zum herrschenden Lohnsatz. Unter Konkurrenzmarktbedingungen und den gegebenen Arbeitsangebots- und nachfragebedingungen ist es bei dem Gleichgewichtslohn w maximal. Bei jedem anderen Lohnsatz wäre es niedriger. Im dargestellten Beispiel ergibt sich ein Sozialprodukt bei Vollbeschäftigung in Höhe von OCBD.

Das Sozialprodukt setzt sich zusammen aus Lohnsumme $\left(\mathrm{OCBw}_{1}\right)$ und Kapitalentlohnung $\left(\mathrm{BDw}_{1}\right)$. Da die Kapitalentlohnung mit sinkenden Lohnsätzen zunimmt, wird sie unter den gegebenen Bedingungen bei $\mathrm{w}_{1}$ maximiert.

40 Diese graphische Darstellung geht auf J.B. Clarke zurück (vgl. Molitor (1988), S. 55), auch bei Samuelson/Nordhaus (1987), S. 252f findet sich diese Darstellung. Dort wird diese Graphik zur Einkommensbestimmung benutzt, wobei von den beiden Produktionsfaktoren Arbeit und Boden ausgegangen wird. Dabei wird darauf hingewiesen, da $\beta$ die gleiche Graphik auch für Kapital und Arbeit Gültigkeit hat (S. 253). Diese graphische Darstellung wird auch genutzt, um Änderungen von Sozialprodukt und Renten infolge von Migrationen zu zeigen, vgl. etwa Straubhaar (1993), S. 29ff. Berry/Sologio (1969) benutzen für die Demonstration der Einkommenseffekte von Auswanderungen eine ähnliche Darstellung. 
Außer der Kapitalentlohnung und dem Sozialprodukt ist auch die Höhe der Beschäftigung bei Realisierung des Gleichgewichtslohns maximal. Lohnsumme und Nutzen der Arbeitnehmer sind es hingegen nicht zwangsläufig. Die Lohnsumme wäre hier nur zufällig am höchsten; sie könnte auch bei einem steigenden Lohnsätzen zunehmen, wenn der Lohnausfall infolge von Entlassungen wegen der Lohnerhöhung für die weiterhin Beschäftigten überkompensiert würde. In diesem Fall wäre die Lohnelastizität der Nachfrage kleiner als eins.

\subsection{Die Ermittlung der Arbeitnehmerrente}

Bei dem Gleichgewichtslohn werden viele Arbeitseinsätze mit einem höheren Lohnsatz als unbedingt notwendig bezahlt, denndie ersten Arbeitsstunden würden auch für einen sehr viel geringeren Lohn geleistet werden. Durch die darüber hinausgehenden Zahlungen erzielen die Arbeiter einen Nutzengewinn in Höhe der Differenz ihrer Mindestforderungen und dem Marktlohn. Dieser Nutzengewinn wird im Folgenden als "Arbeitnehmerrente" bezeichnet ${ }^{41}$. Die Bestimmung der Höhe der Arbeitnehmerrente greift zurück auf Kernelemente des Konzepts der Konsumentenrente.

Das Konzept der Konsumentenrente findet sonst bei der Bewertung finanzpolitischer Projekte Anwendung ${ }^{42}$, insbesondere bei Fragen nach der Belastung der Konsumenten durch neue Steuern oder Tarifänderungen bereits bestehender Steuern. Die Konsumentenrente bestimmt sich bei der Nachfrage nach einem Gut durch das Integral unter der Kurve der marginalen Zahlungswilligkeit abzüglich der Konsumausgaben. Die Nachfragekurve stimmt mit der Kurve der marginalen Zahlungswilligkeit überein, jedoch nur, wenn die Einkommenseffekte einer Preisänderung Null sind. Dieses Konzept gilt es jetzt auf den Arbeitsmarkt zu übertragen.

Um die Arbeitnehmerrente zu bestimmen, sei zunächst noch einmal die LohnFreizeit-Entscheidungssituation eines einzelnen Individuums bei gegebenem Lohnsatz betrachtet, die in Abbildung 6 illustriert wird.

Dargestellt ist das Grenzleid des Arbeitnehmers (GL), das er durch Arbeit erleidet und welches mit zunehmender Arbeit (bzw. abnehmender Freizeit) steigt ${ }^{43}$. Jede zusätzlich aufgegebene Freizeiteinheit wiegt schwerer; die Freizeit wird zunehmend das knappere Gut. Um den Nutzenverlust auszugleichen, der durch

${ }^{41}$ Im Prinzip ist die Arbeitnehmerrente eine Produzentenrente, da es sich um Einkommen von Faktorbesitzern handelt. Vgl. Külp (1982), S. 483.

42 Vgl. Ahlheim/Rose (1989), S. 34.

4. Auf die Problematik dieser Annahme wurde bereits in Abschnitt 2.1.1.1.3 hingewiesen. 
jede weitere Hergabe einer Einheit Freizeit entsteht, muß als Kompensation immer mehr Einkommen gezahlt werden.

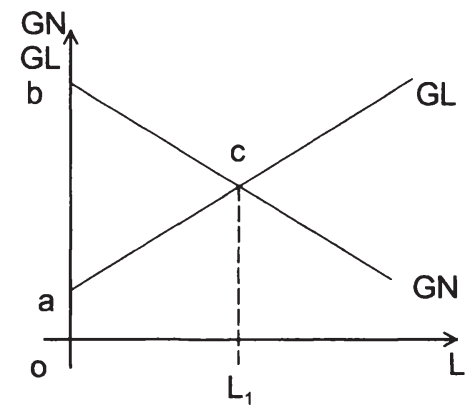

Abbildung 6: Individueller Nutzen bei alternativem Arbeitsangebot ${ }^{44}$

Zum anderen ist der Grenznutzen des Einkommens (GN) abgetragen. Wenn von einem konstanten Lohn ausgegangen wird, kann diese Kurve in das gleiche Diagramm eingetragen werden, denn der Lohnsatz multipliziert mit der auf der Abszisse abgetragenen Arbeitszeit ergibt das Einkommen. Das Einkommen steigt mit zunehmender Arbeitszeit. Der Grenznutzen des Einkommens sei, wie üblicherweise angenommen wird, positiv aber abnehmend. Im Schnittpunkt der beiden Kurven liegt die haushaltsoptimale Arbeitsangebotsmenge: der Grenznutzen entspricht dem Grenzarbeitsleid. Das Arbeitsleid entspricht dem Integral unter der Grenzleidkurve $\left(\mathrm{oL}_{1} \mathrm{ac}\right)$. Bei jeder weiteren Arbeitseinheit würde das entstehende Arbeitsleid den zusätzlichen Nutzen durch das steigende Einkommen übertreffen. Der Nutzen durch das Einkommen (oL1cb) übersteigt im Schnittpunkt der Grenznutzen- und der Grenzarbeitsleidkurve das empfundene Arbeitsleid, denn der Nutzen ist das Integral unter der Grenznutzenkurve. Die gesamte Rente des Arbeiters beträgt acb. Sie setzt sich aus dem zusammen, was dem Arbeiter über das empfundene Arbeitsleid hinaus gezahlt wird, was er aus diesem zusätzlichen Einkommen an Konsumgütern kaufen kann und der Höhe der beim Kauf erzielbaren Konsumentenrente. Dieser gesamte Überschuß soll hier als Arbeitnehmerrente bezeichnet werden.

Übertragen auf das Marktarbeitsdiagramm ergibt sich für die Betrachtung folgendes: die Arbeitnehmerrente ist das Integral zwischen Lohnsumme und dem Betrag, der über die zur Kompensation des Arbeitsleids notwendige Zahlung ausgedrückt durch die Arbeitsangebotskurve- hinausgeht. Dieses Integral umfaßt allerdings nicht die gesamte Arbeitnehmerrente, da die Konsumentenrente im

44 Vgl. Külp (1975) S. 34. 
engeren Sinn nicht berücksichtigt wird. Diese ist im Diagramm nicht darstellbar und wird im folgenden vernachlässigt. Die Arbeitsangebotskurve könnte als Kurve der "marginalen Arbeitswilligkeit" (MAW) interpretiert werden, wenn die „Einkommenseffekte“ von Lohnsatzänderungen gleich Null sind. Hiervon soll aus Vereinfachungsgründen im folgenden ausgegangen werden. Mit dem Einkommen, das durch das Integral unterhalb der MAW ausgedrückt wird, wird gerade das "Arbeitsleid" ausgeglichen, welches bei entsprechenden Lohnsätzen empfunden wird ${ }^{45}$. Das Einkommen zur Kompensation des Arbeitsleids bringt keinen Nutzengewinn gegenüber der Freizeitsituation ohne Einkommen. Die Arbeitnehmerrente - vermindert um die Konsumentenrente im engeren Sinn beträgt in Abbildung $5 \mathrm{ABw}_{1}$.

\subsection{Vergleich der Einkommen mit und ohne Vereinigung}

Nachdem geklärt wurde, wie die funktionale Einkommensverteilung bestimmt werden kann, soll nun unter Zuhilfenahme dieser Methode die Auswirkung der Reallokation der Arbeit infolge einer Vereinigung analysiert werden. In Kapitel 2.1.1.2 wurde dargestellt, daß eine Vereinigung nicht immer zu einer Erhöhung der Summe der regionalen Beschäftigung führen muß. Daran schließt sich die Frage an, ob denn das Sozialprodukt infolge des Wegfalls von Mobilitätsgrenzen stets wächst.

Dies mag zunächst verwundern, da Ökonomen üblicherweise von der These ausgehen, daß das Sozialprodukt steigt, wenn Wanderungen von Orten geringer Produktivität in Regionen mit höherer Produktivität stattfinden. Wird ein solcher Mobilitätsvorgang eines einzelnen Arbeitnehmers betrachtet, so scheint diese Aussage trivial zu sein (vorausgesetzt seine Arbeitszeit verringert sich nicht), denn er erwirtschaftet nach dem Mobilitätsvorgang ein größeres Sozialprodukt. Diese Betrachtung ist aber zu kurz, denn es entstehen noch weitere Effekte aus der Wanderung: Der Gewinn des Unternehmens, dem der Arbeiter kündigt, wird sinken, wenn dort zum bisherigen Lohn kein neuer Arbeiter eingestellt werden kann. Der Gewinn des aufnehmenden Unternehmens wird dagegen steigen. Aber wessen Gewinnänderung ist größer, übersteigt der Gewinnzuwachs des einen die -abnahme bei dem anderen? Und wie hoch ist die Veränderung der Lohnsumme? Eventuell führt der Wechsel des Arbeitnehmers zu steigenden Löhnen in der Abwanderungs- und zu sinkenden in der Zuwanderungsregion. Steigt hierdurch die Lohnsumme insgesamt oder sinkt sie? Die Effekte sind in ihrer Wirkung auf die regionalen Einkommen gegenläufig und in ihrer Gesamtwirkung nicht unmittelbar abzuschätzen.

$45 \mathrm{Vgl}$. zur Annahme des Arbeitsleids Abschnitt 2.1.1.1.3. 
Die einzig eindeutige Wirkung des Mobilitätsprozesses des einzelnen Arbeitnehmers scheint zunächst nur die Zunahme des Sozialprodukts insgesamt zu sein. Aber selbst das ist nicht zwingend.

Die Veränderung des Sozialprodukts ist wesentlich davon abhängig, ob die Beschäftigung zu- oder abnimmt. Zur Illustration der Änderungen von Beschäftigung und Einkommen mit und ohne Vereinigung wird auf zurückgegriffen, wobei zur besseren Anschaulichkeit das dritte Koordinatendiagramm (welches den gemeinsamen Arbeitsmarkt darstellt) weggelassen wird.

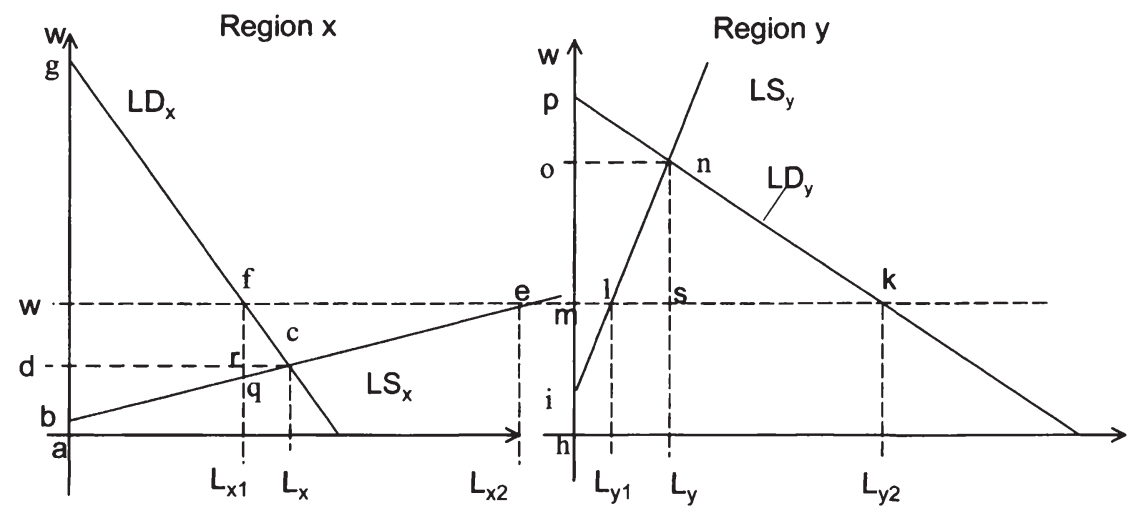

Abbildung 7: Einkommensveränderung durch Vereinigung

Das Sozialprodukt ohne und mit Vereinigung ergibt sich als Summe der in den einzelnen Regionen produzierten Outputs. In Region $\mathrm{x}$ wird ohne Vereinigung ein Sozialprodukt in Höhe von $\mathrm{aL}_{\mathrm{x}} \mathrm{cg}$ erwirtschaftet, mit Vereinigung eines in Höhe von $\mathrm{aL}_{\mathrm{x} 1}$ fg. Der Rückgang der Produktion in $x$ beträgt damit $\mathrm{L}_{x 1} \mathrm{~L}_{\mathrm{x}} \mathrm{cf}$. Das Sozialprodukt in y steigt von $\mathrm{hL}_{\mathrm{y}} \mathrm{np}$ auf $\mathrm{hL}_{\mathrm{y} 2} \mathrm{kp}$. Die Veränderung des Sozialprodukts insgesamt ist in dem abgebildetem Fall positiv, da nicht nur die Beschäftigung zunimmt, bei gleichzeitigem produktiveren Einsatz des Faktors Arbeit.

In Abschnitt 2.1.1.2.3 wurde gezeigt, daß die Beschäftigung ohne Vereinigung unter bestimmten Bedingungen auch höher als mit Vereinigung sein kann. Unter solchen Bedingungen ist die Höhe des Sozialprodukts mit Vereinigung nicht immer höher als ohne Vereinigung, denn das Sozialprodukt sinkt c.p. durch den Mindereinsatz von Arbeit. Gleichzeitig steigt das Sozialprodukt durch die Reallokation der Arbeit und den damit verbundenen effizienteren Einsatz. Jeder der 
beiden Effekte kann dabei überwiegen, so daß der in den beiden Regionen insgesamt produzierte Output zu- oder abnehmen kann.

Die funktionale Einkommensverteilung kann sich sowohl zugunsten von Kapital als auch zugunsten von Arbeit verschieben. Ein Kapitaleinkommensvergleich anhand von Abbildung 7 ergibt folgendes Bild:

Das Kapitaleinkommen ohne Vereinigung beträgt:

$\mathrm{KE}_{\mathrm{ov}}=\mathrm{wfg}+\mathrm{drfw}+\mathrm{rcf}+\mathrm{onp}$

Die ersten drei Summanden bezeichnen das Kapitaleinkommen der Region $\mathrm{x}$, der letzte Summand das Kapitaleinkommen in y.

Das Kapitaleinkommen mit Vereinigung beträgt:

$\mathrm{KE}_{\mathrm{nV}}=\mathrm{wfg}+$ onp + mlno $+\mathrm{lkn}$

Der erste Summand steht für das Kapitaleinkommen der Region $\mathrm{x}$, die letzten drei Summanden für das Kapitaleinkommen in y.

Bei der Berechnung des Kapitaleinkommensunterschieds (Diff ${ }_{K E}$ ) fällt wfg + onp heraus, so daß die Differenz zwischen Ohne- und Mit-Vereinigungsfall lautet:

$\operatorname{Diff}_{\mathrm{KE}}=$ mlno $+\mathrm{lkn}-(\mathrm{drfw}+\mathrm{rcf})=$ mkno $-\mathrm{dcfw}$

Ob die Differenz positiv oder negativ ist, hängt davon ab, ob das zusätzliche Kapitaleinkommen in Region y (mkno) größer ist als die Kapitaleinkommenseinbuße in $\mathrm{x}$ (dcfw). Dieses wiederum ergibt sich aus dem Verlauf der Nachfrageund Angebotskurven. Tendenziell dürfte die Veränderung der Kapitaleinkommen in der Region mit der elastischeren Arbeitsnachfrage niedriger sein als in der mit der unelastischeren. Verläuft die Arbeitsnachfragefunktion in der $\mathrm{Zu}-$ wanderungsregion steiler als in der Abwanderungsregion, ist die Gesamtveränderung an Kapitaleinkommen somit eher positiv.

Für die Lohneinkommen ergibt sich folgendes Bild:

Die Lohnsumme ohne Vereinigung $\left(\mathrm{LE}_{\mathrm{oV}}\right)$ beträgt:

$\mathrm{LE}_{\mathrm{ov}}=\mathrm{aL}_{\mathrm{x} 1} \mathrm{rd}+\mathrm{L}_{\mathrm{xl}} \mathrm{L}_{\mathrm{x}} \mathrm{cr}+\mathrm{hL}_{\mathrm{y}} \mathrm{sm}+\mathrm{msno}$

Die ersten beiden Summanden bilden die Lohnsumme der Region $x$ ab, die letzten beiden die Lohnsumme in $\mathrm{y}$. 
Die Lohnsumme mit Vereinigung $\left(\mathrm{LE}_{\mathrm{mv}}\right)$ beträgt:

$\mathrm{LE}_{\mathrm{mV}}=\mathrm{aL}_{\mathrm{x} 1} \mathrm{rd}+\mathrm{drfw}+\mathrm{hL}_{\mathrm{y}} \mathrm{sm}+\mathrm{L}_{\mathrm{y}}^{\mathrm{mV}} \mathrm{L}_{\mathrm{y}} \mathrm{ks}$

Die ersten beiden Summanden drücken die Lohnsumme, die in der Region $\mathbf{x}$ gezahlt wird und die letzten beiden die in y gezahlt wird, aus. Die Lohnsumme läßt sich graphisch auch als Verdienste der Arbeiter nach Herkunftsregion bestimmen (,gebürtige" $x$-er verdienen nach Vereinigung $L_{x} 2$ ew und „gebürtige“" y-er verdienen $h_{y l} l m$ ), so daß unten auch die Arbeitseinkunftssituation ohne und mit Vereinigung nach Herkunft der Arbeitnehmer verglichen werden kann. Die Lohnsumme insgesamt ergibt sich dann als:

Lohneinkommen mit Vereinigung:

$\mathrm{LE}_{\mathrm{mV}}=\mathrm{aL}_{\mathrm{x} 1} \mathrm{rd}+\mathrm{L}_{\mathrm{x} 1} \mathrm{~L}_{\mathrm{x}} \mathrm{cr}+\mathrm{drfw}+\mathrm{rcf}+\mathrm{cef}+\mathrm{L}_{\mathrm{x}} \mathrm{L}_{\mathrm{x} 2} \mathrm{ec}+\mathrm{hL} \mathrm{L}_{\mathrm{y} 1} \mathrm{~lm}$

Die Differenz der Lohneinkommen (Diff ${ }_{L E}$ ) mit und ohne Vereinigung beträgt:

$\operatorname{Diff}_{\mathrm{LE}}=\mathrm{drfw}+\mathrm{L}_{\mathrm{y}} \mathrm{L}_{\mathrm{y} 2} \mathrm{ks}-\left(\mathrm{L}_{\mathrm{x} 1} \mathrm{~L}_{\mathrm{x}} \mathrm{cr}+\mathrm{msno}\right)$

Die Differenz der Wertschöpfung (Diff ${ }_{w}$ ) ergibt sich als Addition der Differenzen von Lohnsumme und Gewinn:

$\operatorname{Diff}_{W}=\operatorname{Diff}_{L E}+\operatorname{Diff}_{G}$

$\operatorname{Diff}_{w}=d r f w+L_{y} L_{y 2} k s-\left(L_{x 1} L_{x} c r+m s n o\right)+m l n o+l k n-(d r f w+r c f)$

$\operatorname{Diff}_{w}=L_{y} L_{y 2} k n-L_{x 1} L_{x} c f$

Mit Vereinigung kann die Wertschöpfung in der Region y $\left(\mathrm{L}_{\mathrm{y}} \mathrm{L}_{\mathrm{y} z} \mathrm{kn}\right)$ höher oder niedriger sein als die Wertschöpfung in der Region $x\left(\mathrm{~L}_{x 1} \mathrm{~L}_{x} \mathrm{cf}\right)$. . Die Wertschöpfung insgesamt kann mit Vereinigung daher sowohl höher als auchniedriger sein als ohne Vereinigung. Eine Beseitigung von Mobilitätsbarrieren ist bei Zugrundelegung dieses Indikators somit nicht immer positiv zu werten.

Als weiterer Indikator für die Wohlfahrtsänderungen wurde bereits das um das (in Geldeinheiten bewertete) subjektiv empfundene Arbeitsleid bereinigte Sozialprodukt eingeführt. Das bereinigte Sozialprodukt steigt immer dann, wenn sich die Höhe der Beschäftigung durch eine Vereinigung ändert, und zwar nicht nur bei einer Zunahme, sondern auch bei einer Abnahme des Arbeitseinsatzes. Ohne Änderung der Beschäftigungsmenge bleibt das bereinigte Sozialprodukt entweder konstant oder erhöht sich. Das bereinigte Sozialprodukt ist - wie ökonomisch von vornherein zu erwarten war - mit Vereinigung in dem beschriebenen Modell bei Vereinigung mithin nie geringer als ohne Vereinigung. Dieses Ergebnis sei anhand von Abbildung 7 illustriert. Die Arbeitnehmerrenten der ge- 
bürtigen $\mathrm{x}$-er steigen von bcd um dcew auf bew, die der y-er sinken von ino um mlno auf ilm. Das bereinigte Sozialprodukt beträgt vor Vereinigung in Region $\mathbf{x}$ bcg und in Region y inp, nach der Vereinigung beträgt eswfg + mkp + bew +ilm. Die Differenz beträgt cef $+\mathrm{lkn}$. Da beide Größen stets positiv sind, steigt das bereinigte Sozialprodukt.

Durch eine Vereinigung ergeben sich für die regionalen Einkommen folgende Konsequenzen: Die Entlohnung des Kapitals geht in der Abwanderungsregion zurück und steigt in der Zuwanderungsregion. Das gleiche gilt für das Sozialprodukt. Die Richtung der Entwicklung der Lohnsumme hingegen ist abhängig von der Lohnelastizität der Nachfrage. Sie kann in beiden Regionen sowohl jeweils steigen wie auch sinken. Für die aus der Abwanderungsregion stammenden Arbeiter bringt die Vereinigung deutliche Vorteile: Ihre Arbeitnehmerente steigt von bcd auf bew, während die anderen eine Einbuße an Arbeitnehmerrente in Höhe von mlno hinnehmen müssen.

Es läßt sich folgendes Zwischenfazit ziehen: Gewinner einer Vereinigung sind die Arbeiter der Abwanderungsregion und die Kapitalbesitzer der Zuwanderungsregion. Die Auswirkung auf Beschäftigung und Sozialprodukt insgesamt sind unbestimmt und abhängig von den Nachfrage- und Angebotselastizitäten.

Abschließend sollen die Wirkungen der Vereinigung auf die in Abschnitt 2.1.1.2.3 untersuchten Konstellationen a-d beschrieben werden.

Oben wurde gezeigt, daß eine Vereinigung bei gleichen Steigungen der regionalen Arbeitsangebots- und -nachfragekurven, aber unterschiedlichen Achsenabschnitten, nicht zu einer Veränderung der Beschäftigungshöhe insgesamt führt (Pkt. a). Gleichwohl fände aber eine Reallokation der Arbeit statt, wodurch Veränderungen des Sozialprodukts und der Einkommensverteilung ausgelöst werden. Da Arbeit in produktivere Verwendung wechselt und sich die Beschäftigungshöhe nicht ändert, wächst das Sozialprodukt. Für die Änderung der Gewinne ist es ausschlaggebend, ob der potentielle Höchstlohn der Zuwanderungsregion größer oder kleiner als der potentielle Höchstlohnder anderen Region ist. Ist er größer, so steigen die Gewinne, ist er kleiner, so sinken sie. Dies läßt sich anhand von Abbildung 8 veranschaulichen.

Dort sind zwei regionale Nachfragekurven $\left(\mathrm{LD}_{\mathrm{x}}\right.$ und $\left.\mathrm{LD}_{\mathrm{y}}\right)$ mit parallelem Verlauf aber unterschiedlichen Achsenabschnitten abgetragen (Auf die Abbildung der Angebotskurven kann für die Bestimmung der Gewinnänderung verzichtet werden.). In Abschnitt 2.1.1.2.3 wurde ausgeführt, daß sich die Beschäftigungshöhe ohne und mit Vereinigung bei dieser Konstellation insgesamt nicht unterscheidet. Die Beschäftigungserhöhung in der einen Region durch eine 
Vereinigung muß daher der Senkung in der anderen Region entsprechen. Infolge der gleichen Steigungen der Nachfragekurven ist das Lohnniveau der einen Region um denselben Betrag höher, um den es in der anderen Region sinkt. Die Horizontale durch den Gleichgewichtslohn ohne Vereinigung in $\mathrm{x}$ hat daher den gleichen Abstand zur Horizontalen durch den Gleichgewichtslohn mit Vereinigung wie der Abstand dieser zu der Horizontalen durch den Gleichgewichtslohn ohne Vereinigung in Region y. Daher können die Flächen zwischen den Horizontalen direkt miteinander verglichen werden.

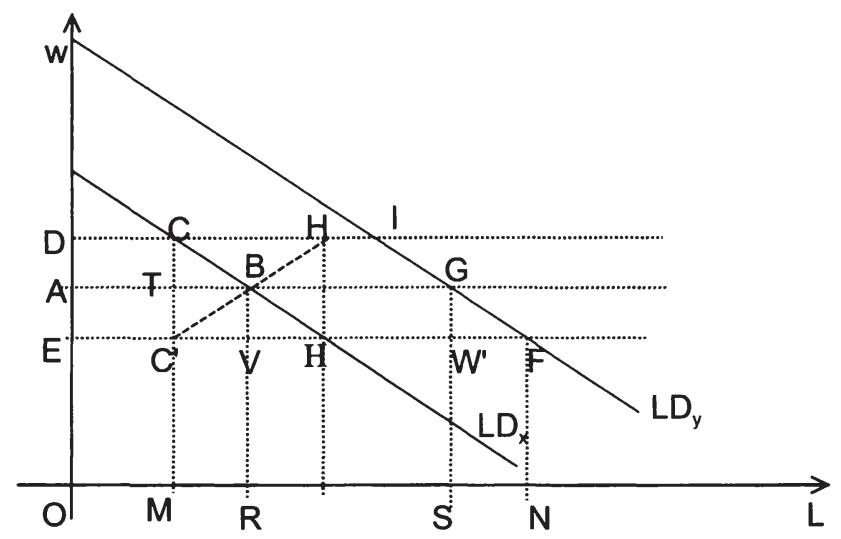

Abbildung 8: Gewinnänderung bei parallel verlaufenden Arbeitsnachfrage- und -angebotskurven

Im dargestellten Beispiel verläuft die Arbeitsnachfragekurve in $\mathrm{x}$ unterhalb der in y. Zunächst sei der Lohn in $x$ ohne Vereinigung höher als in y. Der Lohnsatz in $\mathrm{x}$ habe $\mathrm{D}$ betragen, der in $\mathrm{y}$ E. Das Sozialprodukt wächst infolge einer Vereinigung um CMRB-GSNF, was dem Rechteck TC'VB entspricht. Die Gewinnänderung insgesamt beträgt $\mathrm{ABCD}-\mathrm{EFGA}$. Da $\mathrm{ABCD}$ der Fläche von EC'BA entspricht und da GWF gleich C'BT, entsteht durch die Vereinigung ein Verlust in Höhe von TC'WG für den Unternehmenssektor. Die Lohnsumme erhöht sich entsprechend um den Zuwachs an Sozialprodukt plus dem Gewinnrückgang der Unternehmen. Wäre dagegen der Lohn in $\mathrm{x}$ vorher niedriger gewesen, so hätten sich Gewinnzuwächse bei gleichzeitigem Anstieg des Sozialprodukts und zurückgehender Lohnsumme ergeben. Für das bereinigte Sozialprodukt gilt dasselbe wie oben ausgeführt, nämlich daß es auch bei jeder Konstellation steigt. 
Fall b) war durch gleiche individuelle Arbeitsangebots- und -nachfrageverhältnisse und ihrer Relationen zueinander gekennzeichnet bei unterschiedlicher Größe der Regionen. Eine Vereinigung verursachte keinerlei Veränderungen bezüglich Beschäftigungshöhe, Allokation der Arbeit und Lohnniveau. Infolgedessen bleiben auch Sozialprodukt, Gewinn, Lohnsumme und Arbeitnehmerrente insgesamt und regional konstant.

Bei lohnunelastischem Arbeitsangebot in beiden Regionen (Fall c) bleibt zwar auch die Beschäftigungshöhe konstant, aber die Einkommen steigen, da es zu Mobilitätsbewegungen in Richtung der höheren Produktivität kommt. Der Gewinn der vormaligen Niedriglohnregion schrumpft, da dort die Beschäftigung bei steigenden Löhnen zurückgeht. In der vormaligen Hochlohnregion sinken die Löhne durch die Zuwanderung und der damit einhergehenden höheren Beschäftigung. Das in der vormaligen Hochlohnregion produzierte Sozialprodukt steigt stärker als der Rückgang in der Niedriglohnregion. Für die Lohnsumme gilt folgendes: Ist die Lohnelastizität der Nachfrage in der Zuwanderungsregion kleiner als eins, sinkt die Lohnsumme in dieser Region bei Zuwanderung. Ist die Elastizität in der Abwanderungsregion kleiner als eins, so sinkt dort ebenfalls die Lohnsumme. Zwangsläufig sinkt dann auch die Lohnsumme insgesamt. Die Gewinne steigen absolut stärker als das Sozialprodukt. Verhalten sich die Lohnelastizitäten umgekehrt, steigt die Lohnsumme insgesamt.

Im Fall d (identische Nachfragefunktionen, aber unterschiedliche Angebotsfunktionen bzw. identische Angebotsfunktionen, aber unterschiedliche Nachfragefunktionen) führt eine Vereinigung zu Änderungen der Beschäftigung. Für die Änderung der Einkommen gilt dasselbe wie für den allgemeinen Fall beschrieben.

\subsection{Zusammenfassung}

Im Mit-Vereinigungsfall unterscheidet sich - außer in Sonderfällen - stets die Allokation des Faktors Arbeit von der im Ohne-Vereinigungsfall, Arbeit wird mit Vereinigung produktiver eingesetzt. Durch Wanderungen kommt zu einer Reallokation der Arbeit in Richtung ihres produktiveren Einsatzes. Auch ohne zusätzliche Investitionen oder sonstige Veränderungen des Produktivkapitals ergeben sich in der Abwanderungsregion Arbeitsproduktivitätszuwächse. Diese resultierten jedoch ausschließlich aus dem verringerten Arbeitseinsatz in Kombination mit einem fixen Kapitalstock. Das Sinken der Arbeitsproduktivität in der Zuwanderungsregion ergibt sich entsprechend durch die Zunahme des Arbeitseinsatzes bei konstantem Kapitaleinsatz. Die Beschäftigung, das Sozialprodukt und der Gewinn erhöhen sich im Zuge einer Vereinigung in der Region 
mit den vormals höheren Löhnen, während sie in der Abwanderungsregion zurückgehen. Für die regionalen Lohnsummen dagegen sind die Tendenzen nicht so eindeutig. Infolge der Wanderungen ergeben sich aber nicht nur Einkommensumverteilungen zwischen den Regionen; auch die Relation von Lohn- zu Kapitaleinkommen ändert sich.

Die aggregierten regionalen Kapital- und Lohneinkommen steigen infolge einer Beseitigung der Arbeitsmobilitätsschranken zwar meistens, jedoch nicht grundsätzlich. In Abhängigkeit von den Konstellationen der Arbeitsnachfrage und des -angebots ist ein Rückgang einer oder mehrerer Einkommensgrößen möglich. Ein Rückgang tritt ein, wenn die positiven Wirkungen auf das Sozialprodukts infolge der verbesserten Allokation der Arbeit durch einen Beschäftigungsrückgang überkompensiert werden. Ist dagegen die Beschäftigung mit Vereinigung höher als ohne, so ist stets auch das Sozialprodukt höher. Ist das Arbeitsangebot unelastisch, erhöht sich das Sozialprodukt infolge von vereinigungsbedingten Wanderungen grundsätzlich.

Die Summe des um das Arbeitsleid bereinigten Sozialprodukts beider Regionen ist ohne Vereinigung nie höher als mit Vereinigung. Sofern die Beschäftigungshöhe in den beiden Fällen voneinander abweicht, ist das bereinigte Sozialprodukt im Mit-Vereinigungsfall stets höher. Nur bei einer insgesamt identischen Beschäftigungsmenge ist auch das bereinigte Sozialprodukt insgesamt konstant. Bei Berücksichtigung des Arbeitsleids ist daher die Beseitigung von Mobilitätsbarrieren unabhängig von den jeweiligen Gegebenheiten am Arbeitsmarkt zu begrüßen.

\subsubsection{Modellmäßig zu erwartende Entwicklungen der regionalen deutsch- deutschen Arbeitsmobilität}

In Abschnitt 2.1.1 wurde anhand eines einfachen neoklassischen Modells gezeigt, welche Wanderungsbewegungen und welche Einkommens- und Beschäftigungswirkungen zu erwarten sind, wenn sich zwei Regionen vereinigen und die bezüglich Arbeitsmobilität bestehende Barrieren abbauen. Dieses Modell soll nun auf die deutsche Vereinigung übertragen werden.

Die bisherige Analyse bezog sich auf Reallöhne. In der folgenden Darstellung wird jedoch mit Nominallöhnen operiert. Unterschiede im Preisniveau werden im folgenden zur Vereinfachung ausgeblendet ${ }^{46}$. Dies läßt sich damit rechtfer-

46 Außerdem wird so vorgegangen, als ob es bereits vor Juli 1990 eine einheitliche Währung gab. 
tigen, daß die politische Führung der DDR das Preisgefüge ihrer Waren so gestaltet hatte, daß beide deutschen Währungen ungefähr die gleiche allgemeine Kaufkraft hatten ${ }^{47}$ (Jedoch unterschied sich das ostdeutsche Preisgefüge stark von dem westdeutschen, da in der DDR auf der einen Seite Güter des Grundbedarfs stark subventioniert wurden, während andere Güter - insbesondere technische langlebige Gebrauchsgüter - sehr teuer waren. Die Preise in der Bundesrepublik hingegen bildeten sich im wesentlichen auf dem Markt.).

Zunächst werden die Arbeitsmärkte der früheren Bundesrepublik und der DDR vor der Grenzöffnung skizziert. Im Anschluß werden die modellmäßig zu erwartenden Wanderungen und Entwicklungen am Arbeitsmarkt nach dem November 1989 beschrieben. Danach wird dargestellt, wie die tatsächliche Entwicklung von der modellmäßigen zu erwartenden abwich.

\subsubsection{Stilisierung der west- und ostdeutschen Arbeitsmärkte vor der Grenzöffnung}

Vor dem November 1989 waren der ost- und westdeutsche Arbeitsmarkt voneinander abgeschottet. Wanderungen von Personen, die noch nicht das Rentenalter erreicht hatten, waren wegen der nach Westen geschlossenen DDR-Grenzen (im Prinzip) nicht möglich. Die Annahme der vollkommenen interregionalen Immobilität war somit - zumindest in eine Richtung - nahezu vollständig erfüllt. Eine Angleichung der Löhne über Arbeitskräftewanderungen konnte es daher nicht geben ${ }^{48}$, so daß sich das Lohnniveau der beiden deutschen Staaten dauerhaft sehr stark voneinander unterscheiden konnte: Vor der Wende ${ }^{49}$ wurden in Westdeutschland etwa dreimal so hohe Löhne wie in Ostdeutschland gezahlt.

Zur Stilisierung der beiden deutschen Arbeitsmärkte vor und nach der Grenzöffnung wird zunächst der Verlauf der Arbeitsnachfrage- und -angebotsfunktionen beider deutscher Regionen bestimmt. Dabei sollen in der graphischen Darstellung (Abbildung 9) auf der Abszisse des Arbeitsmarktdiagramms im folgenden abweichend von der bisherigen Darstellung - die jeweilige Bevölkerungszahl

47 Vgl. Sinn/Sinn (1991), S. 42.

48 Auch Kapital war aus verschiedenen Gründen nicht mobil, so daß es auch über diesen Weg nicht zu einem Ausgleich der Faktorentlohnungen kam.

49 Mit „Wende“ wird hier der Herbst/Winter 1989 bezeichnet; in dieser Zeit wurden die Öffnung der DDR und die politischen und wirtschaftlichen Reformen eingeleitet. Mit „Union“ ist im folgenden der 1.7.1990 gemeint, also der Beginn der Wirtschafts-, Währungs- und Sozialunion und mit Vereinigung der 3.10.1990, also der Beitritt der ostdeutschen Länder zur Bundesrepublik. 
anstelle der Erwerbspersonen abgetragen werden ${ }^{50}$. Auf der Ordinate wird das Lohnniveau in Prozent des Lohns der alten Bundesländer abgetragen. Die 100\%Marke entspricht dem im Herbst 1989 in der früheren Bundesrepublik herrschenden Durchschnittslohn.

In der ehemaligen DDR orientierte sich die Arbeitsnachfrage nicht an der Produktivität. Die Wirtschaft war als sozialistische Planwirtschaft organisiert. Die Löhne setzten sich aus einem Grundlohn und einer Lohnprämie zusammen. Die planwirtschaftlich festgelegten Grundlohntabellen waren in Gehaltsgruppen unterteilt. Die einzelnen Wirtschaftsbereiche wurden den Grundlohntabellen so zugeordnet, wie es der von der Staatsführung erwünschten Rangfolge der Lohnhöhe "nach der volkswirtschaftlichen Bedeutung der Zweige" entsprach. Die Differenzierung der Löhne ging jedoch über die Unterschiede in den Grundlohntabellen hinaus, da in den Wirtschaftsbereichen unterschiedliche Lohnprämien gezahlt wurden. Die Festsetzung dieser Lohnprämien, die einen durchschnittlichen Anteil von etwa $22 \%$ am Bruttolohn hatten ${ }^{51}$, lag in der alleinigen Verantwortung der Betriebe.

Da die Betriebe die Höhe der Lohnprämien selbst bestimmten, wird angenommen, daß sich die Lohnprämien an der Produktivität ausrichteten ${ }^{52}$, während die Grundlöhne wohl weitgehend unabhängig von der Produktivität fixiert wurden. Insgesamt ergibt sich ein überwiegend lohnunelastischer, höchstens jedoch ein schwach fallender Verlauf der Arbeitsnachfragekurve. Die damalige DDR-Regierung verfolgte das Ziel, daß jede erwerbsfähige Person einer Arbeit nachging. Dies versuchte sie durch sehr niedrige Löhne zu erreichen, so daß ein Einkommen allein für eine ganze Familie selten ausreichte und beide Partner einem Beruf nachgehen mußten ${ }^{53}$. Die Frauenerwerbstätigkeit wurde obendrein durch ein umfangreiches kostengünstiges Angebot der Kinderbetreuung stark gefördert ${ }^{54}$.

In der DDR gab es eine Beschäftigungsgarantie, d.h. Arbeitswilligen mußte ein Arbeitsplatz nachgewiesen werden. Daher ist die Arbeitsnachfragekurve $\left(\mathrm{LD}_{\mathrm{ol}}\right)$ so zu stilisieren, daß sie nicht die Abszisse schneidet, sondern ab dem festge-

50 Die Zahl der Einwohner erscheint hier sinnvoller als die Zahl der Erwerbspersonen bzw. die Zahl der Arbeitsstunden, da etwa das Rentenalter hinausgeschoben werden kann, so daß die Zahl der Erwerbspersonen flexibler als die Einwohnerzahl ist.

51 Vgl. Stephan/Wiedemann (1990), S. 552.

52 Auch diese Annahme dürfte problematisch sein.

53. Vgl. Friedrich-Ebert-Stiftung (Hrsg.) (1977), S. 14.

54 Je geringer die Kosten für die Kinderbetreuung, desto geringer kann der Lohn sein, bei dem der bisher betreuende Elternteil bereit ist eine Stelle anzunehmen, da die Kinderbetreuungskosten ja erst einmal verdient werden müssen. Durch die Einführung einer derartigen Maßnahme verschiebt sich die Arbeitsangebotskurve nach rechts. 
setzten Mindestlohn horizontal verläuft. Da in der Ausgangssituation bei einer Beschäftigung von knapp 10 Mio. Erwerbspersonen der Durchschnittslohn bei $30 \%$ des Westlohnes lag, dürfte die Arbeitsnachfragekurve durch diesen Punkt verlaufen sein.

Als weiterer Referenzpunkt für die ostdeutsche Arbeitsnachfragekurve wird ein Lohnniveau in Höhe von $60 \%$ des Westlohns gewählt. Bei einem solchen Lohnniveau gab es nach Sinn/Sinn ${ }^{55}$ kaum eine profitable Beschäftigung (Stand April 1990) in der Industrie. Diese Lohn-Beschäftigungskombination bildet die damaligen Verhältnisse in der DDR sicher nicht ganz zutreffend $a b$, zumal sich die Arbeitsnachfrage vor der Systemumstellung nicht an der Produktivität orientierte. Löhne in dieser Höhe wären zudem aus oben genannten Gründen nicht angeboten worden, dieser Bereich der Arbeitsnachfragekurve ist damit illusorisch $^{56}$. Ebensogut hätte der Bereich ausgespart werden können und die Arbeitsnachfrage lediglich mit der 1989 herrschenden Lohn-Beschäftigungskombination dargestellt werden können. Da aber angenommen wird, daß ein Lohnsatz von $60 \%$ des Westlohnniveaus unter marktwirtschaftlichen Bedingungen der höchste angebotene Lohn gewesen wäre, wird er zur Veranschaulichung abgetragen.

In der alten Bundesrepublik orientierte sich die Arbeitsnachfrage an der Produktivität. Daher kann unter Zugrundelegung der üblichen Annahmen bezüglich der Arbeitsproduktivität die westdeutsche Arbeitsnachfragekurve $\left(\mathrm{LD}_{\mathrm{w1}}\right)$ als fallende Funktion des Lohnsatzes dargestellt werden. In Westdeutschland ("West") waren im Herbst 1989 knapp 28 Mio. Menschen beschäftigt.

$\mathrm{LS}_{\mathrm{ol}}\left(\mathrm{LS}_{\mathrm{wl}}\right)$ spiegele das Arbeitsangebot in den neuen (alten) Ländern wider, wie es sich vor dem Reformprozeß in der DDR dargestellt haben könnte. Die Arbeitsangebotskurve der alten Bundesrepublik dürfte im linken Bereich (in dem Teilstück unterhalb von $30 \%$ des Westlohnniveaus) steiler verlaufen sein als die der DDR. Zu solch geringen Lohnsätzen dürften (kurzfristig) nur sehr wenig Menschen in den alten Bundesländern bereit gewesen sein zu arbeiten, da das soziale Netz in Westdeutschland sehr engmaschig ist und nicht nur in Notlagen, sondern sogar bei Arbeitsunwilligkeit eine Grundsicherung gewährt. Bei entsprechend niedrigeren Löhnen hätte es sich kaum noch gelohnt zu arbeiten. In Ostdeutschland wurden dagegen staatliche Unterstützungszahlungen an nicht berufstätige, arbeitsfähige Personen in Form von Arbeitslosengeld und -hilfe nicht gewährt. Sozialfürsorgeunterstützung erhielt nur, wer nicht dazu in der

s5 Vgl. Sinn/Sinn (1991), S. 149.

${ }^{56} \mathrm{Da}$ die Arbeitsnachfrage nicht die Produktivität widerspiegelt, kann das Sozialprodukt nicht durch das Integral unter der Arbeitsnachfragekurve bestimmt werden. 
Lage war, für sich selbst zu sorgen und auch sonst nicht aus anderen Quellen für seinen Unterhalt sorgen konnte. Im rechten Teil der Arbeitsangebotskurve dürfte die ostdeutsche Kurve steiler verlaufen sein als in Westdeutschland, denn in der DDR bedeutete ein höheres Einkommen nicht gleichzeitig höhere Konsummöglichkeiten, da ein Geldüberhang existierte. Höhere Löhne könnten daher ab einem bestimmten Niveau einen geringeren Arbeitsanreiz als in Westdeutschland ausgelöst haben.

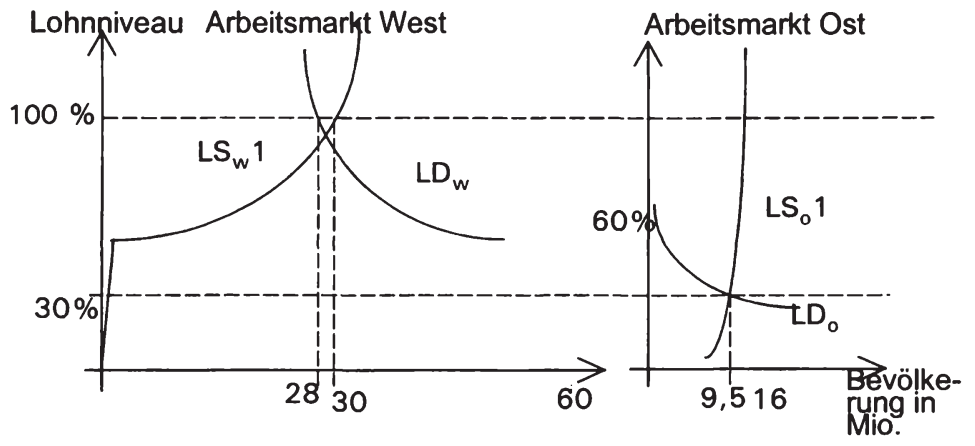

Abbildung 9: Der ost- und der westdeutsche Arbeitsmarkt ohne Vereinigung

Auf dem Arbeitsmarkt in der DDR (in der Abbildung durch "Ost" gekennzeichnet) herrschte ein Gleichgewicht auf dem Arbeitsmarkt in dem Sinne, daß jeder zu dem herrschenden Lohnsatz (30\% des Westlohnniveaus) eine Beschäftigung finden konnte. Das Beschäftigungsniveau lag im Schnittpunkt der Arbeitsangebots- und -nachfragekurve bei knapp 10 Mio. Erwerbstätigen. Auf dem westdeutschen Arbeitsmarkt lag eine Ungleichgewichtssituation mit 28 Mio. Erwerbstätigen vor. Gegenüber einem Gleichgewicht war das Lohnniveau im Jahr $1989 \mathrm{zu}$ hoch. Aufgrund der nach unten rigiden Löhne wurde eine (offene) Arbeitslosigkeit von rund 2 Mio. Arbeitslosen verursacht ${ }^{57}$.

\subsubsection{Modellmäßig zu erwartende Anpassungsprozesse nach der Grenzöffnung}

Im Zuge des politischen Reformprozesses wurden am 9. 11. 1989 die Grenzen geöffnet, jeder konnte quasi seit diesem Tag seinen Wohnort (und damit auch seinen Beschäftigungsort) frei wählen. Wenn unterstellt wird, daß das Kapital

57 "Für die zunehmende und persistente Beschäftigungslosigkeit sind daher in der wissenschaftlichen und öffentlichen Diskussion Lohn- und Preisrigiditäten verantwortlich gemacht worden" (Franz (1991), S. 355). 
kurzfristig immobil ist, hätten - modellgemäß - die erheblichen Lohndifferenzen ostdeutsche Erwerbspersonen in den Westen locken müssen. Dies hätte zu einer Mehrbeschäftigung bei sinkendem Lohnsatz im Westen und zu einer umgekehrten Entwicklung im Osten führen müssen. Ost- und Westlohnniveau hätten sich über die Wanderungsbewegungen aus den neuen in die alten Bundesländer einander anpassen müssen. Nach allen Anpassungsprozessen des Faktors Arbeit hätte der neue Lohnsatz in beiden Regionen gleich hoch sein müssen.

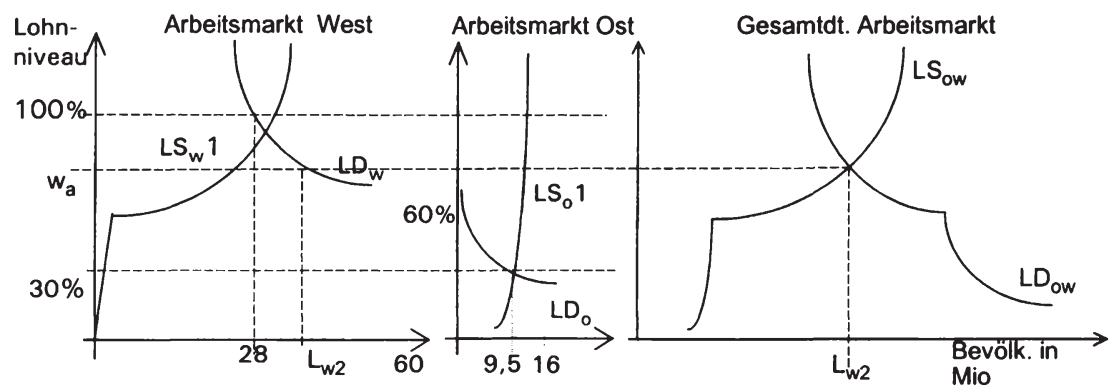

Abbildung 10: Der deutsch-deutsche Arbeitsmarkt vor und nach Vereinigung

Unter Zugrundelegung von Verläufen der Angebots- und Nachfragekurven wie oben stilisiert, wäre modellmäßig zu erwarten gewesen, daß ein großer Teil der ostdeutschen Erwerbspersonen in die alten Bundesländer übergesiedelt wäre und dort gearbeitet hätte. Das Lohnniveau in West- und Ostdeutschland hätte sich auf $\mathrm{w}_{\mathrm{a}}$ angeglichen. Hätte das neue Lohnniveau $\mathrm{w}_{\mathrm{a}}$ allerdings oberhalb von $60 \%$ des alten Westlohnniveaus gelegen, so hätte im Prinzip kein Unternehmen in Ostdeutschland mehr Arbeitskräfte nachfragen dürfen, da unter marktwirtschaftlichen Bedingungen $60 \%$ des Westlohns der höchste von den Unternehmen angebotene Lohn gewesen wäre.

$\mathrm{Zu}$ einer vollständigen Angleichung der Löhne durch Wanderungsbewegungen ist es jedoch in Deutschland bisher nicht gekommen. In Ostdeutschland arbeiten auch einige Jahre nach der Vereinigung die meisten Menschen zu einem niedrigerem Lohn als in Westdeutschland gearbeitet. Es sind zwar viele Menschen aus den neuen in die alten Bundesländer gezogen, jedoch nicht in dem modellmäßig zu erwartendem Umfang. Es stellt sich daher die Frage, welchen Beitrag das neoklassische Modell zur Erklärung der Wirkungen der deutschen Vereinigung leisten kann. Zunächst ist jedoch zu prüfen, ob die Arbeitsnachfrage- und das angebot zutreffend abgebildet wurden. Im nächsten Abschnitt wird unter- 
sucht, wie sich die relevanten Zusammenhänge unter Bezug auf die neoklassische Darstellungsweise stilisieren lassen, wenn von den Erfahrungen, die gemacht wurden, ausgegangen wird.

\subsubsection{Modifikationen und Vergleich mit den tatsächlichen Entwicklungen}

Die ursprüngliche Darstellung des ostdeutschen Arbeitsmarktes ist zunächst deswegen zu modifizieren, weil eine Systemumstellung von einer Planwirtschaft zu einer Marktwirtschaft stattfand. Die ostdeutsche Wirtschaft wurde quasi über Nacht der internationalen Konkurrenz ausgesetzt, nachdem sie vier Jahrzehnte von den westlichen Wettbewerbsmärkten abgeschottet gewesen war.

Daher kann nicht von einer unveränderten ostdeutschen Arbeitsnachfrage ausgegangen werden. Bereits ohne zusätzlichen Einsatz von Kapital sind Veränderungen eingetreten. Unter den in der DDR herrschenden Bedingungen waren viele Arbeitskräfte betriebswirtschaftlich überflüssig eingesetzt worden; Kündigungen waren damals nicht möglich ${ }^{58}$. Die Beschäftigungsgarantie in der DDR verursachte ein hohes Maß an verdeckter Arbeitslosigkeit. Ferner war es häufig zu verlorener Arbeitszeit aufgrund von „Materialpausen“ gekommen ${ }^{59}$. Darüber hinaus haben während der Arbeitszeit politische Aktivitäten stattgefunden. Bereits der Wegfall dieser Faktoren müßte - die Absatzmöglichkeiten vorausgesetzt - den Output pro Arbeitskraft erhöht haben.

Die Höhe der Arbeitsnachfrage ist neben den Löhnen auch abhängig von der Nachfrage nach den produzierten Gütern und Dienstleistungen sowie den Preisen, die hierfür erzielt werden können. Im zugrundegelegten Modell wurde mit Welthandelspreisen operiert. In der DDR wurden dagegen die Preise zentral festgelegt, was zu einer verzerrten Preisstruktur führte. Der Eintritt in den Weltmarkt konfrontierte die Unternehmen mit einer völlig anderen Preisstruktur. Daraufhin brach der Umsatz ein: Im Jahr 1990 ging die Industrieproduktion gegenüber dem Vorjahr um etwa $30 \%$ zurück. Ursächlich für diesen Rückgang war aber nicht ein Ausfall der Inlandsnachfrage, sondern ein Substitutionsprozeß, bei dem westliche Produkte die ostdeutschen verdrängt haben ${ }^{60}$ (Der Nachfrageeinbruch bei ostdeutschen Produkten läßt sich nicht in vollem Umfang mit geringer Qualität und mangelnder Produktvielfalt etc. erklären, sondern war in erheblichem Ausmaß psychologischer Natur). Zusätzlich verloren die neuen

58 Vgl. Deutsche Wirtschaftszeitung (1990).

59 Als „Materialpausen“ wurden solche Arbeitsunterbrechungen bezeichnet, die daraus resultierten, daß die für die Produktion notwendigen Materialien nicht zur Verfügung standen.

${ }^{60}$ Vgl. Egle (1991), S. 514. 
Bundesländer durch den Zusammenbruch des Osthandels ihre traditionellen Absatzmärkte.

Diese Entwicklungen hatten Einfluß auf den Verlauf der Arbeitsnachfragekurve. Abbildung 11 illustriert, wie die Erhöhung der Produktivität einerseits und die Verschlechterung der Nachfrage andererseits die Arbeitsnachfrage verändert haben könnte.

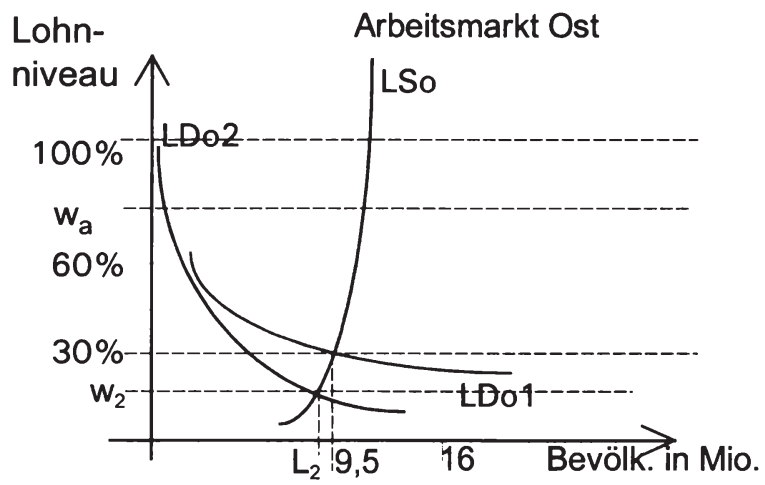

\section{Abbildung 11: Ostdeutscher Arbeitsmarkt nach ersten Modifikationen}

$\mathrm{LS}_{\mathrm{o}}$ und $\mathrm{LD}_{\mathrm{ol}}$ zeigen die Ausgangssituation vor dem Herbst 1989. Zum Vergleich kennzeichne $\mathrm{w}_{\mathrm{a}}$ wiederum das Lohnniveau, welches sich (ohne Modifikationen) bei vollkommener regionaler Arbeitsmobilität zwischen Ost- und Westdeutschland ergäbe. $\mathrm{LD}_{\mathrm{o} 2}$ spiegele die Arbeitsnachfrage Mitte 1990 wider, als bereits eine Umorientierung weg von politischen Lohnfestsetzungen hin zur Produktivität stattgefunden hatte. Da die vor der Wende in der DDR gezahlten Löhne die Produktivität überstiegen, muß die neue Arbeitsnachfrage unterhalb der früheren Lohn- Beschäftigungskombination liegen ${ }^{61}$. Zudem müßte der Nachfrageausfall nach „DDR-Gütern“ einen niedrigeren Verlauf der Arbeitsnachfragekurve bewirkt haben. $\mathrm{Zu}$ berücksichtigen ist aber auch der produktivitätssteigernde Effekt des Wegfalls von Arbeitsausfallzeiten, wodurch die Arbeitsnachfragekurve weniger niedrig verläuft als ohne eine solche Berücksichtigung.

${ }^{61}$ Hierfür spricht z.B. auch der in der DDR vorhanden gewesene Geldüberhang, d.h. die Menschen verfügten über ein die Konsummöglichkeiten - und damit die Produktionsmenge - übersteigendes Einkommen. 
Durch den modifizierten Verlauf der Arbeitsnachfragekurve hätte sich bei einer Systemumstellung ohne Vereinigung ein Lohn von $\mathrm{w}_{2}$ und ein Beschäftigungsniveau von $\mathrm{L}_{2}$ ergeben -flexible Löhne vorausgesetzt. Lohn- und Beschäftigung wären unter solchen Bedingungen niedriger als vorher gewesen, vorausgesetzt, das regionale Arbeitsangebot hätte sich nicht geändert. Aufgrund der neuen Mobilitätsmöglichkeiten war jedoch nicht mit einer Senkung der ostdeutschen Löhne zu rechnen, sondern mit einem Anstieg bei gleichzeitigem Rückgang der Beschäftigung.

Eine derartige Entwicklung stellte sich tatsächlich in Ostdeutschland ein. Bereits vor dem 1. Juli 1990 erhöhten sich die Bruttoverdienste der Arbeitnehmer gegenüber dem ersten Halbjahr 1989 um rund $18 \%{ }^{62} .1991$ betrug das Lohnniveau bereits etwa $47 \%$ des Westlohnniveaus. In den darauffolgenden Jahren waren weitere kräftige Steigerungen um über $30 \%$ (1992) bzw. über $17 \%$ (1993) zu verzeichnen ${ }^{63}$.

Infolge der schnellen Lohnerhöhungen überstiegen die Löhne im 2. Halbjahr 1990 im Verarbeitenden Gewerbe die Erlöse um 38\%, so daß die Unternehmen Verluste machten. Durch Freisetzung von Arbeitskräften versuchen sie, ihre Kosten zu senken ${ }^{64}$. Anfänglich bestand noch ein Kündigungsschutz, der einen entsprechenden Beschäftigungsrückgang zunächst verhinderte. Die Zahl der Beschäftigten verringerte sich von 9,5 Mio. in 1989 auf 8,6 Mio. in 1990 und sank bis Ende 1991 weiter auf 6,4 Mio., d.h., daß innerhalb von zwei Jahren die Beschäftigung um 3 Mio. abgebaut wurde. Tatsächlich war der Abbau von Arbeitsplätzen sogar noch höher. In der Beschäftigtenzahl sind 400.000 Personen in Arbeitsbeschaffungsmaßnahmen und 1,1 Mio. Kurzarbeiter enthalten. Die Zahl der Vollzeitbeschäftigten in "regulären" Arbeitsplätzen in Ostdeutschland schrumpfte daher in weniger als zwei Jahren auf gut die Hälfte.

In Abbildung 12 wird die Entwicklung auf dem ostdeutschen Arbeitsmarkt aufgezeigt. Dabei wurde zusätzlich berücksichtigt, daß es infolge erster Investitionen und verbesserter Arbeitsorganisation zu einer weiteren Verschiebung der Arbeitsnachfragekurve nach außen (auf $\mathrm{LD}_{03}$ ) gekommen sein dürfte. Da insbesondere in kapitalintensive, hochmoderne Anlagen investiert wurde, könnte dies ferner einen steileren Verlauf der Arbeitsnachfragekurve zur Folge gehabt haben ${ }^{65}$.

${ }^{62}$ Vgl. Deutsche Bundesbank (1991).

${ }^{63}$ Vgl. Statistisches Bundesamt (1994), S. 22.

64 Vgl. DIW (1992, 5), S. 50 und S. 54.

65 Waren diese Investitionen Kapitalumschichtungen aus den alten in die neuen Bundesländer, hätte dies eine gleichzeitige Linksverschiebung der Arbeitsnachfragekurve im Westen bedeutet. Handelte es sich um zusätzliche Investitionen hätte dies nur einen Einfluß auf die 


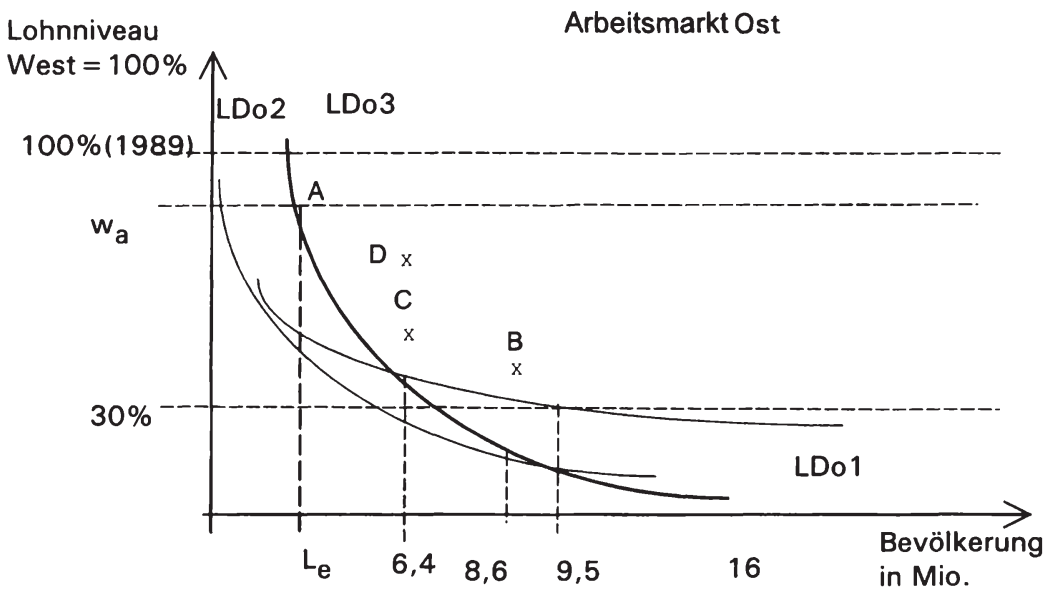

Abbildung 12: Ostdeutscher Arbeitsmarkt nach weiteren Modifikationen

Die Situation in 1990 sei in Abbildung 12 mit Punkt B charakterisiert: die damals gezahlten Löhne waren gemessen an der Produktivität der Beschäftigten zu hoch. Eine weitere Entlassung von Arbeitnehmern war noch nicht möglich. Eine Entlohnung gemäß der Produktivität war zudem nicht für alle Unternehmen gleich dringlich, da Unternehmen, die sich noch im Besitz der öffentlichen Hand befanden, Verluste machen konnten, ohne vom Konkurs bedroht zu sein. Punkt C kennzeichne die im Jahr 1991 herrschende Situation mit 47\% Lohnniveau und 6,4 Mio. Erwerbstätigen. Das Tarifentgelt auf Stundenbasis erreichte in Ostdeutschland im Jahr 1996 knapp 82\% des Westlohnniveaus ${ }^{66}$, die Zahl der Erwerbstätigen betrug 6,3 Mio. (Punkt D). Punkt A zeigt im Vergleich den modellmäßig - bei dem gleichgewichtigen gesamtdeutschen Lohnsatz in Höhe von $\mathrm{w}_{\mathrm{a}}$ zu erwartenden Lohnsatz und die Beschäftigungshöhe in Ostdeutschland.

Eine Lohnangleichung, wie sie annahmegemäß zu erwarten gewesen wäre, ist auch einige Jahre nach der Vereinigung bei weitem nicht realisiert, auch wenn Angleichungsprozesse stattgefunden haben. Gegenüber dem modellmäßig zu erwartendem Gleichgewichtslohn war der ostdeutsche Lohn niedriger und die Beschäftigung höher. Wanderungen hatten nicht im neoklassisch zu erwartenden Umfang stattgefunden. Mitte der neunziger Jahre betrug der Wanderungssaldo zwischen Ost- und Westdeutschland nur noch jährlich rund 25.000 Personen. 
Die meisten Erwerbstätigen boten offenbar nicht simultan auf dem ost- und westdeutschen Arbeitsmarkt ihre Arbeit zu den gleichen Konditionen an. Es existierte somit auch keine ,gemeinsame“ Arbeitsangebotskurve auf einem gemeinsamen Arbeitsmarkt. Gleichwohl haben Mobilitätsprozesse durch die Vereinigung in großem Umfang stattgefunden. 1989 und 1990 siedelten jeweils knapp 400.000 Personen in den Westen über, von denen etwa die Hälfte Erwerbstätige waren. Hinzu kam eine große Zahl an Pendlern: so wurde der ostdeutsche Arbeitsmarkt Ende 1991 durch 0,5. Mio. Pendlern nach Westdeutschland entlastet ${ }^{67}$.

\subsubsection{Fazit}

Gemäß Modell hätten durch die Vereinigung Wanderungsbewegungen in sehr hohem Umfang ausgelöst werden müssen, unter Zugrundelegung der ursprünglichen Arbeitsnachfragekurven sogar eine vollständige Abwanderung aus Ostdeutschland. Die Wanderungen aus den neuen in die alten Länder erreichten jedoch keine derartigen Dimensionen. Aber auch zu einem Ausgleich der regionalen Lohnsätze durch die Mobilitätsprozesse kam es bisher nicht. Die deutschen Lohnniveaus näherten sich zwar einander an, unterschieden sich aber auch einige Jahre nach der Vereinigung noch deutlich voneinander. Theoretisch hätte das Tariflohnniveau in Westdeutschland durch das zusätzliche Arbeitsangebot der Übersiedler und Pendler sinken müssen. Das Reallohnniveau in Westdeutschland hat sich aber sogar noch leicht erhöht.

Die Betrachtung des ostdeutschen Arbeitsmarktes nach der Wende deutet auf das Vorhandensein von arbeitsmarktrelevanten Sachverhalten hin, die im Modell nicht berücksichtigt sind. Viele Änderungen des ostdeutschen Arbeitsmarktes beruhten auf der Umstellung des wirtschaftlichen und politischen Systems. Auf die spezifische Dimension einer solchen Systemänderung ist die Analyse der Arbeitsmobilität im neoklassischen Modell jedoch nicht zugeschnitten.

So wurde im Modell mit Vollbeschäftigungsarbeitsmärkten mit flexibler Lohnbildung operiert. Die Flexibilität der Lohnsätze ist jedoch in Deutschland eingeschränkt. In Ostdeutschland trat nach der Vereinigung massive Arbeitslosigkeit auf. Auch der westdeutsche Arbeitsmarkt war durch gravierende Arbeitslosigkeit gekennzeichnet. Die Bedeutung von Arbeitslosigkeit bzw. die Unsicherheit des Arbeitsplatzes wird in der Literatur neben dem Lohnsatz als wesentliche Determinante für Wanderungsentscheidungen hervorgehoben. Daher wird in Abschnitt 2.3 das Modell entsprechend modifiziert.

${ }^{67}$ Vgl. DIW $(1992,5)$, S. 49f. 
Ferner wurde im Modell mit konstanten regionalen Arbeitsnachfragefunktionen auch bei massiver $\mathrm{Zu}$ - oder Abwanderung operiert. Die Arbeitsnachfrage selbst kann sich aber durch Migrationen ändern. Im Modell wurden nur Unternehmen berücksichtigt, die ausschließlich handelbare Güter produzieren und sie auf dem Weltmarkt verkaufen. Existieren jedoch auch Unternehmen, die nicht-handelbare Güter und Dienstleistungen produzieren, vergrößert (bzw. verkleinert) sich deren Absatzmarkt durch Zuwanderung (Abwanderung) - mit entsprechenden Rückwirkungen für ihre Arbeitsnachfrage. Ebenso dürften längerfristig die Investitionsentscheidungen von den regionalen Absatzmöglichkeiten betroffen werden. Abschnitt 2.3 greift diese Problematik auf.

Wanderungen werden nicht nur von Arbeitsmarktfaktoren beeinflußt, sondern daneben beeinflussen noch weitere Umstände die individuellen Umzugsentscheidungen. Die Abwanderungen aus Ostdeutschland dürften aufgrund dieser Faktoren wesentlich geringer gewesen sein als ohne deren Einfluß. Der Einfluß dieser weiteren Faktoren auf das Ausmaß der Wanderungen und die Lohnanpassungen wird in Abschnitt 2.4 berücksichtigt.

\subsection{Die Berücksichtigung von Arbeitslosigkeit}

Da die Übertragbarkeit der Modellergebnisse auf die deutsche Vereinigung durch die Existenz von Arbeitslosigkeit eingeschränkt wird, wird das Modell im Folgenden entsprechend modifiziert. Zunächst werden einige potentielle Gründe für Arbeitslosigkeit betrachtet. Unter Erweiterung der bisherigen Annahme um nach unten rigide Löhne werden danach Einkommen und Beschäftigung bestimmt. Im Anschluß werden dann für unterschiedliche Vereinbarungen über die Lohnhöhe und die regionalen Lohnunterschiede die Konsequenzen einer Vereinigung von zwei Regionen untersucht.

\subsubsection{Potentielle Gründe für Arbeitslosigkeit}

Ein Ansatz zur Erklärung von Arbeitslosigkeit liefern die Job-Search-Theorien'88. In diesen Modellen werden die Annahmen von homogenen Arbeitsplätzen und kostenlosen Informationen aufgegeben. Heterogene Arbeitsplätze und unvollkommene Informiertheit erzeugen unterschiedliche Entlohnungen, wodurch Arbeitnehmer veranlaßt werden, nach dem für sie optimalen Arbeitsplatz zu suchen $^{69}$. Im Grundmodell der Suchtheorie erfolgt die Suche nach einem neuen

68 Vgl. zu Job-Search-Theorie etwa Stigler (1962) oder König (1979).

69 Vgl. Sesselmeier/Blauermel (1990), S. 42 FN 24. 
Arbeitsplatz aus einer Situation der Arbeitslosigkeit heraus. Begründet wird das damit, daß es effizient ist sich zu spezialisieren - auf die Informationsbeschaffung genauso wie auf andere Produktionsaktivitäten ${ }^{70}$. Während der Zeit der Arbeitslosigkeit könne der Suchende zwar sofort einen Arbeitsplatz finden, er sucht aber nach einem besseren. Er vergleicht die Informationskosten (speziell auch in Form entgangener Arbeitseinkommen) mit den möglichen Gewinnen aus einem besser bezahlten Arbeitsplatz ${ }^{71}$. Der Suchende wird ein Angebot dann akzeptieren, wenn die erwarteten Grenzkosten für die Erlangung eines zusätzlichen Angebots dem damit erwartenden Grenzertrag entsprechen. Je geringer die Informationskosten sind und je höher die Vorstellungen des Suchenden über den erzielbaren Lohn sind, desto länger wird die Suche dauern (und damit auch die Dauer der Arbeitslosigkeit) ${ }^{72}$.

Weitere Gründe für Arbeitslosigkeit werden von den Effizienzlohntheorien beschrieben ${ }^{73}$. Diese gehen davon aus, daß die Arbeitsleistung eines Beschäftigten steigt, wenn ihm ein höherer Reallohn gezahlt wird. Danach würde eine Lohnsenkung die Produktivität der Arbeiter senken und damit zu steigenden Lohnkosten führen. Liegt der Effizienzlohn oberhalb des Vollbeschäftigungslohns werden die Unternehmen die Löhne nicht soweit senken, bis das Gleichgewichtsbeschäftigungsniveau erreicht ist (Es gilt aber weiterhin die Annahme, daß der Lohnsatz dem Wertgrenzprodukt entspricht). Dieses könnte nämlich zu einer so starken Senkung der Arbeitsproduktivität der bereits vor Lohnsenkung beschäftigten Arbeiter führen, die durch die niedrigeren Lohnkostennicht ausgeglichen werden könnte ${ }^{74}$. Liegt der ihnen gezahlten Lohnsatz über dem Gleichgewichtslohnsatz, müssen Arbeitnehmer bei Entlassung befürchten, keinen neuen Arbeitsplatz zu finden oder aber nur einen schlechter bezahlten.

Unternehmen versprechen sich aus Nichtmarkträumungslöhne verschiedene Vorteile ${ }^{75}$ :

1) reduziertes Shirking (Drückebergerei) durch höhere Opportunitätskosten für die Arbeitnehmer bei Entlassung (Shirking-Ansatz) ${ }^{76}$

2) geringere Fluktuationskosten qualifizierter Arbeiter (Labor Turnover-Ansatz)

Vgl. Alchian (1970), S. 29.

Vgl. Rothschild (1988), S. 26.

72 Vgl. Scheuer (1987), S. 85.

${ }^{73}$ Einen Überblick über die verschiedenen Ansätze findet sich etwa bei Yellen (1984), Akerloff/Yellen (1986), Scheuer (1987), S. 111 ff. und Scheuer (1986/87).

${ }^{74} \mathrm{Vgl}$. Sesselmeier/Blauermel (1990), S. 97.

75 Vgl. Yellen (1984), S. $201 \mathrm{ff}$.

${ }^{76}$ Vgl. hierzu auch Bellmann (1986). 
3) höhere Durchschnittsqualität der Arbeitsplatzbewerber (Adverse-SelectionAnsatz)

4) bessere Arbeitsmoral (gift-exchange-Ansatz)

Auch die Modifizierung einer weiteren Annahme liefert einen Grund für Arbeitslosigkeit: Es gibt zwar sehr viele Anbieter und Nachfrager am Arbeitsmarkt, aber es sind in der Regel nur wenige, die in der Bundesrepublik am Lohnbildungsprozeß beteiligt sind. Die Verhandlungen über Lohnsätze werden von den Tarifparteien (Gewerkschaften und Arbeitgeberverbänden) kollektiv geführt. Die Arbeitsmärkte sind kartelliert. Tarifvertragsparteien haben daher eine relativ große Marktmacht, was - wie in jedem anderen Fall von Marktmacht auch - zu Ineffizienzen führt. Wichtige Erklärungsansätze für das gewerkschaftliche Handeln sind das „Monopol-Modell" und das Modell der „,sogenannten effizienten Verhandlungen"77. Nach dem Monopol-Modell verfolgen die Gewerkschaften die miteinander konkurrierenden Ziele Vollbeschäftigung und hohes Lohnniveau. Die Gewerkschaften haben stärker das Interesse ihrer beschäftigten Mitglieder ( $=$ hohe Löhne) im Auge, da die Beschäftigten den größten Teil ihrer Mitglieder ausmacht. Im Ergebnis setzen die Gewerkschaften daher ein Lohnniveau durch, welches oberhalb des Vollbeschäftigungsniveaus liegt. Die Unternehmen begrüßen ein gewisses Maß an Arbeitslosigkeit durchaus (wie bereits oben beschrieben). Nach dem Modell der sogenannten effizienten Verhandlungen kann es für beide Tarifvertragsparteien (in einer kollektiven Perspektive) lohnend sein, sich auf eine Lohnhöhe und ein Beschäftigungsniveau zu einigen, das nicht auf der Nachfragekurve liegt.

Wenn der Lohn dauerhaft über dem Gleichgewichtslohn liegt und Arbeitslosigkeit herrscht, wird dies auch als klassische Arbeitslosigkeit bezeichnet ${ }^{78}$. Für die nachfolgenden Überlegungen wird unterstellt, daß die Löhne nach unten nicht hinreichend flexibel sind, weil die Gewerkschaften mit den Arbeitgeberverbänden kollektive Lohnverträge mit einem Lohnniveau aushandeln, das über dem gleichgewichtigen liegt. Dies entspricht quasi einem bilateralen Monopol79. Die Unternehmen verpflichten sich darin, keinem ihrer Arbeiter weniger als den ausgehandelten Tariflohn zu zahlen. Ferner seien alle Unternehmen Mitglieder der Arbeitgeberverbände, bzw. seien durch Allgemeinverbindlichkeits-

77 Vgl. Franz (1991), S. 239.

${ }^{78}$ Klassische Arbeitslosigkeit ist dadurch gekennzeichnet, daß Arbeitslose zu den gegebenen Löhnen zwar arbeiten würden, die Unternehmen sie jedoch nicht anstellen. Sie würden die Beschäftigung also nur bei sinkenden Löhnen erhöhen. Die Unternehmen werden mithin nicht durch keynesianischen Nachfragemangel nach den von ihnen produzierten Gütern rationiert (vgl. Schettkat (1993), S. 271).

79 Die Ansicht, daß dies die Verhältnisse des deutschen Arbeitsmarktes treffend widerspiegelt, vertritt z.B. Merklein (1993). 
erklärungen zur Zahlung des Tariflohns verpflichtet worden, so daß niemand unterhalb des vereinbarten Lohnniveaus beschäftigt werden kann, auch wenn ein entsprechendes Angebot besteht. Die Auswirkungen einer Beseitigung von Mobilitätsbarrieren sind dann nicht nur von den ursprünglichen regionalen individuellen Grenzproduktivitäts- und Opportunitätskostenbedingungen abhängig, sondern auch von den (kartelliert) vereinbarten Löhnen und Erwartungen darüber, welche Lohnhöhe künftig vereinbart wird. Insgesamt gibt es im wesentlichen 3 Alternativen, auf die sich die Tarifvertragsparteien einigen können:

Alternative 1) Gleiches, nach unten rigides Lohnniveau in beiden Regionen, Alternative 2) Regionale Lohnunterschiede, Alternative 3) Verzicht auf Lohnrigiditäten

$\mathrm{Zu}$ welchen Konsequenzen die Verhandlungsergebnisse im einzelnen führen, wird im folgenden untersucht. Dabei werden nicht nur die Regelungen, die in Deutschland tatsächlich getroffen wurden, betrachtet, sondern auch alternative Vereinbarungen.

Analysiert werden wiederum die Änderungen der Beschäftigung, der Wertschöpfung, der Kapitalentlohnung, der Lohnsumme und des bereinigten Sozialprodukts infolge einer Vereinigung, sowohl aus regionaler als auch aus überregionaler Sicht. Bevor dieses geschehen kann, soll aber zunächst einmal in einem 1-Regionen-Modell betrachtet werden, wie sich die verschiedenen Einkommensgrößen (insbesondere die Arbeitnehmerrente) bei rigiden Löhnen von denen bei flexiblen Löhnen unterscheiden.

\subsubsection{Beschäftigung und Einkommen - insbesondere Arbeitnehmerrenten - bei rigiden Lohnsätzen}

Die Beschäftigung ist c.p. bei nach unten rigiden Löhnen (mit einem Niveau über dem Gleichgewichtslohn) niedriger als bei flexiblen. Die Lohnsumme kann ebenfalls geringer sein; das hängt von der Lohnelastizität der Nachfrage ab. Aufgrund der hier unterliegenden Annahme, nach der Gewerkschaften Monopolrenten für ihre Mitglieder durchsetzen ${ }^{80}$, dürfte die Lohnsumme - jedenfalls kurzfristig $^{81}$ - bei rigiden Löhnen höher sein als bei flexiblen. Die Arbeitneh-

${ }^{80} \mathrm{Vgl}$. Berthold (1987), S. 145ff.

${ }^{81}$ In diesem Zusammenhang ist zu berücksichtigen, daß die Lohnelastizität kurzfristig starrer ist als langfristig. Möglicherweise fördern die Gewerkschaften also eine Politik, die zwar dem kurzfristigen Kollektivinteresse der Arbeitnehmer, aber nicht deren langfristigen Interessen dient. 
merrente kann bei rigiden Löhnen nicht so eindeutig bestimmt werden wie bei flexiblen. Dies wird anhand von Abbildung 13 erläutert.

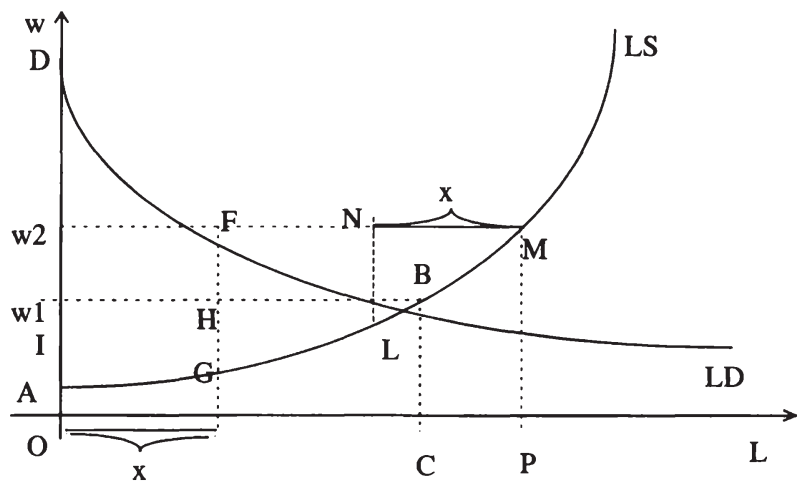

Abbildung 13: Arbeitnehmerrente bei rigiden Löhnen

Nur bis zu dem Gleichgewichtslohnsatz $w_{1}$ ist die Höhe der Arbeitnehmerrente im Sinne der bisherigen Überlegungen exakt berechenbar, bei jedem höheren Lohnsatz ist ihre Höhe davon abhängig, welche Arbeiter beschäftigt werden. Die Unternehmen werden nicht zwangsläufig gerade diejenigen mit der höchsten marginalen Arbeitswilligkeit (also diejenigen, die bei den geringsten Löhnen zu arbeiten bereit wären) einstellen, so daß etwa bei einem Lohn in Höhe vonw2 und einer Beschäftigungsmenge von $\mathrm{x}$ die Fläche AGFw2 nicht die bei diesem Lohn stets realisierte Arbeitnehmerrente widerspiegelt, sondern die maximal mögliche ${ }^{82}, 83$. Die minimale Arbeitnehmerrente bei diesem Lohnniveau betrüge LMN. Sie ergibt sich, wenn genau diejenigen mit der niedrigsten marginalen Arbeitswilligkeit beschäftigt werden. Die Höhe der Arbeitnehmerrente bei sich verändernden Lohnsätzen hängt daher auch davon $a b$, wer vor Lohnänderung und wer nachher beschäftigt wird.

Auch wenn die Argumentation auf die jeweils maximale Arbeitnehmerrente abgestellt wird, ergibt sich bei steigendem Lohnsatz eine Arbeitnehmerrente, die sowohl höher als auch niedriger als die Arbeitnehmerrente bei Gleichgewichtsentlohnung sein kann. Ob sie höher oder niedriger ist, hängt von der Lohnelasti-

${ }^{82}$ Die makroökonomische Arbeitsangebotsfunktion ist eine Aggregation vieler individuellen Arbeitsangebotsfunktionen. Entlang der Kurve kommen immer mehr Arbeitsanbieter hinzu, nämlich diejenigen mit steigenden Reservationslöhnen. Von einer Veränderung der Arbeitsnachfrage muß daher nicht jeder Arbeiter betroffen sein.

${ }^{83}$ Die Auswahl der Arbeiter durch die Unternehmen muß zufällig sein, da Arbeit annahmegemäß homogen ist. 
zität der Nachfrage und des Angebots ab. Der Rückgang an Arbeitnehmerrente infolge eines geringeren Beschäftigungsvolumens kann - muß aber nicht - überkompensiert werden durch ihre Zunahme infolge eines höheren Lohnniveaus.

$\mathrm{Da} ß$ die Arbeitnehmerrente mit steigenden Löhnen sinkt, wenn jeweils die „Minimallösung" realisiert wird, ist dann eindeutig, wenn die Arbeitsangebotskurve linear verläuft. Aus den jeweils zuletzt angebotenen Arbeitsstunden wird dann immer die gleiche Arbeitnehmerrente (und zwar marginal in Höhe von Null) bezogen, unabhängig vom Lohnniveau. Durch die infolge der Lohnerhöhung zurückgehende Beschäftigung sinkt die Arbeitnehmerrente. Bliebe die Beschäftigungshöhe konstant, hätten steigende Löhne keinen Einfluß auf die Arbeitnehmerrente ${ }^{84}$. Verläuft die Arbeitsangebotskurve dagegen progressiv, stiege die Arbeitnehmerrente bei wachsendem Lohn und konstanter Beschäftigung, auch wenn die „Minimallösung“ realisiert wird. Geht die Beschäftigung bei steigenden Lohnsätzen zurück, ist die Veränderung der Arbeitnehmerrente jedoch auch in diesem Fall nicht zwingend positiv.

Daß lediglich die minimal mögliche Arbeitnehmerrente realisiert wird, ist aber unplausibel. Zum einen dürfte die Wahrscheinlichkeit gegen Null gehen, daß die Unternehmen bei ihrer Auswahl gerade immer die Arbeitskräfte auswählen, die das höchste Arbeitsleid empfinden. Auch werden sich diejenigen, die bei den gegebenen Löhnen hohe Arbeitnehmerrenten realisieren würden, stärker um einen Arbeitsplatz bemühen als diejenigen mit nur geringen Arbeitnehmerrenten.

Darüber hinaus ist auch deshalb mit der Minimallösung zu rechnen, da bei einer Mindernachfrage nach Arbeit - speziell bei entsprechenden Arbeitszeitregelungen - gegenüber der Gleichgewichtssituation einige Arbeiter nicht ganz aufhören zu arbeiten, sondern sie nur weniger als im Gleichgewicht arbeiten. Für diese entfallen zwangsläufig gerade die letzten Einheiten Arbeitnehmerrente, also jene, die am geringsten sind. Dies verstärkt die Tendenz zur Annäherung an die maximal möglichen Arbeitnehmerrenten.

Zusammengenommen sprechen die Argumente eher dafür, daß die maximal mögliche Arbeitnehmerrente zustande kommt. Eindeutige Aussagen über die Richtung der Veränderung der Arbeitnehmerrente infolge einer Lohnänderung sind nicht möglich.

${ }^{84}$ Dies gilt nur, wenn stets die Minimallösung realisiert wird, was aber gleichzeitig bedeutete, daß bei Lohnerhöhungen mit konstanter Beschäftigungsmenge andere Arbeiter als vorher beschäftigt werden müssen, nämlich diejenigen, die durch die höheren Löhne erst motiviert werden, ihre Arbeit anzubieten. 
Das bereinigte Sozialprodukt ist bei rigiden Lohnsätzen immer geringer als bei flexiblen, wenn der Verlauf der Arbeitsnachfragekurve nicht vom herrschenden Lohnniveau abhängt. Auch wenn die Arbeitnehmerrente bei rigiden Löhnen höher sein kann als bei flexiblen, wird dies stets durch geringere Kapitaleinkommen überkompensiert. Bei Zugrundelegung der Argumente der Effizienzlohntheorie können jedoch auch die Kapitaleinkommen bei Löhnen über dem Gleichgewichtsniveau höher sein, wie in der folgenden Abbildung illustriert wird:

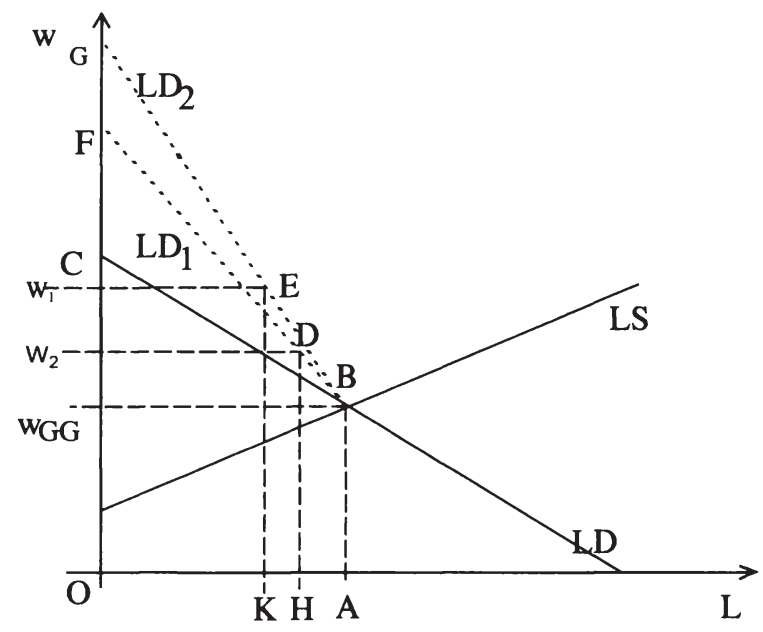

Abbildung 14: Kapitaleinkommen bei Zahlung von Effizienzlöhnen

Die LD-kurven beschreiben die Grenzproduktivität der Arbeiter. Bei jedem Lohnsatz kann sich aber deren Verlauf ändern, wenn sich die Arbeitsmotivation aller Beschäftigten ändert. Bei einem Lohn von $\mathrm{w}_{G \mathrm{G}}$ entspricht die Grenzproduktivität der Funktion LD, bei einem Lohn von w1 verläuft sie bis zum Gleichgewichtslohn oberhalb von LD, in der Graphik dargestellt durch $L_{1}$. Je höher der Lohn, desto steiler verläuft die Kurve. Die Kurven dürfen sich nicht schneiden, da es unplausibel ist, daß bei einem geringeren Lohn die Motivation der Arbeiter höher ist. Die Unternehmen kennen und berücksichtigen die verschiedenen Verläufe. Bei dem in der Graphik unterstelltem Verlauf entspricht der Gewinn bei dem Lohnsatz $w_{1}$ der Fläche $w_{1} D F$. Dieser Gewinn kann über dem bei dem Vollbeschäftigungslohnsatz $\mathrm{w}_{G G}$ realisiertem liegen (Fläche $\mathrm{w}_{G G} \mathrm{BC}$ ). Auch kann das Sozialprodukt bei Löhnen oberhalb des Gleichgewichtniveaus höher sein als bei Vollbeschäftigung. 


\title{
2.2.3 Vergleich der Beschäftigung und der Einkommen mit und ohne Vereinigung
}

Nachdem gezeigt wurde, welchen Einfluß rigide Löhne auf Einkommen und Beschäftigung haben, können nun die Auswirkungen einer Vereinigung bei alternativen Lohnvereinbarungen (Alternative 1-3) beschrieben werden, und zwar der Lohnvereinbarungen, die oben bereits genannt wurden. Dabei wird weiterhin ein 2-Regionen-Modell (mit den Regionen $\mathrm{x}$ und $\mathrm{y}$ ) betrachtet, wobei in der Region y jeweils das höhere Lohnniveau im Ohne-Vereinigungsfall herrschen soll.

\begin{abstract}
Alternative 1) Gleiches, nach unten rigides Lohnniveau in beiden Regionen Wenn sich Arbeitgeber und Arbeitnehmer darauf verständigen, die Lohnniveaus in den beiden Regionen anzugleichen, sind die Auswirkungen von der Ausgangssituation und den konkreten Verhandlungsergebnissen über den zukünftigen gemeinsamen Lohn abhängig. Potentielle Lohnvereinbarungen sind im wesentlichen:
\end{abstract}

a) Eine Region übernimmt das Lohnniveau nach der Vereinigung von der anderen Region,

b) der neue gemeinsame Lohnsatz liegt zwischen den beiden alten

c) der neue Lohn liegt oberhalb oder unterhalb der beiden regionalen Löhne ohne Vereinigung.

Die Verhandlungslösung c) scheint von vornherein nicht durchsetzbar zu sein und soll aus diesem Grund nicht weiter betrachtet werden.

a) Eine Region übernimmt das Lohnniveau nach der Vereinigung von der anderen Region

In der Region mit dem unverändertem Lohnniveau ändert sich nichts an der $\mathrm{Be}$ schäftigungsmenge, da die Unternehmen keinen Anlaß zur Mehr- oder Mindernachfrage haben (vorausgesetzt, sie konnten zu den bisherigen Lohnsätzen ihre Nachfrage befriedigen). Dementsprechend bleiben das regionale Sozialprodukt und die Kapitaleinkommen konstant ${ }^{85}$. Für die Einkommens- und Beschäftigungsentwicklung der anderen Region ist entscheidend, ob das neue Lohnniveau über oder unter dem alten liegt.

Übernähme eine Region einen höheren Lohnsatz, würde dort - gemäß der regionalen Arbeitsnachfragekurve - weniger Arbeit nachgefragt werden. Die Ver-

${ }^{85} \mathrm{Da}$ das Sozialprodukt und die funktionale Einkommensverteilung unverändert bleiben, gilt jedoch nicht in dem Sonderfall, daß der zu übernehmende Lohnsatz niedriger ist als der gemeinsame Gleichgewichtslohn. Dies entspräche einer Aufhebung von Lohnrigiditäten. 
einigung führte zu (erhöhter) Arbeitslosigkeit. Dabei nähme die Zahl der Arbeitslosen nicht nur um die Entlassenen zu, sondern auch um jene, die bei den gestiegenen Lohnsätzen zusätzlich $\mathrm{zu}$ arbeiten bereit wären. Die sinkende Beschäftigung führte zu abnehmendem Sozialprodukt und Kapitaleinkommen in der Region. Wie sich Lohnsumme und Arbeitsleid ändern, hängt von der Elastizität der Arbeitsnachfrage und des -angebots ab. Das bereinigte regionale Sozialprodukt sänke infolge der Vereinigung. Da die Veränderung in dieser Region der gesamtwirtschaftlichen Änderung entspricht, sinkt damit auch das gesamte bereinigte Sozialprodukt. Insofern weichen die Unterschiede des MitVereinigungs-Falls im Vergleich zum Ohne-Vereinigungs-Fall bei rigiden Löhnen signifikant von denen bei flexiblen Löhnen ab, wo das bereinigte Sozialprodukt mit Vereinigung stets höher ist. Durch den Abbau der Mobilitätsbarrieren für Arbeit werden hier keine Wanderungen ausgelöst, da die Menschen, die in der den Lohnsatz übernehmenden Region arbeitslos werden auch in der anderen Region keinen Arbeitsplatz finden.

Es fällt auf, daß die Interessengruppen der Arbeitnehmer jetzt stärker als nur nach dem Merkmal Herkunftsregion zu differenzieren sind. Während bei flexiblen Löhnen die aus der ehemaligen Hochlohnregion stammenden Arbeiter Lohn- und Beschäftigungseinbußen hinnehmen mußten, profitierten die Arbeitnehmer der anderen Region durch die Vereinigung, zumindest aber wurde kein Beschäftigter in der ehemaligen Niedriglohnregion schlechter als vorher entlohnt. Die vorliegende Vereinbarung bewirkt aber, daß Beschäftigung in der ehemaligen Niedriglohnregion bei den höheren Löhnen abgebaut wird und damit auch für einige Arbeiter die Arbeitnehmerrente entfällt, da sie auch in der anderen Region keinen Arbeitsplatz finden. Insofern sind jetzt einige Bürger dieser Region schlechter als vorher gestellt, während andere eine höhere Arbeitnehmerrente als ohne Vereinigung erzielen. Für die Arbeitnehmer der ehemaligen Hochlohnregion steigt durch die zunehmende Arbeitsplatzkonkurrenz ihr Risiko arbeitslos zu werden (was wiederum zu einer Effizienzsteigerung führen könnte), ihre Einkommenssituation bleibt aber unverändert .

Wenn der Lohn einer Region durch eine Vereinigung sinkt, kommt es dort zu einer Mehrnachfrage nach Arbeit; die durch Arbeiter beider Regionen befriedigt werden kann. Die Zahl der Arbeitslosen wird durch die Vereinigung sinken, jedoch nicht ganz abgebaut, sofern der Lohn auch nach der Vereinigung über dem Gleichgewichtsniveau liegt. Diejenigen, die in dieser Region vor der Vereinigung beschäftigt waren, haben Lohneinbußen zu verzeichnen, d.h. sie erleiden Nachteile durch die Vereinigung, während die zusätzlich Beschäftigten von der Vereinigung profitieren. Das Sozialprodukt wächst wegen der Beschäftigungszunahme und damit auch die Gewinne. Die Richtung der Veränderung von 
Lohnsumme und Arbeitnehmerrente hängt von den Elastizitäten ab. Das bereinigte Sozialprodukt wächst.

b) Der neue gemeinsame Lohnsatz liegt zwischen den beiden alten.

Liegt der vereinbarte Lohn über dem Gleichgewichtslohn, wird es weiterhin Arbeitslosigkeit geben. Zu Beschäftigungsanpassungen wird es in beiden Regionen kommen, in der vormaligen Hochlohnregion $\mathrm{zu}$ einem Anstieg und in der vormaligen Niedriglohnregion zu einem Rückgang mit entsprechenden Konsequenzen für die regionalen Wertschöpfungen. $\mathrm{Ob}$ und wie sich die Beschäftigung insgesamt ändert, hängt von dem konkreten Kompromiß und der Ausgangslage ab. Die Arbeitsproduktivität steigt, da die Beschäftigung in der ehemaligen Hochlohnregion zunimmt und in der ehemaligen Niedriglohnregion abnimmt. Daher kann auch bei sinkender Beschäftigung das Sozialprodukt anwachsen.

Alternative 2) Regionale Lohnunterschiede.

Die Tarifvertragsparteien könnten sich darauf einigen, die bisherigen Lohnsätze beizubehalten. In diesem Fall würde sich in keiner Region etwas am Einkommen und der Beschäftigung ändern. Die Unternehmen haben keinen Anlaß ihre Beschäftigungshöhe zu verändern. Die Beseitigung der Mobilitätsbarrieren hätte keine Auswirkungen, auf regionale Lohnunterschiede zurückzuführende Wanderungen fänden nicht statt.

Eine weitere Möglichkeit besteht darin, eine Angleichung des Lohnniveaus zu vereinbaren. In diesem Fall käme es zu den gleichen Anpassungsreaktionen wie sie bei Alternative 1 bereits beschrieben wurden: in der ehemaligen Hochlohnregion nähme die Beschäftigung zu (und damit auch die Wertschöpfung und der Gewinn) und in der anderen Region gingen diese Größen zurück. Die Änderungen insgesamt sind vom Verlauf der Angebots- und Nachfragekurven abhängig.

Theoretisch möglich wäre auch eine Vereinbarung die Lohnabstände noch weiter zu erhöhen. Dies hätte der vorher beschriebenen Vereinbarung gegenläufige Effekte. $\mathrm{Da}$ es für eine derartige Vereinbarung eine Veranlassung geben könnte, ist nicht zu erkennen.

\section{Alternative 3) Aufhebung der Lohnrigiditäten}

Infolge dieser Vereinbarung würden in beiden Regionen Anpassungsvorgänge stattfinden. Die Arbeitslosigkeit in beiden Regionen würde - soweit vorhanden vollständig abgebaut, sofern dem nicht andere Gründe entgegenstehen, wie sie sich etwa gemäß der Effizienzlohntheorie und den Job-Search-Modellen erge- 
ben. Lägen die Löhne beider Regionen ohne Vereinigung über dem gemeinsamen Lohnniveau, so erhöhte sich die Beschäftigung in beiden Regionen und insgesamt. Zumindest aber stiege die eingesetzte Arbeitsmenge in der Region, in der vorher ein Lohn oberhalb des gemeinsamen Gleichgewichtslohns herrschte. Lag der Lohn in einer Region vorher unterhalb des gemeinsamen Gleichgewichtslohns, nimmt die Beschäftigungsmenge dort ab. Der Rückgang in einer Region könnte wohl die Zunahme in der anderen überkompensieren, daher sind die Auswirkungen auf das Sozialprodukt unbestimmt. Ob die Beschäftigung insgesamt zu- oder abnimmt, hängt von den speziellen Gegebenheiten ab. Mit hoher Wahrscheinlichkeit wird es zu Wanderungsbewegungen kommen.

\subsubsection{Zusammenfassung}

Arbeitslosigkeit kann durch vielerlei Gründe entstehen; Erklärungen liefern etwa die Effizienzlohntheorie, die Job-Search-Theorien, das Monopol-Modell und das Modell der „effizienten Verhandlungen“. Analysiert wurden die Folgen eines Abbaus von Mobilitätshindernissen unter der Annahme von nach unten rigiden Löhnen. Existieren mit Vereinigung weiterhin rigide Löhne, so werden nicht im gleichen Ausma $\beta$ wie im Fall flexibler Löhne Wanderungen ausgelöst. Wanderungen führen nicht zu einem regional ausgeglichenen Lohnniveau. Im Extrem unterbleiben sogar jegliche Wanderungen.

Die Höhe der Wanderungen und ihrer Wirkungen auf Einkommen und Beschäftigung hängen von der ursprünglichen Höhe der regionalen Lohnniveaus ab und davon, ob sich an der Höhe der Löhne infolge einer Vereinigung überhaupt etwas ändert. Setzt eine Region ihr bisheriges Lohnniveau für beide Regionen durch, bleiben Einkommen und Beschäftigung in der Region mit dem unveränderten Lohnniveau konstant (vorausgesetzt dieses Lohnniveau liegt über dem gemeinsamen Gleichgewichtslohn). Bei jeder Einigung, die eine Lohnsenkung für eine Region bedeutet, steigen dort die Einkünfte der Kapitaleinkommensbezieher, das regionale Sozialprodukt und die Beschäftigung. Bei jeder Einigung, die eine Lohnerhöhung für eine Region bedeutet, sinken dort die Einkünfte der Kapitaleinkommensbezieher, das regionale Sozialprodukt und die Beschäftigung.

Abgestellt wurde in diesem Abschnitt auf die Bedeutung von Arbeitslosigkeit infolge von rigiden Löhnen für die Wanderungen. Mit Blick auf die deutschen Arbeitsmarktverhältnisse könnten aber auch die Kündigungsschutzvorschriften dazu beigetragen haben, daß die Wanderungen schwächer ausfielen, als neoklassisch zu erwarten war. Durch die westdeutsche Kündigungsschutzpraxis gibt es in eine Beschäftigung führende Wanderungsmöglichkeiten nur in dem Umfang, 
wie neue Betriebe entstehen oder alte ihre Beschäftigung ausdehnen bzw. aus der Labour force ausgeschiedene Arbeitskräfte ersetzt werden. Zudem traten die Bürger aus den neuen Bundesländern in Konkurrenz zu den westdeutschen heimischen Arbeitslosen. Dieser Aspekt soll hier aber nicht weiter vertieft werden.

\subsection{Die Berücksichtigung von Besonderheiten auf der Produktions- seite}

In den vorhergehenden Kapiteln wurde stets die positive Funktion von Mobilität betont, nämlich die Lenkung von Arbeit in ihre produktivere Verwendung. Es kann jedoch auch ökonomische Gründe dafür geben, Wanderungen von Arbeitskräften zu unterbinden. So kann sich die Höhe der regionalen Nachfrage durch Abwanderungen verringern. Außerdem deuten empirische Untersuchungen auf eine Selektivität von Wanderungen hin: meistens wandern die fähigsten und qualifiziertesten Personen ab, da diese am ehesten in einer anderen Region eine Beschäftigung finden. Durch selektive Wanderungen ändert sich mithin die Zusammensetzung des Arbeitsangebots vor Ort. Im Folgenden wird die Bedeutung der Qualifikation von Wanderern für die Arbeitsnachfrage und die Entwicklung der Regionen dargestellt. Dabei wird aber auf die Betrachtung verschiedener spezifischer Produktionsbedingungen wie oligopolistische Märkte oder zunehmende Skalenerträge ${ }^{86}$ verzichtet, da dies den Rahmen der Arbeit gesprengt hätte.

\subsubsection{Die Bedeutung der regionalen Güternachfrage für die Arbeitsnachfrage}

Bisher wurde ein einheitliches Güterpreisniveau in allen Regionen unterstellt, alle Unternehmen konnten zu den gegebenen Preisen unbegrenzt viel absetzen. Transportkosten und Handelshindernisse waren ausgeschlossen. Die Höhe der regionalen Nachfrage war daher für die Unternehmen unerheblich. Diese Annahme ist problematisch. Zumindest Unternehmen, die nicht-handelbare Gütern produzieren, sind von der regionalen Nachfrage abhängig. Zudem errichten

86 Würde die Annahme konstanter Skalenerträge aufgehoben und etwa zugelassen, daß zum einen humankapitalintensive Güter mit zunehmenden Skalenerträgen, zum anderen traditionelle Güter mit konstanten Skalenerträgen produziert werden, würde sich die größere Region auf die Produktion humankapitalintensiver Güter spezialisieren. Die Löhne für qualifizierte Arbeitnehmer in dieser Region würden steigen und einen entsprechenden Wanderungsstrom der qualifizierten Arbeiter zur Folge haben. Hierdurch würde die kleinere Region gegenüber der größeren zurückfallen. Vgl. hierzu etwa Straubhaar/Wolburg (1998), S. 5. 
Unternehmen ihre Produktionsstandorte dort, wo die regionale Nachfrage besonders hoch ist, um ihre Transportkosten zu minimieren ${ }^{87}$. Unter diesen Umständen können Wanderungsbewegungen vorhandene regionale ökonomische Disparitäten verstärken statt sie zu beseitigen:

"Keynesian theory, in contrast, states emigration from an area makes business there less profitable, thus lowering employment and/or wages, and therefore out-migration continues at an even greater rate."88

Zur Verdeutlichung der Wirkungen seien wiederum zwei Regionen betrachtet ${ }^{89}$. Durch eine Beseitigung von Mobilitätsbarrieren für Arbeit werden Wanderungen aus der Niedriglohn- in die Hochlohnregion ausgelöst. Durch die Zuwanderungen wird zusätzliche Nachfrage (nämlich von den Migranten) in der Zielregion entfaltet, die wiederum zu einer steigenden Arbeitsnachfrage führt. Das Lohnniveau steigt hierdurch (bzw. sinkt es weniger stark als ohne Einfluß der regionalen Nachfrage) und die Nachfrage nimmt durch die höheren Einkommen noch weiter zu; es gibt einen Multiplikatoreffekt. Infolge der steigenden Löhne kommt es zu einer zusätzlichen Zuwanderung. Aus diesem Bevölkerungszuwachs entsteht wiederum eine Mehrnachfrage nach Gütern. Infolge der hohen Kapazitätsauslastung kann es zu Erweiterungsinvestitionen kommen, die über die gestiegenen Kapitaleinkommen finanziert werden können. Der Investitionsausgabenmultiplikator verursacht, daß das Wachstum größer ist als die Ausgaben für die Erweiterungsinvestitionen, das Einkommen der Region wächst damit weiter.

Diese Entwicklung könnte gedämpft werden, wenn sich ein Teil der Nachfrage statt auf regional produzierte Güter auf Importgüter richtet. Bei diesen Importgütern kann es sich nämlich auch um Güter der Abwanderungsregion handeln, was sich positiv auf den Absatz dieser Region auswirken könnte. Vor allem die Einwanderer fragen möglicherweise die ihnen vertrauten Güter aus ihrer Heimatregion nach. Es ist aber unwahrscheinlich, daß dieser Umfang so groß ist, daß sich die Richtung der Entwicklung ändert.

In der Abwanderungsregion sind entgegengesetzte Prozesse zu verzeichnen: Die Abwanderungen führen zu einer Verringerung des Einkommen der betroffenen Region. Dies führt zu einem rückläufigen Absatz der lokalen Unternehmen, da durch das gesunkene Einkommen die Nachfrage nach den von ihnen angebotenen Gütern und Dienstleistungen sinkt. Der Markt verliert an Größe und Prosperität. Es werden negative Multiplikatoreffekte ausgelöst. Wenn das Kapital au-

${ }^{87} \mathrm{Vgl}$. Straubhaar (1994), S. 10.

${ }^{88}$ Willis (1974), S. 17.

${ }^{89} \mathrm{Vgl}$. zum folgenden Mieth (1992), S. 124ff. 
Berdem in die gleiche Richtung flie $\beta \mathrm{t}^{90}$, sorgt dies für eine noch weitere Verschärfung der Ungleichgewichte ${ }^{91}$.

Die Menge an Wanderungen wäre nach einer Vereinigung unter solchen Bedingungen höher als ohne Berücksichtigung von Nachfrageeffekten. Entsprechend ist das Einkommen und die Beschäftigung in der Zuwanderungsregion höher, in der Abwanderungsregion niedriger. $\mathrm{Ob}$ die Beschäftigung und das Sozialprodukt insgesamt höher oder niedriger ist als ohne Berücksichtigung der regionalen Nachfrage, ist von der Stärke der Multiplikatoreffekte in den beiden Regionen abhängig.

\subsubsection{Die Bedeutung von Qualifikationsunterschieden}

Migranten verfügen über Kenntnisse und Fähigkeiten, die ihrer Herkunftsregion verlorengehen und ihrer Zielregion zugute kommen. Wenn die Arbeitsnachfrage nicht nur von der Höhe des Sachkapitals, sondern auch vom Humankapital determiniert wird, können Migrationen die regionale Arbeitsnachfrage verändern. Wie sich eine Vereinigung unter solchen Bedingungen auf Lohnsatz, Beschäftigung und Einkommen auswirkt, wird in Abschnitt 2.3.2.1 untersucht.

Im anschließenden Kapitel 2.3.2.2 wird die Bedeutung für Einkommen und Beschäftigung untersucht, wenn wandernde Arbeiter nicht nur Substitute, sondern auch Komplemente für immobile Arbeiter sein können. Im darauf folgenden 2.3.2.3 wird erläutert, inwieweit sich die Zukunftsperspektiven einer Region ändern, wenn nur Arbeiter mit einer bestimmten Qualifikation ab- bzw. zuwandern.

\subsubsection{Das Humankapital als Teil des (die Arbeitsnachfrage determinieren- den) Kapitalstocks}

Der Ansatz, daß Wanderungen zu einer Änderung des regionalen Humankapitals führen und sich die Nachfragekurve nach Arbeit verschiebt, findet sich bei Grubel (1994, S. 78ff). Zum Kapitalstock einer Region werden dort sowohl Sachkapital als auch Humankapital gerechnet. Beide Arten von Kapital haben Einfluß auf die Produktionsfunktion und damit auch auf die Nachfrage nach Arbeit.

\footnotetext{
90 Eine gleichgerichtete Wanderung von Arbeit und Kapital ist wohl typisch für niedergehende Regionen. Vgl. Holland (1976), S. 90.

91 Vgl. Lind (1969), S. 76.
} 
Humankapital ist der Bestand an Wissen und Fertigkeiten von Menschen. Die Zunahme an Humankapital erhöht die Produktivität der betroffenen Personen. Humankapital ist untrennbar mit Menschen verbunden ${ }^{92}$. Bei Wanderungen qualifizierter Arbeitskräfte, also solcher, die mit überdurchschnittlich viel $\mathrm{Hu}$ mankapital ausgestattet sind, verschiebt sich die Arbeitsnachfragekurve der $\mathrm{Zu}$ und der Abwanderungsregion. Je qualifizierter ein Arbeitnehmer ist, desto stärker die Verschiebung. Die Arbeitsnachfragekurve der Abwanderungsregion verschiebt sich nach links, die der Zuwanderungsregion infolge der Zunahme an Humankapital nach rechts.

Grubel zeigt die Lohn- und Beschäftigungswirkungen anhand eines Diagramms, bei dem auf der Abszisse eine gegebene Menge an Arbeit abgetragen wird, auf dem linken Ordinatenabschnitt der Lohnsatz der einen Region, auf dem rechten Ordinatenabschnitt der Lohnsatz der anderen Region. Anhand eines solchen Diagramms kann aber nur der Sonderfall eines unelastischen Arbeitsangebots betrachtet werden.

Die Wirkungen lassen sich unter Bezug auf das oben bereits verwendete Diagramm allgemeiner zeigen. Im Unterschied zu den vorherigen Abschnitten wird jetzt ein Fall von Arbeitsmobilität betrachtet, der gleichzeitig mit Kapitalmobilität verbunden ist, da eine Veränderung des Humankapitals im Rahmen des Modells immer mit Wanderungen des Faktors Arbeit verbunden ist. Bemerkenswert ist vor allem, daß (Human)Kapital in die Region mit dem höheren Lohnniveau wandert.

In Abbildung 15 ist ein einfacher Fall dargestellt, in dem sich die Arbeitsnachfragekurven nach einer Vereinigung durch die stattfindenden Mobilitätsprozesse um den gleichen Betrag (aber mit verschiedenen Vorzeichen) parallel verschieben. In diesem Fall entsprächen die volkswirtschaftlichen Arbeitsnachfrage- und Angebotskurven $\left(\mathrm{LD}_{\mathrm{xy}}\right.$ und $\mathrm{LS}_{\mathrm{xy}}$ ) denen im Referenzfall (Fall ohne Berücksichtigung des Humankapitals), d.h. mit Vereinigung wichen nur die regionalen, aber nicht die gesamtwirtschaftlichen Beschäftigungshöhen voneinander $a b$. Auch der Gleichgewichtslohn mit Vereinigung entspräche dem im Referenzfall. Die Beschäftigung stiege im Referenzfall in der Region $\mathrm{x}$ statt auf $\mathrm{C}$ nur auf $\mathrm{V}$, wäre also um die Differenz von $C$ und $B$ geringer und in der Region y stiege sie auf E. Bei Verschiebung der Arbeitsnachfragekurve infolge von Humankapitalmobilität wäre der Beschäftigungsrückgang in $\mathrm{x}$ um B-A höher; um den gleichen Betrag wäre die Beschäftigungszunahme in y höher (F-E).

92 Vgl. Franz (1991), S.88. 


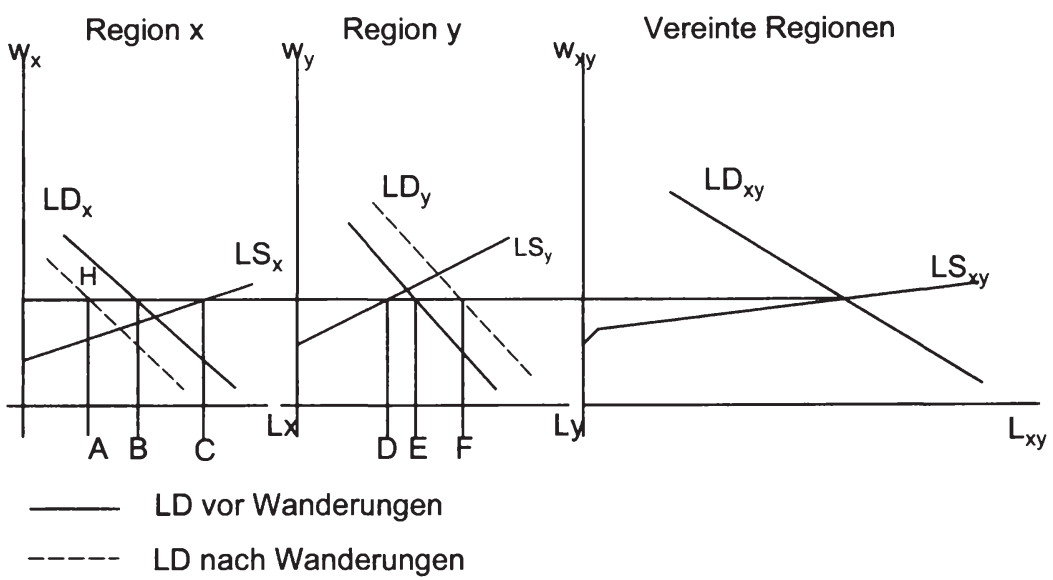

Abbildung 15: Migration bei Einfluß von Humankapital auf die Arbeitsnachfrage

Das Integral unter der Arbeitsnachfragekurve bis zum Gleichgewichtslohnsatz abzüglich der Lohnsumme ergibt die Kapitalentlohnung. Bei Berücksichtigung von Humankapital muß aus dieser Summe nicht nur das Sachkapital, sondern auch das Humankapital entlohnt werden. Die Arbeitnehmer könnten somit zwei Einkommensbestandteile, zum einen Lohn für die normale Arbeitsleistung - für alle Arbeiter gleich hoch - und darüber hinaus eine Entlohnung gemäß ihrer Qualifikation beziehen ${ }^{93}$.

Die Verschiebungen der regionalen Arbeitsnachfragefunktionen müssen vom Betrag her jedoch nicht identisch sein, da das Humankapital in der einen Region möglicherweise produktiver eingesetzt werden kann als in der anderen. Spezifisches, an den Betrieb gebundenes Wissen, geht bei einem Wechsel zu einem anderen Arbeitgeber verloren, dies hätte nur einen Rückgang des Humankapitals in der Abgangsregion zur Folge ohne Erhöhung des Humankapitals in der Zielregion. Ist das Humankapital der Migranten nicht-rival nutzbar, kann das Wissen in der Ursprungsregion erhalten bleiben, sofern auch noch andere immobile Personen dieser Region über das Wissen verfügen, während die Kenntnisse in der Zuwanderungsregion zusätzlich verfügbar werden. Dann haben Wanderungen positive Effekte auf die Arbeitsnachfragefunktion der Zuwanderungsregion, aber keine Auswirkungen auf die der Abwanderungsregion.

93 Eine derartige Betrachtung dürfte allerdings eine Modifikation der Arbeitsangebotskurven notwendig machen. 
Bei stärkerem Einfluß des Humankapitals auf die Arbeitsnachfrage in der Abwanderungsregion als auf die der Zuwanderungsregion gäbe es mit Vereinigung volkswirtschaftlich gesehen niedrigere Löhne, weniger Beschäftigung und damit ein geringeres Sozialprodukt als im Referenzfall. Bei relativ höheren Veränderungen in der Zuwanderungsregion oder Wanderung von Arbeitnehmern, die über nicht-rival nutzbares Humankapital verfügen ${ }^{94}$, wäre mit Vereinigung die Beschäftigung, die Löhne und das Sozialprodukt höher als im Referenzfall.

\subsubsection{Komplementär einsetzbare Qualifikationen}

Migranten sind nicht zwingend nur Substitute für andere Arbeiter, sondern sie können in Abhängigkeit von ihrer Qualifikation auch deren Komplemente sein. Im folgenden werden Wanderungswirkungen unter Berücksichtigung dieser Möglichkeit untersucht. Zur Vereinfachung wird angenommen, daß es nur zwei Arten von Qualifikationen gibt, und es wird mit einer Gruppe qualifizierter Arbeiter $\left(\mathrm{L}_{\mathrm{q}}\right)$ und einer Gruppe weniger qualifizierter Arbeiter $\left(\mathrm{L}_{\mathrm{nq}}\right)$ operiert. Die im vorangegangen Abschnitt beschriebene Annahme einer möglichen Veränderung des Verlaufs der Arbeitsnachfragekurve infolge von Wanderungen qualifizierter Arbeitnehmer wird hier wieder fallengelassen.

Für die Höhe der regionalen Beschäftigung ist aus statischer Sicht entscheidend, ob die Migranten zu den Arbeitern in der Ziel- bzw. Quellregion in komplementärer oder substitutiver Beziehung stehen. Sind die Zuwandernden Substitute für die heimischen Arbeitskräfte, sind sie gleichzeitig deren Konkurrenten. Ihre Zuwanderung kann für die Einheimischen mit Lohnrückgang und/oder Arbeitslosigkeit verbunden sein. Anders ist es, wenn sie deren Komplemente sind. In diesem Fall steigern sie die Produktivität und die Entlohnung der Einheimischen ${ }^{95}$ : Wenn durch die Migranten Lücken gedeckt werden können (etwa bei Facharbeitermangel), so braucht Zuwanderung auch bei einem elastischen Arbeitsangebot nicht mit einem Beschäftigungsrückgang der heimischen Bevölkerung verbunden zu sein. Es könnte sogar zu einer Ausweitung der Beschäftigung von Einheimischen kommen, wenn etwa durch den zusätzlichen Einsatz von Facharbeitern aus der Zuwanderungsregion zusätzlich heimische unqualifizierte Kräfte eingestellt werden können, die vorher arbeitslos waren, bzw. zu den herrschenden Löhnen nicht arbeiten wollten. Andersherum kann aber die Abwanderung dieser Kräfte aus ihrer Heimatregion dazu führen, daß dort Menschen entlassen werden müssen.

94 Vgl. zum Einfluß des nicht-rival nutzbaren Humankapitals Grubel (1994), S. $82 \mathrm{ff}$.

95 Vgl. Straubhaar (1994), S. 11. 
Im günstigsten Fall kommt es zu Wanderungen von Menschen, die über eine Qualifikation verfügen, die in der Abwanderungsregion reichlich und in der Zuwanderungsregion nur knapp vorhanden ist. In diesem Fall könnte in beiden Regionen die Arbeitslosigkeit sinken, es käme nicht zwingend zu einem Produktionsrückgang in der Abwanderungsregion, während es eine Produktionserhöhung und einen Beschäftigungszuwachs in der Zielregion gäbe. Im ungünstigen Fall wandern aber die Menschen mit Qualifikationen, wie sie in der Quellregion als Komplemente gebraucht werden und in der Zuwanderungsregion als Substitute. In diesem Fall könnte es zu einem Beschäftigungsrückgang in der Quellregion über die Abwanderung hinaus kommen und in der Zielregion lediglich zu einer Verdrängung der heimischen Arbeiter.

Wie ein Arbeitsmarkt mit zwei Gruppen von Arbeitern mit unterschiedlicher Qualifikation illustriert werden kann, wird in der folgenden Abbildung deutlich.

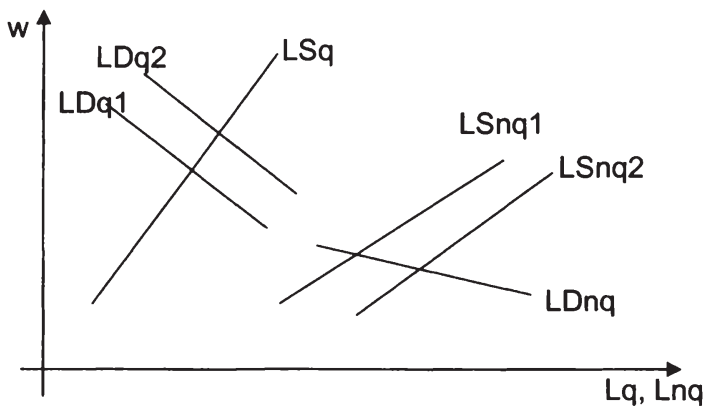

Abbildung 16: Regionale Beschäftigungsentwicklung bei Zuwanderung unqualifizierter Arbeitskräfte ${ }^{96}$

In Abbildung 16 sind in einem Lohn-Beschäftigungsdiagramm das Angebot an und die Nachfrage nach unterschiedlich qualifizierten Arbeitern illustriert. Die Arbeitsnachfragekurven $\mathrm{LS}_{\mathrm{q}}$ (Nachfrage nach qualifizierter Arbeit) und $\mathrm{LS}_{\mathrm{nq}}$ (Nachfrage nach unqualifizierter Arbeit) beschreiben die jeweilige Nachfrage bei gegebenem Lohnsatz der anderen Gruppe von Arbeitern. Betrachtet werde beispielhaft ein Fall von Zuwanderung unqualifizierter Arbeitskräfte. Durch die Zuwanderer verschiebt sich die Arbeitsangebotskurve der unqualifizierten Arbeiter (LSnq1) nach rechts (auf LSnq2). Die Beschäftigung dieser Gruppe steigt, während ihr Lohnsatz sinkt. Dies kann für die Nachfrage nach qualifizierten Arbeitern unterschiedliche Auswirkungen haben. Entweder die unqualifizierten Arbeiter sind Komplemente, dann erhöht sich die Nachfrage nach qualifizierten

96 Vgl. Zimmermann (1993), S. 297. 
Arbeitern, so daß deren Beschäftigung und Löhne steigen. Oder aber die unqualifizierten Arbeiter werden als Substitute eingesetzt (Einstellung von unqualifizierten Arbeiter zu Lasten der qualifizierten), dann sinken die Beschäftigung und die Lohnsätze der qualifizierten Arbeiter. Der erste Fall hätte eine Verschiebung der Arbeitsnachfragekurve nach qualifizierten Arbeitern (LDq) nach oben, der zweite eine nach unten zur Folge.

In der nachfolgenden Abbildung 17 sollen nun Lohnsatz und Einkommen mit und ohne Vereinigung gezeigt werden.

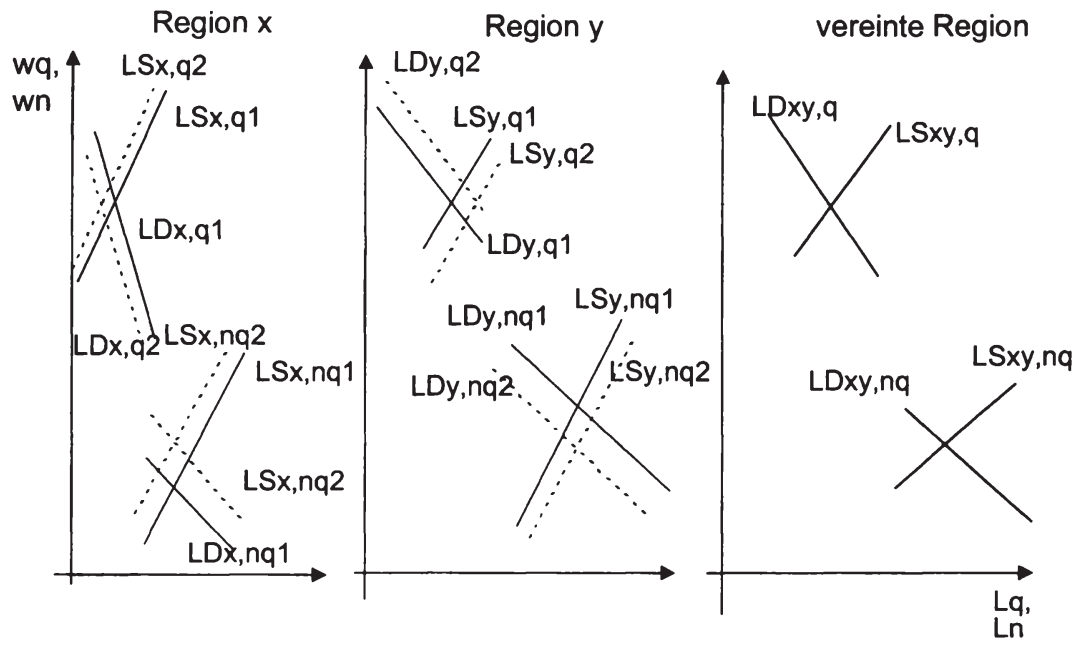

Abbildung 17: Beschäftigungsentwicklung bei Berücksichtigung zweier Arbeitnehmergruppen und Komplementarität

Im dritten Quadranten werden wiederum die aggregierten Angebots- und Nachfragekuven abgebildet. Schwierig zu bestimmen ist die Lage der aggregierten Arbeitsnachfragekurven. Eine einfache Horizontalaggregation der regionalen Kurven vor Vereinigung ist wohl nicht möglich, da eine wechselseitige Abhängigkeit der Arbeitsnachfrage von der Beschäftigung der anderen Arbeitergruppe besteht. Die aggregierten Nachfragekurven vor und nach Vereinigung können sich daher voneinander unterscheiden. Der Einfluß sei anhand eines Beispiels erklärt, wo sich in der Ausgangssituation die Arbeitsmärkte für qualifizierte Arbeit in einem interregionalen Gleichgewicht und die für unqualifizierte Arbeit in einem Ungleichgewicht befinden sollen. Der Lohnsatz für unqualifizierte Arbeit sei in Region y höher. 
Werden die Arbeiter komplementär eingesetzt, so käme es in der Region x aufgrund des durch die Abwanderung steigenden Lohnsatzes für unqualifizierte Arbeit zunächst zu einer sinkenden Nachfrage nach beiden Arten von Arbeit (Abbildung 17: Verschiebung von $\mathrm{LD}_{\mathrm{x}, \mathrm{q} 1}$ auf $\mathrm{LD}_{\mathrm{x}, \mathrm{q} 2}$ und sinkende Nachfrage entlang der $L_{\mathrm{x}, \mathrm{nq}}$-Kurve), in Region y zu einer steigenden Nachfrage (Verschiebung der Nachfragekurve von LDy,q1 auf LDy,q2, steigende Nachfrage entlang der $\mathrm{LD}_{\mathrm{y}, \mathrm{nq} \text { I }}-$ Kurve). Dies induziert Lohnsteigerungen in Region y für qualifizierte Arbeit und führt zu Wanderungen qualifizierter Arbeiter nach y (Verschiebung der Angebotskurve nach außen). Hierdurch sinken die Löhne für die qualifizierte Arbeit in y und das Sinken der Lohnsätze für normale Arbeit in der Niedriglohnregion führt zu einer Erhöhung der Produktivität der dort eingesetzten qualifizierten Arbeit. Die Wanderungen und der Anpassungsprozeß hält solange an, bis sich die Löhne wieder angeglichen haben ${ }^{97}$.

Nun sei angenommen, daß in beiden Regionen qualifizierte Arbeit substitutiv zu unqualifizierter Arbeit eingesetzt werden kann. Bei Beseitigung der Mobilitätsbarrieren wandern unqualifizierte Arbeiter der Region $\mathrm{x}$ in die Region $\mathrm{y}$ (Verschiebung der $\mathrm{LS}_{\mathrm{x}, \mathrm{nq} 1}-$ Kurve nach $\mathrm{LS}_{\mathrm{x}, \mathrm{nq} 2}$ und in Region y Verschiebung der $\mathrm{LS}_{\mathrm{y}, \mathrm{nq} \text { I }}$ auf $\mathrm{LS}_{\mathrm{y}, \mathrm{nq} 2}$ ). Die durch die Wanderungen verursachte Lohnerhöhung für unqualifizierte Arbeit in $\mathrm{x}$ bewirkt eine Erhöhung der Nachfrage nach qualifizierter Arbeit, da sich das Faktorpreisverhältnis geändert hat. In der Region y sinkt dagegen die Nachfrage nach qualifizierter Arbeit, was zu einem sinkenden Lohnsatz für diese Arbeitnehmergruppe führt. Graphisch gesehen kommt es zu einer Verschiebung der $L D_{\mathrm{q}}$-Kurve in Region $\mathrm{x}$ von der in der Abbildung mit $\mathrm{LD}_{\mathrm{x}, \mathrm{q} 2}$ bezeichneten Kurve auf die mit $\mathrm{LD}_{\mathrm{x}, \mathrm{q} 1}$ bezeichnete Kurve und in Region $\mathrm{y}$ entsprechend von $\mathrm{LD}_{\mathrm{y}, \mathrm{q} 2}$ auf $\mathrm{LD}_{\mathrm{y}, \mathrm{q} 1}$. Hierdurch werden wiederum Wanderungen der qualifizierten Arbeitnehmer induziert. Die Wanderungen der Qualifizierten dürften aber wiederum Rückwirkungen auf die Lohn- und Beschäftigungssituation der Unqualifizierten haben, so daß es auch hier wieder zu Anpassungsreaktionen kommt. Nach dem Ende aller Anpassungsprozesse ergibt sich ein interregionaler Ausgleich beider Lohnsätze.

97 Es wurden verschiedene empirische Untersuchungen darüber angestellt, ob Zuwandernde eher Komplemente oder Substitute sind, auch über die Wanderung von Ausländern nach Deutschland. Die Untersuchungen kommen allerdings zu unterschiedlichen Ergebnissen: einige vermuteten daß die Zuwandernden eher Komplemente sind und kaum Einfluß auf Lohn und Beschäftigung hatten, während andere negative Wirkungen feststellten (vgl. hierzu Straubhaar (1994), S. 11f.). 


\subsubsection{Die Tragweite von selektiven Wanderungen für die Entwicklung der Regionen}

Bisher wurde in dieser Arbeit lediglich aus statischer Sicht argumentiert. Da aber Wanderungen auch einen langfristigen Einfluß auf die Entwicklung von Regionen haben, soll in diesem Abschnitt kurz aufgezeigt werden, welche potentiellen Auswirkungen es geben kann.

Die verschiedenen Wachstumsmodelle kommen hinsichtlich der Bewertung von Wanderungen für eine künftige Angleichung der Regionen zu einem unterschiedlichen Ergebnis ${ }^{98}$. Gemäß der exogenen Wachstumstheorie können Auswanderungen von Arbeitskräften aus der weniger entwickelten Region in die besser entwickelte Region zu einer (beschleunigten) Konvergenz der Regionen beitragen. Exogene Wachstumstheorien sind dadurch gekennzeichnet, daß von Produktionsfunktionen mit konstanten Skalenerträgen und abnehmenden Grenzerträgen von Arbeit und Kapital ausgegangen wird. Es werden solange (endogene) Investitionen vorgenommen, bis das Verhältnis von Arbeit zu Kapital optimal ist. Ist die optimale Kapitalintensität erreicht, sinken die Nettoinvestitionen auf Null. Die Rate des technischen Fortschritts ist exogen gegeben und weltweit identisch. Es gibt eine asymptotische Konvergenz der Wachstumsraten (wobei die Wachstumsrate des Sozialprodukts pro Kopf der Rate des technischen Fortschritts entspricht). Gemäß der exogenen Wachstumstheorie kommt es also langfristig zu einer Konvergenz. Durch Wanderungen ändert sich die Kapitalintensität; bei Wanderungen von Arbeitskräften steigt die Kapitalintensität in der Quellregion, während sie in der Zielregion sinkt. Die optimale Kapitalintensität würde mit Vereinigungschneller erreicht als ohne Vereinigung, entsprechend käme es früher zu einer Annäherung der Regionen"9.

Bei Zugrundelegung endogener Wachstumsmodelle ist - im Gegensatz zu exogenen - nicht unbedingt mit einer Konvergenz der Wachstumsraten zu rechnen. Im Rahmen solcher Ansätze wird technischer Fortschritt nicht als exogene Größe, sondern als endogene Größe verstanden. Für die Erklärung endogenen Wachstums gibt es verschiedene Ansätze wie konstante Grenzerträge, endoge

98 Vgl. hierzu etwa Fischer u.a. (1995b).

99 Auf Grundlage des Modells des exogenen Wachstums wurde abgeschätzt, wie lange es dauern könnte, bis sich die Produktivität zwischen Ost- und Westdeutschland angeglichen hat. Nach einer Schätzung von Barro ist eine Angleichung erst Mitte des 21. Jahrhunderts zu erwarten. Eine Schätzung von Dornbusch/Wolf (1992) kam trotz eines etwas optimistischeren Vorgehens auf einen Ausgleichszeitraum, der nur knapp darunter liegt (vgl. Wolf (1994) und Dornbusch/Wolf (1992)). 
ner technischer Fortschritt sowie Unterentwicklungsfallen und deren Überwindung 100 .

Beispielhaft sei ein Fall betrachtet, in dem der Wachstumsprozeß von den privaten Forschungs- und Entwicklungsaktivitäten abhängt ${ }^{101}$. Betrachtet wird eine zwei-Regionen-Welt, in jeder Region gebe es einen traditionellen und einen Forschungssektor. Im traditionellen Sektor werde relativ viel unqualifizierte Arbeit, im Forschungssektor relativ viel qualifizierte Arbeit eingesetzt. Der Erfolg eines Unternehmen habe positive Rückwirkungen auf die Tätigkeiten anderer Unternehmen. Aktionen von Konkurrenten verhelfen also zur Gewinnung neuer eigener Erkenntnisse. Wissen sei ein produktiver Faktor für jede Produktionstätigkeit und darüber hinaus nicht-rival nutzbar. Wie Migration in einer solchen Welt wirkt, hängt wesentlich davon $a b$, wie sich die Wirtschaftsstruktur durch Wanderungen ändert (da ja das Wachstum von dem Forschungssektor abhängt).

Bei Einwanderung unqualifizierter Arbeitskräfte profitiert der Sektor der traditionellen Produktion. Sind die Substitutionselastizitäten zwischen qualifizierter und unqualifizierter Arbeit gering, werden hochqualifizierte Arbeitskräfte aus dem Forschungssektor in Richtung des traditionellen Sektor umverteilt. Hierdurch schrumpft der Forschungssektor, so daß sich der Wachstumsprozeß verlangsamt. Bei einer hohen Substitutionselastizität können dagegen hochqualifizierte Arbeiter im traditionellen Sektor freigesetzt werden. Bei konstanten Weltmarktpreisen dehnt sich der traditionelle Sektor zu Lasten des Forschungssektors aus ${ }^{102}$. Ob sich die Immigration niedrigqualifizierter Arbeit negativ für die Region auswirkt, ist von den Substitutionselastizitäten der Produktion und dem inländischen Produktionsanteil auf den Weltmärkten abhängig: die Wachstumsrate sinkt, wenn die Substitutionselastizitäten der Produktion kleiner sind als der Reziprokwert des inländischen Produktionsanteils auf den Weltmärkten. Für die Abwanderungsregion gilt ein analoger Zusammenhang. Dort verschiebt sich die Struktur bei konstanten Güterpreisen zugunsten des Forschungssektors. Je geringer die Substitutionselastizität ist, desto stärker nimmt der Forschungssektor in seiner Bedeutung zu. Für die beiden Regionen bedeutet dies, daß sie sowohl ohne als auch mit Vereinigung ein höheres oder ein niedrigeres Wachstumspotential aufweisen können.

${ }^{100}$ Vgl. Wolf (1994), S. 189 f.

${ }^{101} \mathrm{Vgl}$. zum folgenden Bretschger (1993) und Bretschger (1993a).

${ }^{102}$ Diese Aussage entspricht dem Rybczynski-Theorem. Das Theorem besagt, daß bei einer Erhöhung der Faktormenge die Produktion des Gutes, daß diesen Faktor relativ stark beansprucht, um mehr als das gesamte Sozialprodukt wächst, während die Erzeugung des anderen Gutes absolut zurückgeht. 
Findet dagegen eine Wanderung qualifizierter Arbeitnehmer statt, sinken in der Zuwanderungsregion die Löhne für qualifizierte Arbeit und damit die Kosten des Forschungssektors. Die Richtung der Auswirkungen einer solchen Wanderung ist von den gleichen Faktoren wie bei unqualifizierter Arbeit abhängig, jedoch mit umgekehrten Vorzeichen.

Von einer gleichmäßiger Einwanderung beider Gruppen profitieren beide Sektoren der Zuzugsregion, während beide Sektoren der Abwanderungsregion von höheren Arbeitskosten betroffen wären. Allerdings entwickelt sich der technische Fortschritt, der auf Rationalisierung der Arbeit ausgerichtet ist, bei Wanderungen anders als ohne: Mit der Einwanderung nimmt der Druck ab, arbeitssparende Innovationen zu entwickeln und einzusetzen, in der Abwanderungsregion nimmt der Druck dagegen zu.

\subsubsection{Zusammenfassung}

Zunächst wurde der potentielle Einfluß der Veränderung der Nachfrage nach nicht-handelbaren Gütern durch Wanderungen dargestellt. Durch Abwanderung kann sich die Absatzsituation vor Ort verschlechtern und zu einer Linksverschiebung der regionalen Arbeitsnachfragekurve führen. Hierdurch käme es über Multiplikatoreffekte zu einer Verringerung der Beschäftigung und des Outputs, die größer wäre als ohne Einfluß der regionalen Nachfrage. Entsprechend nehmen Beschäftigung und Einkommen in der Zuwanderungsregion stärker zu. Die Bedeutung der regionalen Absatzmöglichkeiten scheint aber eher gering zu sein. Das Marktpotential hat nach einer Untersuchung der Prognos-AG (1990) für Europa nur 2\% Gewicht von allen Standortfaktoren.

Wanderungen können in den betroffenen Regionen den Verlauf der Arbeitsnachfragekurven verändern. Ist das Humankapital ein Teil des Kapitalstocks, verschiebt sich die Arbeitsnachfragekurve bei Abwanderung qualifizierter Arbeitnehmer nach links und bei Zuwanderung nach rechts. Die Veränderungen der regionalen Beschäftigung und der Outputs sind höher als ohne solche Berücksichtigung.

Gibt es unterschiedlich qualifizierte Erwerbspersonen, so führt ein regionales Lohnungleichgewicht nicht nur zu Wanderungen von Arbeitnehmern dieser Qualifikation, sondern auch zu Wanderungen andersartig qualifizierter Arbeitnehmer. Die Richtung und Höhe der Wanderungen ist davon abhängig, ob die Arbeiter Substitute oder Komplemente für die jeweils andere Gruppe an Arbeitnehmern ist. 
Ferner wurde gezeigt, daß selektive Wanderungen einen Einfluß auf das wirtschaftliche Wachstum und die Zukunftsaussichten der Regionen haben können. Die unterschiedlichen Wachstumsmodelle kommen zu unterschiedlichen Ergebnissen darüber, ob Wanderungen langfristig zu einer Konvergenz der Regionen beitragen oder ob sie die Unterschiede noch vergrößern.

\subsection{Die Berücksichtigung regionaler Annehmlichkeiten, regionaler Preisunterschiede und Transaktionskosten}

In dem einfachen neoklassischen Modell wurden annahmegemäß ausschließlich aufgrund regionaler Lohnunterschiede Wanderungsentscheidungen getroffen. Im folgenden soll untersucht werden, was es für die Höhe der Wanderungen, das Lohnniveau und die Beschäftigung bedeutet, wenn weitere Wanderungsgründe berücksichtigt werden, nämlich zum einen die Attraktivität von Regionen und zum anderen Wanderungskosten. Offenkundig vermag eine Berücksichtigung solcher Umstände ein andauerndes Bestehen regionaler Reallohnunterschiede zu begründen. Diesbezüglich relevante Zusammenhänge gilt es, im folgenden zu entfalten. Dabei zeigt sich, daß eine Vereinigung unter bestimmten Bedingungen sogar zu einer Umkehrung der interregionalen Reallohnverhältnisse führen kann.

Das Vorgehen in diesem Abschnitt stellt sich wie folgt dar. Zunächst wird nur der Faktor "regionale Annehmlichkeiten"103 einbezogen. Dafür wird eingangs erläutert, was unter regionalen Annehmlichkeiten zu verstehen ist. Im Anschluß werden Indifferenzkurven der Haushalte für Kombinationen von Annehmlichkeiten und Lohnsatz abgeleitet. Ferner werden der potentielle Einfluß regionaler Attraktivität für die Kostensituation der Unternehmen erörtert. Daraufhin wird gezeigt, wann sich eine Region unter dem geänderten Modellrahmen im Gleichgewicht befindet. Anschließend werden im Rahmen einer 2-Regionen-Welt die Auswirkungen einer Vereinigung bestimmt. Danach wird die Bedeutung regionaler Preisunterschiede beschrieben. In Abschnitt 2.4.3 werden persönliche regionale Bindungen und Wanderungskosten berücksichtigt.

${ }^{103}$ Die Begriffe regionale Annehmlichkeiten, bzw. Vorzüge und Attraktivität(sniveau) werden im folgenden synonym verwendet. 


\subsubsection{Regionale Annehmlichkeiten}

\subsubsection{Definition des Begriffs regionale Annehmlichkeiten ${ }^{104}$}

Mit Annehmlichkeiten werden im folgenden bestimmte räumliche Phänomene bezeichnet, die das Leben in einer Region verschönern. Ob etwas angenehm ist, wird hier immer aus Sicht der Haushalte und nicht aus Sicht der Unternehmen bewertet (die Vorzüge von Regionen können daher sowohl kostenerhöhend als auch -senkend auf die Produktion der Unternehmen wirken ${ }^{105}$ ).

Annehmlichkeiten von Regionen können unterschiedlichster Art sein. Ein angenehmes Klima ${ }^{106}$ oder ein hohes Freizeitangebot erfüllen die Kriterien von Annehmlichkeiten, denn sie sind standortgebunden und sie beeinflussen die Lebensqualität positiv. $\mathrm{Zu}$ den regionalen Vorzügen gehört auch die Ausstattung mit Infrastruktur wie Telefon-, Verkehrs-, Versorgungs- und Entsorgungsnetz, Bildungs- und Einkaufsmöglichkeiten ${ }^{107}$. Die Attraktivitätsmerkmale seien an einen bestimmten Standort gebunden. Daher können sie nur dort konsumiert werden, wo sie vorgefunden werden.

Regionale Vorzüge seien für die Haushalte nutzenmäßig substituierbar mit Konsumgütern, und daher auch indirekt mit dem Einkommen. Es wird angenommen, daß sich mit steigendem Einkommen die Nachfrage nach Annehmlichkeiten erhöht. Attraktivitätsniveaus weisen also die Eigenschaften superiorer Gütern auf.

Annehmlichkeiten weisen durchweg Gutseigenschaften öffentlicher Güter auf: Es besteht Nichtrivalität in der Nutzung und es ist nicht möglich jemanden vom Konsum auszuschließen (z.B. bei der Nutzung von Sonnentagen). Jeder, der in der Region ansässig ist, kann damit regionale Vorzüge im gleichenMaße nutzen wie alle anderen auch. Durch seinen Konsum wird die Nutzungsmöglichkeit anderer nicht geschmälert, d.h. das Attraktivitätsniveau ändert sich durch $\mathrm{Zu}$ oder Abwanderung nicht. Diese Annahme ist nicht unproblematisch, da manche Annehmlichkeiten bei hoher Zuwanderung rival in der Nutzung werden können, zur Vereinfachung soll jedoch zunächst mit dieser Annahme gearbeitet wer-

${ }^{104}$ Zum Konzept der Annehmlichkeiten vgl. etwa Diamond/Tolley (1982), S. $5 \mathrm{ff}$.

${ }^{105}$ Vgl. hierzu Abschnitt 2.4.1.2.2.

${ }^{106}$ Zum Einfluß des Klimas auf die Migration vgl. insbesondere Graves (1980) und Graves (1979).

${ }^{107}$ Nicht zu den Annehmlichkeiten zählen die Verbundenheit mit der Heimat oder das Vorhandensein von Freunden und Verwandten vor Ort. Diese Faktoren werden in einem späteren Schritt berücksichtigt (vgl. Abschnitt 2.4.3). 
den $^{108}$. Problematisch ist außerdem, daß die Haushalte regionale Vorzüge unterschiedlich bewerten. Annehmlichkeiten für den einen können Unannehmlichkeiten für einen anderen sein. Auch diese Problemdiemension wird zur Vereinfachung im folgenden ausgeklammert.

\subsubsection{Regionale Annehmlichkeiten und ihre allgemeine Bedeutung für Arbeitsmärkte}

Im folgenden wird gezeigt, welche Standortentscheidung die Haushalte und Unternehmen treffen, wenn zwischen alternativen Lohn- und Attraktivitätsniveaus gewählt werden kann. Es gelten die Annahmen aus Kapitel2.1, d.h.: homogene Arbeit, vollkommene Markttransparenz, keine Transaktions- und Informationskosten, flexibler Lohnsatz und konkurrenzmarktliche Marktverfassung. Bevor in Abschnitt 2.4.1.3 die Konsequenzen für den Fall einer Vereinigung gezeigt werden, werden die Grundzusammenhänge in diesem Abschnitt zunächst anhand einer Volkswirtschaft mit sehr vielen Regionen erläutert, wobei die verschiedenen Regionen mit den unterschiedlichsten Annehmlichkeitsniveaus ausgestattet seien.

\subsection{Einfluß regionaler Annehmlichkeiten auf die Lohnforderungen der Arbeitsanbieter}

Als erstes seien die Standortentscheidungen der Haushalte betrachtet. Der Nutzen U eines Haushaltes hänge außer von der Menge an Konsumgütern X, die er mit seinem Einkommen nachfragen kann, und der Menge an Freizeit (F) auch von dem Attraktivitätsniveau (An) seiner Wohnregion i ab:

$\mathrm{U}=\mathrm{U}\left(\mathrm{X}, \mathrm{F}, \mathrm{An}_{\mathrm{i}}\right)$

Zur Vereinfachung wird auf die weitere Betrachtung der Variable Freizeit verzichtet $^{109}$.

Der Haushalt habe keine Möglichkeit durch sein Verhalten das regionale Lohnniveau zu beeinflussen, da er nur einer von sehr vielen Arbeitsanbietern ist. Auch kann er das Attraktivitätsniveau von Regionen nicht beeinflussen. Er kann

${ }^{108}$ Eine Einbeziehung von bevölkerungsgrößenabhängigen Annehmlichkeiten findet sich etwa bei Mathur/Stein (1991), eine Analyse über die Bedeutung von Zuwanderung für die Nutzungsmöglichkeit öffentlicher Güter (Klubgüter) bei Straubhaar (1992).

${ }^{109}$ Bei der Arbeitszeit-Freizeit-Entscheidung wird bereits der Nutzen aus Freizeit berücksichtigt und geht in die Arbeitsangebotsfunktion ein. 
daher sein Nutzenniveau nur dadurch verändern, indem er umzieht. Er kann zwar theoretisch Ausflüge in andere Regionen machen, um dort vorübergehend die regionalen Vorzüge in Anspruch zu nehmen. Hierfür müßte er jedoch Reisekosten aufwenden, so daß seine sonstigen Konsummöglichkeiten geschmälert würden. Diese Reisekosten seien jedoch so hoch, daß sich ein solches Verhalten für kein Individuum lohne. Die Kosten, die hingegen durch einen Umzug entstehen, fallen nur einmalig an und seien daher zu vernachlässigen ${ }^{110}$.

Der Haushalt biete in jeder Region gemäß seiner Lohn-Freizeit-Präferenzen Arbeit an und erhalte hierfür ein Arbeitseinkommen in Abhängigkeit vom regionalen Lohnniveau $\mathrm{w}_{\mathrm{i}}{ }^{111}$. Es kann daher eine indirekte Nutzenfunktion V in Abhängigkeit vom Lohneinkommen und dem Attraktivitätsniveau formuliert werden ${ }^{112}$, die lautet:

$\mathrm{V}=\mathrm{V}\left(\mathrm{w}_{\mathrm{i}}, \mathrm{An}_{\mathrm{i}}\right)$

Die partiellen Grenznutzen des Lohneinkommens und des Attraktivitätsniveaus seien positiv: $\mathrm{V}_{\mathrm{W}}>0, \mathrm{~V}_{\mathrm{A}}>0$. Lohneinkommen und Attraktivitätsniveau seien substituierbar, so daß unterschiedliche Einkommen bei ungleichen regionalen Vorzügen einen identischen Nutzen stiften können ${ }^{113}$.

Kombinationen von Lohneinkommen und Annehmlichkeiten, welche dem Haushalt den gleichen Nutzen stiften, lassen sich graphisch mit Hilfe von Indifferenzkurven darstellen. Die Indifferenzkurven weisen eine negative Steigung auf (der Nutzenverlust durch einen Lohnrückgang kann ausgeglichen werden durch ein höheres Attraktivitätsniveau) und sind konvex zum Ursprung gekrümmt, wenn mit jeder weiteren Minderung des Einkommens die zum Nutzenausgleich erforderliche Menge an Annehmlichkeiten zunimmt. Die Steigung der Indifferenzkurven drückt auch die Zahlungsbereitschaft der Haushalte für ein angenehmes Wohn- und Arbeitsumfeld aus. ${ }^{114}$

${ }^{110}$ Die Problematik der Umzugskosten wird in den Abschnitten 2.4.3 und 3.1.2.5 und 3.1.2.5 erörtert.

${ }^{\prime \prime \prime}$ Die Arbeitszeit-Freizeit-Entscheidung dürfte in den Regionen unterschiedlich ausfallen. In den attraktiven Gegenden ist die Freizeit attraktiver im Vergleich zu dem Konsum von X als in unattraktiveren Gegenden. Die Indifferenzkurven für Freizeit-Einkommen dürften in attraktiven Gebieten daher steiler verlaufen als in unattraktiven, so daß ein Individuum in schönen Regionen weniger Arbeit anbieten würde, als er dies in einer weniger attraktiven Region zu dem gleichen Lohnsatz machen würde.

${ }^{1 / 2}$ Dies ist allerdings keine echte indirekte Nutzenfunktion, sondern eine Mischung zwischen indirekter und direkter: Sie hängt direkt von den Annehmlichkeiten und indirekt von dem Lohnsatz ab.

${ }^{11.3}$ Vgl. Roback (1982), S. 1260.

${ }^{114} \mathrm{Vgl}$. Rosen (1979), S. 80. 


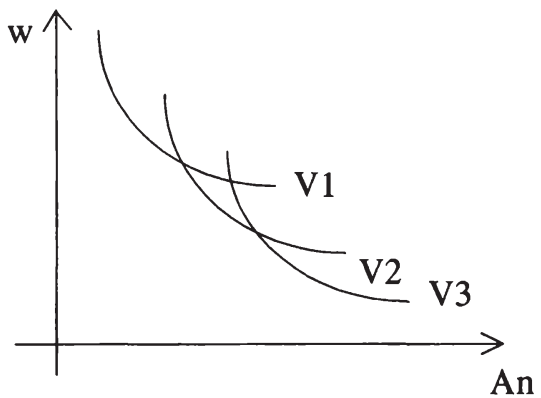

Abbildung 18: Indifferenzkurven für Einkommen und regionale Annehmlichkeiten ${ }^{115}$

Abbildung 18 zeigt jeweils eine Indifferenzkurve (V1,V2 und V3) drei verschiedener Individuen, wobei jedes Individuum andere Präferenzen bezüglich der Kombination von Lohn und Annehmlichkeiten hat. Auf der Ordinate ist der Lohnsatz und auf der Abszisse das Attraktivitätsniveau von Regionen abgetragen. Je weiter die Indifferenzkurven vom Ursprung entfernt sind, desto höher ist ihr Nutzenniveau. Indifferenzkurven dieser Art werden in der Literatur auch als "wage-acceptance" Funktion bezeichnet ${ }^{116}$.

\subsection{Einfluß regionaler Annehmlichkeiten auf die Kosten der Unternehmen}

Annehmlichkeiten haben nicht nur einen Einfluß auf die Standortentscheidung von Individuen, sondern auch auf die Unternehmen, da die Produktionskosten von den Ausstattungsmerkmalen einer Region beeinflußt werden können.

$\mathrm{Zu}$ den regionalen Merkmalen, die einen Einfluß auf die Produktionskosten ausüben, zählen unter anderen Agglomerationsvor- und -nachteile. Dies sind z.B. Konsequenzen, die sich für ansässige Unternehmen durch die Größe des regionalen Absatzmarktes ergeben. Diese Vorteile verstärken sich u.a. durch Bevölkerungswachstum infolge von Wanderungen und der damit verbundenen Steigerung des regionalen Nachfragepotentials. Weitere Agglomerationsvorteile sind das Vorhandensein von Vorleistungs- und Reparaturbetrieben und ein großes Facharbeiterpotential vor Ort sowie die vorhandene Infrastruktur. Zur Vereinfachung werden in der nachfolgende Analyse nur bevölkerungsgrößenunabhängige Faktoren berücksichtigt.

\footnotetext{
${ }^{115}$ Vgl. etwa Henderson (1982), S.40.

${ }^{116}$ Vgl. Clark/Cosgrove (1991), S. 314.
} 
Ein Teil der unternehmensrelevanten Faktoren stiftet auch den Haushalten einen Nutzen. Hierzu gehört im allgemeinen die Infrastruktur. Sie kann einen höheren Output bei gleichem Arbeits- und Kapitaleinsatz bzw. geringere Kosten pro Ausbringungseinheit ermöglichen. Ist eine kostensenkende Infrastruktur vorhanden, kann der Lohnsatz (unter sonst gleichen Umständen) höher sein als bei einer schlechten Infrastrukturausstattung und identischen regionalen Stückkosten.

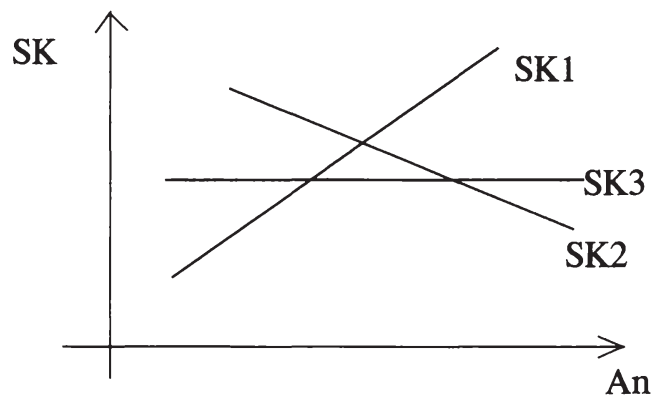

Abbildung 19: Kostenverläufe bei alternativen Wirkungsrichtungen der regionalen Annehmlichkeiten

Abbildung 19 zeigt verschiedenartige Zusammenhänge zwischen Stückkosten (SK) und Attraktivitätsniveau. SK1 zeigt einen Kostenverlauf, in der die Eigenheiten einer Region kostensteigernd für das betreffende Unternehmen wirken, während sie bei SK2 die Kosten senken. In SK3 hat das Attraktivitätsniveau keinen Einfluß auf die Kosten. In der Literatur wird in der Regel davon ausgegangen, daß Annehmlichkeiten, die unabhängig von der Bevölkerungsgröße bzw. dichte sind, kostensteigernd wirken ${ }^{117}$. Dieser Auffassung wird hier nicht gefolgt, da es viele Annehmlichkeiten - wie etwa die Infrastruktur - gibt, die die Produktionskosten senken dürften.

Da die Erlöskurve unter den getroffenen Annahmen in allen Regionen identisch verläuft, ergibt sich bei kostensenkenden Annehmlichkeiten, für die Unternehmen in der attraktivsten Gegend der größte Gewinn. Bei kostensenkenden Annehmlichkeiten wäre eine Unternehmung nur dann bereit, sich in Gegenden mit niedrigerem Attraktivitätsniveau anzusiedeln, wenn sie dort andere kostensenkende Faktoren vorfindet, die es ihr ermöglichen, mindestens den gleichen Gewinn wie in der attraktiveren Region zu erzielen ${ }^{118}$. So ein Faktor können regionale Lohnunterschiede sein. Welche Kombinationen von Lohnsatz und An-

${ }^{117}$ Vgl. etwa Roback (1982) und Mathur/Stein (1991).

118 Dabei wird vernachlässigt, daß die Unternehmer selbst die Annehmlichkeiten als nutzenstiftend empfinden und sich hiervon bei ihrer Standortwahl beeinflussen lassen. 
nehmlichkeiten für die Unternehmen gleichwertig sein können, wird in Abbildung 20 illustriert.

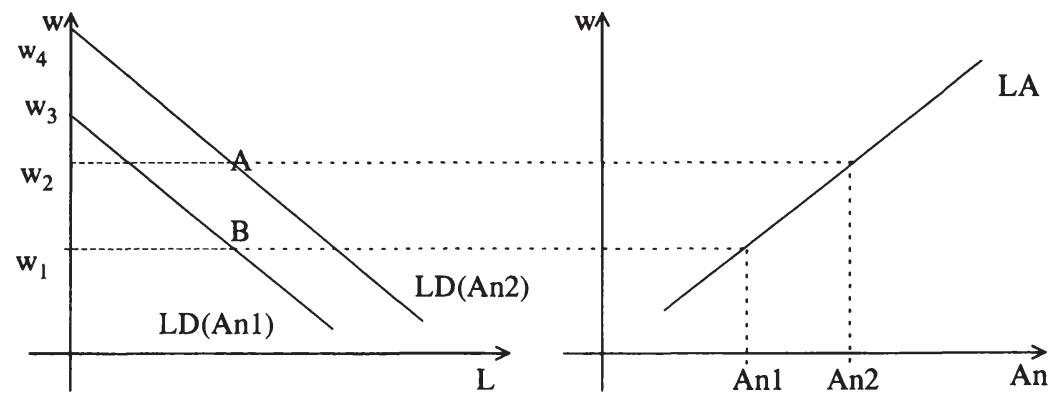

Abbildung 20: Ableitung von Lohn-Angebotskurven

Abbildung 20 zeigt im linken Diagramm zwei Arbeitsnachfragefunktionen für zwei unterschiedliche Attraktivitätsniveaus. Die Annehmlichkeiten An1 seien geringer als An2, Annehmlichkeiten wirken kostensenkend. Bei An2 verläuft die Kostenkurve daher unterhalb von An1, die gewinnmaximale Outputmenge des Unternehmens ist höher und für die größere Produktionsmenge wird mehr Arbeit nachgefragt. Die Arbeitsnachfragekurve bei An2 (LD(AN2)) verläuft daher rechts von $L D(A n 1)$. Wenn bei einem Attraktivitätsniveau Anl der Lohnsatz $w_{1}$ gezahlt wird, machen die Unternehmen genauso viel Gewinn $\left(\mathrm{w}_{1} \mathrm{Bw}_{3}\right)$ wie bei An2 und $w_{2}\left(\right.$ Gewinn $\left.=w_{2} A w_{4}\right)$. Diese Kombinationen können in ein Lohn-Attraktivitäts-Diagramm übertragen werden. Die Verbindung der Kombinationen ergibt eine Isogewinnlinie. Von diesen Kurven gibt es unendlich viele, die jeweils einen anderen Gewinn der Unternehmung kennzeichnen. Diese Kurven können auch als "Lohn-Angebotskurven" (wage-offer function ${ }^{119}$ ) bezeichnet werden, da sie anzeigen, welchen Lohnsatz eine Unternehmung bei unterschiedlichen Attraktivitätsniveaus zahlen kann, ohne daß sich ihr Gewinn ändert. Jeder Punkt unterhalb der Kurve bedeutet einen höheren Gewinn der Unternehmung, jeder Punkt darüber einen geringeren (effizienter Einsatz der Produktionsfaktoren vorausgesetzt).

Die Lohn-Angebotskurven alternativer Unternehmen werden unterschiedlich verlaufen; sie sind abhängig von dem Einfluß des Attraktivitätsniveaus auf die Kostenfunktion. Je geringer der Einfluß der Annehmlichkeiten ist, desto fla-

${ }^{119}$ Vgl. Clark/Cosgrove (1991), S. 314. 
cher wird die Lohn-Angebotskurve verlaufen. Bei kostenneutrale Annehmlichkeiten verlaufen die Isogewinnlinien parallel zur Abszisse. Bei kostenerhöhenden Annehmlichkeiten weist die Lohn-Angebotskurve eine negative Steigung auf.

\subsection{Das Arbeitsmarktgleichgewicht}

Das System befinde sich dann im Gleichgewicht, wenn kein Individuum seinen Nutzen und kein Unternehmen seine Rendite durch eine alternative Standortwahl erhöhen kann und somit keine Wanderungsbewegungen - weder von Arbeit noch von Kapital - stattfinden. Bei welchen Lohn-Annehmlichkeitskombinationen sich das System im Gleichgewicht befindet, soll nun anhand einer Graphik illustriert werden.

Die Indifferenzkurven dreier Individuen $\left(\mathrm{V}_{\mathrm{a}}, \mathrm{V}_{\mathrm{b}}, \mathrm{V}_{\mathrm{c}}\right)$ und die Lohn-Angebotsfunktionen drei verschiedener Unternehmen $\left(\mathrm{LA}_{1}, \mathrm{LA}_{2}, \mathrm{LA}_{3}\right)$, die eine identische Rendite erzielen und für die die regionalen Vorzüge kostensteigernd wirken, können - stellvertretend für alle Wirtschaftssubjekte der Volkswirtschaft - in einem Lohn-Attraktivitätsniveaudiagramm zusammengeführt werden. Die Haushalte versuchen, ihren Nutzen zu maximieren, d.h. die höchstmögliche Indifferenzkurve zu erreichen. Bei gegebenen Lohn-Angebotskurven der Unternehmen wird im Gleichgewicht jeweils der Tangentialpunkt von Indifferenzkurve und Lohn-Angebotskurve realisiert.

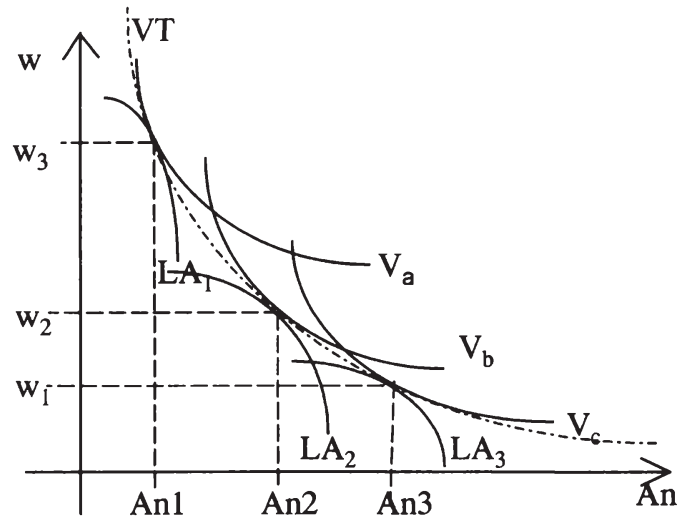

Abbildung 21: Gleichgewichtssituationen bei alternativen LohnAttraktivitätsniveaus 
Die Tangentialpunkte in Abbildung 21 zeigen gleichgewichtige Kombinationen von Löhnen und Annehmlichkeiten an. Kein Arbeiter wäre bereit, eine Arbeit zu Konditionen unterhalb der Verbindungslinie der Tangentialpunkte (VT) zu akzeptieren, weil sein Nutzen dort geringer als sein maximal möglicher Nutzen wäre. Unternehmen des Typs $1\left(\mathrm{LA}_{1}\right)$ würden in jeder anderen Region einen geringeren Gewinn erzielen. Ein Unternehmen, das für ein gegebenes Attraktivitätsniveau einen niedrigeren Lohnsatz anbietet als die Tangentialverbindungslinie anzeigt, würde niemanden finden, der bereit wäre, zu diesem Lohnsatz zu arbeiten.

Als Ergebnis impliziert das Modell - wenn alle möglichen Attraktivitätsniveaus und Präferenzen existieren -, daß im Gleichgewicht in jedem Gebiet nur Individuen mit identischem Geschmack (= identische Indifferenzkurvensysteme) wohnen. Jede Präferenzänderung würde zur Migration des betreffenden Haushalts führen. Änderungen der Annehmlichkeiten eines Ortes können c.p. zur vollständigen Auswanderung der bisherigen Bewohner führen. Gäbe es weniger Attraktivitätsniveaus als individuelle Geschmacksunterschiede, würde es allerdings Regionen geben, in denen Menschen mit unterschiedlichen Geschmäckern leben. Bei gegebenen Lohn werden dann einige Menschen mehr verdienen, als man ihnen bieten müßte, um das vorhandene Attraktivitätsniveau zu akzeptie$\operatorname{ren}^{120}$.

Wenn die Attraktivität von Regionen dagegen eine kostensenkende Wirkung auf die Produktion entfalten würde, sähen die Ergebnisse etwas anders aus. Aufgrund der positiven Steigung der Lohn-Angebotsfunktionen der Unternehmen würden in den attraktivsten Regionen die höchsten Löhne gezahlt werden. Alle Haushalte hätten ein Interesse daran in dieser Gegend zu wohnen.

In Abbildung 22 sei $\mathrm{An}_{\max }$ das maximale Niveau an Annehmlichkeiten in der Modellwelt. Nur bei Realisierung der Lohn-Attraktivitäts-Kombination ( $w_{\mathrm{GG}}$, $\mathrm{An}_{\max }$ ) kommt es zu einem Gleichgewicht. Die Kurven $\mathrm{LA}_{1}$ und $\mathrm{LA}_{2}$ zeigen Lohn-Angebotskurven von zwei alternativen Unternehmen bei einer konstanten Rendite. Unternehmen des Typs 1 (Lohn-AngebotskurveLA $A_{1}$ ) seien die, welche bei $A n_{\max }$ den höchsten Lohn (bei Erzielung der gleichen Rendite wie die Konkurrenzunternehmen) zahlen. Alle anderen bieten bei $\mathrm{An}_{\max }$ einen Lohn, der geringer ist ${ }^{121}$. Alle Unternehmen sind aufgrund des kostensenkenden Charakters der Annehmlichkeiten bereit, bei $\mathrm{An}_{\max }$ den jeweils höchsten Lohn zu zahlen, das Lohnangebot vom Unternehmen des Typs 1 ist jedoch höher als

${ }^{120} \mathrm{Vgl}$. hierzu Henderson (1982), S. 41.

${ }^{121}$ Dabei ist durchaus zulässig, daß sie bei einem geringeren Attraktivitätsniveau einen höheren Lohn als Unternehmen des Typs 1 zu zahlen bereit sind, Bedingung bleibt jedoch, daß die Lohn-Angebotskurve für alle Unternehmen eine positive Steigung aufweist. 
das von Unternehmen des Typs 2. Daher wollen alle Arbeiter bei Unternehmen 1 zum Lohn $\mathrm{w}_{\mathrm{GG}}$ in der Region mit dem Attraktivitätsniveau $\mathrm{An}_{\max }$ arbeiten, da hier alle Individuen - unabhängig davon, ob sich ihre Präferenzen unterscheiden - ihren Nutzen maximieren. Nirgendwo ist es möglich, mehr Lohn und/oder ein höheres Attraktivitätsniveau zu erzielen. Die Unternehmen vom Typ 2 könnten mit der gegebenen Lohn-Angebotsfunktion keine Arbeitnehmer attrahieren, das gesamte Arbeitsangebot würde sich auf Unternehmen des Typs 1 konzentrieren. Unternehmen vom Typ 2 würden - bei den implizit oder explizit getroffenen, aber nicht unbedingt realistischen Annahmen - vom Markt verschwinden und nur noch Unternehmen des Typs 1 übrigbleiben.

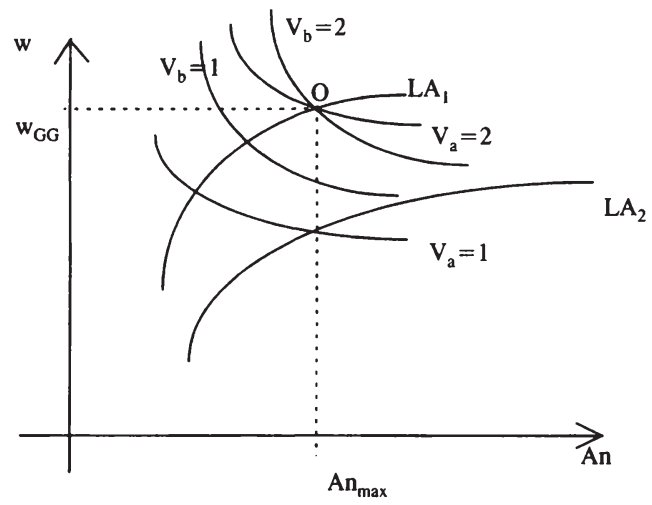

Abbildung 22: Gleichgewicht bei durchgängig kostensenkendem Einfluß der regionalen Annehmlichkeiten

\subsubsection{Vereinigungsbedingte Auswirkungen regionaler Annehmlichkeiten}

Unter Zugrundelegung des oben beschriebenen Modellrahmens werden im folgenden die Auswirkungen einer Vereinigung für Lohnniveau, Beschäftigung und Sozialprodukt bestimmt. Dabei werden nicht die genauen Änderungen berechnet, sondern es wird gezeigt, ob sich die Ergebnisse im Vergleich zum Modell ohne Berücksichtigung von Annehmlichkeiten verstärken oder abschwächen. Auf die explizite Untersuchung der Kapitaleinkommensänderung wird verzichtet, da sie sich immer in die gleiche Richtung wie das Sozialprodukt entwickelt. Die Änderungen der Lohnsummen hängen in hohem Maße von den Arbeitsangebots- und -nachfrageelastizitäten ab. Dies wurde bereits oben ausführlich behandelt; eine erneute Untersuchung brächte keine zusätzlichen Erkenntnisse, daher wurde sie ausgeklammert. 
Im folgenden werden nicht mehr beliebig viele, sondern zwei sich vereinigende Regionen betrachtet. Diese Regionen sollen sich hinsichtlich ihrer Ausstattung mit Annehmlichkeiten unterscheiden, deren jeweiliges Niveau sei aber intraregional identisch. Vor der Vereinigung herrsche in jeder Region ein Gleichgewicht in dem Sinn, daß jedes Individuum bei den gegebenen Bedingungen sein maximal realisierbares Nutzenniveau erreicht.

Oben wurde dargestellt, daß es nach Beseitigung von Mobilitätsbarrieren zu Wanderungen in die Region mit dem höheren Lohnniveau kommt. Eine eindeutige Beziehung dieser Art ist nicht mehr gegeben, wenn zusätzlich der Nutzengewinn aus der potentiellen Nutzungsmöglichkeit regionaler Vorzüge berücksichtigt wird. Es gilt aber weiterhin, daß die Richtung der Wanderungsströme immer davon abhängt, in welcher Region die Haushalte den höheren Nutzen realisieren, und die Menschen solange wandern, bis sich das für sie maximal erreichbare Nutzenniveau in beiden Regionen angeglichen hat. Mit anderen Worten: Wanderungen kommen zum Stillstand, wenn niemand mehr eine Erhöhung des Nutzenniveaus erzielen kann.

Aufgrund der vorangegangenen Untersuchung ist zu vermuten, daß die vereinigungsbedingten Konsequenzen auf den Arbeitsmarkt neben dem Verlauf von Arbeitsangebots- und -nachfragekurven auch von dem Ausmaß der Präferenzen der Haushalte für Annehmlichkeiten sowie von dem Einfluß der regionalen Vorzüge auf die Kostenfunktion der Unternehmen abhängig sein könnten. Dies soll im folgenden mit überprüft werden.

\subsection{Bedingungen, unter denen Wanderungen in die Hochlohnregion induziert werden}

Zunächst wird anhand einer graphischen Betrachtung untersucht, wann eine Vereinigung zu Wanderungen in die Hochlohnregion führt.

Abbildung 23 zeigt Lohn-Annehmlichkeits-Diagramme für die beiden sich vereinigenden Regionen $\mathrm{x}$ und $\mathrm{y}$ im Fall kostensenkender regionaler Vorzüge. Region $x$ sei mit einer geringeren Menge an Annehmlichkeiten $\left(A n_{x}\right)$ ausgestattet als Hochlohnregion y $\left(\mathrm{An}_{\mathrm{y}}\right)$. LA $\mathrm{x}$ sei die Lohn-Angebotskurve vor Vereinigung in $\mathrm{x}, \mathrm{LA}_{\mathrm{y}}$ die der Region $\mathrm{y}$. In beiden Regionen leben identische Individuen, so daß sich die Indifferenzkurvensysteme nicht voneinander unterscheiden. 


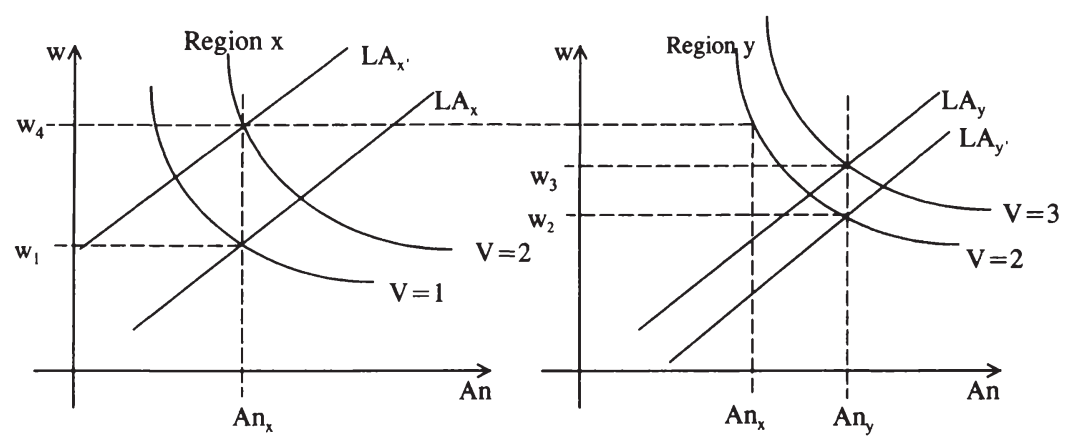

Abbildung 23: Wanderungen in die Hochlohnregion bei kostensenkenden regionalen Annehmlichkeiten

Vor der Vereinigung realisieren die Haushalte in der Region $\mathrm{x}$ ein Nutzenniveau in Höhe von $V=1^{122}$. Die Haushalte in der Region y erreichen das höhere Nutzenniveau $\mathrm{V}=3$. Nicht nur die Annehmlichkeiten, sondern auch das Lohnniveau sei in Region y höher: Es betrage in Region $\mathrm{yw}_{3}$, während in Region x nur ein Lohnsatz in Höhe von $w_{1}$ gezahlt werde.

Werden die bestehenden Wanderungsbarrieren im Zuge einer Vereinigung beseitigt, besteht zunächst für alle Beschäftigten aus x ein Anreiz in die Region y auszuwandern, da dort höhere Löhne gezahlt werden und die Menge an regionalen Vorzügen größer ist. Für die Bewohner der Region y ergeben sich hingegen keine Wanderungsanreize - weder lohn- noch infrastrukturbedingte. Infolge von Abwanderung aus der Region $\mathrm{x}$ in die Region y kommt es zu Lohnanpassungen in beiden Gebieten, die die Wanderungsanreize der Bevölkerung in $\mathbf{x}$ vermindern. In y sinkt der Lohnsatz durch die Erhöhung des Arbeitsangebots bei unveränderter Arbeitsnachfragefunktion. Hierdurch steigt die Beschäftigung, das Sozialprodukt und das Kapitaleinkommen in y. Es wird eine neue LohnAngebotskurve erreicht: statt $\mathrm{LA}_{\mathrm{y}}$ realisieren die Unternehmen die unterhalb verlaufende Lohn-Angebotskurve LA $_{y^{\prime}}$. Als Folge wird relativ mehr Arbeit als vorher eingesetzt. In der Region $\mathrm{x}$ steigt der Lohnsatz, während die Beschäftigung, das Sozialprodukt und die Kapitaleinkommen sinken. Die neue Lohn-Angebotskurve liegt in Region $\mathrm{x}$ links der ursprünglichen.

Ein interregionales Gleichgewicht ist erreicht, wenn alle Haushalte ein einheitliches Nutzenniveau $(\mathrm{V}=2)$ realisieren: Der Lohnsatz $\mathrm{w}_{4}$ in Kombination mit $\mathrm{An}_{\mathrm{x}}$ bringt den Haushalten den gleichen Nutzen wie $\mathrm{An}_{\mathrm{y}}$ und $\mathrm{w}_{2}$. Bei konstanter

${ }^{122}$ Die Werte haben nur ordinale Bedeutung: Eine höhere Zahl zeigt lediglich ein höheres Nutzenniveau an. 
Infrastrukturausstattung können die Anpassungsprozesse nur über den Lohnsatz erfolgen. Ein neues Gleichgewicht ist erst dann erreicht, wenn die Löhne in der Region $\mathrm{x}$ oberhalb des neuen Lohnniveaus der ehemaligen Hochlohnregion liegen. Im Ergebnis verkehrt sich damit die Ausgangssituation: die ehemalige Niedriglohnregion entwickelt sich zur Hochlohnregion.

Wanderungen in die Hochlohnregion finden in einer Welt mit präferenzmäßig identischen Individuen somit immer dann statt, wenn das Nutzenniveau der Hochlohnregion vor Vereinigung höher ist als das Nutzenniveau in der Niedriglohnregion. Das gilt auch, wenn die Hochlohnregion nicht mit regionalen Vorzügen ausgestattet ist. Im Gleichgewicht muß der Nutzen, der aus der Differenz der Einkommensunterschiede gezogen wird, identisch sein mit dem Nutzen aus der Differenz der Unterschiede in den regionalen Vorzügen. Ist das Niveau an Annehmlichkeiten in der Niedriglohnregion vergleichsweise hoch, so bleibt sie auch nach Wanderungen die Niedriglohnregion, andernfalls wird sie zur Hochlohnregion.

Die Art des Einflusses der Infrastrukturausstattung auf die Produktionskosten ist für die Richtung der Anpassung unerheblich. Bevor dieses Resultat für kostensteigernde Infrastruktur erläutert wird, sei zunächst der Fall kostenneutraler Infrastruktur betrachtet.

Ohne Einfluß der Infrastruktur verlaufen die Lohn-Angebotskurven parallel zur Abszisse, und zwar bei einem Lohnsatz, der mit der Grenzproduktivität der Arbeit übereinstimmt. Betrachtet werde ein Fall, bei dem ohne Vereinigung in Region $\mathrm{x}$ ein Lohnsatz von $\mathrm{w}_{1}$ und in Region y ein Lohnsatz in Höhe von $\mathrm{w}_{2}$ herrsche (Abbildung 23 - die Lohn-Angebotskurven entsprechen den Horizontalen durch $w_{1}$ bzw. $w_{2}$, die Indifferenzkurven verlaufen wie eingezeichnet). Infolge einer Vereinigung käme es zu den gleichen Anpassungsprozessen wie im Fall kostensenkender Infrastruktur: Es gäbe massive Abwanderungen von $\mathrm{x}$ nach y (wenn die Regionen wiederum mit Infrastruktur in Höhe von $\mathrm{An}_{\mathrm{x}}$ und $\mathrm{An}_{\mathrm{y}}$ ausgestattet sind), solange bis sich die Nutzenniveaus der Haushalte über den Raum angeglichen hätten. Da die Ausstattung der Regionen mit Annehmlichkeiten unverändert bleibt, ist der Lohnsatz in der Region $\mathrm{x}$ am Ende aller Anpassungsprozesse höher als in der Region y.

Der Fall kostensteigernder Infrastruktur sei anhand vonAbbildung 24 illustriert. Im Vergleich zu Abbildung 23 weisen die Lohn-Angebotskurven hier einen fallenden Verlauf auf. Es fällt auf, daß bei einem gegebenen maximalen Niveau an Annehmlichkeiten ein Gleichgewichtspunkt kein Tangentialpunkt von Indifferenzkurve und Isogewinnlinie sein muß. Mögliche Tangentialpunkte liegen 
rechts des Gleichgewichts, also bei einem höheren als dem gegebenen Annehmlichkeitsniveau und können daher nicht realisiert werden.

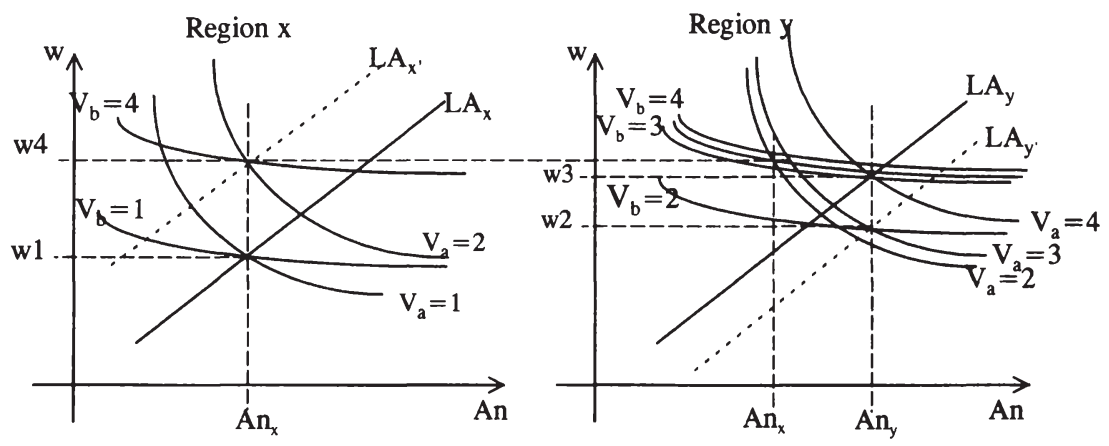

Abbildung 24: Wanderungen in die Hochlohnregion bei kostenerhöhenden regionalen Annehmlichkeiten

Region x (y) weist vor Vereinigung ein Annehmlichkeitsniveau $\mathrm{An}_{\mathrm{x}}\left(\mathrm{An}_{\mathrm{y}}\right)$ auf, die Lohn-Angebotsfunktion ist durch $\mathrm{LA}_{\mathrm{x}}\left(\mathrm{LA}_{\mathrm{y}}\right)$ gekennzeichnet und die Haushalte realisieren bei Löhnen in Höhe von $w_{1}$ respektive $w_{3}$ ein Nutzenniveau von $\mathrm{V}=1(\mathrm{~V}=3)$ auf. Da sich bei dieser Ausgangssituation wiederum die Haushalte in $\mathrm{x}$ durch Wanderung in die Region y nutzenmäßig verbessern können, finden Anpassungsprozesse nach dem oben beschriebenem Muster statt. Die Löhne in x steigen bei sinkender Beschäftigung und sinkendem Sozialprodukt, und zwar solange, bis im neuen Gleichgewicht die Löhne in $\mathrm{x}$ höher als in Region y sind.

\subsection{Bedingungen, unter denen Wanderungen in die Niedriglohnregion bzw. keine Wanderungen induziert werden}

Eine entgegengesetzte Entwicklung der Löhne und der regionalen Beschäftigung kann sich ergeben, wenn die Hochlohnregion weniger regionale Vorzüge als die Niedriglohnregion aufweist. Der maximal erzielbare Nutzen kann dann vor Vereinigung in der Niedriglohnregion höher sein als in der Hochlohnregion. Eine Beseitigung der Wanderungsbarrieren löste dann Migrationen in die Region mit den niedrigeren Löhnen aus, mit der Folge, daß sich die Löhne nicht angleichen, sondern sich noch weiter voneinander entfernen bzw. das Verhältnis sich umkehrt. Die Beschäftigung und das Sozialprodukt in der ehemaligen Niedriglohnregion stiegen, während in der anderen Region eine gegenläufige Entwicklung zu verzeichnen wäre. Die Vereinigung bewirkte eine Abwanderung aus pro- 
duktiven Arbeitsplätzen in unproduktivere, da der Gewinn zusätzlicher Nutzungsmöglichkeiten von Infrastruktur von den Haushalten höher bewertet wird als der Verlust eines Teils ihres Geldeinkommens.

Realisieren alle Haushalte vor Vereinigung das gleiche Nutzenniveau (graphisch gesehen wäre das der Fall, wenn bereits vor Vereinigung alle Haushalte V1 realisieren), so gibt es keine Anpassungsreaktionen.

\subsection{Einfluß der Präferenzstärke für Annehmlichkeiten}

Es bleibt noch, den Einfluß des Verlaufs der Indifferenzkurven zu beschreiben. Je flacher die Indifferenzkurven verlaufen, desto stärker nähern sich die Lohnniveaus nach Vereinigung einander an. Je größer die Vorliebe für regionale Annehmlichkeiten ist, desto stärker werden die Abweichungen der Löhne mit entsprechenden Konsequenzen für Lohn- und Beschäftigungsniveau sein.

Gibt es Gruppen mit unterschiedlichen Präferenzen, so wird nach der Vereinigung die Gruppe mit den starken Präferenzen für Annehmlichkeiten in der Region mit den höheren regionalen Vorzügen leben, während die andere Gruppe in der anderen Region bei höheren Löhnen wohnen wird. Eine Vereinigung führt in diesem Fall zu Wanderungen in beide Richtungen statt wie bisher nur in eine. Dies sei anhand von Abbildung 25 illustriert.

Die Annehmlichkeiten wirken kostensenkend, die Lohn-Angebotskuven LA $_{x}$ und $\mathrm{LA}_{\mathrm{y}}$ haben daher eine positive Steigung. In jeder Region leben jeweils zwei Gruppen von Individuen a und b. Die Präferenzen der Gruppe a (b), die in x leben, seien die gleichen wie die der Gruppe a (b) der Region y. Vor Vereinigung herrsche in Region $\mathrm{x}$ ein Gleichgewicht mit $\mathrm{w}_{1}$ und $A \mathrm{n}_{\mathrm{x}}$. In Region y laute die Gleichgewichtskombination $\mathrm{An}_{\mathrm{y}}$ und $\mathrm{w}_{3}$. Nach einer Vereinigung haben $\mathrm{zu}-$ nächst beide Gruppen von Individuen aus x einen Anreiz nach y zu wandern, da das jeweils zu erreichende Nutzenniveau in y mit $V_{b}=3$ und $V_{a}=4$ höher ist als in Region $\mathrm{x}$ mit $\mathrm{V}_{\mathrm{a}}=2$ und $\mathrm{V}_{\mathrm{b}}=1$. Infolge der Zuwanderungen kommt es in y zu sinkenden Löhnen und steigender Beschäftigung: der Gewinn der Unternehmen in y steigt. Daher verwirklichen sie eine neue Isogewinnlinie. Die LohnAngebotskurve verschiebt sich nach rechts (auf LAy'), die in der Abwanderungsregion aufgrund sinkender Gewinne nach links (auf LAx'). Nach allen Wanderungen stellt sich in Region $x$ ein Lohnniveau von w4, in Region y eines von w2 ein. Individuen der Gruppe b können in Region $x$ nach allen Anpassungsprozessen den Nutzen $\mathrm{V}_{\mathrm{b}}=4$ und in Region y $\mathrm{V}_{\mathrm{b}}=2$ erreichen, sie werden daher Region $\mathrm{x}$ bevorzugen. Personen mit der Präferenzstruktur vom Typ a werden die Region y bevorzugen, wo sie den Nutzen $V_{b}=3$ erzielen können. 


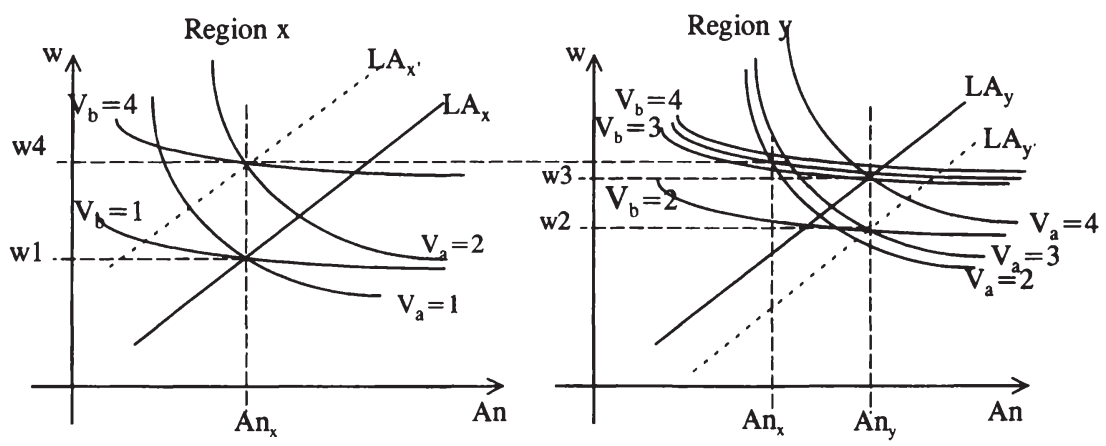

Abbildung 25: Wanderungen bei interindividuellen Unterschieden in den Präferenzen

In dem gewählten Beispiel müssen nach allen Anpassungsprozessen nicht alle gebürtigen y-er eine Nutzeneinbuße infolge der Vereinigung hinnehmen, diejenigen mit Präferenzen vom Typ b erreichen sogar einen höheren Nutzen als ohne Vereinigung. Das sind die Haushalte, die sich nur wenig aus regionalen Vorzügen machen und denen ein hohes Lohnniveau mehr Nutzen erbringt. Sie wandern daher in die Region $\mathrm{x}$ ab, die sich in dem dargestellten Beispiel von der Niedrig- in die Hochlohnregion verwandelt. Dies Ergebnis weicht von dem ohne Berücksichtigung von Annehmlichkeiten ab, wo alle Arbeitnehmer der $\mathrm{Zu}$ wanderungsregion infolge der sinkenden Löhne Nutzeneinbußen hinnehmen mußten.

\subsection{Zwischenfazit}

Unterscheidet sich die Ausstattung der Regionen mit Annehmlichkeiten, kommt es beim Abbau von Mobilitätsbarrieren nicht zu einem regionalen Lohnausgleich. Die sich ergebenden Konsequenzen für Lohnniveau, Beschäftigung und Sozialprodukt verstärken sich durch die Berücksichtigung von Präferenzen für Annehmlichkeiten, wenn die ehemalige Hochlohnregion mehr regionale Vorzüge als die Niedriglohnregion aufweist. Ist dagegen das Niveau in der Niedriglohnregion höher, so vermindern sich die Wanderungen.

Ist das Nutzenniveau der Haushalte in der Niedriglohnregion vor der Vereinigung geringer als in der Hochlohnregion, kommt es $\mathrm{zu}$ Wanderungen in die Hochlohnregion, wodurch der Lohn dort sinkt, während er in der Niedriglohnregion steigt. Das Nutzenniveau der Niedriglohnregion kann vor Vereinigung nur 
dann höher als das der anderen Region sein, wenn die Menge an regionalen Vorzügen größer ist. Eine Vereinigung führt zu Abwanderungen aus der Hochlohnregion und zu einem weiteren Auseinanderdriften der regionalen Lohnsätze. Ist das Attraktivitätsniveau in der ursprünglichen Niedriglohnregion höher, so bleibt das Lohnniveau geringer als das in der anderen Region. Ist es dagegen niedriger, wird die ehemalige Niedriglohnregion zur Hochlohnregion.

Insgesamt lassen sich folgende Bedingungen für die Richtung der Wanderungen nach Vereinigung aufstellen:

Im Gleichgewicht gilt:

$\mathrm{V}\left(\mathrm{An}_{\mathrm{x}}-\mathrm{An} \mathrm{n}_{\mathrm{y}}\right)=\mathrm{V}\left(\mathrm{w}_{\mathrm{y}}-\mathrm{w}_{\mathrm{x}}\right)$

Solange

$\mathrm{V}\left(\mathrm{An}_{\mathrm{x}}-\mathrm{An} \mathrm{n}_{\mathrm{y}}\right)>\mathrm{V}\left(\mathrm{w}_{\mathrm{y}}-\mathrm{w}_{\mathrm{x}}\right)$

gibt es Wanderungen in die Region $\mathrm{x}$.

Solange

$\mathrm{V}\left(A \mathrm{~A}_{\mathrm{x}}-A \mathrm{n}_{\mathrm{y}}\right)<\mathrm{V}\left(\mathrm{w}_{\mathrm{y}}-\mathrm{w}_{\mathrm{x}}\right)$

gibt es Wanderungen in die Region $y$.

\subsubsection{Regionale Preisunterschiede}

In dieser Arbeit wurde bisher weitgehend von regionaler Preisunterschiedslosigkeit ausgegangen. Regionale Preisunterschiede können aber existieren, auch wenn sich die Preise für handelbare Güter nicht (wesentlich) unterscheiden, da es außer handelbaren auch nicht-handelbare (lokale) Güter gibt ${ }^{123}$. Lokale Güter sind alle unbeweglichen Güter wie Boden und Häuser sowie Dienstleistungen, die auf den Absatz in der Umgebung konzentriert sind, wie Wohnungsnutzung, Gaststätten, Friseur etc. ${ }^{124}$. Die Preise nicht-handelbarer Güter können interregional voneinander abweichen.

Angenommen sei, daß die Haushalte handelbare und nicht-handelbare Gütern nachfragen. Das standortungebundene homogene Konsumgut $\mathrm{X}_{\mathrm{H}}$ sei überall $\mathrm{zu}$ identischen Preisen erhältlich. Die lokalen Güter $\mathrm{X}_{\mathrm{NH}}$ können regional unterschiedliche Preise aufweisen. Nicht-handelbare Güter seien superiore Güter, die Nachfrage nach ihnen steigt mit zunehmendem Einkommen. Außerdem seien sie substituierbar durch handelbare Konsumgüter.

${ }^{123}$ Zur Bedeutung der Nachfrage nach lokalen Gütern vgl. auch Abschnitt 2.3.2.
${ }^{124}$ Vgl. Götzinger (1981), S. 109. 
Das Einkommen werde voll für den Konsum der handelbaren und nicht-handelbaren Güter ausgegeben. Der Nutzen eines Haushaltes $(\mathrm{N})$ hänge $a b$ von der nachgefragten Menge an handelbaren Konsumgütern $\mathrm{X}_{\mathrm{H}}$, der nachgefragten Menge an nicht-handelbaren Gütern $\mathrm{X}_{\mathrm{NH}}$ und dem Attraktivitätsniveau der Region $\left(\mathrm{An}_{\mathrm{i}}\right)$ :

$\mathrm{N}=\mathrm{N}\left(\mathrm{X}_{\mathrm{H}}, \mathrm{X}_{\mathrm{NH}}, \mathrm{An}_{\mathrm{i}}\right)$

Der Nutzen eines Haushalts hängt damit indirekt vom regionalen Lohnniveau $\left(w_{i}\right)$, von dem Preisniveau der handelbaren Güter $\left(\mathrm{p}_{\mathrm{H}}, \mathrm{i}\right)$ und dem regionalen Preisniveau $\left(\mathrm{pNH}_{\mathrm{N}} \mathrm{i}\right)$ und vom exogen gegebenen Attraktivitätsgrad $\left(\mathrm{An}_{\mathrm{i}}\right)$ einer Region ab:

$\mathrm{V}=\mathrm{V}\left(\mathrm{w}_{\mathrm{i}}, \mathrm{p}_{\mathrm{H}, \mathrm{i}}, \mathrm{p}_{\mathrm{NH}, \mathrm{i}}, \mathrm{An}_{\mathrm{i}}\right)$

Da der Preis der handelbaren Güter in allen Regionen identisch und exogen gegeben ist, wird er im folgenden aus der Betrachtung weitgehend ausgeklammert.

Die partiellen Grenznutzen von Lohneinkommen und Attraktivitätsniveau seien positiv, aber abnehmend. Je höher das regionale Preisniveau, desto weniger nicht-handelbare Konsumgüter kann der Haushalt bei gegebenem Einkommen nachfragen.

Für die Haushalte lassen sich „Indifferenzkurven“ für regionales Preisniveau und Lohnsatz darstellen. Ein hoher Nominallohnsatz in Verbindung mit einem hohen regionalen Preisniveau stiftet einem Haushalt den gleichen Nutzen wie ein niedriger Nominallohnsatz in Verbindung mit einem geringeren regionalen Preisniveau, wenn er sich mit beiden Güterbündel kaufen kann, die ihm den gleichen Nutzen stiften. (Diese Güterbündel werden sich voneinander unterscheiden, da unterschiedliche Güterpreisverhältnisse vorliegen. In Gegenden mit relativ hohen Preisniveaus werden relativ wenig nicht-handelbare Güter und relativ viel der handelbaren Güter nachgefragt werden. So könnte es etwa in Gegenden mit niedrigen Mieten eine geringere Nachfrage nach handelbaren Gütern geben und eine relativ hohe nach Wohnraum). Die Indifferenzkurven des Haushaltes weisen eine positive Steigung auf. Je höher die Indifferenzkurve für ein gegebenes Attraktivitätsniveau verläuft, desto höher ist der Nutzen des Haushalts.

In Abbildung 26 werden zwei Indifferenzkurven dargestellt, die beide das gleiche Nutzenniveau (in Höhe von k) eines Individuums in unterschiedlichen Regionen zeigen. V2 zeigt eine Indifferenzkurve für Region 2 mit hohem Attraktivitätsniveau, V1 zeigt die Kombinationen gleichen Nutzens für Region 1 mit niedrigem Attraktivitätsniveau. Die niedrigeren lokalen Preise bei gleichem 
Lohnniveau würden den Haushalt für den Annehmlichkeitsnachteil in Region 1 entschädigen.

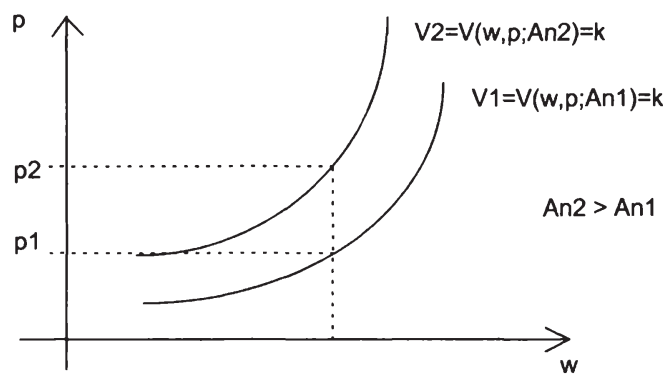

Abbildung 26: Indifferenzkurven bei alternativen Löhnen, Preisen und regionalen Annehmlichkeiten

Haben alle Individuen identische Präferenzen, ist ein räumliches Gleichgewicht erreicht, wenn in allen Regionen der gleiche maximale Nutzen erzielt werden kann. Die Gleichgewichtsbedingung für die Haushalte lautet somit:

$\mathrm{V}\left(\mathrm{w}_{\mathrm{i}}, \mathrm{p}_{\mathrm{i}} ; \mathrm{A}_{\mathrm{i}}\right)=\mathrm{k}$,

mit $\mathrm{k}=$ maximal erreichbares Nutzenniveau jedes Haushalts in allen Regionen ${ }^{125}$.

Die Graphik zeigt einen nicht-linearen Verlauf der Indifferenzkurven. Dieser beruht nicht nur auf dem abnehmendem Grenznutzen des Einkommens, sondern auch auf einer abnehmenden Grenzrate der Substitution zwischen handelbaren und nicht-handelbaren Gütern. Wenn nicht-handelbare Güter und Annehmlichkeiten superior sind, dann steigt die Nachfrage nach diesen Gütern mit zunehmendem Einkommen. Bei Menschen mit hohen Einkommen wird die Standortqualität ein höheres Gewicht bekommen als bei Haushalten mit niedrigen Einkommen. Die Einkommensstarken werden daher überdurchschnittlich häufig in Gegenden mit einer hohen Standortqualität ziehen. Die höhere Nachfrage dieser Einkommensschicht kann zu regionalen Preiserhöhungen, bzw. zu einem höheren Preisniveau führen.

Es zeigt sich wiederum, daß Wanderungen zu einem Ausgleich der interregionalen Nutzen führen. Lohn-, Preis- und Attraktivitätsniveau hingegen können sich auch nach Vereinigung im neuen Gleichgewicht voneinander unterscheiden. Wird durch dirigistische Maßnahmen versucht das Lohn- oder Preisniveau ein-

${ }^{125}$ Vgl. Roback (1982), S. 160. 
ander anzugleichen, würden ohne entsprechende Gegenreaktion der Preise bzw. Löhne alle Haushalte in attraktive Gegenden ziehen, während unattraktive Regionen entvölkert werden.

\subsubsection{Transaktionskosten}

Neben den bisher berücksichtigten Wanderungsdeterminanten können die Kosten, die einem Haushalt durch einen Umzug entstehen, ein weiterer gewichtiger Faktor im Entscheidungskalkül der Haushalte sein. Kosten können durch ein höheres regionales Preisniveau in der Zuzugsregion verursacht werden. Weitere Kosten entstehen durch Bewerbungs- und Umzugskosten ${ }^{126}$, durch Opportunitätskosten und psychische Kosten. Opportunitätskosten sind die entgangenen Erträge durch Reisen, Jobsuche und Anlernzeit in der neuen Stellung. Psychische Kosten entstehen durch Verlassen der gewohnten Umgebung und der Freunde ${ }^{127}$. Es wird daher im folgenden angenommen, daß ein gleiches Einkommen und gleiches Annehmlichkeitsniveau einem Haushalt in seiner Heimatregion mehr Nutzen verschafft als in jedem anderen Ort ${ }^{128}$, da die mit Wanderungen verbundenen Kosten nutzenmindernd wirken.

Angenommen sei, daß alle Menschen die gleichen Präferenzen haben. Die privaten Kosten der Migration können wie folgt berücksichtigt werden: Die Indifferenzkurve eines Arbeitnehmers, die identische Kombinationen von Lohn-Attraktivität bezeichnet, habe in der Heimatregion einen höheren Nutzenindex hat als in jeder anderen Region, so wie in Abbildung 27 dargestellt.

Auf der Indifferenzkurve, die für Region $\mathrm{x}$ eingezeichnet ist und die mit $\mathrm{V}_{\mathrm{x}}=1$, $\mathrm{V}_{\mathrm{y}}=0$ bezeichnet ist, verwirklichen die Arbeiter, die aus Region $\mathrm{x}$ stammen, einen höheren Nutzen $\left(\mathrm{V}_{\mathrm{x}}=1\right)$ als die Arbeiter, die aus Region y stammen $\left(\mathrm{V}_{\mathrm{y}}=\right.$ 0 ). In ihrer Heimatregion würden die aus y stammenden Arbeiter für die gleiche Kombination von Annehmlichkeiten und Lohn einen höheren Nutzen realisieren, nämlich $V_{y}=1$, während die Individuen aus $x$ hier nur einen Nutzen in Höhe von $V_{x}=0$ erzielen würden.

${ }^{126}$ Inwieweit die Kosten im Entscheidungskalkül der Haushalte berücksichtigt werden, ist davon abhängig, von wem die Kosten letztendlich getragen werden. Wenn - wie in Deutschland durchaus üblich - die Reisekosten für Vorstellungsgespräche und die Umzugskosten von den Unternehmen gezahlt werden, so gehen sie nicht in das Entscheidungskalkül der Individuen ein.

${ }^{127} \mathrm{Zu}$ den Umzugskosten vgl. auch Abschnitt 3.1.2.5.

${ }^{128}$ Es mag auch Fälle geben, wo der Nutzen in der anderen Region höher ist, etwa durch die Gewinnung neuer Erfahrungen und neuer Erlebnisse, in der Regel dürfte die Bindung an eine Region aber höher bewertet werden als die Fremde. 


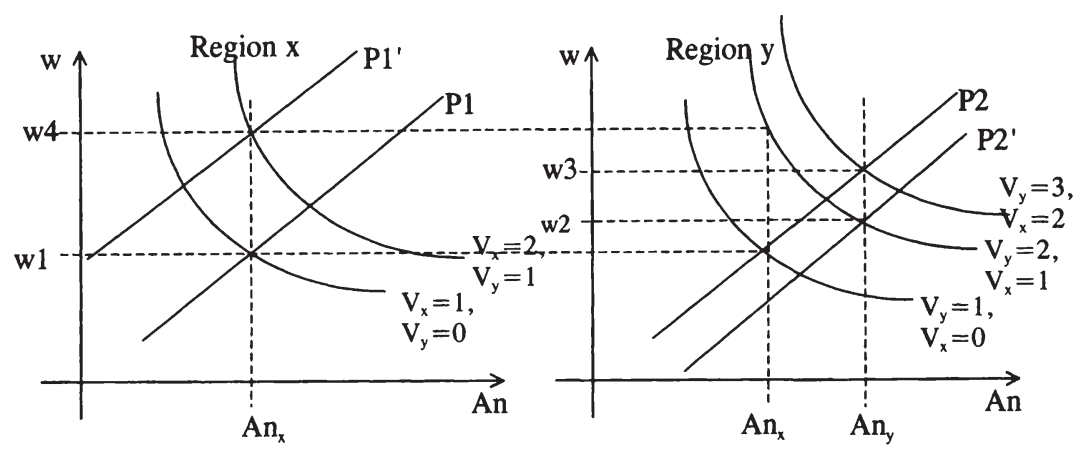

Abbildung 27: Gleichgewicht bei Berücksichtigung von Wanderungskosten

Wenn vor der Vereinigung die Arbeiter in $\mathrm{x}$ das Nutzenniveau $\mathrm{V}_{\mathrm{x}}=1$ erzielten und die Arbeiter der Region y $V_{y}=2$, so würde eine Vereinigung keine Anpassungsreaktionen auslösen, da sich niemand durch eine Wanderung besser stellen könnte, obwohl Nutzendifferentiale bestehen. Die Bewohner der Region y würden sich durch eine Wanderung verschlechtern, die der Region $\mathrm{x}$ könnten durch Wechsel nach y maximal das Nutzenniveau der Heimatregion erreichen, mithin hätte niemand einen Anreiz zum Umzug. In diesem Fall gäbe es daher bei einer Vereinigung keine Wanderungen und damit auch keine Änderung der Beschäftigung, des Lohnniveaus und des Outputs.

Wird aber in Region y das Nutzenniveau $\mathrm{V}_{\mathrm{y}}=3$ erzielt und in Region $\mathrm{x}$ das Nutzenniveau $V_{x}=1$, so ist es für die Einwohner aus $x$ lohnend ihren Wohn- und Beschäftigungsort zu wechseln. Der Lohnsatz in y sinkt daraufhin, während die Beschäftigung und das Sozialprodukt steigen. Die Unternehmen realisieren infolge der Wanderungsbewegungen neue Lohn-Angebotskurven, die in $\mathrm{x}$ liegt links der alten, die in y rechts der ursprünglichen. Das Nutzenniveau der gebürtigen "y-er" sinkt, das Nutzenniveau der zurückgebliebenen "x-er" steigt. Das Nutzenniveau ändert sich solange, bis es für die aus $\mathrm{x}$ stammenden und nach $\mathrm{y}$ wandernden Individuen interregional zum Ausgleich gekommen ist. Das Niveau der "y-er" ist am Ende der Anpassungsprozesse zwar niedriger als ohne Vereinigung, jedoch ist es noch höher als das der "x-er". Die Lohnänderungen (und damit auch die Änderungen der Beschäftigung und des Sozialprodukts) sind in beiden Regionen niedriger als ohne Berücksichtigung von Wanderungskosten.

Bei Berücksichtigung von Wanderungskosten (WK) lassen sich folgende Bedingungen formulieren: 
Wenn

$V_{x}\left(w_{x}, A n_{x}\right) \geq V_{x}\left(w_{y}, A n_{y}\right)+V(W K)$ und

$\mathrm{V}_{\mathrm{y}}\left(\mathrm{w}_{\mathrm{y}}, A \mathrm{An}_{\mathrm{y}}\right) \geq \mathrm{V}_{\mathrm{y}}\left(\mathrm{w}_{\mathrm{x}}, A \mathrm{An}_{\mathrm{x}}\right)+\mathrm{V}(\mathrm{WK})$

dann führt eine Vereinigung nicht zu Anpassungsprozessen.

Wenn

$\mathrm{V}_{\mathrm{x}}\left(\mathrm{w}_{\mathrm{x}}, A \mathrm{n}_{\mathrm{x}}\right)<\mathrm{V}_{\mathrm{x}}\left(\mathrm{w}_{\mathrm{y}}, A \mathrm{An}_{\mathrm{y}}\right)+\mathrm{V}(\mathrm{WK})$ kommt es zu Wanderungen der Bevölkerung aus $x$ in die Region $y$.

Wenn

$\mathrm{V}_{\mathrm{y}}\left(\mathrm{w}_{\mathrm{y}}, A \mathrm{n}_{\mathrm{y}}\right)<\mathrm{V}_{\mathrm{y}}\left(\mathrm{w}_{\mathrm{x}}, A \mathrm{n}_{\mathrm{x}}\right)+\mathrm{V}(\mathrm{WK})$ kommt es zu Wanderungen der Bevölkerung aus $\mathrm{y}$ in die Region $\mathrm{x}$.

Da Wanderungskosten den Nutzen schmälern, ist V(WK) hier als Summand verwendet worden. Die letzte Bedingung liest sich daher etwa als: Wenn der Nutzen, den ein gebürtiger y-er in seiner Heimatregion aus seinem Lohn und den vorhandenen Annehmlichkeiten erzielen kann geringer ist als der Nutzen, den er in der Region $\mathrm{x}$ durch den dort herrschenden Lohn und den Annehmlichkeiten erzielen kann, vermindert um die Einbuße, die er durch die Wanderungskosten erleidet, so ist es für ihn lohnend, nach $\mathrm{x}$ umzusiedeln.

Ein Problem dieser Betrachtung könnte darin liegen, daß ein Teil der Transaktionskosten nur einmalig anfällt (oder im Laufe der Zeit abnimmt) und die Veränderung des Lohnsatzes und der regionalen Annehmlichkeiten dagegen für einen längeren Zeitraum erwartet werden.

\subsubsection{Zusammenfassung}

Regionen können mit unterschiedlichen regionalen Vorzügen ausgestattet sein, aus deren (potentiell möglichen) Inanspruchnahme die Haushalte einen Nutzen ziehen. Sie sind bereit, Lohneinbußen hinzunehmen, um in den Genuß der regionalen Vorzüge zu gelangen. Daher können interregionale Reallohnunterschiede dauerhaft bestehen, ohne Wanderungen auszulösen. Die Region mit dem höchsten Niveau an Annehmlichkeiten wird den niedrigsten Lohnsatz aufweisen, vorausgesetzt die Annehmlichkeiten wirken auf die Produktion kostenerhöhend. Im interregionalen Gleichgewicht wohnen die Individuen mit den stärksten Präferenzen für Annehmlichkeiten in der Region mit den niedrigsten Lohnsätzen, während die Individuen mit den stärksten Präferenzen für ein hohes Geldeinkommen in der Region mit den höchsten Reallohnsätzen und niedrigstem Attraktivitätsniveau leben. 
Vereinigen sich zwei Regionen, von denen die ursprüngliche Niedriglohnregion mit einem geringeren Niveau an Annehmlichkeiten ausgestattet ist als die Hochlohnregion, kommt es zu Wanderungsbewegungen in die Hochlohnregion. Im neuen Gleichgewicht ist der Lohnsatz in der vormaligen Niedriglohnregion höher und die regionale Wertschöpfung und die Beschäftigung niedriger als ohne Einfluß von Annehmlichkeiten. Sind die Annehmlichkeiten dagegen in der Region mit dem niedrigeren Lohnniveau vor Vereinigung höher, sind die Anpassungsreaktionen geringer, die Niedriglohnregion bleibt die Niedriglohnregion. Haben alle Individuen identische Präferenzen für Annehmlichkeiten und Lohn, bewirkt eine Vereinigung die Beseitigung aller interregionaler Nutzenunterschiede.

Im Fall der deutschen Vereinigung dürften die regionalen Annehmlichkeiten Ostdeutschlands keine bedeutende Gegenkraft zu den Wanderungstendenzen des Produktionsfaktors Arbeit von den neuen in die alten Länder gewesen sein. Es ist im Gegenteil zu vermuten, daß die alten Länder ein höheres Attraktivitätsniveau aufwiesen und daher zusätzliche Wanderungsanreize erzeugt wurden.

Wirken die Annehmlichkeiten kostensenkend auf die Produktion, sind die Unternehmen in den attraktivsten Gegenden bereit, die höchsten Löhne zu zahlen. Dann würde es alle Haushalte und Unternehmen in die Gegend mit der höchsten Attraktivität ziehen, während sich die anderen Regionen vollständig entleeren. Infolge solch starker Wanderungen und der damit entstehenden Bevölkerungsdichte dürfte aber die Attraktivität der Zuwanderungsregion sinken.

Starke Wanderungsbewegungen können das regionale Preisniveau beeinflussen. In Regionen mit einem hohen Preis-, Attraktivitäts- und Lohnniveau können Haushalte den gleichen Nutzen erzielen wie in Regionen mit niedrigem Lohn-, Preis- und Attraktivitätsniveau. Steigende Preise infolge starker Nachfrage nach nicht-handelbaren Gütern - wie Wohnraum - können Wanderungsströme versiegen lassen.

Entstehen den Individuen Wanderungskosten - seien sie monetärer oder nichtmonetärer Art - können Nutzenunterschiede zwischen den regionalen Bevölkerungsgruppen bestehen bleiben und zwar derart, daß ein Haushalt durch Wanderung seinen Nutzen nicht mehr erhöhen kann, aber zwischen den Haushalten bestehende interregionale Nutzenunterschiede nicht ausgeglichen werden. Gegenüber dem Beschäftigungsrahmen aus Abschnitt 2.1.1. sind die Wanderungshöhe und -wirkungen geringer. 
Annette Schönherr - 978-3-631-75008-7

Downloaded from PubFactory at 01/11/2019 09:19:06AM

via free access 


\section{Potentielle individuelle Migrationsgewinne und tatsächli- cher Umfang der Wanderungen in Deutschland nach der Vereinigung}

Nachdem im ersten Teil dieser Arbeit die Bedeutung einer Vereinigung zweier Regionen in Hinsicht auf die Wirkungen für den Arbeitsmarkt unter analytisch einfachen Bedingungen betrachtet wurde, steht im folgenden Teil die konkrete deutsch-deutsche Vereinigung im Vordergrund. Oben wurde angenommen, daß ökonomische Unterschiede der Regionen sowie Standortannehmlichkeiten Migrationen auslösen und regionale Differenzen zum Verschwinden bringen können. An den wanderungsauslösenden Faktoren setzt die folgende Analyse an.

Die wirtschaftlichen Unterschiede zwischen Ost- und Westdeutschland waren zum Zeitpunkt der Vereinigung gravierend und ließen sehr große Wanderungsströme erwarten. In Abschnitt 2.1.2 wurde bereits darauf hingewiesen, daß Wanderungen nicht in einem solchen Umfang stattgefunden haben, der eine Angleichung der regionalen Lohnunterschiede bewirkt hätte. Im Folgenden wird untersucht, wie hoch der erwartbare Wanderungsgewinn nach der Vereinigung gewesen ist, um Erklärungen für das Verhalten zu gewinnen. Dafür wird zunächst dargelegt, welche Faktoren in empirischen Untersuchungen üblicherweise berücksichtigt werden. Dabei wird eine Berechnungsmethode vorgestellt, mit der der individuelle Migrationsgewinn bestimmt werden kann und erörtert, wie die einzelnen Faktoren in die Berechnung eingehen sollten. Im Anschluß folgt eine Berechnung der 1990 potentiell erzielbaren Überschüsse aus einem Umzug von Ost- nach Westdeutschland bei gleichzeitigem Arbeitsplatzwechsel. Abschließend wird dargestellt, welche Bevölkerungsgruppen sich als besonders wanderungsfreudig erwiesen haben und inwieweit sie sich mit den Personen decken, für die sich besonders hohe Wanderungsgewinne berechnen lassen.

\subsection{Die empirische Berücksichtigung von Wanderungsdeterminanten}

Bisher berücksichtigte Wanderungsgründe waren im wesentlichen interregionale Unterschiede des Lohnniveaus und der Annehmlichkeiten. Diese Gründe sollen nun in Hinblick auf die Möglichkeit ihrer Berücksichtigung in der späteren Modellrechnung über die Vorteilhaftigkeit eines Umzugs von Ost- nach Westdeutschland mit gleichzeitigem Arbeitsplatzwechsel differenzierter beleuchtet werden. Ferner werden weitere - bisher noch nicht behandelte - mobilitätsfördernde und -hemmenden Faktoren beschrieben. Zunächst wird jedoch ein 
Überblick darüber gegeben, welche Wanderungsursachen in verschiedenen Ansätzen zur Erklärung von Wanderungen üblicherweise berücksichtigt werden.

\subsubsection{Wanderungsdeterminanten in ausgewählten empirisch ausgerichteten Analysen}

Die möglichen Wanderungsursachen sind vielfältig. Inwieweit ein Ansatz zur Erklärung von Migrationsverhalten geeignet ist, ist davon abhängig, ob die relevanten Wanderungsdeterminanten berücksichtigt werden. Je umfassender deren Einbeziehung ist, desto besser können Wanderungsentscheidungen erklärt werden. Allerdings werden durch eine erschöpfende Einbeziehung der verschiedenen Faktoren auch die Bewertungs- und Informationsbeschaffungsprobleme größer und damit eine empirische Überprüfung schwerer ${ }^{129}$.

Nach einer Auswertung von Birg ${ }^{130}$ können die vielfältigen, in empirische Untersuchungen berücksichtigten Faktoren vier verschiedenen Einflußbereichen zugeordnet werden:

1.Grundlagen für die individuelle Güterversorgung:

Einkommenserzielungs- und Beschäftigungsmöglichkeiten

2.Verfügbarkeit über Dienstleistungen, öffentliche und private Güter:

Wohnungssektor, Bildung und Kultur, Gesundheitsdienst, Gesamtangebot an Dienstleistungen

3. Natürliche und soziale Umweltbedingungen:

Klima und Naherholung, Sozialstruktur, Urbanisierungsgrad, Siedlungsstruktur

4.Möglichkeiten der Raumüberwindung:

Verkehrsinfrastruktur und geographische Lage

Nach einem der bekanntesten Ansätze, Migrationsfaktoren zu schematisieren und einem generellen Erklärungsschema unterzuordnen, der push-pull-Hypothese, werden die Faktoren dagegen in zwei Gruppen aufgeteilt. Push-Faktoren sind abstoßende, in der Herkunftsregion wirksame Kräfte, während pull-Faktoren anziehende Kräfte sind, die von der Zielregion ausgehen. Abstoßende Faktoren können nach Bogue (1959, S. 496) sein: ökonomischer Strukturwandel,

${ }^{129}$ Vgl. Delbrück/Raffelhüschen (1993), S. 344f.

${ }^{130}$ Vgl. Birg (1975), S. 45. 
Erschöpfung natürlicher Ressourcen, Beschäftigungsverlust, Diskriminierung (politisch, religiös oder ethnisch), Entfremdung von eigenen sozialen Gruppen durch persönliche Entwicklung, fehlende Entwicklungsmöglichkeiten, Katastrophen. Als anziehende Faktoren werden bessere Beschäftigungsmöglichkeiten, Chancen zur Einkommensverbesserung, bessere Bildungsmöglichkeiten, attraktivere Umgebung und Lebensbedingungen, Abhängigkeit von anderen (Heirat oder Umzug des Lebensgefährten) und Attraktivität der Großstadt aufgeführt. Es fällt auf, daß eine Reihe der genannten Faktoren im ersten Teil der Arbeit nicht erwähnt wurden. Das Erklärungsschema des push-pull-Ansatzes umfaßt auch andere als ökonomische Gründe, die situationsabhängig wesentlich relevanter sein können als die wirtschaftlichen. Die Einbeziehung etwa politischer Motive geht aber über die getroffene Eingrenzung weit hinaus. Auch mit Blick auf die Bestimmung der Vorteilhaftigkeit einer Wanderung nach der deutschen Wiedervereinigung können diese Motive wohl vernachlässigt werden, da hier vermutlich wirtschaftliche Gründe im Vordergrund standen.

Mit anziehenden und abstoßenden Kräften arbeiten auch die Gravitationsmodelle, die auf dem analogen physikalischen Phänomen basieren ${ }^{131}$. Bereits 1885 formulierte Ravenstein ${ }^{132}$ seine „Gesetze“, die besagen, daß die Höhe der Wanderung von einer Region $\mathrm{i}$ in eine Region $\mathrm{j}\left(\mathrm{M}_{\mathrm{ij}}\right)$ direkt proportional der Größe der Bevölkerung (B) in $\mathrm{i}$ und der Anziehungskraft in $\mathrm{j}$ sowie indirekt proportional der Entfernung zwischen Herkunfts- und Zielregion ist (D). Diese Aussage kann durch folgende Gleichung beschrieben werden ${ }^{133}$ :

$M_{i j}=k^{*} D_{i j}-a^{*} B_{i}$

mit

k: Konstante

a: Parameter

Wird außerdem angenommen, daß die Größe der Zielregion $\mathrm{j}$ ebenfalls die Wanderungshöhe beeinflußt, so ergibt sich:

${ }^{131}$ Die Bezeichnung des Modells ist vom Newtonschen Gravitationsgesetz abgeleitet worden: „Im Rahmen dieses Gesetzes wird die Massenanziehung zwischen zwei Körpern im Raum aus der Größe der Massen dieser Körper und aus der Entfernung zwischen ihren Mittelpunkten bestimmt...“ (Kemming (1980), S. 3).

${ }^{132}$ Ravenstein (1885 und 1889) untersuchte Ende des 19. Jahrhunderts zunächst die Daten der englischen Volkszählung, um das Ausmaß der Binnenwanderungen in Großbritannien festzustellen. später Daten aus 20 Ländern und formulierte auf deren Grundlage "laws of Migration". Er merkte jedoch selbst an, daß diese Formulierung zu anspruchsvoll sei.

${ }^{133} \mathrm{Vgl}$. Termote (1972), S.143. 
$\mathrm{M}_{\mathrm{ij}}=\mathrm{k}^{*} \mathrm{D}_{\mathrm{ij}}-\mathrm{a}^{*} \mathrm{~B}_{\mathrm{i}}^{*} \mathrm{~B}_{\mathrm{j}}$

Diese Gleichung bildet die Grundlage der Gravitationsmodelle. Soll in diesem Modell etwa berücksichtigt werden, daß Wanderungen altersabhängig sind, so ist für die entsprechenden Bevölkerungs- und Wanderungszahlen nur die jeweilige Altersgruppe einzusetzen. Ein Ausbau der Gravitationsgleichung um weitere Komponenten, die einen Einfluß auf die Wanderungsentscheidung haben, ist möglich ${ }^{134}$.

In dem Ansatz von Sjaastad (1962) gehen die Zukunftsperspektiven explizit in die Migrationsüberlegungen mit ein. Er differenziert die Einflußfaktoren nach Kosten und Erträge, wobei er diese zusätzlich in monetäre und nicht-monetäre Größen unterscheidet. Unter monetären Kosten versteht er die (Konsum-) Ausgabenerhöhungen, etwa für Nahrungsmittel, Miete, Verkehrsmittel etc., die durch einen Umzug entstehen können. Außerdem zählt er hierzu die Kosten, die durch doppelte Haushaltsführung, Nachhilfe für Kinder etc. anfallen. Die nichtmonetären Kosten werden in zwei Arten aufgespalten: in Opportunitätskosten und psychische Kosten. Opportunitätskosten sind verlorengegangene Erträge durch Reisen, Arbeitsplatzsuche und Anlernzeit auf dem neuen Arbeitsplatz. Psychische Kosten entstehen durch Verlassen der gewohnten Umgebung und der Freunde. Monetäre Erträge sind die erhöhten Geldeinkommen des neuen Arbeitsplatzes gegenüber dem alten. Für nicht-monetäre Erträge müssen die Individuen keine Kostenbeiträge entrichten, etwa für die landschaftlichen Vorzüge oder ein angenehmeres Klima der Zuzugsregion. Sie sind - ebenso wie die nichtmonetären Kosten - schwer zu quantifizieren ${ }^{135}$.

Nach der Kapitalwertmethode kann der Wert einer Investition durch die Summe der noch zu erwartenden abdiskontierten Zahlungsüberschüsse bestimmt werden. Entsprechend können auch Humankapitalgrößen ${ }^{136}$ gemessen werden (bzw. die Rentierlichkeit von Investitionen in Humankapital). Neben Bildungsinvestitionen, die als bedeutsamster Teil von Investitionen in Humankapital angesehen werden ${ }^{137}$, können auch Migrationen solche Investition sein, nämlich dann, wenn infolge einer Wanderung die Produktivität des Humankapitals steigt ${ }^{138},{ }^{139}$.

\footnotetext{
${ }^{134} \mathrm{Vgl}$. hierzu etwa Gatzweiler (1975), S. $117 \mathrm{ff}$.

${ }^{135} \mathrm{Vgl}$. Sjaastad (1962), S. 85f.

136 "The qualification of labor which were relevant for more than one period as production input (thus representing a stock of working capacity) have been defined as human or intangible capital" (Bodenhöfer (1967), S. 435.

${ }^{137} \mathrm{Vgl}$. Bodenhöfer (1969), S. 79.

${ }^{138} \mathrm{Vgl}$. Sjaastad (1962), S. 83.

${ }^{139}$ In den vorhergehenden Abschnitten wurde gezeigt, daß Wanderungen i.d.R. in produktivere Beschäftigungen vorgenommen werden. In diesen Fällen findet eine Steigerung des
} 
Dieser Ansatz wird nun relativ ausführlich dargestellt, da er geeignet erscheint, um den potentiellen Migrationsgewinn von ostdeutschen Erwerbspersonen nach der Wiedervereinigung zu schätzen ${ }^{140}$.

Um zu bestimmen, wie hoch die Einkommensänderung eines Individuums bei einer Wanderung ist, wird das Lebenseinkommen mit und ohne Migration geschätzt. Die entsprechenden Ertragsreihen werden auf den gegenwärtigen Zeitpunkt abgezinst und miteinander verglichen ${ }^{141}$.

Eine Rechnung zur Bestimmung des Migrationsgewinns bei Wanderung aus Region $\mathrm{x}$ in Region $\mathrm{y}(\mathrm{MGy}, \mathrm{x})$ für einen Zeitraum $\mathrm{t}$ könnte daher wie folgt lauten:

$$
M G y, x=\sum_{i=1}^{t} \frac{E m, y-E n m, y-(K m, y+K n m, y)}{(1+r)^{i}}-\sum_{i=1}^{t} \frac{E m, x-E n m, x-(K m, x+K n m, x)}{(1+r)^{i}}
$$

mit:

r: Zeitpräferenzrate

$\mathrm{E}_{\mathrm{m}, \mathrm{a}}$ monetäre Erträge pro Periode in der Region a

Enm,a: nicht-monetäre Erträge pro Periode in der Region a

$\mathrm{K}_{\mathrm{m}, \mathrm{a}}$ : monetäre auftretende Kosten pro Periode in der Region a

$\mathrm{K}_{\mathrm{nm}, \mathrm{a}}$ : nicht-monetäre Kosten pro Periode in der Region a

$\mathrm{a}=\mathrm{x}, \mathrm{y}$.

Kosten und Erträge gehen hier nicht als Differenzbeträge, die durch einen Umzug entstehen, ein, sondern sind die gesamten Einkünfte bzw. Aufwendungen einer Periode in einer bestimmten Region. $t$ ist der Zeithorizont, für den das Individuum vorausplant. Im Prinzip müßte dies eine Planung bis zum Lebensende sein - oder sogar darüber hinaus, wenn die Bedeutung der Wanderung für die Nachkommen in das Wanderungskalkül einbezogen wird.

(mit seinem Ertragswert bewerteten) Werts des Humankapitals statt. Was ist aber, wenn es zu Wanderungen in die andere Richtung kommt, wenn etwa ein Lohnverzicht in Kauf genommen wird, weil der Freizeitwert in der anderen Region höher bewertet wird? Migration ist dann konsequenterweise als Vernichtung von Humankapital einzustufen.

${ }^{140}$ Mit diesem Ansatz kann beurteilt werden, ob sich eine Wanderung für einzelne Bevölkerungsgruppen lohnt. In dem Humankapitalansatz wird angenommen, daß alle Individuen, die einer (identifizierbaren) Gruppe homogener Individuen angehören, die gleiche Migrationsentscheidung treffen (vgl. Molho (1986), S. 401).

${ }^{141}$ Vgl. Delbrück/Raffelhüschen (1993), S. 344. 
Ist die errechnete Differenz $\mathrm{MG}_{\mathrm{y}, \mathrm{x}}$ positiv, lohnt sich für den potentiellen Migranten ein Umzug. Ist sie negativ, ist ein Verbleib in der Ursprungsregion sinnvoll. Bei einem Ergebnis von Null ist der Betroffene indifferent zwischen Umzug und Verharren. Ist die Rechnung für alle Individuen ausgeglichen, besteht ein interregionales Gleichgewicht (vorausgesetzt sämtliche relevanten Kosten und Erträge wurden berücksichtigt).

Diese Gleichgewichtsbedingung geht über die Harris-Todaro-Bedingung ${ }^{142}$ hinaus, bei der in die Wanderungsentscheidung neben den regionalen Lohnsätzen $\left(w_{\mathbf{X}}, w_{y}\right)$ die Arbeitslosenquoten der Regionen $\left(A_{L} Q_{x}, A L Q_{y}\right)$, gewichtet mit der Wahrscheinlichkeit selbst arbeitslos zu werden $(\gamma)$, sowie die psychische Kosten, die beim Umzug entstehen $(\theta$ reflektiert die nichtpekuniären Vorteile der Heimatregion eines marginalen Migranten von x nach y), eingehen:

Harris-Todaro-Bedingung: $\mathrm{w}_{\mathbf{X}}\left(1-\gamma_{\mathrm{X}} \mathrm{ALQ}_{\mathrm{X}}\right) \theta=\mathrm{w}_{\mathbf{y}}\left(1-\gamma_{\mathrm{y}} \mathrm{ALQ}_{\mathrm{y}}\right)^{143}$

In dieser Bedingung werden zwar aktuelle monetäre und nichtmonetäre Kosten und Erträge berücksichtigt, jedoch spiegeln sich die Zukunftserwartungen nicht wider.

Die Humankapitalmethode kann auf vielfältige Arten ergänzt und abgewandelt werden, so ist z.B. auch eine Erweiterung um den Optionswert des Wartens (option value of waiting) möglich. Der Optionswert des Wartens - zunächst für die Bewertung und optimale Anwendung von Finanzoptionen entwickelt - eignet sich für die Analyse von Wahlhandlungen, sofern sie durch folgende Merkmale gekennzeichnet sind:

- fixe sunk costs

- nichtversicherbare Unsicherheit

- es gibt eine Option zu warten, d.h. die Entscheidung und die damit verbundenen fixen Kosten aufzuschieben ${ }^{144}$.

Da diese Bedingungen auf eine Umzugsentscheidung zutreffen, kann das Konzept auch auf Wanderungen angewendet werden ${ }^{145}$.

${ }^{142}$ Harris und Todaro (1970) waren die ersten, die das Problem, daß die internationalen Lohnunterschiede nicht stets von jedem realisiert werden können, formal durch die Einbeziehung der Arbeitslosenquote berücksichtigten.

${ }^{143} \mathrm{Vgl}$. Layard u.a. (1992), S. 34.

144 Vgl. Burda (1993), S. 3.

${ }^{145}$ Eine Anwendung dieses Konzepts für die Untersuchung der ostdeutschen Migrationen findet sich bei Burda (1993). 
Der Optionswert des Wartens drückt den Ertrag aus, der durch Abwarten gegenüber einer Aktion erzielt wird. Er ist positiv, wenn die Verschiebung der (Migrations-)Entscheidung auf einen späteren Zeitpunkt die relative Unsicherheit und das Risiko, sich falsch zu entscheiden, vermindert. In der Wartezeit können zusätzliche Informationen hinzugewonnen werden. Wanderungen finden dann statt, wenn der Gegenwartswert des Einkommensgewinns aus der Wanderung größer ist als die Summe der Fixkosten des Umzugs und des Optionswerts des Wartens. Der Optionswert erhöht sich, wenn die Einkommensunterschiede sich schneller als erwartet angleichen, umgekehrt kann er negativ werden, wenn sich die Einkommen weiter auseinander entwickeln ${ }^{146}$. Dieses Konzept ist erweiterbar um die Einstellung zum Risiko.

\subsubsection{Zur empirischen Erfassung und Erfaßbarkeit von Wanderungsde- terminanten in einer Rechnung zur Messung der Vorteilhaftigkeit einer Migration}

Die einzelnen Faktoren, die Wanderungen beeinflussen, werden im folgenden genauer beleuchtet, insbesondere mit Blick darauf, wie sie in einer Modellrechnung über die Vorteilhaftigkeit einer Wanderung berücksichtigt werden können. Zunächst werden die verschiedenen Komponenten beleuchtet, die bei der Bestimmung der regionalen Einkommen zu beachten sind. Im Anschluß wird dargelegt, auf welche Weise regionale Preisunterschiede in die Rechnung einbezogen werden können. Es folgen dann Überlegungen dazu, wie weitere Kosten und Erträge monetär zu bewerten sind, und zwar die Standortqualität, Transaktions- und Informationskosten, Distanz, psychische Kosten und Erträge sowie wiederholte Wanderungen. Abschließend wird die Bedeutung personenspezifischer Merkmale beleuchtet.

\subsubsection{Regionale Einkommens- und Arbeitslosigkeitsunterschiede}

Der regionale Unterschied der monetären Nettoeinkommen erklärt sich nicht nur durch pauschale regionale Lohndifferenzen, sondern auch durch weitere Faktoren, wie etwa der formalen Qualifikation und Berufserfahrung. Zudem bedeuten statistische regionale Lohndifferenzen nicht gleichzeitig, daß ein Arbeitnehmer diese Lohnunterschiede auch realisieren kann, da Arbeitsmärkte i.d.R. nicht den idealtypischen Annahmen entsprechen, wie sie dem neoklassischen Modell unterliegen, sondern durch Marktunvollkommenheiten gekennzeichnet sind. Im folgenden wird erörtert, welche verschiedenen Einkommensbestandteile besten-

${ }^{146} \mathrm{Vgl}$. Straubhaar (1994), S. 5f. 
falls bei der Abwägung einer Umzugsentscheidung berücksichtigt werden sollten, und im Anschluß wird die Bedeutung der potentiellen Arbeitslosigkeit behandelt.

\subsection{Einkommensbestandteile}

Im folgenden werden nur die Einkommen aus Arbeit betrachtet und Kapitaleinkünfte ausgeklammert. Zur Vereinfachung wird angenommen, daß die Entscheidung, in welcher Region das Kapital investiert wird, von der Wohnsitzentscheidung unabhängig ist. Die Kapitaleinkünfte ändern sich dann bei einem Umzug nicht.

Einbezogen werden in die Rechnung sollte das gesamte Jahresentgelt eines Arbeitnehmers, um betriebliche Sonderzahlungen wie Urlaubs- oder Weihnachtsgeld $\mathrm{zu}$ erfassen. Auch vermögenswirksame Leistungen und Gewinnbeteiligungen sollte nach Möglichkeit berücksichtigt werden.

Bei der Bestimmung der monetären Erträge sollten bei einem Vergleich der Jahreseinkommen berücksichtigt werden, ob sich die Wochenarbeitszeiten, die Zahl der (bezahlten) Urlaubs- und Feiertage voneinander unterscheiden. Monetär einbezogen werden könnte dies durch eine Bewertung der Differenz der Arbeitsstunden zum Stundenlohnsatz.

$\mathrm{Zu}$ den Erträgen zählen außerdem die betrieblichen Zusatzleistungen, etwa Rabatte oder die unentgeltliche Nutzungsmöglichkeit betriebseigener Sportstätten ${ }^{147}$. Zudem muß möglicherweise auch der Verlust einer Anwartschaft auf eine Betriebsrente durch einen Wechsel des Arbeitgebers ${ }^{148}$ berücksichtigt werden. Darüber hinaus sind die künftigen Einkommensperspektiven einzukalkulieren, wenn sich die Karrierechancen regional unterscheiden. So könnte z.B. ein Arbeitnehmer im Stammsitz eines Unternehmen schneller befördert werden, als wenn er in einer Zweigstelle tätig ist.

Für die Höhe des anzusetzenden Einkommens ist auch entscheidend, ob am Zielort ein der Qualifikation entsprechender Arbeitsplatz gefunden werden kann oder ob sich die berufliche Stellung verschlechtert. Zieht etwa ein Facharbeiter

${ }^{147}$ Der Lohn könnte so angesetzt werden, daß er die betrieblichen Annehmlichkeiten einschließt, wobei diese nach Zahlungswilligkeitsgesichtspunkten zu bewerten wären. Dieser Zugang wäre allerdings nicht unproblematisch, da bezüglich der Annehmlichkeiten Präferenzunterschiede bestehen dürften.

${ }^{148} \mathrm{Vgl}$. zu dem Einfluß von Betriebsrenten auf die Wanderungsentscheidung Lansing/Mueller (1973), S.159ff. 
in eine andere Region, weil dort die Facharbeiterlöhne höher sind, und findet dort lediglich eine Stelle als angelernter Arbeiter, hätte er seinen Migrationsgewinn überschätzt, wenn er vor der Wanderung mit dem Lohnniveau eines Facharbeiters in der neuen Region gerechnet hätte.

Schätzungen des Gegenwartswertes der Einkommensdifferenz sind außerdem von den Zukunftserwartungen abhängig. Die Erwartungen darüber, über welchen Zeitraum regionale Lohnunterschiede bestehen oder ob sie überhaupt abgebaut werden, unterscheiden sich individuell. Selbst wenn zwischen den Tarifparteien vereinbart wird, in welchem Zeitraum die Löhne interregional angeglichen werden sollen, hängt es von der Glaubwürdigkeit dieser Gruppen und den Erfahrungen in der Vergangenheit ab, wie diese Daten in das Entscheidungskalkül eingehen.

Entscheidend für die Haushalte ist nicht das Brutto-, sondern das verfügbare Einkommen. Daher sind die regionalen Einkommensteuertarife bei der Berechnung des Wanderungsertrages zu berücksichtigen, ebenso wie alle anderen regionalen steuerlichen Unterschiede, die die Höhe des Nettoeinkommens der Haushalte ${ }^{149}$ beeinflussen, wie etwa - im Fall der Bundesrepublik - die Kirchensteuersätze. Daneben wird das Nettoeinkommen bestimmt von der Art des Steuertarifs. Progressive Steuertarife wirken im Vergleich zu proportionalen Steuertarifen eher mobilitätsmindernd, da sie bei gleichen Steuertarifen zu einer stärkeren Angleichung der Nettoeinkommen der regional unterschiedlichen Bruttolöhne führen als proportionale Tarife. Gibt es in einer Region progressive, in einer anderen proportionale Tarife, könnten die Wanderungsanreize von Beziehern niedriger Einkommen in die Region mit den progressiven Tarifen aber auch stärker sein. Darüber hinaus kann es von dem Familienstand abhängen, wie hoch das Nettogehalt ausfällt. Für einen Ledigen ist die absolute Differenz des Nettogehalts bei einem progressiven Steuertarif i.d.R. niedriger als für Verheiratete gleiche Bruttoeinkommen vorausgesetzt. Außerdem ist zu berücksichtigen, daß sich die Beiträge (und Leistungen) zur Sozialversicherung regional unterscheiden können.

\subsection{Das Arbeitslosigkeitsrisiko}

Empirische Untersuchungen weisen darauf hin, daß Arbeitslose mit einer größeren Wahrscheinlichkeit als Beschäftigte migrieren. Hohe Arbeitslosenquoten in

${ }^{149}$ Interregionale Unterschiede bei Steuern, die die Unternehmen betreffen, dürften für die Haushalte keine Rolle spielen, es sei denn, sie sind selbständig. Diese Steuerarten könnten allerdings Rückwirkungen auf das regionale Preisniveau von nicht-handelbaren Gütern haben. 
der Heimatregion im Vergleich zu anderen Regionen bestärken Arbeitslose in ihren Umzugsabsichten, auf die Beschäftigten sind sie aber nur von einem geringen Einflu $\beta^{150}$. Dieses Ergebnis läßt sich u.a. mit suchtheoretischen Überlegungen erklären: Arbeitslose suchen intensiver nach einer neuen Stelle als Erwerbstätige (Job-Matching-Theorie) ${ }^{151}$. Die Dringlichkeit eines Umzug bei Arbeitslosigkeit wird abgemildert, wenn für die Zeit der Beschäftigungslosigkeit Ersatzeinkommen bezogen werden. Der Ertrag eines Umzugs, der mit einem Wechsel von Arbeitslosigkeit in Erwerbstätigkeit verbunden ist, ist um so geringer, je höher die Transferleistungen sind.

Bei anhaltender Arbeitslosigkeit verschlechtern sich die Aussichten der Arbeitslosen auf eine neue Anstellung. Arbeitslose haben weniger Möglichkeiten als Beschäftigte, ihre beruflichen Fähigkeiten zu erhalten und auf dem neuesten Stand zu bleiben. Mithin tritt häufig eine Entwertung ihrer Qualifizierung auf ${ }^{152}$. Arbeitslosigkeit wird zudem von den Arbeitgebern als "screening device“ genutzt, d.h. die Arbeitslosigkeit und ihre Dauer dienen als Qualifikationserkennungsmerkmal. Deshalb sinkt mit zunehmender Dauer der Arbeitslosigkeit nicht nur die Wahrscheinlichkeit, eine neue Anstellung zu finden, auch die Attraktivität eines schließlich gefundenen Arbeitsplatzes (und damit auch die Entlohnung) ist i.d.R. geringer. In Umbruchperioden (wie bei der deutsche Vereinigung) dürften die Arbeitgeber allerdings davon ausgehen, daß die Zugehörigkeit zur Gruppe der Arbeitslosen stärker zufallsabhängig ist als in „normalen“" Phasen und daraus nicht in gleichem Maße wie sonst üblich auf die Leistungsfähigkeit zurückgeschlossen werden kann $^{153}$.

Bei der Berechnung des Migrationsertrags müssen Transferleistungen und die (individuelle) Wahrscheinlichkeit, arbeitslos zu werden, einbezogen werden. In Gleichung (1) wird dargestellt, wie bei Berücksichtigung dieser Faktoren das verfügbare monetäre Einkommen aus Erwerbstätigkeit bzw. Lohnersatzleistungen in einer Region berechnet werden kann. Für die Bestimmung der Nettoerträge einer potentiellen Migration müßte eine solche Rechnung für alle in Frage kommenden Regionen aufgestellt werden. Die monetären Gewinne können dann durch eine einfache Differenzrechnung ermittelt werden.

Zur Vereinfachung sei bezüglich der Transfers angenommen, daß im ersten Jahr der Arbeitslosigkeit (lohnabhängiges) Arbeitslosengeld, im zweiten Jahr (lohnabhängige) Arbeitslosenhilfe und in den darauf folgenden Jahren (lohnunabhängige) Sozialhilfe gezahlt werde. Die Transfereinkommen seien nicht zu ver-

\footnotetext{
${ }^{150}$ Vgl. DaVanzo (1978).

${ }^{151}$ Vgl. Zimmermann (1993), S. 287.

${ }^{152}$ Vgl. Wagner (1992), S.85.

${ }^{153} \mathrm{Vgl}$. Büchel/Pannenberg (1992), S. 159.
} 
steuern. Die Berechnung des Erwartungswertes der Erträge aus Erwerbstätigkeit $\left(\mathrm{LE}_{\mathrm{x}}\right)$ bzw. Arbeitslosigkeit $\left(\mathrm{AE}_{\mathrm{x}}\right)$ wird in Gleichung (1) für lediglich drei Perioden aufgestellt. Schon so zeigt sich die Komplexität der Berücksichtigung des regionalen Risikos, arbeitslos zu werden.

$$
\begin{aligned}
A E_{x} & =\frac{q_{1} * L E_{x 1}+\left(1-q_{1}\right) A L G_{x 1}}{1+r}+\frac{q_{2} * L E_{x 2}+\left(1-q_{2}\right)\left[q_{1} * A L G_{x 2}+\left(1-q_{1}\right) A L H_{x 2}\right]}{(1+r)^{2}} \\
& +\frac{q_{3} * L E_{x 3}+\left(1-q_{3}\right)\left[q_{2} * A L G_{x 3}+\left(1-q_{2}\right)\left(q_{1} * A L H_{x 3}+\left(1-q_{1}\right) S H_{x 3}\right]\right.}{(1+r)^{3}}
\end{aligned}
$$

mit:

$\mathrm{q}_{\mathrm{i}}$ : Wahrscheinlichkeit, in der Periode $\mathrm{i}$ über einen Arbeitsplatz zu verfügen

$\mathrm{LE}_{\mathrm{xi}}: \quad$ Nettolohneinkommen in einem zum Zeitpunkt 0 der Qualifikation entsprechenden Beruf, berechnet wie in (1) in der Region $\mathrm{x}$ in Periode $\mathrm{i}$.

$\mathrm{ALG}_{\mathrm{xi}}$ : Arbeitslosengeld in Region $\mathrm{x}$ in der Periode $\mathrm{i}$

$\mathrm{ALH}_{\mathrm{xi}}$ : Arbeitslosenhilfe in Region $\mathrm{x}$ in der Periode $\mathrm{i}$

$\mathrm{SH}_{\mathrm{xi}}: \quad$ Sozialhilfe in Region $\mathrm{x}$ in der Periode $\mathrm{i}$

Diese Berechnung gilt nur für Personen, die in der Periode 0 nicht arbeitslos sind. Bei Arbeitslosigkeit in Periode 0 ist die Berechnung entsprechend zu modifizieren.

Für die erste Periode ergibt sich der Erwartungswert für das Einkommen als Lohneinkommen $\left(\mathrm{LE}_{\mathrm{x} 1}\right)$, gewichtet mit der Wahrscheinlichkeit beschäftigt $\mathrm{zu}$ sein $\left(q_{1}\right)$, plus Arbeitslosengeld $\left(A_{L} G_{x 1},\right)$ gewichtet mit der Gegenwahrscheinlichkeit $\left(1-q_{1}\right)$ nicht beschäftigt zu sein. Für die Einkommensermittlung der zweiten Periode wird das potentielle Lohneinkommen wiederum gewichtet mit der Wahrscheinlichkeit beschäftigt zu sein. Mit der Gegenwahrscheinlichkeit wird die Alternative - nämlich arbeitslos zu sein und Transfereinkünfte zu beziehen - gewichtet. Dabei muß jetzt aber noch zusätzlich gewichtet werden. Die Alternative Arbeitslosengeld zu beziehen wird gewichtet mit der Wahrscheinlichkeit in der ersten Periode beschäftigt zu sein, da nur in diesem Fall Arbeitslosengeld bezogen wird. Entsprechend wird die letzte Möglichkeit, nämlich Arbeitslosenhilfe zu beziehen, mit der Wahrscheinlichkeit gewichtet, in der Vorperiode ohne Beschäftigung gewesen zu sein. Für die dritte Periode wird die Berechnung der Transferleistungen entsprechend noch stärker aufgesplittet.

In dieser Rechnung ist der Fall noch nicht enthalten, daß nach einer Phase der Arbeitslosigkeit wieder eine Beschäftigung aufgenommen wird und die Entlohnung des neuen Jobs aufgrund der vorangegangenen Arbeitslosigkeit von einem früher erzielbaren Lohn negativ abweicht. Vorausgesetzt zum Zeitpunkt Null 
liegt keine Arbeitslosigkeit vor, ändert sich an der Einkommensberechnung für die erste Periode nichts. In der zweiten Periode aber weicht die Berechnung von Gleichung $1 \mathrm{ab}$. Der Einkommenserwartungswert für die zweite Periode in Region $\mathrm{x}\left({ }_{2} \mathrm{AE}_{\mathrm{x}}\right)$ berechnet sich als:

$$
{ }_{2} A E_{x}=q_{2, a} * L_{x 2}+q_{2, b} * L_{x 2} * D E Q+\left(1-q_{2, a}-q_{2, b}\right)\left[q_{1} * A L G_{x 2}+\left(1-q_{1}\right) A L H_{x 2}\right]
$$

mit

$\mathrm{q}_{2, \mathrm{a}}$ :

Wahrscheinlichkeit in der zweiten Periode beschäftigt zu sein unter der Bedingung auch in der ersten Periode beschäftigt zu sein

$\mathrm{q}_{2, \mathrm{~b}}$ : Wahrscheinlichkeit in der zweiten Periode beschäftigt zu sein unter der Bedingung Arbeitslosigkeit in der ersten Periode

DEQ: $\quad$ Dequalifizierungsfaktor

$\left(1-q_{2, a}-q_{2, b}\right)$ : Wahrscheinlichkeit in der zweiten Periode arbeitslos zu sein.

Bei Beschäftigung in der ersten und zweiten Periode wird jeweils ein Arbeitslohn entsprechend $L E_{x}$ bezogen. Besteht dagegen in einer früheren Periode Arbeitslosigkeit, kann der Lohn aus oben genannten Gründen geringer sein. Daher ist das Lohneinkommen für diesen Fall mit einem "Dequalifizierungsfaktor“ DEQ, der das Ausmaß der Entwertung des Humankapitals anzeigt und daher kleiner als Eins ist, zu multiplizieren.

Für die dritte Periode ergibt sich entsprechend:

$$
\begin{aligned}
{ }_{3} \mathrm{AE}_{\mathrm{x}}= & \mathrm{q}_{3, \mathrm{a}} * \mathrm{LE}_{\mathrm{x} 3}+\mathrm{q}_{3, \mathrm{~b}} * \mathrm{LE}_{\mathrm{x} 3} * \mathrm{DEQ}_{1}+\mathrm{q}_{3, \mathrm{c}} * \mathrm{LE}_{\mathrm{x} 3} * \mathrm{DEQ}_{1}+\mathrm{q}_{3, \mathrm{~d}} * \mathrm{LE}_{\mathrm{x} 3} * \mathrm{DEQ}_{2} \\
& +\left(1-\mathrm{q}_{3}\right)\left[\mathrm{q}_{2} * \mathrm{ALG}_{\mathrm{x} 3}+\left(1-\mathrm{q}_{2}\right)\left(\mathrm{q}_{1} * \mathrm{ALH}_{\mathrm{x} 3}+\left(1-\mathrm{q}_{1}\right) \mathrm{SH}_{\mathrm{x} 3}\right]\right.
\end{aligned}
$$

mit

$\mathrm{q}_{3}=\mathrm{q}_{3, \mathrm{a}}+\mathrm{q}_{3, \mathrm{~b}}+\mathrm{q}_{3, \mathrm{c}}+\mathrm{q}_{3, \mathrm{~d}}$

$\mathrm{q}_{3}$ : Wahrscheinlichkeit, in Periode 3 über einen Arbeitsplatz zu verfügen

$\mathrm{q}_{3, \mathrm{a}}$ : Wahrscheinlichkeit, in Periode 3 über einen Arbeitsplatz zu verfügen und in den beiden Vorperioden nicht arbeitslos gewesen zu sein

$\mathrm{q}_{3, \mathrm{~b}}: \quad$ wie $\mathrm{q}_{3, \mathrm{a}}$, aber Arbeitslosigkeit in Periode 1

$\mathrm{q}_{3, \mathrm{c}}:$ wie $\mathrm{q}_{3, \mathrm{a}}$, aber Arbeitslosigkeit in Periode 2

$\mathrm{q}_{3, \mathrm{~d}}: \quad$ wie $\mathrm{q}_{3, \mathrm{a}}$, aber Arbeitslosigkeit in Periode 1 und 2

$\mathrm{DEQ}_{\mathrm{i}}$ : Dequalifizierungsfaktor bei einer Dauer der Arbeitslosigkeit von i Perioden

Arbeitslosigkeit ist nicht nur mit einem geringeren monetären Einkommen verbunden, sondern auch mit mehr Freizeit. Freizeit stiftet einen Nutzen. Die hohe Freizeit der Arbeitslosen müßte daher als positive Größe - nämlich als nicht-monetärer Ertrag - berücksichtigt werden. Diese Annahme dürfte aber mit Blick auf Arbeitslosigkeit nicht zutreffen. Zwar verfügen Arbeitslose über viel Freizeit - 
von der allerdings i.d.R. ein nicht unerheblicher Teil für die Arbeitsuche eingesetzt wird -, gleichzeitig geht Arbeitslosigkeit aber häufig mit einer psychischen Belastung einher. Inwieweit Arbeitslosigkeit nicht-monetäre Kosten oder Erträge erbringt, kann im Rahmen dieser Arbeit nicht beurteilt werden und wird daher in der später durchgeführten Migrationsrechnung nicht berücksichtigt.

\subsubsection{Regionale Preisunterschiede}

Neben den nominalen Lohneinkommensdifferenzen sind regionale Preisunterschiede entscheidend für die realen Einkommensdifferenzen und die monetären Erträge einer Wanderung. Die Ermittlung von regionalen Preisen ist jedoch schwierig: regionale Preisindizes sind kaum oder gar nicht verfügbar ${ }^{154}$. Potentielle Migranten müssen daher auf andere Informationsquellen zurückgreifen, die aber häufig nur einen Teil der Preisniveaudifferenzen aufzeigen, wie etwa regionale Mietenspiegel oder eher subjektive eigene Beobachtungen.

Hinzu kommt, daß ein Vergleich der regionalen Preisniveaus von unterschiedlichen Individuen zu voneinander abweichenden Bewertungen führt, auch wenn diese das gleiche Nominaleinkommen erzielen und dieselben Güterpreise zugrunde legen. Das individuelle regionale Preisniveau errechnet sich in Abhängigkeit von dem jeweiligen individuellen Warenkorb. Unterschiedliche Präferenzen für handelbare und lokale Güter können dazu führen, daß bei einem identischen Nominallohn verschiedene Warenkörbe gekauft werden ${ }^{155}$. Es ist daher im Prinzip unzulänglich, wenn bei der Bestimmung von individuellen Migrationsgewinnen für alle Menschen die gleichen regionalen Preisniveaudifferenzen angesetzt werden.

Entscheidend für einen Migranten ist darüber hinaus nicht das durchschnittliche Preisniveau einer Region, sondern ob er die gewünschten (nicht-handelbaren) Güter zu den gleichen Preisen nachfragen kann wie die Einheimischen. Insbesondere auf dem Wohnungsmarkt können sich Differenzen ergeben: Die Preise bei Neuanmietung können - aufgrund von Reglementierungen auf dem Wohnungsmarkt - die durchschnittlichen Mietpreise deutlich übersteigen.

Ebenso wie beim Einkommen sollte in die Berechnung des Migrationsüberschusses eingehen, ob zu erwarten ist, daß die Preisniveaudifferenzen dauerhaft sind oder ob sie im Laufe der Zeit ab- oder zunehmen werden.

${ }^{154}$ Vgl. Götzinger (1981), S. 110.

${ }^{155}$ Im Mai 1990 etwa kostete der DDR-Warenkorb in Ost-Berlin 76 DM, derselbe Warenkorb kostete in West-Berlin 100 DM. Dagegen kostete der westdeutsche Warenkorb in WestBerlin 100 DM und in Ost-Berlin 114 DM (vgl. Busch (1991), S. 226). 
Um die Preisunterschiede für eine Migrationsrechnung bewertbar zu machen, könnte der Warenkorb zugrunde gelegt werden, der in der Heimatregion zum Zeitpunkt Null nachgefragt wird, und es könnten alle alternativen Warenkörbe bestimmt werden, die den gleichen Nutzen brächten. Danach ist zu klären, welcher dieser Warenkörbe für die geringste Geldsumme in der potentiellen $\mathrm{Zu}$ zugsregion gekauft werden könnte, um auf dieser Basis die Preisunterschiede zu bestimmen.

Formelmäßig läßt sich die Einkommensdifferenz nach Berücksichtigung der Preisunterschiede einer Periode $\mathrm{i}$ in der Region $\mathrm{x}$ im Vergleich zu Region y $\left({ }_{i} \mathrm{Em}_{\mathrm{xy}}\right)$ wie folgt bestimmen:

${ }_{i} \mathrm{Em}_{\mathrm{xy}}=\left({ }_{\mathrm{i}} \mathrm{AE}_{\mathrm{x}}-\mathrm{WK}_{\mathrm{pxi}}\right)-\left({ }_{\mathrm{i}} \mathrm{AE} \mathrm{E}_{\mathrm{y}}-\mathrm{WK}_{\mathrm{pyi}}\right)$

mit:

$\mathrm{WK}_{\mathrm{pji}}$ : minimale Ausgabe für einen Warenkorb mit dem Nutzen $\mathrm{k}$ in der $\mathrm{j}=\mathrm{x}, \mathrm{y}$ Region $\mathrm{j}$ in der Periode $\mathrm{i}$

Entsprechend gilt für die preisbereinigte Einkommensdifferenz des gesamten Planungszeitraums $\mathrm{t}=3$ in der Region $\mathrm{x}$ :

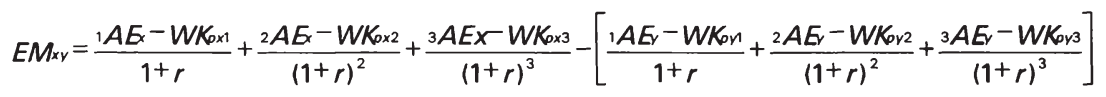

Durch eine derartige Berücksichtigung von Preisniveaudifferenzen wird vermieden, daß Erhöhungen der Konsumausgaben, die durch Einkommensverbesserungen infolge von besserer Entlohnung in der Zuzugsregion verursacht werden, als monetäre Kosten in das Wanderungskalkül eingehen.

\subsubsection{Regionale Annehmlichkeiten}

Die regionalen Annehmlichkeiten einer Region werden - wie bereits in Abschnitt 2.4 erläutert - durch eine Vielzahl von Faktoren bestimmt. Diese Faktoren sind besonders inhomogen und monetär nur schwer zu bewerten. Unter Standortqualität fallen das kulturelles Angebot vor Ort wie Museen, Kino, Theater, Konzerte, Oper, Bildungsmöglichkeiten wie Schule, Hochschule, Volkshochschule, außerdem Sportmöglichkeiten, Gaststättenangebot, Gesundheitsangebot, Landschaft, Klima und Umgebung etc. Es sind die Faktoren, die Birg unter den Punkten „Verfügbarkeit über Dienstleistungen, öffentliche und private Güter“ 
und „natürliche und soziale Umweltbedingungen“ zusammenfaßt ${ }^{156}$. Diese Faktoren treten zum Teil in Form von monetären Erträgen und zum Teil als nicht-monetäre Erträge auf. Ein wärmeres Klima etwa trägt dazu bei, Heizkosten einzusparen und kann obendrein einen nicht-monetären Ertrag durch ein erhöhtes Wohlbefinden erbringen.

Die Verfügbarkeit von Wohnraum könnte eine besonders wichtige Komponente der Standortqualität sein. Je geringer das Angebot an Wohnraum, desto höher sind die Mietkosten und die Transaktionskosten der Wohnraumbeschaffung ${ }^{157}$.

Die Standortfaktoren zu bewerten, ist auch deshalb ein äußerst schwieriges Unterfangen, da diese subjektiv sehr unterschiedlich beurteilt werden. Bei einzelnen Faktoren besteht nicht einmal eine eindeutige Beziehung, ob sie Nutzen stiften oder nicht. Ein Spielkasino vor Ort mag dem einen Freude bereiten, einem anderen gleichgültig und einem dritten unangenehm sein.

Die Bewertung vieler Annehmlichkeiten sollte daher individuell erfolgen. Dabei könnte gefragt werden, welche Annehmlichkeiten dem potentiellen Migranten besonders wichtig sind und welchen Betrag er für das Vorhandensein dieser Faktoren vor Ort zu zahlen bereit wäre. Allerdings wären bei der Beantwortung solcher Fragen viele Menschen wohl überfordert.

\subsubsection{Informationskosten und -probleme}

Menschen verfügen nicht über vollständige Informationen. Sie kennen die Ertragsmöglichkeiten in den verschiedenen Regionen nicht oder nur unvollkommen. Sie können zwar Informationen einholen, dies ist jedoch mit Kosten verbunden. Ökonomisch rational handelnde Individuen beschaffen sich so viele Informationen, bis die marginalen Kosten der Informationsbeschaffung den marginalen Erträgen entsprechen ${ }^{158}$. Vollständige Informationen über alle möglichen Alternativen werden i.d.R. nicht erreicht.

Menschen ziehen eher in Gegenden über die sie bereits viel wissen, als in ihnen unbekannte Gegenden ${ }^{159}$. Informationen aufgrund persönlicher Beziehungen scheinen dabei eine große Rolle zu spielen ${ }^{160}$, da sich der Kenntnisstand erhöht,

${ }^{156}$ Vgl. Birg (1975), S.45.

${ }^{157}$ Dieser Faktor ist aber nicht zwingend als Element der Standortqualität anzusehen, er könnte ebenso gut bei ,regionale Preisunterschiede" eingeordnet werden.

${ }^{158} \mathrm{Vgl}$. Bodenhöfer (1967), S. 443.

${ }^{159} \mathrm{Vgl}$. Greenwood (1970), S. 375.

${ }^{160}$ Vgl. Feithen (1985), S. 66. 
wenn bereits einige Personen aus der Heimatregion umgezogen sind und es zu einem Informationsrückfluß über die von ihnen gemachten Erfahrungen kommt. Das Wissen um die Erfahrungen der Migranten vermindert das Wanderungsrisiko der bisher Immobilien ${ }^{161}$. Ein besonders hoher Informationsrückfluß ist zu erwarten, wenn es sich bei den Migranten um Freunde oder Verwandte handelt. Hierdurch erhöht sich mithin die Wahrscheinlichkeit, daß die Zurückgebliebenen ebenfalls in die Zielregion der vor ihnen gewanderten ziehen. Außerdem erhöhen sich in der Zugangsregion die Informationen über die Abwanderungsregion, was wiederum einen Wanderungsstrom in diese Richtung auslösen kann ${ }^{162}$. Nach Stark und Bloom (1985) wird dieser Prozeß als innovative Annahme (Adoption) und Verbreitung (Diffusion) bezeichnet.

Der Bedarf an Informationen betrifft alle Ertrags- und Kostenkomponenten. Dabei hängt es von den individuellen Präferenzen ab, in welchem Ausmaß Informationen über die einzelnen Faktoren beschafft werden. Die Möglichkeiten, sich über die verschiedenen Kosten und Erträge zu informieren, sind sehr unterschiedlich, hierauf wurde z.T. schon hingewiesen.

Die Informationsbeschaffung wird durch eine schlechte Infrastruktur vor Ort teurer und schwerer. Ferner ist anzunehmen, daß die Kosten, die für die Erlangung von Informationen aufgewandt werden müssen, mit zunehmender Entfernung steigen ${ }^{163}$. Die Menge an Informationen, die sich zu beschaffen lohnt, müßte daher mit zunehmender Distanz sinken.

Informationsbeschaffung nimmt nicht nur Geld, sondern auch Zeit in Anspruch, was dazu führt, daß Menschen auf veränderte Umweltbedingungen erst mit zeitlicher Verzögerung reagieren. Migrationen finden daher erst mit einem time lag statt. Außerdem müssen Haushalte typischerweise einen hohen Grad an Trägheit überwinden, bevor sie (Wanderungs) Entscheidungen treffen. Diese Trägheit kann einhergehen mit der größeren Sicherheit, die gegenwärtigen Um-

${ }^{161}$ Neben dem Informationsfluß beeinflussen bereits stattgefundene Migrationen weitere Wanderungen noch auf andere Weise. Zum einen empfinden die Zurückgebliebenen, daß ihr relatives Einkommen gesunken ist, wenn sie die (erfolgreichen) Migranten in ihrer Referenzsystem einbeziehen. Außerdem vermindern erfolgreiche Migranten das soziale Stigma, welches Wanderungen manchmal anhaftet. Dies macht es für die Zurückgebliebenen leichter, sich für eine Wanderung zu entscheiden. Sind die Migranten dagegen nicht erfolgreich, so gelten diese Zusammenhänge mit umgekehrten Vorzeichen. Vgl. hierzu auch Fischer u.a. (1995a), S.33.

${ }^{162}$ Vgl. Greenwood (1970), S. 375. Bereits Ravenstein (1885) beschrieb in seinen Gesetzen der Wanderung, daß jede Hauptwanderungsströmung eine kompensierende Gegenströmung erzeugt.

${ }^{163}$ Vgl. Schwartz (1973), S. 1159. 
stände zu kennen. Alternativen sind unsicherer und bergen daher Risiken. Je risikoaverser ein Mensch ist, desto mehr wird er in die Informationsbeschaffung investieren ${ }^{164}$.

Informationskosten- und -probleme spielen mithin eine wichtige Rolle bei der Migrationsentscheidung. Es stellt sich die Frage, ob und wieweit diese Kosten bei einer Berechnung des Migrationsgewinns einbezogen werden sollten. Eine Anrechnung dieser Kosten mindert einen eventuellen Überschuß. Auch wenn nach Prüfung aller Möglichkeiten der Entschluß gefällt wird nicht umzuziehen, sind für die Entscheidungsfindung Kosten angefallen. Diese Kosten sollten, da sie unabhängig von der letztlichen Entscheidung für oder gegen einen Umzug entstehen, nicht berücksichtigt werden, da sie für jede mögliche Alternative in gleicher Höhe anzusetzen wären. Spezielle Informationskosten, die erst nach dem Entschluß für einen Umzug anfallen würden, sind dagegen bei der Ermittlung des Migrationsgewinns einzubeziehen.

\subsubsection{Transaktionskosten}

Mit einem Umzug sind verschiedene Transaktionskosten verbunden. Umzugskosten fallen einmalig an und sind verhältnismäßig einfach zu quantifizieren, wenn die Durchschnittskosten zugrunde gelegt werden, die ein Speditionsunternehmen für die Durchführung eines Umzuges berechnen würde. Herangezogen werden könnten aber auch die steuerlichen Vorschriften über die Absetzbarkeit von Umzugskosten. Die Umzugskosten dürften mit zunehmender Distanz der Wanderung steigen. Außerdem gibt es einen positiven Zusammenhang zwischen Umzugskosten und dem Alter der Haushaltsmitglieder. Im Laufe der Zeit sammeln Menschen immer mehr Möbel und andere persönliche Dinge an. Ferner sinkt die Bereitschaft und die Fähigkeit, den Umzug selber durchzuführen, mit zunehmendem Alter ${ }^{165}$. Ein weiterer Einflußfaktor ist die Haushaltsgröße: je mehr Personen er umfaßt, desto umfangreicher ist das anfallende Umzugsgut. Darüber hinaus fallen noch Reisekosten für die Haushaltsmitglieder an.

Teilweise erstatten die Unternehmen die Reisekosten für ein Vorstellungsgespräch und die Umzugskosten (wenn sie es nicht tun und der Betreffende arbeitslos ist, übernimmt z.T. das Arbeitsamt die Kosten), dann dürften die Kosten für Arbeitsuche und Umzug nicht in die Rechnung eingehen. Berücksichtigt werden sollte außerdem, daß die verbleibenden Kosten u.U. steuerlich absetzbar sind.

\footnotetext{
${ }^{164}$ Vgl. Fischer u.a. (1995a), S. 17.

${ }^{165} \mathrm{Vgl}$. Lansing/Mueller (1973), S. 234.
} 
So wie sich ein Arbeitnehmer betriebsspezifisches Humankapital aneignet, so erhöht sich das regionsspezifische Wissen eines Immobilen mit zunehmender Dauer des Aufenthalts. Durch dieses Wissen kann er Vorteile in seiner Freizeit erzielen, etwa weil er weiß, wo er gut und günstig essen gehen oder einkaufen kann ${ }^{166}$. Diese insider Vorteile gehen bei einem Umzug verloren und müssen an einem anderen Ort wieder neu erworben werden, so daß neben den Umzugskosten Kosten, die mit der Integration am neuen Wohnort verbunden sind, entstehen. Die Transaktionskosten können sich verringern, wenn bereits Familienangehörige oder Freunde am künftigen Wohnort leben. Diese können vorübergehend eine Unterkunft gewähren oder Tips über Einkaufsmöglichkeiten, Gaststätten u.ä. geben, so daß sich derartige Suchkosten verringern.

Weitere mögliche Transaktionskosten, die durch einen Umzug entstehen und von größerer Bedeutung sein könnten, sind Kosten, die durch Kauf/Verkauf eines Eigenheimes anfallen. Hausbesitzer haben deshalb regelmäßig wesentlich höhere Transaktionskosten als Mieter.

Im Extremfall können Transaktionskosten Wanderungen verhindern, selbst dann, wenn die Wanderungsrechnung für eine Wanderung spricht. Denkbar ist, daß potentielle Migranten nicht über genügend hohe Rücklagen verfügen, eine Wanderung zu finanzieren, und sie nicht kreditwürdig bzw. in der Lage sind, eine für einen Umzug ausreichend hohe Summe anzusparen.

\subsubsection{Die Länge der Entfernung zwischen Quell- und Zielregion}

Die Bedeutung der Entfernung für die Wanderungsentscheidung wurde bereits bei anderen Aspekten deutlich (z.B. Informationskosten, Transaktionskosten). Die Kosten, die durch die Länge der Distanz verursacht werden, werden im wesentlichen schon bei der Berücksichtigung dieser Faktoren erfaßt. Da diesem Faktor in der Literatur eine sehr große Bedeutung beigemessen wird, sei er hier jedoch noch einmal gesondert genannt. Die Distanz hat folgenden Einfluß:

Mit zunehmender Entfernung

- nehmen die Informationen ab

- steigen die Suchkosten

- steigen die Transportkosten

- unterscheidet sich das soziale System (Unterschiede im sozialen System wirken mobilitätshemmend)

${ }^{166}$ Vgl. hierzu Fischer u.a. (1998), S. 4f, wo die Vorteile als „leisure-oriented location-specific advantages" bezeichnet werden. 
- steigen die regionalen Unterschiede in den klimatischen und natürlichen Bedingungen

- steigt die Zahl der innerhalb dieser Entfernung liegenden möglichen Ziele $^{167}$

Steigende Distanz wirkt grundsätzlich mobilitätshemmend. Auch die mit der Entfernung zunehmenden Unterschiede in den klimatischen und natürlichen Bedingungen werden überwiegend als mobilitätshemmend eingeschätzt. Hierdurch werden weitere Anpassungen an die neue Region erforderlich ${ }^{168}$. Lediglich die Zunahme von Alternativen mit zunehmender Entfernung könnte sich positiv auswirken.

\subsubsection{Die Bedeutung früherer Wanderungen und Rückwanderungen}

Wenn sich die Erwartungen der Akteure, die an eine Wanderung ursprünglich geknüpft waren, nicht erfüllen, kann dies zu (ungeplanten) weiteren Umzügen führen. Nur bei vollständigen Informationen und Sicherheit über die zukünftige Entwicklung könnte ein potentieller Migrant die Migrationserträge stets richtig abschätzen und Fehlentscheidungen vermeiden ${ }^{169}$. Je unzutreffender und unvollkommener die Informationen vor der ursprünglichen Wanderung waren, desto wahrscheinlicher kommt es zu einer „korrigierenden“ Wanderung. Wurde die ursprüngliche Wanderung durch Arbeitslosigkeit hervorgerufen, kommt es häufig zu Rückwanderungen. Diejenigen mit der schlechtesten Ausbildung kehren meistens am ehesten zurück, während die besser ausgebildeten vielfach noch weiter wandern ${ }^{170}$.

Jemand, der bereits einmal gewandert ist, ist über den Prozeß einer Wanderung besser informiert als jemand, der zuvor noch nicht umgezogen ist. Die Wahrscheinlichkeit der Migration ist daher bei Personen, die bereits einmal gewandert sind, höher, da ihre Kosten geringer sind. Außerdem besteht für sie die Möglichkeit, an einen Ort zurückzukehren, an dem sie bereits einmal gelebt haben und einen Teil ihres standortgebundenen Kapitals wieder zu aktivieren (wie Wiederaufnahme bzw. Fortsetzung von Freundschaften, bei Arbeit in dem gleichen Betrieb Nutzung früher erworbener betriebsspezifischer Kenntnisse). Der Migrant wird daher eher wieder an diesen Ort zurückkehren, als sich einen anderen suchen (manchmal aber auch gerade nicht, wenn er ihn in schlechter Erinnerung behalten hat). Da aber das regionenspezifische Kapital im Laufe der Zeit

${ }^{167}$ Vgl. Siebert (1970), S. 60.

${ }^{168}$ Vgl. Harloff (1970), S.140f.

${ }^{169}$ Vgl. DaVanzo (1983), S. 552.

${ }^{170}$ Vgl. DaVanzo (1983), S. 557f. 
abnimmt, sinkt im Laufe der Zeit der Anreiz wieder zurückzukehren ${ }^{171}$. Unter Umständen können einige positive Faktoren am Geburtsort in der Erinnerung überbewertet werden, während negative Aspekte unterbewertet werden, wenn man vor der Wanderung jung und gesund war und viele (lästige) Pflichten im Kindes- bzw. Jugendalter noch nicht übernehmen mußte ${ }^{172}$.

Rückwanderungen (oder weitere Wanderungen) kommen aber nicht nur aufgrund falscher Erwartungen zustande, sondern können bereits Element einer früher getroffenen Wanderungsplanung sein. Eine vorausschauende Wanderungsplanung dürfte die ursprüngliche Wanderung nicht zwingend als dauerhaft ansehen, sondern müßte zusätzlich die Option weiterer Wanderungen beinhalten. Eine Wanderungsrechnung könnte ergeben, daß eine dauerhafte Wanderung in eine andere Region nicht vorteilhaft ist. Wird aber berücksichtigt, daß eine Rückwanderung stattfinden kann, etwa wenn die Lohnunterschiede verschwinden, so könnte eine (vorübergehende) Wanderung doch lohnend sein.

Diese Zusammenhänge wird zur Veranschaulichung anhand eines einfachen Beispiels erläutert:

Das Lohneinkommen betrage in der Region $\mathrm{x}$ in der Periode 160 Geldeinheiten, in der Region y 100 Geldeinheiten. Die Löhne gleichen sich pro Periode um 5 $\%$-Punkte an. In Periode 9 sei ein interregionaler Lohnausgleich erreicht und bleibe in der Zukunft erhalten. Die Zeitpräferenzrate betrage $2 \%$, die Kosten und Erträge werden auf den Zeitpunkt 0 abgezinst. Das Risiko, arbeitslos zu werden sei gleich Null. Der Warenkorb a koste sowohl in Region $\mathrm{x}$ als auch in Region y 60 Geldeinheiten, das Preisniveau sei intertemporal konstant. Die in Geldeinheiten bewerteten psychischen Kosten infolge eines Umzugs übertreffen die entsprechenden Erträge um 10 pro Periode, sie bleiben über den betrachteten Zeitraum konstant. Die Kosten für die Wanderung in Region y betragen 20, für die Rückwanderung 10. Es wird ein Zeitraum von 20 Jahren betrachtet. Es wird untersucht, ob es lohnend ist zu wandern und ob eine Rückwanderung erfolgen sollte; gegebenenfalls wird auch deren Zeitpunkt bestimmt.

Eine Bestimmung des Migrationsertrags ohne Berücksichtigung der Möglichkeit einer Rückwanderung ergäbe, daß ein Umzug nicht lohnt: der errechnete Einkommensverlust bei Berücksichtigung der nächsten 20 Jahre (Planungshorizont) beträgt 13,9 Geldeinheiten (GE). Wird dagegen in Erwägung gezogen, nach einigen Perioden wieder in die Heimatregion zu ziehen, so ergibt sich insgesamt ein Wanderungsgewinn. Das beste Ergebnis erzielt der potentielle Migrant,

\footnotetext{
${ }^{171}$ Vgl. DaVanzo (1983), S. 553ff.

${ }^{172}$ Vgl. Lee (1972), S. 119.
} 
wenn er sofort umzieht und am Ende der Periode zurückwandert, in der die Einkommensdifferenz umkippt, d.h. sie auf Null zusammengeschmolzen ist und in der Folgeperiode negativ würde. In dem betrachteten Beispiel wäre das am Ende der siebten Periode. Die Rückwanderung sollte hier bereits vor einer Angleichung des Lohnniveaus stattfinden, da die psychischen Kosten in der achten Periode höher sind als die Erträge aus den Lohndifferenzen. Insgesamt betrüge der Ertrag des Migranten 71,9 $\mathrm{GE}^{173}$.

Die gleichen Ausgangsdaten vorausgesetzt, aber einen Planungshorizont von 10 Jahren unterstellt, hätte sich bei einer Rechnung ohne Berücksichtigung der Möglichkeit einer Rückwanderung einen Gewinn von 59,8 Geldeinheiten ergeben, die Migration wäre mithin durchgeführt worden. Auch die ideale Verweildauer von 7 Perioden wäre zustande gekommen, vorausgesetzt, die Vorteilhaftigkeit eines Umzugs wäre immer wieder überprüft worden.

Wird für diese Konstellation eine höhere Zeitpräferenzrate von $5 \%$ statt $2 \%$ unterstellt, so ergeben sich nur geringe Änderungen, der Ertrag sänke (bei einer Verweildauer von 7 Perioden und anschließender Rückwanderung) von 71,9 auf 67,7 GE. Werden die psychischen Kosten verdoppelt, so wäre die Wanderung immer noch lohnend, jedoch verkürzt sich die optimale Anzahl der Perioden von 7 auf 5 .

Die einfache Rechnung (ohne Berücksichtigung von Rückwanderungen) käme zu dem Ergebnis, daß ein Umzug nicht lohnend wäre. Eine Erhöhungen der Variablen Lohneinkommen in $\mathrm{x}$ oder des Preisniveaus in $\mathrm{y}$ wirken genauso wie die Erhöhung der psychischen Kosten, Erhöhungen der Löhne in y und des Preisniveaus in $\mathrm{x}$ wirken entgegengesetzt.

\subsubsection{Psychische Kosten und Erträge}

Menschen mögen i.d.R. den Ort, an dem sie aufgewachsen sind, was dazu führt, daß einige Wanderungen, die aus ökonomischen Gründen stattgefunden hätten, unterbleiben ${ }^{174}$. Psychische Erträge entstehen bei einer Wanderung, wenn am potentiellen Zielort bereits Freunde oder (geliebte) Verwandte leben, wenn durch Wegzug der Kontakt zu unliebsamen Freunden/Verwandten gelockert oder abgebrochen werden kann oder wenn die Gewinnung eines neuen Freundeskreises als attraktiv empfunden wird. Psychische Kosten entstehen entsprechend, im

${ }^{173}$ Für die tabellarische Aufstellung seiner Ertragsentwicklung vgl. Anhang 1.

${ }^{174}$ Vgl. Layard u.a. (1992), S. 41. 
Wesentlichen aber durch die Trennung von dem Freundeskreises und der Familie.

Eine monetäre Bewertung der psychischen Kosten und Erträge ist ausgesprochen problematisch. Schwartz (1973) hat in diesem Zusammenhang vorgeschlagen, die psychischen Kosten in Transportkosten zu transformieren. Dafür ist eine Frequenz von Heimfahrten zu ermitteln, die nötig ist, die psychischen Kosten der Trennung auszugleichen. Für die geschätzte Anzahl an Heimfahrten werden die Fahrtkosten, die sie verursachen würden, berechnet.

Die Kosten steigen mit zunehmender Distanz. Die notwendige Heimfahrtfrequenz dürfte altersabhängig sein, da wahrscheinlich mit zunehmendem Alter die Beziehung zu Freunden und Verwandten als wertvoller empfunden werden und mehr investiert wird ${ }^{175}$. I.d.R. ist es in einem späteren Lebensabschnitt schwieriger neue Freundschaften zu schließen als in einem frühen. Die - in Zahlungen ihren Niederschlag findenden - Transportkosten sind - sofern die Fahrten keinen Eigenwert haben - eine (noch zu niedrige) Untergrenze für einen monetären Wertansatz, um die psychischen Kosten auszugleichen. Zusätzlich berücksichtigt werden müßte der Zeitaufwand, der für diese Fahrten notwendig ist ${ }^{176}$. Hier könnten z.B. die Opportunitätskosten angesetzt werden, d.h. die Einnahmen, die sich ergeben hätten, wäre der Migrant in der fraglichen Zeit einer Erwerbstätigkeit nachgegangen. Dies ergäbe allerdings eine monetär zu hohe Bewertung der Fahrtzeit.

Formelmäßig wären die Kosten für ein Jahr dann wie folgt zu ermitteln:

$\mathrm{K}_{\mathrm{nm}}=\mathrm{x}_{*} \mathrm{TK}_{\mathrm{HF}}+\mathrm{x}_{*} \mathrm{st}_{*} \mathrm{LE} / \mathrm{h}$

mit

$\mathrm{x}$ : Anzahl der mindestens notwendigen Heimfahrten pro Jahr

$\mathrm{TK}_{\mathrm{HF}}$ : In Zahlungen ihren Niederschlag findenden Kosten für Hin- und Rückfahrt wie Fahrpreise

st: $\quad$ Fahrtzeit (Hin- und Rückfahrt) in Stunden

LE/h: Lohneinkommen pro Stunde

Angenommen pro Woche würde eine Heimfahrt erfolgen und die Fahrtdauer für eine einfache Fahrt betrage vier Stunden, dann würden die Opportunitätskosten allein bereits einem Arbeitstag pro Woche entsprechen. Betrüge der Lohn in der

${ }^{175}$ Vgl. Schwartz (1973), S. 1160 f.

${ }^{176}$ Es gibt Menschen, denen es Freude bereitet, mit dem Auto oder einem öffentlichen Verkehrsmittel durch die Gegend zu fahren. Nach Auffassung der Verfasserin ist es aber eine Minderheit, die es genießt, regelmäßig dieselbe Strecke zu fahren. Den anderen dürfte es eher unbequem sein. 
Heimat nur etwas mehr als $80 \%$ des auswärtigen, so würde sich ein Umzug schon nicht mehr lohnen ${ }^{177}$. Werden zusätzlich noch die Fahrtkosten berücksichtigt, so könnte diese Differenz noch höher $\operatorname{sein}^{178}$. Verringerten sich allerdings die psychischen Kosten im Laufe der Zeit, kann auch die Lohndifferenz immer geringer werden.

\subsubsection{Sonstige personenspezifische Wanderungsgründe}

Ebenfalls einen Einfluß auf das Wanderungsverhalten haben personenspezifische Merkmale, wie Bildungsstand, Alter, Geschlecht, Familienstand, Stellung im Lebenszyklus und Nationalität.

Eine Vielzahl von empirischen Untersuchungen weist auf einen positiven $\mathrm{Zu}$ sammenhang zwischen Bildungsniveau und Migrationen hin ${ }^{179}$. Zur Erklärung diese Phänomens gibt es verschiedene Ansätze ${ }^{180}$. Ein Ansatz führt dies auf regionale Disparitäten in den Bildungseinrichtungen und den Arbeitsplätzen zurück. Die meisten Arbeitsplätze für Höherqualifizierte gibt es in den Großstädten und die Anzahl von Arbeitsplätzen für spezifische Qualifikationen ist relativ niedrig, Höherqualifizierte müssen daher aus Berufsgründen häufiger umziehen als Geringqualifizierte. Ein anderer Ansatz geht davon aus, daß die lokalen und sozialen Bindungen von Höherqualifizierten niedriger sind als die geringer Qualifizierter, da sie bereits häufig zu Ausbildungszwecken ihren Herkunftsort verlassen mußten. Hierdurch werden lokale Bindungen gesenkt und die psychischen Kosten einer weiteren Wanderung vermindert. Zwei weiteren Erklärungsversuchen liegen handlungstheoretische Ansätze zugrunde. Einmal wird argumentiert, daß mit zunehmender Bildung auch das Informationsniveau über potentielle Wohnorte und Beschäftigungsmöglichkeiten steigt, da die Fähigkeit von gebildeten Menschen Informationen effizient zu verarbeiten, größer ist als bei anderen ${ }^{181}$. Zum anderen wird vermutet, daß sich die Akzeptanz von Werten wie Leistungsbereitschaft und Pflichterfüllung mit steigender Bildung erhöht und damit auch zunehmend Wanderungen in Kauf genommen werden. Auch eine

${ }^{177}$ Dies gilt für das Nettoeinkommen. Werden zusätzlich noch die Einkommensteuereffekte berücksichtigt, könnte die Differenz der Bruttolöhne aufgrund von Progressionseffekten noch größer sein als die Nettolohndifferenzen.

${ }^{178}$ Allerdings wäre außerdem die steuerliche Abzugsfähigkeit der Fahrtkosten für die Heimfahrten zu berücksichtigen. Die Möglichkeit zur Absetzung besteht aber nicht für jeden, sondern nur für diejenigen, deren Lebensmittelpunkt weiterhin am alten Wohnort bleibt, wenn etwa der Ehegatte nicht mit umzieht.

179 Vgl. Wagner (1987), S. 98.

${ }^{180} \mathrm{Vgl}$. zum folgenden Wagner (1987), S. 98ff.

${ }^{181}$ Vgl. DaVanzo (1983), S. 556 und Schultz (1975). 
größere Anpassungsfähigkeit und das schon stärkere Angepaßtsein dürften relevant sein.

Die Rentabilität einer Migration ist von dem Alter der Betroffenen abhängig. Je jünger ein Mensch ist, desto höher ist i.d.R. seine Wanderungsbereitschaft. Junge Leute haben typischerweise relativ viel Zeit in ihre formale Ausbildung investiert, aber erst wenig spezifisches Humankapital ${ }^{182}$ erworben. Der größere Teil des Humankapitals älterer Menschen besteht dagegen in Wissen und Erfahrung in einer bestimmten Stellung. Dieses Humankapital würde - zumindest teilweise - durch einen Wechsel des Arbeitsplatzes entwertet werden. Bei jungen Menschen steigert zudem ihre längere Lebenserwartung den Gegenwartswert der Erträge aus zusätzlichen Investitionen relativ zur Gruppe der Älteren ${ }^{183}$.

Mit zunehmendem Alter wachsen die Anpassungsschwierigkeiten. Ein Umzug ist aber mit der Notwendigkeit einer Sozialisation in der neuen Region verbunden, so daß auch aus diesem Grund das Alter mobilitätshemmend wirkt.

Eine Untersuchung der bundesdeutschen Wanderungsbewegungen deutet darauf hin, daß sich die Gründe der Wanderungsentscheidung geschlechtsspezifisch unterscheiden. Während die Gründe der Frauen überwiegend im persönlichen Bereich lagen (zu knapp 50\%), dominierten bei den Männern berufliche Gründe $(37 \%)^{184}$.

Migrationsanalysen werden zunehmend in einen Familien-(Haushalts-) Zusammenhang gestellt ${ }^{185}$. Ein Verheirateter muß außer seinen eigenen Interessen auch die Belange seiner Familie berücksichtigen, die dann ebenfalls Eingang in seine persönliche Investitionsrechnung finden. Ein Alleinstehender ohne Kinder dagegen wird im wesentlichen nur seine eigenen Interessen berücksichtigen müssen. Ist ein Ehegatte Alleinversorger, so kann seine Migrationsbereitschaft zur Vermeidung von Arbeitslosigkeit größer sein als die eines Alleinstehenden, der keine Familie zu versorgen hat. Sind beide Ehegatten berufstätig, so könnte die Migrationsbereitschaft geringer sein als bei Singles, da bei der Wanderungsentscheidung immer beide einbezogen werden müssen. Was für den einen Ehegatten vorteilhaft ist, mag für den anderen mit Nachteilen verbunden sein. Sandell ${ }^{186}$ beobachtete, daß die Arbeitsmarktorientierung einer Frau ein wichtiger

${ }^{182}$ Spezifisches Humankapital ist der Teil des Humankapitals, der nur bei dem Arbeitgeber produktiv verwendet werden kann, bei dem die Investition erfolgt ist. Der Wert ist bei einem anderen Arbeitgeber gleich Null (vgl. Schaad/Schellenbauer (1993)).

${ }^{183} \mathrm{Vgl}$. Sjaastad (1962), S.88.

${ }^{184} \mathrm{Vgl}$. Prognos (1968), S. 84.

${ }^{185}$ Vgl. Greenwood (1985), S. 527.

${ }^{186} \mathrm{Vgl}$. Sandell (1977), S. 406ff. 
Bestimmungsgrund für die familiäre Migrationsentscheidung ist. Die Wahrscheinlichkeit einer Wanderung nimmt ab, wenn die Ehefrau berufstätig ist. Auch eine langjährige Beschäftigung reduziert die Wahrscheinlichkeit der Migration. Sie steigt mit der Ausbildung des Ehemannes und sinkt mit seinem Alter. Migration führt im allgemeinen dazu, daß das Einkommen des Mannes steigt und das der Frau sinkt (allerdings nur temporär). Die psychischen Kosten von Ehegatten könnten geringer sein als von die Alleinstehenden, da sie der neuen Situation nicht allein gegenüberstehen (vorausgesetzt beide ziehen um). Das Merkmal „verheiratet“ ist daher zwiespältig.

Mincer (1978) kommt in seiner Untersuchung zu dem Ergebnis, daß die Wahrscheinlichkeit einer Wanderung durch intakte Familienstrukturen negativ beeinflußt wird. Verheiratete Personen ziehen seltener um als Ledige, die Mobilität von Geschiedenen und getrennten Paaren ist dagegen die größte. Die Wahrscheinlichkeit eines Umzuges sinkt, je besser die Frau verdient und je dauerhafter sie arbeitet. Migration führt in der Tendenz dazu, daß die Arbeitslosigkeit der Männer sinkt und die der Frauen steigt.

Das Vorhandensein von Kindern erhöht - wie bereits erwähnt - die Transaktionskosten. Ferner ist zu berücksichtigen, daß sich die Zukunftsaussichten der Kinder durch den Umzug verändern: die Qualität der Schulbildung kann regional voneinander abweichen, das soziale Umfeld ändert sich, die Aussichten auf eine Ausbildungsstelle oder einen Arbeitsplatz ändern sich ebenso wie das zu erwartende Lohnniveau.

Auch die Stellung im Lebenszyklus ist für die Migrationswahrscheinlichkeit von Bedeutung. Für Personen, die sich in folgenden Lebensabschnitten befinden, könnte die Migrationsbereitschaft besonders hoch sein:

- Beendigung der Schule

- Eintritt ins Berufsleben

- Beginn einer Karriere

- Heirat/Scheidung

- Geburt und Heranwachsen von Kindern

- Eintritt in den Ruhestand ${ }^{187}$.

Das Ziel der Migranten dürfte sich - je nach Lebensabschnitt - unterscheiden. Bei dem Eintritt ins Berufsleben bzw. bei einem Aufstieg im Beruf werden die Wanderungsgründe stark von beruflichen Aspekten beeinflußt. Bei Ruhestandswanderungen dürften andere Aspekte in den Vordergrund treten, wie etwa Klima oder die Nähe von Freunden und Verwandten. Auch wenn nur Arbeitsmobilität

${ }^{187}$ Vgl. Greenwood (1985), S. 527. 
analysiert werden soll, könnten (geplante) Ruhestandswanderungen ${ }^{188}$ trotzdem von Relevanz sein. Wenn nämlich ein Individuum plant, sich am Ende seines Erwerbslebens in einer bestimmten Region niederzulassen, so wird dies bereits zu einem früheren Zeitpunkt in seine Lebensplanung Eingang finden. Will ein Arbeitnehmer etwa in seiner Heimatregion seinen Lebensabend verbringen, würden ihm Rückkehrkosten entstehen, wenn er vorher noch an einen anderen Ort zieht, um dort zu arbeiten. Kurz vor Erreichen des Rentenalters noch eine arbeitsbedingte Wanderung vorzunehmen, könnte sich dann als unrentabel erweisen.

Die Nationalität kann die (regionale) Wanderungsbereitschaft ebenfalls beeinflussen. Ist der potentielle Migrant in einem anderen Staat geboren worden (und lebt aber bereits in der betrachteten Volkswirtschaft), so wird er i.d.R. keine so starke Bindung an seinen Wohnort haben, als wenn er dort geboren wäre. In dieser Hinsicht sind Ausländer vielleicht vergleichbar mit solchen Menschen, die bereits einmal innerhalb des Landes umgezogen sind. Außerdem ist zu berücksichtigen, daß Ressentiments gegen Ausländer regional unterschiedlich ausgeprägt sein können. Ausländerfeindliche Aktionen können zu Abwanderungen führen, bzw. Zuwanderungen vermindern oder ganz verhindern. Gewicht in der Wanderungsentscheidung von Ausländern könnte ebenfalls haben, ob Menschen aus dem gleichen Kulturkreis bereits am potentiellen Wohnort leben.

\subsubsection{Zusammenfassung}

Es gibt eine Vielzahl potentieller Wanderungsursachen, die bei der Bestimmung des erwartbaren Migrationsgewinns eines Ostdeutschen kurz nach der Vereinigung berücksichtigt werden könnten. Die Qualität der empirischen Untersuchung hängt davon $\mathrm{ab}$, wieweit es gelingt, die relevanten Faktoren zu berücksichtigen. Es wurde beschrieben, daß die monetäre Bewertung der einzelner Faktoren teilweise mit großen Problemen behaftet ist und - wenn überhaupt - nur näherungsweise möglich ist. Wie eine Bewertung der verschiedener Faktoren vorgenommen werden kann, wurde insbesondere mit Blick auf die später durchzuführende Humankapitalrechnung erörtert.

Für einen aussagekräftigen Vergleich der regionalen Arbeitseinkommen ist es nicht ausreichend, die monatlichen Bruttogehälter der betreffenden Gebiete miteinander zu vergleichen. Zugrunde gelegt werden sollte das Jahresgehalt, um

${ }^{188} \mathrm{Da}$ sich der Lebensabschnitt des Ruhestandes erhöht hat aufgrund der höheren Lebenserwartung und des früheren Eintritts gewinnen Ruhestandswanderungen an Bedeutung. Vgl. zur Problematik der Ruhestandswanderung etwa Koch (1976) oder Nestmann (1988). 
betriebliche Sonderleistungen wie ein 13. Monatsgehalt zu erfassen. Daneben sind aber auch alle weiteren betrieblichen Besonderheiten wie Urlaubsregelungen und Rentenansprüche zu berücksichtigen. Bei regionalen Einkommensunterschieden sind nicht nur die aktuellen regionalen Differenzen, sondern auch (die Erwartungen über) die zukünftigen Unterschiede von wesentlicher Bedeutung für die Wanderungsentscheidung. Ferner sollte das Risiko, arbeitslos zu werden, berücksichtigt werden, etwa durch die Einbeziehung regionaler Arbeitslosenquoten.

Auch bezüglich der regionalen Preisunterschiede sollte die voraussichtliche zukünftige Entwicklung in die Untersuchung einbezogen werden. Bei der Bewertung von regionalen Preisunterschieden müssen nicht nur die Preise verglichen werden, sondern zunächst muß ein geeigneter Warenkorb konzipiert werden, anhand dessen sich regionale Preisunterschiede festlegen lassen.

Transaktionskosten sind vergleichsweise leicht abzuschätzen. Sie können sich zwar auch zu einem größeren Betrag summieren, im Gegensatz zu vielen anderen Kosten und Erträgen, die in jeder Periode neu entstehen, fallen sie aber nur einmalig an. Bereits früher vorgenommenen Wanderungen vermindern typischerweise die Kosten einer weiteren Wanderung.

Schier aussichtslos erscheint die Bewertung der Standortqualität. Die Bewertung von psychischen Kosten und Erträgen ist ebenfalls ausgesprochen schwierig und höchst spekulativ. Vorgestellt wurde ein Reisekostenansatz, mit dem eine Schätzung solcher Komponenten annäherungsweise vorgenommen werden kann.

Auch personenspezifische Merkmale, wie Alter und Familienstand beeinflussen die Wanderungsentscheidung. Mit zunehmendem Alter sinken i.d.R. die potentiellen Wanderungsgewinne. Der Einfluß des Familienstands ist nicht eindeutig, da auch die Struktur innerhalb des Familienverbundes eine Rolle spielt (wie Berufstätigkeit des Ehegatten und das Vorhandensein von Kindern). Das Geschlecht des potentiellen Migranten beeinflußt ebenfalls die Wanderungsentscheidung. Ferner wurde die Bedeutung der Stellung im Lebenszyklus dargestellt: In bestimmten Lebensabschnitten ist eher mit Wanderungen zu rechnen.

\subsection{Modellrechnungen zur Beurteilung individueller Vorteilhaftigkeit einer Wanderung aus den neuen in die alten Länder}

In diesem Abschnitt soll ermittelt werden, für welche ostdeutschen Beschäftigten eine Wanderung in die alten Länder lohnend gewesen wäre, wie hoch mithin das Wanderungspotential war. Dafür wird im Folgenden eine Investitionsrechnung 
in Anlehnung an den im vorhergehenden Kapitel vorgestellten Humankapitalansatz durchgeführt. Die einzelnen Faktoren, die eine Wanderungsentscheidung beeinflussen, werden - soweit möglich - so wie beschrieben berücksichtigt. Es wird berechnet, welche Erträge und Kosten verschiedene ostdeutsche Beschäftigte am Ende des Jahres 1990 erwartet haben könnten, wenn sie statt weiter in Ostdeutschland zu wohnen und zu arbeiten, einen Arbeitsplatz in Westdeutschland angenommen hätten und dorthin umgezogen wären. Mit Blick auf diese Erträge wird in dieser Arbeit von ,erwartbaren Erträgen“ gesprochen. Jene ,erwartbaren Erträge“ sagen also etwas über die sich bei einer bestimmten Kalkulation ergebenden Vorstellungen aus. Diese für 1990 erwartbaren Erträge werden dann mit Schätzungen von Migrationsüberschüssen konfrontiert, wie sie auf der Basis der tatsächlichen Daten bis 1994 und deren Fortschreibung zu errechnen sind.

Im Folgenden wird dabei so vorgegangen, daß zunächst nur die potentiellen Erträge aus den regionalen Lohn- und Preisunterschiede berechnet werden. In einem weiteren Schritt werden dann zusätzliche Wanderungskosten und -erträge quantifiziert und wird ihre Bedeutung für den Migrationsertrag dargestellt. Abschließend wird die Bedeutung von Arbeitslosigkeit für die Wanderungsentscheidung untersucht.

Untersuchungszeitpunkt ist das Ende des Jahres 1990. In der Umbruchphase 1989/1990 herrschte Unklarheit darüber, ob die politische Wende unumkehrbar sei. Definitive Sicherheit über die Dauerhaftigkeit des politischen und ökonomischen Wandels bestand wohl erst seit der deutschen Wiedervereinigung am 3. Oktober 1990. Da bis zu diesem Zeitpunkt politische Gründe die ökonomischen Gründe als Wanderungsmotivation überwogen haben könnten ${ }^{189}$, politische Motive aber nicht Thema dieser Arbeit sind, wurde für die Berechnung des Migrationsertrags erst ein Zeitpunkt nach dem Wiedervereinigungsdatum gewählt, um eine möglichst hohe Eingrenzung auf ökonomische Wanderungsmotive zu erreichen $^{190}$.

Alle nach dem Jahr 1990 anfallenden Einkommen und Aufwendungen werden auf den hier unterstellten Entscheidungszeitpunkt am Jahresende 1990 abgezinst. Zur Vereinfachung wird unterstellt, daß alle Einkommen und Aufwendungen

189 „Politische Bedingungen“ und ,persönliche Unfreiheit“" waren zur Jahreswende 1989/1990 die Hauptmotive für die Übersiedlung, diese Gründe wurden von $95 \%$ bzw. $94 \%$ der Übersiedler genannt, vgl. Voigt u.a. (1990), S. 741.

${ }^{190}$ Damit soll nicht verkannt werden, daß durchaus noch politische Gründe für eine Abwanderung bestanden. Im Jahr 1990 existierten zum Beispiel noch viele alte Seilschaften. Außerdem könnte für einige Personen ein Anreiz bestanden haben, in den Westen überzusiedeln, um ihre politische Vergangenheit zu vertuschen. 
jeweils zum Ende eines Jahres anfallen. Alle abgezinsten Größen sind daher dem Betrag nach etwas geringer, als sie bei Erfassung zum Zeitpunkt ihres tatsächlichen Anfalls gewesen wären. Die Abweichungen sind aber als so gering zu vermuten, daß sie den zusätzlichen Rechenaufwand nicht gerechtfertigt hätten.

Es werden ausschließlich Rechnungen für Ledige durchgeführt. Bereits diese Rechnung erweist sich als sehr umfangreich. Es spricht nichts dagegen, daß das, was sich tendenziell für Ledige zeigt, sich im großen und ganzen - unter Berücksichtigung der im Theorieteil erwähnten Unterschiede - nicht auch für Nicht-Ledige gültig sein dürfte. Vor diesem Hintergrund und mit Blick auf den sonst erforderlichen Mehraufwand, der den Rahmen dieser Arbeit gesprengt hätte, dürfte sich die exemplarische Beschränkung auf die Ledigen vertreten lassen. Die Qualifikation der Beschäftigten wird wegen fehlender Daten nur ansatzweise berücksichtigt. Das Alter des potentiellen Migranten wird nicht einbezogen, könnte aber dadurch berücksichtigt werden, daß der Gesamtertrag entsprechend dem Alter des Migranten vermindert wird, wenn nicht mehr der gesamte Ertrag aus den künftigen Lohndifferenzen erzielt werden kann.

Im folgenden werden alle errechneten Beträge ungerundet ausgewiesen, obwohl es sich bei den Rechnungen allenfalls um Näherungswerte handeln kann. Die Zahlen täuschen eine Genauigkeit vor, die sie so nicht einlösen können. Auf eine Rundung der ausgewiesenen Zahlen wird gleichwohl verzichtet, da sie genauso wenig genau oder ungenau ist wie die exakten Werte.

Die Untersuchung soll nicht besagen, daß die errechneten Erträge von allen ostdeutschen Erwerbspersonen hätten erzielt werden können. Die Höhe der individuell erzielbaren Erträge hängt immer auch davon ab, in welchem Ausmaß Wanderungen anderer Personen stattfinden. Die Wanderungen von Ost- nach Westdeutschland hatten vermutlich einen Einfluß auf das Lohnniveau in der Form, daß die Löhne im Westen weniger stark gestiegen sind, als sie ohne $\mathrm{Zu}$ wanderungen gestiegen wären. Die aufgezeigten Ergebnisse für die Jahre 1991 bis 1994 enthalten bereits die Konsequenzen für das Lohnniveau infolge der tatsächlich stattgefundenen Wanderungen. Die im folgenden ausgewiesenen Erträge können also als potentielle Wanderungserträge eines marginal wandernden Individuums verstanden werden.

\subsubsection{Migrationserträge aus regionalen Lohn- und Preisunterschieden}

Nach Darstellung der Datenbasis werden die Wanderungsgewinne berechnet, die sich aus den Unterschieden der regionalen Lohnniveaus und der regionalen Preisunterschiede ergeben. Da eine ausreichende Datenbasis für die Preisunter- 
schiede nicht vorhanden war, werden hilfsweise die Mietpreisniveaus herangezogen. Dies ist nicht unproblematisch, mit Blick darauf, daß der Unterschied in den Mietpreisen den gravierendsten Unterschied in den regionalen Preisen ausmachte, aber wohl zu vertreten ${ }^{191}$. Zunächst wird bestimmt, welche Erträge die Beschäftigten am Ende des Jahres 1990 erwartet haben könnten. Im Anschluß wird auf Basis der tatsächlichen Daten aus den Jahren 1991 bis 1994 und einer Fortschreibung dieser Daten berechnet, welche Erträge tatsächlich erzielbar gewesen wären.

Die regionalen abgezinsten Brutto- und Nettoeinkommensströme vor und nach Mietzahlung werden einander gegenübergestellt und ihre Differenz bestimmt. Diese Differenz drückt aus, wieviel ein Umzug nach Westdeutschland gegenüber dem Status quo mehr an Einkommen gebracht hätte.

Die Abzinsung wird mit zwei verschiedenen Zeitpräferenzraten (r) durchgeführt, zum einen mit $r=0,015$, zum anderen mit $r=0,05$. Diese Sätze stellen die Oberund Untergrenzen von den Zeitpräferenzraten dar, mit denen üblicherweise operiert wird ${ }^{192}$.

Es wird nicht nur ein durchschnittlicher Beschäftigter betrachtet, sondern gleichzeitig die Bedeutung der Branchenzugehörigkeit und - beispielhaft für den Bereich Industrie - die Bedeutung des Status (Angestellter oder Arbeiter) und des Geschlechts eines Beschäftigten. Zur Vereinfachung wird unterstellt, daß ein Arbeitsplatzwechsel nur innerhalb einer Branche möglich ist, und daß Arbeiter und Angestellte ihren jeweiligen Status stets behalten.

\subsubsection{Darstellung der Datenbasis}

Im folgenden wird erläutert, welche Datenbasis der Bestimmung des Einkommens und der regionalen Mietdifferenzen zugrunde liegt.

\subsection{Regionale Brutto- und Nettolohneinkommen}

Für die Bestimmung der Lohneinkommenserträge werden die für verschiedene Beschäftigte erwartbaren Einkommen in Ost- und Westdeutschland für unterschiedliche potentielle Lohnangleichungszeiträume geschätzt und dann deren Differenz ermittelt. Dabei werden die Lohneinkommen zum einen gesplittet

\footnotetext{
191 Vgl. auch Kapitel 3.2.1.1.2.

${ }^{192} \mathrm{Vgl}$. Raffelhüschen (1993).
} 
nach Branchen untersucht und zum anderen für den Bereich Industrie nach Arbeitern und Angestellten sowie nach dem Geschlecht. Den Einkommensdaten liegen verschiedene Quellen zugrunde, auch die Abgrenzung der Einkommen unterscheidet sich.

Für die Branchenbetrachtung wird die tatsächliche Lohnentwicklung zugrundegelegt, so wie sie sich aus den Veröffentlichungen des DIW ergibt, da hier - im Gegensatz zu den Daten des Statistischen Bundesamts - Sonderzahlungen wie das 13. Monatsgehalt berücksichtigt werden.

Problematisch an dieser Betrachtung ist, daß sich die Qualifikation der Beschäftigten in Ost- und Westdeutschland voneinander unterscheiden können. Wenn etwa die Qualifikation der Beschäftigten im Bergbau in Ostdeutschland wesentlich unter (bzw. über) der durchschnittlichen Qualifikation der im westdeutschen Bergbau Beschäftigten liegt, erklärt sich die Einkommensdifferenz zum Teil durch die Qualifikation. Ein nach Ost-Maßstäben durchschnittlich qualifizierter Ostdeutscher könnte dann im Westen nicht das dort herrschende durchschnittliche Lohneinkommen erzielen, sondern es würde darunter (bzw. darüber) liegen.

Für die Auswertung nach Geschlecht und nach Arbeitern und Angestellten werden mangels anderer Daten beispielhaft die Beschäftigten in der Industrie betrachtet. Diese Beschäftigten gehören den Branchen Bergbau, Energie- und Wasserversorgung, Verarbeitendes Gewerbe und Baugewerbe an. Die Einkommen weiblicher und männlicher Arbeiter und Angestellter in der Industrie sind den Veröffentlichungen des Statistischen Bundesamtes entnommen. Nicht enthalten sind daher - im Gegensatz zu der Branchenauswertung - Sonderzahlungen wie ein 13. Monatsgehalt.

\subsection{Regionale (Miet-)Preisniveaus}

Angaben über die Unterschiede der regionalen Preisniveaus finden sich nur sporadisch, so etwa bei Sinn/Sinn (1991, S. 187), die die Preisniveaudifferenz zwischen den alten und neuen Bundesländern im Frühjahr 1991 auf etwa $28 \%$ schätzen. Das statistische Bundesamt legte für den Herbst des Jahres 1993 eine Schätzung vor, wonach die Verbraucherpreise (ohne Miete) in Ostdeutschland $5 \%$ geringer als im Westen waren ${ }^{193}$.

${ }^{193}$ Vgl. Statistisches Bundesamt (1994a), S. 33. 
Um die Unterschiede in den Preisen wenigstens annähernd zu berücksichtigen, wird hilfsweise die Miete als Indikator für die regionalen Preisunterschiede verwendet. Über die Entwicklung der Mieten liegen hinreichend detaillierte Angaben vor. Mietzahlungen machen zwar nur einen Teil der Konsumausgaben aus (im Jahr 1993 betrug die Mietbelastungsquote im Durchschnitt für Westdeutsche ca. 22,2 \% und für Ostdeutsche 16,8 \% ${ }^{194}$ ), aber gerade die Mieten haben sich in den ersten Jahren nach der Vereinigung sehr stark voneinander unterschieden und hatten daher einen bedeutsamen Einfluß auf die Struktur der regionalen Konsumausgaben. So zeigt auch die Schätzung des Statistischen Bundesamtes, daß die Preisunterschiede ohne Mieten im Jahr 1993 nur noch geringfügig waren.

Wie in Tabelle 1 ausgewiesen, waren die Mieten in den neuen Bundesländern auch nach der Wende zunächst noch sehr niedrig. Bis Oktober 1991 betrug der Preis pro Quadratmeter lediglich 2,00 DM. Im Oktober gab es einen Preissprung: die Miete erhöhte sich auf 5,30 DM. Im Jahr 1993 stieg die Miete noch einmal um 2,00 DM pro Quadratmeter.

Tabelle 1: Entwicklung der ostdeutschen Mietpreise

- Angaben in DM pro Quadratmeter -

\begin{tabular}{|l|r|r|r|}
\hline & $\begin{array}{l}\text { Grund- } \\
\text { miete }\end{array}$ & $\begin{array}{l}\text { Nebenkosten } \\
\text { (incl. } \\
\text { Heizung) }\end{array}$ & Gesamt \\
\hline bis 30.09.1991 & 1,00 & 1,00 & 2,00 \\
\hline ab 01.10.1991 & 2,00 & 3,30 & 5,30 \\
\hline ab 01.01.1993 & 4,00 & 3,30 & 7,30 \\
\hline ab 01.01.1994 & 4,40 & 3,20 & 7,60 \\
\hline
\end{tabular}

Quelle: iwd (1991/1993)

Für Westdeutschland waren der Verfasserin nur Preisangaben für das Jahr 1991 (Warmmiete 9,20 DM/qm ${ }^{195}$ ) und für das Jahr 1993 (Grundmiete 7,70 DM/qm und Nebenkosten (incl. Heizung) 3,00 DM/qm ${ }^{196}$ ) verfügbar. Aufgrund dieser beiden Werte werden die Preise für das Jahr 1992 auf 9,95 DM/qm und für das Jahr 1994 auf 11,75 DM/qm geschätzt.

Für die Berechnung der Wohnkosten in den alten und neuen Ländern wurden die durchschnittlichen Mietpreise pro qm zugrunde gelegt. Angenommen wurde, daß die Größe und Ausstattung der Wohnung unabhängig vom Einkommen ist.

\footnotetext{
${ }^{194}$ Vgl. DIW (1994, 21), S. 352.

${ }^{195} \mathrm{Vgl}$. Institut der deutschen Wirtschaft $(1991,40)$.

196 Vgl. Institut der deutschen Wirtschaft (1993, 13).
} 
Diese Annahme ist problematisch, da Wohnen ein superiores Gut ist. Interessant sind für diese Untersuchungen aber nur die Differenzen zwischen den Kosten in Ost- und Westdeutschland. Da höherwertige Wohnungen tendenziell überall teurer und minderwertige billiger sein dürften, dürfte die durchschnittliche Differenz aber trotzdem einen akzeptablen Wert liefern.

Die durchschnittliche Wohnraumgröße pro Person betrug in den neuen Bundesländern zwischen 25 und 31 qm (Stand 1.1.1991) $)^{197}$. Da die Wohnungen von Alleinstehenden im allgemeinen überdurchschnittlich groß sind, wurde für diese ein Wohnraum von $40 \mathrm{gm}$ unterstellt. Die durchschnittliche Wohnfläche für einen Ledigen in Westdeutschland dürfte zwar größer gewesen sein (die durchschnittliche Wohnfläche pro Person betrug in den alten Bundesländern 34-40 $\mathrm{qm}^{198}$ ), gemäß der vorherigen Ausführungen müssen in die Rechnung für beide Regionen aber vergleichbare Wohnungen eingehen. Daher wurde in beiden Regionen eine flächenmäßig gleich große Wohnung von $40 \mathrm{qm}$ berücksichtigt. Problematisch ist allerdings, daß sich die Qualität einer durchschnittlichen Wohnung im Osten von der Qualität einer durchschnittlichen Wohnung im Westen unterschied. Die Wohnungen im Westen waren durchschnittlich in einem besseren Zustand und zudem qualitativ höherwertig ausgestattet. Um gleichwertigen Wohnraum miteinander zu vergleichen, müßte theoretisch eine geringere als die Durchschnittsmiete für Westdeutschland angesetzt werden. Da der Verfasserin entsprechende Schätzungen aber nicht vorliegen, wird hierauf verzichtet.

Bei den Berechnungen des erwartbaren Migrationsertrags am Ende des Jahres 1990 wird angenommen, daß sich die Mieten mit dem gleichen Tempo wie die Löhne aneinander angleichen ${ }^{199}$. Bei Simulation der Mietsteigerung in den alten Ländern, werden die gleichen Wachstumsraten wie bei den Löhnen unterstellt.

Zunächst wird nicht berücksichtigt, daß die Miete bei einer Neuanmietung höher ist als eine Bestandsmiete und nach einem Umzug in den Westen daher mit einer höheren Miete als mit der Durchschnittsmiete gerechnet werden mußte. Eine derartige Berücksichtigung wird später in Abschnitt 3.2.2 vorgenommen.

${ }^{197}$ Vgl. o.V. (1993).

${ }^{198}$ Vgl. o.V. (1993).

${ }^{199}$ Dieses Vorgehen ist nicht unproblematisch, denn möglicherweise wurde mit einem langsameren Tempo bei der Entwicklung der Mietpreise im Vergleich zu den Löhnen gerechnet, da derartige Besitzstände (niedrige Mieten) ungern aufgegeben werden. Bei den Löhnen hingegen war eine Mehrzahl der Ostdeutschen der Überzeugung, einen Anspruch auf die gleiche Entlohnung wie die Westdeutschen zu haben. 


\subsubsection{Im Jahr 1990 erwartbare Migrationserträge aus regionalen Lohn- und Mietunterschieden}

1990 wurde von den Gewerkschaften eine sehr schnelle Angleichung der Löhne (und anderer Bestimmungen wie Arbeitszeit- und Urlaubsregelungen) angestrebt. Die Gewerkschaft HBV wollte etwa eine Tarifunion innerhalb von 2 Jahren verwirklichen. Der Zeitraum, den andere Gewerkschaften im Visier hatten, war nicht wesentlich länger. Die Widerstände der Arbeitgeber gegen solch massive Lohnsteigerungen im Osten waren gering, so vereinbarte z. B. die Metallindustrie 1991 eine Lohnangleichung in mehreren Stufen bis zum 1. April 1994 ${ }^{200}$.

Die Tarifvereinbarungen und auch die Bekenntnisse der Politiker zu einer baldigen Angleichung hatten hohe Erwartungen bei der ostdeutschen Bevölkerung geweckt. So wurden Anfang der 90er Jahre immer wieder ,blühende Landschaften" in den Neuen Bundesländern prognostiziert. Eine Umfrage des Zentralinstituts für Jugendforschung ergab, daß die Bürger in der ehemaligen DDR eine sehr schnelle Anpassung der wirtschaftlichen Situation in Ostdeutschland an die Westdeutschlands erwarteten. $14 \%$ der Befragten rechneten mit einer Angleichungsdauer von drei Jahren, $48 \%$ mit einer Dauer von 4 bis 5 Jahren, $20 \%$ mit einer von 6 bis 9 Jahren und nur $18 \%$ gingen von einer Angleichung in 10 Jahren oder mehr aus ${ }^{201}$. Eine Anpassung innerhalb von 5 Jahren erwartete also die Mehrheit der Bevölkerung, nämlich $62 \%$.

Bei der Berechnung des Erwartungswerts des Migrationsertrags eines Arbeitnehmers in 1990 werden alternative Anpassungszeiträume zugrunde gelegt, und zwar mit einer Länge von 3, 5 und 10 Jahren. Hierdurch werden die unterschiedlichen Erwartungshaltungen der Bevölkerung weitgehend abgedeckt. Es wird jeweils ein degressiver Anpassungsverlauf unterstellt, da wohl davon ausgegangen wurde, daß die Lohnanpassungen in den ersten Jahren überproportional hoch sein würden.

Basis für die Simulation sind die am Ende des Jahres 1990 bekannten Daten, das sind: die Lohneinkommen in Ost- und Westdeutschland für das Jahr 1990 bzw. 1991, der Einkommensteuertarif 1990, die Versorgungsfreibeträge 1991, die Bemessungsgrundlagen und Beitragssätze für die Sozialversicherungen von 1991. Nicht berücksichtigt werden der Solidaritätszuschlag und die Regelung gemäß $\S 32$, Abs. 7 EStG, da diese Steuerregelungen am Ende des Jahres 1990 nicht bekannt waren. Bezüglich des westdeutschen Lohnniveaus wird ange-

${ }^{200}$ Dieser Vertrag wurde allerdings im Jahr 1993 revidiert.

${ }^{201}$ Ergebnisse des Zentralinstituts für Jugendforschung, zitiert nach Neubäumer (1991), S. 136. 
nommen, daß es sich analog zum durchschnittlichen Wachstum in den letzten 10 Jahren vor der Vereinigung entwickeln wird.

\subsection{Erwartbare Migrationserträge gesplittet nach Branchen}

Differenziert nach Branchen lagen 1990 keine Angaben über Einkommen in Ostdeutschland vor. Bei der Untersuchung wird daher so vorgegangen, daß die Westlöhne 1990 in der Branchensplittung zugrunde gelegt werden und für den Osten ein Niveau von jeweils $40 \%$ in 1990 angenommen wird. Dieses Niveau entspricht dem Durchschnitt aller Branchen ${ }^{202}$.

Erwartungsgemäß ergeben sich für die Beschäftigten aller Branchen unter Berücksichtigung der regionalen Einkommen und Mietpreisunterschiede im Westen höhere Erträge als im Osten. Die Erträge unterscheiden sich stark voneinander, je nachdem, welcher Anpassungszeitraum zugrunde gelegt wird und welcher Branche ein Beschäftigter angehört. Die Ergebnisse der Simulation nach Branchen und Anpassungszeiträumen sind in Tabelle 2, Tabelle 3 und Tabelle 4 dargestellt. Mit „Bruttoeinkommensdifferenz" wird im folgenden das (abgezinste) Mehr an Bruttoeinkommen bezeichnet, das im Durchschnitt in Westdeutschland gegenüber Ostdeutschland erzielt wurde. Mit „Nettoeinkommensdifferenz" wird die entsprechende Differenz der Nettoeinkommen (also Bruttoeinkommen nach Steuern und Sozialversicherungsabgaben) bezeichnet. In der „Differenz Nettoeinkommen nach Mietzahlung“ sind neben der Nettoeinkommensdifferenz auch die Unterschiede in den regionalen Ausgaben für Miete enthalten.

Wurde innerhalb von 3 Jahren mit einer Lohnangleichung gerechnet, sind die erwartbaren Migrationserträge - verglichen mit längeren Angleichungsdauern am niedrigsten. Die erwartbaren kumulierten Bruttoerträge liegen überall unter $37.000 \mathrm{DM}$, der geringste beträgt ca. 20.000 DM (Land- und Forstwirtschaft). Netto konnte bei diesen Erwartungen mit einem Ertrag zwischen 11.000 DM bis 19.000 DM gerechnet werden. Nach Berücksichtigung der regionalen Mietdifferenzen vermindert sich der Ertrag um weitere 3.000 DM auf einen erwartbaren Überschuß von durchschnittlich 12.000 DM. Dies ist weniger als das durchschnittliche Jahreseinkommen eines Ostdeutschen im Jahr 1990, dessen Arbeitseinkommen ca. 16.000 DM betrug. Unterschiede in der Zeitpräferenzrate machen sich im Migrationsertrag nur geringfügig bemerkbar: Für einen durchschnittlichen Beschäftigten beträgt der Unterschied in der Differenz der Netto-

${ }^{202}$ Vgl. DIW (1991, 14), S. 189. 
einkommen nach Mietzahlung nur ca. 700 DM. Dies sind rund 6\% der Migrationserträge.

Tabelle 2: Erwartbare Erträge aus regionalen Lohn- und Mietpreisunterschieden nach Branchen bei einer 3jährigen Angleichungsdauer

- Angaben in DM -

\begin{tabular}{|c|c|c|c|c|c|c|}
\hline & \multicolumn{3}{|l|}{$\mathrm{r}=0,015$} & \multicolumn{3}{|l|}{$r=0,05$} \\
\hline Branche & $\begin{array}{l}\text { Bruttoein- } \\
\text { kommens- } \\
\text { differenz }\end{array}$ & $\begin{array}{l}\text { Nettoein- } \\
\text { kommens- } \\
\text { differenz }\end{array}$ & $\begin{array}{l}\text { Differenz } \\
\text { Nettoeink. } \\
\text { nach Miet- } \\
\text { zahlung }\end{array}$ & $\begin{array}{l}\text { Bruttoein- } \\
\text { kommens- } \\
\text { differenz }\end{array}$ & $\begin{array}{l}\text { Nettoein- } \\
\text { kommens- } \\
\text { differenz }\end{array}$ & $\begin{array}{l}\text { Differenz } \\
\text { Nettoeink. } \\
\text { nach Miet- } \\
\text { zahlung }\end{array}$ \\
\hline Land- und Forstw. & 20.767 & 11.914 & 9.051 & 19.659 & 11.286 & 8.576 \\
\hline Bergbau & 36.543 & 18.843 & 15.981 & 34.594 & 17.849 & 15.139 \\
\hline Energiewirtsch. & 31.725 & 16.952 & 14.089 & 30.033 & 16.055 & 13.345 \\
\hline Verarb. Gewerbe & 31.953 & 17.050 & 14.187 & 30.248 & 16.148 & 13.438 \\
\hline Baugewerbe & 27.262 & 15.002 & 12.139 & 25.808 & 14.206 & 11.496 \\
\hline Handel & 23.680 & 13.312 & 10.450 & 22.417 & 12.606 & 9.896 \\
\hline Verkehr & 27.353 & 15.041 & 12.179 & 25.894 & 14.243 & 11.533 \\
\hline Dienstleistungen & 24.294 & 13.616 & 10.754 & 22.998 & 12.894 & 10.184 \\
\hline alle Branchen & 27.232 & 14.989 & 12.126 & 25.779 & 14.193 & 11.483 \\
\hline
\end{tabular}

Quelle: DIW (1990-1991), iwd (1991/1993), eigene Berechnungen

Der größte Überschuß ist im Bergbau erwartbar gewesen, mit einigen Abstand folgen die Energiewirtschaft und das Verarbeitende Gewerbe. Die Branchen Verkehr und Baugewerbe entsprechen nahezu dem Durchschnitt. Die Erträge in den Branchen Dienstleistungen, Handel sowie Land- und Forstwirtschaft sind unterdurchschnittlich. Die sich ergebende Rangfolge entspricht der Rangfolge der Branchen nach absoluter Lohnhöhe in Westdeutschland 1990. Da für alle Branchen ein Anfangsniveau der Ostlöhne von $40 \%$ der Westlöhne unterstellt wurde und identische Anpassungsverläufe in allen Branchen zugrunde gelegt wurden, ist dies allerdings eine Konsequenz der für die Berechnung zugrunde gelegten Annahmen.

Bei einer Anpassungsdauer von 5 Jahren sind die Erträge etwas höher als bei einer Dauer von 3 Jahren. Netto betragen die Überschüsse in diesem Fall mindestens 12.000 DM (nach Abzug der Miete). Im Durchschnitt beträgt der Bruttoüberschuß ca. $37.000 \mathrm{DM}$, netto 20.000 und nach Berücksichtigung der regionalen Mietdifferenzen 16.000 DM bis 17.000 DM. Gegenüber einem Anpassungszeitraum von 3 Jahren sind das etwa $40 \%$ mehr. Damit beläuft sich der Migrationsertrag auf ein Bruttojahreseinkommen in den neuen Ländern im Jahr 1990. Auch Unterschiede in der Zeitpräferenzrate machen sich absolut gesehen 
etwas stärker bemerkbar, sie betragen jetzt ca. $1.000 \mathrm{DM}$; prozentual bleibt der Unterschied aber in etwa konstant.

Tabelle 3: Erwartbare Erträge aus regionalen Lohn- und Mietpreisunterschieden nach Branchen bei einer 5jährigen Angleichungsdauer

- Angaben in DM -

\begin{tabular}{|c|c|c|c|c|c|c|}
\hline & \multicolumn{3}{|l|}{$r=0,015$} & \multicolumn{3}{|l|}{$\mathrm{r}=0,05$} \\
\hline Branche & $\begin{array}{l}\text { Bruttoein- } \\
\text { kommens- } \\
\text { differenz }\end{array}$ & \begin{tabular}{|l|} 
Nettoein- \\
kommens- \\
differenz
\end{tabular} & $\begin{array}{l}\text { Differenz } \\
\text { Nettoeink. } \\
\text { nach Miet- } \\
\text { zahlung }\end{array}$ & \begin{tabular}{|l|} 
Bruttoein- \\
kommens- \\
differenz
\end{tabular} & $\begin{array}{l}\text { Nettoein- } \\
\text { kommens- } \\
\text { differenz }\end{array}$ & $\begin{array}{l}\text { Differenz } \\
\text { Nettoeink. } \\
\text { nach Miet- } \\
\text { zahlung }\end{array}$ \\
\hline Land- und Forstw. & 29.299 & 16.843 & 12.804 & 27.375 & 15.754 & 11.980 \\
\hline Bergbau & 51.557 & 26.511 & 22.472 & 48.171 & 24.801 & 21.027 \\
\hline Energiewirtsch. & 44.760 & 23.811 & 19.772 & 41.820 & 22.267 & 18.493 \\
\hline Verarb. Gewerbe & 45.081 & 23.945 & 19.906 & 42.120 & 22.393 & 18.619 \\
\hline Baugewerbe & 38.462 & 21.166 & 17.128 & 35.937 & 19.791 & 16.017 \\
\hline Handel & 33.409 & 18.807 & 14.768 & 31.215 & 17.583 & 13.809 \\
\hline Verkehr & 38.590 & 21.225 & 17.186 & 36.056 & 19.846 & 16.072 \\
\hline Dienstleistungen & 34.276 & 19.202 & 15.163 & 32.025 & 17.952 & 14.179 \\
\hline alle Branchen & 38.420 & 21.147 & 17.108 & 35.897 & 19.773 & 15.999 \\
\hline
\end{tabular}

Quelle: DIW (1990-1991), iwd (1991/1993), eigene Berechnungen

Tabelle 4: Erwartbare Erträge aus regionalen Lohn- und Mietpreisunterschieden nach Branchen bei einer 10jährigen Angleichungsdauer

- Angaben in DM -

\begin{tabular}{|c|c|c|c|c|c|c|}
\hline & \multicolumn{3}{|l|}{$r=0,015$} & \multicolumn{3}{|l|}{$r=0,05$} \\
\hline Branche & \begin{tabular}{|l|} 
Bruttoein- \\
kommens- \\
differenz
\end{tabular} & $\begin{array}{l}\text { Nettoein- } \\
\text { kommens- } \\
\text { differenz }\end{array}$ & $\begin{array}{l}\text { Differenz } \\
\text { Nettoeink. } \\
\text { nach Miet- } \\
\text { zahlung }\end{array}$ & \begin{tabular}{|l|} 
Bruttoein- \\
kommens- \\
differenz
\end{tabular} & $\begin{array}{l}\text { Nettoein- } \\
\text { kommens- } \\
\text { differenz }\end{array}$ & $\begin{array}{l}\text { Differenz } \\
\text { Nettoeink. } \\
\text { nach Miet- } \\
\text { zahlung }\end{array}$ \\
\hline Land- und Forstw. & 78.722 & 45.272 & 34.420 & 69.292 & 39.967 & 30.415 \\
\hline Bergbau & 138.525 & 70.154 & 59.302 & 121.931 & 62.037 & 52.485 \\
\hline Energiewirtsch. & 120.262 & 63.347 & 52.495 & 105.856 & 55.969 & 46.417 \\
\hline Verarb. Gewerbe & 121.125 & 63.684 & 52.832 & 106.615 & 56.268 & 46.716 \\
\hline Baugewerbe & 103.342 & 56.397 & 45.545 & 90.963 & 49.796 & 40.244 \\
\hline Handel & 89.765 & 50.383 & 39.531 & 79.012 & 44.474 & 34.922 \\
\hline Verkehr & 103.686 & 56.544 & 45.692 & 91.265 & 49.926 & 40.374 \\
\hline Dienstleistungen & 92.093 & 51.447 & 40.595 & 81.061 & 45.414 & 35.862 \\
\hline alle Branchen & 103.558 & 56.348 & 45.496 & 90.862 & 49.753 & 40.201 \\
\hline
\end{tabular}

Quelle: DIW (1990-1991), iwd (1991/1993), eigene Berechnungen 
Tabelle 4 zeigt, daß Arbeitnehmer, die erst nach 10 Jahren einen Lohnausgleich erwarteten, im Durchschnitt mit Überschüssen von über 40.000 DM gerechnet haben könnten. Sie könnten damit Migrationserträge erwartet haben, die etwa zweieinhalb mal so hoch waren, wie die der Personen, die mit Lohn- und Mietangleichungen innerhalb von 5 Jahren rechneten, und die fast viermal so hoch waren wie die der dritten Vergleichsgruppe. Selbst für einen Beschäftigten in der Land- und Forstwirtschaft mit einer hohen Zeitpräferenzrate wäre ein Migrationsertrag von brutto fast $70.000 \mathrm{DM}$ erwartbar gewesen. Netto ohne Miete hätte er immerhin noch mit einem Ertrag von 30.000 DM kalkuliert.

Im Durchschnitt beträgt die 1990 erwartbare Bruttodifferenz 100.000 DM bzw. 91.000 DM. Dies entspricht dem sechsfachen Bruttojahreseinkommen eines Beschäftigten in Ostdeutschland im Jahr 1990, bzw. mehr als dem doppelten Bruttojahreseinkommens in Westdeutschland. Auch in der Nettobetrachtung nach Mietzahlung ist der Ertrag durchweg höher als das Bruttojahreseinkommen von 1990 im Westen.

\subsection{Erwartbare Migrationserträge gesplittet nach Status und Geschlecht}

Bei der Simulation der am Ende des Jahres 1990 erwartbaren Migrationserträge nach Geschlecht und Status werden als Basis nicht wie bei den Branchenauswertungen die Einkommen aus dem Jahr 1990 zugrunde gelegt, sondern hilfsweise die tatsächlichen Werte des Jahres 1991, da der Verfasserin entsprechende Daten von 1990 nicht vorlagen. Ferner wird nicht wie oben die durchschnittliche Quote „Einkommen im Osten in \% des Westniveaus“ zugrunde gelegt, sondern die tatsächlichen Lohneinkommen des Jahres 1991. Dieses Vorgehen erscheint hier sinnvoll, da Einkommensunterschiede zwischen den Qualifikationen und den Geschlechtern in der DDR geringer waren als in Westdeutschland und daher deutliche Unterschiede bezüglich der Quote „Ostlohn in \% des Westlohns“ bestanden haben.

Wie Tabelle 5 zeigt, sind die erwartbaren Erträge der Beschäftigten in der Industrie höher als die der Beschäftigten insgesamt, mit Ausnahme der Arbeiterinnen. Die erwartbaren Einkommensdifferenzen der Angestellten sind nahezu doppelt so hoch wie die der Arbeiter, wobei die Differenzen zwischen den männlichen Arbeitern und Angestellten größer sind als zwischen den weiblichen.

Die erwartbaren Erträge sind für die männlichen Angestellten die höchsten. Ihre Entlohnung war am höchsten und ihr Lohnniveau gemessen am Westlohnniveau war 1991 mit $42 \%$ unterdurchschnittlich (im Vergleich zu $50 \%$ bei den Arbeitern und weiblichen Angestellten). Ihr erwartbarer Bruttoertrag von über 70.000 DM schmilzt allerdings netto nach Berücksichtigung der Mietzahlungen 
auf gut 30.000 DM ab. Der Nettoertrag übersteigt damit das Bruttojahresarbeitseinkommen des Jahres 1991 im Osten (26.340), liegt aber deutlich unter dem im Westen (63.317). Der Migrationsertrag liegt um fast 20.000 DM über dem eines durchschnittlichen Arbeitnehmers mit den gleichen Erwartungen über die Lohnanpassungsdauer. Da bei der Berechnung für die Industriebeschäftigten Sonderzahlungen nicht berücksichtigt wurden, die aber einen zusätzlichen Migrationsertrag bedeutet hätten, dürfte der tatsächliche Unterschied noch höher sein. Männliche Industrieangestellte haben mithin im Jahr 1990 besonders hohe Migrationsanreize gehabt. Ebenfalls höher als für die Durchschnittsbeschäftigten sind die errechneten Wanderungsüberschüsse für die männlichen Industriearbeiter, jedoch beträgt die Differenz nur ca. 5.000 DM.

Tabelle 5: Erwartbare Erträge aus regionalen Lohn- und Mietpreisunterschieden nach Status und Geschlecht bei einer 3jährigen Angleichungsdauer

- Angaben in DM -

\begin{tabular}{|l|r|r|r|r|r|r|}
\hline & \multicolumn{2}{|c|}{$\mathrm{r}=0,015$} & \multicolumn{2}{l|}{$\mathrm{r}=0,05$} \\
\hline & $\begin{array}{l}\text { Bruttoein- } \\
\text { kommens- } \\
\text { differenz }\end{array}$ & $\begin{array}{l}\text { Nettoein- } \\
\text { kommens- } \\
\text { differenz }\end{array}$ & $\begin{array}{l}\text { Diff. der } \\
\text { Nettoeink. } \\
\text { nach Miet- } \\
\text { zahlung }\end{array}$ & $\begin{array}{l}\text { Bruttoein- } \\
\text { kommens- } \\
\text { differenz }\end{array}$ & $\begin{array}{l}\text { Nettoein- } \\
\text { kommens- } \\
\text { differenz }\end{array}$ & $\begin{array}{l}\text { Diff. der } \\
\text { Nettoeink. } \\
\text { nach Miet- } \\
\text { zahlung }\end{array}$ \\
\hline Arbeiter & 37.291 & 20.703 & 16.966 & 35.406 & 19.666 & 16.117 \\
\hline Angestellte & 68.177 & 35.289 & 30.534 & 64.570 & 33.451 & 28.947 \\
\hline Arbeiter, männl. & 39.231 & 21.545 & 17.807 & 37.247 & 20.466 & 16.918 \\
\hline $\begin{array}{l}\text { Angestellte, } \\
\text { männl. }\end{array}$ & 74.806 & 37.975 & 33.220 & 70.849 & 36.001 & 31.497 \\
\hline Arbeiter, weibl. & 28.185 & 16.376 & 12.589 & 26.692 & 15.519 & 11.933 \\
\hline Angestellte, weibl. & 39.424 & 21.627 & 17.890 & 37.430 & 20.545 & 16.996 \\
\hline
\end{tabular}

Quelle: Statistisches Bundesamt (1994), iwd (1991/1993), eigene Berechnungen

Die - an den erwartbaren Erträgen gemessenen - Migrationsanreize für Frauen waren deutlich niedriger als für Männer. Zwar ist der errechnete erwartbare Migrationsertrag für die weiblichen Angestellten höher als für den Durchschnittsbeschäftigten, jedoch erreicht der Überschuß bei weitem nicht die Höhe des Überschusses ihrer männlichen Kollegen. Die Arbeiterinnen konnten nur den Ertrag eines Durchschnittsbeschäftigten erwarten.

Besonders stark verändern sich die erwartbaren Erträge der männliche Angestellten, wenn statt 3 Jahren 5 Jahre Anpassungszeit zugrunde gelegt wird: Brutto bedeutet der längere Zeitraum einen zusätzlichen Ertrag von 25.000 DM. Dies entspricht einem Zuwachs von ca. 35 \%. Für die Arbeiterinnen ist die Zuwachsrate fast ebenso hoch, absolut beträgt der Zugewinn aber lediglich 9.000 
DM. Für die Arbeiter insgesamt ergibt sich ein geringerer Zuwachs von unter 20 $\%$.

Tabelle 6: Erwartbare Erträge aus regionalen Lohn- und Mietpreisunterschieden nach Status und Geschlecht bei einer 5jährigen Angleichungsdauer

- Angaben in DM -

\begin{tabular}{|l|r|r|r|r|r|r|}
\hline & \multicolumn{2}{|l|}{$\mathrm{r}=0,015$} & \multicolumn{2}{l|}{$\mathrm{r}=0,05$} \\
\hline & $\begin{array}{l}\text { Bruttoein- } \\
\text { kommens- } \\
\text { differenz }\end{array}$ & $\begin{array}{l}\text { Nettoein- } \\
\text { kommens- } \\
\text { differenz }\end{array}$ & $\begin{array}{l}\text { Diff. der } \\
\text { Nettoeink. } \\
\text { nach Miet- } \\
\text { zahlung }\end{array}$ & $\begin{array}{l}\text { Bruttoein- } \\
\text { kommens- } \\
\text { differenz }\end{array}$ & $\begin{array}{l}\text { Nettoein- } \\
\text { kommens- } \\
\text { differenz }\end{array}$ & $\begin{array}{l}\text { Diff. der } \\
\text { Nettoeink. } \\
\text { nach Miet- } \\
\text { zahlung }\end{array}$ \\
\hline Arbeiter & 44.643 & 24.590 & 20.116 & 41.852 & 23.076 & 18.882 \\
\hline Angestellte & 92.497 & 47.211 & 40.759 & 86.059 & 44.004 & 38.001 \\
\hline Arbeiter, männl. & 46.965 & 25.578 & 21.104 & 44.029 & 24.004 & 19.810 \\
\hline Angestellte, männl. & 101.491 & 50.717 & 44.265 & 94.427 & 47.282 & 41.279 \\
\hline Arbeiter, weibl. & 37.382 & 21.564 & 16.542 & 34.843 & 20.121 & 15.440 \\
\hline Angestellte, weibl. & 47.196 & 25.675 & 21.201 & 44.245 & 24.096 & 19.901 \\
\hline
\end{tabular}

Quelle: Statistisches Bundesamt (1994), iwd (1991/1993), eigene Berechnungen

Mit Ausnahme der Arbeiterinnen dürfte für alle Industriebeschäftigten, die von einer Lohnangleichung nach 10 Jahren oder mehr ausgingen, ein Migrationsertrag von brutto über $100.000 \mathrm{DM}$ erwartbar gewesen sein. Nach Zahlung von Steuern, Sozialabgaben und Miete wäre mindestens ein erwartbarer Überschuß von $44.000 \mathrm{DM}$ verblieben.

Tabelle 7: Erwartbare Erträge aus regionalen Lohn- und Mietpreisunterschieden nach Status und Geschlecht bei einer 10jährigen Angleichungsdauer

- Angaben in DM -

\begin{tabular}{|l|r|r|r|r|r|r|}
\hline & \multicolumn{2}{|c|}{$r=0,015$} & \multicolumn{2}{r|}{$r=0,05$} \\
\hline & $\begin{array}{l}\text { Bruttoein- } \\
\text { kommens- } \\
\text { differenz }\end{array}$ & $\begin{array}{l}\text { Nettoein- } \\
\text { kommens- } \\
\text { differenz }\end{array}$ & $\begin{array}{l}\text { Diff. der } \\
\text { Nettoeink. } \\
\text { nach Miet- } \\
\text { zahlung }\end{array}$ & $\begin{array}{l}\text { Bruttoein- } \\
\text { kommens- } \\
\text { differenz }\end{array}$ & $\begin{array}{l}\text { Nettoein- } \\
\text { kommens- } \\
\text { differenz }\end{array}$ & $\begin{array}{l}\text { Diff. der } \\
\text { Nettoeink. } \\
\text { nach Miet- } \\
\text { zahlung }\end{array}$ \\
\hline Arbeiter & 107.850 & 58.216 & 47.408 & 94.893 & 51.388 & 41.878 \\
\hline Angestellte & 170.228 & 84.695 & 72.822 & 150.597 & 75.307 & 64.803 \\
\hline Arbeiter, männl. & 113.460 & 60.534 & 49.726 & 99.829 & 53.446 & 43.936 \\
\hline Angestellte, männl. & 186.779 & 90.694 & 78.820 & 165.240 & 80.692 & 70.188 \\
\hline Arbeiter, weibl. & 74.820 & 42.507 & 32.455 & 65.545 & 37.336 & 28.530 \\
\hline Angestellte, weibl. & 114.018 & 60.761 & 49.953 & 100.320 & 53.648 & 44.138 \\
\hline
\end{tabular}

Quelle: Statistisches Bundesamt (1994), iwd (1991/1993), eigene Berechnungen 
Am lohnendsten wäre ein Umzug für die männlichen Angestellten gewesen, ihr potentieller Ertrag lag brutto über 160.000 DM. Für männliche Arbeiter und weibliche Angestellte ergibt sich in etwa ein Ergebnis von gut 100.000 DM. Eine Industriearbeiterin hätte brutto einen Überschuß von über $65.000 \mathrm{DM}$ erzielen können.

Mit zunehmender Länge der Anpassungszeit steigt der Einfluß der Zeitpräferenzrate. Für die Angestellten mit einer hohen Zeitpräferenzrate ist der erwartbare abgezinste Bruttoertrag um 20.000 DM geringer als für Angestellte mit einer niedrigen Zeitpräferenzrate.

Zusammenfassend ist festzustellen, daß alle Beschäftigten in der Industrie 1990 mit einem Überschuß gerechnet haben könnten, wenn sie eine Arbeit im Westen aufgenommen hätten, statt in den neuen Ländern zu arbeiten. Das gilt auch dann, wenn die regionalen Unterschiede in den Mietpreisen berücksichtigt werden und die Erwartungen über die Nivellierungsdauer sehr zuversichtlich waren. Die höchsten erwartbaren Erträge errechnen sich für die männlichen Beschäftigten und für solche Angestellte, die lange Lohnangleichungsdauern vermuteten.

\subsection{Zusammenfassung}

Für alle hier betrachteten ostdeutschen Arbeitnehmersegmente hätte sich ein Überschuß ergeben, wenn sie auf Basis des Ende 1990 vorherrschenden Meinungsklimas die Lohn- bzw. realen Einkommensdifferentiale einer Wanderung für sich berechnet hätten. Einen besonders hohen Einfluß auf den potentiellen Wanderungsertrag hat die Erwartung über die Lohnanpassungsdauer wie Abbildung 28 zeigt. Bei einem 5jährigen Anpassungszeitraum steigt der erwartbare Ertrag durchschnittlich um $40 \%$ gegenüber einem 3jährigen Zeitraum; bei einem 10jährigen sogar um $280 \%$. Der Nettoertrag nach Miete beläuft sich bei einer erwarteten Angleichungsdauer von 3 Jahren auf ca. 75\% eines Bruttojahreseinkommens aus dem Jahr 1990 im Osten ${ }^{203}$, bei einer Angleichungsdauer von 5 Jahren auf ein Bruttojahreseinkommen und bei einer Dauer von 10 Jahren auf 2,5 bis 3 Jahresgehälter.

${ }^{20.3}$ Zur besseren Vergleichbarkeit wurde in der Branchenbetrachtung ein Vergleich mit den Jahreseinkommen 1990 vorgenommen, da für 1991 unterschiedliche Jahreseinkommen (in Abhängigkeit von der unterstellten Angleichungsdauer) erwartet wurden. 
Abbildung 28: Erwartbare Erträge aus regionalen Lohn- und Mietpreisdifferenzen eines durchschnittlichen Beschäftigten

- Zeitpräferenzrate 0,015 -

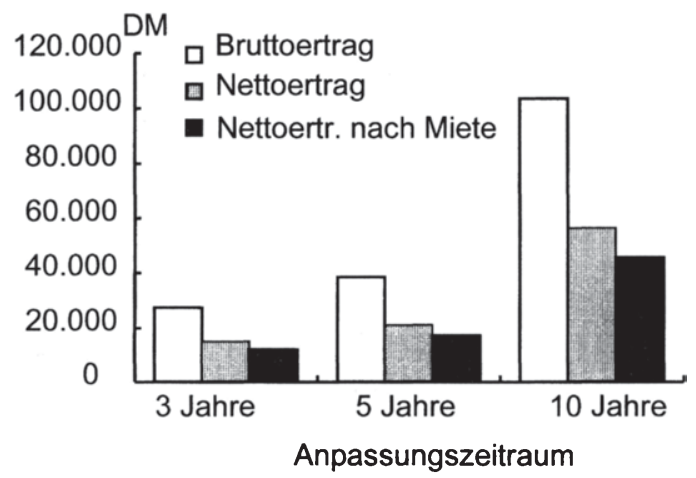

Quelle: Tabelle 2, Tabelle 3, Tabelle 4

Aufgrund der zu zahlenden Steuern und Sozialabgaben ist der Nettoüberschuß nur gut halb so hoch wie die Bruttodifferenz. Die regionalen Unterschiede im Mietniveau verringern den Migrationsertrag für einen durchschnittlichen Beschäftigten um rund 3.000 DM (bei einer erwarteten Anpassungsdauer von 3 Jahren), 4.000 DM (Anpassungsdauer 5 Jahre) bzw. 10.000 DM (Anpassungsdauer 10 Jahre). Der Einfluß der Zeitpräferenzrate ist bei kurzen Anpassungszeiträumen eher gering, macht sich aber bei einer längeren Anpassungsperiode stärker bemerkbar.

Die Migrationserträge unterscheiden sich nach Branchenzugehörigkeit sowie nach Status und Geschlecht. Die Differenzen, die sich in der Branchenbetrachtung ergeben, sind geringer als zwischen den Industriebeschäftigten nach Status und Geschlecht, wie Abbildung 29 zeigt. Dort sind von allen Branchen nur diejenigen mit den höchsten und niedrigsten Erträgen abgebildet. Besonders hoch sind die Überschüsse für Beschäftigte im Bergbau sowie für die männlichen Beschäftigten, insbesondere die Angestellten. Vergleichsweise gering sind die Überschüsse für Beschäftigte in der Land- und Forstwirtschaft sowie für die Frauen, insbesondere für Arbeiterinnen. 
Abbildung 29: Ausgewählte erwartbare Nettomigrationserträge nach Mietzahlung bei alternativen Zeitpräferenzraten und 10jähriger Lohnanpassungsdauer

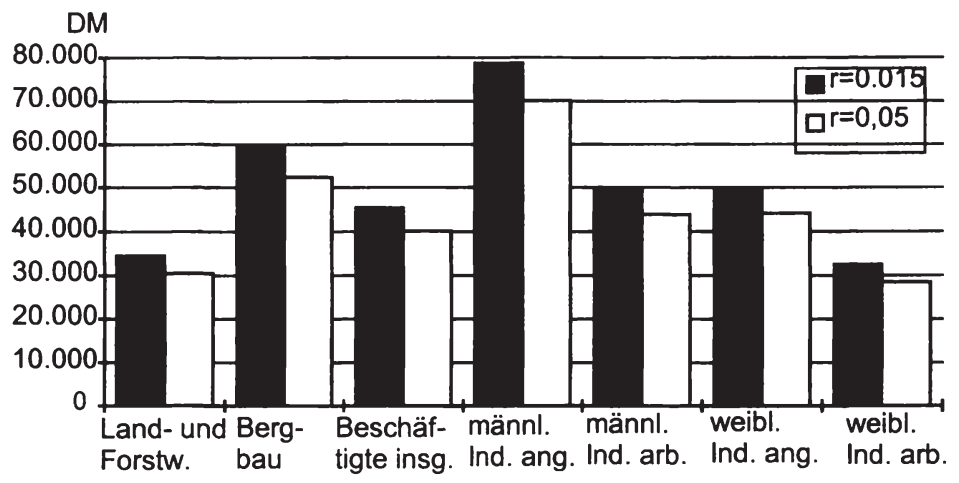

Quelle: Tabelle 4, Tabelle 7

\subsubsection{Im Jahr 1990 erwartbare Migrationserträge aus regionalen Lohn- und Mietunterschieden unter Berücksichtigung der tatsächlichen Entwick- lung bis 1994}

Nach der Vorlage ökonomisch fundierter Schätzungen, was sich bei der Ende 1990 herrschenden Stimmungslage ein Bewohner Ostdeutschlands von einer Wanderung an Wanderungsertrag versprechen konnte, werden in diesem Abschnitt die erzielbaren Migrationserträge auf Basis der tatsächlichen Daten bis 1994 bestimmt. Zunächst wird berechnet, welcher Überschuß sich für die ersten vier Jahre ergeben hätte und im Anschluß auf Basis dieser Daten eine Simulation für die Jahre nach 1994 durchgeführt. Später (unter 3.2.1.4) werden dann diese Werte mit den zuvor ermittleten erwartbaren Erträgen verglichen.

\subsection{Korrigierte erwartbare Migrationserträge aus regionalen Lohn- und Mietunterschieden bis 1994}

Zugrunde gelegt wird für die Branchenbetrachung die tatsächliche Lohnentwicklung, so wie sie sich aus den quartalsweisen Veröffentlichungen des DIW ergibt. Die jeweiligen Quartalswerte gehen in die Berechnung ein. Die ostdeutschen Lohneinkommen wurden für das Jahr 1991 nicht explizit ausgewiesen, sie werden von der Verfasserin aufgrund der vorliegenden Lohnwachstumsrate von 1992 gegenüber 1991 und den absoluten Werten von 1992 errechnet. Die Zahlen für die beiden letzten Quartale 1994 lagen zum Untersuchungszeitpunkt noch 
nicht vor. Sie werden geschätzt aufgrund der Löhne in den letzten beiden Quartalen 1993 und der Wachstumsraten der ersten Hälfte 1994 gegenüber der ersten Hälfte 1993. Bei den Daten des DIW über die Lohnentwicklung handelt es sich häufig um Schätzungen, die später revidiert wurden. In dieser Arbeit werden die Daten zugrundegelegt, die relativ zeitnah veröffentlicht wurden. Absolute bereinigte Daten werden kaum veröffentlicht. Eine Möglichkeit bereinigte Daten zu berücksichtigen, wäre gewesen, aus den (korrigierten) Wachstumsraten die relevanten Daten zu gewinnen. Da dies aber nicht für alle Jahre möglich ist, wird darauf verzichtet.

Für die Auswertung nach Geschlecht und Status lagen zum Zeitpunkt der Rechnung nur Daten für die Jahre 1991 bis 1993 vor, die Daten für 1994 werden geschätzt aufgrund der Vorjahresdaten und der durchschnittlichen Lohnwachstumsrate, so wie sie sich aus den Lohneinkommensdaten des DIW ergibt.

In Ostdeutschland mußten im Durchschnitt für eine 40qm große Wohnung im Zeitraum 1991 bis 1994 abgezinst auf das Jahresende 1990 insgesamt 10.593 DM (mit $r=0,015$ ) bzw. 9.627 DM (mit $r=0,05$ ) an Miete bezahlt werden. Für Westdeutschland betrugen die entsprechenden Kosten 19.212 DM bzw. 17.615 DM. Insgesamt ergibt sich daher für eine Wohnung in Westdeutschland eine um 8.619 DM $(r=0,015)$ bzw. $7.987 \mathrm{DM}(r=0,05)$ höhere Mietbelastung als in Ostdeutschland (Die Ausgaben für die Wohnungsmiete sind annahmegemäß nur von der Region und nicht vom Einkommen abhängig).

Für die Berechnung der Nettoeinkommen wird der Einkommensteuertarif 1990 zugrunde gelegt. Nicht berücksichtigt werden die Abrundungsvorschriften des Steuertarifs. In die Berechnung der Steuerzahlung gehen die für die jeweiligen Jahre bestehenden Regelungen bezüglich des Versorgungsfreibetrages und des zum Teil erhobenen Solidaritätszuschlag ein. Berücksichtigt wird auch der Arbeitnehmerfreibetrag in Höhe von $2.000 \mathrm{DM}$ sowie die Regelung gemäß $\S 32$, Abs. 7 EStG, die für die Jahre 1991 bis 1993 einen Tariffreibetrag in Höhe von 600 DM pro Person mit Wohnsitz in Ostdeutschland vorsieht. Die Beitragssätze und Bemessungsgrundlagen für die Sozialversicherung gehen mit den jeweils geltenden Werten in die Rechnung ein ${ }^{204}$.

\subsection{Migrationserträge gesplittet nach Branchen}

Im Durchschnitt ergeben sich Brutto- bzw. Nettojahreseinkommen pro Beschäftigten gem. Tabelle $8^{205}$ :

\footnotetext{
${ }^{204} \mathrm{Vgl}$. zu den Bemessungsgrundlagen und den Beitragssätzen Anhang 2.

${ }^{205} \mathrm{Vgl}$. für Bruttoeinkommen gesplittet nach Branchen sowie Arbeiter/Angestellte in der In-
} 
Tabelle 8: Durchschnittliches Jahreseinkommen von Beschäftigten in Ost- und Westdeutschland

- Angaben in DM -

\begin{tabular}{|l|r|r|r|r|r|r|}
\hline & \multicolumn{2}{|l|}{ Ostdeutschland } & \multicolumn{2}{l|}{ Westdeutschland } & \multicolumn{2}{l|}{ regionale Eink.differenz } \\
\hline Jahr & $\begin{array}{l}\text { Bruttoein- } \\
\text { kommen }\end{array}$ & $\begin{array}{l}\text { Nettoein- } \\
\text { kommen }\end{array}$ & $\begin{array}{l}\text { Bruttoein- } \\
\text { kommen }\end{array}$ & $\begin{array}{l}\text { Nettoein- } \\
\text { kommen }\end{array}$ & $\begin{array}{l}\text { Bruttoein- } \\
\text { kommen }\end{array}$ & $\begin{array}{l}\text { Nettoein- } \\
\text { kommen }\end{array}$ \\
\hline 1991 & 24.729 & 17.591 & 44.511 & 28.549 & 19.782 & 10.958 \\
\hline 1992 & 29.997 & 20.582 & 47.016 & 29.526 & 17.019 & 8.945 \\
\hline 1993 & 34.077 & 23.076 & 48.291 & 30.162 & 14.214 & 7.086 \\
\hline 1994 & 35.178 & 23.380 & 49.461 & 30.676 & 14.283 & 7.296 \\
\hline
\end{tabular}

Quelle: DIW (1991-1994), eigene Berechnungen

Bis 1993 waren erhebliche Lohnsteigerungen in Ostdeutschland zu verzeichnen, bei einem relativ moderaten Lohnanstieg in den alten Ländern. Daher war die regionale Lohndifferenz sowohl brutto als auch netto 1991 am höchsten. Das Bruttoeinkommen in Westdeutschland war in diesem Jahr nahezu doppelt so hoch wie in Ostdeutschland, das Nettoeinkommen in den neuen Ländern betrug gut $60 \%$ des Westeinkommens. Die Einkommensdifferenz baute sich in den folgenden beiden Jahren ab. 1994 war allerdings ein leichter absoluter Anstieg der regionalen Einkommensdifferenz zu beobachten. Der stärkere Anstieg der Nettoeinkommensdifferenz 1994 gegenüber der Bruttoeinkommensdifferenz erklärt sich aus dem Wegfall des Sonderfreibetrags für Personen mit Wohnsitz in Ostdeutschland von $600 \mathrm{DM}$.

Die sich auf dieser Basis errechneten Einkommensdifferenzen sind für den Zeitraum 1991-1994 gesplittet nach Branchen in Tabelle 9 dargestellt. Die Tabelle enthält sowohl die Bruttoerträge, die Nettoerträge als auch die Nettoerträge nach geleisteter Mietzahlung. Spalte 2 bis 4 zeigen den Migrationsertrag, der sich bei Zugrundelegung einer Zeitpräferenzrate von 1,5\% ergibt, die letzten drei Spalten zeigen die Einkommensdifferenzen bei einer Zeitpräferenzrate von $5 \%$.

Die Differenzen im Bruttoeinkommen sind bereits für den untersuchten Zeitraum von 4 Jahren recht hoch: Durchschnittlich beträgt der erwartbare Bruttomigrationsertrag rund $60.000 \mathrm{DM}$, dies ist mehr als das Doppelte eines Jahreseinkommens eines ostdeutschen Beschäftigten in 1991. Für Beschäftigte des verarbeitenden Gewerbes ist der Bruttomgrationsertrag der höchste, er beträgt rund 90.000 DM. Die geringsten Bruttodifferenzen ergeben sich im Baugewerbe mit knapp 40.000 DM und im Dienstleistungsbereich mit bis zu 45.000 DM.

dustrie nach Geschlecht Anhang 3. 
Tabelle 9: Korrigierte erwartbare Erträge aus regionalen Lohn- und Mietpreisunterschieden bis 1994 nach Branchen

- Angaben in DM -

\begin{tabular}{|c|c|c|c|c|c|c|}
\hline Branche & $r=0,015$ & & & $r=0,05$ & & \\
\hline & $\begin{array}{l}\text { Bruttoein- } \\
\text { kommens- } \\
\text { differenz }\end{array}$ & $\begin{array}{l}\text { Nettoein- } \\
\text { kommens- } \\
\text { differenz }\end{array}$ & $\begin{array}{l}\text { Differenz } \\
\text { Nettoeink. } \\
\text { nach Miet- } \\
\text { zahlung }\end{array}$ & $\begin{array}{l}\text { Bruttoein- } \\
\text { kommens- } \\
\text { differenz }\end{array}$ & $\begin{array}{l}\text { Nettoein- } \\
\text { kommens- } \\
\text { differenz }\end{array}$ & $\begin{array}{l}\text { Differenz } \\
\text { Nettoeink. } \\
\text { nach Miet- } \\
\text { zahlung }\end{array}$ \\
\hline Land- und Forstw. & 53.345 & 30.090 & 21.471 & 49.320 & 27.848 & 19.861 \\
\hline Bergbau & 72.286 & 35.604 & 26.985 & 67.628 & 33.397 & 25.409 \\
\hline Energiewirtsch. & 72.011 & 36.043 & 27.424 & 66.339 & 33.236 & 25.249 \\
\hline Verarb. Gewerbe & 93.137 & 48.702 & 40.084 & 86.008 & 45.033 & 37.045 \\
\hline Baugewerbe & 40.361 & 20.705 & 12.086 & 37.214 & 19.113 & 11.126 \\
\hline Handel & 61.052 & 32.996 & 24.377 & 56.359 & 30.494 & 22.507 \\
\hline Verkehr & 66.514 & 34.789 & 26.170 & 61.488 & 32.200 & 24.213 \\
\hline Dienstleistungen & 45.356 & 23.884 & 15.265 & 42.091 & 22.198 & 14.211 \\
\hline alle Branchen & 63.060 & 33.129 & 24.511 & 58.306 & 30.673 & 22.686 \\
\hline
\end{tabular}

Quelle: DIW (1991-1994), iwd (1991/1993), eigene Berechnungen

Die hohen Differenzen im verarbeitenden Gewerbe erklären sich dadurch, daß der Bruttolohn zwar in beiden Teilen Deutschlands über dem Durchschnittslohn lag, gemessen am westdeutschen Einkommen der ostdeutsche Lohnabstand jedoch in allen betrachteten Jahren der höchste war (im Jahr 1991 betrugen die Ostlöhne 45 \% des Westniveaus gegenüber 55 \% im Durchschnitt, im Jahr 1994 $64 \%$ gegenüber $71 \%$ ). Die vergleichsweise niedrige Differenz der kumulierten Einkommen im Bergbau erklärt sich ebenfalls durch den Lohnangleichungsprozeß, denn dieser war im Bergbau am schnellsten fortgeschritten. In 1991 betrugen die Ostlöhne bereits $70 \%$ des Westlohnniveaus und zwei Jahre später $82 \%$. 1994 schrumpfte das Niveau allerdings auf $77 \%$.

Die Nettoeinkommensdifferenzen sind sehr viel geringer als die Bruttodifferenzen. Sie sind nur etwa halb so groß, so daß die Bandbreite der Erträge nur noch zwischen 20.000 DM und 48.000 DM liegt (bei $r=0,015$ ). Diese Einkommensdifferenz ist zwar längst nicht so attraktiv wie die Bruttoeinkommensdifferenz, der Überschuß übersteigt aber immer noch ein ostdeutsches Jahreseinkommen in 1991. An der Rangfolge der Branchen ändert sich selbstverständlich nichts.

Werden nun noch die unterschiedlichen Mietkosten in die Betrachtung einbezogen, so sinken die Differenzen nochmals jeweils um rund 8.000 DM. In den Branchen Bau und Dienstleistungen verbleiben (nur) noch Überschüsse von 12.000 DM bzw. 15.000 DM. Gegenüber dem Bruttoertrag sind das etwa 
30.000 DM weniger. Recht hoch bleibt der erwartbare Migrationsertrag eines Beschäftigten im Verarbeitenden Gewerbe mit 40.000 DM.

Die Auswirkungen der unterschiedlichen Zeitpräferenzraten ist für den betrachteten kurzen Zeitraum relativ gering. Brutto beträgt die Differenz zwischen einer Zeitpräferenzrate von $1,5 \%$ und $5 \%$ etwa $5.000 \mathrm{DM}$, netto $2.500 \mathrm{DM}$ und ohne Miete 2.000 DM.

Die errechneten Überschüsse sind bereits für die Jahre 1991 bis 1994 höher als einige der vorher berechneten Migrationserträge, bei denen die Erwartungen von 1990 über die künftige Entwicklung zugrunde lagen. Sofern eine Anpassungsdauer von 5 Jahren und weniger zugrunde gelegt wird, sind die erwartbaren Erträge niedriger als die Erträge, die sich aufgrund der tatsächlichen Entwicklung bis 1994 ergaben, da die tatsächliche Lohnanpassung nicht so schnell vorangeschritten ist.

\subsection{Migrationserträge gesplittet nach Status und Geschlecht}

Werden die zwischen 1991 und 1994 potentiell erzielbaren Überschüsse aus den regionalen Lohn- und Mietpreisunterschieden gesplittet nach Status und Geschlecht betrachtet, so zeigt sich, daß nicht nur die Ertragserwartungen stark voneinander abwichen, sondern auch die Erträge, die tatsächlich hätten erzielt werden können.

Der korrigierte erwartbare Migrationsertrag eines Angestellten in der Industrie ist wesentlich höher als der eines Arbeiters; er beträgt etwa das Doppelte, sowohl brutto als auch netto. Brutto ergibt sich für einen durchschnittlichen Industrieangestellten ein korrigierter erwartbarer Ertrag von über 110.000 DM. Dies übersteigt den maximalen Bruttoüberschuß, der sich in der Branchensplittung errechnete, um etwa 30.000 DM. Der Überschuß, den ein Industriearbeiter erzielt hätte, entspricht dem eines durchschnittlichen Beschäftigten.

Ebenfalls sehr hoch sind die geschlechtsspezifischen Ertragsdifferenzen. Der korrigierte erwartbare Migrationsertrag eines männlichen Arbeiter hätte den einer Arbeiterin um brutto 25.000 DM überstiegen, netto verbleibt eine Differenz von ca. 11.000 DM. Für eine Arbeiterin hätte sich nach der Mietzahlung lediglich ein Migrationsertrag zwischen 16.000 und 17.000 DM ergeben. Damit ist der Überschuß einer Industriearbeiterin etwa so hoch wie der aller Beschäftigten im Dienstleistungsgewerbe. Zwischen den Angestellten ist die Ertragsdifferenz noch höher. Der korrigierte erwartbare Bruttomigrationsertrag eines männlichen Angestellten hätte zwischen den Jahren 1991 und 1994 bei 55.000 DM über dem 
einer weiblichen Angestellten gelegen, netto beträgt die Differenz 26.000 DM. Für weibliche Angestellte liegt der Migrationsertrag knapp über dem männlicher Arbeiter.

Tabelle 10: Korrigierte erwartbare Erträge aus regionalen Lohn- und Mietpreisunterschieden bis 1994 nach Status und Geschlecht

- Angaben in DM -

\begin{tabular}{|c|c|c|c|c|c|c|}
\hline & \multicolumn{3}{|l|}{$\mathrm{r}=0,015$} & \multicolumn{3}{|l|}{$r=0,05$} \\
\hline & $\begin{array}{l}\text { Bruttoein- } \\
\text { kommens- } \\
\text { differenz }\end{array}$ & \begin{tabular}{|l} 
Nettoein- \\
kommens- \\
differenz
\end{tabular} & $\begin{array}{l}\text { Diff. der } \\
\text { Nettoeink. } \\
\text { nach Miet- } \\
\text { zahlung }\end{array}$ & $\begin{array}{l}\text { Bruttoein- } \\
\text { kommens- } \\
\text { differenz }\end{array}$ & $\begin{array}{l}\text { Nettoein- } \\
\text { kommens- } \\
\text { differenz }\end{array}$ & $\begin{array}{l}\text { Diff. der } \\
\text { Nettoeink. } \\
\text { nach Miet- } \\
\text { zahlung }\end{array}$ \\
\hline Arbeiter & 64.821 & 34.307 & 25.688 & 60.052 & 31.831 & \begin{tabular}{|r|}
23.844 \\
\end{tabular} \\
\hline Angestellte & 120.231 & 60.904 & 52.286 & 111.079 & 56.358 & 48.371 \\
\hline Arbeiter, männl. & 69.914 & 36.804 & 28.185 & 64.754 & 34.135 & 26.148 \\
\hline Angestellte, männl. & 128.015 & 64.031 & 55.412 & 118.345 & 59.285 & 51.298 \\
\hline Arbeiter, weibl. & 45.266 & 25.583 & 16.964 & 41.909 & 23.708 & 15.721 \\
\hline Angestellte, weibl. & 73.063 & 38.383 & 29.765 & 67.597 & 35.563 & 27.576 \\
\hline
\end{tabular}

Quelle: Statistisches Bundesamt (1994), iwd (1991/1993), eigene Berechnungen

\subsection{Zusammenfassung}

Abbildung 30: Korrigierte erwartbare Migrationserträge für unterschiedliche Beschäftigte bis 1994

- Zeitpräferenzrate 0,015 -

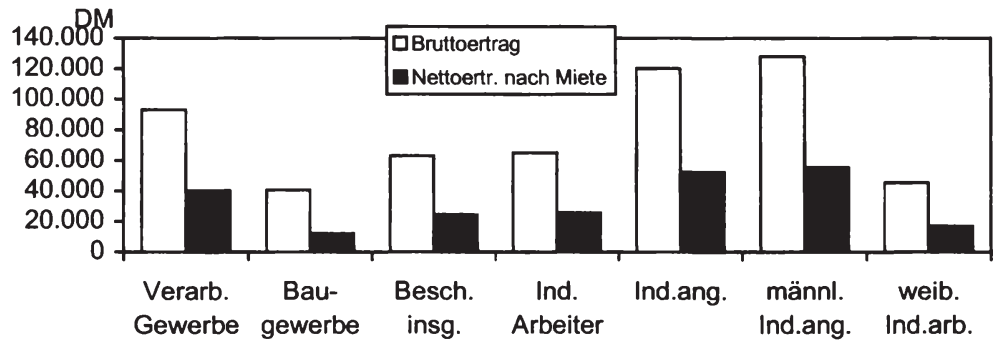

Quelle: Tabelle 9 und Tabelle 10

Ein Umzug aus den neuen in die alten Länder hätte, die Möglichkeit eines entsprechenden Arbeitsplatzwechsels unterstellt, bereits für die Jahre 1991 bis 1994 
einen Überschuß aus den regionalen Lohn- und Mietpreisunterschieden erbracht, der ein Bruttojahreseinkommen in Ostdeutschland überstiegen hätte. Der absoluten Höhe nach wären die Erträge von männlichen Angestellten im Verarbeitenden Gewerbe besonders hoch gewesen, während die Erträge von Arbeiterinnen eher gering gewesen wären. Im Dienstleistungssektor und im Baugewerbe waren die zu erzielenden Überschüsse am geringsten, wie Abbildung 30 zeigt:

\subsection{Simulation der korrigierten erwartbaren Migrationserträge aus regionalen Lohn- und Mietunterschieden nach 1994}

Da bis einschließlich 1994 noch keine vollkommene Lohnangleichung vollzogen wurde, konnte auch in den darauf folgenden Jahren noch ein Überschuß erzielt werden. Wie hoch dieser Überschuß ist, konnte zum Zeitpunkt der Untersuchung $^{206}$ nur geschätzt werden. Die Ergebnisse dieser Schätzung werden in diesem Abschnitt präsentiert.

Basis der Kalkulation sind die Einkommen und Mieten von 1994. Als Lohnsteigerungsrate wird für Westdeutschland die durchschnittliche Lohnwachstumsrate dieses Gebietes in den Jahren 1980 bis 1990 zugrunde gelegt. Die durchschnittliche jährliche Steigerung der Nominallöhne betrug 3,52\%. Diese Wachstumsrate wird ebenfalls für die Erhöhung der Bemessungsgrundlagen der Sozialversicherung verwendet, während die Beitragssätze konstant gehalten werden. Gerechnet wird mit dem Einkommensteuertarif von 1990. Angenommen wird ferner, daß der Solidarzuschlag, der im Jahr 1995 eingeführt wurde, 5 Jahre lang erhoben wird und dann gänzlich abgeschafft wird.

Bezüglich der Lohneinkommensentwicklung in Ostdeutschland werden zwei unterschiedliche Szenarien betrachtet, und zwar werden Lohnangleichungszeiträume von 5 (d. h. bis 1999) und von 10 Jahren (bis 2004) untersucht. Für beide Anpassungspfade werden degressive Verläufe gewählt. Dabei müssen für die verschiedenen Branchen, Statusse und Geschlechter unterschiedliche Anpassungsverläufe simuliert werden, da sich die jeweilige Ausgangsposition 1995 unterschied. 1994 betrug das Lohnniveau im Bergbau bereits $90 \%$ des Westniveaus, ein Anpassungszeitraum von 5 Jahren bedeutet nur geringe Änderungen pro Jahr. Beim Verarbeitenden Gewerbe dagegen, wo die Löhne im Jahr 1994 erst $64 \%$ des Westlohnniveaus erreichten, müssen wesentlich höhere jährliche Sprünge bei gleichem Anpassungszeitraum zugrunde gelegt werden.

${ }^{206}$ Die Datensammlung für die Löhne und Mieten wurde 1995 abgeschlossen. Die Entwicklungen, wie sie sich nach dieser Zeit vollzogen, fielen weniger günstig aus, als mit den angenommenen Lohnsteigerungsraten unterstellt wurde. 
Die Mieten in den neuen Ländern hatten im Jahr 1994 eine Höhe erreicht, die 65 $\%$ des Mietniveaus in den alten Ländern entsprach. Für die Mieten werden den Lohnanpassungen identische Anpassungszeiträume simuliert, d.h. 5 und 10 Jahre. Bezüglich der Mietpreissteigerungen im Westen wird die gleiche Wachstumsrate wie für die Westlöhne unterstellt. Die Preissteigerungsrate der Lebenshaltung der privaten Haushalte in Westdeutschland lag zwar in den Jahren 1980 bis 1990 etwas niedriger als der Lohnanstieg, jedoch nahmen die Mietpreise stets überdurchschnittlich zu. Abgezinst auf 1990 ergibt sich für die Zeit nach 1994 bei einem degressiv verlaufenden Anpassungszeitraum von 5 Jahren eine Differenz in den kumulierten Mietzahlungen von 2.800 DM (bzw. 2.300 DM). Bei einer Anpassungsdauer von 10 Jahren wären die Ausgaben für die Miete in Ostdeutschland insgesamt sogar um 7.200 DM (bzw. 5.700 DM) niedriger gewesen als in Westdeutschland.

\subsection{Simulation gesplittet nach Branchen}

Die Migrationserträge, die bei den getroffenen Annahmen nach 1994 noch erwartbar waren, sind deutlich niedriger als die, die zwischen 1991 bis 1994 hätten erzielt werden können, selbst wenn ein Anpassungszeitraum von 10 Jahren zugrunde gelegt wird. Tabelle 11 zeigt die Erträge, die sich bei einem Anpassungszeitraum von 5 Jahren ergäben, Tabelle 12 die Erträge bei einem Anpassungszeitraum von 10 Jahren.

Tabelle 11: Korrigierte erwartbare Erträge aus regionalen Lohn- und Mietpreisunterschieden nach 1994 nach Branchen bei einer Angleichung bis 1999

- Angaben in DM -

\begin{tabular}{|c|c|c|c|c|c|c|}
\hline Branche & $r=0,015$ & & & $\mathrm{r}=0,05$ & & \\
\hline & $\begin{array}{l}\text { Bruttoein- } \\
\text { kommens- } \\
\text { differenz }\end{array}$ & $\begin{array}{l}\text { Nettoein- } \\
\text { kommens- } \\
\text { differenz }\end{array}$ & $\begin{array}{l}\text { Differenz } \\
\text { Nettoeink. } \\
\text { nach Miet- } \\
\text { zahlung }\end{array}$ & $\begin{array}{l}\text { Bruttoein- } \\
\text { kommens- } \\
\text { differenz }\end{array}$ & $\begin{array}{l}\text { Nettoein- } \\
\text { kommen } \\
\text { s- } \\
\text { differenz }\end{array}$ & $\begin{array}{l}\text { Differenz } \\
\text { Nettoeink. } \\
\text { nach Miet- } \\
\text { zahlung }\end{array}$ \\
\hline Land- und Forstw. & 13.743 & 6.983 & 4.172 & 11.295 & 5.739 & 3.433 \\
\hline Bergbau & 9.853 & 4.261 & 1.450 & 8.073 & 3.493 & 1.188 \\
\hline Energiewirtsch. & 21.614 & 9.764 & 6.953 & 17.764 & 8.031 & 5.725 \\
\hline Verarb. Gewerbe & 30.267 & 14.053 & 11.242 & 24.819 & 11.534 & 9.228 \\
\hline Baugewerbe & 14.974 & 7.230 & 4.419 & 12.271 & 5.928 & 3.622 \\
\hline Handel & 23.698 & 11.834 & 9.023 & 19.432 & 9.707 & 7.402 \\
\hline Verkehr & 18.490 & 8.873 & 6.062 & 15.196 & 7.297 & 4.992 \\
\hline Dienstleistungen & 13.708 & 6.808 & 3.997 & 11.233 & 5.582 & 3.276 \\
\hline alle Branchen & 17.862 & 8.673 & 5.862 & 14.681 & 7.132 & 4.827 \\
\hline
\end{tabular}

Quelle: DIW (1994), iwd (1991/1993), eigene Berechnungen 
In den Branchen Dienstleistungen, Baugewerbe, Land- und Forstwirtschaft und vor allem im Bergbau sind bei einer Anpassung der Löhne und Mieten bis 1999 für die letzten fünf Jahre nur noch relativ geringe Migrationserträge erwartbar verglichen mit den Erträgen in den ersten vier Jahren. Durchschnittlich betragen die (abgezinsten) Erträge nach Berücksichtigung der unterschiedlichen Mietniveaus ca. 5.000 DM, brutto etwa das Dreifache. Die korrigierten erwartbaren Erträge im Verarbeitenden Gewerbe sind etwa doppelt so hoch. Relativ bedeutend sind auch die erwartbaren Erträge im Handel mit ca. 8.000 DM. Äußerst gering sind die Differenzen im Bergbau mit gut 1.000 DM.

Die korrigierten erwartbaren Erträge, die sich ab 1995 bei einer Lohn- und Mietangleichung bis 2004 noch ergeben, sind der Höhe nach mit denen der ersten vier Jahren vergleichbar. Bei allen Branchen (mit Ausnahme des Bergbaus) sind die Erträge bei einem Anpassungszeitraum von 10 Jahren erheblich höher als bei einer Angleichung innerhalb von 5 Jahren. Die Bruttoeinkommen liegen um über 30.000 DM höher. Im Durchschnitt errechnet sich für die Zeit nach 1994 ein korrigierter erwartbarer Nettomigrationsertrag nach Berücksichtigung der regionalen Mietdifferenzen von 20.000 DM. Dies ist etwa das Vierfache des Überschusses bei einer Anpassung bis 1999.

Im Bergbau geht der nach 1995 noch erwartbare Migrationsertrag bei einem weiteren Anpassungszeitraum von 10 Jahren gegen Null. Das liegt daran, daß der Bruttolohn im Bergbau 1994 bereits $90 \%$ des Westniveaus erreicht hatte, die Mieten jedoch erst $65 \%$. Von den geringeren Mieten in den neuen Bundesländern profitieren Beschäftigte im Bergbau aber ebenso wie Beschäftigte anderer Branchen, wenn sie in Ostdeutschland bleiben. Ihre Erträge sind aufgrund der langsameren Mietanpassung sogar niedriger, als wenn ein Anpassungszeitraum von 5 Jahren unterstellt wird.

Unterdurchschnittlich sind die noch erwartbaren Erträge im Baugewerbe und bei den Dienstleistungen, sie liegen unter 10.000 DM. Die größten Erträge ergeben sich wiederum für Beschäftigte des Verarbeitenden Gewerbes, deren Bruttolohn $199464 \%$ des Westniveaus entsprach.

Der Einfluß der Zeitpräferenzrate ist bei einem Anpassungszeitraum von 10 Jahren recht bemerkenswert. Brutto beträgt die Differenz im Durchschnitt 15.000 DM, nach Mietzahlung verbleibt noch eine Differenz von über 5.000 DM. Lediglich im Bergbau spielt sie kaum eine Rolle, da hier die Erträge nur gering sind. 
Tabelle 12: Korrigierte erwartbare Erträge aus regionalen Lohn- und Mietpreisunterschieden nach 1994 nach Branchen bei einer Angleichung bis 2004

- Angaben in DM -

\begin{tabular}{|c|c|c|c|c|c|c|}
\hline Branche & $\mathrm{r}=0,015$ & & & $r=0,05$ & & \\
\hline & $\begin{array}{l}\text { Bruttoein- } \\
\text { kommens- } \\
\text { differenz }\end{array}$ & $\begin{array}{l}\text { Nettoein- } \\
\text { kommens- } \\
\text { differenz }\end{array}$ & $\begin{array}{l}\text { Differenz } \\
\text { Nettoeink. } \\
\text { nach Miet- } \\
\text { zahlung }\end{array}$ & $\begin{array}{l}\text { Bruttoein- } \\
\text { kommens- } \\
\text { differenz }\end{array}$ & $\begin{array}{l}\text { Nettoein- } \\
\text { kommens- } \\
\text { differenz }\end{array}$ & $\begin{array}{l}\text { Differenz } \\
\text { Nettoeink. } \\
\text { nach Miet- } \\
\text { zahlung }\end{array}$ \\
\hline Land- und Forstw. & 48.515 & 24.943 & 17.644 & 37.515 & 19.296 & 13.645 \\
\hline Bergbau & 17.831 & 7.557 & 258 & 13.804 & 5.862 & 21 \\
\hline Energiewirtsch. & 76.301 & 34.301 & 27.002 & 59.001 & 26.584 & 20.932 \\
\hline Verarb. Gewerbe & 78.171 & 35.976 & 28.678 & 60.662 & 27.982 & 22.331 \\
\hline Baugewerbe & 34.278 & 16.415 & 9.116 & 26.788 & 12.846 & 7.195 \\
\hline Handel & 61.204 & 30.458 & 23.160 & 47.496 & 23.660 & 18.008 \\
\hline Verkehr & 65.271 & 31.224 & 23.925 & 50.472 & 24.186 & 18.535 \\
\hline Dienstleistungen & 31.379 & 15.476 & 8.178 & 24.522 & 12.109 & 6.458 \\
\hline alle Branchen & 63.056 & 30.530 & 23.231 & 48.760 & 23.646 & 17.995 \\
\hline
\end{tabular}

Quelle: DIW (1994), iwd (1991/1993), eigene Berechnungen

\subsection{Simulation gesplittet nach Status und Geschlecht}

Tabelle 13 und Tabelle 14 zeigen die korrigierten erwartbaren Migrationserträge, die sich nach 1994 für die Beschäftigten in der Industrie ergeben, zunächst für einen Anpassungszeitraum von 5 Jahren, im Anschluß für eine Anpassung innerhalb von 10 Jahren.

Auch bei den Beschäftigten in der Industrie sind die korrigierten erwartbaren Migrationserträge, die sich ab 1995 für einen Lohnanpassungszeitraum bis 1999 errechnen, relativ gering - verglichen mit den Erträgen in den ersten Jahren. Am niedrigsten sind sie für Arbeiterinnen, für die nach Berücksichtigung der Mietdifferenzen noch etwa 3.000 DM erwartbar waren.

Die Erträge der Angestellten insgesamt ab 1995 stimmen nahezu überein mit den Erträgen der männlichen Angestellten, obwohl die Erträge der weiblichen Angestellten deutlich niedriger sind. Dies vermittelt den Eindruck, daß die Männer die Gruppe der Angestellten in der Industrie deutlich dominieren. Hier tritt jedoch ein Struktureffekt auf, der sich anhand der Bruttoeinkommen von 1994 verdeutlichen läßt: Die weiblichen Angestellten verdienten 1994 brutto $69 \%$ des Westniveaus, die männlichen $62 \%$. Das Ostlohnniveau der Angestellten insgesamt betrug aber nur $60 \%$ des Westniveaus. Daraus ist zu schließen, daß der 
Anteil der Frauen (die in beiden Regionen weniger verdienen als ihre männlichen Kollegen) an den Angestellten in der Industrie im Osten höher ist als im Westen. Auf diese Weise kann das Lohnniveau der Angestellten insgesamt niedriger sein als die Lohnniveaus differenziert nach Geschlecht.

Tabelle 13: Korrigierte erwartbare Erträge aus regionalen Lohn- und Mietpreisunterschieden nach 1994 nach Status und Geschlecht bei einer Angleichung bis 1999

- Angaben in DM-

\begin{tabular}{|c|c|c|c|c|c|c|}
\hline & \multicolumn{3}{|c|}{$\mathrm{r}=0,015$} & \multicolumn{3}{|c|}{$r=0,05$} \\
\hline & $\begin{array}{l}\text { Bruttoein- } \\
\text { kommens- } \\
\text { differenz }\end{array}$ & \begin{tabular}{|l|}
$\begin{array}{l}\text { Nettoein- } \\
\text { kommens- } \\
\text { differenz }\end{array}$ \\
\end{tabular} & \begin{tabular}{|l|} 
Diff. der \\
Nettoeink. \\
nach Miet- \\
zahlung \\
\end{tabular} & \begin{tabular}{|l|} 
Bruttoein- \\
kommens- \\
differenz
\end{tabular} & \begin{tabular}{|l|} 
Nettoein- \\
kommens- \\
differenz
\end{tabular} & $\begin{array}{l}\text { Diff. der } \\
\text { Nettoeink. } \\
\text { nach Miet- } \\
\text { zahlung }\end{array}$ \\
\hline Arbeiter & 17.538 & 8.567 & \begin{tabular}{|l|}
5.756 \\
\end{tabular} & 14.414 & 7.045 & $\begin{array}{l}4.740 \\
\end{array}$ \\
\hline Angestellte & 30.393 & 12.928 & 10.117 & 25.085 & 10.683 & 8.378 \\
\hline Arbeiter, männl. & 18.375 & 8.837 & 6.026 & 15.102 & 7.267 & 4.962 \\
\hline Angestellte, männl. & 31.700 & 12.940 & 10.129 & 26.137 & 10.683 & 8.378 \\
\hline Arbeiter, weibl. & 13.079 & 6.686 & 3.875 & 10.749 & 5.496 & 3.191 \\
\hline Angestellte, weibl. & 19.046 & 9.045 & 6.234 & 15.654 & 7.439 & 5.134 \\
\hline
\end{tabular}

Quelle: Statistisches Bundesamt (1994), iwd (1991/1993), eigene Berechnungen

Der potentielle Ertrag der männlichen Angestellten ist mit 9.000 DM bis 10.000 DM der höchste (wie bereits in 1991 bis 1994).

Tabelle 14: Korrigierte erwartbare Erträge aus regionalen Lohn- und Mietpreisunterschieden nach 1994 nach Status und Geschlecht bei einer Angleichung bis 2004

- Angaben in DM -

\begin{tabular}{|l|r|r|r|r|r|r|}
\hline & \multicolumn{3}{|c|}{$\mathrm{r}=0,015$} & \multicolumn{3}{c|}{$\mathrm{r}=0,05$} \\
\hline & $\begin{array}{l}\text { Bruttoein- } \\
\text { kommens- } \\
\text { differenz }\end{array}$ & $\begin{array}{l}\text { Nettoein- } \\
\text { kommens- } \\
\text { differenz }\end{array}$ & $\begin{array}{l}\text { Diff. der } \\
\text { Nettoeink. } \\
\text { nach Miet- } \\
\text { zahlung }\end{array}$ & $\begin{array}{l}\text { Bruttoein- } \\
\text { kommens- } \\
\text { differenz }\end{array}$ & $\begin{array}{l}\text { Nettoein- } \\
\text { kommens- } \\
\text { differenz }\end{array}$ & $\begin{array}{l}\text { Diff. der } \\
\text { Nettoeink. } \\
\text { nach Miet- } \\
\text { zahlung }\end{array}$ \\
\hline Arbeiter & 61.910 & 30.148 & 22.849 & 47.873 & 23.348 & 17.697 \\
\hline Angestellte & 99.470 & 43.123 & 35.824 & 77.321 & 33.583 & 27.932 \\
\hline Arbeiter, männl. & 64.861 & 31.098 & 23.799 & 50.155 & 24.088 & 18.437 \\
\hline Angestellte, männl. & 108.624 & 45.467 & 38.168 & 84.437 & 35.437 & 29.786 \\
\hline Arbeiter, weibl. & 46.169 & 23.989 & 16.690 & 35.701 & 18.562 & 12.911 \\
\hline Angestellte, weibl. & 67.243 & 31.821 & 24.522 & 51.997 & 24.651 & 19.000 \\
\hline
\end{tabular}

Quelle: Statistisches Bundesamt (1994), iwd (1991/1993), eigene Berechnungen 
Bei einer Anpassungszeit bis zum Jahr 2004 sind bei allen betrachteten Gruppen die Erträge deutlich höher als bei der um fünf Jahre kürzeren Angleichungsdauer. Nach Mietzahlung sind die Erträge - wie auch in der Branchensplittung etwa viermal so hoch wie bei einer Anpassung innerhalb von fünf Jahren.

\subsection{Zusammenfassung}

Abbildung 31: Korrigierte erwartbare Überschüsse aus regionalen Lohn- und Mietdifferenzen nach 1994

- Zeitpräferenzrate 0,015 -

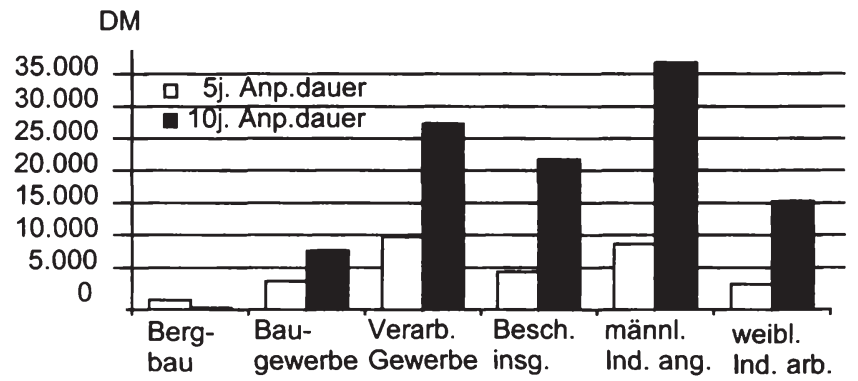

Quelle: Tabelle 11, Tabelle 12, Tabelle 13, Tabelle 14

Die korrigierten erwartbaren Migrationserträge für die Zeit nach 1994 sind wenn von einer Angleichung bis 1999 ausgegangen wird - mit durchschnittlich gut 5.000 DM relativ gering, verglichen mit den Erträgen, die zwischen den Jahren 1991 und 1994 bei einem entsprechenden Arbeitsplatzwechsel faktisch erzielt werden konnten. Die Bandbreite in der Branchengliederung geht von 1.000 DM (Bergbau) bis 10.000 DM (Verarbeitendes Gewerbe). Sollte der Angleichungsprozeß dagegen 5 Jahre länger dauern, so vervierfachen sich im Durchschnitt die Erträge, die nach 1994 erwartbar waren. Im Bereich Bergbau allerdings verringern sich in diesem Fall die Ertragserwartungen.

\subsection{Korrigierte erwartbare Migrationserträge aus regionalen Lohn- und Mietunterschieden insgesamt unter Berücksichtigung der tatsächlichen Entwicklung bis 1994}

Nachdem die Migrationserträge in Abschnitt 3.2.1.3.1. für die Jahre 1991 bis 1994 berechnet wurden und in Abschnitt 3.2.1.3.2 die Erträge ab 1995 simuliert 
worden sind, wird hieraus nun der gesamte Migrationsertrag errechnet und in diesem Abschnitt präsentiert. Im folgenden wird zur Vereinfachung mit „tatsächliche" Entwicklung die reale Lohn- und Mietpreisentwicklung bis 1994 und die für die darauf folgenden Jahre für die Simulation angenommene Entwicklung bezeichnet.

\subsection{Migrationserträge nach Branchen}

Gesplittet nach Branchen ergibt sich bei einer Anpassung bis 1999 folgendes Bild: In allen Branchen hätten positive Migrationserträge erzielt werden können, deren absolute Höhe sich jedoch deutlich voneinander unterscheidet. Die Bandbreite der potentiellen Bruttomigrationserträge liegt zwischen 123.000 und 50.000 DM. Der Mindestnettolohnüberschuß liegt bei $28.000 \mathrm{DM}$, so daß nach Berücksichtigung der Mietdifferenzen mindestens noch 15.000 DM Überschuß verbleiben. Die Nettoeinkommensdifferenzen sind lediglich gut halb so hoch wie die Bruttodifferenzen, nach Mietzahlung belaufen sie sich sogar nur auf $40 \%$ der Bruttoeinkommensdifferenzen.

Tabelle 15: Korrigierte erwartbare Erträge aus regionalen Lohn- und Mietpreisunterschieden insgesamt nach Branchen bei einer Angleichung bis 1999

- Angaben in DM -

\begin{tabular}{|l|r|r|r|r|r|r|}
\hline Branche & \multicolumn{3}{|c|}{$\mathrm{r}=0,015$} & \multicolumn{3}{c|}{$\mathrm{r}=0,05$} \\
\hline & $\begin{array}{l}\text { Bruttoein- } \\
\text { kommens- } \\
\text { differenz }\end{array}$ & $\begin{array}{l}\text { Nettoein- } \\
\text { kommens- } \\
\text { differenz }\end{array}$ & $\begin{array}{l}\text { Differenz } \\
\text { Nettoeink. } \\
\text { nach Miet- } \\
\text { zahlung }\end{array}$ & $\begin{array}{l}\text { Bruttoein- } \\
\text { kommens- } \\
\text { differenz }\end{array}$ & $\begin{array}{l}\text { Nettoein- } \\
\text { kommens- } \\
\text { differenz }\end{array}$ & $\begin{array}{l}\text { Differenz } \\
\text { Nettoeink. } \\
\text { nach } \\
\text { Mietzahlung }\end{array}$ \\
\hline Land- und Forstw. & 67.088 & 37.073 & 25.643 & 60.615 & 33.587 & 23.294 \\
\hline Bergbau & 82.139 & 39.865 & 28.435 & 75.701 & 36.890 & 26.597 \\
\hline Energiewirtsch. & 93.625 & 45.807 & 34.377 & 84.103 & 41.267 & 30.974 \\
\hline Verarb. Gewerbe & 123.404 & 62.755 & 51.326 & 110.827 & 56.567 & 46.273 \\
\hline Baugewerbe & 55.335 & 27.935 & 16.505 & 49.485 & 25.041 & 14.748 \\
\hline Handel & 84.750 & 44.830 & 33.400 & 75.791 & 40.201 & 29.909 \\
\hline Verkehr & 85.004 & 43.662 & 32.232 & 76.684 & 39.497 & 29.205 \\
\hline Dienstleistungen & 59.064 & 30.692 & 19.262 & 53.324 & 27.780 & 17.487 \\
\hline alle Branchen & 80.922 & 41.802 & 30.373 & 72.987 & 37.805 & 27.513 \\
\hline
\end{tabular}

Quelle: DIW (1991-1994), iwd (1991/1993), eigene Berechnungen

Den höchsten Nettoertrag konnten die Beschäftigten im Verarbeitenden Gewerbe mit 63.000 DM (bzw. $57.000 \mathrm{DM}$ ) erzielen. Sie übertrafen den Durchschnittswert um $50 \%$, nach Berücksichtigung der Mietdifferenz sogar um knapp 
$70 \%$. Im Bau- und Dienstleistungsgewerbe sind die Erträge dagegen stark unterdurchschnittlich.

Tabelle 16: Korrigierte erwartbare Erträge aus regionalen Lohn- und Mietpreisunterschieden insgesamt nach Branchen bei einer Angleichung bis 2004

- Angaben in DM -

\begin{tabular}{|l|r|r|r|r|r|r|}
\hline & \multicolumn{3}{|c|}{$\mathrm{r}=0,015$} & \multicolumn{3}{c|}{$\mathrm{r}=0,05$} \\
\hline & $\begin{array}{l}\text { Branche } \\
\text { kommens- } \\
\text { differenz }\end{array}$ & $\begin{array}{l}\text { kommens- } \\
\text { differenz }\end{array}$ & $\begin{array}{l}\text { Nettoeink. } \\
\text { nach Miet- } \\
\text { zahlung }\end{array}$ & $\begin{array}{l}\text { Bruttoein- } \\
\text { kommens- } \\
\text { differenz }\end{array}$ & $\begin{array}{l}\text { Nettoein- } \\
\text { kommens- } \\
\text { differenz }\end{array}$ & $\begin{array}{l}\text { Diff. der } \\
\text { Nettoeink. } \\
\text { nach Miet- } \\
\text { zahlung }\end{array}$ \\
\hline Land- und Forstw. & 101.860 & 55.033 & 39.115 & 86.835 & 47.144 & 33.506 \\
\hline Bergbau & 90.117 & 43.161 & 27.243 & 81.432 & 39.259 & 25.620 \\
\hline Energiewirtsch. & 148.312 & 70.344 & 54.426 & 125.340 & 59.820 & 46.181 \\
\hline Verarb. Gewerbe & 171.308 & 84.678 & 68.762 & 146.670 & 73.015 & 59.376 \\
\hline Baugewerbe & 74.639 & 37.120 & 21.202 & 64.002 & 31.959 & 18.321 \\
\hline Handel & 122.256 & 63.454 & 47.537 & 103.855 & 54.154 & 40.515 \\
\hline Verkehr & 131.785 & 66.013 & 50.095 & 111.960 & 56.386 & 42.748 \\
\hline Dienstleistungen & 76.735 & 39.360 & 23.443 & 66.613 & 34.307 & 20.669 \\
\hline alle Branchen & 126.116 & 63.659 & 47.742 & 107.066 & 54.319 & 40.681 \\
\hline
\end{tabular}

Quelle: DIW (1991-1994), iwd (1991/1993), eigene Berechnungen

Die Relationen „Brutto- zu Nettoerträgen“ und „Brutto- zu Nettoerträgen ohne Miete" auf Basis der tatsächlichen Entwicklung bis 1994 und einer simulierten Anpassung bis 2004 verhalten sich zueinander wie die entsprechenden Relationen bei einer fünf Jahre kürzeren Lohnanpassungszeit. Die Rangfolge der Branchen dagegen zeigt sich leicht verändert. In der Land- und Forstwirtschaft sind die Erträge höher als im Bergbau; bei einer Anpassungszeit bis 1999 sind sie niedriger. Das gleiche gilt für die Branchen Handel und Verkehr, die Veränderung der Abstände ist allerdings weniger kraß.

Die korrigierten erwartbaren Migrationserträge für einen im Bergbau beschäftigten Arbeitnehmer liegen für den Zeitraum 1991-1994 um etwa 5.000 DM über denen in der Land- und Forstwirtschaft. Im Jahr 1991 betrug der Lohn in beiden Branchen $51 \%$ des Westniveaus, bis zum Ende des Jahres 1994 hatte sich der Unterschied im Bergbau wesentlich stärker abgebaut (auf $90 \%$ ) als in der Landund Forstwirtschaft. Aufgrund der absolut höheren Einkommen im Bergbau sind die Migrationserträge dort aber zunächst insgesamt höher. Bereits 1994 wirkte sich die stärkere Lohnangleichung dahingehend aus, daß die Migrationserträge dieses Jahres absolut im Bergbau geringer sind als in der Land- und Forstwirtschaft. Entsprechendes gilt für die Folgejahre. Für die Branchen Handel und Verkehr ergibt sich ein analoger Effekt. 
In der Branche Verarbeitendes Gewerbe führt sowohl das hohe Lohnniveau als auch der höchste Abstand aller Branchen zum Westlohnniveau zu den höchsten erzielbaren Migrationserträgen.

\subsection{Migrationserträge nach Status und Geschlecht}

Für die Industriebeschäftigten ergeben sich bei einer Anpassung der Mieten und Löhne an das Westniveau bis zum Ende des Jahres 1999 die inTabelle 17 aufgeführten Erträge.

Die Angestellten in der Industrie hätten beachtliche Migrationserträge erzielen können, und zwar brutto über 130.000 DM. Netto nach Abzug der Mietkosten verbleiben über 50.000 DM. Sie übertreffen damit die Erträge eines Industriearbeiters um das Doppelte.

Die Männer hätten deutlich höhere Erträge als die Frauen erzielen können: Sie liegen etwa $60 \%$ darüber. Relativ gesehen ist der geschlechtsspezifische Unterschied in der Gruppe der Arbeiter größer, absolut ist er bei den Angestellten höher.

Tabelle 17: Korrigierte erwartbare Erträge aus regionalen Lohn- und Mietpreisunterschieden insgesamt nach Status und Geschlecht bei einer Angleichung bis 1999

- Angaben in DM -

\begin{tabular}{|l|r|r|r|r|r|r|}
\hline & \multicolumn{3}{|c|}{$\mathrm{r}=0,015$} & \multicolumn{3}{c|}{$\mathrm{r}=0,05$} \\
\hline & $\begin{array}{l}\text { Bruttoein- } \\
\text { kommens- } \\
\text { differenz }\end{array}$ & $\begin{array}{l}\text { Nettoein- } \\
\text { kommens- } \\
\text { differenz }\end{array}$ & $\begin{array}{l}\text { Diff. der } \\
\text { Nettoeink. } \\
\text { nach Miet- } \\
\text { zahlung }\end{array}$ & $\begin{array}{l}\text { Bruttoein- } \\
\text { kommens- } \\
\text { differenz }\end{array}$ & $\begin{array}{l}\text { Nettoein- } \\
\text { kommens- } \\
\text { differenz }\end{array}$ & $\begin{array}{l}\text { Diff. der } \\
\text { Nettoeink. } \\
\text { nach Miet- } \\
\text { zahlung }\end{array}$ \\
\hline Arbeiter & 82.359 & 42.874 & 31.444 & 74.466 & 38.876 & 28.584 \\
\hline Angestellte & 150.624 & 73.832 & 62.403 & 136.164 & 67.041 & 56.749 \\
\hline Arbeiter, männl. & 88.289 & 45.641 & 34.211 & 79.856 & 41.402 & 31.110 \\
\hline Angest., männl. & 159.715 & 76.971 & 65.541 & 144.482 & 69.968 & 59.676 \\
\hline Arbeiter, weibl. & 58.345 & 32.269 & 20.839 & 52.658 & 29.204 & 18.912 \\
\hline Angest., weibl. & 92.109 & 47.428 & 35.999 & 83.251 & 43.002 & 32.710 \\
\hline
\end{tabular}

Quelle: Statistisches Bundesamt (1994), iwd (1991/1993), eigene Berechnungen

Bei einer Zeitpräferenzrate von 1,5\% entsprechen die Bruttoerträge der Angestellten in etwa den Bruttoerträgen der Beschäftigten in der Energiewirtschaft bei 
einem Anpassungszeitraum der Löhne und Mieten von $10 \mathrm{Jahren}^{207}$. Aufgrund der höheren absoluten Einkommen und damit höheren Grenzsteuersätze der Beschäftigten in der Energiewirtschaft unterscheiden sich die Nettodifferenzen etwas stärker. Werden die Differenzen nach Berücksichtigung der Mieten verglichen, läßt sich eine noch höhere Differenz feststellen. Die Differenz beträgt bei diesem Vergleich 8.000 DM (brutto 2.000 DM, netto 3.000 DM). Der Grund dafür liegt darin, daß bei einer längeren Anpassungszeit der Mieten die Erträge einer Migration schmelzen.

Wird die Lohnanpassung erst bis 2004 vollendet, hätte ein männlicher Industrieangestellter im Durchschnitt durch einen Umzug einen Bruttoüberschuß von über 200.000 DM erzielen können. Selbst für Industriearbeiterinnen beträgt der Überschuß noch über $70.000 \mathrm{DM}$, ihr korrigierter erwartbarer Ertrag liegt aber deutlich unter den durchschnittlichen aller Beschäftigten. Ihr erwartbarer Bruttoüberschuß liegt knapp unter dem der Beschäftigten im Bergbau. Da ihr Grenzsteuersatz aber deutlich niedriger ist, liegt die Nettodifferenz um 4.000 DM höher.

Tabelle 18: Korrigierte erwartbare Erträge aus regionalen Lohn- und Mietpreisunterschieden insgesamt nach Status und Geschlecht bei einer Angleichung bis 2004

- Angaben in DM -

\begin{tabular}{|l|r|r|r|r|r|r|}
\hline & \multicolumn{3}{|c|}{$\mathrm{r}=0,015$} & \multicolumn{3}{c|}{$\mathrm{r}=0,05$} \\
\hline & $\begin{array}{l}\text { Bruttoein- } \\
\text { kommens- } \\
\text { differenz }\end{array}$ & $\begin{array}{l}\text { Nettoein- } \\
\text { kommens- } \\
\text { differenz }\end{array}$ & $\begin{array}{l}\text { Diff. der } \\
\text { Nettoeink. } \\
\text { nach Miet- } \\
\text { zahlung }\end{array}$ & $\begin{array}{l}\text { Bruttoein- } \\
\text { kommens- } \\
\text { differenz }\end{array}$ & $\begin{array}{l}\text { Nettoein- } \\
\text { kommens- } \\
\text { differenz }\end{array}$ & $\begin{array}{l}\text { Diff. der } \\
\text { Nettoeink. } \\
\text { nach Miet- } \\
\text { zahlung }\end{array}$ \\
\hline Arbeiter & 121.962 & 61.979 & 46.693 & 107.925 & 55.179 & 41.541 \\
\hline Angestellte & 210.549 & 99.481 & 84.195 & 188.400 & 89.941 & 76.303 \\
\hline Arbeiter, männl. & 129.615 & 65.233 & 49.947 & 114.909 & 58.223 & 44.585 \\
\hline Angest., männl. & 226.969 & 104.752 & 89.466 & 202.782 & 94.722 & 81.084 \\
\hline Arbeiter, weibl. & 88.078 & 47.697 & 32.411 & 77.610 & 42.270 & 28.632 \\
\hline Angest., weibl. & 134.840 & 67.384 & 52.098 & 119.594 & 60.214 & 46.576 \\
\hline
\end{tabular}

Quelle: Statistisches Bundesamt (1994), iwd (1991/1993), eigene Berechnungen

Die weiblichen Industrieangestellten hätten einen höheren Ertrag erzielen können als ein durchschnittlicher Arbeitnehmer. Die Industriearbeiter insgesamt

${ }^{207}$ Ein Vergleich von Erträgen, für die unterschiedliche Anpassungszeiträume zugrunde gelegt werden, ist unproblematisch, da bei einem kürzeren Zeitraum die gegenüber dem längeren Zeitraum fehlenden Jahre quasi mit Null in die Rechnung eingehen, da in diesen Jahren keine Unterschied mehr besteht. 
kommen auf den gleichen Ertrag wie die durchschnittlichen Arbeitnehmer aller Branchen.

\subsection{Zusammenfassung}

Die ostdeutschen Beschäftigten hätten durch einen Arbeitsplatzwechsel bei einer Orientierung an den durchschnittlichen regionalen Unterschieden im Erfolgsfall erhebliche Überschüsse erzielen können. Bei einer Angleichung der Löhne bis zum Jahr 1999 (2004) und einer Zeitpräferenzrate von 1,5\% beträgt die Bruttoeinkommensdifferenz 81.000 DM (126.000 DM). Diese Differenz ist mehr als dreimal (fünfmal) so hoch wie das durchschnittliche Bruttojahreseinkommen in Ostdeutschland im Jahr 1991. Die Nettoeinkommensdifferenz beträgt knapp 42.000 DM (knapp 64.000 DM), durch die geringeren Mieten in Ostdeutschland verringert sie sich um weitere 11.500 DM (16.000 DM). Die Erträge sind in den ersten Jahren besonders hoch und bauen sich im Zeitablauf kontinuierlich ab. Bei einer Angleichung bis 2004 hätte in den letzten zehn Jahren etwa noch einmal der gleiche Ertrag erzielt werden können wie in den ersten vier Jahren.

Abbildung 32: Ausgewählte korrigierte erwartbare Migrationserträge aus Lohnund Mietunterschieden insgesamt

- Zeitpräferenzrate 0,015 -

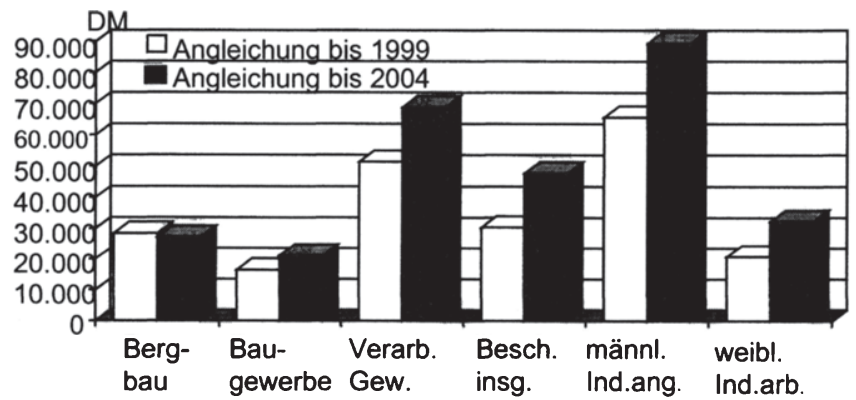

Quelle: Tabelle 15, Tabelle 16, Tabelle 17 und Tabelle 18

Für die unterschiedlichen Gruppen der Beschäftigten ergibt sich eine große Bandbreite bezüglich der potentiellen Migrationserträge, wie Abbildung 32 zeigt. In der Branche Verarbeitendes Gewerbe führt sowohl das hohe Lohnniveau als auch der große Abstand zum Westlohnniveau zu den höchsten Migrationserträgen. Die Bruttoeinkommensdifferenz beträgt das fünf- bis sechsfache des ostdeutschen Bruttojahreseinkommens im Jahr 1991 bei einer Angleichung bis 1999, bei einem fünf Jahre längerem Lohnangleichungszeitraum sogar das 
sechs- bis siebenfache. Die Erträge der männlichen Industrieangestellten übertreffen die Durchschnittserträge im Verarbeitenden Gewerbe zwar absolut, bei einem Vergleich mit ihrem Bruttojahreseinkommen im Jahr 1991 schneiden sie aber etwas schlechter ab. Absolut und im Vergleich zum Bruttojahreseinkommen schneiden die korrigierten erwartbaren Migrationserträge in den Branchen Baugewerbe und Dienstleistungen stark unterdurchschnittlich ab.

Ein wesentlicher Einflußfaktor für die Höhe des Einkommens und damit auch des Migrationsertrags ist die Qualifikation. Aufgrund der fehlenden Datenbasis konnte die Qualifikation allerdings quasi nicht berücksichtigt werden. Nur für 1993 lagen der Verfasserin Einkommensdaten der Industriebeschäftigten nach Leistungsgruppen vor ${ }^{208}$. Die Daten weisen darauf hin, daß bei höher qualifizierten Angestellten und Arbeitern die Lohnangleichung nicht so weit fortgeschritten war wie bei den geringer qualifizierten Beschäftigten. Besonders deutlich traf dies auf die Angestellten zu. In Leistungsstufe II betrug die Verdienstrelation zu Westdeutschland 58\%, während sie in Leistungsstufe $\mathrm{V}$ bereits 79 \% betrug. Da die Löhne der Qualifizierten außerdem auch absolut höher waren, dürften die erwartbaren Migrationserträge für die Qualifizierten wesentlich höher gewesen sein als für die Unqualifizierten.

\subsubsection{Vergleich der 1990 erwartbaren mit den korrigierten erwartbaren Migrationserträgen aus regionalen Lohn- und Preisunterschieden}

Unter Zugrundelegung der bisherigen Entwicklung zeigt sich, daß die in 1990 erwartbaren Migrationserträge erheblich von dem abwichen, womit aufgrund der tatsächlichen jüngeren Entwicklung und ihrer Fortschreibung zu rechnen wäre ${ }^{209}$. Je nach Erwartungen und Anpassungszeitraum ist die Abweichung positiv oder negativ. Auffällig ist die starke Streuung der potentiellen Migrationserträge zwischen den verschiedenen Branchen.

Wurde 1990 eine Lohnangleichung innerhalb von drei Jahren erwartet, so liegt in allen untersuchten Fällen der damals erwartbare Ertrag unter dem, der tatsächlich hätte realisiert werden können. Gleiches gilt für eine Erwartung einer fünfjährigen Angleichungsdauer Jahren (mit Ausnahme der Beschäftigten in der Baubranche).

Bei einer tatsächlichen Angleichung der Löhne bis 1999 hätten die Beschäftigten, die mit Lohnanpassungen nach 3 Jahren rechneten, den Nettomigrationser-

${ }^{208} \mathrm{Vgl}$. Statistisches Bundesamt (1994), S. $25 \mathrm{f}$.
${ }^{209} \mathrm{Zu}$ den Differenzen vgl. Anhang 4. 
trag nach Mietzahlung um durchschnittlich gut 15.000 DM unterschätzt. Der Differenzbetrag ist sogar höher als der im Jahr 1990 erwartbare Überschuß. Dieses Ergebnis gilt allerdings nicht für alle Branchen. Im Bergbau, im Baugewerbe und bei den Dienstleistungen sind zwar ebenfalls hohe Differenzen zu verzeichnen, jedoch sind sie niedriger als der erwartbare Ertrag. Die absolut höchsten Differenzen liegen im Verarbeitenden Gewerbe vor, der Bruttoertrag übersteigt den 1990 erwartbaren Ertrag um über 80.000 DM. Die Vorteile des niedrigeren Mietniveaus wären durchschnittlich um 8.000 DM unterschätzt worden.

Für die Beschäftigten, die eine 5jährige Anpassungsdauer erwarteten, sind die Abweichungen etwas geringer. Im Durchschnitt liegen sie brutto zwischen 37.000 DM und 43.000 DM und netto ohne Miete zwischen 12.000 DM und 13.000 DM. Brutto übertrifft die Differenz auch bei dieser Personengruppe die Erwartung um das Doppelte, netto ist sie etwas geringer. Im Baugewerbe ergibt sich zwar sowohl brutto als auch netto ein den Erwartungen übersteigender Überschuß, bei Einbeziehung der Mietdifferenzen ergibt sich aber ein etwas geringerer Ertrag als der 1990 erwartbare. Das liegt daran, daß die Mietdifferenz länger anhielt, als der Kalkulation zugrunde gelegt wurde und dies den Migrationsertrag schmälert. Im Baugewerbe entsprechen mithin die Erwartungen am ehesten der tatsächlichen Entwicklung. Besonders kräftig schmilzt bei der 5jährigen gegenüber der 3jährigen Anpassungserwartung der die Erwartungen übertreffende Migrationsertrag. Netto ohne Miete verringert sich die Differenz von über 11.000 DM auf unter 6.000 DM. Bei anderen Branchen ist die Differenz absolut zwar ähnlich hoch, jedoch ist die relative Bedeutung nicht ganz so hoch.

Die Beschäftigten, die eine Lohnangleichung erst nach 10 Jahren erwartet hatten, hätten die potentiellen Erträge dagegen überschätzt, wenn sich die Lohnsätze bis Ende 1999 angleichen, und zwar durchschnittlich um rund 15.000 DM (netto nach Mietzahlung). Die Differenz durch die Berücksichtigung der Mieten beträgt für die Migranten circa $600 \mathrm{DM}$ bzw. $700 \mathrm{DM}$. Die Entwicklung der Mieten wäre damit im wesentlichen zutreffend antizipiert worden. Der Grund für die Überschätzung liegt im Einkommensbereich.

Lediglich die 1990 im Verarbeitenden Gewerbe erwartbaren Erträge entsprechen in etwa den ,tatsächlich“ zu erzielenden Überschüssen. Brutto liegen die Ertragserwartungen zwar etwas höher, aber netto leicht darunter. In allen anderen Branchen werden die Erträge überschätzt, im Bergbau und im Baugewerbe sogar um über 40.000 DM brutto. Die Nettoerträge nach Miete wären über 25.000 DM höher als 1990 erwartbar gewesen. Im Handel ist die Abweichung netto höher als brutto. Bei geringen absoluten Abweichungen können die Steuervorteile 
der Ostdeutschen durchschlagen, die bei der Berechnung der Erwartungswerte nicht berücksichtigt wurden und die sich zu Lasten des Migrationsertrags auswirken.

Bei Zugrundelegung der faktischen Entwicklung bis zum Jahr 1994 und der Annahme einer Lohnangleichung bis zum Jahr 1999 betrüge der Lohnangleichungszeitraum 9 Jahre und wäre damit vergleichbar mit einer 1990 erwarteten Anpassungsdauer von 10 Jahren. Daraus könnte theoretisch gefolgert werden, daß jemand, der am Ende des Jahres 1990 mit einem zehnjährigen Anpassungszeitraum rechnete, seinen potentiellen Migrationsertrag in etwa zutreffend antizipiert haben müßte. Warum wichen aber die erwartbaren Erträge unter $\mathrm{Zu}$ grundelegung des Kenntnisstands von 1990 von den korrigierten erwartbaren Erträgen unter Zugrundelegung eines Kenntnisstands von 1994 voneinander ab, wenn der Lohnanpassungszeitraum in etwa identisch war? Ein Grund liegt darin, daß 1994 (durchschnittlich) keine Lohnangleichung stattfand. Dies wird bei der Berechnung der „tatsächlichen“ Erträge berücksichtigt. Bei der Berechnung des Erwartungswerts unter Zugrundelegung der Kenntnisse von 1990 wird demgegenüber eine stetige Annäherung der Einkommen zugrunde gelegt. Zum anderen wirkt sich aus, daß in dem einen Fall zehn und im anderen neun Jahre bis zur Lohnangleichung unterstellt wurden.

Ist die völlige Nivellierung der Löhne und Mieten erst in der Mitte des nächsten Jahrzehnts abgeschlossen, weichen die erwartbaren Erträge noch stärker von den tatsächlich erzielbaren Überschüssen ab. Die Beschäftigten mit 3jährigen Anpassungserwartungen hätten den Bruttomigrationsertrag um über $80.000 \mathrm{DM}$ unterschätzt, d.h. der Überschuß wäre knapp fünfmal so hoch gewesen wie 1990 erwartbar. Der Nettoüberschuß nach Berücksichtigung der Miete wäre viermal so hoch gewesen. Die regionalen Mietunterschiede verringern die Nettodifferenz um über 10.000 DM. Die Erwartungen von 1990 bezüglich des Migrationsertrags deckt sich bei Beschäftigten in der Baubranche, im Bergbau und im Dienstleistungssektor dann am ehesten mit den real erzielbaren Erträgen, netto ohne Miete beträgt die Abweichung rund 10.000 DM. Analog zu den Ergebnissen bei Zugrundelegung einer Angleichung bis Ende der neunziger Jahre sind die größten Differenzen im Verarbeitenden Gewerbe zu verzeichnen, sie liegen brutto über 110.000 DM und netto über 50.000 DM.

Bei 5jährigen Anpassungserwartungen am Ende des Jahres 1990 liegen auch die Erwartungen von Beschäftigten der Bauindustrie tendenziell etwas zu niedrig, vorausgesetzt, die Löhne haben sich bis zum Jahr 2004 angeglichen. Die Abweichungen sind vergleichbar zu denen mit 3jähriger Erwartung, nur daß die Differenzen durchgängig niedriger sind. 
Von den Beschäftigten mit 10jährigen Nivellierungserwartungen hätten bei diesem Vergleich nur die Beschäftigten dreier Branchen ihren Migrationsertrag überschätzt, und zwar die im Bergbau, im Baugewerbe und im Dienstleistungsgewerbe.

Die zu optimistische Einschätzung des Migrationsertrags bei diesen Angleichungsvarianten für Beschäftigte des Baugewerbes erklärt sich - neoklassisch gesehen - aus der relativen Knappheit als Folge des durch den Staat angeheizten Baubooms. Nach der Vereinigung wurden massive Förderungen und Steuervergünstigungen für Bauinvestitionen in den Neuen Ländern gewährt, die eine immense Bautätigkeit zur Folge hatten (nach Auslaufen vieler Förderungen am Ende der 90er Jahre entstanden allerdings erhebliche Probleme auf dem Immobilienmarkt). Auch die Überschätzungen für den Dienstleistungssektor dürften sich allokativ-güterwirtschaftlich, also aus relativen Knappheiten, erklären. Die hohen Überschätzungen im Bergbau könnten dagegen die Folge des staatlichen Protektionismus in Verbindung mit monopolistischen Strukturen sein. Diese Rahmenbedingungen gelten auch für den Energiesektor, so daß sich hieraus ebenfalls Gründe für die Tendenzen zur Überschätzung für Beschäftigte dieser Branche finden lassen.

In den anderen Branchen entspricht der erwartbare Ertrag dem realisierbaren, teilweise liegt er auch darunter. Insbesondere im Verarbeitenden Gewerbe wären selbst bei diesen vergleichsweise pessimistischen Erwartungen über die Lohnanpassung die Erträge noch unterschätzt worden. Für die Beschäftigten insgesamt klaffen die Erwartungen zwischen den Bruttoerträgen um circa 20.000 DM auseinander, netto nach Miete entsprechen sie aber in etwa den korrigierten erwartbaren Erträgen. Die langsamere Angleichung der Mieten im Vergleich zu den Erwartungen drückt die Differenz um 4.000 DM bis 5.000 DM.

Für die Auswertung nach Status und Geschlecht ergibt sich ein analoges Bild, daher wird im folgenden nur auf Auffälligkeiten hingewiesen. Auch hier sind bei denjenigen, bei denen die erwartbaren Migrationserträge besonders groß sind, die Abweichungen zu den korrigierten erwartbaren Erträgen ebenfalls auffallend hoch, während bei niedrigen Migrationserträgen auch die Abweichungen relativ gering sind. Entsprechend sind die 1990 erwartbaren Überschüsse aus regionalen Lohn- und Mietpreisunterschieden für die Gruppe der Arbeiter zutreffender als für die Angestellten und für die Frauen zutreffender als für die Männer. Diejenigen Industriebeschäftigten, die mit einer Lohnangleichung in 10 Jahren rechneten, überschätzen die Erträge, wenn die tatsächlichen Lohnangleichung bis Ende der neunziger Jahre stattgefunden hätte. Die Erwartungen übertreffen dann für alle Gruppen die Erträge um etwa 10.000 DM bis 15.000 DM. Dauert die Lohnnivellierung 5 Jahre länger, treffen die Erwartungen bezüglich der 
Nettoerträge ohne Miete hingegen in etwa zu, hätten lediglich die männlichen Angestellten höhere Erträge erzielen können.

\subsubsection{Bewertung und Berücksichtigung weiterer Migrationskosten und - erträge}

In den vorhergehenden Abschnitten wurde berechnet, welchen Ertrag ein Umzug aufgrund von regionalen Lohn- und Mietpreisdifferenzen brutto und netto erbracht hätte. Die Ergebnisse deuten auf einen Überschuß hin, der sich durch einen Umzug nach Westdeutschland ergibt. Theoretisch wäre es nach diesen Ergebnissen für alle Beschäftigten lohnend gewesen, in den alten Bundesländern $\mathrm{zu}$ arbeiten. Bereits in früheren Teilen der Arbeit wurde darauf hingewiesen, daß neben Löhnen und Mieten noch weitere Migrationsmotive bestehen. Im folgenden wird ein Teil der weiteren Migrationskosten und -erträge zu quantifizieren versucht, und anhand dieser Quantifizierung wird dann geprüft, ob es für einen marginalen Beschäftigten - und wenn ja für welchen - vorteilhaft gewesen wäre zu migrieren.

\subsubsection{Monetäre Bewertung weiterer Migrationskosten und -erträge}

Mit „Kosten“ oder „Abschlag“ wird im folgenden ein in monetären Größen ausgedrückter Sachverhalt bezeichnet, der das erwartbare Einkommen in Westdeutschland mindert und damit den Migrationsertrag schmälern würde. Entsprechend werden mit „Zuschlag" solche Umstände bezeichnet, die die Einkommensdifferenz im Vergleich zur einfachen Rechnung, in der nur Löhne und durchschnittliche Mieten berücksichtigt wurden, erhöhen.

Die $\mathrm{Zu}$ - und Abschläge werden in drei Kategorien aufgeteilt. Die erste Kategorie sind Kosten und Erträge, die unabhängig von der Person und dem Ziel des Migranten entstehen. Solche Kosten werden hier in Form von Abschlägen für die erhöhten Mietkosten bei Neuanmietung einer Wohnung (die im Durchschnitt höher ist als eine Bestandsmiete) berücksichtigt. In die zweite Kategorie fallen solche Kosten, die von der Wanderungsdistanz abhängig sind. Dies sind zum einen Umzugskosten und zum anderen die psychischen Kosten. In die dritte und letzte Kategorie fallen solche Kosten und Erträge, deren Höhe von der Tätigkeit des Migranten abhängen. Aus dieser Kategorie werden solche Abschläge berücksichtigt, die sich ergeben, wenn ein Ostdeutscher im Westen eine Verschlechterung seiner beruflichen Position hinnehmen muß. Ferner werden Zuschläge für die im Westen niedrigere jährliche Arbeitszeit kalkuliert. 
Unterschiede in der Standortqualität werden hier nicht berücksichtigt. Die neuen und alten Bundesländer sind in sich sehr heterogen, so daß solche Schätzungsversuche wenig sinnvoll erscheinen. Pauschalierend ist aber festzustellen, daß die Standortqualität in Westdeutschland für die privaten Haushalte in den ersten Jahren nach der Vereinigung höher war, wenn man an Faktoren wie Straßenzustand, Luftverschmutzung oder Verfügbarkeit über Telefonanschlüsse denkt. Aufgrund der immensen Investitionen in die Infrastruktur verbessert sich die Standortqualität in den neuen Bundesländer allerdings zügig, trotzdem bestand auch einige Jahre nach der Vereinigung noch ein erheblicher Vorsprung der alten Länder. Im Prinzip müßte daher ein Zuschlag auf das Westeinkommen vorgenommen werden. Das unterbleibt hier jedoch, weil eine differenzierende Bewertung den Rahmen dieser Arbeit sprengt, und ein einfaches Ansetzen von Pauschalbeträgen zu willkürlich erscheint. Ferner müßten auch dienatürlichen Gegebenheiten berücksichtigt werden; auch deren Bedeutung läßt sich auf eine zum Rahmen dieser Arbeit noch passende Weise nicht ermitteln.

Bei der Suche nach einem Arbeitsplatz entstehen Kosten. Bewerbungskosten fallen aber auch dann an, wenn eine neue Arbeitsstelle in der gleichen Region gesucht wird. Da viele der ostdeutschen Beschäftigten von einem Fortfall ihres Arbeitsplatzes bedroht oder betroffen waren und Bewerbungskosten dann sowieso anfielen, soll von ihrer Berücksichtigung abgesehen werden.

Auswirkungen auf die Höhe der Altersrente infolge eines Arbeitsplatzwechsels werden nicht berechnet. Eine solche Berücksichtigung hätte den Rahmen dieser Arbeit gesprengt.

\subsection{Mehrkosten durch Neuanmietung einer Wohnung}

Bei einem Wohnungswechsel ist die Miete der neuen Wohnung, auch wenn sie von der Ausstattung, Größe und Lage der alten entspricht, in der Regel höher als die Miete der alten ${ }^{210}$. Dies gilt jedoch nur in den alten und nicht in den neuen Ländern ${ }^{211}$. Bei einem Umzug nach Westdeutschland sollte daher nicht die dort herrschende Durchschnittsmiete für die Errechnung des Migrationsertrags zugrunde gelegt werden, sondern eine etwas höhere Miete. Oben wurden bereits die regionalen durchschnittlichen Bestandsmieten berücksichtigt. Einzubeziehen ist jetzt noch die Differenz zwischen einer Bestandsmiete und einer „Neumiete“

${ }^{210}$ Unterschiede in der Qualität des Wohnraums sollen nicht betrachtet werden. Vgl. Abschnitt 3.2.1.1.2.

${ }^{211}$ Vgl. DIW $(1994,21)$, S. 351. Bei einem Umzug von West- nach Ostdeutschland fielen solche Kosten nicht an. Die Miete bei einer Wohndauer von unter 5 Jahren ist im Osten sogar ganz geringfügig niedriger als die Durchschnittsmiete. 
in den alten Bundesländern. Gemäß einer Umfrage im Rahmen des sozialökonomischen Panels beträgt die Differenz pro Quadratmeter und Monat 1,10 $\mathrm{DM}^{212}$. Da sich ein Unterschied nur in den ersten 5 Jahren nach einem Umzug ergibt, wird dieser Betrag im folgenden nur für diesen Zeitraum berücksichtigt.

Tabelle 19: Mehrkosten bei Neuanmietung einer Wohnung

- Angaben in DM -

\begin{tabular}{|l|r|r|}
\hline Jahr & $r=0,015$ & $r=0,05$ \\
\hline 1991 & 520 & 503 \\
\hline 1992 & 513 & 479 \\
\hline 1993 & 505 & 456 \\
\hline 1994 & 497 & 434 \\
\hline 1995 & 490 & 414 \\
\hline Summe & 2.525 & 2.286 \\
\hline
\end{tabular}

Quelle: iwd (1991/1993), DIW (1994, 21), eigene Berechnungen

Gemäß Tabelle 19 ergeben sich durch die höheren Kosten einer Neuanmietung gegenüber einer Bestandsmiete abgezinste Mehrkosten von über 2.000 DM.

\subsection{Entfernungsabhängige Kosten}

\subsection{Umzugskosten ${ }^{2 / 3}$}

Die Kosten eines Umzugs hängen wesentlich von der Entfernung zwischen dem alten und dem neuen Wohnsitz ab. In einem früheren Abschnitt wurde darauf hingewiesen, daß noch andere Faktoren von Bedeutung sind, wie etwa das Alter der Migranten. Diese Faktoren werden hier vernachlässigt.

Die Umzugskosten werden für unterschiedliche Entfernungen geschätzt, dabei ist die längste berücksichtigte Distanz $1.000 \mathrm{~km}$. Viel länger kann die maximale Entfernung aus geographischen Gründen nicht sein. Die kürzeste untersuchte Distanz beträgt $100 \mathrm{~km}$; noch kürzere Entfernungen dürften durch Pendeln überwunden werden und sind nicht Untersuchungsgegenstand dieser Arbeit. Ferner wird noch eine mittlere Distanz von $400 \mathrm{~km}$ berücksichtigt.

${ }^{212}$ Vgl. DIW (1994, 21).

${ }^{213}$ Die Umzugskosten werden z.T. von dem neuen Arbeitgeber erstattet, sie wären in einer Berechnung des Migrationsertrags dann nicht zu berücksichtigen. Dies ist jedoch nicht immer der Fall, so wird etwa bei Berufsanfängern argumentiert, daß es sich nicht um einen beruflich bedingten Umzug handelt. 
Die Kosten werden in Anlehnung an die Kosten ermittelt, die bei einem Umzug aus beruflichem Anlaß steuerlich absetzbar sind. Die einzelnen Positionen und ihre Höhe sind in Tabelle 20 aufgeführt.

Tabelle 20: Umzugskosten

\begin{tabular}{|c|c|c|c|c|}
\hline Kostenart & Berechnung & $100 \mathrm{~km}$ & $400 \mathrm{~km}$ & $\begin{array}{l}1000 \\
\mathrm{~km}\end{array}$ \\
\hline $\begin{array}{l}\text { Kosten für Beförderung des Hausrats } \\
\text { durch eine Spedition }\end{array}$ & & 2.500 & 4.500 & 6.500 \\
\hline Fahrtkosten beim Umzug & $\begin{array}{l}\text { Fahrkm } * 0,42 \mathrm{DM} \\
\text { (Auto) }\end{array}$ & 42 & 168 & 420 \\
\hline $\begin{array}{l}\text { Mehraufwendung für Verpflegung am } \\
\text { Umzugstag }\end{array}$ & pauschal $64 \mathrm{DM}$ & 64 & 64 & 64 \\
\hline Übernachtungskosten am Umzugstag & circa $100 \mathrm{DM}$ & 100 & 100 & 100 \\
\hline $\begin{array}{l}\text { Kosten für Wohnungssuche und - } \\
\text { besichtigung } \\
\text { - Reisekosten ( } 2 * \text { An- und Abfahrt) } \\
\text { - Übernachtung ( } 2 \text { mal je } 100 \mathrm{DM}) \\
\text { - Mehraufwand. für Verpflegung (4 } \\
\text { Tage) } \\
\text { - Kosten der Wohnungssuche vor Ort } \\
\text { (Zeitungen/Telefonate/Fahrten) }\end{array}$ & $\begin{array}{l}\text { Fahrkm *0,42 DM } \\
2.100 \mathrm{DM} \\
4.64 \mathrm{DM} \\
\text { pauschal }\end{array}$ & $\begin{array}{r}168 \\
200 \\
256 \\
100\end{array}$ & $\begin{array}{l}672 \\
200 \\
256 \\
100\end{array}$ & $\begin{array}{r}1.680 \\
200 \\
256 \\
100\end{array}$ \\
\hline Fahrt zur Vorbereitung des Umzugs & Fahrkm * 0,42 DM & 42 & 168 & 420 \\
\hline doppelte Miete (1 Monat Miete Ost) & $40 \mathrm{qm}$ bei $2 \mathrm{DM} / \mathrm{qm}$ & 80 & 80 & 80 \\
\hline $\begin{array}{l}\text { Maklergebühren, durchschnittlich das } \\
1,5 \text { fache der Monatsmiete }\end{array}$ & $\begin{array}{l}1,5 * \text { Miete West } \\
(1,5 * 40 * 9,20 \mathrm{DM}) \\
\end{array}$ & 552 & 552 & 552 \\
\hline Herd, Öfen u.a. & pauschal & 200 & 200 & 200 \\
\hline $\begin{array}{l}\text { sonstiges (Trinkgelder, Ânderung von } \\
\text { Gardinen, Telefonanschluß etc.) }\end{array}$ & pauschal & 500 & 500 & 500 \\
\hline Insgesamt & & 4.804 & 7.560 & 11.072 \\
\hline
\end{tabular}

Quelle: Einkommensteuergesetz Stand 1990, Slomma (1990), S. 29 ff., o.V. (1995), eigene Berechnungen

Der größte Posten der Umzugskosten ist die Beförderung des Hausrats. Die Kosten für die Spedition wurden geschätzt anhand einer Preiserhebung der Stiftung Warentest (1995) für einen Umzug über eine Distanz von $400 \mathrm{~km}$. Dabei wurde u.a. ein Umzugsvolumen unterstellt, daß einer Zwei-Zimmer-Wohnung entspricht, das Transportvolumen umfaßte sämtliche Einrichtungsgegenstände sowie 40 Umzugskartons und 4 Kleiderbehälter. Die mittleren Kosten betragen je nach Obergeschoß und Service zwischen 4.276 DM und 5.395 DM (für einen Nahumzug über $50 \mathrm{~km}$ lagen die Preise zwischen $2.204 \mathrm{DM}$ und $3.366 \mathrm{DM})^{214}$. Für die Ermittlung der Migrationsüberschüsse dürfte es angemessen sein, Um-

${ }^{214}$ Vgl. o.V. (1995), S. $28 f$. 
zugskosten mit Packservice und Auf- und Abbau zugrunde zu legen. Auch wenn diese Arbeiten vom Migranten selbst durchgeführt werden, wäre diese Tätigkeit als Opportunitätskosten zu berücksichtigen ${ }^{215}$. Die Bandbreite der Kosten bei einem Service durch die Umzugsunternehmen liegt zwischen 5.113 DM und 5.395 DM. Da die Angaben aus dem Jahr 1995 stammen, der relevante Umzug aber am Jahresende 1990 stattfinden sollte, und davon auszugehen ist, daß die Preise zu dieser Zeit niedriger waren, werden die Preise abgerundet auf 4.500 DM. Für die nähere bzw. weitere Entfernung wird eine Pauschale von 2.000 DM abgezogen bzw. zugeschlagen. (Dies entspricht in etwa dem Unterschied zwischen den Preisen gemäß Stiftung Warentest von 1995 für Nahumzug gegenüber dem Umzug über die längere Distanz).

Für den Umzug über $100 \mathrm{~km}$ hätte angenommen werden können, daß keine Übernachtungen anfielen, sondern die betreffende Person abends wieder nach Hause fährt. Die Kosten für die Rückfahrt und Wiederkehr entsprechen jedoch in etwa den Übernachtungskosten, so daß sich an der Summe nichts geändert hätte.

Die Umzugskosten sind auf Basis eines solchen Ansatzes mit rund 7.500 DM anzusetzen, wenn die Entfernung $400 \mathrm{~km}$ beträgt, ein Speditionsunternehmen mit dem Umzug beauftragt wird und alle Posten gemäß steuerlichen Regelungen berücksichtigt werden. Die tatsächlichen Umzugskosten können deutlich von den errechneten Kosten abweichen. Wenn der Umzug wesentlich mehr kostet, ist es fraglich, ob diese Kosten unvermeidlich sind und ob sie wirklich in der entstandenen Höhe in die Migrationsrechnung eingehen sollten ${ }^{216}$. Wird der Umzug hingegen selbst durchgeführt, entstehen zwar weniger Ausgaben, aber höhere Opportunitätskosten.

\subsection{Psychische Kosten}

Die Abschätzung der psychischen Kosten dürfte von allen hier quantifizierten Kosten- und Ertragsarten die spekulativste sein. Um diesen Verlust monetär zu bewerten, wird die in Kapitel 3.1.2.8 erwähnte Reisekostenmethode verwendet. Dabei wird angenommen, daß der Migrant in der Regel die Trennung von seinen Freunden und Verwandten als Verlust empfindet.

${ }^{215}$ Es stellt sich dann aber die Frage der Angemessenheit einer Bewertung in Höhe der Geldbeträge, die professionelle Unternehmen fordern.

${ }^{216}$ So können die Übernachtungskosten unter Umständen sehr viel höher sein, dies könnte aber ein persönlicher Luxus sein, der nicht mit dem Umzug zusammenhängt, sondern darüber hinaus einen Nutzen stiftet. 
In der folgenden Modellrechnung wird davon ausgegangen, daß sich die Migranten nach etwa 5 Jahren so gut eingelebt haben, daß sie sich im neuen Wohnort ebenso wohl fühlen wie in der alten Umgebung, und zwar in dem Sinne, daß durch den zusätzlichen Nutzen des neuen Bekanntenkreises die lockerer werdenden Kontakte in der Heimat ausglichen werden. Dabei wird angenommen, daß die psychischen Kosten im ersten Jahr am höchsten sind, da die Umgebung noch fremd ist und sich neue Freundschaften noch nicht gefestigt haben. Daher werden für das erste Jahr wöchentliche Heimfahrten veranschlagt, die gewährleisten sollen, daß sich der Migrant psychisch so wohl fühlt wie ohne Umzug, so daß die Zahl der notwendigen Heimfahrten im ersten Jahr 46 betrage (in der Urlaubszeit sollen keine Kosten anfallen). Im zweiten Jahr seien Heimfahrten nur noch alle zwei Wochen nötig, im dritten alle drei, im vierten Jahr alle vier und im fünften Jahr alle acht Wochen.

Es werden nur die reinen Fahrtkosten berechnet und auf den Ausweis von Opportunitätskosten (für die Fahrtzeit) verzichtet. Die Höhe der Fahrtkosten ist abhängig von dem benutzten Verkehrsmittel, der Distanz und der Zeitpräferenzrate. Bei der Bundesbahn kostete der einfache Kilometer im Jahr 1990 etwa 20 Pfennig (in den neuen Bundesländern etwas weniger). Die Kosten bei Pkw-Benutzung beliefen sich auf ca. 42 Pfennig pro Entfernungskilometer ${ }^{217}$. Die Kosten für einen Fahrtkilometer waren für Bahn und PKW somit in etwa identisch. In die Rechnung gehen die Kosten für Heimfahrten mit einem PKW ein. Mögliche Ersparnisse durch Sondertarife für längere Strecken wie das Sparticket bei der Bahn oder die Möglichkeit von Fahrgemeinschaften bei Benutzung eines Pkws werden zur Vereinfachung nicht berücksichtigt. Bezüglich der Entfernungen werden die gleichen Distanzen wie bei der Schätzung der Umzugskosten untersucht.

Aufgrund der unterstellten notwendigen Besuchsfrequenz nehmen die Kosten Jahr für Jahr deutlich ab. Tabelle 21 zeigt die Entwicklung dieser Kosten.

Bei einer Entfernung von $100 \mathrm{~km}$ betragen die psychischen Kosten rund 4.000 $\mathrm{DM}$, für jede weiteren $100 \mathrm{~km}$ nehmen die Kosten jeweils um diesen Betrag zu, so daß sie sich bei einer Entfernung von $1.000 \mathrm{~km}$ auf rund $40.000 \mathrm{DM}$ belaufen.

Unter bestimmten Bedingungen können (tatsächlich realisierte) Kosten für Heimfahrten steuerlich geltend gemacht werden. Netto wären die Kosten dann geringer als in Tabelle 21 ausgewiesen. Bei ledigen Migranten erkennt aber das Finanzamt in der Regel diese Kosten nicht an. Die Kosten können von Ledigen

${ }^{217}$ In Anlehnung an die Absetzbarkeit von Kosten für Heimfahrten bei doppelter Haushaltsführung gemäß Einkommensteuergesetz. 
nur in bestimmten Fällen abgesetzt werden, etwa wenn der Umzug beruflich bedingt und nur vorübergehend ist. Angenommen wird im folgenden, daß die Kosten nicht abgesetzt werden können und damit in voller Höhe vom Nettoeinkommen abzuziehen sind.

Tabelle 21: Psychische Kosten einer Migration bewertet mit der Reisekostenmethode

- Angaben in DM -

\begin{tabular}{|l|r|r|r|r|r|r|}
\hline & \multicolumn{3}{|c|}{$\mathrm{r}=0,015$} & \multicolumn{3}{c|}{$\mathrm{r}=0,05$} \\
\hline Jahr & $100 \mathrm{~km}$ & $400 \mathrm{~km}$ & $1000 \mathrm{~km}$ & $100 \mathrm{~km}$ & $400 \mathrm{~km}$ & $1000 \mathrm{~km}$ \\
\hline 1991 & 1.903 & 7.614 & 19.034 & 1.840 & 7.360 & 18.400 \\
\hline 1992 & 938 & 3.751 & 9.377 & 876 & 3.505 & 8.762 \\
\hline 1993 & 616 & 2.463 & 6.159 & 556 & 2.225 & 5.563 \\
\hline 1994 & 455 & 1.820 & 4.551 & 397 & 1.589 & 3.974 \\
\hline 1995 & 224 & 897 & 2.242 & 189 & 757 & 1.892 \\
\hline insges. & 4.136 & 16.545 & 41.362 & 3.859 & 15.436 & 38.591 \\
\hline
\end{tabular}

Quelle: Eigene Berechnungen

\subsection{Arbeitsplatzabhängige Kosten und Erträge}

\subsection{Differenzen der regionalen Jahresarbeitszeiten}

Bisher wurde nicht berücksichtigt, daß sich die Wochenarbeitszeiten und die Länge des Urlaubs in den alten und neuen Bundesländern unterscheiden. Um diese Unterschiede zu bewerten, wird in diesem Abschnitt ein Ausgleichsbetrag in Abhängigkeit vom Bruttomonatslohn errechnet.

Die Regelungen zur Wochenarbeitszeit sind allerdings nicht nur zwischen alten und neuen Bundesländern, sondern auch in den einzelnen Branchen und Tarifbezirken unterschiedlich. Es wird aber für alle Beschäftigten die gleiche Abweichung zwischen Ost- und Westarbeitszeiten unterstellt, da die Ergebnisse durch Berücksichtigung aller Einzelregelungen den Mehraufwand wohl nicht gerechtfertigt hätten. Das Statistische Bundesamt weist für die Industriearbeiter $1992 \mathrm{im}$ Osten eine durchschnittliche Wochenarbeitszeit von 40,9 Stunden und im Westen von 39,0 Stunden aus ${ }^{218}$. 1993 betrug sie 41,2 Stunden im Osten und 38,0 Stunden im Westen. Die Differenz hat sich also nicht abgebaut, sondern ist sogar noch gestiegen (im Handwerk allerdings blieb die Differenz von knapp zwei

${ }^{218}$ Vgl. Statistisches Bundesamt (1994) S. 24. 
Stunden bestehen). Für das Jahr 1991 lagen der Verfasserin solche Durchschnittswerte nicht vor. Die Übersicht des WSI über die Tarifregelungen der Branchen und Tarifgebiete in $1991^{219}$ vermittelt den Eindruck, daß sich die Wochenarbeitszeiten 1991 durchschnittlich um 2 bis 3 Stunden unterschieden. Bei einer wöchentlichen Arbeitszeit von 40 Stunden machen 2 Stunden einen circa 5\%igen Unterschied in der Wochenarbeitszeit aus. Werte für 1994 und später lagen der Verfasserin zum Zeitpunkt der Bearbeitung nicht vor.

Der den Arbeitnehmern zustehende Urlaub war in den neuen Ländern kürzer als in den alten ${ }^{220} .1991$ betrug der Unterschied bis zu 10 Tage, im Durchschnitt 4 Tage. 1992 baute sich die Spanne etwas ab, der Unterschied betrug maximal 6 Tage und im Durchschnitt 2 Tage. 1993 waren die Unterschiede ähnlich wie im Vorjahr. Wird im Jahr effektiv an 220 Tagen gearbeitet, dann sind 2 Tage knapp $1 \%$ der Arbeitszeit. Für 1991 wird daher der erwartbare Westlohn um $2 \%$, für 1992 und 1993 um $1 \%$ erhöht.

Über die zukünftige Entwicklung der Arbeitszeit kann nur spekuliert werden, sicher scheint jedoch, daß die Gewerkschaften gleiche Arbeitszeiten in Ost- und Westdeutschland anstreben. Für die Berechnung wird daher angenommen, daß sich die 1993 noch bestehende Differenz der Wochenarbeitszeit und des Urlaub von $6 \%$ in jedem Jahr um $1 \%$-Punkt abbaut. Die Jahresarbeitszeiten würden sich dann 1999 nicht mehr voneinander unterscheiden, wie Tabelle 22 zeigt:

Tabelle 22: Abweichung der ostdeutschen Jahresarbeitszeit von der westdeutschen

\begin{tabular}{|c|c|c|c|c|c|c|c|c|c|}
\hline Jahr & $1991^{*}$ & $1992^{*}$ & $1993^{*}$ & $1994^{* *}$ & $1995^{* *}$ & $1996^{* *}$ & $1997^{* *}$ & $1998^{* *}$ & $1999^{* *}$ \\
\hline Abw. in \% & 7 & 6 & 6 & 5 & 4 & 3 & 2 & 1 & 0 \\
\hline
\end{tabular}

* Schätzung, ** Prognose

Quelle: Statistisches Bundesamt (1994), Bispinick u.a. (1992), dies. (1993), dies. (1994), eigene Berechnung

Die Abweichungen werden nun monetär bewertet, und zwar zunächst für Jahre 1991 bis 1994. Dafür wird das jeweilige Arbeitseinkommen in Westdeutschland mit der prozentualen Abweichung bewertet, so daß sich das Bruttomehreinkommen ergibt. Ferner wird bestimmt, wie hoch das zusätzliche Einkommen netto, d.h. nach Steuern und Sozialabgaben, gewesen wäre. Die Ergebnisse sind differenziert nach Branchen in Tabelle 23 aufgeführt. Die Differenzen sind nicht unerheblich, sie betragen netto zwischen 4.000 und 7.000 DM.

${ }^{219} \mathrm{Vgl}$. Bispinck u.a. (1992), S. 131.

${ }^{220}$ zu den Urlaubsregelungen vgl. Bispinck u.a. (1992), dies. (1993) und dies. (1994). 
Tabelle 23: Monetäre Bewertung der geringeren Arbeitszeiten in den alten Ländern 1991-1994 nach Branchen

- Angaben in DM -

\begin{tabular}{|l|r|r|r|r|}
\hline & \multicolumn{2}{|c|}{$\mathrm{r}=0,015$} & \multicolumn{2}{c|}{$\mathrm{r}=0,05$} \\
\hline Branche & $\begin{array}{l}\text { Bruttoein- } \\
\text { kommens- } \\
\text { differenz }\end{array}$ & $\begin{array}{l}\text { Nettoein- } \\
\text { kommens- } \\
\text { differenz }\end{array}$ & $\begin{array}{l}\text { Bruttoein- } \\
\text { kommens- } \\
\text { differenz }\end{array}$ & $\begin{array}{l}\text { Nettoein- } \\
\text { kommens- } \\
\text { differenz }\end{array}$ \\
\hline Land- und Forstwirtschaft & 8.326 & 4.529 & 7.680 & 4.180 \\
\hline Bergbau & 14.260 & 7.225 & 13.160 & 6.682 \\
\hline Energiewirtschaft & 13.093 & 6.302 & 12.075 & 5.817 \\
\hline Verarbeitendes Gewerbe & 12.421 & 6.094 & 11.455 & 5.625 \\
\hline Baugewerbe & 10.694 & 5.501 & 9.862 & 5.076 \\
\hline Handel & 9.801 & 5.163 & 9.040 & 4.765 \\
\hline Verkehr & 11.330 & 5.730 & 10.452 & 5.290 \\
\hline Dienstleistungen & 10.052 & 5.261 & 9.274 & 4.857 \\
\hline alle Branchen & 10.909 & 5.581 & 10.064 & 5.152 \\
\hline
\end{tabular}

Quelle: Statistisches Bundesamt (1994), Bispinick u.a. (1992), dies. (1993), dies. (1994), DIW (1991-1994), eigene Berechnungen

In der Industrie ist der Ertrag etwas höher als im Durchschnitt aller Branchen, die Differenzen betragen netto zwischen 4.000 DM und 8.000 DM. Besonders hoch ist die Bedeutung der geringeren Arbeitszeiten in den alten Ländern für die männlichen Angestellten, da ihr Lohneinkommen absolut das höchste und die Lohnangleichung verglichen mit den anderen Gruppen am wenigsten weit fortgeschritten war.

Tabelle 24: Monetäre Bewertung der geringeren Arbeitszeiten in den alten Ländern 1991-1994 nach Status und Geschlecht

- Angaben in DM -

\begin{tabular}{|c|c|c|c|c|}
\hline & \multicolumn{2}{|c|}{$\mathrm{r}=0,015$} & \multicolumn{2}{|c|}{$\mathrm{r}=0,05$} \\
\hline Branche & \begin{tabular}{|l|}
$\begin{array}{l}\text { Bruttoaus- } \\
\text { gleich }\end{array}$ \\
\end{tabular} & $\begin{array}{l}\text { Nettoaus- } \\
\text { gleich }\end{array}$ & \begin{tabular}{|l|}
$\begin{array}{l}\text { Bruttoaus- } \\
\text { gleich }\end{array}$ \\
\end{tabular} & \begin{tabular}{|l|}
$\begin{array}{l}\text { Nettoaus- } \\
\text { gleich }\end{array}$ \\
\end{tabular} \\
\hline Arbeiter & 10.737 & 5.519 & 9.906 & 5.095 \\
\hline Angestellte & 15.576 & 7.971 & 14.367 & 7.357 \\
\hline Arbeiter, männlich & 11.279 & 5.713 & 10.407 & 5.275 \\
\hline Angestellte, männlich & 17.049 & 8.379 & 15.727 & 7.735 \\
\hline Arbeiter, weiblich & 8.003 & 4.266 & 7.383 & 3.942 \\
\hline Angestellte, weiblich & 11.523 & 5.796 & 10.627 & 5.349 \\
\hline
\end{tabular}

Quelle: Bispinick u.a. (1992), dies. (1993), dies. (1994), Statistisches Bundesamt (1994), eigene Berechnungen 
Die geringste Bedeutung hat es für die Arbeiterinnen. Sie verdienen absolut am wenigsten und die regionale Lohnangleichung war am höchsten. In etwa die gleiche Bedeutung ergibt sich für die weiblichen Angestellten und die männlichen Arbeiter, deren absolutes Gehalt und Abstand zum Westlohn sehr ähnlich waren.Diese Zahlungen müssen noch um einen Ausgleich für die Zeit nach 1994 ergänzt werden. Ab 1995 sind auch die Arbeitseinkommen nur noch geschätzt, ebenso wie die Arbeitszeitdifferenzen. Auf eine differenzierte Rechnung wird daher verzichtet und ein identischer pauschaler Zuschlag für alle Arbeitnehmer auf Basis des durchschnittlichen Arbeitseinkommens vorgenommen. Brutto beträgt der sich ergebende Zuschlag 4.848 DM (3.956 $\mathrm{DM})$ und netto $2.282 \mathrm{DM}(1.862 \mathrm{DM})$. Dies sind $44 \%$ bzw. gut $40 \%$ des Zuschlags für den Zeitraum 1991-1994. Die Zuschläge gemäß Tabelle 23 und Tabelle 24 werden jeweils um diesen Prozentsatz erhöht. Der Zuschlag für einen Arbeiter etwa beträgt brutto $15.461 \mathrm{DM}$ und netto $7.726 \mathrm{DM}$.

Welcher Anpassungszeitraum bis zum regionalen Lohnausgleich unterstellt wird, ist für die Höhe des Zuschlags unerheblich, da sich die Rechnung nur auf die Einkommen in Westdeutschland beziehen und unterstellt wird, daß Lohnanpassung und Arbeitszeitanpassung nicht miteinander korreliert sind.

\subsection{Verschlechterung der beruflichen Stellung}

Einige Übersiedler konnten in der Bundesrepublik nicht sofort einen ihrer bisherigen beruflichen Stellung entsprechenden Arbeitsplatz finden und haben zunächst einen Arbeitsplatz angenommen, der eine geringere Qualifikation erforderte. Aufgrund des engen Arbeitsmarktes in den alten Ländern und der Tatsache, daß die in der DDR erworbenen Erfahrungen und Fähigkeiten zum Teil in den alten Ländern nicht nachgefragt wurden (sie aber in den neuen Ländern noch entsprechend ihrer Qualifikation beschäftigt wurden), war diese Alternative nicht selten. So ergab etwa eine Umfrage aus der Jahreswende 1989/1990 bei Übersiedlern, daß $40 \%$ der Befragten einer Tätigkeit nachgingen, die nur zum Teil bzw. überhaupt nicht ihrer Qualifikation entsprach ${ }^{221}$ :

${ }^{221}$ Die Befragten könnten allerdings ihre Qualifikation falsch beurteilt und insbesondere die qualifikatorische Bedeutung von Erfahrungen auf dem konkreten Arbeitsplatz nur unangemessen berücksichtigt haben. 
Tabelle 25: Umfrageergebnis: Inwieweit entspricht der Arbeitsplatz der Qualifikation der Übersiedler?

- Angaben in \% -

\begin{tabular}{|l|r|r|r|}
\hline & \multicolumn{1}{|c|}{ Gesamt } & \multicolumn{1}{|c|}{ Männer } & \multicolumn{1}{|c|}{ Frauen } \\
\hline voll & 38 & 40 & 35 \\
\hline weitgehend & 22 & 21 & 24 \\
\hline nur zum Teil & 18 & 18 & 17 \\
\hline überhaupt nicht & 22 & 21 & 23 \\
\hline
\end{tabular}

Quelle: Umfrage des Zentralinstituts für Jugendforschung, zitiert nach Köhler (1990), S. 429.

Gibt es eine positive Korrelation zwischen Qualifikation und Lohn, so würde jemand, der eine nicht seiner Qualifikation entsprechende Stelle annimmt, schlechter entlohnt werden, als wenn er qualifikationsadäquat beschäftigt ist ${ }^{222}$. Wenn nur in den alten Ländern die Gefahr besteht keinen adäquaten Arbeitsplatz zu finden, so muß dies in Form von Kosten in die Migrationsrechnung eingehen $^{223}$.

Wie hoch diese Kosten sind, ist wiederum recht spekulativ. Entspricht der neue Arbeitsplatz voll der bisherigen beruflichen Stellung muß kein Abschlag, entspricht er weitgehend der bisherigen Stellung muß nur ein geringer Abschlag vom erwartbaren Lohneinkommen vorgenommen werden. Entspricht der Arbeitsplatz der Qualifikation überhaupt nicht, kann der Abschlag dagegen recht hoch sein.

Als Berechnungsbasis für den Abschlag werden die Einkommen der westdeutschen Beschäftigten herangezogen. Grundlage der Schätzung bilden Lohneinkommensdaten der Industriearbeiter gesplittet nach Qualifikation. 1993 verdiente ein Arbeiter in der Leistungsgruppe 1 (Facharbeiter) brutto 4.340 DM monatlich, in der Leistungsgruppe 2 (angelernte Arbeiter) $3.691 \mathrm{DM}$ und in der Leistungsgruppe 3 (Hilfsarbeiter) $3.179 \mathrm{DM}^{224}$. Ein Facharbeiter, der nur eine Beschäftigung in Leistungsgruppe 2 fände, müßte daher einen um $15 \%$ niedrigeren Lohn hinnehmen, als wenn er gemäß seiner formalen Qualifikation angestellt wird. In Leistungsgruppe 3 betrüge die Lohneinbuße sogar 27\%. (In der

${ }^{222}$ Es dürfte angemessen sein, davon auszugehen, daß ein „nicht der Qualifikation entsprechend" Beschäftigter nicht überqualifiziert, sondern unterqualifiziert eingesetzt wird.

${ }^{223}$ Damit wird implizit unterstellt, daß die Arbeitnehmer in den neuen Ländern gemäß ihrer Qualifikation arbeiten. Dies mag für die Zeit vor der Wende gegolten haben. Später wurden viele Arbeitnehmer entlassen und mußten sich eine neue Arbeitsstelle suchen. Die neue Arbeitsstelle muß nicht immer adäquat gewesen sein. Aus diesem Grund ist die Berechnung des hier vorgenommen Abschlags nicht unproblematisch.

${ }^{224}$ Vgl. Statistisches Bundesamt (1994). 
Gruppe der Angestellten fällt die Differenz noch höher aus, der Unterschied zwischen Leistungsgruppe II und Leistungsgruppe IV beträgt fast $50 \%$.)

Ferner wird angenommen, daß innerhalb des ersten Jahres nach der Migration eine adäquate Stelle gefunden wird, die zu Beginn des Jahres 1992 angetreten wird. Bei Verallgemeinerung der Umfrageergebnisse gemäß Tabelle 25 ergibt sich, daß mit einer Wahrscheinlichkeit von $38 \%$ kein Abschlag vorzunehmen ist und mit 22\%iger Wahrscheinlichkeit (Arbeitsplatz ist weitgehend der Qualifikation entsprechend) der Lohnabzug nur gering ist (Annahme Bruttolohnabzug 5\%). Der Abschlag für ,nur zum Teil“" wird mit dem Unterschied einer Leistungsklasse bewertet (Lohnabzug 15\%) und ein Abschlag in Höhe von zwei Leistungsklassen für „überhaupt nicht“ (Lohnabzug 27\% mit einer Wahrscheinlichkeit von $22 \%$ ).

Das erwartbare Bruttoarbeitseinkommen eines durchschnittlichen Beschäftigten aus Ostdeutschland in Westdeutschland 1991 berechnet sich unter diesen Annahmen wie folgt:

$$
\begin{aligned}
\mathrm{E}\left(\mathrm{y}_{91}\right)= & 0,38 * \mathrm{BL}_{91}+0,22 *(1-0,05) * \mathrm{BL}_{91}+0,18 *(1-0,15) * \mathrm{BL}_{91}+ \\
& 0,22 *(1-0,27) * \mathrm{BL}_{91}
\end{aligned}
$$

mit

$\mathrm{E}\left(\mathrm{y}_{9_{1}}\right)=$ Erwartungswert des Bruttoeinkommens eines Ostdeutschen in Westdeutschland in 1991

$\mathrm{BL}_{91}=$ durchschnittlicher Bruttolohn eines Beschäftigten in Westdeutschland 1991

Bei Zugrundelegung der tatsächlichen Lohneinkommen von 1991 und Berücksichtigung der Möglichkeit einer Verschlechterung der beruflichen Stellung ergeben sich Einkommensdifferenzen, die um die in Tabelle 26 bzw. in Tabelle 27 ausgewiesenen Beträge niedriger wären als ohne die Einbeziehung dieser Möglichkeit.

Die Abschläge, die sich infolge der - annahmegemäß - vorübergehenden Verschlechterung der beruflichen Stellung ergeben, sind relativ gering. Die Spanne liegt netto zwischen 1.700 DM und 2.800 DM nach Branchen und zwischen $1.700 \mathrm{DM}$ und 3.500 DM nach Status und Geschlecht. Durchschnittlich beträgt der Abschlag pro Beschäftigten 4.000 DM brutto, bzw. gut 2.000 DM netto. 
Tabelle 26: Monetäre Bewertung der Verschlechterung der beruflichen Stellung 1991-1994 nach Branchen

- Angaben in DM -

\begin{tabular}{|l|r|r|r|r|}
\hline & \multicolumn{2}{|c|}{$\mathrm{r}=0,015$} & \multicolumn{2}{c|}{$\mathrm{r}=0,05$} \\
\hline Branche & $\begin{array}{l}\text { Bruttoein- } \\
\text { kommens- } \\
\text { differenz }\end{array}$ & $\begin{array}{l}\text { Nettoein- } \\
\text { kommens- } \\
\text { differenz }\end{array}$ & $\begin{array}{l}\text { Bruttoein- } \\
\text { kommens- } \\
\text { differenz }\end{array}$ & $\begin{array}{l}\text { Nettoein- } \\
\text { kommens- } \\
\text { differenz }\end{array}$ \\
\hline Land- und Forstwirtschaft & -3.272 & -1.790 & -3.163 & -1.730 \\
\hline Bergbau & -5.581 & -2.789 & -5.395 & -2.696 \\
\hline Energiewirtschaft & -5.082 & -2.616 & -4.913 & -2.529 \\
\hline Verarbeitendes Gewerbe & -4.832 & -2.524 & -4.670 & -2.439 \\
\hline Baugewerbe & -4.128 & -2.244 & -3.991 & -2.169 \\
\hline Handel & -3.819 & -2.111 & -3.692 & -2.041 \\
\hline Verkehr & -4.436 & -2.370 & -4.288 & -2.291 \\
\hline Dienstleistungen & -3.938 & -2.163 & -3.806 & -2.091 \\
\hline alle Branchen & -4.271 & -2.303 & -4.129 & -2.226 \\
\hline
\end{tabular}

Quelle: DIW (1991-1994), Umfrage des Zentralinstituts für Jugendforschung, zitiert nach

Köhler (1990), S. 429, eigene Berechnungen

Tabelle 27: Monetäre Bewertung der Verschlechterung der beruflichen Stellung 1991-1994 nach Status und Geschlecht

- Angaben in DM -

\begin{tabular}{|l|r|r|r|r|}
\hline & \multicolumn{2}{|c|}{$\mathrm{r}=0,015$} & \multicolumn{2}{c|}{$\mathrm{r}=0,05$} \\
\hline Branche & $\begin{array}{l}\text { lruttoaus- } \\
\text { gleich }\end{array}$ & $\begin{array}{l}\text { Nettoaus- } \\
\text { gleich }\end{array}$ & $\begin{array}{l}\text { Bruttoaus- } \\
\text { gleich }\end{array}$ & $\begin{array}{l}\text { Nettoaus- } \\
\text { gleich }\end{array}$ \\
\hline Arbeiter & -4.228 & -2.285 & -4.087 & -2.209 \\
\hline Angestellte & -6.076 & -3.235 & -5.873 & -3.127 \\
\hline Arbeiter, männlich & -4.449 & -2.375 & -4.300 & -2.296 \\
\hline Angestellte, männl. & -6.666 & -3.520 & -6.444 & -3.403 \\
\hline Arbeiter, weiblich & -3.154 & -1.717 & -3.049 & -1.660 \\
\hline Angestellte, weibl. & -4.470 & -2.383 & -4.321 & -2.304 \\
\hline
\end{tabular}

Quelle: Umfrage des Zentralinstituts für Jugendforschung, zitiert nach Köhler (1990), S. 429, Statistisches Bundesamt (1994), eigene Berechnungen

Bei der Bestimmung des Nettoabschlags wurde die Einkommensteuerschuld so berücksichtigt, daß zunächst der Bruttoerwartungswert der Einkommen berechnet wurde und auf dieser Grundlage die Steuern berechnet wurden. Eigentlich hätten - wegen der Progression des Einkommensteuertarifs - für die verschiedenen Einzelkomponenten die Steuern berechnet werden müssen. Ein derartiges Vorgehen wäre aber wesentlich aufwendiger gewesen und die geringen Abweichungen, die wohl zu erwarten sind, dürften den Aufwand nicht rechtfertigen. 


\subsection{Zusammenfassung}

Die erwartbaren Migrationserträge sind aufgrund der Mehrkosten bei Neuanmietung einer Wohnung im Vergleich zu einer Bestandsmiete um 2.525 DM ( $\mathrm{r}=$ $0,015)$ bzw. 2.286 DM $(r=0,05)$ zu vermindern.

In Abhängigkeit von der Entfernung ergeben sich ausschließlich Kosten, die den Migrationsertrag um bis zu $50.000 \mathrm{DM}$ verringern, wie Tabelle 28 zeigt:

Tabelle 28: Zusammenfassung der entfernungsabhängigen Kosten

\begin{tabular}{|l|r|r|r|r|r|r|}
\hline & \multicolumn{2}{|c|}{$100 \mathrm{~km}$} & \multicolumn{2}{c|}{$400 \mathrm{~km}$} & \multicolumn{2}{c|}{$1000 \mathrm{~km}$} \\
\hline & $\mathrm{r}=0,015$ & $\mathrm{r}=0,05$ & $\mathrm{r}=0,015$ & $\mathrm{r}=0,05$ & $\mathrm{r}=0,015$ & $\mathrm{r}=0,05$ \\
\hline Umzug & -4.804 & -4.804 & -7.560 & -7.560 & -11.072 & -11.072 \\
\hline psy. Kosten & -4.136 & -3.859 & -16.545 & -15.436 & -41.362 & -38.591 \\
\hline insgesamt & -8.940 & -8.663 & -24.105 & -22.996 & -52.434 & -49.663 \\
\hline
\end{tabular}

Quelle: Tabelle 20 und Tabelle 21, eigene Berechnung

Für die dritte Kategorie ergeben sich zum einen Erträge durch die geringere Arbeitszeit in Westdeutschland, zum anderen Kosten bei einer Verschlechterung der beruflichen Position nach dem Umzug. Saldiert ergeben sich gesplittet nach Branchen Erträge, wie sie in Tabelle 29 ausgewiesen sind.

Tabelle 29: Zusammenfassung der Kosten und Erträge durch Verschlechterung der beruflichen Stellung und Arbeitszeitdifferenzen nach Branchen

- Angaben in DM -

\begin{tabular}{|l|r|r|r|r|}
\hline & \multicolumn{2}{|c|}{$\mathrm{r}=0,015$} & \multicolumn{2}{c|}{$\mathrm{r}=0,05$} \\
\hline Branche & $\begin{array}{l}\text { Bruttoein- } \\
\text { kommens- } \\
\text { differenz }\end{array}$ & $\begin{array}{l}\text { Nettoein- } \\
\text { kommens- } \\
\text { differenz }\end{array}$ & $\begin{array}{l}\text { Bruttoein- } \\
\text { kommens- } \\
\text { differenz }\end{array}$ & $\begin{array}{l}\text { Nettoein- } \\
\text { kommens- } \\
\text { differenz }\end{array}$ \\
\hline Land- und Forstwirtschaft & 8.717 & 4.551 & 7.896 & 4.122 \\
\hline Bergbau & 14.953 & 7.326 & 13.555 & 6.659 \\
\hline Energiewirtschaft & 13.772 & 6.207 & 12.475 & 5.615 \\
\hline Verarbeitendes Gewerbe & 13.054 & 6.008 & 11.825 & 5.436 \\
\hline Baugewerbe & 11.271 & 5.457 & 10.210 & 4.937 \\
\hline Handel & 10.294 & 5.117 & 9.326 & 4.630 \\
\hline Verkehr & 11.879 & 5.652 & 10.763 & 5.115 \\
\hline Dienstleistungen & 10.537 & 5.202 & 9.549 & 4.709 \\
\hline alle Branchen & 11.438 & 5.510 & 10.363 & 4.987 \\
\hline
\end{tabular}

Quelle: Tabelle 22 und Tabelle 25, eigene Berechnung

Für einen durchschnittlichen Arbeitnehmer ergibt sich ein Überschuß von rund 11.000 DM brutto und rund 5.000 DM netto. Brutto betragen die Differenzen 
zwischen den Branchen bis zu 6.000 DM, netto schrumpfen sie bis auf höchstens $3.000 \mathrm{DM}$ zusammen. Im Bergbau ist der zusätzliche Ertrag am höchsten, in der Land- und Forstwirtschaft am geringsten.

Für die Beschäftigten in der Industrie ergeben sich folgende, inTabelle 30 ausgewiesene, Ertragszuwächse:

Tabelle 30: Zusammenfassung der Kosten und Erträge durch Verschlechterung der beruflichen Stellung und Arbeitszeitdifferenzen nach Status und Geschlecht - Angaben in DM -

\begin{tabular}{|l|r|r|r|r|}
\hline & \multicolumn{2}{|c|}{$r=0,015$} & \multicolumn{2}{c|}{$r=0,05$} \\
\hline & $\begin{array}{l}\text { Bruttoein- } \\
\text { kommens- } \\
\text { differenz }\end{array}$ & $\begin{array}{l}\text { Nettoein- } \\
\text { kommens- } \\
\text { differenz }\end{array}$ & $\begin{array}{l}\text { Bruttoein- } \\
\text { kommens- } \\
\text { differenz }\end{array}$ & $\begin{array}{l}\text { Nettoein- } \\
\text { kommens- } \\
\text { differenz }\end{array}$ \\
\hline Arbeiter & 11.233 & 5.442 & 10.178 & 4.924 \\
\hline Angestellte & 16.353 & 7.924 & 14.815 & 7.173 \\
\hline Arbeiter, männlich & 11.793 & 5.623 & 10.686 & 5.089 \\
\hline Angestellte, männlich & 17.885 & 8.211 & 16.203 & 7.426 \\
\hline Arbeiter, weiblich & 8.370 & 4.255 & 7.583 & 3.859 \\
\hline Angestellte, weiblich & 12.123 & 5.731 & 10.982 & 5.185 \\
\hline
\end{tabular}

Quelle: Tabelle 22 und Tabelle 25, eigene Berechnung

Sowohl für die weiblichen als auch die männlichen Arbeiter und Angestellten wäre ein Überschuß der Erträge über die Kosten der dritten Kategorie erwartbar gewesen. Der Nettoüberschuß der Angestellten übertrifft den der Arbeiter um über 2.000 DM. Die geschlechtsspezifische Differenz ist bei den Angestellten höher als bei den Arbeitern.

Bei Zusammenfassung aller drei Kategorien von „weiteren“ Kosten und Erträgen überwiegen die Kosten, wie Abbildung 33 zeigt.

Die niedrigsten Kosten entstehen bei einem Umzug in die nähere Umgebung, die höchsten bei einem Fernumzug. Die Höhe der weiteren Kosten und Erträge wird dominiert durch die psychischen Kosten. Die Ermittlung der psychischen Kosten ist jedoch - wie bereits erläutert - sehr spekulativ. Da aber in Befragungen der Faktor „Freunde und Verwandte vor Ort" immer wieder als der wichtigste Faktor im Wanderungskalkül angegeben wird, muß auch seine monetäre Bewertung relativ hoch ausfallen. Ohne Berücksichtigung der psychischen Kosten würden die weiteren Kosten die weiteren Erträge aber ebenfalls übersteigen. Die Erträge aus Lohn- und Mietpreisunterschieden, die in Abschnitt 3.2.1 ermittelt wurden, spiegeln somit in jedem Fall einen zu hohen Migrationsertrag wider. 
Abbildung 33: Zusammenfassung der weiteren Migrationskosten und -erträge

- Zeitpräferenzrate 0,015 -

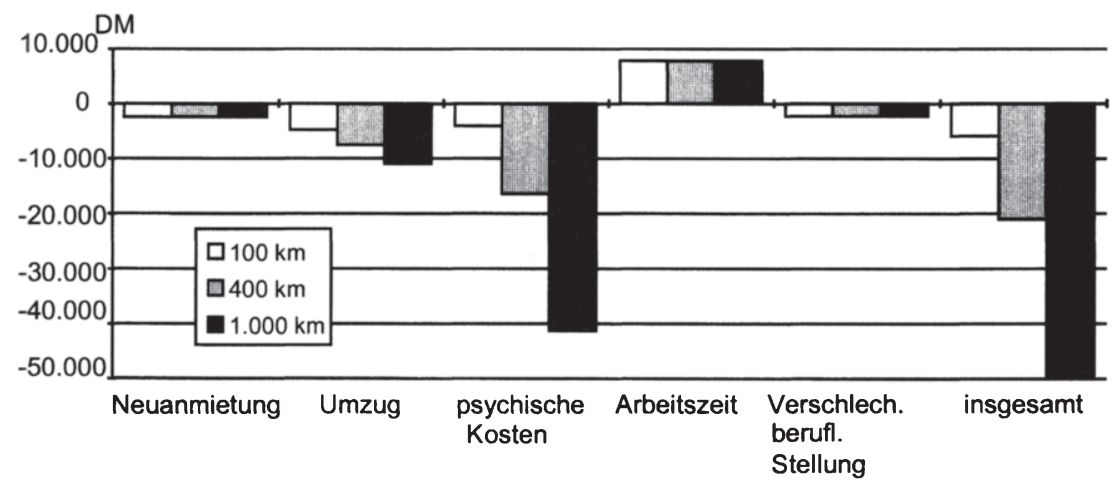

Quelle: Tabelle 19, Tabelle 20, Tabelle 21, Tabelle 23, Tabelle 26

Für die Beschäftigten der verschiedenen Branchen ergeben sich aus der Berücksichtigung der weiteren Migrationskosten und -erträge Abzüge beim erwartbaren Migrationsertrag gemäß Tabelle 31 .

Tabelle 31: Zusammenfassung der weiteren Migrationskosten und -erträge nach Branchen

- Angaben in DM -

\begin{tabular}{|l|r|r|r|r|r|r|}
\hline & \multicolumn{2}{|c|}{$100 \mathrm{~km}$} & \multicolumn{2}{c|}{$400 \mathrm{~km}$} & \multicolumn{2}{c|}{$1.000 \mathrm{~km}$} \\
\hline & $\mathrm{r}=0,015$ & $\mathrm{r}=0,05$ & $\mathrm{r}=0,015$ & $\mathrm{r}=0,05$ & $\mathrm{r}=0,015$ & $\mathrm{r}=0,05$ \\
\hline Land- und Forstwirt. & -6.914 & -6.827 & -22.079 & -21.160 & -50.408 & -47.827 \\
\hline Bergbau & -4.139 & -4.290 & -19.304 & -18.623 & -47.633 & -45.290 \\
\hline Energiewirtschaft & -5.258 & -5.334 & -20.423 & -19.667 & -48.752 & -46.334 \\
\hline Verarb. Gewerbe & -5.457 & -5.513 & -20.622 & -19.846 & -48.951 & -46.513 \\
\hline Baugewerbe & -6.008 & -6.012 & -21.173 & -20.345 & -49.502 & -47.012 \\
\hline Handel & -6.348 & -6.319 & -21.513 & -20.652 & -49.842 & -47.319 \\
\hline Verkehr & -5.813 & -5.834 & -20.978 & -20.167 & -49.307 & -46.834 \\
\hline Dienstleistungen & -6.263 & -6.240 & -21.428 & -20.573 & -49.757 & -47.240 \\
\hline alle Branchen & -5.955 & -5.962 & -21.120 & -20.295 & -49.449 & -46.962 \\
\hline
\end{tabular}

Quelle: Tabelle 19, Tabelle 20, Tabelle 21, Tabelle 23, Tabelle 26

Mindestens abzuziehen vom Migrationsertrag, wie er sich nach Berücksichtigung von Einkommens- und Mietpreisdifferenzen ergibt, sind 4.000 DM. Erfolgt die Wanderung über eine sehr weite Distanz, so kann sich der Ertrag um über 
40.000 DM mindern. Zwischen den verschiedenen Branchen gibt es keine gravierenden Unterschiede bezüglich des Abzugsbetrags, da die Distanz die Kosten bei weitem dominieren. Das gleiche gilt für die Industriearbeiter gesplittet nach Status und Geschlecht, wie Tabelle 32 zeigt.

Tabelle 32: Zusammenfassung der weiteren Migrationskosten- und -erträge nach Status und Geschlecht

- Angaben in DM -

\begin{tabular}{|l|r|r|r|r|r|r|}
\hline & \multicolumn{2}{|c|}{$100 \mathrm{~km}$} & \multicolumn{2}{c|}{$400 \mathrm{~km}$} & \multicolumn{2}{c|}{$1000 \mathrm{~km}$} \\
\hline & $\mathrm{r}=0,015$ & $\mathrm{r}=0,05$ & $\mathrm{r}=0,015$ & $\mathrm{r}=0,05$ & $\mathrm{r}=0,015$ & $\mathrm{r}=0,05$ \\
\hline Arbeiter & -6.023 & -6.025 & -21.188 & -20.358 & -49.517 & -47.025 \\
\hline Angestellte & -3.541 & -3.776 & -18.706 & -18.109 & -47.035 & -44.776 \\
\hline Arbeiter, männlich & -5.842 & -5.860 & -21.007 & -20.193 & -49.336 & -46.860 \\
\hline Angestellte, männl. & -3.254 & -3.523 & -18.419 & -17.856 & -46.748 & -44.523 \\
\hline Arbeiter, weiblich & -7.210 & -7.090 & -22.375 & -21.423 & -50.704 & -48.090 \\
\hline Angestellte, weibl. & -5.734 & -5.764 & -20.899 & -20.097 & -49.228 & -46.764 \\
\hline
\end{tabular}

Quelle: Tabelle 19, Tabelle 20, Tabelle 21, Tabelle 24, Tabelle 27

Die Industriebeschäftigten mit den größten erwartbaren Migrationserträgen infolge der Lohndifferenzen - nämlich die männlichen Angestellten - haben die geringsten Abzüge, während die Arbeiterinnen, die am wenigsten voneinem Umzug profitieren würden, die höchsten Abzüge von ihrem Migrationsertrag aufweisen. Das liegt daran, daß der fiktive Ertrag, der sich für die männlichen Angestellten durch die kürzere Arbeitszeit im Westen ergibt, infolge ihres überdurchschnittlichen Jahreseinkommens höher ist als die der Arbeiterinnen.

\subsubsection{Erwartbare Migrationserträge nach Berücksichtigung weiterer Kosten und Erträge}

Nachdem die Migrationserträge aus regionalen Lohn- und Mietdifferenzen und die weiteren Kosten und Erträge bisher isoliert betrachtet wurden, werden die Ergebnisse im folgenden zusammengefaßt. Dafür werden die jeweiligen Migrationserträge, so wie sie in Abschnitt 3.2.1 und 3.2.2.1 bestimmt wurden, aufaddiert. Es ergibt sich dann ein Migrationsertrag insgesamt, wie er für einen Ostdeutschen bei einem erfolgreichen Arbeitsplatzwechsel 1990 erwartbar gewesen sein könnte, wenn die dieser Arbeit zugrundeliegenden Annahmen zutreffen. Da die in Abschnitt 3.2.2.1 ermittelten weiteren Kosten und Erträge netto anfallen (eine steuerliche Absetzbarkeit wurde ausgeschlossen) und die Nettogrößen die für die Entscheidung relevanten Größen sind, werden im folgenden nur noch die Nettolohneinkommen betrachtet. 
Zur Erinnerung seien hier noch einmal alle im folgenden berücksichtigten Faktoren genannt:

- regionale Lohnunterschiede zwischen den alten und neuen Ländern und ihre Entwicklung,

- regionale Unterschiede des Einkommensteuertarifs, der Bemessungsgrundlage der Sozialversicherung und der Beitragssätze der Sozialversicherung,

- regionale Mietpreisunterschiede zwischen den alten und neuen Ländern und ihre Entwicklung,

- Mehrkosten bei Neuanmietung einer Wohnung im Vergleich zu einer Bestandsmiete,

- Umzugskosten,

- psychische Kosten,

- regionale Unterschiede der Jahresarbeitszeit,

- potentielle Verschlechterung der beruflichen Stellung.

\subsection{Erwartbare Migrationserträge insgesamt}

In diesem Abschnitt werden die erwartbaren Migrationserträge bestimmt, die sich aufgrund der Kenntnisse und Erwartungen am Ende des Jahres 1990 ergeben. Für die Berechnung der Erträge werden für Lohnangleichungserwartungen innerhalb von 5 Jahren die weiteren Erträge und Kosten im Vergleich zu Abschnitt 3.2.2.1 geringfügig modifiziert. Es wird angenommen, daß in diesem Fall auch mit schnelleren Angleichungen der Arbeitszeit gerechnet wird. Die Zuschläge auf den Migrationsertrag sind dadurch geringer. Tabelle 33 zeigt die erwartbaren Erträge aus einer Wanderung bei einem vermuteten Anpassungszeitraum von 3 Jahren in der Branchensplittung.

Bei einem 3jährigen Anpassungszeitraum wäre nur ein Umzug in die nähere Umgebung lohnend gewesen. Im Durchschnitt hätte lediglich ein Überschuß von 3.000 bis 4.000 DM erzielt werden können. Für die Beschäftigten in der Landund Forstwirtschaft hätten sich die Kosten und Erträge sogar die Waage gehalten. Bei einem Umzug über eine mittlere oder ferne Distanz wäre für alle Erwerbstätigen ein Verlust von mindestens 6.000 DM erwartbar gewesen. Bei einem Umzug über $1.000 \mathrm{~km}$ hätte der erwartbare Verlust $30.000 \mathrm{DM}$ und mehr betragen. 
Tabelle 33: Erwartbare Migrationserträge insgesamt bei einer 3jährigen Angleichungsdauer nach Branchen

- Angaben in DM -

\begin{tabular}{|l|r|r|r|r|r|r|}
\hline & \multicolumn{2}{|c|}{$100 \mathrm{~km}$} & \multicolumn{2}{c|}{$400 \mathrm{~km}$} & \multicolumn{2}{c|}{$1.000 \mathrm{~km}$} \\
\hline & $\mathrm{r}=0,015$ & $\mathrm{r}=0,05$ & $\mathrm{r}=0,015$ & $\mathrm{r}=0,05$ & $\mathrm{r}=0,015$ & $\mathrm{r}=0,05$ \\
\hline Land- und Forstw. & 325 & 77 & -14.840 & -14.256 & -43.169 & -40.923 \\
\hline Bergbau & 8.952 & 8.176 & -6.213 & -6.157 & -34.542 & -32.824 \\
\hline Energiewirtschaft & 6.310 & 5.684 & -8.855 & -8.649 & -37.184 & -35.316 \\
\hline Verarb. Gewerbe & 6.292 & 5.675 & -8.873 & -8.658 & -37.202 & -35.325 \\
\hline Baugewerbe & 3.931 & 3.454 & -11.234 & -10.879 & -39.563 & -37.546 \\
\hline Handel & 2.037 & 1.671 & -13.128 & -12.662 & -41.457 & -39.329 \\
\hline Verkehr & 4.074 & 3.583 & -11.091 & -10.750 & -39.420 & -37.417 \\
\hline Dienstleistungen & 2.387 & 2.001 & -12.778 & -12.332 & -41.107 & -38.999 \\
\hline alle Branchen & 3.939 & 3.460 & -11.226 & -10.873 & -39.555 & -37.540 \\
\hline
\end{tabular}

Quelle: Tabelle 2, Tabelle 31

Ohne Berücksichtigung der psychischen Kosten ergäben sich zwischen den verschiedenen Distanzen nur geringe Unterschiede in Höhe von 3.000 bis 4.000 DM. Umzüge hätten in diesem Fall für die Beschäftigten bei einer Nahdistanz Überschüsse bis zu 10.000 DM erbracht. Bei einer mittleren Distanz berechnete sich für die Beschäftigten insgesamt ein leichter Überschuß von etwa 4.000 DM. Bei einem Umzug über $1.000 \mathrm{~km}$ Entfernung glichen sich in diesem Fall die Kosten und Erträge im Durchschnitt gerade aus, nur in wenigen Branchen (wie im Bergbau) hätte ein Überschuß erzielt werden können.

Tabelle 34: Erwartbare Migrationserträge insgesamt bei einer 5jährigen Angleichungsdauer nach Branchen

- Angaben in DM -

\begin{tabular}{|l|r|r|r|r|r|r|}
\hline & \multicolumn{2}{|c|}{$100 \mathrm{~km}$} & \multicolumn{2}{c|}{$400 \mathrm{~km}$} & \multicolumn{2}{c|}{$1.000 \mathrm{~km}$} \\
\hline & $\mathrm{r}=0,015$ & $\mathrm{r}=0,05$ & $\mathrm{r}=0,015$ & $\mathrm{r}=0,05$ & $\mathrm{r}=0,015$ & $\mathrm{r}=0,05$ \\
\hline Land- und Forstw. & 4.078 & 3.481 & -11.087 & -10.852 & -39.416 & -37.519 \\
\hline Bergbau & 15.443 & 14.064 & 278 & -269 & -28.051 & -26.936 \\
\hline Energiewirtschaft & 11.993 & 10.832 & -3.172 & -3.501 & -31.501 & -30.168 \\
\hline Verarb. Gewerbe & 12.011 & 10.856 & -3.154 & -3.477 & -31.483 & -30.144 \\
\hline Baugewerbe & 8.920 & 7.975 & -6.245 & -6.358 & -34.574 & -33.025 \\
\hline Handel & 6.355 & 5.584 & -8.810 & -8.749 & -37.139 & -35.416 \\
\hline Verkehr & 9.081 & 8.122 & -6.084 & -6.211 & -34.413 & -32.878 \\
\hline Dienstleistungen & 6.796 & 5.996 & -8.369 & -8.337 & -36.698 & -35.004 \\
\hline alle Branchen & 8.921 & 7.976 & -6.244 & -6.357 & -34.573 & -33.024 \\
\hline
\end{tabular}

Quelle: Tabelle 3 und Tabelle 31 
Bei einer vermuteten Lohnangleichung nach fünf Jahren hätte ein Nahumzug für die Beschäftigten aller Branchen einen Gewinn von mindestens 3.000 DM und höchstens 15.000 DM bedeutet, wie in Tabelle 34 ausgewiesen wird. Ab einer mittleren Distanz wären Verluste (Ausnahme Bergbau) zu erwarten gewesen. Die Verluste bei einer Fernwanderung wären zwar niedriger als bei einer 3jährigen Angleichungsdauer, sie sind mit über $30.000 \mathrm{DM}$ aber trotzdem hoch. Ohne Berücksichtigung psychischer Kosten ergeben sich für die Beschäftigten aller Branchen und über alle Distanzen leichte Überschüsse, die durchschnittlich zwischen 5.000 DM bis 10.000 DM betragen.

Tabelle 35: Erwartbare Migrationserträge insgesamt bei einer 10jährigen Angleichungsdauer nach Branchen

- Angaben in DM -

\begin{tabular}{|l|r|r|r|r|r|r|}
\hline & \multicolumn{2}{|c|}{$100 \mathrm{~km}$} & \multicolumn{2}{c|}{$400 \mathrm{~km}$} & \multicolumn{2}{c|}{$1.000 \mathrm{~km}$} \\
\hline & $\mathrm{r}=0,015$ & $\mathrm{r}=0,05$ & $\mathrm{r}=0,015$ & $\mathrm{r}=0,05$ & $\mathrm{r}=0,015$ & $\mathrm{r}=0,05$ \\
\hline Land- und Forstw. & 27.506 & 23.588 & 12.341 & 9.255 & -15.988 & -17.412 \\
\hline Bergbau & 55.163 & 48.195 & 39.998 & 33.862 & 11.669 & 7.195 \\
\hline Energiewirtschaft & 47.237 & 41.083 & 32.072 & 26.750 & 3.743 & 83 \\
\hline Verarb. Gewerbe & 47.375 & 41.203 & 32.210 & 26.870 & 3.881 & 203 \\
\hline Baugewerbe & 39.537 & 34.232 & 24.372 & 19.899 & -3.957 & -6.768 \\
\hline Handel & 33.183 & 28.603 & 18.018 & 14.270 & -10.311 & -12.397 \\
\hline Verkehr & 39.879 & 34.540 & 24.714 & 20.207 & -3.615 & -6.460 \\
\hline Dienstleistungen & 34.332 & 29.622 & 19.167 & 15.289 & -9.162 & -11.378 \\
\hline alle Branchen & 39.541 & 34.239 & 24.376 & 19.906 & -3.953 & -6.761 \\
\hline
\end{tabular}

Quelle: Tabelle 4 und Tabelle 31

Tabelle 35 zeigt, daß bei einem Anpassungszeitraum von 10 Jahren ein regionaler Arbeitsplatzwechsel über eine nahe oder mittlere Distanz für die Beschäftigten aller untersuchten Branchen lohnend gewesen wären. Erträge von bis zu $50.000 \mathrm{DM}$ waren erwartbar. Über eine weite Entfernung wäre aber selbst bei diesem verhältnismäßig langen Anpassungszeitraum ein Umzug für viele Beschäftigte nicht lohnend gewesen, im Durchschnitt errechnen sich Verluste von rund 5.000 DM. Ohne psychische Kosten hätten sich Überschüsse ergeben, selbst bei einer Fernwanderung wären Überschüsse von bis zu 50.000 DM angefallen.

Werden die Beschäftigten in der Industrie betrachtet, zeigen sich ähnliche Ergebnisse. Die Spannweite der erwartbaren Erträge ist jedoch größer als in der Branchenbetrachtung. 
Tabelle 36: Erwartbare Migrationserträge insgesamt bei einer 3jährigen Angleichungsdauer nach Status und Geschlecht

- Angaben in DM -

\begin{tabular}{|l|r|r|r|r|r|r|}
\hline & \multicolumn{2}{|c|}{$100 \mathrm{~km}$} & \multicolumn{2}{c|}{$400 \mathrm{~km}$} & \multicolumn{2}{c|}{$1000 \mathrm{~km}$} \\
\hline & $\mathrm{r}=0,015$ & $\mathrm{r}=0,05$ & $\mathrm{r}=0,015$ & $\mathrm{r}=0,05$ & $\mathrm{r}=0,015$ & $\mathrm{r}=0,05$ \\
\hline Arbeiter & 8.735 & 8.054 & -6.430 & -6.279 & -34.759 & -32.946 \\
\hline Angestellte & 23.805 & 22.228 & 8.640 & 7.895 & -19.689 & -18.772 \\
\hline Arbeiter, männlich & 9.680 & 8.948 & -5.485 & -5.385 & -33.814 & -32.052 \\
\hline Angestellte, männl. & 26.614 & 24.880 & 11.449 & 10.547 & -16.880 & -16.120 \\
\hline Arbeiter, weiblich & 3.673 & 3.266 & -11.492 & -11.067 & -39.821 & -37.734 \\
\hline Angestellte, weibl. & 9.838 & 9.092 & -5.327 & -5.241 & -33.656 & -31.908 \\
\hline
\end{tabular}

Quelle: Tabelle 5, Tabelle 32

Für die Angestellten - speziell die männlichen Angestellten - ergibt sich mit $25.000 \mathrm{DM}$ bei einer Distanz von $100 \mathrm{~km}$ ein recht hoher Migrationsüberschuß, der alle Erträge, die in der Branchengliederung ausgewiesen wurden, deutlich übersteigt. Sie sind die einzige Arbeitnehmergruppe, für die sich ein Umzug über eine mittleren Distanz gelohnt hätte. Nur ein Umzug über eine Ferndistanz wäre für sie - wie für alle anderen auch - mit höheren Kosten als Erträgen verbunden gewesen. Die erwartbaren Erträge der Frauen sind deutlich niedriger als die der Männer. Der Überschuß der weiblichen Angestellten ist um etwa 15.000 DM geringer als der ihrer männlichen Kollegen und beläuft sich auf knapp 10.000 DM. Für die Arbeiterinnen war eine Wanderung noch weniger attraktiv: Selbst für die Nahdistanz betragen die erwartbaren Überschüsse nur etwa 3.000 DM.

Tabelle 37: Erwartbare Migrationserträge insgesamt bei einer 5jährigen Angleichungsdauer nach Status und Geschlecht

- Angaben in DM -

\begin{tabular}{|l|r|r|r|r|r|r|}
\hline & \multicolumn{2}{|c|}{$100 \mathrm{~km}$} & \multicolumn{2}{c|}{$400 \mathrm{~km}$} & \multicolumn{2}{c|}{$1000 \mathrm{~km}$} \\
\hline & $\mathrm{r}=0,015$ & $\mathrm{r}=0,05$ & $\mathrm{r}=0,015$ & $\mathrm{r}=0,05$ & $\mathrm{r}=0,015$ & $\mathrm{r}=0,05$ \\
\hline Arbeiter & 11.885 & 10.819 & -3.280 & -3.514 & -31.609 & -30.181 \\
\hline Angestellte & 34.030 & 31.282 & 18.865 & 16.949 & -9.464 & -9.718 \\
\hline Arbeiter, männlich & 12.977 & 11.840 & -2.188 & -2.493 & -30.517 & -29.160 \\
\hline Angestellte, männl. & 37.659 & 34.662 & 22.494 & 20.329 & -5.835 & -6.338 \\
\hline Arbeiter, weiblich & 7.626 & 6.773 & -7.539 & -7.560 & -35.868 & -34.227 \\
\hline Angestellte, weibl. & 13.149 & 11.997 & -2.016 & -2.336 & -30.345 & -29.003 \\
\hline
\end{tabular}

Quelle: Tabelle 6, Tabelle 32

Bei einer um 2 Jahre längeren Anpassungszeit der Löhne und Mieten ändert sich das Ergebnis nur marginal. Bei einem Umzug über eine Strecke von $100 \mathrm{~km}$ war 
auch in diesem Fall für alle Arbeitnehmer ein Migrationsüberschuß erwartbar, und zwar von durchschnittlich $3.000 \mathrm{DM}$ mehr als bei einer Anpassung innerhalb von 3 Jahren. Eine Wanderung über die mittlere Distanz ist nach wie vor nur für die männlichen Angestellten attraktiv, für alle anderen wäre sie von Nachteil. Wie bereits bei der kürzeren Angleichungsdauer errechnen sich für eine Fernwanderung durchweg Verluste. Entstehen keine psychischen Kosten, wären zum Teil erhebliche Überschüsse entstanden, bei den männlichen Angestellten bis zu $35.000 \mathrm{DM}$ über die Ferndistanz. Selbst für die Arbeiterinnen wäre hier noch einen Gewinn von circa 6.000 DM erwartbar gewesen.

Tabelle 38: Erwartbare Migrationserträge insgesamt bei einer 10jährigen Angleichungsdauer nach Status und Geschlecht

- Angaben in DM -

\begin{tabular}{|l|r|r|r|r|r|r|}
\hline & \multicolumn{2}{|c|}{$100 \mathrm{~km}$} & \multicolumn{2}{c|}{$400 \mathrm{~km}$} & \multicolumn{2}{c|}{$1000 \mathrm{~km}$} \\
\hline & $\mathrm{r}=0,015$ & $\mathrm{r}=0,05$ & $\mathrm{r}=0,015$ & $\mathrm{r}=0,05$ & $\mathrm{r}=0,015$ & $\mathrm{r}=0,05$ \\
\hline Arbeiter & 41.385 & 35.853 & 26.220 & 21.520 & -2.109 & -5.147 \\
\hline Angestellte & 69.281 & 61.027 & 54.116 & 46.694 & 25.787 & 20.027 \\
\hline Arbeiter, männlich & 43.884 & 38.076 & 28.719 & 23.743 & 390 & -2.924 \\
\hline Angestellte, männl. & 75.566 & 66.665 & 60.401 & 52.332 & 32.072 & 25.665 \\
\hline Arbeiter, weiblich & 25.245 & 21.440 & 10.080 & 7.107 & -18.249 & -19.560 \\
\hline Angestellte, weibl. & 44.219 & 38.374 & 29.054 & 24.041 & 725 & -2.626 \\
\hline
\end{tabular}

Quelle: Tabelle 6, Tabelle 32

Alle Beschäftigten in der Industrie, die eine Lohnangleichung innerhalb von 10 Jahren erwarteten, müßten - bei Durchführung einer entsprechenden Rechnung mit hohen Migrationserträgen zwischen 20.000 DM und 75.000 DM bei einer Nahwanderung gerechnet haben. Auch eine Wanderung über eine Distanz von rund $400 \mathrm{~km}$ wäre für alle Beschäftigten lohnend gewesen. Selbst bei einer Fernwanderung ergeben sich für die männlichen Angestellten hohe Migrationserträge. Für die männlichen Arbeiter und die weiblichen Angestellten halten sich die Kosten und Erträge die Waage. Lediglich für die Arbeiterinnen waren hohe Verluste erwartbar.

Zusammenfassend ist festzustellen, daß sich bei Berücksichtigung aller hier untersuchten Kosten und Erträge ein Umzug mit gleichzeitigem Arbeitsplatzwechsel aus Ost- nach Westdeutschland für jeden Beschäftigten gelohnt hätte, sofern das Ziel der Wanderung die nähere Umgebung gewesen wäre. Die erwartbaren Migrationsgewinne sind um so höher, je pessimistischere Erwartungen bezüglich des Anpassungszeitraums zugrunde gelegt werden. Während für einen durchschnittlichen Arbeitnehmer, der mit einer Angleichungsdauer von 3 Jahren rechnete, ein Überschuß von unter 4.000 DM erwartbar war, war bei 10jähriger Angleichungsdauer ein zehnmal so hoher Überschuß erwartbar. Die Migrati- 
onserträge entsprechen bei einer Angleichungsdauer von 3 Jahren 3 Bruttomonatslöhnen in Ostdeutschland im Jahr 1990, bei einer Angleichungsdauer von 10 Jahren 30 Bruttomonatslöhnen. Verglichen mit den Löhnen von 1991 hätte der Migrationsertrag sogar nur weniger als 2 Bruttomonatslöhne (bzw. 20) betragen.

Bei einem Umzug über eine Strecke von $400 \mathrm{~km}$ war für alle Beschäftigten ein Gewinn erwartbar, die mit Lohnangleichungsdauern von deutlich über 5 Jahre rechneten. Die männlichen Industrieangestellten konnten sogar bei einem 3jährigen Anpassungszeitraum mit Überschüssen rechnen, der erwartbare Ertrag belief sich auf etwa 4 Monatslöhne des Jahres 1991 in Ostdeutschland. Ein Umzug über eine Distanz von $1.000 \mathrm{~km}$ war ausschließlich bei 10jährigen Lohnangleichungserwartungen lohnend, und selbst dann nicht für alle Beschäftigten.

Die Bewertung der subjektiv anfallenden Kosten, nämlich der psychischen, hat einen starken Einfluß auf das Ergebnis. Fallen derartige Kosten nicht an, wäre für sämtliche Beschäftigte über jede Distanz einen monetären Überschuß bei einem Umzug erwartbar gewesen. Männliche Industrieangestellte, die von einer Lohnanpassung in 10 Jahren ausgingen, hätten bei jeder Entfernung mit Erträgen von über $70.000 \mathrm{DM}$ rechnen können. Für Arbeiterinnen mit gleichen Erwartungen wären Überschüsse von circa $20.000 \mathrm{DM}$ erwartbar gewesen. Wenn ein Umzug nicht mit Mühen und psychischen Kosten (und Erträgen) verbunden ist, hätte die Entscheidung mithin für alle Beschäftigten zugunsten eines Umzugs ausfallen müssen - immer vorausgesetzt, sie hätten in den alten Bundesländern zu den herrschenden Durchschnittslöhnen einen Arbeitsplatz gefunden.

\subsection{Korrgierte erwartbare Migrationserträge insgesamt}

In der Branchengliederung ergeben sich aufgrund der tatsächlichen Entwicklung bis zum Jahr 1994 und geschätzter Angleichung bis 1999 unter Berücksichtigung aller untersuchten Einflußfaktoren die in Tabelle 39 dargestellten erwartbare Migrationserträge.

Die Simulation ergibt für einen durchschnittlichen Arbeitnehmer einen positiven Migrationsertrag sowohl für eine nahe als auch eine mittlere Entfernung. Eine Nahwanderung hätte einen Ertrag gebracht, der in etwa einem Bruttojahreslohn eines ostdeutschen Beschäftigten entsprach. Bei einer Fernwanderung hätte sich dagegen ein Verlust von knapp 20.000 DM ergeben. Lediglich im Verarbeitenden Gewerbe entsprechen sich die Kosten und Erträge bei einer Fernwanderung in etwa. Für Beschäftigte im Dienstleistungssektor und im Baugewerbe hätte sogar ein Umzug über $400 \mathrm{~km}$ einen Wanderungsverlust bedeutet. 
Tabelle 39: Korrigierte erwartbare Migrationserträge insgesamt nach Branchen bei einer Angleichung bis 1999

- Angaben in DM -

\begin{tabular}{|l|r|r|r|r|r|r|}
\hline & \multicolumn{2}{|c|}{$100 \mathrm{~km}$} & \multicolumn{2}{c|}{$400 \mathrm{~km}$} & \multicolumn{2}{c|}{$1.000 \mathrm{~km}$} \\
\hline & $\mathrm{r}=0,015$ & $\mathrm{r}=0,05$ & $\mathrm{r}=0,015$ & $\mathrm{r}=0,05$ & $\mathrm{r}=0,015$ & $\mathrm{r}=0,05$ \\
\hline Land- und Forstw. & 18.729 & 16.467 & 3.564 & 2.134 & -24.765 & -24.533 \\
\hline Bergbau & 24.296 & 22.307 & 9.131 & 7.974 & -19.198 & -18.693 \\
\hline Energiewirtschaft & 29.119 & 25.640 & 13.954 & 11.307 & -14.375 & -15.360 \\
\hline Verarb. Gewerbe & 45.869 & 40.760 & 30.704 & 26.427 & 2.375 & -240 \\
\hline Baugewerbe & 10.497 & 8.736 & -4.668 & -5.597 & -32.997 & -32.264 \\
\hline Handel & 27.052 & 23.590 & 11.887 & 9.257 & -16.442 & -17.410 \\
\hline Verkehr & 26.419 & 23.371 & 11.254 & 9.038 & -17.075 & -17.629 \\
\hline Dienstleistungen & 12.999 & 11.247 & -2.166 & -3.086 & -30.495 & -29.753 \\
\hline alle Branchen & 24.418 & 21.551 & 9.253 & 7.218 & -19.076 & -19.449 \\
\hline
\end{tabular}

Quelle: Tabelle 9, Tabelle 31

Die höheren Migrationsüberschüsse bzw. geringeren -verluste inTabelle 40 gegenüber Tabelle 39 ergeben sich aufgrund einer um fünf Jahre längeren Anpassungsdauer. Für einen durchschnittlichen Arbeitnehmer erhöht sich der Migrationsertrag hierdurch um über 13.000 DM. Für Beschäftigte im Verarbeitenden Gewerbe wird bei dieser Anpassungsdauer sogar eine Fernwanderung lohnend.

Tabelle 40: Korrigierte erwartbare Migrationserträge insgesamt nach Branchen bei einer Angleichung bis 2004

- Angaben in DM -

\begin{tabular}{|l|r|r|r|r|r|r|}
\hline & \multicolumn{2}{|c|}{$100 \mathrm{~km}$} & \multicolumn{2}{c|}{$400 \mathrm{~km}$} & \multicolumn{2}{c|}{$1.000 \mathrm{~km}$} \\
\hline & $\mathrm{r}=0,015$ & $\mathrm{r}=0,05$ & $\mathrm{r}=0,015$ & $\mathrm{r}=0,05$ & $\mathrm{r}=0,015$ & $\mathrm{r}=0,05$ \\
\hline Land- und Forstw. & 32.201 & 26.679 & 17.036 & 12.346 & -11.293 & -14.321 \\
\hline Bergbau & 23.104 & 21.330 & 7.939 & 6.997 & -20.390 & -19.670 \\
\hline Energiewirtschaft & 49.168 & 40.847 & 34.003 & 26.514 & 5.674 & -153 \\
\hline Verarb. Gewerbe & 63.305 & 53.863 & 48.140 & 39.530 & 19.811 & 12.863 \\
\hline Baugewerbe & 15.194 & 12.309 & 29 & -2.024 & -28.300 & -28.691 \\
\hline Handel & 41.189 & 34.196 & 26.024 & 19.863 & -2.305 & -6.804 \\
\hline Verkehr & 44.282 & 36.914 & 29.117 & 22.581 & 788 & -4.086 \\
\hline Dienstleistungen & 17.180 & 14.429 & 2.015 & 96 & -26.314 & -26.571 \\
\hline alle Branchen & 41.787 & 34.719 & 26.622 & 20.386 & -1.707 & -6.281 \\
\hline
\end{tabular}

Quelle: Tabelle 10, Tabelle 31

Bei Antizipation der tatsächlichen Entwicklung bis 1994 und den hier für die darauf folgenden Jahre angenommenen Anpassungsverlauf hätten alle Beschäftigten davon ausgehen können, daß sich eine Nahwanderung für sie lohnt (das gilt um so mehr, wenn keine psychischen Kosten anfallen). Auch Wanderungen 
über eine Distanz von bis zu $400 \mathrm{~km}$ hätten sich für die Beschäftigten der meisten Branchen gelohnt (mit Ausnahme des Dienstleistungs- und des Baugewerbes), nur Fernwanderungen hätten für die meisten Beschäftigten einen Verlust bedeutet.

Die potentiellen Migrationserträge der Industriebeschäftigten nach Status und Geschlecht unter der Annahme einer Lohn- und Preisangleichung bis 1999 werden in Tabelle 41 abgebildet.

Tabelle 41: Korrigierte erwartbare Migrationserträge insgesamt nach Status und Geschlecht bei einer Angleichung bis 1999

- Angaben in DM -

\begin{tabular}{|l|r|r|r|r|r|r|}
\hline & \multicolumn{2}{|c|}{$100 \mathrm{~km}$} & \multicolumn{2}{c|}{$400 \mathrm{~km}$} & \multicolumn{2}{c|}{$1000 \mathrm{~km}$} \\
\hline & $\mathrm{r}=0,015$ & $\mathrm{r}=0,05$ & $\mathrm{r}=0,015$ & $\mathrm{r}=0,05$ & $\mathrm{r}=0,015$ & $\mathrm{r}=0,05$ \\
\hline Arbeiter & 25.421 & 22.559 & 10.256 & 8.226 & -18.073 & -18.441 \\
\hline Angestellte & 58.862 & 52.973 & 43.697 & 38.640 & 15.368 & 11.973 \\
\hline Arbeiter, männlich & 28.369 & 25.250 & 13.204 & 10.917 & -15.125 & -15.750 \\
\hline Angestellte, männl. & 62.287 & 56.153 & 47.122 & 41.820 & 18.793 & 15.153 \\
\hline Arbeiter, weiblich & 13.629 & 11.822 & -1.536 & -2.511 & -29.865 & -29.178 \\
\hline Angestellte, weibl. & 30.265 & 26.946 & 15.100 & 12.613 & -13.229 & -14.054 \\
\hline
\end{tabular}

Quelle: Tabelle 10, Tabelle 32

Die Resultate ähneln denen der Branchenauswertung. Für einen marginalen durchschnittlichen Angestellten hätte sich ein Umzug über jede Entfernung gelohnt. Selbst im schlechtesten Fall (bei einer hohen Zeitpräferenzrate und einer Distanz von $1.000 \mathrm{~km}$ ) hätte sein Migrationsertrag mindestens einem halben Bruttojahreseinkommen eines Ostdeutschen im Jahr 1991 entsprochen. Es zeigen sich aber deutliche Unterschiede zwischen den Geschlechtern. Eine weibliche Angestellte hätte bestenfalls (Umzug über $100 \mathrm{~km}$ und eine niedrige Zeitpräferenzrate) das 16fache ihres Bruttomonatslohns von 1991 erzielen können, während ein männlicher Angestellter fast das 26 fache seines Bruttomonatslohns hätte erreichen können. Auch bei den Arbeitern zeigen sich deutliche geschlechtsspezifische Unterschiede. Während ein Arbeiter bei einem Umzug über eine Entfernung von $400 \mathrm{~km}$ einen Ertrag von mehr als 8.000 DM erzielt hätte, hätte eine Arbeiterin einen Verlust gemacht. Die korrigierten erwartbaren Erträge der Angestellten übertreffen die der Arbeiter um durchschnittlich 30.000 DM.

Bei der Berechnung der Ergebnisse, die in Tabelle 42 ausgewiesen sind, ist eine fünf Jahre längere Anpassungsdauer zugrunde gelegt. 
Tabelle 42: Korrigierte erwartbare Migrationserträge insgesamt nach Status und Geschlecht bei einer Angleichung bis 2004

- Angaben in DM -

\begin{tabular}{|l|r|r|r|r|r|r|}
\hline & \multicolumn{2}{|c|}{$100 \mathrm{~km}$} & \multicolumn{2}{c|}{$400 \mathrm{~km}$} & \multicolumn{2}{c|}{$1.000 \mathrm{~km}$} \\
\hline & $\mathrm{r}=0,015$ & $\mathrm{r}=0,05$ & $\mathrm{r}=0,015$ & $\mathrm{r}=0,05$ & $\mathrm{r}=0,015$ & $\mathrm{r}=0,05$ \\
\hline Arbeiter & 40.669 & 35.516 & 25.504 & 21.183 & -2.825 & -5.484 \\
\hline Angestellte & 80.654 & 72.526 & 65.489 & 58.193 & 37.160 & 31.526 \\
\hline Arbeiter, männlich & 44.105 & 38.725 & 28.940 & 24.392 & 611 & -2.275 \\
\hline Angestellte, männl. & 86.211 & 77.561 & 71.046 & 63.228 & 42.717 & 36.561 \\
\hline Arbeiter, weiblich & 25.202 & 21.542 & 10.037 & 7.209 & -18.292 & -19.458 \\
\hline Angestellte, weibl. & 46.364 & 40.811 & 31.199 & 26.478 & 2.870 & -189 \\
\hline
\end{tabular}

Quelle: Tabelle 11, Tabelle 32

Bei dieser Anpassungsdauer wären die Erträge eines Umzugs um 10.000 DM bis $20.000 \mathrm{DM}$ höher gewesen als bei der kürzeren Anpassungsdauer. Dies führt u.a. dazu, daß ein Umzug über eine Distanz von $400 \mathrm{~km}$ auch für die Arbeiterinnen einträglich gewesen wäre. Den Ausschlag für die Vorteilhaftigkeit einer Fernwanderung gibt bei den weiblichen Angestellten und den männlichen Arbeitern die Höhe der Zeitpräferenzrate.

Ohne Berücksichtigung psychischer Kosten wären sogar Fernwanderungen für alle Industriebeschäftigten vorteilhaft gewesen.

\subsubsection{Zusammenfassung und Vergleich}

Bei Berücksichtigung weiterer Migrationskosten und -erträge schmälert den am Ende des Jahres 1990 erwartbaren Ertrag eines Umzugs erheblich. Wanderungen in die nähere Umgebung wären zwar weiterhin für alle Beschäftigten vorteilhaft gewesen, jedoch ist der erwartbare Ertrag mit maximal 9.000 DM relativ gering (ein Abschluß des Anpassungsprozesses innerhalb von 5 Jahren vorausgesetzt). Wird eine deutlich längere Anpassungsdauer zugrunde gelegt, errechnen sich Erträge, die mindestens das Vierfache betragen. In Abhängigkeit von der Anpassungsdauer, der Branchenzugehörigkeit, dem Status und dem Geschlecht war eine Wanderung über eine mittlere Distanz nicht für alle Arbeitnehmer vorteilhaft. Bei Fernwanderungen waren nur für Beschäftigte des Bergbaus, mit Einschränkungen für Beschäftigte der Energiewirtschaft und des Verarbeitenden Gewerbes sowie für die männlichen Industrieangestellten bei 10jährigen Anpassungserwartungen Erträge aus einem Umzug erwartbar. 
Abbildung 34: Ausgewählte erwartbare Migrationserträge bei alternativen Angleichungszeiträumen und Wanderungsdistanzen

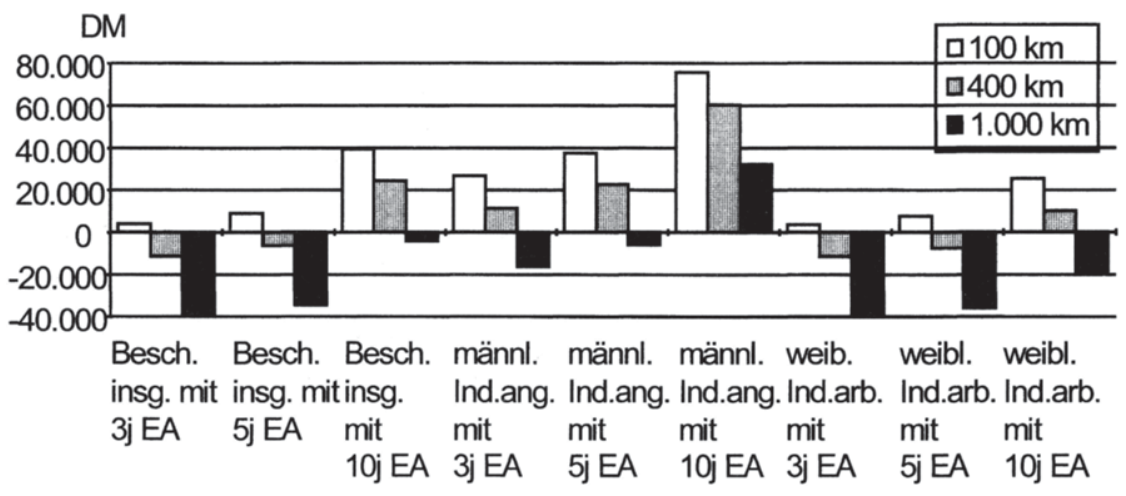

mit EA $=$ Erwartung über die Angleichungsdauer

Quelle: Tabelle 33, Tabelle 34, Tabelle 35, Tabelle 36, Tabelle 37, Tabelle 38

Der Ertrag für einen durchschnittlichen Beschäftigten beläuft sich bei Zugrundelegung der tatsächlichen Entwicklung bis 1994 und deren Fortschreibung für eine Nahwanderung auf 21.000 DM bis $42.000 \mathrm{DM}$, für eine mittlere Entfernung auf einen Betrag zwischen 7.000 DM und 27.000 DM, für eine Fernwanderung ergab sich ein Verlust. Während sich für Nahwanderungen durchgängig für alle Branchen Wanderungsgewinne errechneten, wurde für Beschäftigte einiger Branchen ein Verlust bei einem Umzug über $400 \mathrm{~km}$ festgestellt. Für Arbeitnehmer im Verarbeitenden Gewerbe wäre sogar eine Fernwanderung ertragreich gewesen.

Die Unterschiede zwischen den 1990 erwartbaren und den auf Basis der tatsächlichen Daten von 1991-1994 und deren Fortschreibung berechneten Migrationserträgen wurden bereits in Abschnitt 3.2.1.4 dargestellt. Dort wurde deutlich, daß die zuletzt genannten Migrationserträge die 1990 erwartbaren übertreffen. Die absolute Differenz zwischen den Migrationserträgen ändert sich nur geringfügig durch die zusätzliche Berücksichtigung weiterer Kosten und Erträge, da diese Faktoren im wesentlichen nicht von der Anpassungsdauer abhängen. Lediglich bei den Berechnungen, denen Erwartungen über eine Anpassung innerhalb von 5 Jahren zugrunde liegen, weichen die Differenzen etwas ab. Der Grund für die Abweichungen liegt darin, daß hier die Erträge aus den geringeren Arbeitszeiten in den alten Ländern gekürzt wurden, da annahmegemäß auch die Erwartungen bezüglich einer Angleichung der Arbeitszeiten zu optimistisch waren. 
Die bei Zugrundelegung der „tatsächlichen“ Entwicklung der Löhne und Mieten errechneten höheren Migrationsüberschüsse als gegenüber den 1990 erwartbaren sind in einigen Fällen so viel größer, daß sich die betreffenden Erwerbspersonen bei richtiger Antizipation der Entwicklung für statt gegen einen Umzug entschieden hätten. Welche Personen hiervon betroffen waren, wird im folgenden analysiert.

Da Wanderungen in die nähere Umgebung für die Beschäftigten aller Branchen bei sämtlichen untersuchten Erwartungshaltungen lohnend gewesen wären, hätten auch die Erwartungen, die 1990 wohl bestanden, nicht zu falschen Wanderungsentscheidungen geführt. Am stärksten betroffen von Fehlentscheidungen waren Wanderungen über eine mittlere Distanz. Von der Höhe der Abweichungen zwischen 1990 erwartbaren und „tatsächlich“ zu erzielenden Erträgen vermittelt Abbildung 35 einen Eindruck.

Mit Ausnahme des Dienstleistungs- und des Baugewerbes (nicht abgebildet) hätten sich Wanderungen über eine mittlere Distanz für alle Beschäftigten bei Zugrundelegung der tatsächlichen Entwicklung gelohnt. Bei bis zu 5jährigen Lohnangleichungserwartungen berechnet sich aber ein Verlust, so daß aufgrund der Fehleinschätzung der künftigen Entwicklung eine ablehnende Wanderungsentscheidung getroffen wurde.

Abbildung 35: Ausgewählte erwartbare Migrationserträge unter alternativen Angleichungsverläufen in ausgewählten Branchen bei einer Wanderungsdistanz von $400 \mathrm{~km}$

- Zeitpräferenzrate $=0,015$

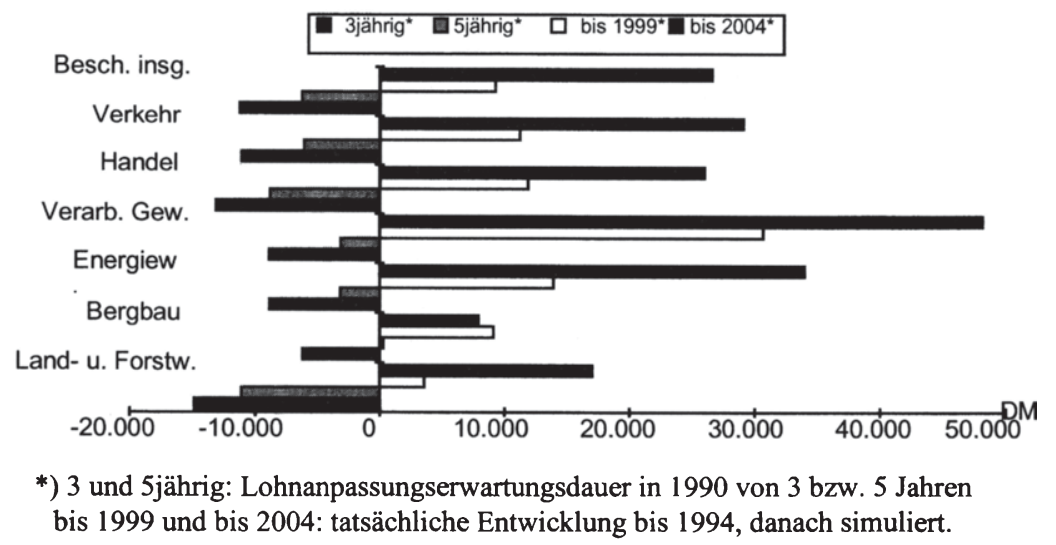

Quelle: Tabelle 33, Tabelle 34, Tabelle 39, Tabelle 40 
Bei einer Entfernung von $1.000 \mathrm{~km}$ führt die Rechnung nur selten zur falschen Entscheidung, da bei zutreffenden und unzutreffenden Erwartungen in der Regel die Kosten überwiegen. Allerdings errechnet sich für Arbeitnehmer, die im Bergbau und im Verarbeitenden Gewerbe beschäftigt waren und die 1990 eine Lohnangleichung innerhalb von zehn Jahren vermuteten, ein positiver Ertrag. Bei Zugrundelegung der realen Entwicklung zeigt sich, daß ein positiver Ertrag im Verarbeitenden Gewerbe tatsächlich erzielt werden konnte, während dies im Bergbau hingegen nicht möglich war.

Bei der Erwägung eines Umzugs in die nähere Umgebung hätten Industriebeschäftigte im Jahr 1990 - sofern sie eine Migrationsrechnung durchgeführt hätten - keine „falschen“ Schlußfolgerungen gezogen: Bei allen untersuchten Erwartungshaltungen und möglichen Entwicklungen war für einen potentiellen Migranten ein positiver Migrationsertrag erwartbar. Bei einer mittleren Distanz dagegen war auch bei dieser Arbeitnehmergruppe ein Verlust bei der Kalkulation in 1990 erwartbar, während sich tatsächlich Migrationsüberschüsse ergeben hätten, wie Abbildung 36 zeigt.

Abbildung 36: (Korrigierte) erwartbare Migrationserträge bei alternativen Angleichungsverläufen nach Status und Geschlecht bei einer Wanderungsdistanz von $400 \mathrm{~km}$

Zeitpräferenzrate 0,015 -

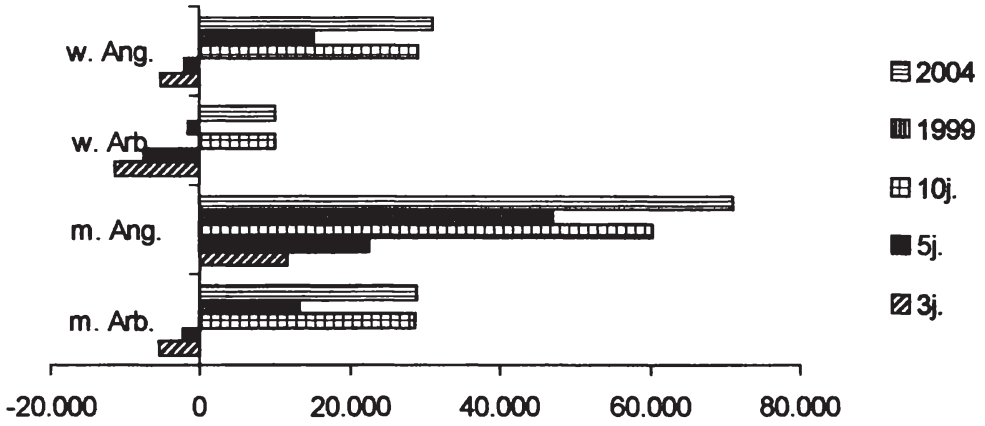

3,5 und $10 \mathrm{j} .:$ Lohnanpassungserwartungsdauer in 1990 von 3, 5 und 10 Jahren bis 1999 und bis 2004: tatsächliche Entwicklung bis 1994, danach simuliert.

Quelle: Tabelle 36, Tabelle 37, Tabelle 38, Tabelle 41, Tabelle 42

Bei einer Distanz von $1.000 \mathrm{~km}$ ergibt sich für alle betrachteten Arbeitnehmergruppen, daß sich aufgrund der Erwartungen im Jahr 1990 eine Wanderung nicht gelohnt hätte; dieses Ergebnis stimmt mit der Berechnung auf Grundlage der tatsächlichen Entwicklung überein. Lediglich bei den männlichen Angestellten 
mit Lohnanpassungserwartungen von höchstens 5 Jahren hätte die Durchführung einer derartigen Migrationsrechnung bei der Ferndistanz zu einer falschen Entscheidung geführt, nämlich zu einem Wanderungsablehnung, obwohl tatsächlich ein Umzug lohnend gewesen wäre.

\subsubsection{Migrationserträge nach Berücksichtigung von Arbeitslosigkeit}

Im Zusammenhang mit der deutschen Vereinigung wurde häufig darauf hingewiesen, daß Arbeitslosigkeit mehr Menschen zu Wanderungen veranlassen könnte als regionale Lohnunterschiede. Der Einfluß von Arbeitslosigkeit auf den potentiellen Migrationsertrag wird im folgenden untersucht. Dafür wird exemplarisch der Zeitraum 1991-1994 betrachtet. Eine Untersuchung in der bisherigen Vertiefung wird allerdings nicht vorgenommen. Da nur für die Branchengliederung Einkommensdaten für 1994 vorliegen, werden sie der Auswertung von Industriebeschäftigten nach Status und Geschlecht vorgezogen, um die Schätzfehler möglichst gering zu halten. Zudem sind in den Branchendaten mehr Einkommensbestandteile (wie das 13. Gehalt) erfaßt.

In Kapitel 3.1.2.1.2 wurde erläutert, daß die Möglichkeit arbeitslos zu werden gemäß Gleichung (1) bei der Berechnung des Einkommensstroms berücksichtigt werden kann. Ausgangspunkt der Betrachtung ist eine Erwerbsperson, diein der Periode vor dem Umzug beschäftigt war. Da es in der ehemaligen DDR quasi keine Arbeitslosigkeit gab, erscheint diese Vorgehen gerechtfertigt.

(1) $A E, x=\frac{q 1 * L E \times 1+(1-q 1) * A L G \times 1}{1+r}+\frac{q 2 * L E \times 2+(1-q 2) *[q 1 * A L G \times 2+(1-q 1) * A L H \times 2]}{(1+r)^{2}}$

$$
+\frac{q 3 * L E \times 3+(1-q 3) *[q 2 * A L G \times 3+(1-q 2) *(q 1 * A L H \times 3+(1-q 1) * S H \times 3]}{(1+r)^{3}}
$$

mit:

AE,x: Erwartungswert des Erwerbseinkommen (Einkommen aus Beschäftigung, Lohnersatzleistungen, laufende Hilfe zum Lebensunterhalt) in der Region $\mathrm{x}$

qi: Wahrscheinlichkeit, in der Periode i über einen Arbeitsplatz zu verfügen

LE: Nettolohneinkommen in einem zum Zeitpunkt 0 der Qualifikation entsprechenden Beruf

ALG: Arbeitslosengeld

ALH: Arbeitslosenhilfe

SH: laufende Hilfe zum Lebensunterhalt (Sozialhilfe)

r: Zeitpräferenzrate

$\mathrm{x}$ : Region

i: $\quad$ Periode 
Die Wahrscheinlichkeit arbeitslos zu werden, wird im folgenden hilfsweise mit der herrschenden Arbeitslosenquote gleichgesetzt, und zwar global ohne Berücksichtigung etwa bestehender branchenspezifischer Unterschiede. Es wird angenommen, daß eine Wanderung nach Westdeutschland auch in ein nicht gesichertes Beschäftigungsverhältnis erfolgen kann $^{225}$ (bzw. könnte der Migrant nach kurzer Zeit wieder entlassen werden). Unter ,normalen“ Umständen wäre die herrschende Arbeitslosenquote tendenziell zu hoch, um das Risiko arbeitslos zu werden, auszudrücken, da die von Arbeitslosigkeit betroffenen schwerer einen neuen Job finden als Beschäftigte arbeitslos werden bzw. einen neuen Arbeitsplatz finden ${ }^{226}$. Bei der ungewöhnlichen Situation in Ostdeutschland kurz nach der Wende dürfte dies allerdings höchstens eingeschränkt zutreffen.

Die Erwartungswerte des Einkommens in Ost- und Westdeutschland in den Jahre 1991 bis 1994 lassen sich dann wie folgt bestimmen:

(2) $\mathrm{AE}, \mathrm{x}=\frac{(1-\mathrm{ALQ91})^{\star} \mathrm{LE} 91+\mathrm{ALQ91*} \mathrm{ALG} \mathrm{G}_{0}}{1+\mathrm{r}}$

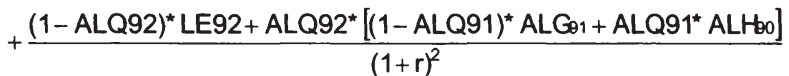

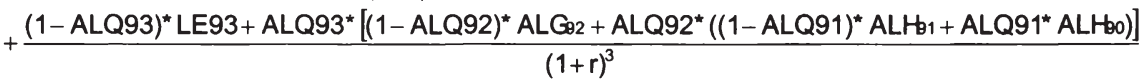

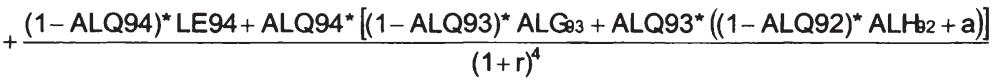

$\left.a=A L Q 92(1-A L Q 91)^{\star} A L H_{1}+A L Q 91^{\star} A L H_{0}\right)$

mit:

AE,x: Erwartungswert des Erwerbseinkommen (Einkommen aus Beschäftigung und Lohnersatzleistungen) in der Region $\mathrm{x}$

ALQi:Arbeitslosenquote im Jahr i

LEi: Lohneinkommen im Jahr i

$\mathrm{ALG}_{\mathrm{i}}$ : Arbeitslosengeld, berechnet aufgrund des Einkommens in Periode i

$\mathrm{ALH}_{\mathrm{i}}$ : Arbeitslosenhilfe, berechnet aufgrund des Einkommens in Periode i

Ist der Index i nicht tiefgestellt, bezieht sich der jeweilige Wert direkt auf das mit i ausgedrückte Jahr, wie etwa ALQ91 = Arbeitslosenquote in 1991. Ist der Index dagegen tiefgestellt, so berechnet sich der Wert das Lohneinkommen in dem

${ }^{225}$ Wanderungen dieser Art sind allerdings nur unter speziellen Bedingungen vorstellbar, etwa wenn besondere persönliche Motive vorliegen.

${ }^{226} \mathrm{Vgl}$. hierzu auch Kapitel 3.1.2.1.2. 
Jahr, den der Index anzeigt, die jeweiligen Lohnersatzleistungen fallen aber in einem anderen Jahr an.

Die Lohneinkommen gehen als Nettogrößen in die Berechnung ein, d.h. nach Steuern und Sozialabgaben. Mietdifferenzen und weitere Kosten und Erträge werden vorerst nicht berücksichtigt, da zunächst der Einfluß von Arbeitslosigkeit auf das erwartete Nettoeinkommen isoliert bestimmt werden soll. Aufgrund des Nettolohneinkommens wird das Arbeitslosengeld bzw. die Arbeitslosenhilfe berechnet. Die betrachtete Person habe im Falle von Arbeitslosigkeit einen Anspruch auf Zahlung von Arbeitslosengeld für eine Dauer von 12 Monaten ${ }^{227}$. Das Arbeitslosengeld für Ledige ohne Kinder betrug bis einschließlich 1993 63\% des Nettolohns aus dem Durchschnitt der letzten 3 Monatseinkommen (ohne Sonderzahlungen wie 13. Gehalt), seit dem Jahr 199460 v.H. auf Grundlage der letzten 6 Monate. Die Arbeitslosenhilfe für Personen ohne Kinder betrug bis 199356 \%, seit $199453 \%$ der Bemessungsgrundlage. Für die Bemessungsgrundlage des Arbeitslosengeldes und der Arbeitslosenhilfe wird hier das letzte Jahresnettoeinkommen zugrunde gelegt, auch wenn dies tendenziell zu hoch ist, da es Sonderzahlungen enthält. Dies wird dadurch etwas abgemildert, daß das Monatseinkommen der letzten 6 Monate aufgrund von Lohnsteigerungen tendenziell höher ist als das der vorhergehenden Monate. Das westdeutsche Arbeitslosengeld wird wegen der umfangreicheren Sonderleistungen in den alten Ländern wohl stärker überschätzt als das ostdeutsche.

Für die Berechnung des Arbeitslosengeldes $\left(\mathrm{ALG}_{90}\right)$ bzw. der -hilfe $\left(\mathrm{ALH}_{90}\right)$ bei Arbeitslosigkeit im Jahr 1991 und später wird - unabhängig von dem Wohnort in 1991 - in beiden Regionen das Nettoeinkommen aus dem Jahr 1990 aus Ostdeutschland zugrunde gelegt, da annahmegemäß der potentielle Migrant 1990 noch in Ostdeutschland gewohnt hat.

Arbeitslosenhilfe wird nach geltendem Recht bei Bedürftigkeit unbegrenzt lange gewährt. Im Gegensatz zum Arbeitslosengeld muß zunächst auf eigenes Einkommen und Vermögen zur Bestreitung des Lebensunterhaltes zurückgegriffen werden. Nur wenn dies nicht vorhanden ist, wird Arbeitslosenhilfe gewährt. Im folgenden wird zunächst eine Person mit Anspruch auf Arbeitslosenhilfe betrachtet und dann im Anschluß die Bedeutung des Anspruchs auf Arbeitslosenhilfe für den Migrationsertrag untersucht. In der Rechnung werden Wohngeld oder sonstige staatliche Transfers nicht berücksichtigt.

${ }^{227}$ Die betreffende Person wäre also jünger als 42 und in den letzten Jahren beschäftigt gewesen. Wäre sie älter und hätte sie lange gearbeitet, kann sich der Anspruch bis auf insgesamt 3 Jahre verlängern. 
Es scheint keine sinnvolle Verfahrensweise zu sein, die offizielle Arbeitslosenquote für die Rechnung zugrunde zu legen, da sich die verdeckte Arbeitslosigkeit nicht in dieser Quote widerspiegelt. Für die Rechnung müssen daher zunächst die Arbeitslosenquoten bestimmt werden, die sich ergeben, wenn zusätzlich die Personen zu den Arbeitslosen gerechnet werden, die infolge von staatlichen Maßnahmen wie Arbeitsbeschaffungsmaßnahmen (ABM) oder Vorruhestand als Arbeitslose aus der Arbeitsmarktstatistik herausfallen.

Die Höhe der offenen und verdeckten Arbeitslosigkeit wurde in Anlehnung an den Sachverständigenrat zur Begutachtung der gesamtwirtschaftlichen Entwicklung (1994/95) ermittelt ${ }^{228}$, so daß sich die später verwendete „Arbeitslosenquote" wie folgt zusammensetzt:

Zähler: Arbeitslose

+ Personen in ABM

+ Kurzarbeiter (Arbeitslosenäquivalente)

+ sonstige nichtbeschäftigte Erwerbspersonen

Nenner: beschäftigte Arbeitnehmer

+ Arbeitslose

+ sonstige nichtbeschäftigte Erwerbspersonen

In der Größe „,beschäftigte Arbeitnehmer“ sind die in ABM beschäftigten Erwerbspersonen und die Kurzarbeiter enthalten. „Sonstige nichtbeschäftigte Erwerbspersonen" sind Leistungsempfänger nach $§ 105$ AFG $^{229}$, Personen im Vorruhestand, Bezieher von Altersübergangsgeld und Personen in (Vollzeit-)Fortbildung und Umschulungsmaßnahmen (FuU). Auf Grundlage dieser Abgrenzung ergeben sich Arbeitslosenquoten, so wie sie in Tabelle 43 und Tabelle 44 dargestellt sind.

\footnotetext{
${ }^{228} \mathrm{Vgl}$. Sachverständigenrat zur Begutachtung der gesamtwirtschaftlichen Entwicklung (1994/95), S. 100.

${ }^{229}$ Leistungsempfänger nach $\S 105$ AFG sind u.a. Empfänger von Nahtlosigkeitsleistungen und Personen, die 58 Jahre und älter sind und daher der Arbeitsvermittlung nicht zur Verfügung stehen müssen.
} 
Tabelle 43: Entwicklung der Beschäftigung und der Arbeitslosigkeit in Ostdeutschland

- Angaben in 1.000 -

\begin{tabular}{|c|c|c|c|c|}
\hline $\begin{array}{l}\text { beschäftigte Arbeitnehmer } \\
\text { darin: } \\
\text { - } A B M\end{array}$ & $\begin{array}{r}1991 \\
7.219 \\
183\end{array}$ & $\begin{array}{r}1992 \\
6.344\end{array}$ & $\begin{array}{l}1993 \\
6.125\end{array}$ & $\begin{array}{l}1994 \\
6.125\end{array}$ \\
\hline $\begin{array}{l}\text { - Kurzarbeiter (Arbeits- } \\
\text { losenäquivalent) } \\
\text { Arbeitslose }\end{array}$ & $\begin{array}{l}900 \\
913\end{array}$ & $\begin{array}{r}197 \\
1.170\end{array}$ & $\begin{array}{r}81 \\
1.149\end{array}$ & $\begin{array}{r}45 \\
1.250\end{array}$ \\
\hline $\begin{array}{l}\text { Leistungsempfänger nach } \\
\S 105 \mathrm{AFG}\end{array}$ & & 9 & 18 & 28 \\
\hline Vorruhestand & 365 & 295 & 214 & 129 \\
\hline Altersübergangsgeld & 189 & 516 & 639 & 488 \\
\hline Vollzeit-FuU-Maßnahmen & 169 & 425 & 345 & 235 \\
\hline Quote in \% & 30,7 & 34,25 & 31,9 & 29,7 \\
\hline
\end{tabular}

Quelle: Sachverständigenrat zur Begutachtung der gesamtwirtschaftlichen Entwicklung, (1994/95), S. 100, Engelen-Kefer u.a. (1995), S.324, S.79 u.a.S.

Tabelle 44: Entwicklung der Beschäftigung und der Arbeitslosigkeit in Westdeutschland

- Angaben in 1.000 -

\begin{tabular}{|c|c|c|c|c|}
\hline $\begin{array}{l}\text { beschäftigte Arbeitnehmer } \\
\text { darin: } \\
\text { - } A B M\end{array}$ & $\begin{array}{l}1991 \\
25.920\end{array}$ & $\begin{array}{l}1992 \\
26.063\end{array}$ & $\begin{array}{l}1993 \\
25.601\end{array}$ & $\begin{array}{l}1994 \\
25.195\end{array}$ \\
\hline $\begin{array}{l}\text {-Kurzarbeiter (Arbeits- } \\
\text { losenäquivalent) } \\
\text { Arbeitslose }\end{array}$ & $\begin{array}{r}43 \\
1.689\end{array}$ & $\begin{array}{r}88 \\
1.808\end{array}$ & $\begin{array}{r}227 \\
2.270\end{array}$ & $\begin{array}{r}90 \\
2.560\end{array}$ \\
\hline $\begin{array}{l}\text { Leistungsempfänger nach } \\
\S 105 \mathrm{AFG}\end{array}$ & 85 & 125 & 180 & 197 \\
\hline Vorruhestand & 59 & 39 & 18 & 2 \\
\hline Altersübergangsgeld & . & . & 2 & 3 \\
\hline Vollzeit-FuU-Maßnahmen & 195 & 205 & 195 & 180 \\
\hline Quote in \% & 7,7 & 8,3 & 10,4 & 11,0 \\
\hline
\end{tabular}

Quelle: Sachverständigenrat zur Begutachtung der gesamtwirtschaftlichen Entwicklung, (1994/95), S.100, Engelen-Kefer u.a. (1995), S.324, S.79 u.a.

Die so ermittelten Arbeitslosenquoten unterscheiden sich erwartungsgemäß stärker voneinander als die auf dem üblichen Weg bestimmten Arbeitslosenquoten. Insbesondere in Ostdeutschland weicht die hier ermittelte Quote von der „offiziellen" Arbeitslosenquote, die nur die offene Arbeitslosigkeit berücksichtigt, erheblich ab. Die Wahrscheinlichkeit, in Ostdeutschland arbeitslos zu sein, betrug 
im untersuchten Zeitraum etwa ein Drittel, während sie in Westdeutschland bei circa $10 \%$ lag. In Ostdeutschland erreichte die Arbeitslosenquote 1992 ihren (vorläufigen) Höhepunkt und nahm seitdem innerhalb des untersuchten Zeitraums geringfügig ab (allerdings ohne gleichzeitigen Anstieg der Beschäftigung). Die Arbeitslosenquote in Westdeutschland nahm dagegen kontinuierlich zu.

Bei der unten angestellten Berechnung der regionalen Einkommen wird angenommen, daß alle Personen in ABM, alle Kurzarbeiter(Arbeitslosenäquivalente) und alle sonstigen nichtbeschäftigten Erwerbspersonen ein niedrigeres Einkommen bezogen als regulär beschäftigte Arbeitnehmer, und zwar wurde ein Einkommen in Höhe des Arbeitslosengeldes bzw. der -hilfe angesetzt (das Unterhaltsgeld für Teilnehmer an beruflichen Weiterbildungsmaßnahmen beispielsweise entspricht nach geltenden Regelungen der Höhe nach dem Arbeitslosengeld).

Unter Zugrundelegung der jeweils geltenden Steuergesetze und Sozialversicherungsbestimmungen ergibt sich für die abgezinsten Einkommensströme 19911994 für Ost- und Westdeutschland das in Tabelle 45 aufgezeigte Bild:

Tabelle 45: Erwartungswerte für das Nettoeinkommen 1991-1994 bei Berücksichtigung von Arbeitslosigkeit

- Angaben in DM -

\begin{tabular}{|l|r|r|r|r|r|r|}
\hline & \multicolumn{2}{|c|}{ Erwartungswert Ost } & \multicolumn{2}{|c|}{ Erwartungswert West } & \multicolumn{2}{|c|}{ Differenz } \\
\hline Branche & $\mathrm{r}=0,015$ & $\mathrm{r}=0,05$ & $\mathrm{r}=0,015$ & $\mathrm{r}=0,05$ & $\mathrm{r}=0,015$ & $\mathrm{r}=0,05$ \\
\hline Land- und Forstw. & 52.401 & 47.960 & 87.574 & 80.468 & 35.173 & 32.508 \\
\hline Bergbau & 91.425 & 83.586 & 133.264 & 122.384 & 41.839 & 38.798 \\
\hline Energiewirt. & 81.411 & 74.601 & 124.393 & 114.175 & 42.982 & 39.574 \\
\hline Verarb. Gewerbe & 66.427 & 60.755 & 119.279 & 109.475 & 52.852 & 48.720 \\
\hline Bau & 78.728 & 72.179 & 105.891 & 97.188 & 27.162 & 25.008 \\
\hline Handel & 61.427 & 56.255 & 98.394 & 90.314 & 36.967 & 34.060 \\
\hline Verkehr & 70.240 & 64.287 & 110.820 & 101.742 & 40.580 & 37.455 \\
\hline Dienstleistungen & 70.688 & 64.708 & 100.452 & 92.228 & 29.764 & 27.520 \\
\hline insgesamt & 69.082 & 63.244 & 107.382 & 98.581 & 38.300 & 35.337 \\
\hline
\end{tabular}

Quelle: DIW (1990-1994), Arbeitslosenquoten gemäß Tabelle 43 und Tabelle 44, Anpassungsfaktoren für die Bemessungsgrundlage gemäß Anhang, eigene Berechnungen

Wie bereits in den vorangegangenen Untersuchungen zeigt sich, daß die Höhe der Zeitpräferenzrate keine gravierende Bedeutung für die Einkommenshöhe hat, die Differenz beträgt circa 3.000 DM. Durch eine Wanderung in den Westen ist das abgezinste Einkommen für 1991-1994 zwischen 50-70 \% höher als bei einem Verbleib im Osten. 
Die Bedeutung von Arbeitslosigkeit für die Wanderungsentscheidung zeigt sich in Tabelle 46, in der die Überschüsse aus Arbeitseinkommen mit Arbeitslosigkeit den Überschüssen aus Arbeitseinkommen ohne Berücksichtigung von Arbeitslosigkeit gegenübergestellt werden.

Der Vergleich zeigt, daß sich die Migrationserträge mit Berücksichtigung von Arbeitslosigkeit (erwartungsgemäß) vergrößern, da die Arbeitslosigkeit in Ostdeutschland höher als in Westdeutschland war. Je nach Branche steigen die erwartbaren Migrationserträge um 4.000 DM bis 7.500 DM. Die größten Differenzen ergeben sich für das Baugewerbe und im Energiesektor. In der Baubranche überzeichnen die errechneten Ergebnisse aber die realen Verhältnisse, da die Arbeitslosigkeit in dieser Branche deutlich unter dem Durchschnitt lag. Der Ertrag erhöht sich im Branchendurchschnitt um gut 5.000 DM. Verglichen mit diesem Ertrag ist der durch die regionalen Lohnunterschiede wesentlich größer: für einen durchschnittlichen Beschäftigten beträgt der Ertrag in den Jahren 19911994 gut 30.000 DM, d.h. die erwartbaren positiven Einkommenszuwächse durch Berücksichtigung der regionalen Arbeitslosenquoten betragen rund $15 \%$ der potentiellen Mehreinkommen durch die höheren Löhne in Westdeutschland. Diese Ergebnisse sprechen dafür, daß die Lohnunterschiede im Vergleich zur Arbeitslosigkeit der wichtigere Faktor bei einem Wanderungskalkül gewesen sein könnten.

Tabelle 46: Vergleich der erwartbaren Überschüsse aus regionalen Lohndifferenzen mit und ohne Berücksichtigung von Arbeitslosigkeit

- Angaben in DM -

\begin{tabular}{|l|r|r|r|r|r|r|}
\hline & \multicolumn{2}{|l|}{$\begin{array}{l}\text { Überschuß aus Arbeits- } \\
\text { einkommen mit Berück- } \\
\text { sichtigung von Arbeits- } \\
\end{array}$} & $\begin{array}{l}\text { Überschuß aus Arbeits- } \\
\text { losinkommen ohne } \\
\text { Berücksichtigung von } \\
\text { Arbeitslosigkeit }\end{array}$ & \multicolumn{2}{c|}{ Differenz } \\
\hline & $\mathrm{r}=0,015$ & $\mathrm{r}=0,05$ & $\mathrm{r}=0,015$ & $\mathrm{r}=0,05$ & $\mathrm{r}=0,015$ & $\mathrm{r}=0,05$ \\
\hline Land- und Forstw. & 35.173 & 32.508 & 30.090 & 27.848 & 5.083 & 4.660 \\
\hline Bergbau & 41.839 & 38.798 & 35.604 & 33.397 & 6.235 & 5.401 \\
\hline Energiewirtschaft & 42.982 & 39.574 & 36.043 & 33.236 & 6.939 & 6.338 \\
\hline Verarb. Gewerbe & 52.852 & 48.720 & 48.702 & 45.033 & 4.150 & 3.687 \\
\hline Bau & 27.162 & 25.008 & 20.705 & 19.113 & 6.457 & 5.895 \\
\hline Handel & 36.967 & 34.060 & 32.996 & 30.494 & 3.971 & 3.566 \\
\hline Verkehr & 40.580 & 37.455 & 34.789 & 32.200 & 5.791 & 5.255 \\
\hline Dienstleistungen & 29.764 & 27.520 & 23.884 & 22.198 & 5.880 & 5.322 \\
\hline alle Branchen & 38.300 & 35.337 & 33.129 & 30.673 & 5.171 & 4.664 \\
\hline
\end{tabular}

Quelle: DIW (1990-1994), Arbeitslosenquoten gemäß Tabelle 43 und Tabelle 44, eigene Berechnungen 
Es wurde bereits darauf hingewiesen, daß nicht jeder Arbeitslose Anspruch auf die Zahlung von Arbeitslosenhilfe hat. Die erwartbaren Migrationserträgen sind um 2.000 DM höher, wenn kein solcher Anspruch besteht (vgl. Tabelle 47). Durch die Leistungen der Bundesanstalt für Arbeit (BfA) wird der Anreiz zur Wanderung in die alten Länder mithin abgemildert. Zur Abschätzung der Wanderungswirkungen sei abschließend der theoretische Fall betrachtet, daß die BfA auf alle Leistungen und Maßnahmen verzichtet ${ }^{230}$. Die Einkommenserwartungen reduzieren sich entsprechend. Da hiervon die Erwerbspersonen in den neuen Ländern stärker als die in den alten betroffen wären, erhöhte sich der Anreiz, in den Westen zu wandern. Der Migrationsertrag stiege um 7.000 DM bis 8.000 DM. Dies entspricht gut einem Drittel der durch regionale Lohndifferenzen erwartbaren Erträge.

Tabelle 47: Differenzen der Erträge aus regionalen Lohndifferenzen bei alternativen Annahmen über die Zahlung von Lohnersatzleistungen im Vergleich zum Ohne-Arbeitslosigkeits-Fall

- Angaben in DM -

\begin{tabular}{|l|r|r|r|r|r|r|}
\hline & \multicolumn{2}{|l|}{$\begin{array}{l}\text { Differenz mit ALG } \\
\text { und ALH }\end{array}$} & \multicolumn{2}{l|}{$\begin{array}{l}\text { Differenz ohne } \\
\text { ALH, mit ALG }\end{array}$} & \multicolumn{2}{l|}{$\begin{array}{l}\text { Differenz ohne ALG, } \\
\text { ohne ALH }\end{array}$} \\
\hline Branche & $\mathrm{r}=0,015$ & $\mathrm{r}=0,05$ & $\mathrm{r}=0,015$ & $\mathrm{r}=0,05$ & $\mathrm{r}=0,015$ & \multicolumn{1}{r|}{$\mathrm{r}=0,05$} \\
\hline Land- und Forstwirtschaft & 5.083 & 4.660 & 7.383 & 6.733 & 10.891 & 10.004 \\
\hline Bergbau & 6.235 & 5.401 & 9.331 & 8.185 & 13.219 & 11.700 \\
\hline Energie & 6.939 & 6.338 & 10.166 & 9.238 & 15.916 & 14.561 \\
\hline Verarbeitendes Gewerbe & 4.150 & 3.687 & 6.847 & 6.116 & 10.739 & 9.750 \\
\hline Bau & 6.457 & 5.895 & 10.031 & 9.115 & 16.690 & 15.284 \\
\hline Handel & 3.971 & 3.566 & 6.696 & 6.023 & 11.117 & 10.138 \\
\hline Verkehr & 5.791 & 5.255 & 8.894 & 8.053 & 13.945 & 12.754 \\
\hline Dienstleistungen & 5.880 & 5.322 & 8.926 & 8.065 & 14.440 & 13.174 \\
\hline insgesamt & 5.171 & 4.664 & 8.098 & 7.301 & 13.054 & 11.902 \\
\hline
\end{tabular}

Quelle: DIW (1990-1994), Arbeitslosenquoten gemäß Tabelle 43 und Tabelle 44, eigene Berechnungen

Die arbeitsmarktpolitischen Maßnahmen wirkten also mobilitätshemmend. Dies gilt nicht nur für die Lohnersatzleistungen, sondern auch für Weiterqualifizierungsmaßnahmen und Arbeitsbeschaffungsmaßnahmen, da sich durch diese Maßnahmen die Perspektiven Menschen eine neue Tätigkeit vor Ort zu finden, verbessern (Dieser Effekt wurde in der Rechnung allerdings nicht aufgefangen $)^{231}$.

${ }^{230}$ Zur Vereinfachung wurde der Beitrag zur Arbeitslosenversicherung konstant gehalten, einkommens- und beschäftigungswirksame Auswirkungen aus dem Fortfall der Versicherung wurden vernachlässigt.

${ }^{231}$ Andererseits ist nicht auszuschließen, daß die Qualifizierung erst bestehende Wande- 
Die erwartbaren Migrationserträge werden zwar durch die Unterschiede in den Arbeitslosenquoten beeinflußt, der Einfluß ist jedoch offenbar - selbst bei der hier vorgenommenen pauschalen Sicht - wesentlich geringer als der durch die Unterschiede im Lohnniveau. Die Wahrscheinlichkeit, arbeitslos zu sein, war trotz der hohen Arbeitslosenquoten immer noch geringer als die Wahrscheinlichkeit, über einen Arbeitsplatz zu verfügen.

Die Lohnunterschiede hatten sich bis 1994 deutlich abgebaut. Bei den Arbeitslosenquoten gab es zwar auch eine leichte Annäherung, jedoch waren die Differenzen 1994 noch erheblich. Mit zunehmenden Lohnangleichungen könnte die Bedeutung von Unterschieden in der Arbeitslosigkeit für den Migrationsertrag zunehmen.

Die Berechnung zeigt nur den durchschnittlichen Einfluß der Arbeitslosenquote. Das individuelle Risiko, arbeitslos zu sein, kann sich davon deutlich unterscheiden. So betrug z.B. die offizielle Arbeitslosenquote in Ostdeutschland 1993 insgesamt $16 \%$, wobei die der Männer bei 10,6\% und die der Frauen bei $21,9 \%$ $\operatorname{lag}^{232}$. Ältere Arbeitnehmer finden schwerer einen neuen Arbeitsplatz als jüngere, unqualifizierte schlechter als qualifizierte. Die Nichtberücksichtigung der unterschiedlichen Wahrscheinlichkeiten arbeitslos zu sein, verfälscht jedoch die Ergebnisse nur wenig, da Frauen und Unqualifizierte auch in den alten Ländern stärker von Arbeitslosigkeit betroffen sind.

Arbeitslosigkeit dürfte vor allem für schon Arbeitslose ein Wanderungsgrund sein. Für eine Person, die vor der Alternative steht, in Ostdeutschland arbeitslos zu sein, in Westdeutschland jedoch arbeiten könnte, ist der erwartbare Migrationsertrag höher als oben berechnet. In die Berechnung des Migrationsertrags für diese Personen dürfte für die Ermittlung der Lohneinkommensdifferenzen für Ostdeutschland nur das Arbeitslosengeld, in die für Westdeutschland jedoch das Lohneinkommen in vollem Umfang eingehen. Der potentielle Migrationsertrag wäre aber auch bereits höher, wenn in diesem Fall in den alten Ländern damit gerechnet werden mußte, mit einer Wahrscheinlichkeit in Höhe der durchschnittlichen Arbeitslosenquote arbeitslos zu werden. In so einem Fall wäre der Migrationsertrag um circa 7.000 DM höher, als oben ausgewiesen.

Da sich die Migrationserträge durch die zwischen 1991 und 1994 existierende Arbeitslosigkeit nicht im gleichen Maße ändern wie durch die vorhandenen regionalen Lohnunterschiede, dürften sich die Ergebnisse der Abschnitte 3.2.1 und

rungssperren (wie Angst vor unbekannten Anforderungen bzw. einer Arbeit im Westen) reduziert.

${ }^{232} \mathrm{Vgl}$. Engelen-Kefer u.a. (1995), S. 327. 
3.2.2 durch Berücksichtigung von Arbeitslosigkeit nicht grundlegend ändern. Dies gilt insbesondere für die Ertragserwartungen wie sie im Jahr 1990 bestanden. Über die Hälfte der ostdeutschen Bevölkerung hatte eine Angleichung der wirtschaftlichen Situation in weniger als sechs Jahren erwartet ${ }^{233}$; diese Erwartungen dürften sich auch auf die Höhe der durchschnittlichen Arbeitslosigkeit bezogen haben. Daher dürften sich die Ergebnisse auch bei Einbeziehung von Arbeitslosigkeit kaum ändern. Erwerbspersonen, die einen längeren Anpassungszeitraum erwartet hatten, könnten evtl. eher mit abweichenden Arbeitslosenquoten gerechnet haben. Fraglich ist jedoch, ob sie auch in der tatsächlichen Höhe erwartet wurden. Die Berücksichtigung von Arbeitslosigkeit hätte dazu führen können, daß auch Wanderungen über hohe Distanzen lohnend werden.

Hätten zutreffende Erwartungen bezüglich der Entwicklung in den Jahren 19911994 bestanden, hätte sich am Ergebnis durchschnittlich wenig geändert - dazu waren die zusätzlichen Erträge zu gering. Eventuell wäre für einige Gruppen eine Wanderung lohnend geworden, wie etwa für die im Baugewerbe Beschäftigten über eine mittlere Distanz oder für die Arbeiter eine Fernwanderung. Bei Berücksichtigung der branchenspezifischen Risiken arbeitslos zu werden, müßte diese Aussage allerdings relativiert werden.

\subsubsection{Zusammenfassung und Schlußfolgerungen}

Im folgenden werden die Ergebnisse dieses Abschnittes zusammengefaßt. Im Anschluß folgen Überlegungen dazu, welches Wanderungspotential bei $\mathrm{Zu}-$ grundelegung der obigen Berechnungen über die Vorteilhaftigkeit einer Wanderung am Ende des Jahres 1990 bestand. Abschließend wird dargestellt, daß die potentiellen Wanderungserträge fünf Jahre nach der Wende in Ostdeutschland deutlich geschrumpft waren.

\subsubsection{Zusammenfassung}

Eingangs wurde geschätzt, welche Erträge für die Ostdeutschen bei einem Umzug in die alten Länder durch die regionalen Miet- und Lohndifferenzen aus der Perspektive von 1990 erwartbar gewesen wären. Da Umfragen darauf hin wiesen, daß die Mehrzahl der Ostdeutschen zu diesem Zeitpunkt noch von einer sehr schnellen ökonomischen Angleichung an die alten Länder ausgingen, wurden relativ kurze Anpassungszeiten von 3, 5 und 10 Jahren simuliert.

${ }^{233} \mathrm{Vgl}$. Abschnitt 3.2.1.2. 
Bei ausschließlicher Berücksichtigung der regionalen Miet- und Lohndifferenzen ergab sich für jeden Beschäftigten ein positiver erwartbarer Ertrag durch einen Umzug. Die Migrationserträge bei einer Lohnanpassungserwartung von höchstens fünf Jahren betrugen für alle Beschäftigten weniger als 20.000 DM, mit Ausnahme der männlichen Industrieangestellten, deren verfügbares Einkommen im Westen insgesamt bis zu 44.000 DM höher gewesen wäre. Bei einer zehnjährigen Anpassungszeit betrugen die erwartbaren Überschüsse im Durchschnitt brutto rund $95.000 \mathrm{DM}$, netto 50.000 DM und nach Berücksichtigung der Mietpreisunterschiede zwischen 40.000 DM und 45.000 DM, je nach Höhe der Zeitpräferenzrate.

Im Anschluß wurden im Abschnitt 3.2.1.3 die korrigierten erwartbaren Erträge ermittelt. Zugrunde gelegt wurden dafür die realen Daten für die Jahre 1991 bis 1994. Da die Löhne und Mietkosten für die darauf folgenden Jahre zum Untersuchungszeitpunkt noch nicht bekannt waren, wurden sie auf Basis der vorliegenden Daten fortgeschrieben. Dabei wurden zwei unterschiedliche Anpassungsverläufe simuliert. Es ergaben sich durchweg positive Migrationserträge. Der durchschnittliche Nettoertrag eines Beschäftigten belief sich nach Berücksichtigung der Lohneinkommen und der Mietzahlungen in Abhängigkeit vom ökonomischen Anpassungsverlauf auf rund $30.000 \mathrm{DM}$ bzw. 40.000 bis $48.000 \mathrm{DM}$ (bei einer Zeitpräferenzrate von 1,5\%, bei einer höheren Zeitpräferenzrate sind die Erträge etwas niedriger).

Bei Erwartungen einer 3jährigen Anpassungsdauer wurden die Nettoerträge durchschnittlich auf höchstens die Hälfte des tatsächlichen Ertrags geschätzt. Eine ostdeutsche Erwerbsperson, die mit einer Angleichung innerhalb von 5 Jahren rechnete, hätte ihren Migrationsertrag mithin um mindestens $50 \%$ unterschätzt. Selbst bei erwarteten Lohnangleichungen nach mindestens 10 Jahren wurden die Migrationserträge in den meisten Branchen ebenfalls unterschätzt. Die Migrationsneigung der Ostdeutschen dürfte somit bei zutreffenderen Antizipation der künftigen Entwicklung wohl größer gewesen sein als bei den im Jahre 1990 bestehenden Erwartungen.

In Abschnitt 3.2.2 wurden weitere Migrationskosten und -erträge monetär bewertet, und zwar die Umzugskosten, die geringeren Arbeitszeiten in den alten Ländern, die psychischen Kosten, die potentielle Lohneinbußen durch eine Verschlechterung der beruflichen Stellung und die Mehrkosten bei Neuanmietung einer Wohnung im Vergleich zur Bestandsmiete. Im Durchschnitt überstiegen die weiteren Kosten die weiteren Erträge bei einer Nahwanderung von $100 \mathrm{~km}$ um knapp 6.000 DM und bei einer Fernwanderung $(1.000 \mathrm{~km})$ um knapp 50.000 DM. 
Die einzelnen Kosten und Ertragsarten haben ein unterschiedliches relatives Gewicht. Um dieses zu ermitteln, ist es sinnvoll, die absoluten Beträge aller Komponenten aufzuaddieren ( $=$ Gesamtergebnis) und die jeweiligen Anteile der einzelnen Komponenten daran zu ermitteln. Die Summe der -absolut genommenen - einzelnen Komponenten ist stets höher als der Migrationsertrag, da Kosten nicht abgezogen, sondern zum Gesamtergebnis dem Betrag nach aufaddiert werden. Alle (negativen) Vorzeichen fallen auf diese Weise bei der Anteilsberechnung weg.

Für die Darstellung (s. Abbildung 37) und Erläuterung wird die Aufteilung der Kosten und Erträge für einen durchschnittlichen Beschäftigten herausgegriffen, der am Ende des Jahres 1990 davon ausging, die Löhne und Mieten würden sich innerhalb von drei Jahren angleichen. Daneben gestellt werden die Ergebnisse bei Berücksichtigung der tatsächlichen Entwicklung bis zum Jahr 1994 und einer vermuteten Angleichung bis zum Jahr 2004. In Abbildung 37 werden alle drei untersuchten Entfernungen abgebildet.

Abbildung 37: Anteil der verschiedenen Ertrags- und Kostenkomponenten am Gesamtergebnis

- Zeitpräferenzrate $r=0,015$ -

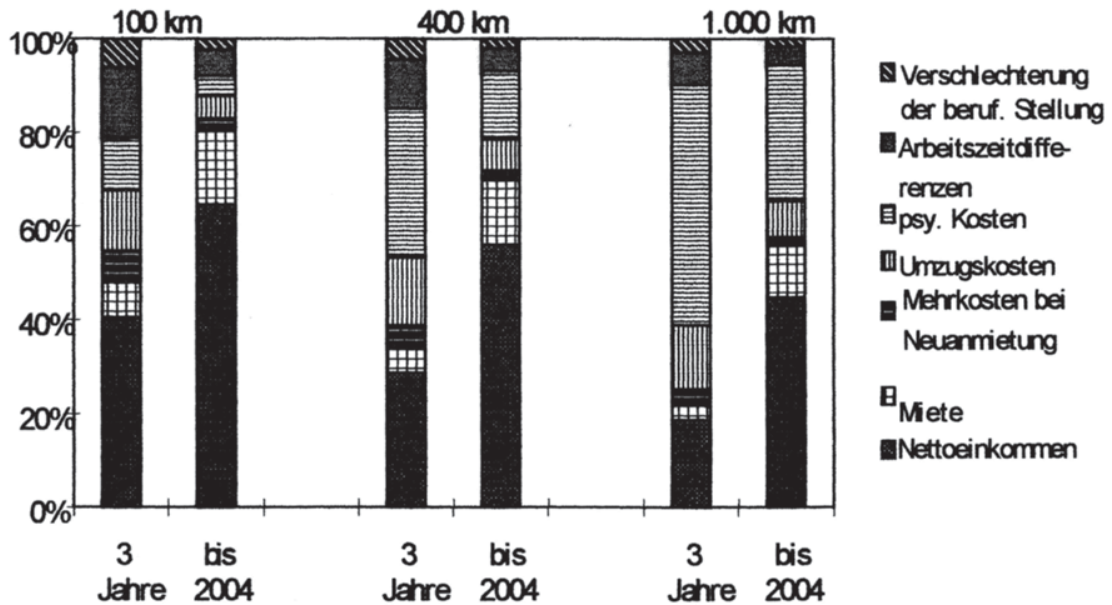

Quelle: Tabelle 2, Tabelle 16, Tabelle 20, Tabelle 21, Tabelle 23 und Tabelle 26, eigene Berechnung 
Abbildung 37 zeigt, daß bei einer Distanz von $100 \mathrm{~km}$ der regionale Lohnunterschied der wichtigste Einflußfaktor ist. Sein Anteil an den gesamten Kosten und Erträgen beträgt $40 \%$ bzw. $64 \%$. Mit zunehmender Entfernung steigt die Bedeutung der psychischen Kosten (absolut nehmen sie für jede weiteren $100 \mathrm{~km}$ um rund 4.000 DM zu). Bei 3jährigen Lohnangleichungserwartungen erhöht sich der Anteil der psychischen Kosten von $11 \%$ am Gesamtergebnis bei einer Nahwanderung auf $51 \%$ bei einer Fernwanderung. Unter Zugrundelegung der tatsächlichen Entwicklung ist der Anteil der psychischen Kosten am erwartbaren Gesamtergebnis deutlich geringer (er beträgt $4 \%$ bzw. $29 \%$ ), hier sind die Lohnunterschiede bei allen Entfernungen am bedeutendsten.

Das Gewicht der Umzugskosten ist unabhängig von der Entfernung relativ konstant. Zwar steigen die Umzugskosten mit zunehmender Distanz, der Anteil am absoluten Gesamtergebnis ändert sich durch die gleichzeitige noch stärkere Erhöhung der psychischen Kosten aber nur wenig. Den geringsten Einfluß haben die Kosten, die sich durch eine potentielle Verschlechterung der beruflichen Stellung ergeben. Ihr Anteil liegt zwischen $2 \%$ und $6 \%$ und ist am höchsten bei einer schnellen Angleichung und gleichzeitiger Nahwanderung. Nur wenig bedeutender sind die Kosten durch Neuanmietung einer Wohnung im Vergleich zur Bestandsmiete mit einem Anteil zwischen 2\% und 7\% am absoluten Gesamtbetrag.

Eine Berücksichtigung der weiteren Kosten und Erträge bedeutet für den durchschnittlichen Beschäftigten, der mit Anpassungen innerhalb von 10 Jahren rechnete, einen Verlust bei einer Fernwanderung. Bei Anpassungserwartungen von höchstens 5 Jahren ergibt sich für eine Distanz von mindestens $400 \mathrm{~km}$ ebenfalls ein Verlust. Nahwanderungen dagegen waren stets lohnend. Die höchsten Wanderungsüberschüsse schienen - aus der Perspektive von 1990 - die Beschäftigten im Bergbau bei einer Nahwanderung realisieren zu können. Bei den Industriebeschäftigten war der erwartbare Ertrag der männlichen Angestellten am höchsten.

Bei Zugrundelegung der tatsächlichen Daten von 1991-1994 und deren Fortschreibung hätten Fernwanderungen für einen durchschnittlichen Beschäftigten einen Verlust bedeutet. Dieses Ergebnis war auch 1990 erwartbar. Es konnten aber nicht - wie damals zu erwarten war - im Bergbau, sondern im Verarbeitenden Gewerbe die höchsten Migrationsgewinne erzielt werden. Die geringsten Wanderungsüberschüsse hätten die im Baugewerbe Beschäftigten bei einem Umzug erzielen können, obwohl ihre Überschüsse bei der Simulation mit den Erwartungen des Jahres 1990 im Durchschnitt zu liegen schienen. Für die männlichen Angestellten ergaben sich von allen Industriebeschäftigten erwartungsgemäß die höchsten Erträge. 
Eine hohe Zeitpräferenzrate schmälert den Gewinn gegenüber einer niedrigen um maximal 7.000 DM. In einigen wenigen Fällen war die Zeitpräferenzrate ausschlaggebend dafür, ob eine Wanderung lohnend war oder nicht. Bei längeren Anpassungsverläufen ist ihr Anteil bedeutender als bei kürzeren. Die Differenz des Migrationsertrags zwischen einer niedrigen und hohen Rate ist etwa so hoch wie die Summe der regionalen Mietdifferenzen bis zur Angleichung.

Abschließend wurde in Abschnitt 3.2.3 die Bedeutung von regionalen Unterschieden in der Arbeitslosigkeit bewertet. Exemplarisch wurden nur die Jahre 1991 bis 1994 betrachtet. Im Durchschnitt ergab sich für eine durchschnittliche Erwerbsperson ein um 5.000 DM höherer Migrationsertrag als ohne Berücksichtigung der regionalen Arbeitslosenquoten. Da der Nettoertrag aus den regionalen Nettolohnunterschieden im vergleichbaren Zeitraum mehr als sechsmal so hoch waren, dürften für ostdeutsche Erwerbstätige die regional unterschiedlich hohen Arbeitslosenquoten weit weniger gewichtig gewesen sein als die Lohndifferentiale. Von größerer Bedeutung dürfte Arbeitslosigkeit nur für die tatsächlich davon Betroffenen gewesen sein.

\subsubsection{Schlußfolgerungen und Weiterführendes}

\subsection{Fazit aus der Modellrechnung}

Im folgenden soll auf Basis der obigen Berechnungen abgeschätzt werden, wie hoch die Zahl der Ostdeutschen gewesen sein könnte, für die Ende 1990 positive Erträge aus einem Umzug erwartbar gewesen waren. Diese Zahl informiert über das mögliche „ökonomisch begründete“ Wanderungspotential.

Migrationsanreize hatten unter den getroffenen Annahmen ausschließlich Erwerbspersonen. Für nicht erwerbstätige Personen ergeben sich nach der hier angewandten Methode keine Vorteile durch einen Umzug, sie zählen daher nicht zum Wanderungspotential.

Nach der bereits oben zitierten Umfrage erwarteten $14 \%$ der ostdeutschen Bevölkerung innerhalb von 3 Jahren eine ökonomische Anpassung zwischen den alten und neuen Ländern. Wichen die durchschnittlichen Erwartungen der Bevölkerung nicht von denen der Erwerbstätigen ab, hätten - übertragen auf die oben durchgeführte Berechnung - 14\% der Beschäftigten der neuen Länder $(=1,1$ Mio. Personen) damit gerechnet, daß sich für sie am Ende des Jahres 1990 eine Wanderung über Entfernungen von mehr als $100 \mathrm{~km}$ nicht lohnt. Lediglich über 
noch kürzere Wanderungsdistanzen hätte sich für sie ein kleiner Wanderungsüberschuß ergeben.

$42 \%$ der Ostdeutschen rechneten mit Angleichungen in 4 bis 5 Jahren. Übertragen auf die Beschäftigten ergibt sich daraus eine Zahl von 3,2 Mio. Personen, die höchstens bei einer Wanderung in einen Umkreis von bis zu $200 \mathrm{~km}$ mit positiven Erträgen gerechnet hätten.

Für die $20 \%$ der ostdeutschen Beschäftigten (=1,5 Mio.), die mit Anpassungen innerhalb von 6 bis 9 Jahren rechneten, wären Wanderungen über eine Entfernung bis zu $200 \mathrm{~km}$ ebenfalls lohnend gewesen. Bei weiteren Entfernungen war die Vorteilhaftigkeit davon abhängig, welcher Branche dieBeschäftigten angehörten und ob sie eher mit 6 oder mit 9 Jahren Angleichungszeit rechneten. Bei einer Entfernung von $1.000 \mathrm{~km}$ dürfte aber keiner mehr mit einem Wanderungsgewinn gerechnet haben.

Die restlichen 1,4 Mio. Beschäftigten, die eine Anpassungszeit von mindestens 10 Jahren erwarteten, hätten damit rechnen können, daß eine Wanderung über eine kurze oder mittlere Distanz grundsätzlich lohnend gewesen wären. Bei einer Fernwanderung dürften hingegen nur die $18 \%$ der Beschäftigten der Branchen Bergbau, Energiewirtschaft und Verarbeitendes Gewerbe mit Angleichungserwartungen von mindestens zehn Jahren bei Durchführung einer entsprechenden Rechnung einen Überschuß vermutet haben. Dies waren rund 0,5 Mio. Menschen. Bei noch differenzierterer Betrachtung dieses Personenkreises engt sich das Potential für Fernwanderungen abermals ein. Für die weiblichen Industriearbeiterinnen waren Fernwanderungen nicht lohnend. Damit entfallen von den 0,5 Mio. potentiellen Fernwanderern 130.000 Personen ${ }^{234}$. Für die 208.000 männlichen Arbeiter und die 62.000 weiblichen Angestellten ergaben sich nur bei einer sehr niedrigen Zeitpräferenzrate kleine Wanderungsgewinne, bei einer mittleren Rate aber Verluste. Damit verbleiben im wesentlichen die 100.000 männlichen Angestellten als potentielle Anwärter für eine Fernwanderung am Jahresende 1990.

Ein durchschnittlicher Beschäftigter hat bei allen untersuchten Erwartungen über den Anpassungszeitraum stets mit einem positiven Wanderungsertrag gerechnet, wenn er in die nähere Umgebung gezogen wäre. Das Potential für Nahwanderungen umfaßte mithin sämtliche 7,6 Mio. Beschäftigte. Das Potential für eine Entfernung von $200 \mathrm{~km}$ war um 14\% geringer, d.h. es umfaßte 6,5 Mio.

${ }^{234}$ Ausgewiesen wird in den Veröffentlichungen des Statistischen Bundesamts nur die Frauenquote insgesamt von 38,3\%. Hilfsweise wird hier davon ausgegangen, daß die Quote bei Angestellten und Arbeitern der durchschnittlichen entspricht. 
Erwerbstätige. Über eine mittlere Entfernung ergibt sich ein Potential zwischen $18 \%$ und $38 \%$ der Beschäftigten, d.h. die Bandbreite lag zwischen 1,37 Mio. und 2,89 Mio. Für eine Fernwanderung war die sich ergebende Zahl potentieller Migranten mit rund 100.000 deutlich geringer.

Die Zahl der potentiellen Migranten könnte sich durch die Berücksichtigung der Familienangehörigen der Migranten weiter erhöhen, sofern diese mit ihnen umziehen. Andererseits kann aber auch gerade die Berücksichtigung der Kosten und Erträge von Familienmitgliedern eine Wanderung unrentabel machen.

Das Wanderungspotential dürfte sich - zumindest für Nahwanderungen - um die rund 700.000 Arbeitslosen und etwa 100.000 Menschen in Fortbildungsmaßnahmen zum Kalkulationszeitpunkt erhöhen. Da sie höhere Wanderungserlöse erzielen konnten als ein durchschnittlicher Beschäftigter (vorausgesetzt es besteht ein Arbeitsplatzangebot bzw. eine größere Chance im Westen Arbeit zu finden), ist ihre Wanderungswilligkeit - auch für größere Distanzen - höher als die eines Beschäftigten.

$15 \%$ aller Erwerbstätigen waren über $55 \mathrm{Jahre} \mathrm{alt}^{235}$. Einige dieser Personen hätten aufgrund ihres Alters nur noch von einem Teil der regionalen Lohnunterschiede profitieren können, die Kosten wären ihnen jedoch im vollen Umfang entstanden. Ihre Wanderungsneigung ist mithin geringer als die durchschnittliche. Hinzu kommt, daß ältere Arbeitnehmer nur noch schwer einen neuen Arbeitsplatz finden.

Wanderungen sind nicht nur eine Folge von Wohlstandsunterschieden, sondern hängen auch davon ab, ob es den Umzugswilligen gelingt, den Wohlstandsunterschied für sich zu nutzen. Die Möglichkeiten zur Realisierung eines Migrationsüberschusses sind vielleicht objektiv nicht vorhanden, wenn z. B. kein Arbeitsplatz in einer Distanz gefunden werden kann, bei der eine Wanderung gerade noch einträglich wäre. Entfernungen von $100 \mathrm{~km}$ und weniger wären schon aus rein geographischen Gründen für einen Teil der Beschäftigten nicht realisierbar gewesen. Für einen weiteren Teil wäre das mögliche Ziel bei dieser Entfernung lediglich West-Berlin oder die ehemaligen Zonenrandgebiete. Die ehemaligen Zonenrandgebiet waren aber wirtschaftlich eher schwach entwickelt; dort gab es kaum freie Arbeitsplätze. War eine Nahwanderung dagegen potentiell möglich, kam als Alternative zum Umziehen aber auch Pendeln zu einem westdeutschen Arbeitsplatz mit Beibehaltung des Wohnsitzes in Ostdeutschland in Betracht.

${ }^{235}$ Diese Angabe stammt allerdings vom 30.9.1989 (vgl. Institut für Arbeitsmarkt- und Berufsforschung (1990), Tabelle 3). Der Anteil dürfte sich jedoch nicht wesentlich geändert haben. 
Selbst wenn die hier aufgezeigten Migrationsgewinne hätten realisiert werden können, wird der Betreffende aber nicht grundsätzlich einen Umzug durchführen. Einige Kosten und Erträge wurden in der Rechnung nicht berücksichtigt. Zudem können hier zwar berücksichtigte Kosten und Erträge im Einzelfall deutlich höher (oder niedriger) sein. Insbesondere die psychischen Kosten dürften individuell unterschiedlich ausfallen.

Ein identischer potentieller Migrationsertrag wird individuell unterschiedlich bewertet werden. Bei abnehmendem Grenznutzen des Einkommens hätte ein absolut gleicher Migrationsertrag bei den geringer Verdienenden eher einen Umzug ausgelöst als bei den Besserverdienenden. Ein „kleiner“ Migrationsertrag braucht noch keine Wanderungsbereitschaft auszulösen. Ein potentieller Migrant könnte zu dem Schluß kommen, daß seine Mühen nicht hinreichend entlohnt werden. Risikoaverse Menschen benötigen für das Eingehen eines Risikos (in diesem Fall der Umzug und gleichzeitiger Arbeitsplatzwechsel) einen Ausgleich.

Die Situation in Ostdeutschland weist im Vergleich zu sonstigen Wanderungsentscheidungen zudem Besonderheiten auf: Die Bevölkerung erlebte einen radikalen Wechsel des Systems und ihrer Lebensumstände. Diesen Wechsel galt es zu verkraften ${ }^{236}$. Das Erleben einer solchen Schocksituation könnte die Migrationsbereitschaft verringert haben, da in der Heimat zumindest noch Freunde und ein bekanntes Umfeld vorhanden waren - wenngleich auch dort grundlegende Veränderungen stattfanden. Die Vorstellung, die neuen Lebensumstände auch noch in einer anderen Region zu erleben, könnte manchen potentiellen Migranten abgeschreckt haben.

\subsection{War ein Umzug aus der Perspektive von 1994 noch lohnend?}

Abschließend wird berechnet, wie hoch die erwartbaren Migrationserträge fünf Jahre nach der Vereinigung waren und diskutiert, ob die zu diesem Zeitpunkt erwartbaren Überschüsse so hoch waren, daß weiterhin ein größeres Wanderungspotential bestanden hat.

Im Jahr 1994 erreichten die Ostlöhne circa 70 \% des Westlohnniveaus. Über das weitere Anpassungstempo bestand nach wie vor Unsicherheit. Aufgrund der Revidierung von Lohnabschlüssen im Jahr 1993 mußte zudem mit weiteren nachträglichen Änderungen von Tarifvereinbarungen gerechnet werden. Für die Berechnung der erwartbaren Erträge wird aufgrund dieser Unsicherheit davon

${ }^{236} \mathrm{Zu}$ den psychischen Folgen der Bewältigung einer solchen Schocksituation vgl. etwa Busch (1991), S. 222f. 
ausgegangen, daß - analog zur obigen Berechnung - bis zur wirtschaftlichen Angleichung weitere fünf bzw. zehn Jahre verstreichen.

In den folgenden Tabellen finden sich die Erträge durch regionale Lohn- und Mietdifferenzen, die sich Ende 1994 auf Basis des damaligen Kenntnisstands erwartbar waren. Die Berechnung erfolgt analog zu Abschnitt 3.2.1.3.2 gesplittet nach Branchen, Status und Geschlecht.

Wurde im Jahr 1994 vermutet, daß sich die Löhne und Mieten innerhalb der nächsten fünf Jahre angleichen, waren zwar regionale Einkommensdifferenzen von brutto circa 18.000 DM, netto jedoch nur von 6.000 DM erwartbar. Dieser potentielle Ertrag wäre von den weiteren Kosten und Erträgen eines Umzug aufgezehrt worden. Attraktiv wäre ein Umzug in die nähere Umgebung dann gewesen, wenn auch die bestehenden Unterschiede in der Wahrscheinlichkeit arbeitslos zu sein berücksichtigt werden. Wanderungen über $400 \mathrm{~km}$ und mehr wären aber weiterhin unattraktiv gewesen.

Tabelle 48: 1994 erwartbare Erträge aus regionalen Lohn- und Mietunterschiede nach Branchen bei einer Angleichung bis 1999

- Angaben in DM -

\begin{tabular}{|l|r|r|r|r|r|r|}
\hline & \multicolumn{3}{|c|}{$r=0,015$} & \multicolumn{3}{c|}{$r=0,05$} \\
\hline Branche & $\begin{array}{l}\text { Bruttoein- } \\
\text { kommens- } \\
\text { differenz }\end{array}$ & $\begin{array}{l}\text { Nettoein- } \\
\text { kommens- } \\
\text { differenz }\end{array}$ & $\begin{array}{l}\text { Differenz } \\
\text { Nettoeink. } \\
\text { nach Miet- } \\
\text { zahlung }\end{array}$ & $\begin{array}{l}\text { Bruttoein- } \\
\text { kommens- } \\
\text { differenz }\end{array}$ & $\begin{array}{l}\text { Nettoein- } \\
\text { kommens- } \\
\text { differenz }\end{array}$ & $\begin{array}{l}\text { Differenz } \\
\text { Nettoeink. } \\
\text { nach Miet- } \\
\text { zahlung }\end{array}$ \\
\hline Land- und Forstw. & 14.586 & 7.411 & 4.428 & 13.729 & 6.975 & 4.173 \\
\hline Bergbau & 10.457 & 4.522 & 1.539 & 9.812 & 4.246 & 1.444 \\
\hline Energiewirtsch. & 22.940 & 10.363 & 7.379 & 21.593 & 9.762 & 6.959 \\
\hline Verarb. Gewerbe & 32.125 & 14.915 & 11.932 & 30.167 & 14.020 & 11.217 \\
\hline Baugewerbe & 15.893 & 7.673 & 4.690 & 14.915 & 7.205 & 4.403 \\
\hline Handel & 25.152 & 12.560 & 9.577 & 23.620 & 11.799 & 8.997 \\
\hline Verkehr & 19.624 & 9.417 & 6.434 & 18.471 & 8.870 & 6.067 \\
\hline Dienstleistungen & 14.549 & 7.226 & 4.242 & 13.654 & 6.784 & 3.982 \\
\hline alle Branchen & 18.958 & 9.205 & 6.222 & 17.844 & 8.670 & 5.867 \\
\hline
\end{tabular}

Quelle: wie Tabelle 11, eigene Berechnungen

Im Vergleich zu den Ergebnissen auf Basis des Kenntnisstands von 1990 ergibt sich ein deutlich geringerer Ertrag. Der Migrationsüberschuß ist sogar kleiner als der niedrigste 1990 erwartbare Ertrag (3jährige Anpassungserwartungen im Jahr 1990). Die Migrationsanreize dürften somit deutlich zurückgegangen sein. Für einen durchschnittlichen Beschäftigten, der über einen sicheren Arbeitsplatz ver- 
fügte, war bei dem hier angenommenen Angleichungsverlauf keinen Wanderungsüberschuß erwartbar.

Wurde ein um fünf Jahre längere Dauer für die Anpassung vermutet, waren die im Jahr 1994 noch erwartbaren Migrationsüberschüsse höher als die 1990 erwartbaren Überschüsse unter Zugrundelegung einer 3jährigen wirtschaftlichen Angleichungsdauer, wie Tabelle 49 zeigt.

Die Nettoerträge aus den regionalen Lohndifferenzen hätten nach Mietzahlung über 20.000 DM betragen (Für Beschäftigte im Bergbau allerdings spiegelt die Bruttobetrachtung zwar einen Überschuß wider, netto nach Berücksichtigung der regionalen Mietpreisunterschiede hingegen ist ein solcher Überschuß nicht mehr erkennbar). In den Branchen Land- und Forstwirtschaft, Energiewirtschaft, Verarbeitendes Gewerbe, Handel und Verkehr ergeben sich auch nach Abzug der Umzugskosten noch Überschüsse. Werden aber psychische Kosten analog zu Abschnitt 3.2.2.1.2.2 berücksichtigt, errechnet sich nur noch für einen Umzug, dessen Distanz $400 \mathrm{~km}$ nicht übertrifft, in den erwähnten Branchen ein geringer Überschuß.

Tabelle 49: 1994 erwartbare Erträge aus regionalen Lohn- und Mietunterschiede nach Branchen bei einer Angleichung bis 2004

- Angaben in DM -

\begin{tabular}{|l|r|r|r|r|r|r|}
\hline & \multicolumn{3}{|c|}{$\mathrm{r}=0,015$} & \multicolumn{3}{c|}{$\mathrm{r}=0,05$} \\
\hline & $\begin{array}{l}\text { Bruttoein- } \\
\text { kommens- } \\
\text { differenz }\end{array}$ & $\begin{array}{l}\text { Nettoein- } \\
\text { kommens- } \\
\text { differenz }\end{array}$ & $\begin{array}{l}\text { Differenz } \\
\text { Nettoeink. } \\
\text { nach Miet- } \\
\text { zahlung }\end{array}$ & $\begin{array}{l}\text { Bruttoein- } \\
\text { differenz- } \\
\text { differens- }\end{array}$ & $\begin{array}{l}\text { Nettoein- } \\
\text { kommens- } \\
\text { differenz }\end{array}$ & $\begin{array}{l}\text { Differenz } \\
\text { Nettoeink. } \\
\text { nach Miet- } \\
\text { zahlung }\end{array}$ \\
\hline Land- und Forstw. & 51.492 & 26.474 & 18.727 & 45.600 & 23.455 & 16.585 \\
\hline Bergbau & 18.926 & 8.020 & 274 & 16.779 & 7.125 & 256 \\
\hline Energiewirtsch. & 80.983 & 36.406 & 28.659 & 71.716 & 32.313 & 25.443 \\
\hline Verarb. Gewerbe & 82.967 & 38.184 & 30.437 & 73.736 & 34.012 & 27.143 \\
\hline Baugewerbe & 36.382 & 17.422 & 9.676 & 32.561 & 15.615 & 8.745 \\
\hline Handel & 64.960 & 32.327 & 24.581 & 57.732 & 28.759 & 21.889 \\
\hline Verkehr & 69.276 & 33.140 & 25.393 & 61.349 & 29.399 & 22.529 \\
\hline Dienstleistungen & 33.304 & 16.426 & 8.679 & 29.806 & 14.719 & 7.850 \\
\hline alle Branchen & 66.926 & 32.403 & 24.656 & 59.268 & 28.742 & 21.873 \\
\hline
\end{tabular}

Quelle: wie Tabelle 12, eigene Berechnung 
Tabelle 50: 1994 erwartbare Erträge aus regionalen Lohn- und Mietunterschieden nach Status und Geschlecht bei einer Angleichung bis zum Jahr 1999

- Angaben in DM -

\begin{tabular}{|l|r|r|r|r|r|r|}
\hline & \multicolumn{3}{|c|}{$\mathrm{r}=0,015$} & \multicolumn{3}{c|}{$\mathrm{r}=0,05$} \\
\hline & $\begin{array}{l}\text { Bruttoein- } \\
\text { kommens- } \\
\text { differenz }\end{array}$ & $\begin{array}{l}\text { Nettoein- } \\
\text { kommens- } \\
\text { differenz }\end{array}$ & $\begin{array}{l}\text { Diff. der } \\
\text { Nettoeink. } \\
\text { nach Miet- } \\
\text { zahlung }\end{array}$ & $\begin{array}{l}\text { Bruttoein- } \\
\text { kommens- } \\
\text { differenz }\end{array}$ & $\begin{array}{l}\text { Nettoein- } \\
\text { kommens- } \\
\text { differenz }\end{array}$ & $\begin{array}{l}\text { Diff. der } \\
\text { Nettoeink. } \\
\text { nach Miet- } \\
\text { zahlung }\end{array}$ \\
\hline Arbeiter & 18.615 & 9.092 & 6.109 & 17.521 & 8.563 & 5.761 \\
\hline Angestellte & 32.258 & 13.721 & 10.738 & 30.491 & 12.986 & 10.184 \\
\hline Arbeiter, männl. & 19.502 & 9.379 & 6.396 & 18.356 & 8.834 & 6.032 \\
\hline Angestellte, männl. & 33.646 & 13.734 & 10.751 & 31.769 & 12.985 & 10.183 \\
\hline Arbeiter, weibl. & 13.882 & 7.097 & 4.114 & 13.066 & 6.680 & 3.878 \\
\hline Angestellte, weibl. & 20.215 & 9.600 & 6.617 & 19.027 & 9.042 & 6.240 \\
\hline
\end{tabular}

Quelle: wie Tabelle 13, eigene Berechnungen

Auch die Industriebeschäftigten konnten im Jahr 1994 nur noch mit deutlich niedrigeren Erträgen als 1990 rechnen, wie Tabelle 50 zeigt.

Ein Umzug über eine größere Entfernung wäre für keinen der Industriebeschäftigten lohnend gewesen, wenn weitere Kosten und Erträge berücksichtigt werden. Dies gilt auch dann, wenn zusätzliche Überschüsse von mehreren tausend DM infolge der regional unterschiedlichen Arbeitslosigkeit auf den Migrationsertrag einkalkuliert werden. Dagegen errechnet sich für einen Umzug in die nähere Umgebung noch ein kleiner Wanderungsgewinn.

Tabelle 51: 1994 erwartbare Erträge aus regionalen Lohn- und Mietunterschieden nach Status und Geschlecht bei einer Angleichung bis zum Jahr 2004

- Angaben in DM -

\begin{tabular}{|c|c|c|c|c|c|c|}
\hline & \multicolumn{3}{|c|}{$r=0,015$} & \multicolumn{3}{|c|}{$r=0,05$} \\
\hline & \begin{tabular}{|l|} 
Bruttoein- \\
kommens- \\
differenz
\end{tabular} & $\begin{array}{l}\text { Nettoein- } \\
\text { kommens- } \\
\text { differenz }\end{array}$ & $\begin{array}{l}\text { Diff. der } \\
\text { Nettoeink. } \\
\text { nach Miet- } \\
\text { zahlung }\end{array}$ & $\begin{array}{l}\text { Bruttoein- } \\
\text { kommens- } \\
\text { differenz }\end{array}$ & $\begin{array}{l}\text { Nettoein- } \\
\text { kommens- } \\
\text { differenz }\end{array}$ & \begin{tabular}{|l|} 
Diff. der \\
Nettoeink. \\
nach Miet- \\
zahlung
\end{tabular} \\
\hline Arbeiter & 65.709 & 31.998 & 24.251 & 58.190 & 28.380 & 21.511 \\
\hline Angestellte & 105.573 & 45.769 & 38.022 & 93.984 & 40.820 & 33.951 \\
\hline Arbeiter, männl. & 68.842 & 33.006 & 25.259 & 60.964 & 29.279 & 22.410 \\
\hline Angestellte, männl. & 115.290 & 48.257 & 40.510 & 102.633 & 43.074 & 36.205 \\
\hline Arbeiter, weibl. & 49.002 & 25.462 & 17.715 & 43.395 & 22.562 & 15.693 \\
\hline Angestellte, weibl. & 71.369 & 33.773 & 26.026 & 63.203 & 29.963 & 23.094 \\
\hline
\end{tabular}

Quelle: wie Tabelle 14, eigene Berechnungen 
Tabelle 51 zeigt, daß die erwartbaren Erträge aus einem Umzug bei einer um weitere fünf Jahre längeren Angleichungsdauer, netto nach Mietzahlung rund viermal so hoch sind wie bei einer kürzeren Angleichungsdauer.

Dauert die Lohnanpassung bis 2004 belaufen sich die auf 1994 abgezinsten kumulierten Bruttoeinkommensdifferenzen für die Beschäftigten in der Industrie auf bis zu 106.000 DM. Der Bruttoertrag der Arbeiter beträgt knapp zwei, der der Angestellten gut zwei Bruttojahreseinkommen eines ostdeutschen Industriearbeiters (bzw. -angestellten). Die Nettoeinkommensdifferenzen nach Mietzahlungen liegen zwischen 15.000 DM und 40.000 DM. Werden die regional unterschiedlichen Wahrscheinlichkeiten arbeitslos zu werden berücksichtigt, sind die erwartbaren Erträge noch höher. Bei dem unterstellten Lohnanpassungsverlauf hätte ein Umzug für alle Industriebeschäftigten im Jahr 1994 über eine kurze oder mittlere Distanz einen Überschuß bedeutet, selbst bei Berücksichtigung der psychischen Kosten. Eine Fernwanderung wäre hingegen nur für männliche Angestellte attraktiv gewesen.

Fünf Jahre nach der Grenzöffnung der ehemaligen DDR waren die potentiellen Wanderungsgewinne, die bei einem Umzug mit gleichzeitigem Arbeitsplatzwechsel aus den neuen in die alten Länder erwartbar waren, erheblich geschrumpft. Daher dürfte das zu diesem Zeitpunkt noch vorhandene Wanderungspotential deutlich geringer gewesen sein als 1990. Zudem dürften diejenigen, deren Wanderungsneigung aufgrund bestehender Einkommensunterschiede besonders hoch war, einen Umzug bereits realisiert haben. Im Jahr 1994 war daher nicht mehr mit vielen Umzügen bis dahin immobiler Personen zu rechnen. $\mathrm{Zu}$ einer Wanderung entschlossen haben dürften sich im wesentlichen nur noch Personen, an deren persönlicher oder beruflicher Situation sich etwas geändert hat.

\subsection{Ausmaß der Wanderungen zwischen den neuen und alten Bun- desländern}

Oben wurde gezeigt, daß 1990 für alle ostdeutschen Arbeitnehmer bei einem Umzug in die alten Länder Wanderungsüberschüsse erwartbar waren - falls sie in ihrer näheren Umgebung einen westdeutschen Standards entsprechenden Arbeitsplatz hätten finden können. Bei Umzügen über eine weite Entfernung dagegen wäre nur für wenige Beschäftigte ein positiver Ertrag erwartbar gewesen. Bei diesen Ergebnissen könnte es naheliegen zu folgern, daß im Prinzip jeder Beschäftigte versucht haben müßte, in den alten Ländern einen Arbeitsplatz zu finden, der zudem möglichst nah an seinem bisherigen Wohnsitz sein sollte. 
Im folgenden wird dargestellt, in welchem Umfang tatsächlich Wanderungen zwischen den alten und neuen Bundesländern stattfanden. Aufgrund fehlender Daten kann eine so weit wie in Kapitel 3.2 getriebene Differenzierung allerdings nicht vorgenommen werden. Der Verfasserin lagen lediglich Daten über Wanderungsbewegungen gesplittet nach Bundesländern, Alter und Geschlecht vor. Untersuchungen etwa darüber, ob die Migranten erwerbstätig oder arbeitslos waren oder welcher Branche sie angehörten, waren daher nicht möglich. Auch eine Analyse des Einflusses der Wanderungsdistanz konnte nicht durchgeführt werden. Anhaltspunkte über die Entfernung der Zielregion liefert aber die Auswertung der Wanderungen nach Bundesländern. Über die Wanderungsgründe können aus zwei Befragungen Hinweise gewonnen werden.

\subsubsection{Entwicklung der Wanderungen}

Zunächst setzten immense Wanderungsbewegungen ein, nachdem die ehemalige DDR-Grenze im Jahr 1989 durchlässig wurde. Während im Jahr zuvor lediglich 40.000 Menschen abwanderten, siedelten im Jahr 1989 insgesamt 388.400 Personen aus der DDR in die alte Bundesrepublik über. Diese Anzahl wurde im Jahr 1990 mit rund 395.300 Abwanderern noch übertroffen. In diesem Jahr erreichten die Fortzüge ihren Höhepunkt. Spätestens mit der Vereinigung am 3. Oktober 1990 entfielen weitgehend die politische Gründe für eine Abwanderung aus den neuen Bundesländern. Nach der Vereinigung waren die Abwanderungen aus den neuen Ländern stark rückläufig; im Jahr 1992 hatten sich die Wanderungen gegenüber 1990 bereits halbiert. Seit dem Jahr 1995 zeichnet sich jedoch wieder eine leichte Zunahme der Abwanderungen ab.

Seit der Grenzöffnung bis einschließlich September 1996 sind knapp 1,9 Mio. Menschen aus den neuen in die alten Bundesländer gezogen, wie Tabelle 52: Wanderungen zwischen den neuen und alten Bundesländern zeigt. Damit hat etwa jeder neunte Einwohner die neuen Bundesländer im Betrachtungszeitraum verlassen.

Die Zuwanderungen aus den alten in die neuen Bundesländer entwickelten sich gegenläufig zu den Abwanderungen. Während 1989 lediglich 5.000 Menschen zuzogen, waren es 1995 bereits 143.000. Bis zum Ende des drittes Quartal 1996 sind seit der Wende insgesamt 740.000 Personen aus Westdeutschland zugewandert. Der Wanderungssaldo sank von $383.000 \mathrm{im}$ Jahr 1989 auf $25.000 \mathrm{im}$ Jahr 1995. Seit der Grenzöffnung bis zum September 1996 betrug der Bevölkerungsverlust infolge von Wanderungen zwischen den alten und neuen Bundesländern über 1 Mio. 
Tabelle 52: Wanderungen zwischen den neuen und alten Bundesländern

\begin{tabular}{|l|r|r|r|r|r|r|}
\hline Jahr & $\begin{array}{c}\text { Fortzüge aus } \\
\text { den neuen } \\
\text { Ländern in } \\
\text { Tsd. }\end{array}$ & $\begin{array}{c}\text { Veränd. } \\
\text { geg. } \\
\text { Vorj. in } \\
\%\end{array}$ & $\begin{array}{c}\text { Zuzüge in } \\
\text { die neuen } \\
\text { Länder in } \\
\text { Tsd. }\end{array}$ & $\begin{array}{c}\text { Veränd. } \\
\text { geg. } \\
\text { Vorj. in } \\
\%\end{array}$ & $\begin{array}{c}\text { Wande- } \\
\text { rungssal- } \\
\text { do in Tsd. }\end{array}$ & $\begin{array}{c}\text { Veränd. } \\
\text { geg. } \\
\text { Vorj. in } \\
\%\end{array}$ \\
\hline 1989 & 388,4 & & 5,1 & & $-383,3$ & \\
\hline 1990 & 395,3 & 1,8 & 36,2 & 609,8 & $-359,1$ & $-6,3$ \\
\hline 1991 & 249,7 & $-36,8$ & 80,3 & 121,8 & $-169,4$ & $-52,8$ \\
\hline 1992 & 199,2 & $-20,2$ & 111,3 & 38,6 & $-87,9$ & $-48,1$ \\
\hline 1993 & 172,4 & $-13,4$ & 119,1 & 7,0 & $-53,3$ & $-39,4$ \\
\hline 1994 & 163,0 & $-5,5$ & 135,8 & 14,0 & $-27,2$ & $-48,9$ \\
\hline 1995 & 168,3 & 3,3 & 143,1 & 5,4 & $-25,2$ & $-7,3$ \\
\hline $1 .-3$. Quartal 1996 & 125,3 & $\left.0,2^{*}\right)$ & 108,8 & $\left.3,2^{*}\right)$ & $-16,5$ & $\left.-15,8^{*}\right)$ \\
\hline 1989 -Sept.1996 & $1.861,6$ & & 739,7 & & $-1.121,9$ & \\
\hline
\end{tabular}

*) geg. anteiligem Vorjahreszeitraum

Quelle: Statistisches Bundesamt (1994c) S. 14, Fleischer/Sommer (1995), S. 33, Statistisches Bundesamt (1996 und 1997), Tabelle 1.7, S. 8f

Bezüglich der Abwanderungshäufigkeit unterschieden sich die einzelnen neuen Bundesländer nur wenig voneinander; lediglich die Ost-Berliner sind weit überdurchschnittlich abgewandert, wie Tabelle 53 zeigt:

Tabelle 53: Fort- und Zuzüge zwischen den neuen und den alten Länder je 1.000 der Bevölkerung

\begin{tabular}{|l|r|r|r|r|r|r|r|r|r|r|}
\hline & \multicolumn{9}{|c|}{ Fortzüge aus den neuen Länder } & \multicolumn{4}{|c|}{ Zuzüge in die neuen Ländern } \\
\hline & 1991 & 1992 & 1993 & 1994 & 1995 & 1991 & 1992 & 1993 & 1994 & 1995 \\
\hline Brandenburg & 15,6 & 14,7 & 12,1 & 10,2 & 10,9 & 4,6 & 6,0 & 7,7 & 10,2 & 11,2 \\
\hline $\begin{array}{l}\text { Mecklenburg- } \\
\text { Vorpommern }\end{array}$ & 16,9 & 13,8 & 12,4 & 11,4 & 11,4 & 4,3 & 6,6 & 7,3 & 7,8 & 8,8 \\
\hline Sachsen & 15,2 & 11,2 & 9,0 & 8,3 & 8,2 & 4,6 & 6,1 & 6,1 & 6,6 & 6,9 \\
\hline Sachsen-Anh. & 16,6 & 12,0 & 10,0 & 10,1 & 10,2 & 4,4 & 5,5 & 6,4 & 6,9 & 7,3 \\
\hline Thüringen & 16,0 & 12,2 & 10,2 & 9,7 & 10,1 & 5,4 & 7,9 & 6,9 & 7,2 & 7,0 \\
\hline Ost-Berlin & 11,9 & 14,0 & 17,7 & 19,7 & 22,1 & 9,7 & 15,5 & 17,8 & 21,5 & 22,7 \\
\hline Neue Ld. insg. & 15,6 & 12,6 & 11,0 & 10,4 & 10,8 & 5,1 & 7,1 & 7,6 & 8,7 & 9,2 \\
\hline
\end{tabular}

Quelle: Statistisches Bundesamt (1997), Tabelle 1.7, S. 8, eigene Berechnungen

Während die Anzahl der Fortzüge pro 1.000 Einwohner von knapp 16 auf gut 10 im Durchschnitt der neuen Länder sank, stieg sie in Ost-Berlin von 12 auf über 22. Dauerhaft über dem Durchschnitt lag die Abwanderung aus MecklenburgVorpommern, während die Quote der Fortzüge aus Sachsen stets unterdurchschnittlich war. Trotzdem stammten aus Sachsen die meisten Wanderer, da Sachsen das bevölkerungsreichste der neuen Bundesländer ist. Bezogen auf die 
Anzahl der Einwohner war Ost-Berlin die beliebteste Zuwanderungsregion, gefolgt von Brandenburg.

Zur Bestimmung des Ziels der ostdeutschen Wanderer wurde das alte Bundesgebiet in vier Regionen eingeteilt, und zwar in die Regionen Nord, Mitte, Süd ${ }^{237}$ und West-Berlin. Tabelle 54 zeigt die Verteilung der ostdeutschen und zum Vergleich der westdeutschen Wanderungen und der Wohnbevölkerung nach Regionen der alten Länder:

Tabelle 54: Verteilung der Wanderungen auf Regionen der alten Länder - Anteile in \% von Wanderern der jeweiligen Herkunftsregion -

\begin{tabular}{|l|r|r|r|r|r|r|r|r|}
\hline & \multicolumn{4}{|c|}{ Zielgebiet 1991 } & \multicolumn{4}{c|}{ Zielgebiet 1994 } \\
\hline Herkunftsregion & Nord & Mitte & Süd & $\begin{array}{c}\text { Berlin- } \\
\text { West }\end{array}$ & Nord & Mitte & Süd & $\begin{array}{c}\text { Berlin- } \\
\text { West }\end{array}$ \\
\hline neue Länder ohne Berlin-Ost & 26 & 33 & 40 & 2 & 30 & 32 & 33 & 5 \\
\hline alte Länder & 28 & 40 & 27 & 6 & 31 & 39 & 26 & 4 \\
\hline $\begin{array}{l}\text { nachrichtlich: } \\
\text { Anteil Einw. der Regionen } \\
\text { an Einw. alte Länder insg. }\end{array}$ & 19 & 44 & 33 & 4 & & & & \\
\hline
\end{tabular}

Quelle: Statistisches Jahrbuch 1993 und 1996, eigene Berechnungen

In die Regionen Nord, Mitte und Süd wanderten im Jahr 1994 jeweils ein knappes Drittel der ostdeutschen Wanderer, nach West-Berlin 6\%. Die Verteilung der Wanderungen wich deutlich von der Einwohnerverteilung in dem Gebiet der früheren Bundesrepublik ab, wo circa 19\% der aller Einwohner im Norden, $44 \%$ in der Mitte, 33\% im Süden und 4\% in West-Berlin lebten. Im Vergleich zur Verteilung der Einwohner waren die Wanderungen Ostdeutscher in die nördlichen Bundesländer überdurchschnittlich hoch. Diese Tendenz verstärkte sich im Laufe der Zeit, der Wanderungsanteil stieg von 26\% im Jahr 1991 auf $30 \%$ im Jahr $1994^{238}$. Ebenfalls überdurchschnittlich war im Jahr 1991 der Anteil

$\begin{array}{ll}{ }^{237} \text { Nord: } & \text { Hamburg, Schleswig-Holstein, Bremen, Niedersachsen } \\ \text { Mitte: } & \text { Nordrhein-Westfalen, Saarland, Rheinland-Pfalz, Hessen } \\ \text { Süd: } & \text { Baden-Württemberg, Bayern }\end{array}$

${ }^{238}$ Eine Umfrage, die um die Jahreswende 1989/1990 durchgeführt wurde, zeigt, daß die bis zu diesem Zeitpunkt übergesiedelten Menschen in den nördlichen Bundesländern schlechtere Erfahrungen machten als in den übrigen alten Bundesländern. Nach dieser Umfrage haben $60 \%$ der Übersiedler durchweg positive Erfahrungen in den alten Ländern gemacht, aber nur $50 \%$ der in den Norden übergesiedelten. Insgesamt positive, aber auch negative Erfahrungen machten $39 \%$ aller Befragten und $47 \%$ der in den Norden übergesiedelten Menschen. $3 \%$ dieser Übersiedler machten insgesamt eher negative Erfahrungen, während es bei insgesamt nur 1 \% war (vgl. Köhler (1990), S. 427). Diese Erfahrungen spiegeln sich jedoch nicht in den Wanderungsdaten wider, die überdurchschnittliche 
der ostdeutschen Abwanderungen in den Süden mit $40 \%$, er sank aber bis zum Jahr 1994 auf den Bevölkerungsanteil dieser Region. Durchgängig unterdurchschnittlich waren die Wanderungen in die Mitte des früheren Bundesgebiets, der Anteil betrug $32 \%$ bzw. 33\%.

Das ostdeutschen Wanderungsverhalten unterschied sich deutlich von dem Wanderungsverhalten innerhalb der alten Länder. Im früheren Bundesgebiet waren die Wanderungen in die nördliche Region mit einem Anteil von etwa 30\% zwar ebenfalls überdurchschnittlich im Vergleich zu der Verteilung der Einwohner, die Wanderungen in den Süden waren jedoch unterdurchschnittlich. Die Wanderungen in die mittlere Region waren mit einem Anteil von circa $40 \%$ höher als die aus den neuen Ländern (33\%), sie waren jedoch ebenfalls niedriger als der Einwohneranteil.

Die Ziele der ostdeutschen Wanderer unterschieden sich deutlich voneinander je nachdem aus welchem Bundesland die Migranten stammten. In Abbildung 38 ist die Verteilung der Abwanderungen aus den fünf neuen Ländern auf die Regionen der alten Länder exemplarisch für das Jahr 1994 dargestellt ${ }^{239}$.

Der Anteil der Umzüge in die Mitte des früheren Bundesgebiets war durchgängig für alle neuen Bundesländer niedriger als der Bevölkerungsanteil dieser Region. Lediglich der Thüringer Anteil erreichte nahezu den Wert des Bevölkerungsanteils der Region an den Einwohnern insgesamt. Der Wanderungsanteil in die nördlichen alten Bundesländer lag nur bei den Thüringern und den Sachsen unter dem Anteil der Bevölkerung. Bezüglich der südlichen alten Länder verhielt es sich entgegengesetzt: Nur der Anteil der Thüringer und der Sachsen war überdurchschnittlich.

Nach Berlin waren die Wanderungen aus allen neuen Bundesländern unterdurchschnittlich - mit Ausnahme von Brandenburg, bei denen Umzüge ins angrenzende West-Berlin überdurchschnittlich beliebt waren. Brandenburg grenzt nur zu einem kleinen Teil an die alten Ländern an, die zu überwindenden Distanzen bei einem Umzug in das übrige frühere Bundesgebiet sind relativ hoch. Nach Baden-Württemberg, Rheinland-Pfalz und ins Saarland beträgt die Distanz von hier aus mehr als $500 \mathrm{~km}$, teilweise auch $1.000 \mathrm{~km}$.

Wanderungen in die nördlichen Bundesländer zeigen. Wären die Erfahrungen im Norden erfreulicher gewesen, wären die Wanderungen in diese Region u.U. noch höher gewesen.

${ }^{239}$ Die Struktur der Abwanderungen in die Regionen der alten Länder hat sich im Laufe der Zeit nur wenig geändert. 
Abbildung 38: Verteilung der Abwanderungen aus den neuen Ländern auf Regionen der alten Länder

- Abwanderungen in die Regionen an Abwanderungen in alte Länder insgesamt in \%-

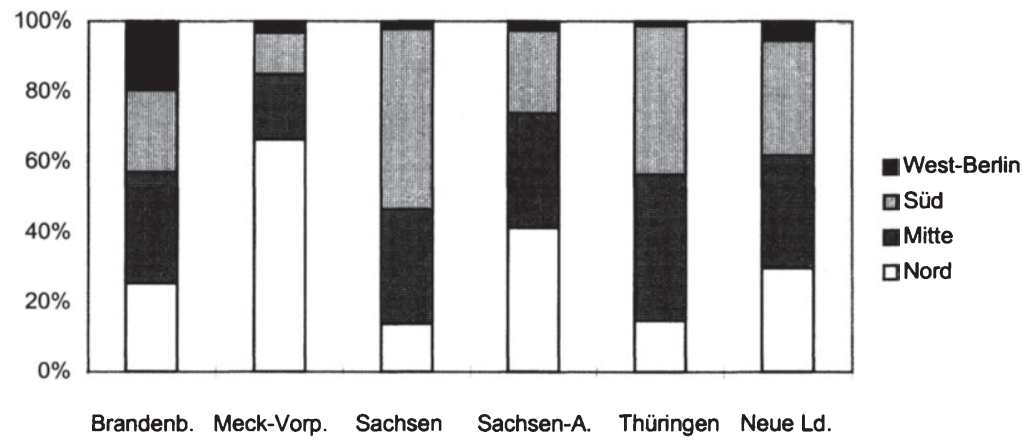

Quelle: Statistisches Jahrbuch (1996), eigene Berechnungen

Auffällig ist auch die Verteilung der mecklenburg-vorpommerischen Umzüge. Die Abwanderer aus dem nördlichsten der neuen Bundesländer hat es überwiegend in den Norden der alten Länder gezogen. Für die Mecklenburg-Vorpommerer beträgt die Entfernung in südliche und westliche Bundesländer häufig 500 $\mathrm{km}$ und mehr. Die Sachsen - als südlichstes Bundesland neben Thüringen - zog es hauptsächlich in den Süden des alten Bundesgebiets. Die Umzüge der Sachsen-Anhaltiner ins angrenzende Niedersachsen waren mit einem Wanderungsanteil von $31 \%$ (alle neuen Bundesländer $17 \%$ ) überdurchschnittlich.

Wesentlich für die Vorteilhaftigkeit einer Wanderung ist das Lebensalter. Über die Altersstruktur der Migranten lagen der Verfasserin nur Werte für das Jahr 1989 und den Anfang des Jahres 1990 vor $^{240}$. Die Altersstruktur zeigt Tabelle 55.

${ }^{240}$ Für 1989 wiesen sowohl das Statistische Jahrbuch der DDR (1990) als auch das Statistische Jahrbuch der Bundesrepublik (1991) 296.958 Wegzüge aus der DDR aus. 255.756 davon waren Wanderungen in die alten Bundesländer. Die Zahlen wurden später erheblich nach oben korrigiert. Die Auswertung nach Alter der Migranten ist daher nur bedingt aussagekräftig. In späteren Jahren erfolgte kein Ausweis nach Alter der Migranten mehr. 
Tabelle 55: Wanderungen 1989 in die alten Bundesländer nach Alter

\begin{tabular}{|l|r|r|r|r|}
\hline Altersgruppe & $\begin{array}{c}\text { Anzahl } \\
\text { Wanderer } \\
1989\end{array}$ & $\begin{array}{c}\text { Anteil Abwan-de- } \\
\text { rer an Wand. 1989 } \\
\text { insg. in \% }\end{array}$ & $\begin{array}{c}\text { Anteil Abwan-de- } \\
\text { rer an Wand. } \\
01 .-02.1990 \text { in \% }\end{array}$ & $\begin{array}{c}\text { Ant. der Altersgr. } \\
\text { an Bevölk. insg. in } \\
\%\end{array}$ \\
\hline unter 15 & 59.463 & 23,2 & 21,0 & 19,5 \\
\hline $15-17$ & 8.036 & 3,1 & 2,2 & 3,2 \\
\hline $18-25$ & 49.490 & 19,4 & 24,9 & 10,2 \\
\hline $25-40$ & 94.984 & 37,1 & 37,0 & 23,6 \\
\hline $40-50$ & 26.524 & 10,4 & 8,5 & 12,1 \\
\hline $50-60 / 65^{1}$ & 9.816 & 3,8 & 3,4 & 15,2 \\
\hline $60 / 65$ und älter & 2 & 2,9 & 2,1 & 16,2 \\
\hline insgesamt & 255.756 & 100,0 & 100,0 & 100,0 \\
\hline
\end{tabular}

1 Frauen bis unter 60 , Männer bis unter 65

2 Frauen über 59, Mănner über 64

Quelle: Statistisches Jahrbuch der DDR (1990), Grundmann (1990), S. 1423, eigene Berechnungen

Ausgewiesen werden neben den absoluten Wanderungszahlen aus dem Jahr 1989 die Anteile der Wanderer einer Altersklasse an den Wanderern insgesamt in dem Jahr 1989 und in den ersten beiden Monaten des Jahres 1990, sowie der Einwohneranteil in einer Altersklasse an den Einwohnern insgesamt.

Menschen, die jünger als 40 Jahre waren, sind überdurchschnittlich häufig gewandert. Am stärksten war die Wanderungsneigung in der Gruppe der 18 bis 25jährigen, ihr Anteil an den Wanderern übersteigt ihren Anteil an der Bevölkerung um nahezu das Doppelte. Auch die 25 bis 40jährigen weisen eine stark überdurchschnittliche Wanderungshäufigkeit auf. Kinder und Jugendliche unter 15 Jahren sind nur leicht überdurchschnittlich umgezogen. Angehörige dieser Altersklasse treffen in der Regel keine eigenen Umzugsentscheidungen, sondern ihre Eltern entscheiden für sie. Ihre Eltern gehören im wesentlichen der Gruppe der 18 bis 40jährigen an. Da die relative Häufigkeit der Kinder zu wandern geringer war als die der Gruppe der 18 bis 40jährigen, ist zu vermuten, daß Personen mit Kindern seltener abgewandert sind als ihre kinderlosen Altersgenossen.

Lediglich 7.500 Personen im Rentenalter sind umgezogen. Während jeder sechste Einwohner im entsprechenden Alter war, gehörte nur jeder fünfzigste Wanderer dieser Altersgruppe an. Für die Rentner entfielen die wichtigsten ökonomischen Gründe für eine Wanderung, da sie Gewinne aus regionalen Lohndifferenzen in der Regel nicht mehr realisieren konnten. Ferner waren sie nicht von Arbeitslosigkeit betroffen bzw. bedroht. Darüber hinaus konnten sie bereits vor dem Jahr 1989 aus der DDR ausreisen, so daß sich für sie durch die Grenzöffnung in Hinsicht auf ihre Wanderungsmöglichkeiten nur wenig änderte. 
Menschen, die das Rentenalter noch nicht erreicht hatten, aber mindestens 50 Jahre alt waren, sind unterdurchschnittlich häufig gewandert. Sie dürften größere Schwierigkeiten als junge Menschen bei der Arbeitsplatzsuche gehabt haben. Lohndifferenzen in vollem Umfang konnten sie zum Teil wegen des vorhergehenden Eintritts in den Ruhestand nicht mehr voll realisieren. Zudem waren ihre psychischen Kosten durch einen Umzug sowie ihre Umzugskosten tendenziell höher als die jüngerer Menschen ${ }^{241}$.

Die Altersstruktur der Wanderer unterschied sich im Jahr 1989 und zu Beginn des Jahres 1990 wenig voneinander, lediglich der Anteil der jüngeren Wanderer gegenüber den älteren ist tendenziell noch gestiegen. Auch die Ergebnisse der Befragungen im Rahmen des Sozialökonomische Panels (SOEP) ${ }^{242}$ weisen darauf hin, daß jüngere Menschen im Vergleich zu älteren deutlich häufiger gewandert $\sin ^{243}$.

Die Auswertungen des SOEP zeigen ferner, daß Männer eine etwas höhere Wanderungsbereitschaft als Frauen aufwiesen. Aus dem 4. Quartal 1990 liegen statistische Daten über die gesamten Wanderungen nach Geschlecht vor.

Tabelle 1 : Wanderungen nach Geschlecht im 4. Quartal 1990

- Angaben in \% -

\begin{tabular}{|l|r|r|r|r|r|r|}
\hline & \multicolumn{1}{|c|}{$\begin{array}{c}\text { Anteil } \\
\text { Männer an } \\
\text { Wohnbe- }\end{array}$} & \multirow{2}{*}{$\begin{array}{c}\text { Anteil } \\
\text { Männer an } \\
\text { Wälkerung }\end{array}$} & & \multicolumn{2}{|l|}{ Abwanderungs-quote*) } & \multicolumn{2}{|l|}{$\begin{array}{l}\text { Zuwanderungs- } \\
\text { quote**) }\end{array}$} \\
\cline { 5 - 8 } & & & \multicolumn{1}{c|}{ insg. } & \multicolumn{1}{c|}{ männl. } & \multicolumn{1}{c|}{ insg. } & männl. \\
\hline Brandenburg & 48 & 51 & 0,26 & 0,28 & 0,22 & 0,24 \\
\hline Mecklenb.-Vorp. & 49 & 53 & 0,30 & 0,33 & 0,20 & 0,21 \\
\hline Sachsen & 47 & 55 & 0,32 & 0,37 & 0,11 & 0,12 \\
\hline Sachsen-Anhalt & 48 & 53 & 0,34 & 0,38 & 0,15 & 0,16 \\
\hline Thüringen & 48 & 54 & 0,37 & 0,42 & 0,13 & 0,14 \\
\hline Berlin-Ost & 48 & 50 & 0,23 & 0,25 & 0,33 & 0,38 \\
\hline neue Ld. insg. & 48 & 53 & 0,31 & 0,35 & 0,17 & 0,18 \\
\hline
\end{tabular}

*) Abwanderungsquote $=($ Fortzüge aus den neuen in die alten Länder*100)/ Einwohner in den neuen Ländern

**) Zuwanderungsquote $=($ Zuzüge aus den alten in die neuen Ländern*100) $/$ Einwohner in den neuen Ländern

Quelle: Statistisches Bundesamt (1990) und (1991), eigene Berechnungen

${ }^{241}$ Vgl. hierzu Kapitel 3.1.2.

${ }^{242}$ Das SOEP ist eine "empirische Langzeituntersuchung zur repräsentativen Erfassung und Interpretation des gesellschaftlichen und wirtschaftlichen Wandels" (Schermet/Schupp (1991), S. 4). Die Studie wurde erstmals 1984 in den alten Ländern eingeführt, seit Juni 1990 wurde sie auf die neuen Länder erweitert.

${ }^{243}$ Vgl. etwa Schermet/Schupp (1991) und DIW $(1995,46)$. 
Im 4. Quartal 1990 waren Männer etwas wanderungsfreudiger als Frauen. Während der männliche Anteil an der Bevölkerung $48 \%$ ausmacht, liegt ihr Anteil an den Wanderungen aus den neuen in die alten Länder bei $53 \%$. Besonders ausgeprägt ist der geschlechtsspezifische Unterschied in Sachsen. Bei den Wanderungen aus den alten in die neuen Länder sind die geschlechtsspezifischen Unterschiede geringer. Die Zuwanderungsquote der Männer unterscheidet sich mit Ausnahme von Ost-Berlin - nur geringfügig von der Zuwanderungsquote insgesamt.

Sachsen weist im 4. Quartal nicht nur die höchsten Abwanderungsquoten, sondern auch die niedrigsten Zuwanderungsquoten auf. Die Zahlen sind daher anscheinend nur bedingt repräsentativ für die spätere Entwicklung, denn die Abwanderung aus Sachsen ist seit dem Jahr 1991 unterdurchschnittlich.

Über weitere Merkmale der Migranten liegen keine Daten für die Gesamtheit der Wanderer vor, sondern lediglich die Stichprobenergebnisse aus dem SOEP. Danach waren Qualifizierte im Vergleich zu den Nichtqualifizierten häufiger zur Wanderung bereit. Besonders hoch war die Abwanderungsbereitschaft außerdem bei den Westpendlern ${ }^{244}$. Die Daten deuten darauf hin, daß Arbeitslose nicht häufiger als andere gewandert sind. Dagegen war die Umzugswahrscheinlichkeit von Personen, die von Arbeitslosigkeit bedroht waren, anscheinend deutlich erhöht ${ }^{245}$.

Über die Gründe der Wanderungen geben die Wanderungszahlen keinen Aufschluß. Daher werden im folgenden verschiedene Umfrageergebnissen zu den Motiven zusammengefaßt.

Als Hauptgrund für die Wanderungsüberlegung wurde in der Befragung von Akerlof et al $(1991)^{246}$ die Unsicherheit des Arbeitsplatzes genannt. Dagegen

${ }^{244}$ Vgl. Schermet/Schupp (1991) und dies. (1992).

${ }^{245}$ Vgl. DIW $(1994,9)$.

${ }^{246}$ Ein Manko der Umfrage von Akerlof et al (1991) ist allerdings, daß die Stichprobe nicht repräsentativ ist. Es wurden persönliche Interviews durchgeführt. Die Interviewten wurden willkürlich in Cafés, Einkaufszentren und Bahnstationen in verschiedenen ostdeutschen Städten angesprochen. Befragt wurden ausschließlich Personen, die mindestens 16 Jahre alt waren, in Ostdeutschland aufgewachsen waren, die in dem Jahr vor der Untersuchung gearbeitet hatten und zum Zeitpunkt des Interviews zum Erwerbspersonenpotential gehörten. Darüber hinaus wurden Studenten verschiedener Universitäten befragt, die in der DDR aufgewachsen waren und planten, nach Abschluß des Studiums eine Arbeit aufzunehmen. Zusätzlich wurden identische Interviews in Arbeitsämtern durchgeführt, zumeist mit Arbeitslosen. Ergänzt wurde die Umfrage durch eine schriftliche Befragung. Die Auswahl der Nichtstudenten war keine repräsentative Stichprobe der ostdeutschen Arbeitskräfte, Landbewohner und Frauen waren unterrepräsentiert, überproportional vertreten 
wurden keine systematischen Beweise dafür gefunden, daß die Lohnunterschiede an sich ein wichtiger Faktor für Migrationen waren. Im Durchschnitt erwarteten die Befragten einen um 154\% höheren Verdienst im Westen. Es wurde auch geprüft, ob der Einfluß der Lohndifferenzen so gering war, weil die Menschen eine schnelle Angleichung der Löhne erwarteten. Nach den Umfrageergebnissen nahmen $46 \%$ der Interviewten an, daß ihre Löhne schnell steigen werden, während $31 \%$ dies nicht annahmen.

Die psychischen Kosten hatten nach dieser Umfrage einen starken Einfluß auf die Wanderungsentscheidung: die räumliche Nähe zu Freunden und der Familie erwies sich als wichtigster wanderungshemmender Faktor. Dieser Faktor wurde von Nichtstudenten häufiger (78\%) als von Studenten (54\%) genannt. Menschen ohne Kontakte in die alten Länder waren weniger wanderungsbereit als Menschen mit solchen Verbindungen. Dabei war die Zahl derer mit solchen Kontakten relativ hoch: $53 \%$ der Befragten gaben an, sie hätten viele Freunde und Verwandte im Westen. Wanderungshemmend wirkte sich ferner aus, daß viele der Ostdeutschen (44\%) meinten, in Westdeutschland nicht willkommen $\mathrm{zu}$ sein. $17 \%$ der Befragten gaben an, daß hohe Mieten sie von einer Wanderung abhielten.

Burda (1993) kam bei der Auswertung des SOEP zu dem Ergebnis, daß die Nettoumzugskosten erheblich geringer waren, wenn Freunde und Bekannte am potentiellen Zuzugsort lebten. Mit zunehmender Familiengröße stiegen die Migrationskosten. Auch der Besitz eines Eigenheims hemmte die Wanderungswilligkeit. Beschäftigte mit einer langen Betriebszugehörigkeit verfügten über viel firmenspezifisches Humankapital, was im Westen weniger wert war und daher wanderungshemmend wirkte. Daneben wurde eine starke Abhängigkeit der Migrationsbereitschaft mit dem Alter festgestellt. Gegenwärtige Löhne und deren Wachstumsraten waren nach der Untersuchung für die Migrationswilligkeit weniger relevant, sondern sie waren stärker mit Fähigkeiten und Kenntnissen korreliert. Signifikant waren dagegen der Besitz eines Arbeitsplatzes, die Höhe der Wohnungsmieten und die antizipierte Arbeitslosigkeit.

\subsubsection{Zusammenfassende Schlußfolgerungen}

Insgesamt wurden zwischen 1989 und dem Ende des 3. Quartals 1996 knapp 1,9 Mio. Fortzüge aus den neuen in die alten Länder registriert, denen $740.000 \mathrm{Zu}$ züge gegenüberstanden. Daraus ergibt sich in dem genannten Zeitraum ein Wanderungssaldo von 1,1 Mio. Der Schwerpunkt der Abwanderungen aus den

waren Personen mit überdurchschnittlicher Ausbildung sowie Arbeitslose. 
neuen Ländern war in den ersten zwei Jahren nach der politischen Wende in Ostdeutschland zu verzeichnen. Bereits im Jahr 1991 gingen die Wanderungen zurück, während die Zuzüge in den Osten zunahmen. Im Jahr 1995 war der Wanderungssaldo auf 25.000 abgeschmolzen.

Die in Kapitel 3.2 durchgeführte Berechnung des erwartbaren Migrationsertrags ergab, daß am Ende des Jahres 1990 unter Zugrundelegung der damaligen Kenntnisse und Erwartungen für alle Erwerbspersonen Wanderungsüberschüsse erwartbar waren, sofern die Umzugsdistanz relativ gering war. Die Höhe der tatsächlichen Wanderungen war wesentlich geringer als die Schätzung des Wanderungspotentials auf Basis der Modellrechnung. Aus den Abweichungen könnte der Schluß gezogen werden, daß sich die durchgeführte Modellrechnung entweder nicht dafür eignet, die Vorteilhaftigkeit einer Migration zu bestimmen, oder daß die relevanten Migrationskosten und -erträge oder andere Tatbestände nicht bzw. falsch berücksichtigt wurden.

Ein Erklärungsgrund für die „geringen“ Wanderungszahlen könnte der Mangel an verfügbaren Arbeitsplätzen in den alten Ländern und damit die fehlende Möglichkeit zur Realisierung der errechneten potentiellen Wanderungsüberschüsse sein. Dieser Grund dürfte angesichts der hohen Arbeitslosigkeit in den alten Ländern gewichtig sein. Die Umfrageergebnisse aus dem SOEP zeigen, daß von den Personen, die bei der Befragung im Jahr 1991 sehr gerne in die alten Länder ziehen wollten, innerhalb der nächsten zwei Jahre lediglich $17 \%$ ihr Vorhaben realisiert hatten ${ }^{247}$. Dieses Ergebnis dürfte mit der fehlenden Mobilitätsfähigkeit zusammenhängen. Damit läßt sich aber nicht vollständig erklären, warum nicht nur die durchgeführten Wanderungen, sondern auch die Bereitschaft dazu, offenbar wesentlich geringer war, als die Ergebnisse der Modellrechnungen erwarten lassen. Nach einer Umfrage von Akerlof et al (1991) lehnten ein Jahr nach der Wende $22 \%$ der Ostdeutschen einen Umzug in die alten Länder kategorisch ab und über die Hälfte der Befragten stand einem Umzug eher ablehnend gegenüber. Nach den Ergebnissen des SOEP wollte sogar mindestens die Hälfte der Bevölkerung unter keinen Umständen umziehen.

Höchstens ein Bruchteil der Bevölkerung wird eine Rechnung durchgeführt haben, in der nicht nur die gegenwärtigen Lohndifferenzen, sondern auch die $\mathrm{Zu}$ kunftserwartungen monetär bewertet und einbezogen worden sind. Viele ostdeutsche Erwerbstätige dürften unterschätzt haben, welchen monetären Überschuß sie bei einer Wanderung in die alten Länder aus einer Lebenszeitperspektive hätten erzielen können. Hat beispielsweise ein durchschnittlicher Beschäftigter nur die Überschüsse aus den regionalen Lohndifferenzen eines Jahres

${ }^{247}$ Vgl. DIW $(1994,9)$. 
berücksichtigt, wäre er wohl häufig zu dem Ergebnis gekommen, daß diese Erträge von den Umzugskosten aufgezehrt werden. Wird angenommen, daß die relevanten Kosten und Erträge in etwa zutreffend berücksichtigt wurden, so wäre zu vermuten, daß Menschen ihre Entscheidungen nicht rational planen und daher Entscheidungen treffen, die nicht dazu geeignet sind, den Nutzen aus einer Lebenszeitperspektive zu maximieren. Selbst bei den viel zu optimistischen Erwartungen über die Angleichungsgeschwindigkeit hätten fast alle Beschäftigten eine Wanderung in Betracht ziehen müssen.

Als besonders starkes Wanderungshemmnis kristallisierte sich in verschiedenen Umfragen die Nähe zu Freunden und der Familie heraus. Die Bestimmung der psychischen Kosten war in der durchgeführten Modellrechnung in hohem Maße spekulativ und die errechneten Werte mithin besonders problematisch. Sie könnten individuell noch sehr viel höher (aber auch niedriger) gewesen sein, als hier angenommen. Bei einem Umzug über $100 \mathrm{~km}$ wurde angenommen, daß die Kosten lediglich rund 4.000 DM betragen, dies hätte einem Anteil an der Summe der absoluten Kosten und Erträge von 5 bis $11 \%$ bedeutet. Wenn die psychischen Kosten Wanderungen am stärksten verhinderten, könnten sie in der Modellrechnung eventuell zu niedrig bewertet worden sein. Die Ansätze für die Kosten bei einem Umzug über eine größere Entfernung könnten zutreffender gewesen sein. Besonders problematisch dürfte bei der Bewertung der psychischen Kosten der unterstellte starke Zusammenhang mit der Entfernung sein. Die Behandlung eines Teils der psychischen Kosten als Fixkostenblock wäre eventuell problemadäquat gewesen, wobei sich dann aber wieder die Frage gestellt hätte, wie hoch diese Fixkosten angesetzt werden sollten.

Befragungen im Rahmen des SOEP zeigten, daß die Unsicherheit des Arbeitsplatzes offenbar ein wichtiger Wanderungsgrund war, der sogar schwerwiegender war als Arbeitslosigkeit selbst. Die Bedeutsamkeit von Lohnunterschieden wurde dagegen geringer bewertet. Dieses Ergebnis spiegelt sich in der durchgeführten Rechnung so nicht wider, die berechneten Erträge für die regionalen Unterschiede in der Arbeitslosigkeit waren vergleichsweise gering. Die Berücksichtigung der Arbeitsplatzunsicherheit durch die durchschnittlichen Wahrscheinlichkeit, arbeitslos zu werden, könnte für die Situation nach der deutschen Wiedervereinigung nicht adäquat gewesen sein. Andererseits könnten die ostdeutschen Beschäftigten möglicherweise das monetäre Risiko der Arbeitslosigkeit überschätzt haben bzw. - wie oben schon erwähnt - die anderen Ertragsfaktoren wie die regionalen Lohnunterschiede unterschätzt haben.

In der Umfrage von Akerlof hatten $17 \%$ der Befragten angegeben, daß hohe Mieten sie von einer Wanderung abhielten. Die Mietdifferenzen wären jedoch für die Beschäftigten - von den regionalen Lohnunterschieden bei weitem über- 
troffen worden. Die Antwort der Befragten zeigt, daß unzutreffende Vorstellungen über die Lohnunterschiede im Vergleich zu den Mietdifferenzen und den zu erwartenden Entwicklungen vorlagen.

Einige Ergebnisse der Modellrechnungen haben sich bestätigt. So sind die Menschen offenbar bevorzugt in benachbarte Regionen gewandert. Die Mecklenburg-Vorpommerer zogen vorzugsweise in den Norden der alten Länder, die Sachsen in den Süden. Die Thüringer bevorzugten eher die mittlere und die südliche Region, während die Brandenburger überdurchschnittlich häufig nach Berlin zogen. Der erwartbare Migrationsertrag war für männliche Beschäftigte im Vergleich zu den Frauen höher. Tatsächlich sind Männer auch öfter als Frauen gewandert.

In Kapitel 3.1 wurde erläutert, warum die Wanderungsneigung jüngerer Menschen höher als die älterer Menschen ist. Die Wanderungszahlen zeigen, daß jüngere Menschen häufiger als ältere aus den neuen in die alten Bundesländer gewandert sind. In den Modellrechnungen wurde das Alter der Beschäftigten nicht explizit berücksichtigt. Die Erträge aus den Lohndifferenzen hätten unter Umständen durch einen vorherigen Eintritt in den Ruhestand nicht in vollem Umfang realisiert werden können. Sämtliche Kosten wären aber trotzdem entstanden, die psychischen Kosten und die Umzugskosten wären eventuell sogar höher gewesen. Die erwartbaren Migrationserträge insgesamt wären mithin für ältere Menschen tendenziell niedriger als die ausgewiesenen gewesen und die Wanderungswahrscheinlichkeit geringer. 
Annette Schönherr - 978-3-631-75008-7

Downloaded from PubFactory at 01/11/2019 09:19:06AM

via free access 


\section{Schlußbetrachtung}

Nach einer Zusammenfassung der wichtigsten Ergebnisse soll abschließend kurz betrachtet werden, durch welche Eingriffe in das Marktgeschehen die Wanderungen aus den neuen in die alten Länder beeinflußt worden sein könnten.

\subsection{Zusammenfassung}

Im ersten Teil der Arbeit (Kapitel 2) wurden vereinigungsbedingte Folgen von regionaler Arbeitsmobilität unter analytisch einfachen Bedingungen behandelt. Eingangs wurde anhand eines einfachen 2-Regionen-Vollbeschäftigungsmodells gezeigt, daß Arbeit nach Aufhebung von Mobilitätsbarrieren an den Ort des effizienten Einsatzes wandert und sich die Löhne interregional angleichen. Die Reallokation der Arbeit führt zwar in der Regel zu einer erhöhten Gesamtbeschäftigung, jedoch kann es auch Konstellationen geben, bei denen weniger als zuvor gearbeitet wird. Bei konstanter oder zunehmender Arbeitseinsatzmenge steigt grundsätzlich das Sozialprodukt der betroffenen Regionen insgesamt. Bei sinkender Beschäftigung ist dagegen entscheidend, ob der Produktionsrückgang infolge der Beschäftigungsabnahme durch die verbesserte Allokation der Arbeit überkompensiert wird. Gleicht die Produktionszunahme infolge der effizienteren Allokation den Rückgang durch den verringerten Arbeitseinsatz nicht aus, kann der Output nach einer Vereinigung auch geringer als ohne Vereinigung sein. Das um das Arbeitsleid bereinigte Sozialprodukt dagegen sinkt durch den Abbau von Mobilitätsbarrieren grundsätzlich nicht, sondern es bleibt entweder konstant oder es erhöht sich.

Nach der Darstellung des Modells mit und ohne Vereinigung wurden die ostund westdeutschen Arbeitsmarktverhältnisse zum Zeitpunkt der Grenzöffnung im Jahr 1989 skizziert und auf das Modell übertragen. Danach wäre bei der damaligen Konstellation eine vollständige Abwanderung der ostdeutschen $\mathrm{Be}$ schäftigten zu erwarten gewesen. Die tatsächlichen Wanderungen waren jedoch niedriger, zudem war der Wanderungssaldo fünf Jahre nach der Vereinigung nahezu ausgeglichen, während sich die regionalen Lohnsätze zwar angenähert hatten, sich aber noch deutlich unterschieden. Das zugrunde gelegte Modell war offenbar nicht geeignet, Ausmaß und Wirkungen der regionalen Arbeitsmobilität richtig abzubilden. Aufgrund der Ergebnisse wurde in den folgenden Kapiteln untersucht, wie das Modell zu modifizieren ist, um Ereignisse wie die deutsche Vereinigung zutreffender abzubilden. 
Zunächst wurde Arbeitslosigkeit berücksichtigt, die durch nach unten rigide Löhne entsteht. Es zeigte sich, daß unter solchen Bedingungen weniger Wanderungen stattfinden und auch der Abbau von Mobilitätsbarrieren nicht zu einer optimalen Allokation der Arbeit führt. Die Lohnsätze können sich nicht durch die Wanderungen aneinander anpassen. Entsprechend sind die Einkommensund Beschäftigungsanpassungen bei Beseitigung der Mobilitätsbarrieren in der Regel geringer als bei flexiblen Lohnsätzen.

In Kapitel 2.3 wurden verschiedene Modifikationen hinsichtlich der Produktionsseite vorgenommen. Ist neben dem Sachkapital auch das Humankapital der Wanderer ein Teil des die Arbeitsnachfrage determinierenden Kapitalstocks, sind größere Veränderungen der regionalen Beschäftigung und des Outputs zu erwarten als ohne einen solchen Einfluß. Ein weiteres Ergebnis dieses Kapitels ist, daß bei unterschiedlicher Qualifikation der Arbeitnehmer regionale Lohndifferenzen auf dem Arbeitsmarkt für eine bestimmte Qualifikation nicht nur zu Wanderungen der entsprechend qualifizierten Arbeitnehmern führen, sondern auch zu Wanderungen von Erwerbstätigen mit einer anderen Qualifikation. Die Richtung und Höhe der Wanderungen ist davon abhängig, ob die Migranten substitutiv oder komplementär für die jeweils andere Gruppe an Arbeitnehmern einsetzbar sind. Ferner wurde der Einfluß selektiver Wanderungen auf das wirtschaftliche Wachstum beleuchtet. Dabei zeigte sich, daß je nach den zugrundegelegten Annahmen Wanderungen zu einer Konvergenz von Regionen beitragen oder auch bestehende Unterschiede weiter verstärken können. Darüber hinaus wurde beschrieben, daß sich durch Abwanderungen die Absatzsituation vor Ort verschlechtern kann. Die Verringerung der Beschäftigung und des Outputs der Abwanderungsregion kann größer sein als ohne Einfluß der regionalen Nachfrage.

In Kapitel 2.4 wurden regionale Annehmlichkeiten von Regionen berücksichtigt und gezeigt, daß bei einer unterschiedlichen Ausstattung der Regionen interregionale Reallohnunterschiede dauerhaft bestehen können, ohne Wanderungen auszulösen. Durch eine Vereinigung können sich Lohnunterschiede verstärken oder auch umkehren. Die Regionen mit dem höchsten Niveau an Annehmlichkeiten weisen im Mit-Vereinigungsfall die niedrigsten Lohnsätze auf, wenn die Annehmlichkeiten auf die Produktion kostenerhöhend wirken. Wirken die Annehmlichkeiten kostensenkend, sind die Unternehmen in den attraktivsten Gegenden bereit, die höchsten Löhne zu zahlen. Alle Haushalte und Unternehmen würden in die Gegend mit der höchsten Attraktivität ziehen, während sich die andere Regionen vollständig entleerte. Infolge solch starker Wanderungen dürfte aber die Attraktivität der Zuwanderungsregion durch die zunehmende Rivalität in der Nutzung der Annehmlichkeiten oder über Preissteigerungen für lokale Güter sinken. Abschließend wurde gezeigt, daß bei Berücksichtigung von 
Wanderungskosten Nutzenunterschiede zwischen den regionalen Bevölkerungsgruppen auch mit Vereinigung bestehen bleiben können.

Eine ins Einzelne gehende Übertragung des Modells und seiner Modifikationen auf die deutsch-deutsche Vereinigung hätte den Rahmen der Arbeit gesprengt. Daher beschränkte sich der nachfolgende empirische Teil auf einen Aspekt der deutsch-deutschen Arbeitsmobilität nach der Vereinigung. Nach der Vereinigung gab es weniger Wanderungen, als aufgrund der Lohnunterschied neoklassisch zu vermuten gewesen wäre. Betrachtet wurde daher die Entscheidungssituation der ostdeutschen Erwerbspersonen nach der Vereinigung, um zu beleuchten, warum die meisten Beschäftigten die bestehenden Lohnunterschiede nicht für sich genutzt haben. Um diese Frage zu beantworten wurde zunächst in Kapitel 3.1 beschrieben, welche Wanderungsursachen in der Entscheidungsfindung berücksichtigt werden und wie die Faktoren monetär bewertet werden können, um in eine Rechnung zur Messung der Vorteilhaftigkeit eines Umzugs einzugehen. So zeigte sich z.B., daß bei einem Vergleich der regionalen Löhne nicht nur die Jahreseinkommen zu berücksichtigen sind, sondern auch diverse andere Einkommensbestandteile wie die Urlaubslänge oder betriebliche Sonderleistungen. Entscheidend sind dabei nicht nur die aktuellen, sondern auch die erwarteten künftigen Unterschiede. Da zudem das reale Einkommen maßgeblich ist, müssen zusätzlich zu den regionalen Arbeitseinkommen die regionalen Preisniveaus in die Entscheidungsfindung einbezogen werden. Ein besonderes Problem stellt die Bewertung von Unterschieden in der Lebensqualität der Regionen dar, deren Quantifizierung schier aussichtslos ist. Die Bewertung von psychischen Kosten und Erträgen erwies sich ebenfalls als ausgesprochen problematisch. Vorgestellt wurde der Reisekostenansatz, mit dem eine Schätzung solcher Komponenten vorgenommen wird. Ferner wurden noch diverse andere Merkmale, wie Alter und Familienstand, erläutert, die ebenfalls von Bedeutung für die Höhe des erwartbaren Migrationsgewinns sind. So sinken etwa mit zunehmendem Alter i.d.R. die potentiellen Wanderungsgewinne.

Im anschließenden Kapitel 3.2 wurden dann die verschiedenen Kosten und Erträge geschätzt, die sich für einen ostdeutschen Beschäftigten am Ende des Jahres 1990 ergeben hätten, wenn er in die alten Länder gezogen wäre bei einem gleichzeitigem Wechsel des Arbeitsplatzes. Darauf basierend wurde berechnet, ob durch eine Migration ein Überschuß unter Zugrundelegung des damaligen Kenntnisstandes erwartbar war. Gegenübergestellt wurde eine Rechnung in der die Daten der tatsächlichen Entwicklung bis einschließlich 1994 eingingen.

Zunächst wurde gezeigt, welche Überschüsse aus den regionalen Nettolohndifferenzen für Beschäftigten unterschiedlicher Branchen erwartbar gewesen waren. Zusätzlich wurden Rechnungen gesplittet nach Geschlecht und nach Arbei- 
tern/Angestellten durchgeführt. Zugrunde gelegt wurden Lohnangleichungszeiträume von drei, fünf und zehn Jahren, da Umfragen darauf hindeuteten, daß kurz nach der Vereinigung eine schnelle Angleichung erwartet wurde. Ferner wurden stellvertretend für die regionalen Preisunterschiede die Mieten berücksichtigt. Aus den regionalen Lohndifferenzen ergab sich für alle Beschäftigten ein positiver erwartbarer Ertrag aus einem Umzug, der durch die regionalen Mietunterschiede allerdings geschmälert wurde. Die abgezinsten kumulierten Erträge lagen bei Erwartungen über eine Lohnanpassung von höchstens fünf Jahren für die meisten Beschäftigten unter 20.000 DM. Bei einer Anpassungszeit von zehn Jahren waren die errechneten Überschüsse deutlich höher: Sie betrugen im Durchschnitt zwischen 40.000 DM und 45.000 DM, je nach Höhe der Zeitpräferenzrate.

Die korrigierten erwartbaren Erträge, die sich auf Basis der realen Lohn- und Mietpreisentwicklung bis 1994 und deren Fortschreibung berechneten, waren ebenfalls durchweg positiv. Der durchschnittliche Nettoertrag eines Beschäftigten belief sich (in Abhängigkeit vom ökonomischen Anpassungsverlauf) auf rund 30.000 DM bzw. 48.000 DM. Dieser Betrag entsprach 15 bzw. 23 Bruttomonatslöhnen eines Ostdeutschen im Jahr 1991.

Die korrigierten erwartbaren Migrationserträge waren höher als die erwartbaren, sofern Lohnangleichungserwartungen von 5 Jahren und weniger zugrunde gelegt wurden. Bei Erwartungen einer 3jährigen Anpassungsdauer wurden die Nettoerträge durchschnittlich auf höchstens die Hälfte des korrigierten erwartbaren Ertrags geschätzt. Selbst bei Lohnangleichungen nach mindestens 10 Jahren waren die erwartbaren Migrationserträge für die meisten Beschäftigten geringer als die korrigierten.

Die Bewertung weiterer Migrationskosten und -erträge, und zwar der Umzugskosten, der geringeren Jahresarbeitszeiten in Westdeutschland, psychischer Kosten, Lohneinbußen durch eine Verschlechterung der beruflichen Stellung und der Mehrkosten bei Neuanmietung einer Wohnung im Vergleich zur Bestandsmiete, ergab im Durchschnitt bei einer Nahwanderung insgesamt einen Kostenüberschuß von knapp 6.000 DM und bei einer Fernwanderung von knapp $50.000 \mathrm{DM}$.

Eine Berücksichtigung der weiteren Kosten und Erträge in die Berechnung des erwartbaren Migrationsertrags eines Ostdeutschen führte für einen durchschnittlichen Beschäftigten grundsätzlich zu einem Verlust bei einer Fernwanderung. Bei einer mittleren Distanz ergab sich für Anpassungserwartungen von höchstens 5 Jahren ebenfalls ein Verlust. Für alle Beschäftigten war dagegen ein Überschuß bei einer Nahwanderung erwartbar. 
Bei einem Umzug in die nähere Umgebung war die Bedeutung der regionalen Lohnunterschiede von allen berücksichtigten Faktoren am höchsten. Mit zunehmender Entfernung stieg der rechnerische Einfluß der psychischen Kosten, insbesondere wenn sehr optimistische Erwartungen über die wirtschaftliche Angleichung bestanden. Unter Zugrundelegung der tatsächlichen Entwicklung war der Anteil der psychischen Kosten deutlich geringer als bei der 1990 erwarteten Entwicklung, dagegen waren die Lohnunterschiede bei allen Entfernungen am bedeutendsten.

Die Berücksichtigung der unterschiedlich hohen Arbeitslosigkeit in Ost- und Westdeutschland ergab einen zusätzlichen erwartbaren Migrationsertrag. Da der Nettoertrag aus den regionalen Lohnunterschieden im Durchschnitt aber deutlich höher war, dürften für ostdeutsche Erwerbstätige die regional unterschiedlich hohen Arbeitslosenquoten weit weniger gewichtig gewesen sein als die Lohndifferentiale. Von weit größerer Bedeutung ist Arbeitslosigkeit für die tatsächlich davon Betroffenen gewesen.

Für einen durchschnittlichen Beschäftigten ergab sich bei allen untersuchten Erwartungen über den Anpassungszeitraum stets ein positiver erwartbarer Wanderungsertrag bei einem Umzug in die nähere Umgebung. Das Potential für Nahwanderungen müßte mithin sämtliche 7,6 Mio. Beschäftigte umfaßt haben. Für eine Entfernung von $200 \mathrm{~km}$ könnte das Potential um 14\% geringer gewesen sein, d.h. es umfaßte 6,5 Mio. Erwerbstätige. Über eine mittlere Entfernung ergab sich ein rechnerisches Potential von Wanderungswilligen zwischen $18 \%$ und 38\% der Beschäftigten, d.h. 1,37 Mio. bis zu 2,89 Mio. Personen. Für eine Fernwanderung war die Zahl der Wanderungswilligen gemäß Hochrechnung mit rund 100.000 deutlich geringer.

Abschließend wurde gezeigt, daß die erwartbaren Wanderungsgewinne für die Ostdeutschen fünf Jahre nach der Wende erheblich geschrumpft waren. Die Wanderungsneigung müßte daher stark zurückgegangen sein und mit Umzügen bis dahin immobiler Personen kaum noch zu rechnen gewesen sein, sofern sich an deren persönlicher oder beruflicher Situation keine Veränderungen ergeben haben. Dies harmoniert auch deutlich mit den faktischen Entwicklungen des Wanderungsverhaltens.

Anschließend wurden in Kapitel 3.3 die Höhe der tatsächlichen Wanderungen zwischen den alten und neuen Ländern aufgezeigt. Der Schwerpunkt der Fortzüge aus Ostdeutschland lag in den ersten zwei Jahren nach der Grenzöffnung. Bereits im Jahr 1991 war ein Rückgang der Wanderungen zu verzeichnen, während gleichzeitig die Zuzüge aus den alten Ländern in den Osten zunahmen. Insgesamt wurden zwischen 1989 und dem Ende des 3. Quartals 1996 knapp 1,9 
Mio. Fortzüge aus den neuen in die alten Länder registriert. Dem standen 740.000 Zuzüge gegenüber. Daraus ergab sich in dem genannten Zeitraum ein Wanderungssaldo von 1,1 Mio.

Einige Ergebnisse der in Kapitel 3.2 durchgeführten Berechnung über die Vorteilhaftigkeit eines Umzugs aus den neuen in die alten Länder wurden durch die tatsächliche Entwicklung bestätigt. So zeigte sich, daß die Wanderungsziele offenbar mit der räumlichen Nähe zusammen hingen. Jüngere Menschen sind häufiger als ältere von Ost- nach Westdeutschland gezogen, Frauen seltener als Männer. Dieses Verhalten deckt sich mit dem Wanderungsverhalten zu anderen Zeiten und zwischen anderen Orten.

Die Wanderungszahlen waren wesentlich niedriger als das errechnete Potential. Dies muß aber nicht bedeuten, daß die aufgestellte Rechnung falsch ist. Vielen ostdeutschen Beschäftigten dürfte nicht bewußt gewesen sein, welchen monetären Überschuß sie bei einer Wanderung in die alten Länder aus einer Lebenszeitperspektive hätten erzielen können. Außerdem dürften einige Menschen, die wanderungswillig waren, keinen Arbeitsplatz in Westdeutschland in einer für sie noch lohnenden Entfernung gefunden haben und daher nicht umgezogen sein.

\subsection{Abschließende Bemerkungen}

Im ersten Teil der vorliegenden Arbeit wurde dargestellt, daß sich die Faktorallokation infolge einer Vereinigung durch die Faktorwanderungen verbessert. Das hohe Wanderungspotential und die massiven Abwanderungen in den ersten Jahren nach der Grenzöffnung riefen aber offenbar Befürchtungen hervor, daß die negativen Folgen von Wanderungen die positiven überwiegen könnten. In der öffentlichen Diskussion und nicht zuletzt von politischer und ökonomischer Seite wurde immer wieder unterstrichen, daß massive selektive Wanderungsbewegungen von Ost- nach Westdeutschland unerwünscht waren, wie folgende Zitate zeigen:

"Er (der Umstrukturierungsprozeß, Anm. der Verfasserin)...führt über Wanderungen und deren Selektivität zur Gefahr passiver Sanierung der Abwanderungsregionen" (Blaschke u.a. (1992), S. 128)

" Da gerade qualifizierte Arbeitskräfte für den Neuaufbau der ostdeutschen Wirtschaft unverzichtbar sind, ist für diese eine schnelle Lohnangleichung unerläßlich...Es ist also erwünscht, daß qualifizierte Arbeitskräfte von vornherein in Ostdeutschland bleiben." (Horn (1992), S. 140)

"Für eine Übergangszeit geht es darum, das Humankapital im Lande zu 
halten (und zu verbessern) und so schnell wie möglich um das fehlende physische Kapital zu ergänzen, so daß ein hohes Produktivitätsniveau erreicht werden kann" (Schumacher (1993), S. 134)

Eine passive Sanierung Ostdeutschlands, d.h. eine „Gesundschrumpfung“ der regionalen Lebensverhältnisse über Abwanderungen, war offenbar nicht gewollt, vielmehr wurde eine Angleichung der Lebensverhältnisse angestrebt.

Hierfür wurden verschiedene Gründe genannt. So wurde in Frage gestellt, ob das politische System noch jahrelang eine anhaltende Ungleichheit der Einkommens- und Lebensverhältnisse ertragen konnte ${ }^{248}$. Daneben wurden auch moralische Gründe angeführt. Ferner wurden Artikel 20 und 28 des Grundgesetzes (Sozialstaatsprinzip) sowie Artikel 72 und 106 (Einheitlichkeit der Lebensverhältnisse) zur Begründung von staatlichen Eingriffen herangezogen. Auch auf das Raumordnungsgesetz (ROG) und das Bundesraumordnungsprogramm (BROP), in denen das Ziel der gleichwertigen Lebensbedingungen festgeschrieben ist, wurde hingewiesen.

Alle Eingriffe, mit denen die Lebensbedingungen in der potentiellen Abwanderungsregion verbessert werden sollten, beeinflußten indirekt auch die Wanderungsneigung der Bevölkerung. Theoretisch hätte der Staat auch ein vielfältiges direktes Instrumentarium zur Steuerung derWanderungsmöglichkeiten einsetzen können. Einige dieser Instrumente, die zur Verhinderung internationaler Wanderungen eingesetzt werden könnten, stehen jedoch auf nationaler Ebene aufgrund des Freizügigkeitsgebots nicht zur Verfügung. Dazu zählen Maßnahmen wie Wanderungsverbote, administrative Mengenregelungen oder Einwanderungszertifikate. Es verblieb aber eine Vielzahl anderer Instrumente, wie etwa die steuerliche Absetzmöglichkeiten von Umzugskosten. Am bedeutsamsten für die Beeinflussung der ostdeutschen Abwanderungen waren aber wohl die Maßnahmen zur Unterstützung der ökonomischen Angleichung der neuen an die alten Länder.

Für die Schaffung von Lebensbedingungen in den neuen Ländern, die denen der alten Länder vergleichbar ist, waren immense Investitionen in Ostdeutschland notwendig. Zum Zeitpunkt der Vereinigung war der Kapitalstock der neuen Länder stark veraltet. Nach dem IAB-Westphal-Modell belief sich das benötigte Nettoinvestitionsvolumen auf 1,3 Billionen DM, damit die Arbeitsproduktivität pro Stunde in Ostdeutschland im Jahre $200080 \%$ des Westniveaus erreicht ${ }^{249}$. Schumacher (1993) kam zu dem Ergebnis, daß bei einem Anpassungszeitraum von 5 Jahren jährliche Investitionen von 170 - $250 \mathrm{Mrd}$. DM notwendig gewesen

${ }^{248}$ Vgl. Spahn (1991), S. 79.

${ }^{249}$ vgl. Klauder (1990), S. 22ff und Blaschke u.a. (1992), S. $125 f$. 
wären ${ }^{250}$. Auch die Schätzungen von anderen Stellen (OECD, Prognos, Ifo-Institut u.a.) über den Investitionsbedarf belief sich auf ähnliche Größenordnungen zwischen 1 und 2 Billionen DM $^{251}$. Bei einem Anpassungszeitraum von 15 Jahren bis zur Erreichung des westdeutschen Niveaus, wären jährlich Nettoinvestitionen in Höhe von 80 Mrd. DM erforderlich gewesen ${ }^{252}$.

Zur Förderung von Investitionen wurden Kapitalsubventionen in hohem Ausmaß an die ostdeutschen Unternehmen gewährt. Im Einigungsvertrag (Art. 28) wurde das bereits in der alten Bundesrepublik bestehende System zur Regionalförderung, die "Gemeinschaftsaufgabe zur Verbesserung der regionalen Wirtschaftsstruktur" (GRW), für zunächst fünf Jahre auf die neuen Bundesländer ausgedehnt. Aufgrund der Probleme in Ostdeutschland wurden zahlreiche Sonderregelungen bezüglich der GRW-Mittel getroffen ${ }^{253}$. Hinzu kamen ERP-Mittel und das Sonderprogramm "Aufschwung Ost"254. Die große Zahl von Programmen der Kapitalsubventionen sollte mithelfen eine wettbewerbsfähige Wirtschaftsstruktur im Osten zu schaffen. Tatsächlich hat die Förderpolitik offenbar auch dazu beigetragen, daß mehr investiert wurde als ohne eine solche Politik ${ }^{255}$ und damit indirekt mobilitätssenkend gewirkt. Alles andere wäre auch erstaunlich gewesen, es sei denn die Investitionen haben das Lohnniveau im Osten in die Höhe getrieben. Allerdings dürften die Neuinvestitionen auch eine massive Vernichtung (,Ersatz“) von vorhandenem Altkapital bewirkt haben.

Ferner wurde von der öffentlichen Hand selbst Infrastrukturinvestitionen in großem Ausmaß vorgenommen. Die Unterschiede in der Ausstattung mit Infrastruktur zwischen den alten und neuen Bundesländern waren im Jahr 1989 beträchtlich. Die Infrastruktur war in einem desolaten Zustand und der Umfang verglichen mit Westdeutschland - sehr gering. Das Bruttoanlagevermögen an den Infrastrukturbereichen Energie- und Wasserversorgung, Nachrichtenübermittlung, Verkehr und Abwasserbeseitigung wurde für Ostdeutschland auf knapp $11 \%$ der westdeutschen Infrastruktur geschätzt. Die Intensität (Bruttoanlagevermögen/Erwerbspersonenpotential) betrug $40 \%$ des Westniveaus ${ }^{256}$.

Neben diesen Maßnahmen, die zu einer Angleichung der Lebensverhältnisse beitrugen, haben noch weitere Regelungen die Umzugsneigung herabgesetzt. Besonderes Gewicht hatten dabei die Lohnverhandlungen zwischen den Tarif-

\footnotetext{
${ }^{250} \mathrm{Vgl}$. Schumacher (1993), S. 136f.

${ }^{251}$ Vgl. Buttler/Klauder (1992), S. 253.

${ }^{252}$ Vgl. Lübbering (1993).

${ }^{253} \mathrm{Zu}$ den Sonderregelungen vgl. Fritsch u.a. (1991), S. $628 \mathrm{f}$.

${ }^{254}$ Vgl. Fritsch u.a. (1991), S. 627.

${ }^{255}$ Vgl. hierzu DIW (1997, 3), S. 52ff.

${ }^{256}$ Vgl. DIW (1994, 27), S. 461.
} 
parteien, die eine sehr schnelle Lohnangleichung vereinbarten. Der Einfluß der Löhne und der erwarteten Lohnangleichungen wurde oben bereits ausführlich dargestellt.

Mobilitätssenkend dürften nach der Vereinigung auch arbeitsmarktpolitische Maßnahmen gewirkt haben. Durch die aktive Arbeitsmarktpolitik sind nach Aussage des Bundesinnenministeriums bis zu zwei Millionen Arbeitnehmer in Ostdeutschland vor Arbeitslosigkeit bewahrt worden ${ }^{257}$. Auf die besonders hohe Mobilität von Personen, die erwarten arbeitslos zu werden, wurde bereits oben hingewiesen.

Die Beschäftigung in einer Arbeitsbeschaffungsmaßnahme (ABM) bietet den Betroffenen allerdings nicht die gleichen Perspektiven wie eine reguläre Anstellung, denn sie ist zeitlich befristet und weniger gut bezahlt. ABM werden auch Modernisierungseffekte zugeschrieben, die den Strukturwandel der Region beschleunigen, etwa wenn die Betroffenen in Maßnahmen der investiven Infrastruktur, bei Versorgungsanlagen, in der Gebäudeerschließung und im Hochbau eingesetzt werden ${ }^{258}$. Gleichzeitig können sie aber auch Konkurrenz für ansässige Betriebe bedeuten.

Qualifizierungsmaßnahmen verbessern die Aussichten auf eine Anstellung und auf ein höheres Gehalt. Diejenigen Ostdeutschen, die eine Fortbildung erhielten, waren aber i.d.R. nicht die hochqualifizierten Arbeitnehmer, deren Abwanderung verhindert werden sollte. Eine zusätzliche Qualifikation konnte im Gegenteil sogar einen Umzug erst möglich machen, wenn nämlich hierdurch Kenntnisse erworben wurden, die in der potentiellen Zielregion gefragt waren.

Durch die Zahlung von Arbeitslosengeld wird der Unterhalt der Betroffenen gesichert und der Druck verringert, eine neue Stelle finden zu müssen. Hierdurch sinkt die Mobilitätsbereitschaft. Die Zahlung von Vorruhestandsgeld könnte ebenfalls die Umzugsbereitschaft vermindert haben, allerdings gehören ihre Bezieher keiner besonders mobilen Gruppe an. Sie hätten aufgrund ihres Alters bei einem Umzug nur noch kurz von den Lohndifferenzen profitiert. Zudem hatten ältere Arbeitnehmer große Schwierigkeiten eine neue Stellung - gleich in welcher Region - zu finden. Scheiden durch die Zahlung von Vorruhestandsgeld einige Erwerbspersonen aus dem Arbeitsmarkt aus, wirkt dies allerdings lohnerhöhend. Gleichzeitig werden Arbeitsplätze frei, was wiederum mobilitätssenkend auf die restlichen Beschäftigten wirkt.

${ }^{257}$ Zitiert nach o.V. (1997).

${ }^{258}$ Vgl. Engelen-Kefer u.a. (1995), S. 500. 
Mobilitätsmindernden Einfluß hatten auch die regionalen Unterschiede der Mieten, die durch Reglementierungen zustande kamen. Die Mieten waren in der DDR auf einem sehr niedrigen Niveau festgeschrieben. Nach der Vereinigung wurden die Mieten nicht schlagartig freigegeben, sondern die Anpassung der Mieten wurde gesetzlich geregelt und erfolgte stufenweise ${ }^{259}$. Zugunsten eines Verbleibs in den neuen Bundesländern wirkte auch der Einkommensteuerfreibetrag in Höhe von 600 DM in den Jahren 1991 bis 1993 für Bürger mit Wohnsitz in Ostdeutschland. Für Besserverdienende hat sich zudem die niedrigere Sozialversicherungsgrenze in den neuen Bundesländern positiv bemerkbar gemacht.

In welchem Ausmaß die Summe der Eingriffe die Wanderungsvorhaben beeinflußt haben, kann hier nicht beurteilt werden. Einige Regelungen sind offenbar nur durch den massiven Druck der angedrohten Abwanderungen so schnell zustande gekommen, etwa die Vereinbarungen über die Lohnangleichung und die Einführung der DM in den neuen Ländern. Starkes Aufsehen erzielten etwa die Massendemonstrationen, in denen immer wieder der folgende Spruch zu hören war: „Kommt die DM bleiben wir, kommt sie nicht, gehen wir zu ihr.“

Die Entwicklung der Wanderungszahlen zeigen, daß die massiven Wanderungsströme zum Erliegen gekommen sind. Die Abwanderungen in den ersten Jahren nach der Vereinigung haben zwar den ostdeutschen Arbeitsmarkt entlastet, gleichzeitig sind aber viele qualifizierte Arbeitnehmer abgewandert, was nicht nur die Zukunftsperspektiven der betroffenen Regionen negativ beeinflußt haben könnte, sondern auch die regionale Nachfragehöhe verringert hat.

Obwohl die Abwanderungen stark zurückgegangen sind und nicht mehr zur Begründung von hohen Lohnabschlüssen herangezogen werden, werden trotz der hohen Arbeitslosigkeit die Lohnangleichungen weiter vorangetrieben. Mitte des Jahres 1996 betrug das ostdeutsche Bruttoeinkommen pro Erwerbstätigen 75\% des Niveaus im früheren Bundesgebiet ${ }^{260}$ und war damit um 20\%-Punkte höher als das Niveau der Wertschöpfung je Erwerbstätigen im Vergleich zu den alten Ländern ${ }^{261}$. Die hohen Lohnabschlüsse führten offenbar dazu, daß das Tarifvertragssystem anscheinend nicht mehr voll funktionsfähig ist, denn in der Jahresmitte 1997 zahlten bereits $40 \%$ der ostdeutschen Industrieunternehmen weniger als den tariflichen Mindestlohn ${ }^{262}$.

\footnotetext{
${ }^{259}$ Vgl. hierzu Kapitel 3.2.1.1.2.

${ }^{260}$ Vgl. DIW (1997, 7), S. 122.

${ }^{261}$ Vgl. DIW (1997, 3), S. 47.

${ }^{262} \mathrm{Vgl}$. Paqué (1997).
} 
Trotz vieler noch bestehender Probleme ist die Angleichung der ökonomischen Verhältnisse in den vergangenen Jahren deutlich vorangekommen. Allein bis zum Frühjahr 1997 wurden nach Abzug aller Rückflüsse knapp tausend Milliarden DM in die neuen Länder transferier ${ }^{263}$. Ein Ende des Angleichungsprozesses ist aber noch lange nicht in Sicht. Die zukünftigen ökonomische Angleichung von Ost- und Westdeutschland wird voraussichtlich weniger durch Arbeitsmobilität, sondern im wesentlichen durch Kapitaltransfers voranschreiten.

${ }^{263}$ Bericht zum Stand der deutschen Einheit, zusammengestellt vom Bundesinnenministerium, zitiert nach o.V. (1997). 
Annette Schönherr - 978-3-631-75008-7

Downloaded from PubFactory at 01/11/2019 09:19:06AM

via free access 


\section{Literaturverzeichnis}

Ahlheim, Michael und Manfred Rose (1989): Messung individueller Wohlfahrt, Heidelberg

Akerlof et al (1991): East Germany in from the Cold: The Economic Aftermath of Currency Union, in Brookings Papers of Economic Activity, S. 1-105

Akerlof, George A. und Janet L. Yellen (1986): Introduction, in: Akerlof/Yellen (eds.): Efficiency Wage Models of the Labor Market, Cambridge u.a.O., S. 1-21

Alchian, Armen A. (1970): Information Costs, Pricing, and Resource Unemployment, in: Phelps, E.S u.a. (eds.): Microenonomic Foundations of Employment and Inflation Theory, New York

Barro, Robert J. (1986): Makroökonomie, Regensburg

Bartels, Dietrich (1982):Wirtschafts- und Sozialgeographie, in: HdWW, Bd. 9, Stuttgart u.a.O., S. 44-55

Bellmann, Lutz (1986): Ökonomische Begründungen rigider Löhne: Der Beitrag der Shirking-Theorie, Arbeitskreis Sozialwissenschaftliche Arbeitsmarktforschung (SAMF), Arbeitspapier 1986-3

Berry, R.A. und Soligo, R. (1969): Some Welfare Aspects of International Migration, in: Journal of Political Economy, Sept./Oct., 77(5), S. 778-794

Berthold, Norbert (1987): Lohnstarrheit und Arbeitslosigkeit, Freiburg

Birg, H. (1975): Analyse und Prognose der Bevölkerungsentwicklung in der Bundesrepublik Deutschland und ihren Regionen bis zum Jahr 1990, DIW, Heft 35

Bispinick, Reinhard und WSI-Tarifarchiv(1992): Tarifpolitik in der Transformationskrise, in WSI-Mitteilungen 3, 45. Jg

Bispinick, Reinhard und WSI-Tarifarchiv (1993): Sind die Löhne schuld? - Die Tarifpolitik in den neuen Ländern im Jahr 1992, in WSI-Mitteilungen 3, 46. Jg

Bispinick, Reinhard und WSI-Tarifarchiv (1994): Zwischen Revision und Angleichung - der schwierige Spagat der Tarifpolitik in Ostdeutschland im Jahr 1993, in WSI-Mitteilungen 3, 47. Jg, S. 154-165

Blaschke, Dieter u.a. (1992): Der Arbeitsmarkt in den neuen Ländern - Zwischenbilanz und Herausforderungen, in: MittAB 2, S. 119-135

Blümle, Gerold (1975): Theorie der Einkommensverteilung, Berlin u.a.O. 
Bodenhöfer, Hans-Joachim (1967): The Mobility of Labor and the Theory of Human Capital, in: The Journal of Human Ressources, II-4, S. 431-448

Bodenhöfer, Hans-Joachim (1969): Arbeitsmobilität und regionales Wachstum, Berlin

Bombach, G. (1988): Beschäftigung und Arbeitsproduktivität im Konjunkturverlauf. 25 Jahre Okun'sches Gesetz, in: Franz, W. u.a. (Hrsg.): Theoretische und angewandte Wirtschaftsforschung, Heidelberg

Bouge, Donald. J. (1959): Internal Migration, in: Philip Hauser und Otis Duncan: The Study of Population - An Inventory and Appraisal, Chicago

Bretschger, Lucas (1993): Labour Migration and Long-Term Growth, Diskussionsbeitrag NR. 9302 des Instituts für empirische Wirtschaftsforschung der Universität Zürich, Februar

Bretschger, Lucas (1993a): Migration und internationale Wettbewerbsfähigkeit, in: Schweizerische Zeitschrift für Volkswirtschaftslehre und Statistik 129, S. 473 $-489$

Brinkmann, Gerhard (1981): Ökonomik der Arbeit, Bd.2, Stuttgart.

Büchel, Felix und Markus Pannenberg (1992): Erwerbsbiographische Folgerisiken von Kurzarbeit und Arbeitslosigkeit, in: MittAB 2/92, S. 158-167

Burda, Michael (1993) The Determinants of East-West-Migration: Some First Results, Diskussionspapier FS I 93 -306, März

Busch, Ulrich (1991): Zur Entwicklung des Lebensstandards in den neuen Bundesländern, in: Westphal, A . (Hrsg): Wirtschaftspolitische Konsequenzen, S. 215-235.

Buttler, Friedrich und Wolfgang Klauder(1992): Investitionsförderung - Schlüssel zur Lösung des Beschäftigungsproblems, in: MittAB 3, S. 252-255

Christaller, Walter (1933): Die zentralen Orte in Süddeutschland, Jena

Clark, David E. und James C. Cosgrove(1991): Amenities Versus Labor Market Opportunities: Choosing the Optimal Distance to Move, in: Journal of Regional Science, Vol. 31, N. 3, S. 311- 328

DaVanzo, Julie (1978): Does Unemployment Affect Migration? - Evidence from Micro Data, Review of Economics and Statistcs, 60, S.504-514

DaVanzo, Julie (1983): Repeat Migration in the United States: Who Moves Back and Who Moves On?, in: The Review of Economics and Statistics, 65, S. 552559 
Dedering, Heinz (1972): Arbeitsmobilität - Diskussion und Systematik ihrer Begriffsinhalte, in: Kölner Zeitschrift für Soziologie \& Sozialpsychologie, Heft 1, S. 46-67

Delbrück, Christopher und Bernd Raffelhüschen(1993): Die Theorie der Migration, in: Jahrbücher für Nationalökonomie und Statistik, Bd. 212/3-4, S. 341-355

Deutsche Bundesbank (1991): Die Wirtschaftslage in der Bundesrepublik Deutschland um die Jahreswende 1990/91, S. 5-46, in: Monatbericht der Deutschen Bundesbank Februar, Frankfurt, S. 5-46

Deutsche Bundesbank (1997, 10): Zur Entwicklung der Arbeitseinkommen seit Anfang der neunziger Jahre, in: Monatbericht der Deutschen Bundesbank Oktober, Frankfurt, S. 19-32

Diamond, Douglas B. und George Tolley(1982): The Economic Role of Urban Amenities, in Diamond/Tolley(Hrsg.): The Economics of Urban Amenities, New York, S. 3-54

$D I W(1990,32)$ : DDR: Hohe pauschale Lohnforderungen gefährden die Wettbewerbsfähigkeit, in: DIW-Wochenbericht 32, S. 441-446

DIW (1990-1991):

DIW (1990, 41): Gespaltener Arbeitsmarkt, in: DIW-Wochenbericht 41, S. 583-590

DIW (1991, 14): Der Arbeitsmarkt in Deutschland, in: DIW-Wochenbericht 14 , S. $185-190$

DIW (1991, 14): Der Arbeitsmarkt in Deutschland, in: DIW-Wochenbericht 14, S. $185-190$

DIW (1991-1994):

DIW (1991, 30): Der Arbeitsmarkt ein Jahr nach Beginn der Währungsunion, in: DIW-Wochenbericht 30, S. 427-433

DIW (1992, 5): Der Arbeitsmarkt in Deutschland, in: DIW-Wochenbericht 5-6, S. 49-57

DIW $(1992,28)$ : Der Arbeitsmarkt in Deutschland, in: DIW-Wochenbericht 28 - 29, S. 355-363

$D I W(1992,41)$ : Arbeitsmärkte in Deutschland im Zeichen konjunktureller Schwäche, in: DIW-Wochenbericht 41, S. 509 - 514

$D I W(1993,4)$ : Sinkende Beschäftigung und steigende Arbeitslosigkeit in Deutschland, in: DIW-Wochenbericht 4, S. 35-42 
$D I W(1993,15)$ : Weiterhin sinkende Beschäftigung in Deutschland, in: DIW-Wochenbericht 15, S. 190-197

$D I W(1993,28)$ : Beschäftigungsabbau setzt sich fort, in: DIW-Wochenbericht 28, S. 377-384

DIW (1993, 40): Arbeitsmarkt: Keine Entspannung in Sicht, in: DIW-Wochenbericht 40 , S. 541-547

DIW $(1994,4):$ Arbeitsmarkt: Die Talsohle ist noch nicht durchschritten, in: DIW-Wochenbericht 4, S. 47-54

$D I W(1994,14)$ : Steigende Arbeitslosigkeit - sinkende Realeinkommen, in: DIW-Wochenbericht 14, S. 193-201

$D I W$ (1994): in: DIW-Wochenbericht 1/94 fehlt

$D I W$ (1994, 40): Beschäftigungsrückgang abgeschwächt, 40, in: DIWWochenbericht S. 675-681

$D I W(1992,5):$ Der Arbeitsmarkt in Deutschland, in: DIW-Wochenbericht 5-6, 59. Jg., S. 49-57

DIW (1994, 21): Wohnungsmieten in Ost- und Westdeutschland 1993, in: : DIW-Wochenbericht 21, S. 350-355

DIW (1994, 27): Wirtschaftsnahe Infrastruktur und Wirtschaftsentwicklung: Konsequenzen für Ostdeutschland, in: DIW-Wochenbericht 27, S. 457-464

$D I W(1994,9):$ Wanderungen von Ost- nach Westdeutschland, in: : DIW-Wochenbericht 9, S. 128-132

$D I W(1995,46)$ : Bilanz der Erwerbschancen fünf Jahre nach der Wende in Ostdeutschland), in: DIW-Wochenbericht 46, S. 789-795

$D I W$ (1997, 3): Gesamtwirtschaftliche und unternehmerische Anpassungsprozesse in Ostdeutschland, 15. Bericht, in: DIW-Wochenbericht 3/97, S. 45-64

$D I W(1997,7)$ : Weitere Annäherung des Arbeitseinkommens in Ost- und Westdeutschland - ostdeutsche Lohnstückkosten nach wie vor über Westniveau, DIW-Wochenbericht 7/97, S. 119-125

Dornbusch, Rüdiger und Holger C. Wolf(1992): Economic Transition in Eastern Germany, in: Brookings Papers on Economic Activity, S. 235-272

Egle, Franz (1991): Der Ost-West-Konflikt auf dem deutschen Arbeitsmarkt, in: Informationen für die Beratungs- und Vermittlungsdienste der Bundesanstalt für Arbeit, 12, S. 513-521 
Engelen-Kefer, Ursula u.a. (1995): Beschäftigungspolitik, Köln

Feithen, Rosemarie (1985): Arbeitskräftewanderungen in der Europäischen Gemeinschaft, Bielefeld

Fischer, Peter A. u.a. (1995a): Should I stay or should I go?:(micro)economic contributions towards and interdisciplinary theory of South-North migration and migration dynamics, Diskussionsbeiträge zur Wirtschaftspolitik Nr. 48, Hamburg

Fischer, Peter A. u.a. (1995b): Developement an Migration or migration and developement? Macroeconomic contributions towards an interdisciplinary theory of South-North migration, Diskussionsbeiträge zur Wirtschaftspolitik Nr. 57, Hamburg

Fischer, Peter A. u.a. (1998): Why do People stay? The Insider Approach - Empirical Evidence from Swedish Labour Markets, CEPR-Discussion Paper No. 1952, London

Fleischer, Henning und Bettina Sommer (1995): Bevölkerungsentwicklung 1993, in: Wirtschaft und Statistik 1, S. 30-38

Franz, Wolfgang (1991): Arbeitsmarktökonomik, Berlin u.a.O.

Friedrich-Ebert-Stiftung (Hrsg.) (1977): Warum gibt es in der DDR keine Arbeitslosigkeit?, Bonn - Bad Godesberg

Fritsch, Michael u.a. (1991): Regionalpolitik in Ostdeutschland, in: Wirtschaftsdienst XII, S. 626-631

Fürst, Dietrich und Joachim Jens Hesse (1981): Landesplanung, Düsseldorf

Gatzweiler, Hans Peter (1975): Zur Selektivität interregionaler Wanderungen, Forschungen zur Raumentwicklung Bd. 1.1975, Bonn-Bad Godesberg

Götzinger, Hermann J.M. (1981): Die Bedeutung von preisniveauunterschieden für die regionale Wirtschaftspolitik, Raumforschung und Raumordnung, Heft 23, S. 109-116.

Graves, Philip E. (1979): A Life-Cycle Empirical Analyses of Migration and Climate, by Race in: Journal of Urban Economics, 6, S. 135-147

Graves, Philip E. (1980): Migration and Climate, in: Journal of Regional Science, 20, S. 223-232

Greenwood, M.J. (1970): Lagged Response in the Decision to Migrate, in: Journal of Regional Science, Vol 10, No.5, S. 375-384 
Greenwood, M.J. (1985): Human Migration: Theory, Models, and Empirical Studies, in: Journal of Regional Science, Vol. 25, No.4, S. 521-544

Grubel, Herbert G. (1994): The Economics of International Labor and Capital Flows, in: Herbert Giersch (Hrsg.): Economic Aspects of International Migration, Berlin u.a.O.

Grundmann, Siegfried (1990): Außen- und Binnenmigration der DDR 1989, in: Deutschland-Archiv, 22. Jg., H9, S. 1422-1432

Harris, J.R. und M.P. Todaro (1970): Migration, unemployment and developement: a two-sector analysis, in: American Economic Review, Vol. 60, S. 126142

Harloff, Hans Joachim (1970): Der Einfluß psychischer Faktoren auf die Mobilität der Arbeit, Berlin

Henderson, J. Vernon (1982): Evaluating Consumer Amenities and Interregional Welfare Differences, in: Journal of Urban Economics 11, S. 32-59

Hoffman, Saul D. (1986): Labor Market Economics, Englewood Cliffs/New Jersey

Holland, S. (1976): Capital versus the Regions, London u.a.O.

Horn, Gustaf (1992): Lohnentwicklung in Ostdeutschland - ein Schrecken ohne Ende?, in: Konjunkturpolitik, 38. Jg., H.3, S. 139ff.

Institut der deutschen Wirtschaft(1991, 40): Wohnungsmarkt Ost - Mieten bleiben unter Marktniveau, in: iwd (40/1991), S. 2.

Institut der deutschen Wirtschaft (1993, 13): Ost-Miete wiegt weniger, in iwd 13/1993, S. 1

Institut für Arbeitsmarkt und Berufsforschung (1990): Eckzahlen der Erwerbstätigkeit in den neuen Bundesländern, IAB-Kurzbericht vom 26.11.1990

iwd (1991/1993):

Institut der deutschen Wirtschaft (1993, 13): Ost-Miete wiegt weniger, in iwd 13/1993, S. 1

Institut der deutschen Wirtschaft (1991, 40): Wohnungsmarkt Ost - Mieten bleiben unter Marktniveau, in iwd (40/1991), S. 2

Kemming, Herbert (1980): Raumwirtschaftstheoretische Gravitationsmodelle, Berlin 
Klauder, Wolfgang (1990): Auswirkungen der politischen Entwicklung seit 1989 auf die Arbeitsmarktperspektiven. Ein quantitatives Szenario bis 2000 unter veränderten Rahmenbedingungen, in: MittAB 1, S. 22-33

Koch, Reinhold (1976): Altenwanderung und räumliche Konzentration alter Menschen, Forschungen zur Raumentwicklung Band 4, Bonn-Bad Godesberg

Köhler, Anne (1990): Ist die Übersiedlerwelle noch zu stoppen?, in: Deutschland-Archiv, 23. Jg., H.3, März, S. 425-431

König, H. (1979): Job-Search-Theorien, in: Bombach, G. u.a.: Neuere Entwicklungen in der Beschäftigungstheorie und -politik, Tübingen

Külp, Bernhard (1975): Wohlfahrtsökonomik I: Die Wohlfahrtskriterien, Tübingen und Düsseldorf

Külp, Bernhard (1982): Wohlfahrtsökonomik I: Grundlagen, in: HdWW, Bd. 9, Stuttgart u.a.O., S. 469-486

Lansing, John B. und Eva Mueller (1973): The Geographik Mobility of Labor, Ann Arbor, 4. Aufl.

Layard, Richard u.a. (1992): East-West Migration: the Alternatives, Cambridge (Massachusetts) und London

Lee, E. (1972): Eine Theorie der Wanderung, in: G. Szell (Hrsg.): Regionale Mobilität, München, S. 117-129

Lind, Harold (1969): Internal Migration in Britain, in: Jackson, J.A. (Hrsg.): Migration, Cambridge

Lübbering, Marcus (1993): Mit der Förderung wächst in den neuen Ländern die Gefahr von Fehlinvestitionen, in: Handelsblatt vom 22.04, S. B2

Mackensen, R., Vanberg, M. und Krämer, K. (1975): Probleme regionaler Mobilität, Göttingen

Mathur, Vijay und Sheldon H. Stein (1991): A Dynamic Interregional Theory of Migration and Population Growth, in: Land Economics, 67(3), August, S. 292298

Merklein, Renate (1993): Nach der Vereinigung zeigt sich die Gefährlichkeit einer antimarktwirtschaftlichen Arbeitsverfassung, Handelsblatt Nr. 120 vom 25./26.6.93. 
Mieth, Wolfram (1992): Die zweigeteilte Konjunktur im vereinigten Deutschland und das ostdeutsche Lohnniveau, in: Kleinhenz, Gerhard (Hrsg.): Sozialpolitik im vereinten Deutschland II, Schriften des Vereins für Socialpolitik, Bd. 208/II, Berlin, S. 121-172

Mincer, Jacob (1978): Family Migration Decisions, in: Journal of Political Economy, vol. 86, No. 5, S. 749-773

Molho, Ian (1986): Theories of Moigration: A Review, in: Scottish Journal of Political Economy, Vol 33, No.4, S. 396-419

Molitor, Bruno (1969): Zur Politik der Arbeitsmobilität, in: Hamburger Jahrbuch für Wirtschafts- und Gesellschaftspolitik, S. 87-110

Molitor, Bruno (1988): Lohn- und Arbeitsmarktpolitik, München

Müller, J. Heinz (1977): Ballung, in: HdWW, Bd. 1, Stuttgart u.a.O., S. 454-463

Nestmann, Eckhard (1988): Bedeutung der Infrastruktur für die Ruhestandswanderung, Dissertation Karlsruhe

Neubäumer, Renate (1991): Der ostdeutsche Arbeitsmarkt - Bestandsaufnahme und Ansatzpunkte einer mehr auf Beschäftigung ausgerichteten Wirtschaftspolitik, in: Görgens u.a. (Hrsg.): Wirtschaftspolitische Probleme der Integration der ehemaligen DDR in die Bundesrepublik, Schriften des Vereins für Socialpolitik N.F., Bd. 212, Berlin

Nierhaus (1991): Realeinkommen und Lohnpolitik in den neuen Bundesländern, in: IFO-Schnelldienst 12/1991, S. 3-5

o. V. (1993): Wohnungsgrößen, in: Handelsblatt vom 13./14.8., S. 4

o. $V$. (1995): Bis zu 10.000 Mark gespart, in: Stiftung Warentest (Hrsg.): Test, Heft 2, S. 28-29

o. V. (1997): Bisher tausend Milliarden Mark für die neuen Länder, in: Frankfurter Allgemeine Zeitung vom 24. 7.

Paqué, Karl-Heinz (1997): Neue Wege der neuen Länder, in: Frankfurter Allgemeine Zeitung vom 26.7., S. 13

Parnes, Herbert S. (1954): Research on Labor Mobility, New York

Pencavel, John (1986): Labor Supply of Men: A Survey, in: Ashenfelter,O. und Layard, R. (Hrsg.): Handbook of Labor Economic, Amsterdam u.a.O., S. 3-102.

Priewe, Jan (1984): Zur Kritik konkurrierender Arbeitsmarkt- und Beschäftigungstheorien und ihren politischen Implikationen, Frankfurt/Main. 
Prognos $A G$ (1968): Struktur und Motive der Wanderungsbewegungen in der Bundesrepublik Deutschland, Basel

Raffelhüschen, Bernd (1993): Wanderungen von Erwerbspersonen im vereinigten Deutschland, in: Zeitschrift für Wirtschafts- und Sozialwissenschaften 113, 2, S. 273-295

Raschke, Freddy (1985): Bevölkerungsstruktur, Mobilität und Lohn, Frankfurt am Main, Bern, New York

Ravenstein, E.G. (1885): The Laws of Migration, in: Journal of the Statistical Society, 48, S. 167-227

Ravenstein, E.G. (1889): The Laws of Migration, Part 2, in: Journal of the Statistical Society, 52, S. 241-301

Roback, Jennifer (1982): Wages, Rents, and Quality of Life, in: Journal of Political Economy, 90, S. 1257 - 1278

Röder, Horst (1974): Ursachen, Erscheinungsformen und Folgen regionaler Mobilität, Münster

Rosen, Sherwin (1979): Wage-based Indexes of Urban Quality of Life, in: Mieszkowski, Peter und Straszheim, Mahlon (Hrsg.): Current Issues in Urban Economics, Balimore, Maryland, S. 74-105

Rothschild, Kurt (1988): Theorien der Arbeitslosigkeit, München

Sachverständigenrat zur Begutachtung der gesamtwirtschaftlichen Entwicklung (1994/1995): Den Aufschwung sichern - Arbeitsplätze schaffen, Bonn

Samuelson, Paul A. und William D. Nordhaus (1987): Volkswirtschaftslehre Bd.2, 8. Aufl., Köln

Sandell, Steven H. (1977): Women and the Economics of Family Migration, in: Review of Economics and Statistics, 59, S. 406- 414

Schaad, Jakob und Patrik Schellenbauer (1993): Spezifisches Humankapital, implizite Verträge und ausländische Arbeitskräfte, in: Schweizerische Zeitschrift für Volkswirtschaft und Statistik, Vol 129 (3), S. 437-454

Scheremet, Wolfgang und Jürgen Schupp (1991): Pendler und Migranten - Zur Arbeitskräftemobilität in Ostdeutschland, DIW Diskussionspapier Nr. 36, Berlin

Scheremet, Wolfgang und Jürgen Schupp (1992): Pendler und Migranten - Zur Arbeitskräftemobilität in Ostdeutschland, in: DIW- Wochenbericht 3/92, S. 2126. 
Schettkat, Ronald (1993): Neuere Entwicklungen in der Arbeitsmarkt- und Beschäftigungstheorie, in: Wirtschaftsdienst 1993/5, S. 268-276

Scheuer, Markus (1986/87): Die Effizienzlohntheorien - ein Beitrag zur mikroökonomischen Fundierung der Erklärung unfreiwilliger Arbeitslosigkeit, in: RWI-Mitteilungen, Jg. 37/38, S. 407-431

Scheuer, Markus (1987): Zur Leistungsfähigkeit neoklassischer Arbeitsmarkttheorien, Bonn

Schmitt-Rink, Gerhard (1978): Verteilungstheorie, Tübingen/Düsseldorf

Schultz, Theodore (1975): The Value of the Ability to Deal with Disequilibria, in: Journal of Economic Literature, Vol. XIII, N. 3, S. 827-846.

Schumacher, Dieter (1993): Lohnerhöhungen, internationale Wettbewerbsfähigkeit und Aufholprozeß in Ostdeutschland, in: Konjunkturpolitik, H3, S. 121-147.

Schwartz, Aba (1973): Interpreting the Effect of Distance on Migration, in: Journal of Political Economy, 81(5), S. 1153-1169

Sengenberger, Werner (1987): Struktur und Funktionsweise von Arbeitsmärkten die Bundesrepublik Deutschland im internationalen Vergleich, Franfurt/Main, New York

Sesselmeier, Werner und Gregor Blauermel(1990): Arbeitsmarkttheorien, Heidelberg

Sheldon, George (1985): Berufliche und geographische Flexibilität, Beiträge zur Arbeitsmarkt- und Berufsforschung 92, Nürnberg

Siebert, Horst (1970): Regionales Wirtschaftswachstum und interregionale Mobilität, Tübingen

Sinn Gerlinde und Hans-Werner Sinn (1991): Kaltstart - Volkswirtschaftliche Aspekte der deutschen Vereinigung, Tübingen

Sjaastad, Larry A. (1962): The Costs and Returns of Human Migration, Journal of Political Economy, Bd. 70, Suppl. 1962, S. 80-93

Slomma, Hans (1990): Steuerzahler kontra Finanzamt: 218 „unbezahlbare“ Steuertips, 5. Aufl., Freiburg

Spahn, Heinz-Peter (1991),: Das erste und das zweite deutsche Wirtschaftswunder, in: Wirtschaftsdienst II, 71. Jg., S. 73-79

Staatliche Zentralverwaltung für Statistik: Statistisches Jahrbuch der DDR, verschiedene Jahrgänge 
Stark, Oded und David E. Bloom (1985): The New Economics of Labor Migration, in: American Economic Journal, Vol. 75, S. 173-178

Statistisches Bundesamt (1990): Fachserie 1, Reihe 1, 4. Quartal, Wiesbaden

Statistisches Bundesamt (1991): Statistisches Jahrbuch 1991 für die Bundesrepublik Deutschland, Wiesbaden

Statistisches Bundesamt (1993): Statistisches Jahrbuch 1993 für die Bundesrepublik Deutschland, Wiesbaden

Statistisches Bundesamt (1994): Zur wirtschaftlichen und sozialen Lage in den neuen Bundesländern, Juni

Statistisches Bundesamt (1994a): Zur wirtschaftlichen und sozialen Lage in den neuen Bundesländern, August

Statistisches Bundesamt (1994c): Zur wirtschaftlichen und sozialen Lage in den neuen Bundesländern, März

Statistisches Bundesamt (1996): Tabellensammlung zur wirtschaftlichen und sozialen Lage in den neuen Bundesländern, Arbeitsunterlage, Ausgabe 1, Wiesbaden

Statistisches Bundesamt (1996a): Statistisches Jahrbuch 1996 für die Bundesrepublik Deutschland, Wiesbaden

Statistisches Bundesamt (1997): Tabellensammlung zur wirtschaftlichen und sozialen Lage in den neuen Bundesländern, Arbeitsunterlage, Ausgabe 2, Wiesbaden

Stephan, Helga und Eberhard Wiedemann (1990): Lohnstruktur und Lohndifferenzierung in der DDR, in: MittAB, 23.Jg, 4, S. 550-562

Stigler, G.J. (1962): Information in the Labor Market, The Economics of Information, in: Journal of Political Economy 70, S. 94-105.

Straubhaar, Thomas (1992): Migration und öffentliche Güter, in: Buttler, G. u.a. (Hrsg.): Acta Demographica 1992, Heidelberg, S. 177-188

Straubhaar, Thomas (1993): Druck und/oder Sog: Migration aus ökonomischer Sicht, Diskussionsbeiträge zur Wirtschaftspolitik Nr. 33, Hamburg

Straubhaar, Thomas (1994): Neuere Entwicklungen in der Migrationstheorie, Diskussionsbeiträge zur Wirtschaftspolitik Nr. 41, Hamburg

Straubhaar, Thomas (1998): Brain Drain and Brain Gain in Europe - An Evaluation of the East-European Migration to Germany, Center for German and European Studies, Working Paper 4.16, University of California, Berkeley 
Szell, Gyoergy (Hrsg.) (1972): Regionale Mobilität, München

Termote, Marc (1972): Wanderungsmodelle, in Gyoergy Szell (Hrsg.): Regionale Mobilität, München, S. 141-175

Voigt, Dieter u.a. (1990): Die innerdeutsche Wanderung und der Vereinigungsprozeß, in: Deutschland-Archiv, 5, Mai, 23. Jg., S. 732-746

Wagner, Gert (1992): Arbeitslosigkeit, Abwanderung und Pendeln von Arbeitskräften der neuen Bundesländer, in: Sozialer Fortschritt, Heft 4, S. 84-89

Wagner, Michael (1987): Bildung und Migration, in: Raumforschung und Raumordnung, Heft 3, S. 97-105

Willis, K.G. (1974): Problems in Migration Analysis, Westmead, Farnborough 1974

Wolf, Holger C. (1994): Wachstumstheorie im Widerstreit - Konvergenz oder Divergenz?, in: WiSt, Heft 4, S. 187-193

Yellen, Janet (1984): Efficiency Wage Models of Unemployment, in: American Economic Review, vol. 74, Papers and Porceedings, S. 200ff

Zimmermann, Klaus F. (1993): Ökonomische Konsequenzen der Migration für den heimischen Arbeitsmarkt, in: Schweizerische Zeitschrift für Volkswirtschaft und Statistik, Vol 129 (3), S. 283-301 


\section{Symbolverzeichnis}

ALGxi: Arbeitslosengeld in Region $\mathrm{x}$ in der Periode $\mathrm{i}$

ALHxi: Arbeitslosenhilfe in Region $\mathrm{x}$ in der Periode $\mathrm{i}$

An: Annehmlichkeiten

Ax, Ay: Lohnsatz, den die Unternehmen in der Region $\mathrm{x}$ bzw. y maximal zu zahlen bereit sind.

ax, ay: Steigung der Arbeitsnachfragefunktion

Bx, By: Reservationslohn in der Region $\mathrm{x}$ bzw. y

bx, by: Steigung der Arbeitsangebotsfunktion

DEQi: Dequalifizierungsfaktor bei einer Dauer der Arbeitslosigkeit von i Perioden

Em,a: monetäre Erträge in der Region a

Enm,a: nicht-monetäre Erträge in der Region a

F: $\quad$ Freizeit

K: $\quad$ Kapital

KE: Kapitaleinkommen

$\mathrm{Km}$,a: monetäre Kosten in der Region a

Knm,a: nicht-monetäre Kosten in der Region a

L: $\quad$ Arbeitsstunden

LA: Lohn-Angebotskurve

LD: Arbeitsnachfrage

LE/h: Lohneinkommen pro Stunde

LE: Lohneinkommen

LExi: Nettolohneinkommen in einem zum Zeitpunkt 0 der Qualifikation entsprechenden Beruf, berechnet wie in (1) in der Region $\mathrm{x}$ in Periode i.

LGG: gleichgewichtige Arbeitsmenge

LS: Arbeitsangebot 
MAW: marginale Arbeitswilligkeit

q2,a: Wahrscheinlichkeit in der zweiten Periode beschäftigt zu sein unter der Bedingung Beschäftigung in der ersten Periode

q2,b: Wahrscheinlichkeit in der zweiten Periode beschäftigt zu sein unter der Bedingung Arbeitslosigkeit in der ersten Periode

q3,a: Wahrscheinlichkeit, in Periode 3 über einen Arbeitsplatz zu verfügen und in den beiden Vorperioden nicht arbeitslos gewesen zu sein.

q3,b: wie q3,a, aber Arbeitslosigkeit in Periode 1

q3,c: wie q3,a, aber Arbeitslosigkeit in Periode 2

q3,d: wie q3,a, aber Arbeitslosigkeit in Periode 1 und 2

qi: Wahrscheinlichkeit, in der Periode $\mathrm{i}$ über einen Arbeitsplatz zu verfügen

r: Zeitpräferenzrate

SHxi: Sozialhilfe in Region $\mathrm{x}$ in der Periode $\mathrm{i}$

SK: $\quad$ Stückkosten

st: $\quad$ Fahrtzeit (Hin- und Rückfahrt) in Stunden

TK: Transaktionskosten

TKHF: Transaktionskosten für Hin- und Rückfahrt

U: $\quad$ Nutzen

V: indirekter Nutzen

w: Reallohnsatz

wGG: gleichgewichtiger Reallohnsatz

WK: Wanderungskosten

WKpji: Preis eines Warenkorbs mit dem Nutzen $\mathbf{k}$ in der Region $\mathrm{j}$ in der Periode i

WKx, WKy: Ausgaben für Warenkorb in Region x, bzw. in Region y

wx, wy: Reallohnsatz in der Region x bzw. y

Y: Output 


\section{Anhang 1}

\begin{tabular}{|r|r|r|r|r|r|r|}
\hline $\begin{array}{l}\text { Peri- } \\
\text { ode }\end{array}$ & $\begin{array}{c}\text { Lohneink. } \\
\text { in Region } \\
\mathrm{x}\end{array}$ & $\begin{array}{c}\text { Um- } \\
\text { zugs- } \\
\text { kosten }\end{array}$ & $\begin{array}{c}\text { Migrations- } \\
\text { ertrag der } \\
\text { jew. Periode, } \\
\text { abgez. auf } \\
\mathrm{t}=0, \mathrm{r}=0,02\end{array}$ & $\begin{array}{c}\text { kummul. } \\
\text { Migra- } \\
\text { tions- } \\
\text { ertrag, } \\
\mathrm{r}=0,02\end{array}$ & $\begin{array}{c}\text { Migrations- } \\
\text { ertrag der } \\
\text { jew. Periode, } \\
\text { abgez. auf } \\
\mathrm{t}=0, \mathrm{r}=0,05\end{array}$ & $\begin{array}{c}\text { kummul. } \\
\text { Migra- } \\
\text { tions- } \\
\text { ertrag } \\
\mathrm{r}=0,05\end{array}$ \\
\hline 1 & 60 & 20 & 10,39 & 10,39 & 10,95 & 10,95 \\
\hline 2 & 65 & & 24,03 & 34,42 & 22,68 & 33,63 \\
\hline 3 & 70 & & 18,85 & 53,27 & 17,28 & 50,90 \\
\hline 4 & 75 & & 13,86 & 67,13 & 12,34 & 63,25 \\
\hline 5 & 80 & & 9,06 & 76,18 & 7,84 & 71,08 \\
\hline 6 & 85 & & 4,44 & 80,62 & 3,73 & 74,81 \\
\hline 7 & 90 & 10 & $-8,71$ & 71,92 & $-7,11$ & 67,70 \\
\hline 8 & 95 & & $-4,27$ & 67,65 & $-3,38$ & 64,32 \\
\hline 9 & 100 & & $-8,37$ & 59,28 & $-6,45$ & 57,87 \\
\hline 10 & 100 & & $-8,20$ & 51,08 & $-6,14$ & 51,74 \\
\hline 11 & 100 & & $-8,04$ & 43,04 & $-5,85$ & 45,89 \\
\hline 12 & 100 & & $-7,88$ & 35,15 & $-5,57$ & 40,32 \\
\hline 13 & 100 & & $-7,73$ & 27,42 & $-5,30$ & 35,02 \\
\hline 14 & 100 & & $-7,58$ & 19,84 & $-5,05$ & 29,97 \\
\hline 15 & 100 & & $-7,43$ & 12,41 & $-4,81$ & 25,16 \\
\hline 16 & 100 & & $-7,28$ & 5,13 & $-4,58$ & 20,58 \\
\hline 17 & 100 & & $-7,14$ & $-2,01$ & $-4,36$ & 16,21 \\
\hline 18 & 100 & & $-7,00$ & $-9,02$ & $-4,16$ & 12,06 \\
\hline 19 & 100 & & $-6,86$ & $-15,88$ & $-3,96$ & 8,10 \\
\hline 20 & 100 & & $-6,73$ & $-22,61$ & $-3,77$ & 4,33 \\
\hline
\end{tabular}

\section{Konstante Größen:}

Lohneinkommen in Region y in allen Perioden 100

Ausgaben in Region $\mathrm{x}$ und in Region y für Warenkorb a in allen Perioden: 60 psychische Kosten in allen Perioden 10 


\begin{tabular}{|r|r|r|r|r|r|r|}
\hline $\begin{array}{l}\text { Peri- } \\
\text { ode }\end{array}$ & $\begin{array}{c}\text { Lohneink. } \\
\text { in Region } \\
\mathrm{x}\end{array}$ & $\begin{array}{c}\text { Um- } \\
\text { zugg- } \\
\text { kosten }\end{array}$ & $\begin{array}{c}\text { Migrations- } \\
\text { ertrag der } \\
\text { jew. Periode, } \\
\text { abgez. auf } \\
\mathrm{t}=0, \mathrm{r}=0,02\end{array}$ & $\begin{array}{c}\text { kummul. } \\
\text { Migra- } \\
\text { tions- } \\
\text { ertrag, } \\
\mathrm{r}=0,02\end{array}$ & $\begin{array}{c}\text { Migrations- } \\
\text { ertrag der } \\
\text { jew. Periode, } \\
\text { abgez. auf } \\
\mathrm{t}=0, \mathrm{r}=0,05\end{array}$ & $\begin{array}{c}\text { kummul. } \\
\text { Migra- } \\
\text { tionsertrag } \\
\mathrm{r}=0,05\end{array}$ \\
\hline 1 & 60 & 20 & 0,39 & 0,39 & 0,95 & 0,95 \\
\hline 2 & 65 & & 14,42 & 14,81 & 13,61 & 14,56 \\
\hline 3 & 70 & & 9,42 & 24,23 & 8,64 & 23,20 \\
\hline 4 & 75 & & 4,62 & 28,85 & 4,11 & 27,31 \\
\hline 5 & 80 & 10 & $-9,06$ & 19,79 & $-7,84$ & 19,47 \\
\hline 6 & 85 & & $-4,44$ & 15,35 & $-3,73$ & 15,74 \\
\hline 7 & 90 & & $-8,71$ & 6,65 & $-7,11$ & 8,64 \\
\hline 8 & 95 & & $-12,80$ & $-6,15$ & $-10,15$ & $-1,52$ \\
\hline 9 & 100 & & $-16,74$ & $-22,89$ & $-12,89$ & $-14,41$ \\
\hline 10 & 100 & & $-16,41$ & $-39,30$ & $-12,28$ & $-26,69$ \\
\hline 11 & 100 & & $-16,09$ & $-55,38$ & $-11,69$ & $-38,38$ \\
\hline 12 & 100 & & $-15,77$ & $-71,15$ & $-11,14$ & $-49,52$ \\
\hline 13 & 100 & & $-15,46$ & $-86,61$ & $-10,61$ & $-60,12$ \\
\hline 14 & 100 & & $-15,16$ & $-101,77$ & $-10,10$ & $-70,22$ \\
\hline 15 & 100 & & $-14,86$ & $-116,63$ & $-9,62$ & $-79,84$ \\
\hline 16 & 100 & & $-14,57$ & $-131,20$ & $-9,16$ & $-89,01$ \\
\hline 17 & 100 & & $-14,28$ & $-145,48$ & $-8,73$ & $-97,73$ \\
\hline 18 & 100 & & $-14,00$ & $-159,48$ & $-8,31$ & $-106,04$ \\
\hline 19 & 100 & & $-13,73$ & $-173,21$ & $-7,91$ & $-113,96$ \\
\hline 20 & 100 & & $-13,46$ & $-186,67$ & $-7,54$ & $-121,50$ \\
\hline
\end{tabular}

Konstante Größen:

Lohneinkommen in Region y in allen Perioden 100 Ausgaben in Region $\mathrm{x}$ und in Region y für Warenkorb a in allen Perioden 60 psychische Kosten in allen Perioden 20 


\section{Anhang 2}

\begin{tabular}{|l|r|r|r|r|}
\hline \multicolumn{5}{|c|}{ Bruttojahreseinkommen in den neuen Ländern nach Branche } \\
& 1991 & 1992 & 1993 & 1994 \\
\hline Land- und & & & & \\
Forstwirtschaft & 17.370 & 21.831 & 26.646 & 23.565 \\
\hline Bergbau & 29.970 & 38.193 & 56.760 & 48.786 \\
\hline Energiewirtschaft & 32.865 & 37.794 & 41.445 & 39.189 \\
\hline Verarbeitendes & & & & \\
Gewerbe & 22.758 & 28.644 & 36.573 & 32.250 \\
\hline Baugewerbe & 30.255 & 36.849 & 38.124 & 38.508 \\
\hline Handel & 21.630 & 25.686 & 30.213 & 29.667 \\
\hline Verkehr & 25.656 & 31.218 & 36.843 & 34.146 \\
\hline Dienstleistungen & 25.242 & 30.207 & 35.973 & 36.222 \\
\hline Insgesamt & 24.729 & 29.997 & 35.178 & 34.155 \\
\hline
\end{tabular}

\begin{tabular}{|l|r|r|r|r|}
\hline \multicolumn{5}{|c|}{ Bruttojahreseinkommen in den alten Ländern nach Branche } \\
& 1991 & 1992 & 1993 & 1994 \\
\hline \begin{tabular}{l|r|r|} 
Land- und \\
Forstwirtschaft
\end{tabular} & 34.098 & 35.514 & 38.055 & 37.137 \\
\hline Bergbau & 58.164 & 62.931 & 62.976 & 62.916 \\
\hline Energiewirtschaft & 52.962 & 56.277 & 59.850 & 58.620 \\
\hline $\begin{array}{l}\text { Verarbeitendes } \\
\text { Gewerbe }\end{array}$ & 50.349 & 53.268 & 57.348 & 55.479 \\
\hline Baugewerbe & 43.020 & 46.092 & 49.404 & 47.952 \\
\hline Handel & 39.798 & 41.991 & 44.901 & 43.827 \\
\hline Verkehr & 46.224 & 48.732 & 51.198 & 50.721 \\
\hline Dienstleistungen & 41.034 & 43.365 & 45.225 & 44.955 \\
\hline Insgesamt & 44.511 & 47.016 & 49.461 & 48.660 \\
\hline
\end{tabular}

Quelle: DIW (1991-1994), eigene Berechnungen 


\begin{tabular}{|l|r|r|r|r|}
\hline \multicolumn{5}{|c|}{ Bruttojahreseinkommen der Industriebeschäftigten in den neuen } \\
\multicolumn{5}{|c|}{ Ländern } \\
& 1991 & 1992 & 1993 & 1994 \\
\hline Arbeiter & 22.164 & 28.764 & 33.576 & 34.650 \\
\hline Angestellte & 26.340 & 35.016 & 41.568 & 42.898 \\
\hline Männer, Arbeiter & 23.040 & 29.736 & 34.680 & 35.790 \\
\hline Männer, Angestellte & 29.244 & 39.204 & 46.680 & 48.174 \\
\hline Frauen, Arbeiter & 17.784 & 22.428 & 25.464 & 26.279 \\
\hline Frauen, Angestellte & 22.824 & 30.060 & 35.232 & 36.359 \\
\hline
\end{tabular}

\begin{tabular}{|c|c|c|c|c|}
\hline \multicolumn{5}{|c|}{$\begin{array}{l}\text { Bruttojahreseinkommen der Industriebeschäftigten in den alten } \\
\text { Ländern }\end{array}$} \\
\hline & 1991 & 1992 & 1993 & \\
\hline Arbeiter & 44.064 & 46.170 & 47.424 & 48.56 \\
\hline Angestellte & 63.317 & 66.824 & 69.280 & 70.94 \\
\hline Männer, Arbeiter & 46.358 & 48.668 & 49.685 & 50.87 \\
\hline Männer, Angestellte & 69.463 & 73.279 & 75.656 & 77.472 \\
\hline Frauen, Arbeiter & 32.872 & 34.346 & 35.367 & 36.21 \\
\hline Frauen, Angestellte & 46.580 & 49.279 & 51.509 & 52.74 \\
\hline
\end{tabular}

Quelle: Statistisches Bundesamt (1994), eigene Berechnungen 


\section{Anhang 3}

Beitragsbemessungsgrenzen und Beitragssätze der Sozialversicherung

Beitagsbemessungsgrenzen in den neuen Ländern:

2.Hj. 1991: 3400 DM (für die Rentenversicherung) /2550 DM (für die Krankenversicherung)

1992: 3900/2925 DM

1993: 5300/3975 DM

1994: 5700/4275 DM

Beitragsbemessungsgrenzen in den alten Ländern:

1991: 6500/4875 DM

1992: $6800 / 5100 \mathrm{DM}$

1993: $7200 / 5400 \mathrm{DM}$

1994: $7600 / 5700 \mathrm{DM}$

Beitragssätze in den neuen Ländern:

\begin{tabular}{|l|l|l|l|l|}
\hline & 1991 & 1992 & 1993 & 1994 \\
\hline Rentenversicherung & $17,7^{*}$ & 17,7 & 17,5 & 19,2 \\
\hline Krankenversicherung & $12,8^{* *}$ & 12,8 & 12,5 & 12,5 \\
\hline Arbeitslosenversicherung & $6,8^{*}$ & 6,3 & 6,5 & 6,5 \\
\hline Pflegeversicherung &. &. &. &. \\
\hline
\end{tabular}

* ab April 1991, ** ab 2. Hj. 1991

Beitragssätze in den alten Ländern:

\begin{tabular}{|l|l|l|l|l|}
\hline & 1991 & 1992 & 1993 & 1994 \\
\hline Rentenversicherung & 17,7 & 17,7 & 17,5 & 19,2 \\
\hline Krankenversicherung & 12,2 & 12,2 & 13,4 & 13,4 \\
\hline Arbeitslosenversicherung & $5,3 * / 6,8 * *$ & 6,3 & 6,5 & 6,5 \\
\hline Pflegeversicherung &. &. &. &. \\
\hline
\end{tabular}

* bis April 1991, ** ab April 1991 


\section{Anhang 4}

\begin{tabular}{|c|c|c|c|c|c|c|}
\hline \multirow[b]{2}{*}{ Branche } & \multicolumn{3}{|c|}{$r=0,015$} & \multicolumn{3}{|c|}{$r=0,05$} \\
\hline & \begin{tabular}{|l|} 
Bruttoein- \\
kommens- \\
differenz
\end{tabular} & \begin{tabular}{|l|} 
Nettoein- \\
kommens- \\
differenz
\end{tabular} & $\begin{array}{l}\text { Diff. Netto- } \\
\text { einkommen } \\
\text { nach Miete }\end{array}$ & \begin{tabular}{|l|} 
Bruttoein- \\
kommens- \\
differenz \\
\end{tabular} & \begin{tabular}{|l|} 
Nettoein- \\
kommens- \\
differenz
\end{tabular} & $\begin{array}{l}\text { Diff. Netto- } \\
\text { einkommen } \\
\text { nach Miete }\end{array}$ \\
\hline \multicolumn{7}{|c|}{$\begin{array}{l}\text { Differenz zwischen dem korrigierten erwartbaren Migrationsertrag bei Angleichung bis } \\
1999 \text { und dem erwartbaren Migrationsertrag bei Angleichung in } 3 \text { Jahren }\end{array}$} \\
\hline Land- & 46.321 & 25.159 & 16.592 & 40.956 & 22.301 & 14.718 \\
\hline Berg & & 21.022 & 454 & 107 & 41 & 11.458 \\
\hline Ener & & 855 & 88 & 070 & & 17.629 \\
\hline Verarb. Gewe & .451 & 45.705 & 37.139 & 0.579 & & 32.835 \\
\hline Bauge & & .933 & 366 & & & 3.252 \\
\hline Han & & 18 & & 374 & & 20.013 \\
\hline Verkehr & & 621 & 053 & 0.790 & & 17.672 \\
\hline Dien & 770 & 076 & 508 & 326 & & 7.303 \\
\hline Insge & 690 & 26.813 & 18.247 & 47.208 & 12 & 16.030 \\
\hline \multicolumn{7}{|c|}{$\begin{array}{l}\text { Differenz zwischen dem korrigierten erwartbaren Migrationsertrag bei Angleichung bis } \\
1999 \text { und dem erwartbaren Migrationsertrag bei Angleichung in } 5 \text { Jahren }\end{array}$} \\
\hline Land & & 20.230 & 12.839 & .240 & & 11.314 \\
\hline Berg & 582 & 354 & 5.963 & .530 & 089 & 5.570 \\
\hline Energ & 865 & 21.996 & 14.605 & .283 & .000 & 12.481 \\
\hline era & & 38.810 & 31.420 & 707 & 34.174 & 27.654 \\
\hline Baug & 3 & 6.769 & -623 & .548 & 5.250 & -1.269 \\
\hline Hand & 341 & .023 & 632 & .576 & 618 & 16.100 \\
\hline erk & & .437 & & 628 & 651 & 13.133 \\
\hline ien & & .490 & 4.099 & 299 & & 3.308 \\
\hline Insgesamt & 42.502 & 20.655 & 13.265 & 37.090 & 18.032 & 11.514 \\
\hline \multicolumn{7}{|c|}{$\begin{array}{l}\text { Differenz zwischen dem korrigierten erwartbaren Migrationsertrag bei Angleichung bis } \\
1999 \text { und dem erwartbaren Migrationsertrag bei Angleichung in } 10 \text { Jahren }\end{array}$} \\
\hline and & -1 & -8.199 & -8.777 & -8.677 & & -7.121 \\
\hline erg & -56.386 & -30.289 & -30.867 & -46.230 & -25.147 & -25.888 \\
\hline ner & -26.63 & -17.540 & -18.118 & -21.753 & -14.702 & -15.443 \\
\hline Verarb. Gewerbe & 2.279 & -929 & -1.506 & 4.212 & 299 & -443 \\
\hline Baugewerbe & -48.007 & -28.462 & -29.040 & -41.478 & -24.755 & -25.496 \\
\hline andel & & & & -3.221 & -4.273 & -5.013 \\
\hline Verkehr & -18.682 & -12.882 & -13.460 & -14.581 & -10.429 & -11.169 \\
\hline Dienstleistungen & -33.029 & -20.755 & -21.333 & -27.737 & -17.634 & -18.375 \\
\hline Insgesamt & -22.636 & -14.546 & -15.123 & -17.875 & -11.948 & -12.688 \\
\hline
\end{tabular}

Quelle: Tabellen 2, 3, 4 und 15 


\begin{tabular}{|c|c|c|c|c|c|c|}
\hline \multirow[b]{2}{*}{ Branche } & \multicolumn{3}{|c|}{$r=0,015$} & \multicolumn{3}{|c|}{$r=0,05$} \\
\hline & $\begin{array}{l}\text { Bruttoein- } \\
\text { kommens- } \\
\text { differenz }\end{array}$ & $\begin{array}{l}\text { Nettoein- } \\
\text { kommens- } \\
\text { differenz }\end{array}$ & $\begin{array}{l}\text { Diff. Netto- } \\
\text { einkommen } \\
\text { nach Miete }\end{array}$ & \begin{tabular}{|l|} 
Bruttoein- \\
kommens- \\
differenz
\end{tabular} & \begin{tabular}{|l|} 
Nettoein- \\
kommens- \\
differenz
\end{tabular} & $\begin{array}{l}\text { Diff. Netto- } \\
\text { einkommen } \\
\text { nach Miete }\end{array}$ \\
\hline \multicolumn{7}{|c|}{$\begin{array}{l}\text { Differenz zwischen dem korrigierten erwartbaren Migrationsertrag bei Angleichung bis } 2004 \\
\text { und dem erwartbaren Migrationsertrag bei Angleichung in } 3 \text { Jahren }\end{array}$} \\
\hline Land- und Forstw. & 81.093 & 43.119 & 30.064 & 67.176 & 35.858 & 24.930 \\
\hline Bergbau & 53.574 & 24.318 & 11.262 & 46.838 & 21.410 & 10.481 \\
\hline Energiewirtschaft & 116.587 & 53.392 & 40.337 & 95.307 & 43.765 & 32.836 \\
\hline Verarb. Gewerbe & 139.355 & 67.628 & 54.575 & 116.422 & 56.867 & 45.938 \\
\hline Baugewerbe & 47.377 & 22.118 & 9.063 & 38.194 & 17.753 & 6.825 \\
\hline Handel & 98.576 & 50.142 & 37.087 & 81.438 & 548 & 30.619 \\
\hline Verkehr & 104.432 & 50.972 & 37.916 & 86.066 & 42.143 & 31.215 \\
\hline Dienstleistungen & 52.441 & 25.744 & 12.689 & 43.615 & & 10.485 \\
\hline Insge: & 98.884 & 48.670 & 35.616 & 81.287 & 40.126 & 29.198 \\
\hline \multicolumn{7}{|c|}{$\begin{array}{l}\text { Differenz zwischen dem korrigierten erwartbaren Migrationsertrag bei Angleichung bis } 2004 \\
\text { und dem erwartbaren Migrationsertrag bei Angleichung in } 5 \text { Jahren }\end{array}$} \\
\hline Land- und Forstw. & 72.561 & 38.190 & 26.311 & 59.460 & 31.390 & 21.526 \\
\hline Bergbau & 38.560 & 16.650 & 4.771 & 33.261 & 14.458 & 4.593 \\
\hline Energiewirtschaft & 103.552 & 46.533 & 34.654 & 83.520 & 37.553 & 27.688 \\
\hline Verarb. Gewerbe & 126.227 & 60.733 & 48.856 & 104.550 & 50.6 & 40.757 \\
\hline Baugewerbe & 36.177 & 15.954 & 4.074 & 28.065 & 12.168 & 2.304 \\
\hline Handel & 88.847 & 44.647 & 32.769 & 72.640 & 36.571 & 26.706 \\
\hline Verkehr & 93.195 & 44.788 & 32.909 & 75.904 & 36.540 & 26.676 \\
\hline Dienstleistungen & 42.459 & 20.158 & 8.280 & 34.588 & 16.355 & 6.490 \\
\hline Insgesamt & 87.696 & 42.512 & 30.634 & 71.169 & 34.546 & 24.682 \\
\hline \multicolumn{7}{|c|}{$\begin{array}{l}\text { Differenz zwischen dem korrigierten erwartbaren Migrationsertrag bei Angleichung bis } 2004 \\
\text { und dem erwartbaren Migrationsertrag bei Angleichung in } 10 \text { Jahren }\end{array}$} \\
\hline Land- und Forstw. & 23.138 & 9.761 & 4.695 & 17.543 & 7.177 & 3.091 \\
\hline & -48.408 & -26.993 & -32.059 & -40.499 & -22.778 & -26.865 \\
\hline Energiewirtschaft & 28.050 & 6.997 & 1.931 & 19.484 & 3.851 & -236 \\
\hline Verarb. Gewerbe & 50.183 & 20.994 & 15.930 & 40.055 & 16.747 & 12.660 \\
\hline Baugewerbe & -28.703 & -19.277 & -24.343 & -26.961 & -17.837 & -21.923 \\
\hline Handel & 32.491 & 13.071 & 8.006 & 24.843 & 9.680 & 5.593 \\
\hline Verkehr & 28.099 & 9.469 & 4.403 & 20.695 & 6.460 & 2.374 \\
\hline Dienstleistungen & -15.358 & -12.087 & -17.152 & -14.448 & -11.107 & -15.193 \\
\hline Insgesamt & 22.558 & 7.311 & 2.246 & 16.204 & 4.566 & 480 \\
\hline
\end{tabular}

Quelle: Tabellen 2, 3, 4 und 16 


\begin{tabular}{|c|c|c|c|c|c|c|}
\hline \multirow[b]{2}{*}{ Branche } & \multicolumn{3}{|c|}{$r=0,015$} & \multicolumn{3}{|c|}{$\mathrm{r}=0,05$} \\
\hline & \begin{tabular}{|l|} 
Bruttoein- \\
kommens- \\
differenz \\
\end{tabular} & $\begin{array}{l}\text { Nettoein- } \\
\text { kommens- } \\
\text { differenz }\end{array}$ & $\begin{array}{l}\text { Diff. Netto- } \\
\text { einkommen } \\
\text { nach Miete }\end{array}$ & $\begin{array}{l}\text { Bruttoein- } \\
\text { kommens- } \\
\text { differenz }\end{array}$ & $\begin{array}{l}\text { Nettoein- } \\
\text { kommens- } \\
\text { differenz }\end{array}$ & $\begin{array}{l}\text { Diff. Netto- } \\
\text { einkommen } \\
\text { nach Miete }\end{array}$ \\
\hline \multicolumn{7}{|c|}{$\begin{array}{l}\text { Differenz zwischen dem korrigierten erwartbaren Migrationsertrag bei Angleichung bis } 1999 \\
\text { und dem erwartbaren Migrationsertrag bei Angleichung in } 3 \text { Jahren }\end{array}$} \\
\hline Arbeiter & 45.068 & 22.171 & 14.478 & 39.060 & 19.210 & 12.467 \\
\hline Angestellte & 82.447 & 38.543 & 31.8 & 594 & 33.590 & 27.802 \\
\hline Arbeiter, männlich & 49.058 & 24.096 & 16.404 & 42.609 & 20.936 & 14.192 \\
\hline Angestellte, männl. & 84.909 & 38.996 & 32.321 & 73.633 & 33.967 & 28.179 \\
\hline Arbeiter, weiblich & 30.160 & 15.893 & 8.250 & 25.966 & 13.685 & 6.979 \\
\hline Angestellte, weibl. & 52.685 & 25.801 & 18.109 & 45.821 & 22.457 & 15.714 \\
\hline \multicolumn{7}{|c|}{$\begin{array}{l}\text { Differenz zwischen dem korrigierten erwartbaren Migrationsertrag bei Angleichung bis } 1999 \\
\text { und dem erwartbaren Migrationsertrag bei Angleichung in } 5 \text { Jahren }\end{array}$} \\
\hline Arbeiter & 37.716 & 18.284 & 11.328 & 32.614 & 15.800 & 9.702 \\
\hline Angestellte & 58.127 & 26.621 & 21.644 & 50.105 & 23.037 & 18.748 \\
\hline Arbeiter, männlich & 41.324 & 20.063 & 13.107 & 35.827 & 17.398 & 11.300 \\
\hline Angestellte, männl. & 58.224 & 26.254 & 21.276 & 50.055 & 22.686 & 18.397 \\
\hline Arbeiter, weiblich & 20.963 & 10.705 & 4.297 & 17.815 & 9.083 & 3.472 \\
\hline Angestellte, weibl. & 44.913 & 21.753 & 14.798 & 39.006 & 18.906 & 12.809 \\
\hline \multicolumn{7}{|c|}{$\begin{array}{l}\text { Differenz zwischen dem korrigierten erwartbaren Migrationsertrag bei Angleichung bis } 1999 \\
\text { und dem erwartbaren Migrationsertrag bei Angleichung in } 10 \text { Jahren }\end{array}$} \\
\hline Arbeiter & -25.491 & -15.342 & -15.964 & -20.427 & -12.512 & -13.294 \\
\hline Angestellte & -19.604 & -10.863 & -10.419 & -14.433 & -8.266 & -8.054 \\
\hline Arbeiter, männlich & -25.171 & -14.893 & -15.515 & -19.973 & -12.044 & -12.826 \\
\hline Angestellte, männl. & -27.064 & -13.723 & -13.279 & -20.758 & -10.724 & -10.512 \\
\hline Arbeiter, weiblich & -16.475 & -10.238 & -11.616 & -12.887 & -8.132 & -9.618 \\
\hline Angestellte, weibl. & -21.909 & -13.333 & -13.954 & -17.069 & -10.646 & -11.428 \\
\hline
\end{tabular}

Quelle: Tabellen 5, 6, 7 und 17 


\begin{tabular}{|c|c|c|c|c|c|c|}
\hline \multirow[b]{2}{*}{ Branche } & \multicolumn{3}{|c|}{$r=0,015$} & \multicolumn{3}{|c|}{$r=0,05$} \\
\hline & $\begin{array}{l}\text { Bruttoein- } \\
\text { kommens- } \\
\text { differenz }\end{array}$ & $\begin{array}{l}\text { Nettoein- } \\
\text { kommens- } \\
\text { differenz }\end{array}$ & $\begin{array}{l}\text { Diff. Netto- } \\
\text { einkommen } \\
\text { nach Miete }\end{array}$ & $\begin{array}{l}\text { Bruttoein- } \\
\text { kommens- } \\
\text { differenz }\end{array}$ & \begin{tabular}{|l|} 
Nettoein- \\
kommens- \\
differenz
\end{tabular} & $\begin{array}{l}\text { Diff. Netto- } \\
\text { einkommen } \\
\text { nach Miete }\end{array}$ \\
\hline \multicolumn{7}{|c|}{$\begin{array}{l}\text { Differenz zwischen dem korrigierten erwartbaren Migrationsertrag bei Angleichung bis } 2004 \\
\text { und dem erwartbaren Migrationsertrag bei Angleichung in } 3 \text { Jahren }\end{array}$} \\
\hline Arbeiter & 84.671 & 41.276 & 29.727 & 72.519 & 35.513 & 25.424 \\
\hline Angestellte & 142.372 & 64.192 & 53.661 & 123.830 & 56.490 & 47.356 \\
\hline Arbeiter, männlich & 90.384 & 43.688 & 32.140 & 77.662 & 37.757 & 27.667 \\
\hline Angestellte, männl. & 152.163 & 66.777 & 56.246 & 131.933 & 58.721 & 49.587 \\
\hline Arbeiter, weiblich & 59.893 & 31.321 & 19.822 & 50.918 & 26.751 & 16.699 \\
\hline Angestellte, weibl. & 95.416 & 45.757 & 34.208 & 82.164 & 39.669 & 29.580 \\
\hline \multicolumn{7}{|c|}{$\begin{array}{l}\text { Differenz zwischen dem korrigierten erwartbaren Migrationsertrag bei Angleichung bis } 2004 \\
\text { und dem erwartbaren Migrationsertrag bei Angleichung in } 5 \text { Jahren }\end{array}$} \\
\hline Arbeiter & 77.319 & 37.389 & 26.577 & & 32.103 & 22.659 \\
\hline Angestellte & 118.052 & 52.270 & 43.436 & 102.341 & 45.937 & 38.302 \\
\hline Arbeiter, männlich & 82.650 & 39.655 & 28.843 & 70.880 & 34.219 & 24.775 \\
\hline Angestellte, männl. & 125.478 & 54.035 & 45.201 & 108.355 & 47.440 & 39.805 \\
\hline Arbeiter, weiblich & 50.696 & 26.133 & 15.869 & 42.767 & 22.149 & 13.192 \\
\hline Angestellte, weibl. & 87.644 & 41.709 & 30.897 & 75.349 & 36.118 & 26.675 \\
\hline \multicolumn{7}{|c|}{$\begin{array}{l}\text { Differenz zwischen dem korrigierten erwartbaren Migrationsertrag bei Angleichung bis } 2004 \\
\text { und dem erwartbaren Migrationsertrag bei Angleichung in } 10 \text { Jahren }\end{array}$} \\
\hline Arbeiter & 14.112 & 3.763 & -715 & 13.032 & 3.791 & -337 \\
\hline Angestellte & 40.321 & 14.786 & 11.373 & 37.803 & 14.634 & 11.500 \\
\hline Arbeiter, männlich & 16.155 & 4.699 & 221 & 15.080 & 4.777 & 649 \\
\hline Angestellte, männl. & 40.190 & 14.058 & 10.646 & 37.542 & 14.030 & 10.896 \\
\hline Arbeiter, weiblich & 13.258 & 5.190 & -44 & 12.065 & 4.934 & 102 \\
\hline Angestellte, weibl. & 20.822 & 6.623 & 2.145 & 19.274 & 6.566 & 2.438 \\
\hline
\end{tabular}

Quelle: Tabellen 5, 6, 7 und 18 


\section{SCHRIFTEN ZUR WIRTSCHAFTSTHEORIE UND WIRTSCHAFTSPOLITIK}

Herausgegeben von Rolf Hasse, Jörn Kruse, Wolf Schäfer, Thomas Straubhaar, Klaus W. Zimmermann

Band 1 Lars Bünning: Die Konvergenzkriterien des Maastricht-Vertrages unter besonderer Berücksichtigung ihrer Konsistenz. 1997.

Band 2 Andreas Henning: Beveridge-Kurve, Lohnsetzung und Langzeitarbeitslosigkeit. Eine theoretische Untersuchung unter Berücksichtigung des Insider-Outsider-Ansatzes und der Entwertung des Humankapitals. 1997.

Band 3 Iris Henning: Die Reputation einer Zentralbank. Eine theoretische Untersuchung unter besonderer Berücksichtigung der Europäischen Zentralbank. 1997.

Band 4 Rüdiger Hermann: Ein gemeinsamer Markt für Elektrizität in Europa. Optionen einer Wettbewerbsordnung zwischen Anspruch und Wirklichkeit. 1997.

Band 5 Alexander Tiedtke: Japan und der Vorwurf des Trittbrettfahrerverhaltens in der US-amerikanisch-japanischen Allianz. 1997.

Band 6 Wolfgang Grimme: Ordnungspolitisches Konzept der Regionalpolitik. Darstellung der Defizite und des Reformbedaris der Regionalpolitik am Beispiel Mecklenburg-Vorpommerns. 1997.

Band 7 Christian Ricken: Determinanten der Effektivität der Umweltpolitik. Der nationale Politikstil im Spannungsfeld von Ökonomie, Politik und Kultur. 1997.

Band 8 Christian Schmidt: Real Convergence in the European Union. An Empirical Analysis. 1997.

Band 9 Silvia Marengo: Exchange Rate Policy for MERCOSUR: Lessons from the European Union. 1998.

Band 10 Jens Kleinemeyer. Standardisierung zwischen Kooperation und Wettbewerb. Eine spieltheoretische Betrachtung. 1998.

Band 11 Stefan M. Golder: Migration und Arbeitsmarkt. Eine empirische Analyse der Performance von Ausländem in der Schweiz. 1999.

Band 12 Stefan Kramer: Die Wirkung einer Intemationalisierung des Yen auf die japanischen Finanzmärkte, die japanische Geldpolitik und die Usancen der Fakturierung. 1999.

Band 13 Antje Marielle Gerhold: Wirtschaftliche Integration und Kooperation im asiatisch-pazifischen Raum. Die APEC. 1999.

Band 14 Tamim Achim Dawar: Deutsche Direktinvestitionen in Australien. Eine Evaluation der theoretischen Erklärungsansätze und der Standortattraktivität des Produktions- und Investitionsstandortes Australien. 1999.

Band 15 Hans-Markus Johannsen: Die ordnungspolitische Haltung Frankreichs im Prozeß der europäischen Einigung. 1999.

Band 16 Annette Schönherr: Vereinigungsbedingte Dimensionen regionaler Arbeitsmobilität. Wirkungen unter analytisch einfachen Bedingungen und potentielle individuelle Migrationsgewinne in Deutschland nach der Vereinigung. 1999. 
Annette Schönherr - 978-3-631-75008-7

Downloaded from PubFactory at 01/11/2019 09:19:06AM

via free access 
Annette Schönherr - 978-3-631-75008-7

Downloaded from PubFactory at 01/11/2019 09:19:06AM

via free access 